\title{
Portsmouth Gaseous Diffusion Plant Environmental Report for 1989
}

OPERATED BY

MARTIN MARIETTA ENERGY SYSTEMS, INC.

FOR THE UNITED STATES

DEPARTMENT OF ENERGY 
This report has been reproduced directly from the best available copy.

Available to DOE and DOE contractors trom the Office of Scientific and Technical Information, P.O. Box 62, Oak Ridge, TN 37831: prices available from (615) 576-8401, FTS 626-8401.

t.vailable to the public from the National Technical Information Service, U.S. Department of Commerce, 5285 Port Royal Rd., Springfield, VA 22161.

NTIS price codes-Printed Copy: A1I Microfiche A01

This report was prepared as an account of work sponsored by an agency of the United States Government. Neither the United States Government nor any agency thereot, nor any of their employoes, makes any warranty, express or implied, or assumes any legal liability or responsibility for the accurecy, completeness, or usefulness of any information, apparafus, product, or process disclosed, or represents that its use would not intringe privately owned rights. Reference herein to any specific commercial product, process, or service by trade name, trademark, manufacturer, or otherwise, does not necessarily constitute or imply its endorsement, recommendation, or favoring by the United States Government or any agency thereof. The views and opinions of authors expressed herein do not necessarily state or reflect those of the United States Government or any agency thereof 


\title{
PORTSMOUTH GASEOUS DIFFUSION PLANT ENVIRONMENTAL REPORT FOR 1989
}

\author{
Project director \\ F. C. Kornegay \\ Project coordinator \\ D. C. West \\ Technical coordinator \\ R. L. Grant \\ Coordinating editor \\ J. W. Turner
}

Date published: October 1990

Prepared by the

Office of Environmental Compliance Documentation and the Environmental Management Staff

MARTIN MARIETTA ENERGY SYSTEMS, INC.

P.O. Box 2008

Oak Ridge, Tennessee 37831

and the

Environmental Control Department

Portsmouth Gaseous Diffusion Plant

MARTIN MARIETTA ENERGY SYSTEMS, INC.

P.O. Box 628

Piketon, Ohio 45661

for the

U.S. DEPARTMENT OF ENERGY

under contract DE-AC05-84OR21400 and DE-AC05-76OR00001 


\section{SUMMARY OF 1989 ENVIRONMENTAL SURVEILLANCE DATA}

\section{Airborne Discharges and Air Monitoring}

Many point and nonpoint sources in the plant are permitted by the state of Ohio and the EPA for the release of pollutants into the atmosphere. These pollutants include both standard industrial pollutants such as smoke (i.e.,

fly ash), sulfur dioxide $\left(\mathrm{SO}_{2}\right)$, gaseous fluorides, gasoline and diesel fuel vapors, cleaning solvent vapors, and process coolants (i.e., chlorofluorocarbons) as well as small amounts of radionuclides. Airborne radionuclides are the main source of radiation dose to the public from plant operations.

A total of $0.213 \mathrm{Ci}$ of radionuclides was released to the air in $1989,45 \%$ of which was ${ }^{99} \mathrm{Tc}$ (a weak beta emitter) and $51 \%$ of which was uranium isotopes; all are alpha emitters. The remainder (i.e., $4 \%$ ) comprised the various shortlived uranium daughters (also beta emitters). The trend in airborne radionuclide emissions is shown in Fig. 1. As this figure shows, the total radionuclides (in curies) discharged to the

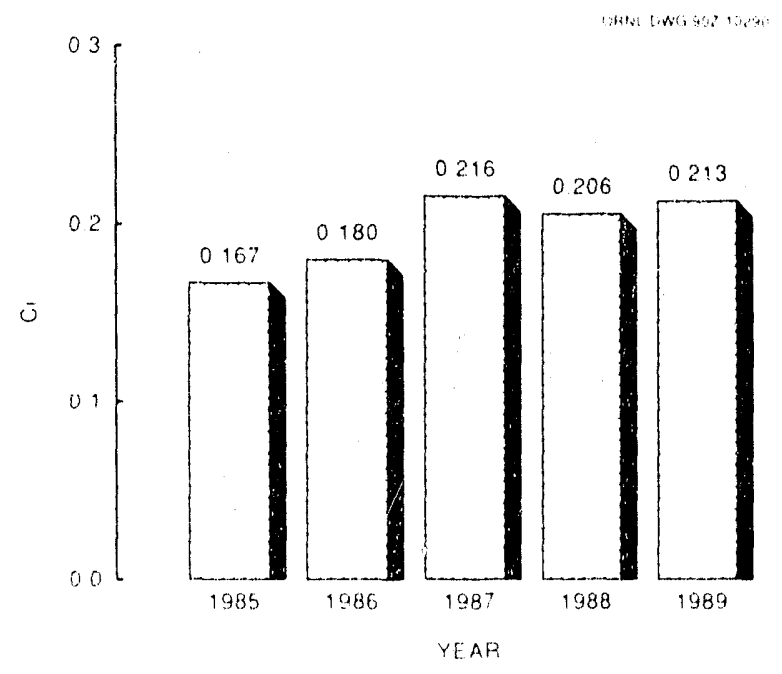

Fig. 1. Total radionuclides discharged to the air at PORTS, 1985-1989. atmosphere over the last 5 years has remained relatively constant.

Of the nonradiological air pollutants released from PORTS, smoke and $\mathrm{SO}_{2}$ from the coal-fired steam plants are the most significant. In 1989 the X-600 steam plant achieved $99.97 \%$ compliance for opacity and $100 \%$ compliance for $\mathrm{SO}_{2}$ with its Ohio Environmental Protection Agency (OEPA) permit limits. In April 1990, however, the OEPA informed PORTS that the regularity limits for $\mathrm{SO}_{2}$ emissions from the steam plant had been lowered effective November 1989. The PORTS steam plant did exceed these new limits in November and December.

\section{Waterborne Discharges and Surface Water Monitoring}

All plant-site liquid effluents are regulated by the plant's NPDES permit and either discharge to surface streams that pass through the reservation or are piped directly to the Scioto River. Overall plant-wide NPDES compliance was $97.5 \%$ for CY 1989. Figure 2 is a 5 -year trend chart on NPDES compliance at PORTS.

Radiological analyses are performed at all NPDES sampling locations and at other routine water locations that can better contribute to assessment of the impact of plant discharges on the receiving stream through upstream and downstream measurements. Data and graphs presented in this report show that the effects of discharges on the receiving streams have been minimal and are comparable to past annual discharges. Discharges to Little Beaver Creek appear to have only slight impact on radioactivity levels downstream, primarily because X-705 operations were "redirected" to new treatment facilities and to outfall 004 and the Scioto River. Plant discharges appear to have no noticeable effect on radioactivity levels in Big Run Creek or 


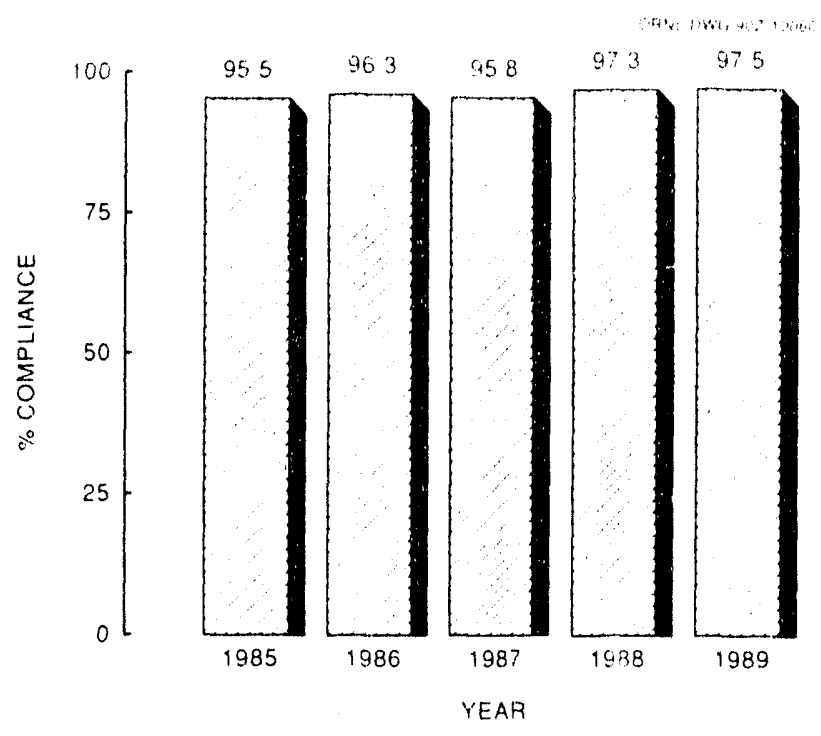

Fig. 2. National Pollutant Discharge Elimination System compliance at PORTS, 1985-1989.

the Scioto River. Figure 3 shows the 5-year trend in waterborne emissions at PORTS.

A number of pollution abatement projects and activities were initiated or completed in 1989. They include second-year optimization efforts for the biodenitrification facility; optimization of the new X-705 waste treatment facility (NPDES 605); conversion of a portion of the recirculating cooling water system from chromates to phosphates; continued NPDES permitting

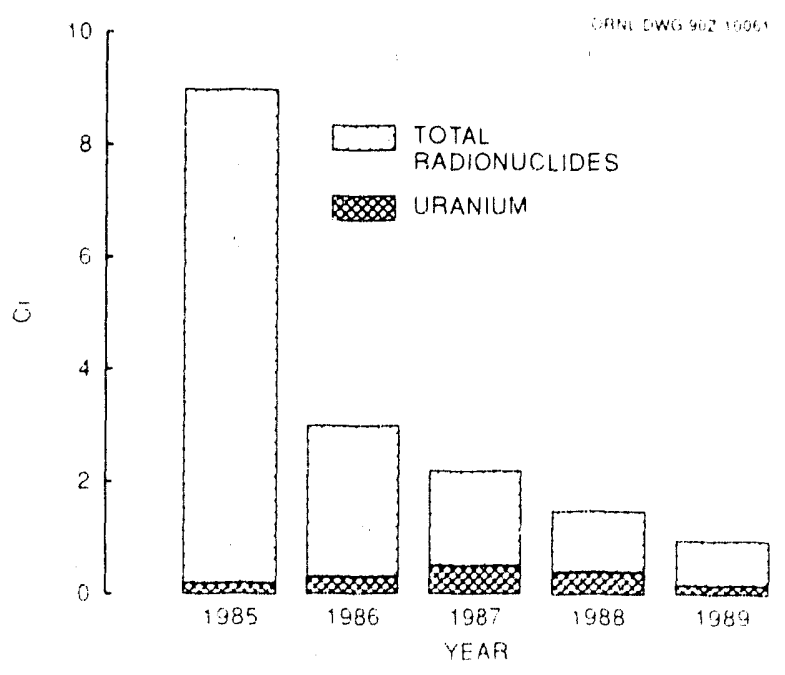

Fig. 3. Waterborne emissions at PORTS, 1985-1989. negotiations with the U.S. Environmental Protection Agency (EPA); initiation of new improvement projects for the $X-621$ coal pile treatment facility (NPDES 602), the south holding pond (NPDES 002), and the chromate treatment facility (NPDES 004); and continued algae control at Gas Centrifug: Enrichment Plant ponds (NPDES (F001 and G002). The most significant activity in 1989 was the formation of an NPDES task team and the appointment of a full-time NPDES program manager.

\section{Groundwater Monitoring}

The Off-site Residential Groundwater Monitoring Program was continued at PORTS in 1989. A review of the Off-Site Monitoring Program by a DOE/Martin Marietta Energy Systems, Inc., committee determined that historical and recent data indicate that plant operations at PORTS have had no adverse chemical or radiological effects on residential drinking water supplies in the area.

\section{Other Monitoring}

External gamma radiation. The whole-body external gamma exposure at and around PORTS is given in Fig. 4. There is no significant difference between the gamma levels around PORTS and

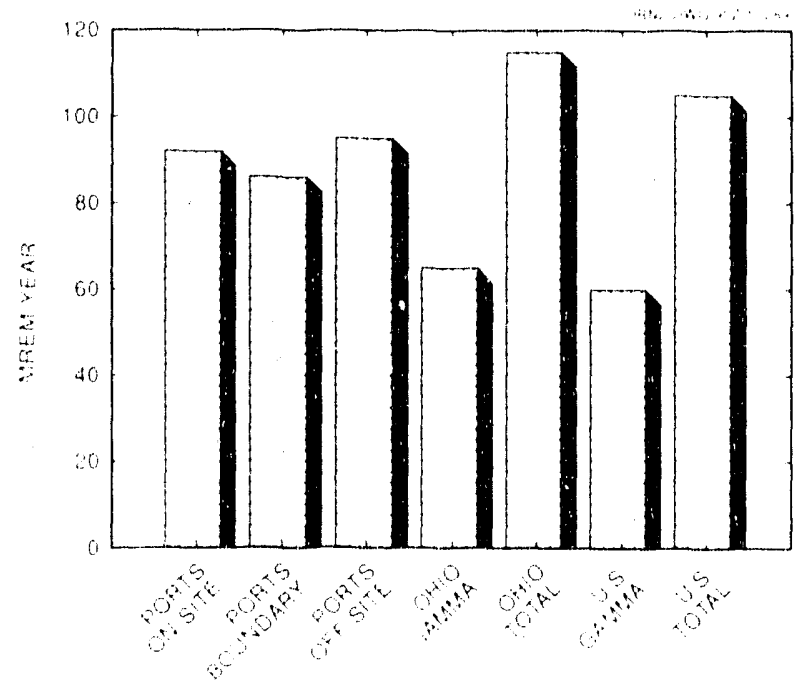

Fig. 4. Whole-body external gamma exposures at and around PORTS. 
those throughout the rest of Ohio. These values include gamma radiation attributable to PORTS operations, natural terrestrial gamma radiation, and natural cosmic radiation. It is not possible to separate these contributions because the average off-site gamma levels are higher than the average on-site and property-line levels. This is because of higher concentrations of natural radionuclides in the shale and sandstone hills surrounding PORTS than in the clays and silts directly under the plant. None of the radiation levels found are significantly different from the average radiation levels throughout Ohio.

Biological monitoring. Analyses of fish showed no significant pattern of contamination. Food crops showed only one sample with a higher than background concentration of uranium. This was a background sample from a soybean field $16.09 \mathrm{~km}$ (10 miles) north of the plant site. Technetium concentrations were all lower than the lower analytical confidence level. Grass samples were collected and analyzed for radionuclide and fluoride contamination. Results showed no significant contamination; alpha activities were at or below normal background levels. Uranium and technetium were all below the lower analytical confidence level.

Soil and sediment sampling. Soil samples are collected in parallel with the grass sampling at the same locations to allow estimation of uptake rates of radionuclides by vegetation. As in the case of the grass samples themselves, no significant contamination was found. Sediment samples are taken from local surface waters as a check for any buildup of waterborne radionuclides. Results have consistently shown slight and variable radionuclide concentrations immediately downstream of the plant discharge point that diminish to no detectable contamination in the Scioto River. There is no indication of an ongoing buildup of radionuclides at any specific point.

\section{Radiation doses to the public}

The maximum 50-year committed effective dose equivalent to an individual from PORTS activities during 1989 is shown in Table 1. A comparison of maximum effective doses due to
Table 1. Summary of estimated radiation doses to an adult during 1989 at locations of maximum exposure

\begin{tabular}{ccc} 
Emission & Location & $\begin{array}{c}\text { Effective dose } \\
\text { (mrem) }\end{array}$ \\
\hline Gaseous effluents & $\begin{array}{c}\text { Maximally exposed } \\
\text { individual } \\
(1770 \mathrm{~m} \text { ENE) }\end{array}$ & 0.07 \\
Liquid effluents & Scioto River & 0.002 \\
\hline
\end{tabular}

airborne emissions over the last 5 years is shown in Fig. 5. A comparison of the maximum effective dose due to waterborne emissions over the last 5 years is shown in Fig. 6. None of the doses resulting from PORTS operations are significant to public health. The maximum 50-year committed effective dose equivalent (EDE) to an individual during 1989 from airborne emissions was only 0.07 mrem. This dose is much lower than the EPA newly established (December 1989) standard of $10 \mathrm{mrem} /$ year of plant operation.

The calculated effective dose equivalent from drinking water and eating fish from the Scioto River for one year by a hypothetical maximum individual was 0.002 mrem. This dose is below the EPA drinking water limit of $4 \mathrm{mrem} /$ year that applies to a public water supply and the DOE-

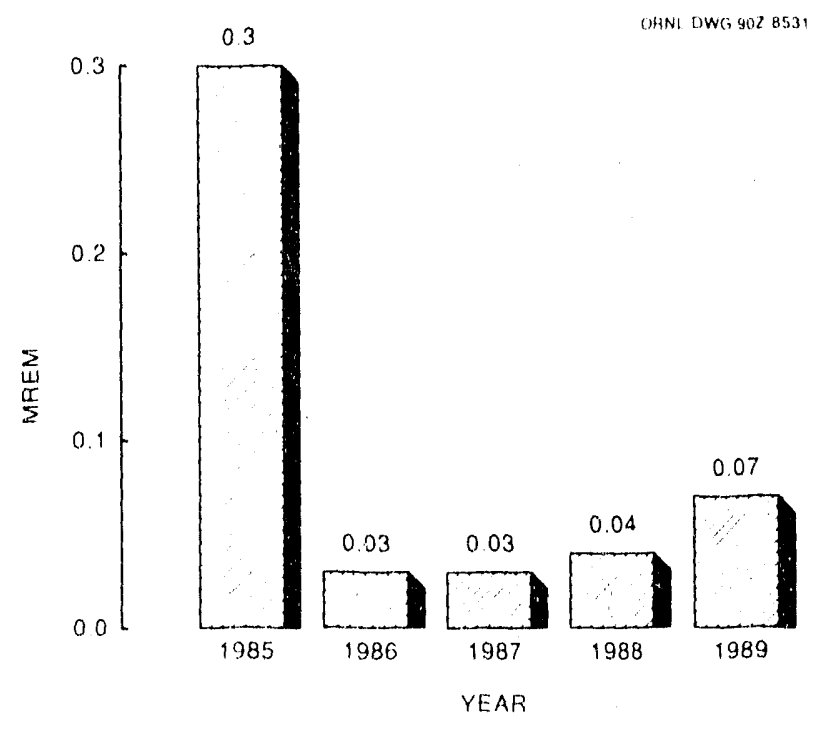

Fig. 5. Maximum predicted individual effective dose equivalent from airborbe radionuclides, 1985-1989. 


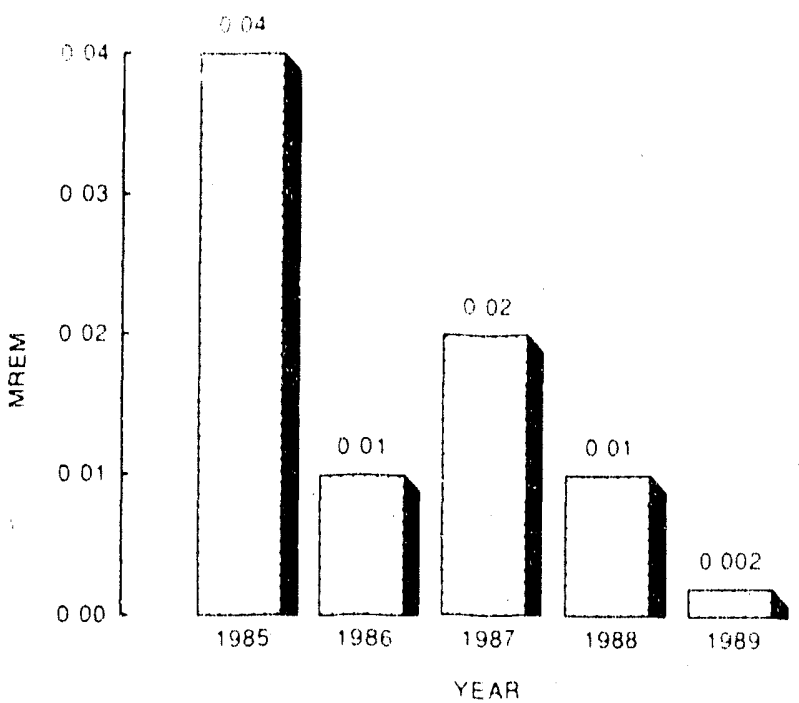

Fig. 6. Maximum predicted indiridual effective dose equivalent from waterborne radionuclides, 1985-1989. proposed limit of $100 \mathrm{mrem} / \mathrm{year}$ EDE for all exposures (i.e., waterborne plus airborne). No public water supply and no known private water supply draws from the Scioto River, and only occasional sport fishing takes place on the river downstream of PORTS. Consequently, this dose is hypothetical and should be very conservative. Also note that this hypothetical maximum waterborne dose is located on the opposite side of the plant (i.e., southwest) from the maximum airborne dose (i.e., east-northeast); this makes it unlikely that one individual could be exposed to both doses.

The population doses (i.e., collective effective dose equivalent) from airborne radionuclides were 0.01 person-rem to the nearest community and 0.4 person-rem to the total population within $80 \mathrm{~km}$ (50 miles) of the plant. No waterborne population dose was calculated because the population exposed is essentially zero. 


\section{SUMMARY OF 1989 ENVIRONMENTAL ACTIVITIES}

\section{Chemical Release}

In compliance with the Superfund Amendments and Reauthorization Act (SARA), storage and releases of hazardous chemicals have been reported to the appropriate agencies. The storage of hazardous chemicals is required by SARA Sect. 312 for listed chemicals above the threshold-planning quantities for the chemicals. Typical chemicals included are chlorine, Freon-114, gasoline, fluorine, hydrogen fluoride, various acids, and uranium hexafluoride.

Chemical releases under SARA are covered under Sect. 313. If a Sect. 313 chemical is manufactured, processed, or otherwise used in excess of a set amount, then releases to the environment of that chemical must be reported to the EPA and the OEPA. Approximately $94,000 \mathrm{~kg}$ $(207,000 \mathrm{lb})$ of listed chemicals were released to the environment.

\section{Audits and Reviews}

The PORTS Environmental Protection Program undergoes rignrous annual appraisals, audits, and reviews by organizations both internal and external. All appraisals, audits, and reviews may result in recommendations requiring operating contractor management attention.

Recommendations on environmental issues result in the development and implementation of corrective actions formulated to improve overall site environmental protection.

Appraisals, audits, and/or reviews of the site Environmental Protection Program conducted during 1989 included an Environmental Prutection Appraisal by the Environmental Protection Division in April, a review of the 1986 Environmental Survey by the Office of Environmental Audit in May, a pre-Tiger Team review by Lee Wan Associates in July, a follow-up to the Lee Wan Associates review by Analysas
Corporation in August, and the DOE Tiger Team Appraisal in October and November.

\begin{abstract}
Abnormal Occurrences
PORTS incident reports revealed two abnormal occurrences. The first involved the rupture of an expansion joint on a chilled water pump. This resulted in a loss of several thousand gallons of a $30 \%$ ethylene glycol solution. A portion of the solution escaped from the building to the storm sewer system. The second occurrence involved the operation of the X-104A indor firing range facility. Incorrect filters were used on the ventilation exhaust ducts resulting in an exceedence of the lead emissions to the air. The firing range was shut down until the appropriate type of filters (i.e., high-efficiency particulate air) could be installed. In both instances there was no significant impact to PORTS or the surrounding communities.
\end{abstract}

\section{Environmental Restoration Program}

Closure plans developed in 1988 for the four RCRA-regulated sites at PORTs (i.e., X-616, X-231B, X-701B, and X-749) were approved by the OEPA on July 14, 1989. Closure activities on the X-701 B site began in late 1989. Activities on the other sites are scheduled to start in 1990. Groundwater cleanup of essentially pure chlorinated solvents near X-701B (which began in late summer 1988) is continuing. The solvent recovered will be incinerated, and any contaminated groundwater recovered will go through an activated charcoal system to remove the volatile organic compounds. Other remedial actions include the removal of two $X-750$ underground storage tanks (USTs) and three other USTs (i.e., X-611, X-611C W, and X-611C NW) during December 1989. Petroleum-contaminated soils from these sites was, or will be, removed for 
the post-treatment and disposal. All the sites will be cleaned up and returned to a stable condition.

Negotiations that began between DOE, the OEPA, and the EPA in 1988 were concluded in August and October 1989, respectively, with the signing of the Ohio Consent Decree and the EPA Administrative Consent Order. In accordance with the requirements of these documents, DOE is tasked to perform a corrective action cleanup at the PORTS site over approximately the next 10 years. An RCRA Facility Investigation General Work Plan describing the lasic approach to the first phase of this process was submitted in December 1988 and was approved by the OEPA in April 1989. Review by the EPA is in progress. The site was divided into four quadrants based predominantly on groundwater flow. These quadrants were numbered in order of priority to be cleaned up based on preliminary reviews (i.e., Quadrant I most important, etc.). Thus resources will be utilized to the fullest to ensure environmental restoration at the most potentially significant locations first. Site-specific RFI work plans for Quadrants I and II were submitted to both agencies in May and November 1989 and are still undergoing review. A groundwater quality assessment report for the four RCRA-regulated sites mentioned above was submitted to the OEPA and the EPA during 1989. The results of the assessment concluded that there was no off-site contamination of groundwater. It did, however, conclude that three out of the four ch-site areas showed groundwater contamination.

\section{Solid Waste Management}

In accordance with RCRA and the Ohio Solid Waste Act, the Solid Waste Management Program at PORTS encompasses solid, liquid, semisolid, or contained gas that is being discarded. The purpose of the program is to manage the accumulation, treatment, storage, and disposal of all discarded solid waste and to ensure compliance with solid waste regulations and policies. The administration of the Solid Waste Management Program is accomplished by two departments: the Waste Management Department and the Environmental Control Department.
The goals of PORTS' solid waste management are:

- to ensure compliance with all federal, state, and local regulations;

- to minimize the volume and hazard of all hazardous and radioactive wastes produced;

- to protect the environment from unintentional releases of hazardous or radioactive materials;

- to protect personnel from hazards associated with handling solid wastes; and

- to minimize the long-term risks and liability for waste disposals.

Waste minimization strategy currently includes segregation, material substitution, process changes, mechanical compression volume reduction, and recycle and reuse.

Environmental protection is provided through the use of specification packaging and by storage in properly engineered and permitted facilities. An extensive inspection and audit program is in place to identify and to correct deficiencies. Personnel protection is provided through standard industrial protection equipment applied in accordance with requirements determined from Materials Safety Data Sheets and Industrial Hygiene/Health Physics recommendations. Long-term risks and liabilities for disposals are minimized by destroying or removing the hazardous constituent whenever possible, as opposed to burial or storage.

PORTS solid waste is managed in five different categories: radioactive waste; classified wastes; hazardous wastes as defined by the RCRA; polychlorinated biphenyls and asbestos, which are regulated by the Toxic Substances Control Act (TSCA); and conventional solid waste, which is generally managed by sanitary landfill. Medical and infectious wastes are a special subcategory of conventional solid wastes. Trends in the generation of PORTS waste streams are indicated $\iota_{11}$ Figs. 7 through 18.

PORTS stores RCRA, TSCA, radioactive, and nonregulated wastes in accordance with applicable regulations and DOE orders. PORTS disposal facilities are a sanitary landfii, a classified landfill, and a radioactive landfill. 


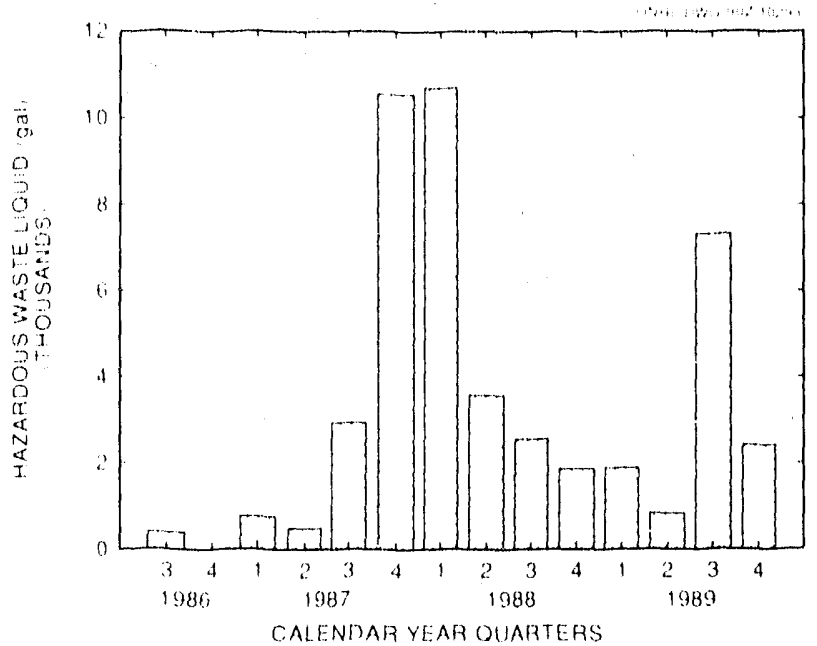

Fig. 7. Liquid hazardous waste by quarters.

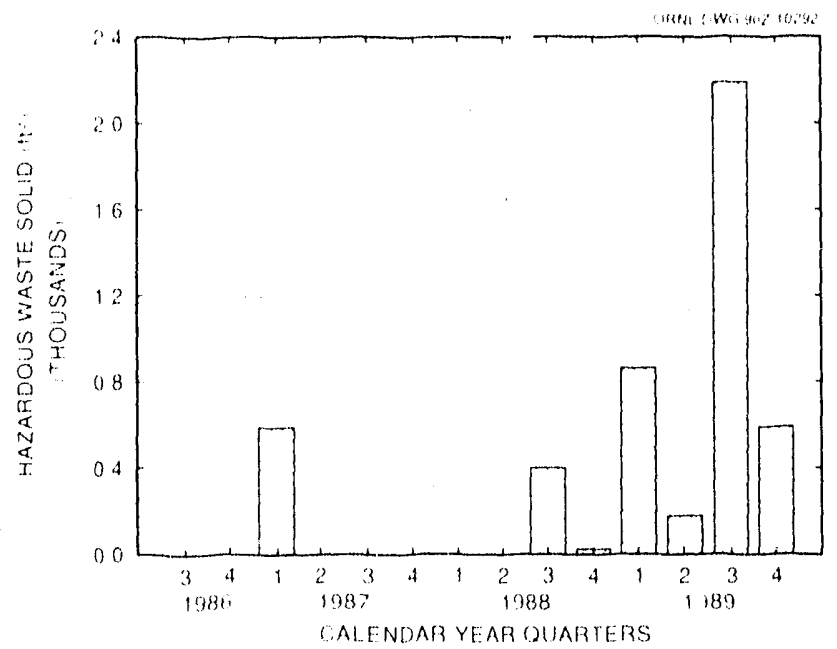

Fig. 8. Solid hazardous waste by quarters.

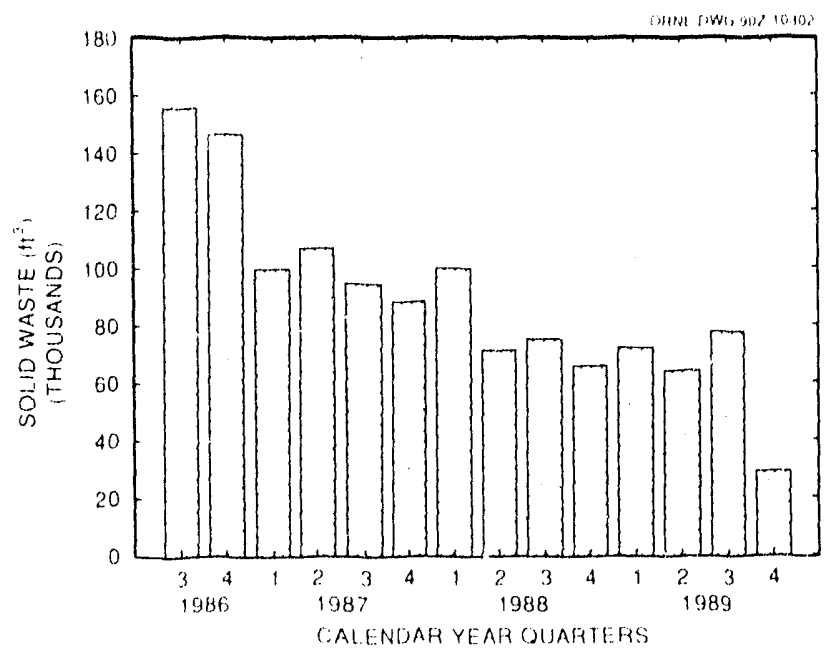

Fig. 9. Sanitary waste generation by quarters.

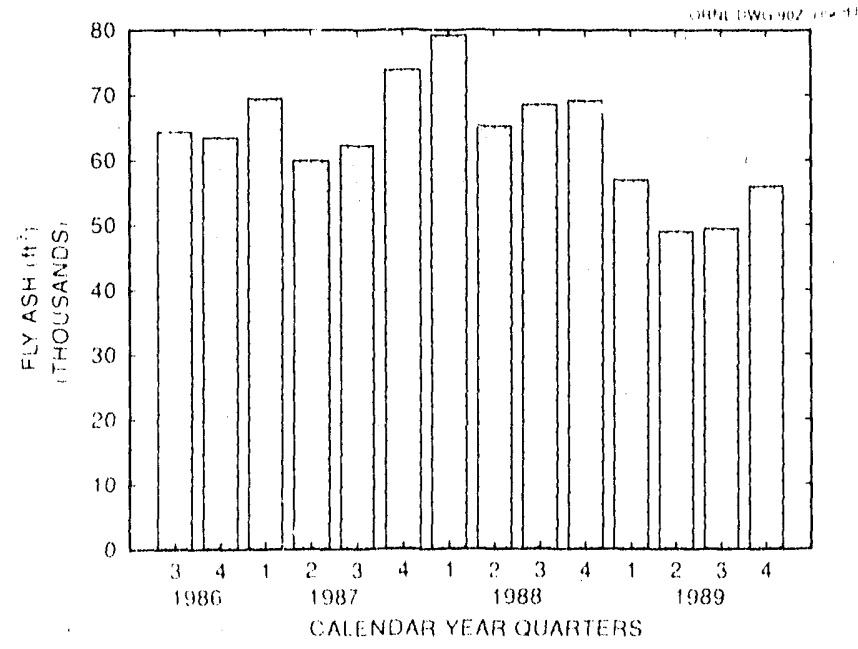

Fig. 10. Fly ash by quarters.

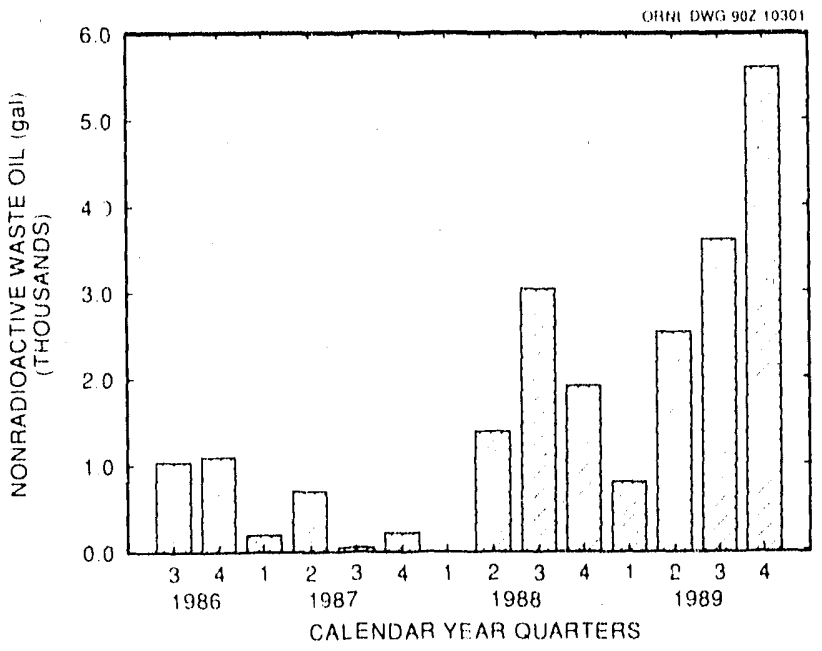

Fig. 11. Nonradioactive waste oll by quarters.

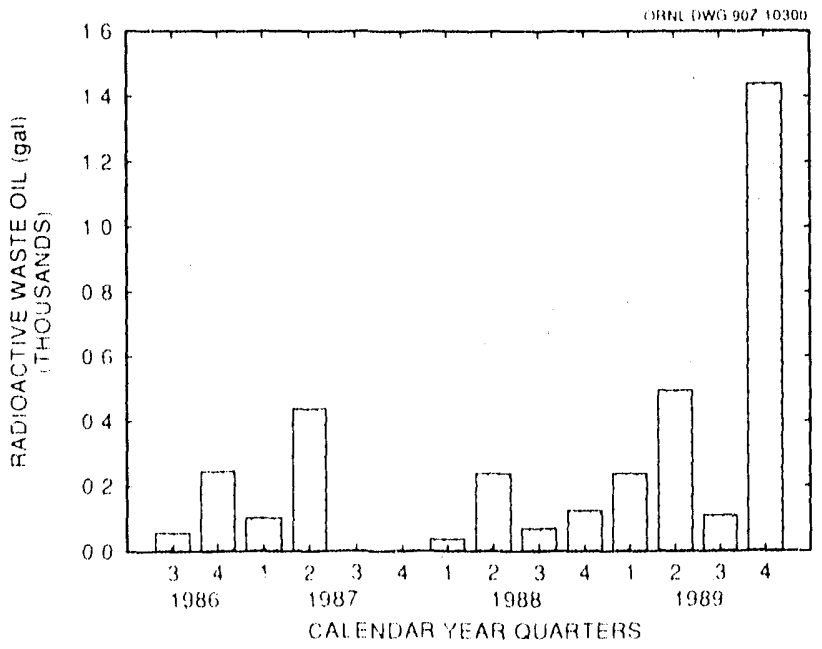

Fig. 12. Radioactive waste oil by quarters. 


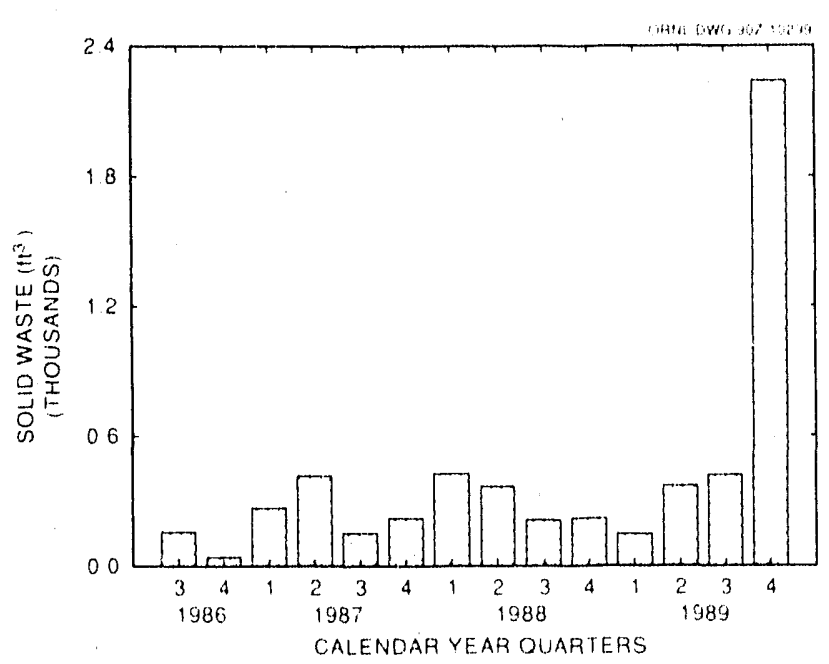

Fig. 13. Solid mixed hazardous waste by quarters.

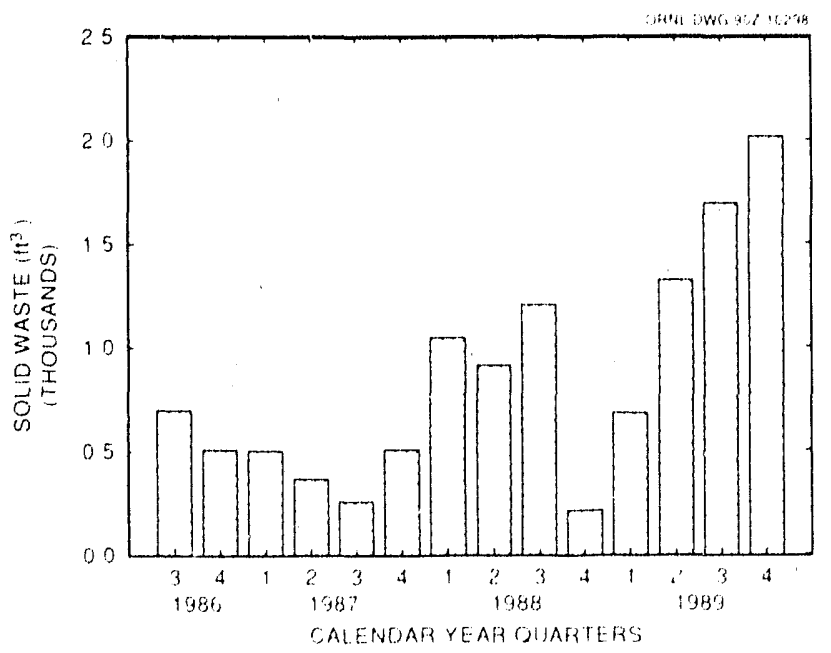

Fig. 14. Solid mixed polychlorinated biphenyl waste by quarters.

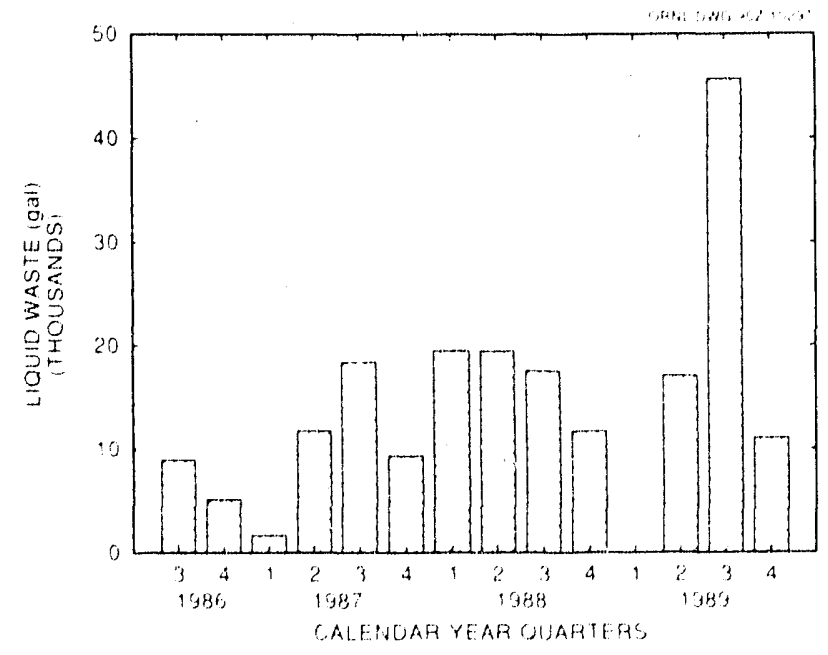

Fig. 15. Liquid polychlorinated biphenyl waste by quarters.

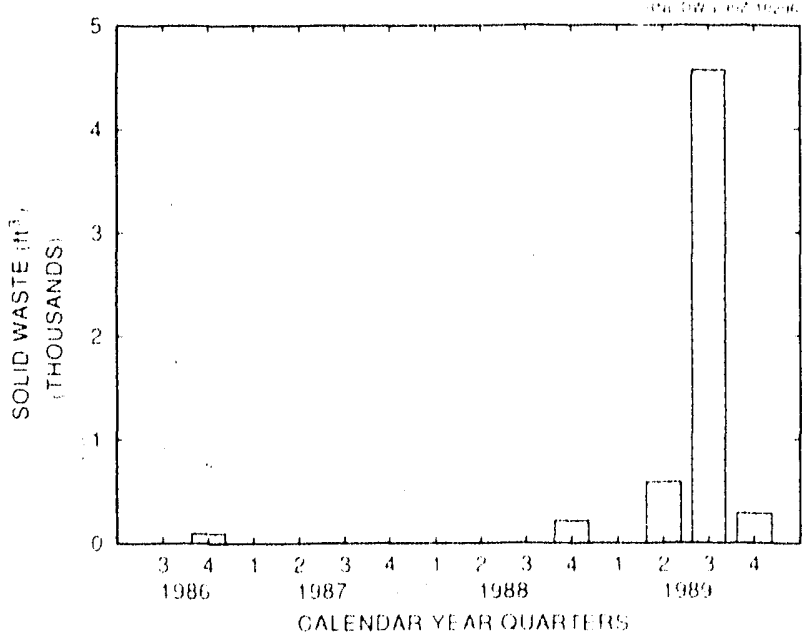

Fig, 16. Solid polychlorinated biphenyl waste by quarters.

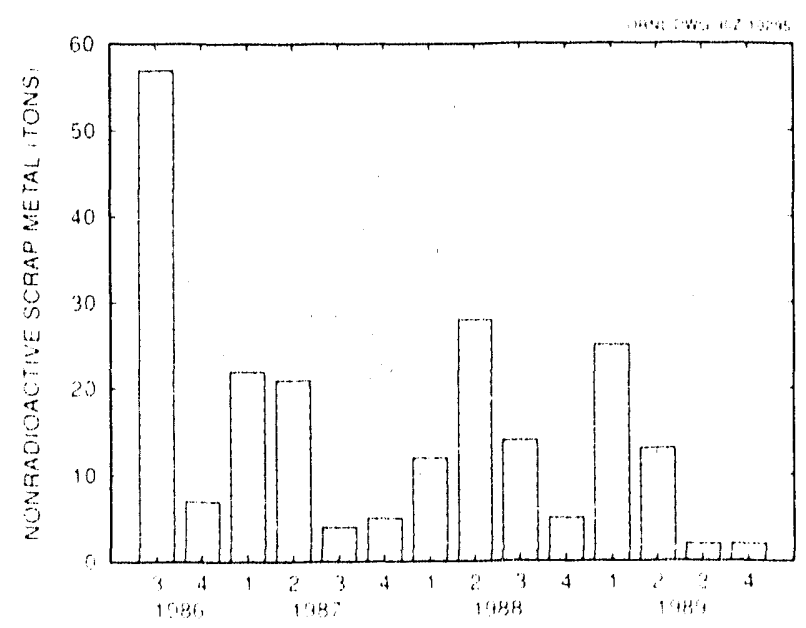

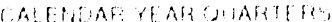

Fig. 17. Nonradioactive scrap metal by quarters.

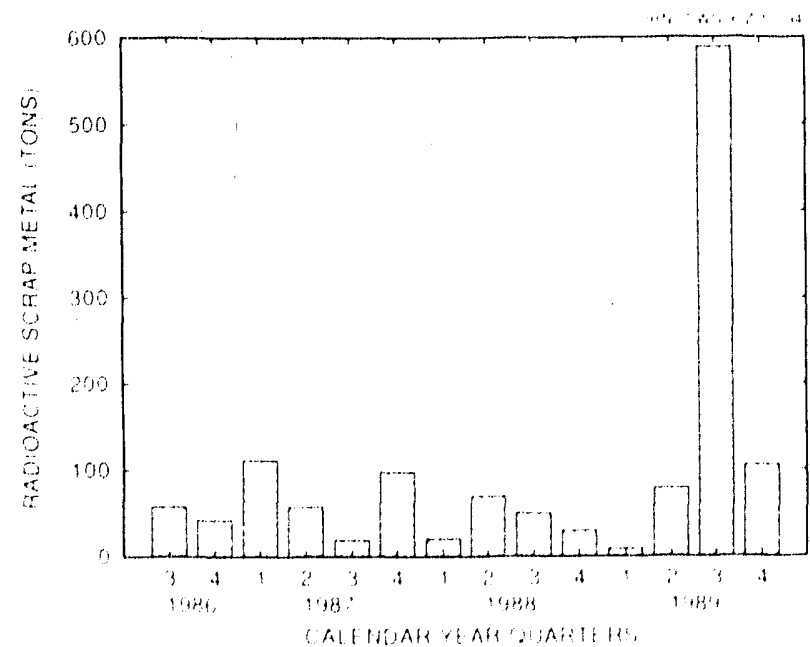

Fig. 18. Radioactive scrap metal by quarters. 
Construction spoils landfills are used for the disposal of nonregulated waste to conserve the area dedicated for use by the sanitary landfill.

The facilities administered by the DOE Oak Ridge Operations Office including PORTS have undertaken a Low-Level Waste Disposal Development and Demonstration (LLWDDD) Program. The objective of this program is to develop, to demonstrate, and to evaluate low-level waste disposal options for the future. The LLWDDD Program is defining performance parameters that the future waste disposal facilities must achieve. These are being developed to be consistent with Nuclear Regulatory Commission regulations and with the advice of area EPA regulators.
The Hazardous Waste Development, Demonstration, and Disposal Program is similar to the LLWDDD Program but addresses hazardous and mixed wastes for Energy Systems installations. PGRTS uses commercial disposal of RCRA- and TSCA-regulated wastes to the maximum extent possible for nonradicactive wastes. Thermal or chemical destruction of the hazardous constituents is used whenever possible. Use of chemical waste landfills is minimized. The use of commercial facilities for treating radioactive wastes is being investigated. The 1989 off-site waste shipments are also summarized in this report. 


\section{SUMMARY ASSESSMENT ENVIRONMENTAL COMPLIANCE ACTIVITY U.S. DEPARTMENT OF ENERGY}

\section{PORTSMOUTH GASEOUS DIFFUSION PLANT}

\section{BACKGROUND AND OVERVIEW}

The Portsmouth Gaseous Diffusion Plant (PORTS) must operate in conformance with environmental (and other) requirements established by a number of federal and state statutes and regulations, Executive Orders, U.S. Department of Energy (DOE) Orders, and Compliance and Settlement Agreements. Compliance status with major environmental statutes is summarized below.

\section{Clean Air Act (CAA) and National Emission Standards for Hazardous Air Pollutants (NESHAP)}

PORTS has 9 state air permits with an additional 76 air emission sources registered with the Ohio Environmental Protection Agency (OEPA). Nine radiological stacks are equipped with continuous stack samplers to monitor radiological emissions. Seventeen field airmonitoring stations are used to monitor environmental air emissions (i.e., 6 on-site, 8 property line, and 3 off-site). PORTS radiological air emissions are well within the newest NESHAP standards. Stack monitors are being added, and field samplers are being upgraded to comply with the new NESHAP monitoring requirements. Permit applications have now been submitted to the OEPA for all identified air emission sources. Unpermitied services and procedural violations had been identified in the past; however, recent improvements should result in full compliance.

\section{Clean Water Act (CWA)}

The PORTS National Pollutant Discharge Elimination System (NPDES) permit encompasses 20 monitored outfalls, 6 of which are classified as point source discharges. Three wastewater treatment facilities have been constructed and brought on-line since 1983. NPDES excursions and spills have resulted in some parameters exceeding NPDES permit limits; however, progress continues in minimizing these incidents and their effects on receiving streams.

\section{Resource Conservation and Recovery Act (RCRA)}

PORTS operates three interim status RCRA storage facilities and has four RCRA facilities currently with approved closure plans and a fifth RCRA facility that has been closed and inspected and is awaiting OEPA approval. Additionally, seven 90-day storage areas have been established for ic.nporary storage of hazardous wastes.

Seventy-seven solid waste management units have been identified for further study under the 3004(u) Remedial Action Program. RCRA violations have oscurred, and citations have been received in the past; however, recent improvements have resulted in a substantially upgraded program. A Consent Decree with the state of Ohio was negotiated and signed on August 31, 1989.

\section{Toxic Substances Control Act (TSCA)}

PORTS currently operates six waste storage areas for polychlorinated biphenyls (PCBs). All storage areas meet the storage requirements of TSCA regulations. Current plant-site inventory of $\mathrm{PCBs}$ in electrical equipment (and spare equipment) is approximately 1.2 million $\mathrm{kg}$. Compliance issues do exist regarding systems that are contaminated with $\mathrm{PCBs}$ and the storage of uranium-contaminated $\mathrm{PCB}$ wastes beyond the 1-year TSCA limit (see Current Issues below for details). 
Comprehensive Environmental Response, Compensation, and Liability Act (CERCLA)

Negotiations between DOE and the U.S. Environmental Protection Agency (EPA) were completed on a Consent Order that addresses CERCLA and RCRA corrective attions. The Consent Order signed by the EPA and DOE on October 26. 1989. has an effective date of November 2, 1989.

\section{Underground Storage Tanks (UST)}

During 1989 a survey was conducted of all be jw-grade petroleum storage tanks greater than $189.3 \mathrm{~L} \mathrm{(50} \mathrm{gal)} \mathrm{in} \mathrm{volume.} \mathrm{The} \mathrm{survey} \mathrm{listed}$ locations, status, capacity, installation date, material of construction, product stored, and leak detection requirement dates. Furthermore, the survey listed recommendations for upgrade, removal, or consolidation of tanks.

A UST Management Plan was prepared to ensure that the daily operation of the USTs remains in compliance with EPA, OEPA, state fire marshal, and DOE regulations. An Inventory Control Program was instituted to establish a record of daily operations at each tank and to indicate any abnormal operating conditir is requiring further investigation.

Four USTs and their associated piping were integrity-tested by the tracer leak detection method. A fifth UST, X-720, was air-pressuretested by Martin Marietta Energy Systems, Inc., personnel. Five USTs were removed from the ground. Corrective actions were undertaken at the UST sites where contamination occurred.

\section{CURRENT ISSUES}

In addition to the general trends discussed above, a number of specific compliance issues have been identified. These significant issues are addressed below. Aithough these issues remain unresolved, they are well-defined, and necessary actions for their resolution have been initiated.

\section{PCB-Contaminated Oil}

Some unit hydraulic/lube oil systems constitute PCB-contaminated nontotally enclosed systems. A project is under way to remove PCBs from the contaminated lube oil systems.

\section{PCB-Contaminated Ventilation Sy'tem Gaskets}

Oil drips from $P C B$ ventilation gasket.s are considered PCB spills. The TSCA requires documentation and immediate cleanup action and verification after discovery. At present, PORTS conducts daily inspections to idertify leaks and initiates cleanup within $24 \mathrm{~h}$ as required by the EPA Spill Cleanup Policy contained in the TSCA. Sampling and cleanup verification, also required by this policy, is not being performed at present because of the large number of drips occurring. $A$ statistical sampling system is being used. A drip collection system has been installed under all known leaks. DOE and the EPA are negotiating a Federal Facility Agreement (FFA) to bring the facility into compliance with TSCA regulations for use, storage, and disposal of PCBs.

\section{Unapproved Containers for PCB/Uranium Mixed Wastes}

Containers that are not approved by the U.S. Department of Transportation (DOT) arc being used to store PCB liquids that may contair highassay uranium. Containers of $5 \mathrm{in}$. diam are required to satisfy nuclear criticality safety requirements for unknown or high-assay uraniumbearing materials. No DOT-approved containers of these dimensions are commercially available in the types authorized by the TSCA. This issue is being incorporated into an FFA that also addresses the storage of mixed $\mathrm{PCB} /$ radioactive waste.

\section{Mixed TSCA Waste Storage}

Uranium-contaminated $\mathrm{PCB}$ wastes (i.e., mixed wastes) are being stored in excess of the 1-year limit imposed by the TSCA because of the lack of mixed waste treatment and disposal capabilities. A compliance agreement with the EPA is being pursued.

\section{Land Disposal Restricted Waste}

Currently, PORTS is storing liquid, low-level radioactive waste that also contains RCRA 
hazardous waste. This waste is subject to the RCRA land disposal restriction regulations that allow storage only for accumulation of sufficient quantities for treatment and disposal. This waste is being stored because of the lack of treatment or disposal facilities for the material. DOE and the EPA are continuing to discuss the issue, and the inventory of such materials is reported to the regulators on an annual basis. DOE expects to negotiate with the EPA an FFA that would detail the steps needed to attain compliance.

\section{Unpermitted Air Emissions}

Small point sources, purge cascade emissions, process vents, and cooling tower emissions have not been permitted or exempted by the OEPA at PORTS. Permit applications for cooling tower emissions, small point sources, purge cascade emissions, and process vents have been submitted to the OEPA for their determination of regulatory compliance status.

NESHAF ragulations require $i$ :monstration of compliance vith the airborne radionuclide standard by March 15, 1990. PORTS is awaiting the EPA's approval of expanded source monitoring and an ambient-air-monitoring plan submitted on March 7, 1990, to demonstrate compliance with the regulation. Plans are being developed so that implementation of the program can proceed as soon as possible after EPA approval.

\section{Parameters in Excess of NiPDES Permit Limits}

Rainfall runoff and algae growth have routinely resulted in $\mathrm{pH}$ and total suspended solids exceedances. New NPDES permit limits are still being negotiated for $\mathrm{pH}$ and total suspended solids due to these causes. Draining of ponds to reduce algae growth and decrease $\mathrm{pH}$ values, dredging of ponds to reduce total suspended solids, and other measures are being implemented to eliminate these occurrences. Efforts to improve compliance are showing significant progress. During the six months from Octobe1 1, 1989, to March 31, 1990, the number of exceedances was reduced to 28 from 56 in the prior 6 months, a $50 \%$ reduction.

\section{DOE Environmental Assessment (Tiger Team)}

In October and November 1989 a team of DOE environmental specialists conducted a comprehensive assessment of the environmental practices at PORTS. The assessment covered the CWA, the CAA, the RCRA, the TSCA, remedial action programs, NESHAP, the National Environmental Policy Act (NEPA), USTs, and environmental monitoring and surveillance. The assessment identified 35 compliance issues including 6 for the CWA, 5 for the CAA, 6 for the RCRA, 7 for the TSCA, 2 for NESHAP, 6 for NEPA, 2 for environmental monitoring and surveillance, and 1 relating to radiological dose assessments. Thirty-six other findings relating to Best Management Practices were also identified. A draft compliance assessment report has been prepared that describes each Tiger Team finding. This report has been submitted to sıate of Ohio environmental regulatory agencies and to the EPA and has been made available to the U.S. Congress. The report is also available to the public on request. An action plan has been developed and is being implemented to address each finding. 


\section{CONTENTS}

PART 1: NARRATIVE, SUMMARIES, AND CONCLUSIONS

1. INTRODUCTION AND GENERAL INFORMATION $\ldots \ldots \ldots \ldots \ldots \ldots \ldots \ldots$

1.1 OPERATIONS OF THE PORTSMOUTH SITE $\ldots \ldots \ldots \ldots \ldots \ldots \ldots \ldots \ldots$

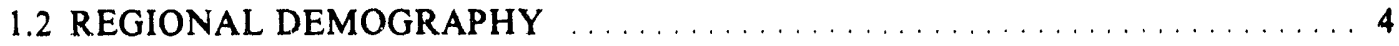

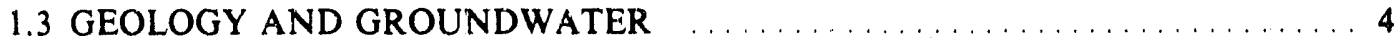

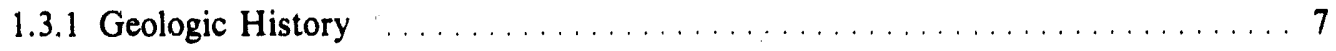

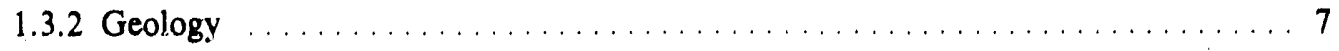

1.3.3 Regional Groundwater Conditions $\ldots \ldots \ldots \ldots \ldots \ldots \ldots \ldots \ldots \ldots$

1.3.4 Site Groundwater Conditions . .......................... 9

1.4 TOPOGRAPHY AND SURFACE WATER $\ldots \ldots \ldots \ldots \ldots \ldots \ldots \ldots$

1.4.1 Regional Drainage ...................................... 9

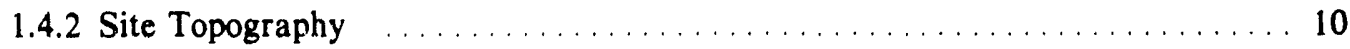

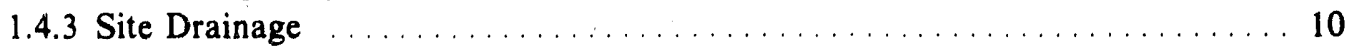

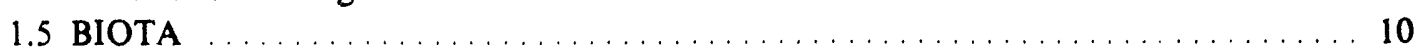

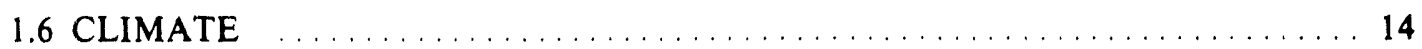

2. ENVIRONMENTAL MONITORING AND SAMPLING

SUMMARY .......................................... 15

2.1 AIRBORNE DISCHARGES AND AIR AND

METEOROLOGICAL MONITORING $\ldots \ldots \ldots \ldots \ldots \ldots \ldots \ldots \ldots$

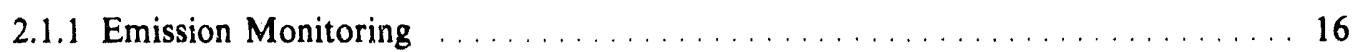

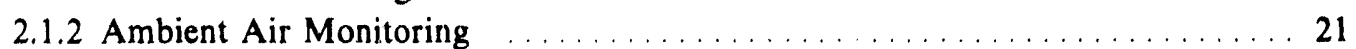

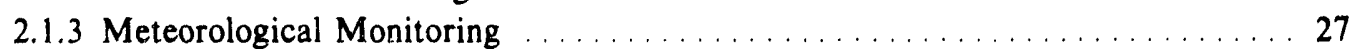

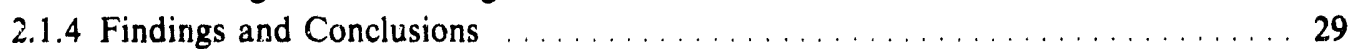

2.2 SURFACE WATER . . . . . . . . . . . . . . . . . . . . . . . . 29

2.2.1 Surface Water Monitoring . . . . . . . . . . . . . . . . . . . . . . . . . 29

2.2.2 National Pollutant Discharge Elimination System Monitoring . . . . . . . . . 33

2.2.3 Pollution Abatement Projects and Activities in $1989 \ldots \ldots \ldots \ldots \ldots$

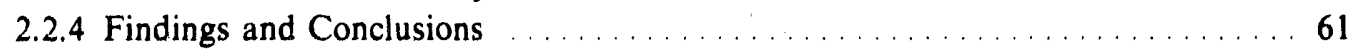

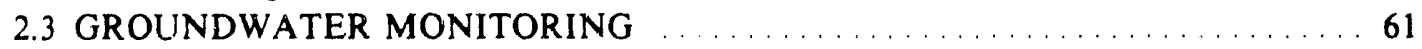

2.3.1 Groundwater Monitoring Program Descriptions $\ldots \ldots \ldots \ldots \ldots \ldots \ldots 6 . \ldots 6$

2.3.2 Geologic Setting ................................... 70

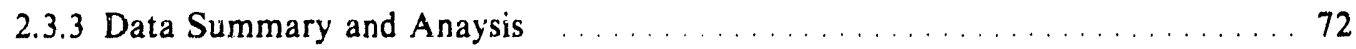

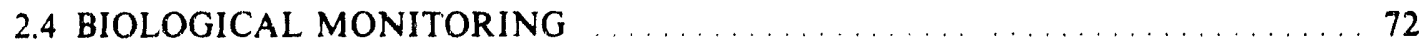

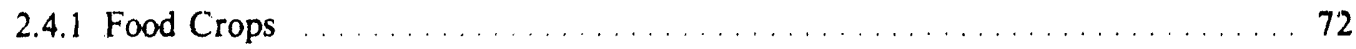

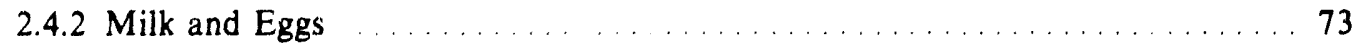

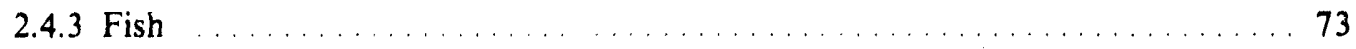

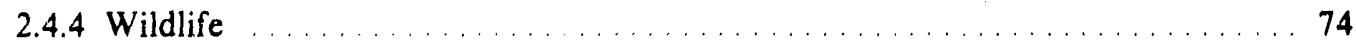

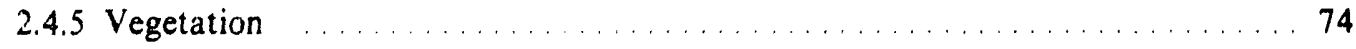


2.5 SOIL AND SEDIMENT MONITORING $\ldots \ldots \ldots \ldots \ldots \ldots \ldots \ldots \ldots$

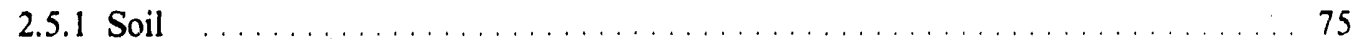

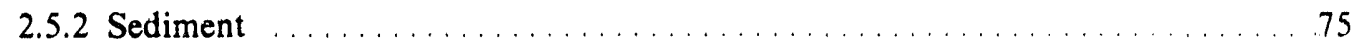

2.6 EXTERNAL GAMMA RADIATION $\ldots \ldots \ldots \ldots \ldots \ldots \ldots \ldots \ldots \ldots \ldots$

2.6.1 Program Description $\ldots \ldots \ldots \ldots \ldots \ldots \ldots \ldots \ldots \ldots \ldots \ldots \ldots \ldots \ldots \ldots$

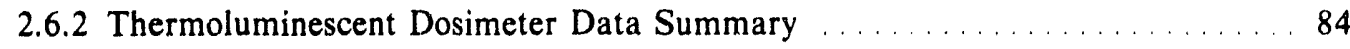

3. POTENTIAL RADIATION AND CHEMICAL DOSE

TO THE PUBLIC $\ldots \ldots \ldots \ldots \ldots \ldots \ldots \ldots \ldots \ldots \ldots \ldots \ldots \ldots \ldots$

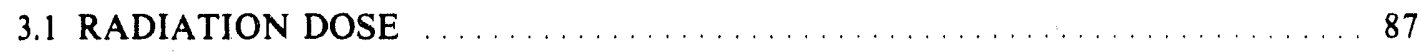

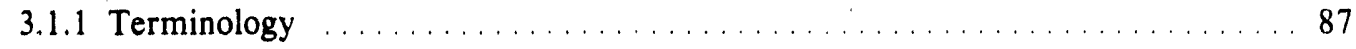

3.1 .2 Methods of Evaluation $\ldots \ldots \ldots \ldots \ldots \ldots \ldots \ldots \ldots \ldots \ldots \ldots$

3.1.3 Current Year Summary $\ldots \ldots \ldots \ldots \ldots \ldots \ldots \ldots \ldots \ldots \ldots \ldots \ldots$

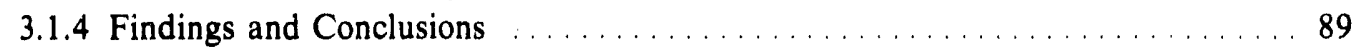

3.2 CHEMICAL DOSE $\ldots \ldots \ldots \ldots \ldots \ldots \ldots \ldots \ldots \ldots \ldots \ldots \ldots \ldots \ldots$

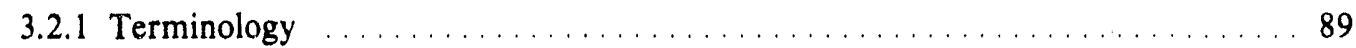

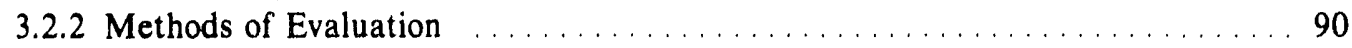

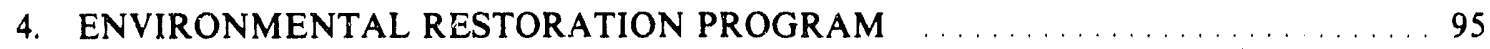

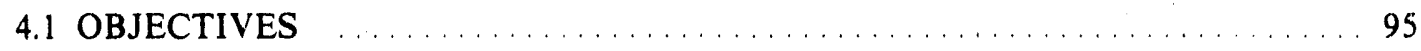

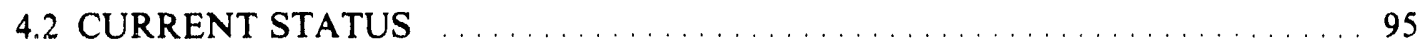

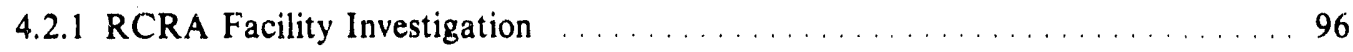

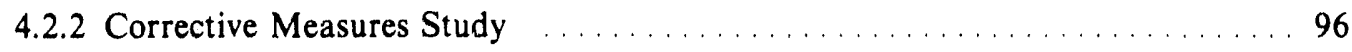

4.2.3 Corrective Measures Implementation $\ldots \ldots \ldots \ldots \ldots \ldots \ldots \ldots \ldots \ldots \ldots$

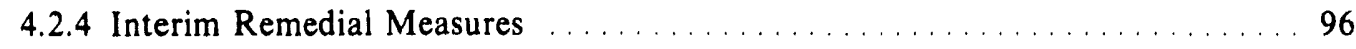

4.3 UNDERGROUND STORAGE TANK(S) $\ldots \ldots \ldots \ldots \ldots \ldots \ldots \ldots \ldots \ldots$

5. SOLID WASTE MANAGEMENT $\ldots \ldots \ldots \ldots \ldots \ldots \ldots \ldots \ldots \ldots \ldots \ldots \ldots \ldots \ldots$

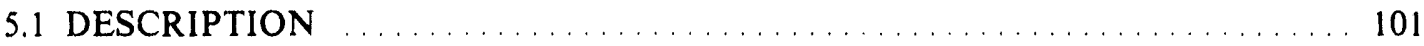

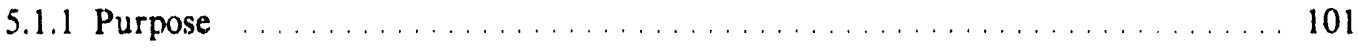

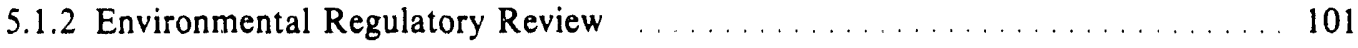

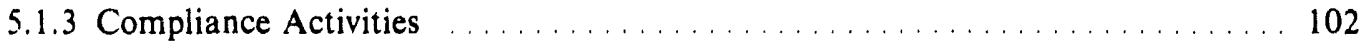

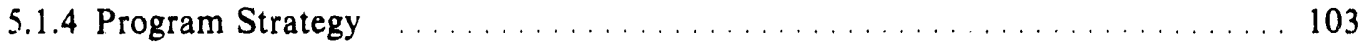

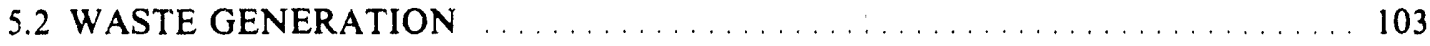

5.2 .1 Types of Waste Generated $\ldots \ldots \ldots \ldots \ldots \ldots \ldots \ldots \ldots \ldots \ldots$

5.2 .2 Waste-Generating Activities $\ldots \ldots \ldots \ldots \ldots \ldots \ldots \ldots \ldots \ldots \ldots \ldots$

5.3 WASTE MANAGEMENT ACTIVITIES $\ldots \ldots \ldots \ldots \ldots \ldots \ldots \ldots \ldots \ldots$

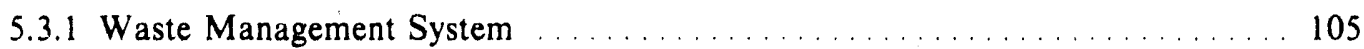

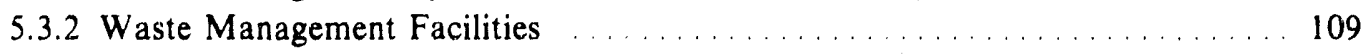

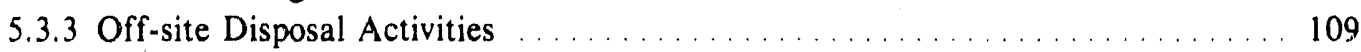

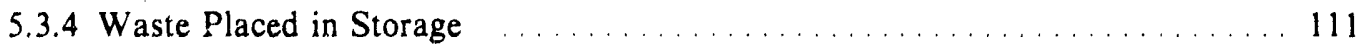

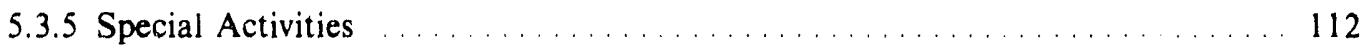

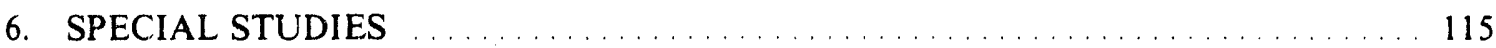

6.1 PORTSMOUTH GASEOUS DIFFUSION PLANT SPILL REPORT _. . . . . . 115

7. QUALITY ASSURANCE AND GENERAL REVIEWS $\ldots \ldots \ldots \ldots \ldots \ldots \ldots$

7.1 FIELD SAMPLING AND MONITORING $\ldots \ldots \ldots \ldots \ldots \ldots \ldots \ldots \ldots$ 


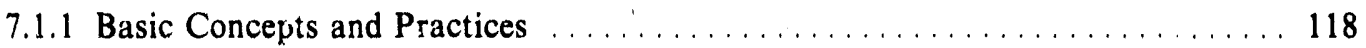

7.1 .2 Air Monitoring .................................... 118

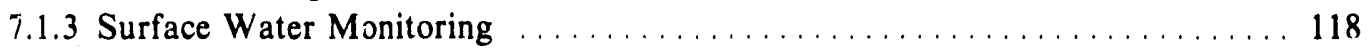

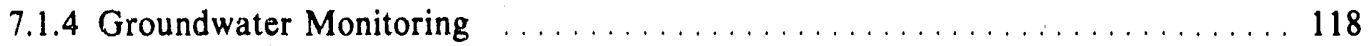

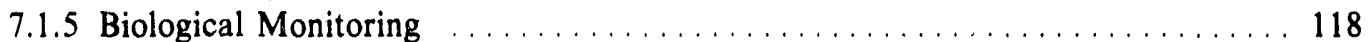

7.1.6 Soil and Sediment .................................. 119

7.1.7 External Gamma Radiation ................................ 119

7.1.8 Waste-Monitoring Program .............................. 119

7.2 ANALYTICAL QUALITY ASSURANCE $\ldots \ldots \ldots \ldots \ldots \ldots \ldots \ldots \ldots \ldots$

7.2 .1 Internal Quality Control .................................. 119

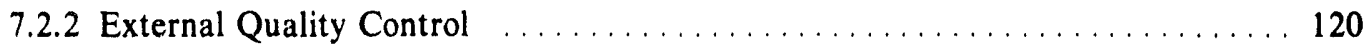

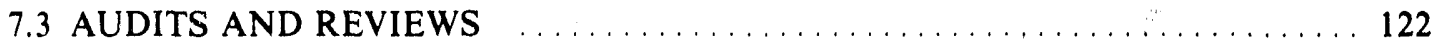

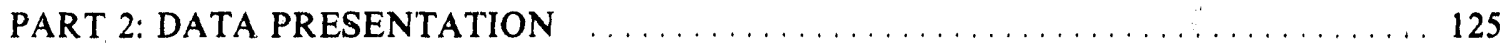

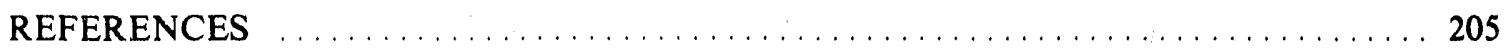

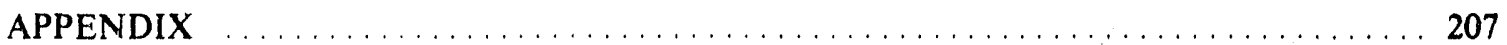




\section{ABBREVIATIONS, ACRONYMS, AND INITIALISMS}

\begin{tabular}{|c|c|}
\hline ADI & acceptable daily intake \\
\hline APG & Analytical Products Group \\
\hline BMP & best management practices \\
\hline Btu & British thermal unit \\
\hline${ }^{\circ} \mathrm{C}$ & degrees celsius \\
\hline CAA & Clean Air Act \\
\hline $\mathrm{CDI}$ & calculated daily intake \\
\hline $\mathrm{Ci}$ & curie \\
\hline $\mathrm{cm}$ & centimeter \\
\hline $\mathrm{cm}^{2}$ & square centimeter \\
\hline $\mathrm{cm}^{3}$ & cubic centimeter \\
\hline CMI & Corrective Measures Implementation \\
\hline CMS & Corrective Measures Study \\
\hline $\operatorname{COS}$ & Closure Option Study \\
\hline $\mathrm{CPF}$ & carcinogen potency factor \\
\hline${ }^{137} \mathrm{Cs}$ & cesium -137 \\
\hline CWA & Clean Water Act \\
\hline CWMO & Central Waste Management Office \\
\hline d & day \\
\hline DCF & dose conversion factor \\
\hline DMR & Discharge Monitoring Report \\
\hline DOE & U.S. Department of Energy \\
\hline DOT & U.S. Department of Transportation \\
\hline EML & Environmental Measurements Laboratory \\
\hline EMSL-LV & Environmental Monitoring System Laboratory at Las Vegas \\
\hline EP & extraction procedure \\
\hline EPA & Environmental Protection Agency \\
\hline ERP & Environmental Restoration Program \\
\hline ES\&H & Environmental Safety and Health \\
\hline${ }^{\circ} \mathrm{F}$ & degrees Fahrenheit \\
\hline FFA & Federal Facility Agreement \\
\hline $\mathrm{ft}$ & foot \\
\hline $\mathrm{ft}^{3}$ & cubic foot \\
\hline $\mathrm{g}$ & gram \\
\hline gal & gallon \\
\hline GCEP & Gas Centrifuge Enrichment Plant \\
\hline GDP & gaseous diffusion plant \\
\hline GWQA & Groundwater Quality Assessment \\
\hline $\mathrm{h}$ & hour \\
\hline ha & hectare \\
\hline HASA & high-assay sampling area \\
\hline HAZWDDD & Hazardous Waste Development, Demonstration, and Disposal \\
\hline
\end{tabular}




\begin{tabular}{|c|c|}
\hline $\mathrm{HF}$ & hydrofluoric acid \\
\hline in. & inch \\
\hline IRIS & Integration Risk Information System \\
\hline IRM & interim remedial measures \\
\hline $\mathrm{kg}$ & kilogram \\
\hline $\mathrm{km}$ & kilometer \\
\hline $\mathrm{L}$ & liter \\
\hline $1 \mathrm{~b}$ & pound \\
\hline LCSG & Laboratory Controls and Standards Group \\
\hline LLWDDD & Low-Level Waste Disposal Development and Demonstration \\
\hline $\mathrm{m}$ & meter \\
\hline M & million \\
\hline $\mathrm{m}^{3}$ & cubic meter \\
\hline$\mu \mathrm{Ci}$ & microcurie \\
\hline$\mu \mathrm{g}$ & microgram \\
\hline $\mathrm{mCi}$ & millicurie \\
\hline $\mathrm{mL}$ & milliliter \\
\hline $\mathrm{mg}$ & milligram \\
\hline Mgd & million gallons per day \\
\hline mrem & millirem \\
\hline $\min$ & minute \\
\hline NIST & National Institute for Standards and Testing \\
\hline NEPA & National Environmental Policy Act \\
\hline NESHAP & National Emissions Standards for Hazardous Air Pollutants \\
\hline NIOSH & National Institute of Occupational Safety and Health \\
\hline NPDES & National Pollutant Discharge Elimination System \\
\hline NVLAP & National Voluntary Accreditation Program \\
\hline $\mathrm{O} \& \mathrm{G}$ & oil and grease \\
\hline OEPA & Ohio Environmental Protection Agency \\
\hline ORGDP & Oak Ridge Gaseous Diffusion Plant \\
\hline ORO & Oak Ridge Operations \\
\hline${ }^{234 m} \mathrm{~Pa}$ & protoactinium-2.34 molal \\
\hline PAT & proficiency analytical testing \\
\hline $\mathrm{PCB}$ & polychlorinated biphenyl \\
\hline $\mathrm{pCi}$ & picocurie \\
\hline PET & proficiency environmental testing \\
\hline PETC & Pittsburgh Energy Technology Center \\
\hline pH & hydrogen ion concentration \\
\hline PORTS & Portsmouth Gaseous Diffusion Plant \\
\hline $\mathrm{ppb}$ & parts per billion \\
\hline ppm & parts per million \\
\hline PTI & Permit to Install \\
\hline QA & quality assurance \\
\hline QC & quality control \\
\hline RCRA & Resource Conservation and Recovery Act \\
\hline $\mathrm{RCW}$ & recirculating cooling water \\
\hline RFA & RCRA Facility Assessment \\
\hline RFI & RCRA Facility Investigation \\
\hline
\end{tabular}




$\begin{array}{ll}\text { RW } & \text { routile water } \\ \text { S } & \text { secorld } \\ \text { SARA } & \text { Superfund Amendments and Reauthorization Act } \\ \mathrm{SO}_{2} & \text { sulfur dioxide } \\ \mathrm{SWMU}_{99} \mathrm{Tc} & \text { Solid Waste Management Unit } \\ \text { tectinetium-99 } \\ \mathrm{TCA} & 1,1,1 \text {-trichloroethane } \\ \mathrm{TCE} & \text { trichloroethylene } \\ { }^{231} \mathrm{Th} & \text { thorium-231 } \\ \mathrm{TLD} & \text { thermoluminescent dosimeter } \\ \text { TRCL } & \text { total residual chlorine } \\ \text { TSCA } & \text { Toxic Substances Control Act } \\ \text { TSS } & \text { total suspended solids } \\ 234 \mathrm{U} & \text { uranium-234 } \\ \text { UF } 6 & \text { uranium hexafluoride } \\ \text { USGS } & \text { U.S. Geological Survey } \\ \text { UST } & \text { underground storage tank } \\ \text { VOC } & \text { volatile organic compound }\end{array}$




\section{PART 1}

Narrative, Summaries, and Conclusions 


\section{INTRODUCTION AND GENERAL INFORMATION}

\subsection{OPERATIONS OF THE PORTSMOUTH SITE}

This report presents environmental data and supporting narratives for the U.S. Department of Energy's (DOE's) Portsmouth Gaseous Diffusion Plant (PORTS) site Environmental Monitoring Program during 1989. Part 1 includes all narrative descriptions, summaries, and conclusions and is intended to be a "stand-alone" report for the reader who does not want to review in detail all of the 1989 data. Part 2 includes the detailed data summarized in a format that ensures that all environmental data are represented in the tables. Narratives are generally not included in Part 2.

PORTS is cwned by DCE and is managed by Martin Marietta Energy Systems, Inc. The location of the plant site is shown on the map of Ohio in Fig. 1.1.1. The facility is located in sparsely populated, rural Pike County, Ohio, on a $16.2-\mathrm{km}^{2}\left(6.3-\mathrm{mile}^{2}\right)$ site about $1.6 \mathrm{~km}$ (1 mile) east of the Scioto River Valley at an elevation approximately $36.6 \mathrm{~m}(120 \mathrm{ft})$ above the Scioto River floodplain. Figure 1.1.2 depicts the plant site and its immediate environs. The terrain surrounding the plant, except for the Scioto River floodplain, consists of marginal farmland and densely forested hills. The Scioto River floodplain is farmed extensively, particularly with grain crops.

The principal site process is the separation of uranium isotopes through gaseous diffusion. The gaseous diffusion process for uranium enrichment is based on the fact that lighter molecules diffuse slightly faster than heavier molecules through the walls of a porous tube called a barrier. The portion of gas passing through the barrier wall is slightly richer in ${ }^{235} U$. Support operations include the feed and withdrawal of material from the primary process, treatment of water for both potable and cooling purposes, steam generation for heating purposes, decontamination of equipment removed

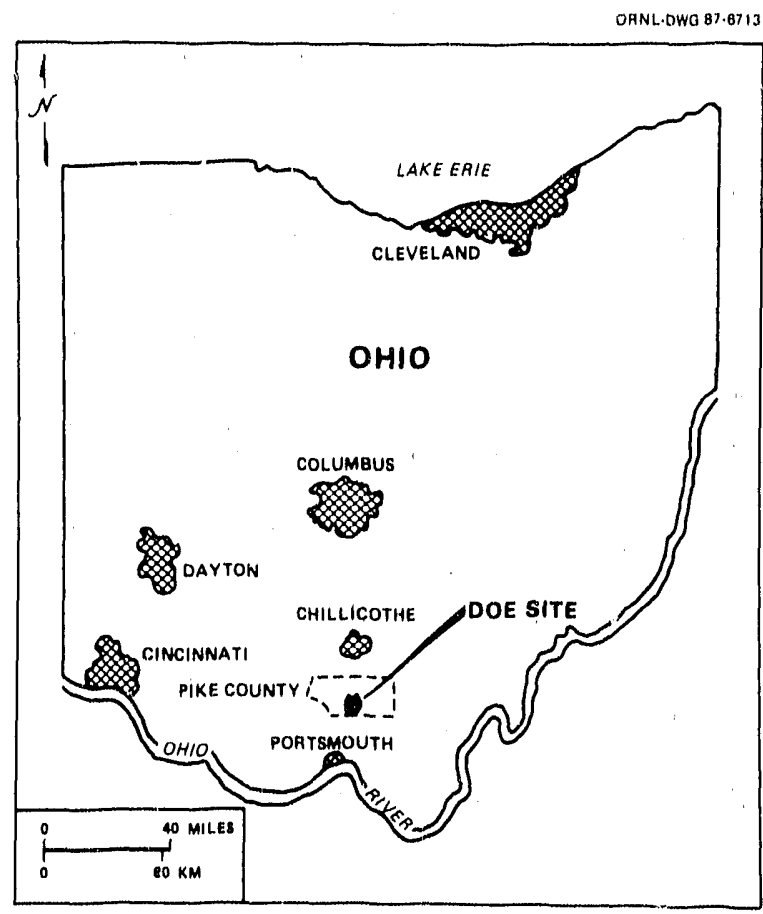

Fig. 1.1.1. Map showing the location of DOE's installation in the state of Ohio.

from the plant for maintenance or replacement, recovery of uranium from various waste materials, and treatment of industrial wastes.

Local tributaries flowing into the Scioto River are relatively unpolluted except for contamination introduced by agricultural nonpoint source runoff. PORTS discharges into Little Beaver Creek, Big Run Creek, two pipelines, and the west drainage ditch-all of which ultimately flow into the Scioto River (Fig. 1.4.1). The two pipelines carry the effluent from the sewage treatment plant and the treated blowdown from the recirculating cooling water (RCW) system. Sludge from the water treatment plant is discharged into a lagoon where the sludge settles and the slupernatant water is recycled to the water treatrnent plant. The other 


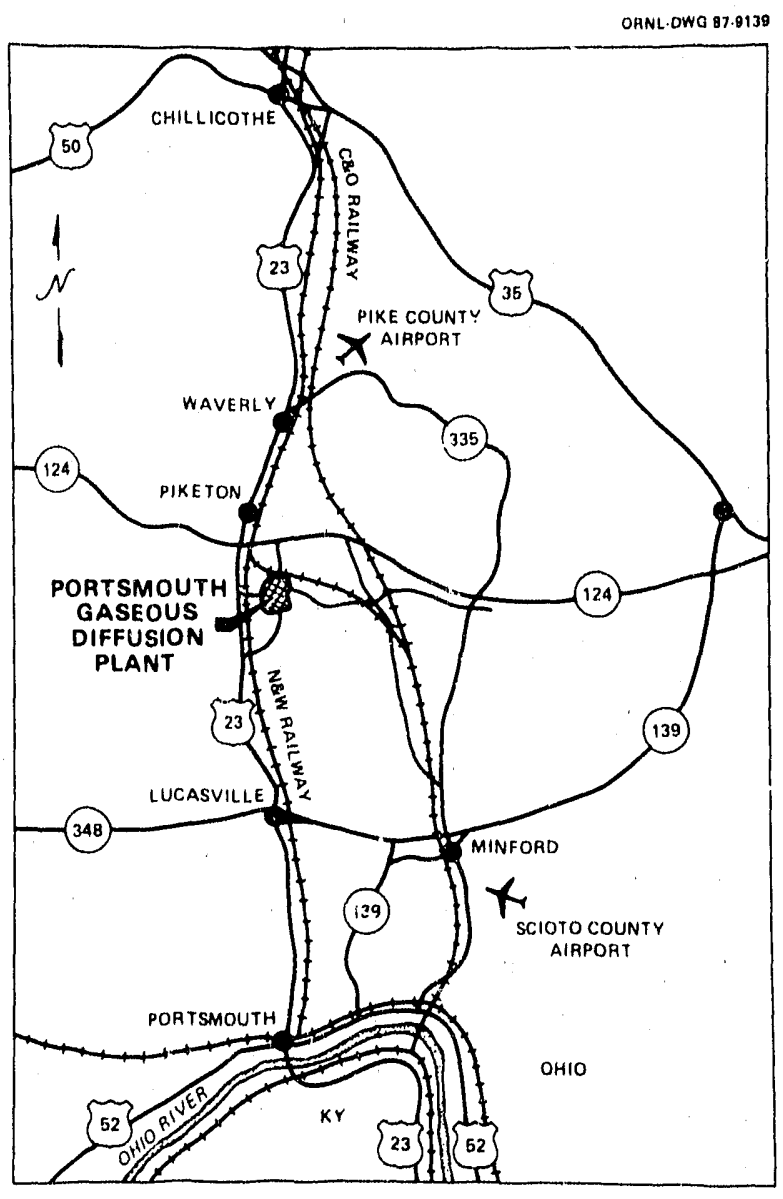

Fig. 1.1.2. Map showing the location of the Portsmouth site in relation to the geographic regicn.

effluent streams contain primarily storm runoff and water used for once-through cooling purposes.

\subsection{REGIONAL DEMOGRAPHY}

Pike County has approximately 23,000 residents. Scattered rural development is typical; however, the county contains numerous small villages such as Piketon, Wakefield, and Jasper, which lie within a few kilometers of the plant. The county's largest community, Waverly, is about $19 \mathrm{~km}$ (12 miles) north of the plant site and has a population of approximately 5100 residents. An estimate of the population distribution within an $8-\mathrm{km}(5-$ mile) radius of the plant is presented in Fig. 1.2.1, which shows a division of the area into sectors. The only residential center in this area is
Piketon, which is about $8 \mathrm{~km}$ (4.97 miles) north of the plent on U.S. Route 23; its 1986 population was 1900 . Several residences are adjacent to the southern half of the eastern boundary and along the Wakefield Mound Road (Old U.S. 23) directly west of the plant. Two nursing homes with a combined capacity of 60 persons are located along Wakefield Mound Road.

Additional population centers within $80 \mathrm{~km}$ (50 miles) of the plant are Portsmouth (population $25,500), 43 \mathrm{~km}$ (27 miles) south; Chillicothe (population 23,420), $43 \mathrm{~km}$ (27 miles) north; and Jackson (population 6675), $29 \mathrm{~km}$ (i 8 miles) east. The total population of the area lying within an $80-\mathrm{km}$ (50-mile) radius of the plant is approximately 600,000 .

\subsection{GEOLOGY AND GROUNDWATER}

\subsubsection{Geologic History}

The site is located within the Appalachian Plateau physiographic province near its northwestern boundary. Surficial rock units in this region were deposited in an inland sea during the Paleozoic era. At the end of the Paleozoic era (i.e., 230 million years ago), the region was uplifted and gently folded to form a shallow basin that trenćs parallel to the Appalachian Mountains. Subsequent erosion of the uplifted sediments produced the deeply dissected, knobby terrain that characterizes the region today.

During the Quaternary period (i.e., 1 million years ago), continental glaciation spread from a base near Hudson Bay to as far south as Chillicothe, Ohio (see Fig. 1.3.1). These glaciers indirectly influenced conditions south of this boundary and altered drainage patterns. The major surface water body in the region prior to glaciation was the Teays River. A major tributary of this river, the Portsmouth River, flowed northward through the area now occupied by the plant site and joined the Teays River near Piketon. During the Pleistocene age the Teays River was dammed at a point north of the site (presumably by another glacial event). This formed a large lake named Lake Tight. Alluvium deposited in the Teays and Portsmouth River valleys and lacustrine material 


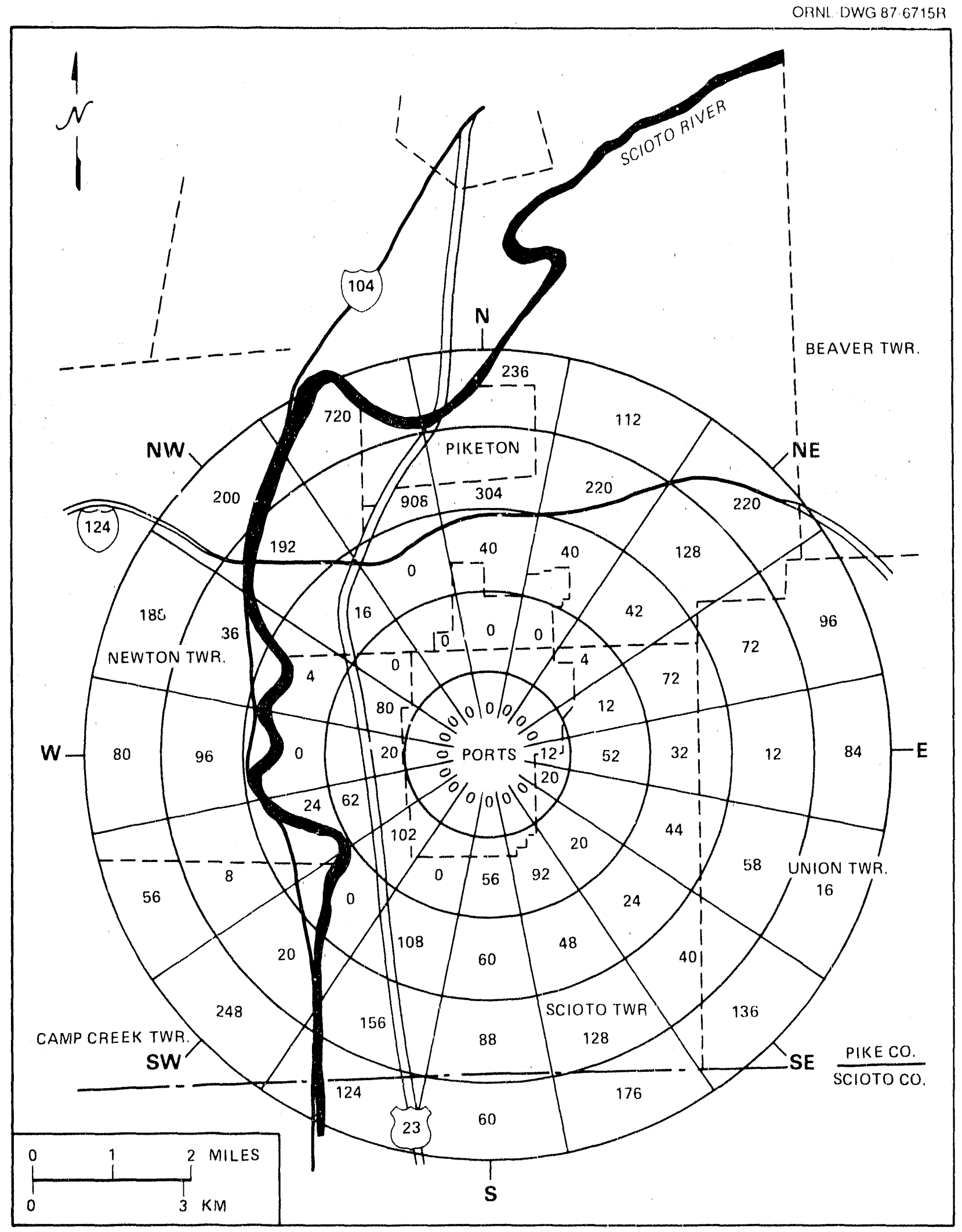

Fig. 1.2.1. Population distribution in an 8-km (5-mile) radius. 
CANL. DWG 89.10468

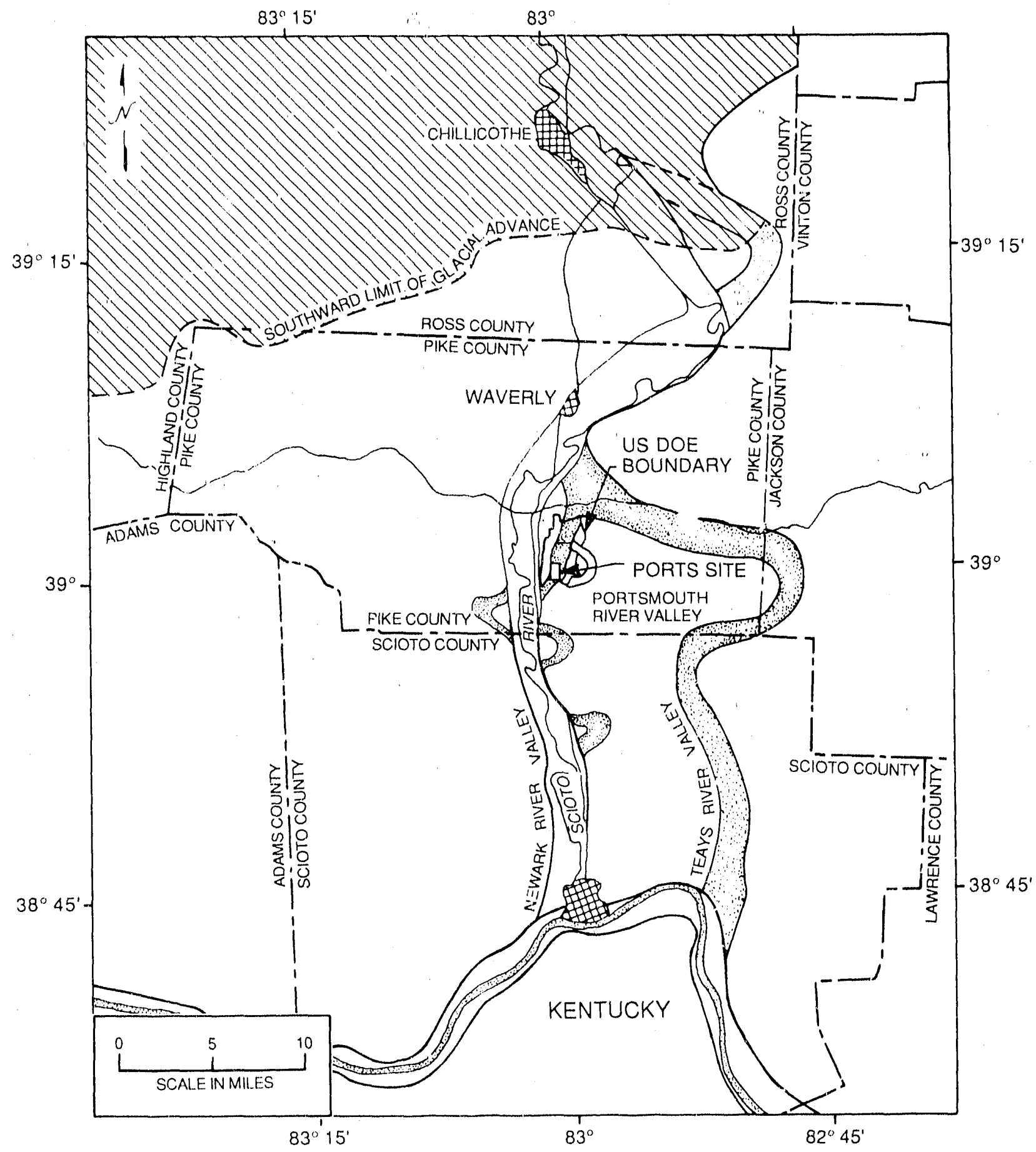

Fig. 1.3.1. Regional geography of ancient Teays and Portsmonth rivers. 
deposited within Lake Tight are represented in the Teays Formation, which unconformably overlies the Paleozoic units in the site area.

Modification to the regional drainage system occurred as Lake Tight filled and sought another drainage outlet. Thus, the Newark River rose and flowed southwestwardly from Chillicothe to Portsmouth where it joined the Cincinnati River, which flowed through the valley now occupied by the Ohio River. The Newark River was a major channel for alluvium-bearing meltwater from the Pleistocene continental glaciations. The deep Newark River valley was partially filled with sand, silt, and gravel outwash from the north; this was deposited unconformably over the clay unit formerly deposited by Lake Tight.

\subsubsection{Geology}

The geology of the site vicinity, like that of the region, is dominated by the Paleozoic shales and sandstones that are unconformably overlain by unconsolidated Pleistocene fluvial and lacustrine deposits. Man-made fill and recent alluvium and colluvium are locally present also.

The Mississippian Waverly Group crops out at and near the site vicinity and dips gently to the east. Because th Naverly Group and unconsolidated Pleistocene strata comprise the near-surface materials most likely to be affected by waste disposal operations, they have been studied more extensively than other strata present within the site vicinity. In ascending order the Waverly Group consists of the Bedford Shale, Berea Sandstone, Sunbury Formation, Cuyahoga Formation, Logan Formation, and the Maxville Limestone. The latter two units are not present at PORTS and thus are not discussed here.

The Berea Sandstone underlies the unconsolidated sediments in the western half of the plant site. This formation is a fine-grained saindstone with thin shale beds and is approximately $8 \mathrm{~m}(26 \mathrm{ft})$ thick. The Sunbury Shale overlies the Berea Sandstone and underlies the unconsolidated sediments along the eastern half of the PORTS site. It is highly carbonaceous black shale approximately $6 \mathrm{~m}(20 \mathrm{ft})$ thick.
Relatively smooth topographic features in the site vicinity generally represent unconsolidated Pleistocene fluvial and lacustrine deposits from the ancestral Portsmouth River and Lake Tight. As much as $16 \mathrm{~m}(52 \mathrm{ft})$ of fluvial material from the Portsmouth River system and lacustrine material from Lake Tight unconformably overlie the Waverly Group. The most common sequence of unconsolidated strata in the buried Portsmouth River valley consists of a thin layer of silty sand and gravel (representing fluvial deposition) overlain by a thicker layer of lacustrine fine sand, silt, and clay.

These unconsolidated near-surface sediments at the plant site consist of the Teays Formation; it has two members-the Gallia Sand and the Minford Clays. The Gallia Sand is the basal unit, and its contact with the underlying bedrock is sharp. The Gallia Sand at the facility consists of sands and gravels, varies in thickness 2 to $3 \mathrm{~m} \mathrm{(7}$ to $10 \mathrm{ft}$ ), and is usually found at depths ranging from 6 to $9 \mathrm{~m} \mathrm{(20} \mathrm{to} 30 \mathrm{ft}$ ). Overlying the Gallia Sand is the Minford Clay; this varies in thickness from 0 to $12 \mathrm{~m}(0$ to $39 \mathrm{ft})$ at the site.

Recent alluvium and colluvium are present in isolated portions of the site vicinity. Such deposits are associated with small streams that cross the site vicinity. Man-made fill that was placed during the construction of the facility occupies a significant portion of the site vicinity [maximum depth is 5 to $6 \mathrm{~m}$ (16 to $20 \mathrm{ft}$ )], particularly within the Perimeter Road.

\subsubsection{Regional Groundwater Conditions}

The availability, quantity, and quality of groundwater at any location are controlled by the geologic conditions beneath the surface of the land. Sand and gravel deposits in particular store and transmit sizable quantities of water. Clay, silt, and shale may contain as much water per cubic meter as sand and gravel; however, the water is held in pores so small that it generally cannot be transmitted in usable quantities. Water yields in limestone or sandstone depend upon the particular nature of the formation being examined (e.g., grain size, fractures, joint bedding planes, etc.). 
Geologic formations that occur near the surface in Pike County form two general classes: (1) consolidated layers of limestone, shale, and sandstone and (2) unconsolidated alluvial deposits of sand, gravel, clay, and silt. Eastern Pike County is underlain by Mississippian age sandstone and shale formations that normally do not yield enough water for domestic use. Actual yields from wells developed in these sandstone and shale formations depend on the presence of fractures and bedding planes and can vary widely over comparatively short disicances.

The unconsolidated alluvial deposits are found in the old Teays River valley and its tributaries including the Portsmouth and Newark River valleys. The primary source of groundwater in Pike County is the alluvial deposits in the former Newark River valley. These deposits consist of interbedded layers of sand and gravel beneath recent silt deposits and range from 18 to $30 \mathrm{~m}$ ( 59 to $98 \mathrm{ft}$ ) thick. Large supplies of groundwater are available from the alluvium because of the thickness of the sand and gravel and its proximity to the Scioto River. Pumping from wells near the river induces infiltration from the river into the gravels supplying the wells.

The Teays River valley from Beaver to Piketon is partially filled with clay, silt, sand, and fine gravel. The alluvium is thinnest in the western portion of the valley and thickens to the east in the vicinity of Beaver. The most productive sources of grouadwater in this valley are located near the village of Beaver and in the lower portion of Beaver Creek valley where a layer of fine sand and gravel is encountered above the bedrock surface. Elsewhere, the alluvium is primarily silt and clay with occasional lenses of sand and gravel on top of the shale bedrock. Water supplies in these areas are meager unless one of these lenses is encountered. Underlying both the Newark and Teays valleys is shale bedrock that, unless fractured, yields virtually no water. Regional groundwater flow is north to south, primarily along the sand and gravel deposits within the old Newark River valley.

\subsubsection{Site Groundwater Conditions}

The hydrogeolopic conditions underlying the DOE site are similar to those of the Teays River valley. The shale and sandstone bedrock underlies the entire property and outcrops in the northsouth-trending hills along the east and west portions of the facility. These units contain little or no water. The unconsolidated alluvial deposits are the Minford Clays and the Gallia Sand formations. A moderate amount of free vater is contained in the gravelly Gallia Sand but is not easily obtainable because of the large percentage of clay mixed in the gravel. The Minford Clays are essentially impermeable except in the weathered surface layers.

Three aquifers or aquifer types can be distinguished at the PORTS site: (1) the bedrock aquifer consisting of shallow Mississippian shales and sandstones, (2) the Scioto River alluvial aquifer, and (3) other alluvial aquifers. The latter two aquifer types have similar characteristics and properties; thus they can be described as a single aquifer system.

The bedrock aquifer includes the Cuvahoga Shale, Sunbury Shale, Berea Sandstone, and Bedford Shale. Near the top of the bedrock surface, permeabilities of these units are mostly the result of fractures and fissures. Based on analysis of well yields for bedrock wells located near the site vicirity, no significant difference is evident in permeability between shale and sandstone units. As depth increases, fractures influence overall permeability to a decreasing extent, and porosity, grain shape, and packing arrangement exert a greater influence on permeability. At greater depths the Berea Sandstone is probably more permeable than the shale units, which would act as confining layers. No well-defined lower limit to the bedrock aquifer exists, but because pumping costs increase and because permeability decreases with depth, only the shallow portions of the bedrock aquifer have been exploited in the site vicinity. The depth to bedrock in the site vicinity varies from near zero on hills and ridges to about $16 \mathrm{~m}$ 
(52 ft) in the lowlands. In some parts of the Scioto River valley, the depth to bedrock is greater than $32 \mathrm{~m}$ (104 ft).

Bedrock aquifers are commonly recharged (or discharged) by alluvial aquifers that overlie the bedrock. Groundwater in the bedruck aquifer may discharge via vertical leakage to deeper strata or via springs in hillsides where the bedrock is exposed. Past studies on water levels have shown that the bedrock aquifer is hydraulically connected to the alluvial aquifer system and that there is little or no vertical hydraulic gradient to induce mixing of the aquifers. Leakage between the shallow bedrock aquifer and underlying strata is possible; but the direction and rate of such leakage has not yet been verified.

The Alluvial Aquifer System consists of alluvium in the Scioto River valley, the Portsmouth River valley, the Teays River valley, and other smaller tributary river valleys. In general, a thick coarse-grained glacial outwash deposit is overlain by finer-grained modern alluvium. Of all of these, the Scioto River valley is the most productive aquifer. The thickness of the Scioto River alluvium ranges up to about $32 \mathrm{~m}$ (104 ft). This aquifer is coextensive with other alluvial aquifers near the site vicinity; however, grain size and hydraulic conductivity are smaller in tributary valleys than in the Scioto River valley.

As stated earlier, other alluvial aquifers occur discontinuously in alluvial valleys other than the Scioto River alluvial aquifer; these include the Portsmouth River valley, the Teays River valley, Big Run, Little Beaver Creek, and Beaver Creek. In general, these aquifers are extensive in the direction parallel to stream flow, but their widths and thicknesses are limited by the sides and bottoms of the valleys they occupy.

Alluvial aquifers may be recharged by, or discharge to, the streams that occupy their valleys. Highs in the potentiometric surface (i.e., an imaginary surface representing the total head of groundwater in a confined aquifer that is defined by the level to which the water will rise in a well) generally correspond to topographic high points, and lows generally occur beneath low points in the ground surface. This indicates that alluvial groundwater flows toward, and discharges to, hydraulic boundaries such as ponds and streams that occur at low elevations.

An analysis of topographical maps, surface water drainage, and past aerial photos of the site led to the detection of groundwater divides and an interpretation of groundwater flow directions. In general, groundwater in the northern part of the site flows toward Little Beaver Creek. In the vicinity of X-701B, it flows eastward toward the creek. Little Beaver Creek eventually discharges into Big Beaver Creek. The flow direction at $\mathrm{X}-616$ is westward toward a small, unnamed, intermittent tributary of the Scioto River. Subsurface flow at X-749 is divided between a westward component and an eastward component conforming to the upper reaches of the Big Run drainage basin. The upper tributaries of the Big Run drain the area of X-231B. Groundwater flow in this flat area is probably toward the south.

At this time, because of concentrated well placement at each of the four Resource Conservation and Recovery Act (RCRA) units, the four areas can be related to one another only by comparing the ranges of water level elevations among the four units. The higher values are found in the central area of the plant site, and the lower values are near the edges. This distribution of values implies that rainfall infiltrates in the flat central area of the plant site and recharges underlying groundwater. Flow is then outward from the central high area toward lower elevations The central area contains a complex divide that is probably continually changing location because of seasonal changes in infiltration and major recharge events.

\subsection{TOPOGRAPHY AND SURFACE WATER}

\subsubsection{Regional Drainage}

The DOE site lies within the Scioto River drainage basin, the second largest in Ohio. Both groundwater and surface water are drained from the plant site by a network of tributaries of the Scioto and Ohio rivers. A U.S. Geological Survey (USGS) gauging station is located at Higby, which is just north of the county line between Pike and Ross counties. The Scioto River flows measured at 
Higby from 1930 to 1973 range from $5310 \mathrm{~m}^{3} / \mathrm{s}$ $\left(187,550 \mathrm{ft}^{3} / \mathrm{s}\right)$ to $7.32 \mathrm{~m}^{3} / \mathrm{s}\left(260 \mathrm{ft}^{3} / \mathrm{s}\right)$ and average $133 \mathrm{~m}^{3} / \mathrm{s}\left(4700 \mathrm{ft}^{3} / \mathrm{s}\right)$. The estimated 7-day, 10-year low-flow discharge at Higby is $8.58 \mathrm{~m}^{3} / \mathrm{s}\left(303 \mathrm{ft}^{3} / \mathrm{s}\right)$. The USGS has also measured extremes, averages, and daily concentrations of chemical contaminants, particle size, distribution of suspended sediment, water temperature, dissolved oxygen, specific conductance, suspended sediment discharge, and radiochemical contaminants at Higby, which is upstream of the gaseous diffusion facilities, and at a recording station at Lucasville, which is downstream. Those data have been published (USGS, 1986).

\subsubsection{Site Topography}

The predominant landform in the site area is the relatively level, broad, filled valley of the preglacial Portsmouth River. The valley is oriented north to south and is bounded on the east and west by ridges or low-lying hills that have been deeply dissected by present and past drainage features. These ridges consist of Mississippian age formations of Sunbury and Cuyahoga shales. Another significant landform is the small valley formed by Little Beaver Creek; this creek flows in a northwesterly direction across the middle of the DOE property just north and east of the main plant area (see Fig. 1.4.1).

Other significant landforms consist of several small valleys formed by streams that have cut into the relatively level lak: and river deposits of the Portsmouth River vailey and (in some areas) into the Portsmouth River valley floor. One of these valleys is that of a northwestward-flowing stream near the west-central area of plant site whose. valley has been significantly altered by grading in the area. Two more streams are located in the southern portion of the plant area. In the southeast portion of the site, the southerly flowing stream named Big Run Creek is situated in a relatively broad, gently sloping valley where significant deposits of recent alluvium have been laid down by the stream. An unnamed southerly and westerly flowing stream in the southwest portion of the site has formed a narrow, steep-walled valley with relatively little recent alluvium. The Mississippian rocks are exposed to a greater extent here than in the eastern valley created by Big Run Creek.

\subsubsection{Site Drainage}

The PORTS site is drained by several small tributaries of the Scioto River (see Fig. 1.4.1). The largest stream on the plant property is Little Beaver Creek, which drains the northern and northeastern portions of the site before discharging into Big Beaver Creek (see Fig. 1.4.2). Upstream of the plant, Little Beaver Creek has intermittent flow throughout the year. On-site, Little Beaver Creek received treated process wastewater from the $\mathrm{X}-701 \mathrm{~B}$ holding pond via the east drainage ditch (until November 1988 when waste streams were stopped from entering the X-701 B pond) and stormwater runoff from the northwestern and northern portions of the plant via several storm sewers, watercourses, and the north holding pond. Shortly after leaving the reservation, Little Beaver Creek flows into Big Beaver Creek, which also receives runoff from the northeastern portion of the plant directly. Big Beaver Creek flows to the Scioto River.

Storm sewers on the south end of the plant site discharge to the south holding pond, which overflows to Big Run Creek, another intermittent stream. A small, unnamed, intermittent watercourse drains the southwest corner of the facility via the southwest holding pond. Farther north on the property is another intermittent watercourse that receives runoff from the central and western portions of the site via the west drainage ditch. All of these streams flow directiy to the Scioto River and carry only stormwater runoff.

\subsection{BIOTA}

Pike County consists primarily of farmland (including cropland, woodlot, and pasture) and forest (including Pike State Forest and portions of Wayne National Forest); urban and suburban areas occupy about $1 \%$ of the total land area. Figure 1.5.1 shows the land use within an $8-\mathrm{km}$ (5-mile) radius of the plant. 


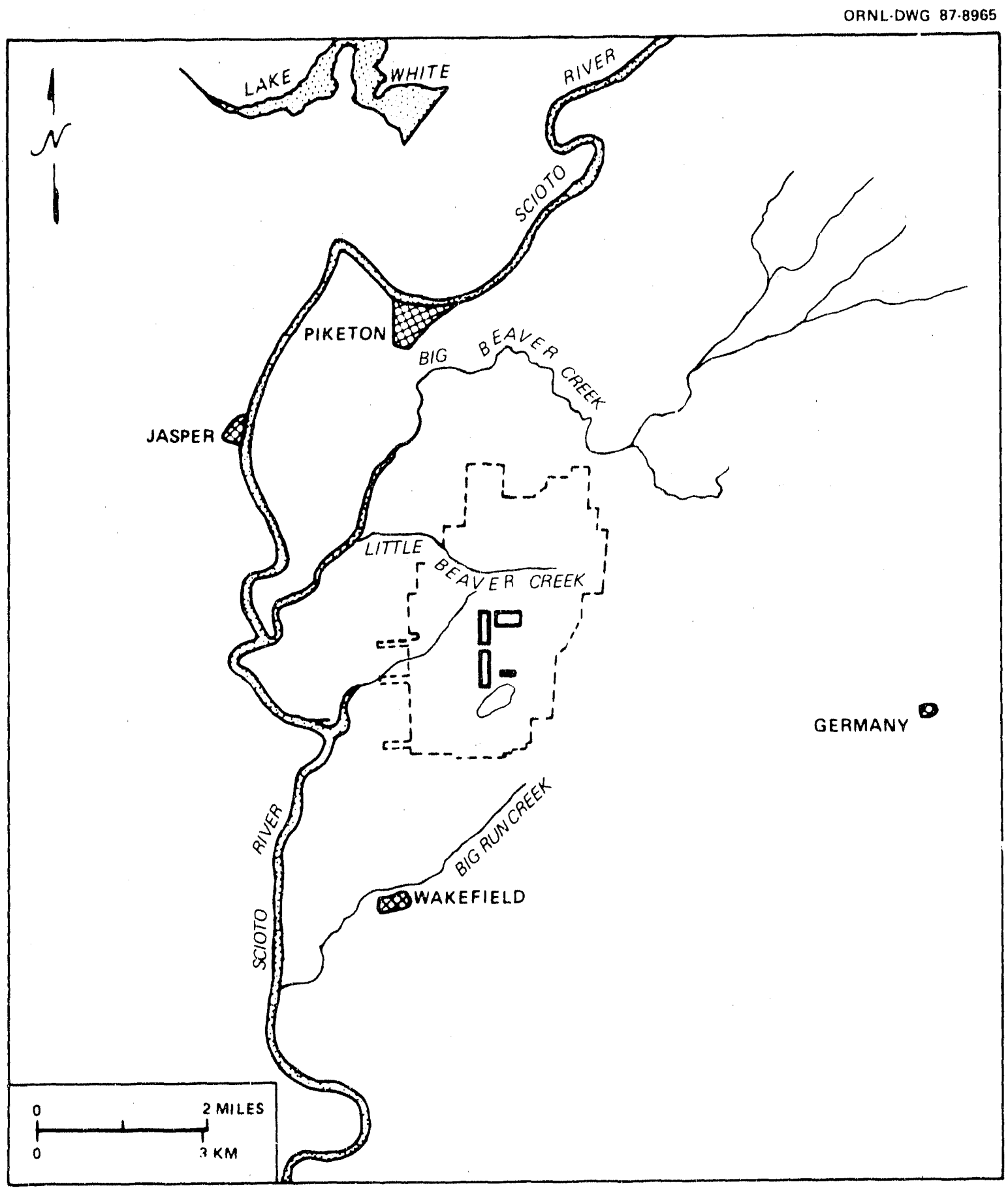

Fig. 1.4.1. Location of surface water bodies on and in the vicinity of the PORTS site.

The croplands lie mostly on or adjacent to the Scioto River floodplain and are farmed intensively, particularly with grain crops such as corn and soybeans. Other produce such as tomatoes, cabbage, and fruits are also cultivated in the area. Hillsides and terraces are more commonly used for cattle pasture. Both dairy and beef cattle are raised in the area of the Portsmouth site. Other 


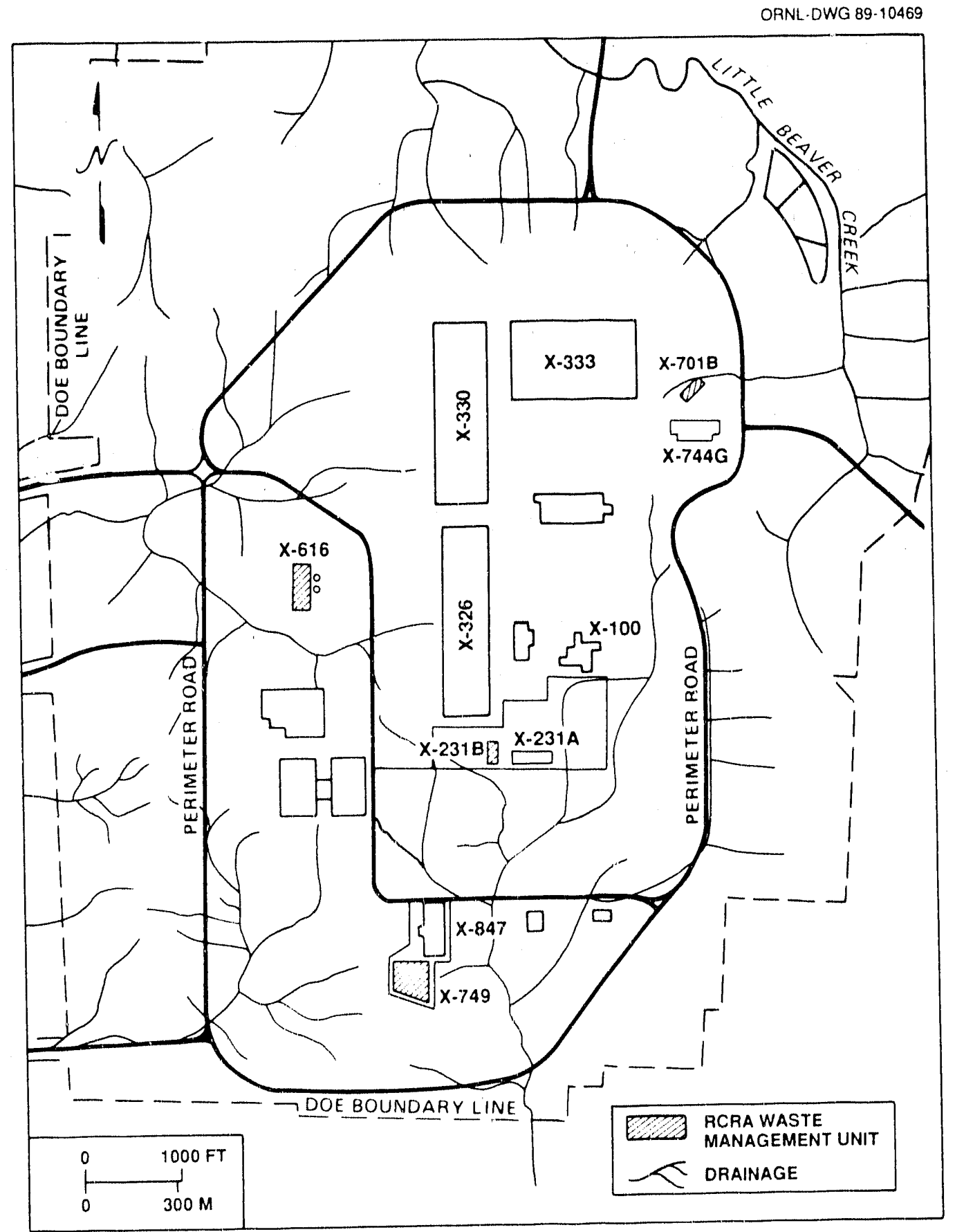

Fig. 1.4.2. Local drainage.

farm animals such as horses, pigs, sheep, goats, and chickens are raised to a lesser extent. Commercial woodlands (excluding sapling-seedling stands) are predominately sawtimber stands; poletimber stands are of lesser porportion.
About 9890 ha (24,430 acres) of farmland (including cropland, woodlot, and range pasture) and 9885 ha $(24,416$ acres) of forest lie within an $8-\mathrm{km}$ (5-mile) radius of the plant. There are also 206 ha ( 510 acres) in urban areas within the same 
ORNL ... DWG 87.6716

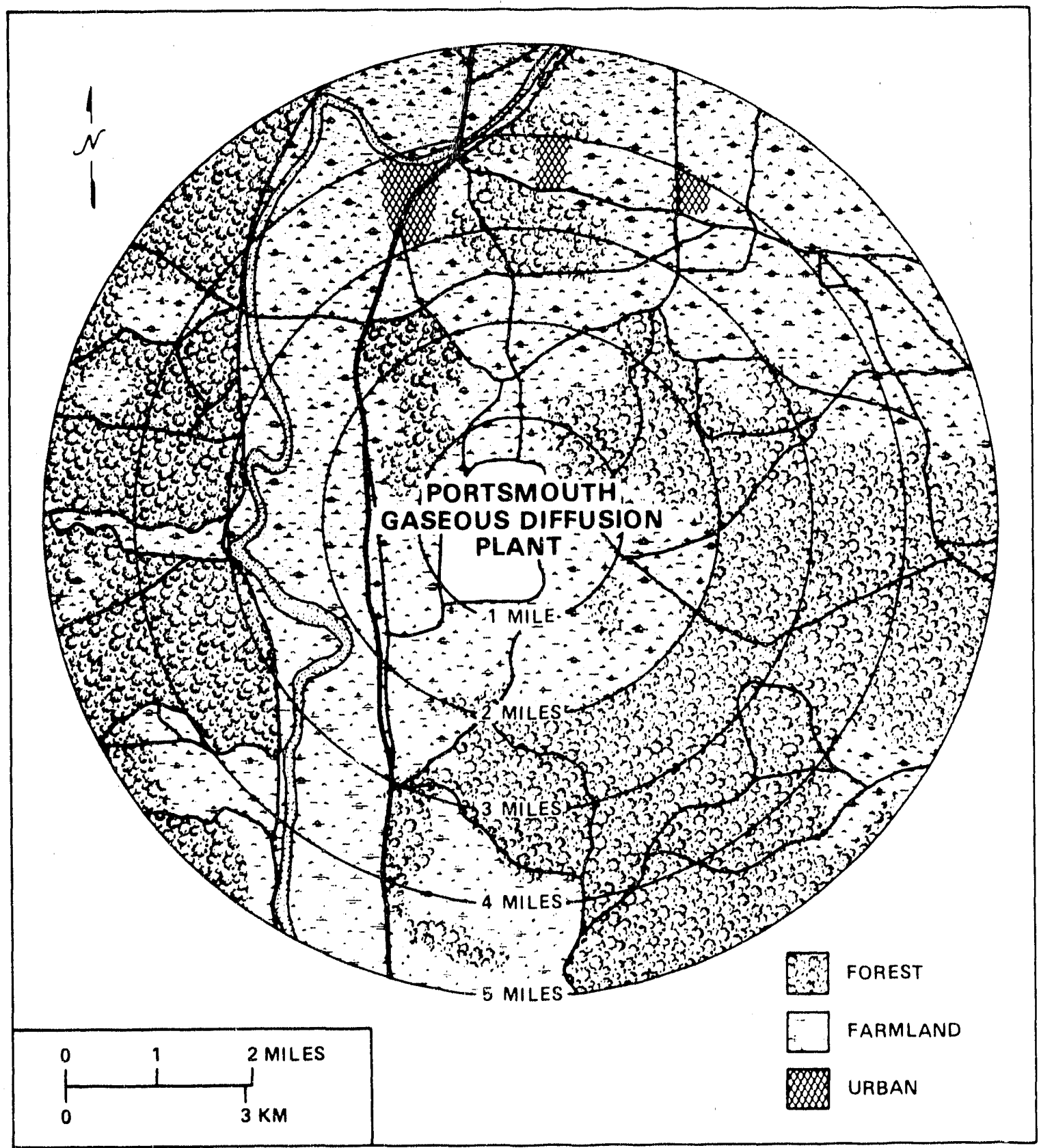

Fig. 1.5.1. Land use within 8-km (5 miles) of PORTS.

distance. The distribution of forest property in Pike County is similar to that of surrounding counties. Pike County is also typical from the standpoint of productivity. Land use is important in determining the impact of plant operations.
Natural forests are dominated by a tree cover of white oak, red oak, and hickory, mixed with lesser numbers of maple, ash, pine, and sycamore. Very dry or infertile soils are dominated by small stands of pine or black locust. Logged areas 
support scattered young oaks, hickories, and an abundance of brambles. Other subcanopy species vary throughout the area; sumac, poison ivy, and blackberry are most common.

Local animal species represent a typical southern Ohio fauna. The most important species include white-tailed deer, eastern cottontail rabbit, squirrels, muskrats, bullfrogs, mallards and wood ducks, bobwhites, pigeons, and mourning doves. Deer are rather more common on-site than off-site, presumably because of lower hunting pressure onsite. Numerous species of snakes (most commonly the black rat snake and northern black racer), turtles, frogs, and toads are also present. Fish populations in local small streams are typical of headwater-type streams and include carp, shad, bluegill, and various species of minnows and darters. Populations in larger streams (i.e., Big Beaver Creek and the Scioto River) are more abundant and diverse and also include various catfish, suckers, sunfish, trout, bass, and bullheads. There are no threatened or endangered species known to be present on or near the PORTS site.

\subsection{CLIMATE}

Pike County has a generally moderate climate. The National Oceanic and Atmospheric Administration maintained a weather station (temperature and precipitation only) in Waverly about $16 \mathrm{~km}$ (10 miles) north of the plant from
1889 until the early 1980s. An on-site station operated by the Portsmouth Emergency Preparedness Department has monitored air temperature; humidity; and wind direction, speed, and stability at both $10 \mathrm{-m}(32.8-\mathrm{ft})$ and $40-\mathrm{m}$ (131.2- $\mathrm{ft}$ ) levels (as well as solar radiation, precipitation, and soil temperatures at ground level) since December 1983.

Winters in Pike County are moderately cold. On the average, there are $112 \mathrm{~d} /$ year at or below $32^{\circ} \mathrm{F}\left(0^{\circ} \mathrm{C}\right)$ but only $3 \mathrm{~d} /$ year below $0^{\circ} \mathrm{F}$ $\left(-17.8^{\circ} \mathrm{C}\right)$. Summers are moderately warm and humid [i.e., an average of $27 \mathrm{~d} /$ year at or above $\left.90^{\circ} \mathrm{F}\left(32.2^{\circ} \mathrm{C}\right)\right]$. Annual precipitation averages $39.8 \mathrm{in}$. $(101.1 \mathrm{~cm})$. The precipitation is usually well distributed - fall being the driest season. Average annual snowfall at Waverly is 20.4 in. $(51.8 \mathrm{~cm})$.

Prevailing winds at the site are out of the southwest to south. Average wind speeds are about $5 \mathrm{mph}(8 \mathrm{~km} / \mathrm{h})$, although winds of up to $75 \mathrm{mph}$ $(120 \mathrm{~km} / \mathrm{h})$ have been recorded at the plant site. Usually, high winds are associated with thunderstorms that occur in spring and summer. Daytime wind stabilities are most commonly class D (i.e., neutral) followed by class $C$ (i.e., slightly unstable). Nighttime winds are predominately class F (i.e., moderately stable). Southern Ohio is within the midwestern tornado belt. No tornados have struck the plant site to date. 


\section{ENVIRONMENTAL MONITORING AND SAMPLING SUMMARY}

Routine monitoring and sampling for radiation, radioactive materials, and chemical substances on and off the PORTS site are used to document compliance with appropriate standards, to identify trends, to provide information for the public, and to contribute to general environmental knowledge. The surveillance program assists in fulfilling the DOE policy of protecting the public, employees, and the environment from harm that could be caused by its activities and reducing negative environmental impacts to the greatest degree practicable.

Monitoring and sampling locations for various types of measurements are organized into three groups:

- off-site stations located some distance from the DOE Reservation in residential and community areas to document conditions in areas occupied and visited by the public and potentially affected by DOE installations,

- perimeter stations located on the boundaries of the DOE Reservation, and

- on-site stations located in PORTS areas accessible only to employees or authorized visitors.

In 1989, approximately 100,000 measurements were taken in air, groundwater, streams, lakes, drinking water, grasses, garbage, sewage, soil, sediment, and sludge.

A summary of the Superfund Amendments and Reauthorization Act (SARA) Title III report is given in the appendix. The SARA report provides the community with the opportunity to learn about estimated quantities of certain toxic chemicals used at a facility that are routinely or potentially could be released during an accident into the environment. The appendix includes some additional "large quantity" chemicals used at the facility that are not required to be reported by SARA Title III but are known to be emitted from the facility. The appendix is not all inclusive but provides information on the major chemical emissions to the air, water, or land from processes at the facilities.

\subsection{AIRBORNE DISCHARGES AND AIR AND METEOROLOGICAL MONITORING}

This subsection deseribes the air-related environmental programs at PORTS. These programs are the Emission Monitoring and Control Programs, the Ambient Air Monitoring Program, and the Meteorological (Weather) Monitoring Program (described in Subsects. 2.1.1, 2.1.2, and 2.1.3 respectively). Each subsection is divided into two units: the first describes each program; the second summarizes and interprets the data collected by that program in 1989. Subsection 2.1.4 summarizes the overall findings and conclusions in this area. A more detailed presentation of the data without discussion is located in Part 2 of this report. This subsection does not discuss the Direct Radiation Monitoring Prcgram or the calculation of doses to the general public. The Direct Radiation Monitoring Program is described and discussed separately in Subsect. 2.6. The potential dose to members of the public (including that from airborne discharges) is summarized and discussed in Sect. 3.

Table 2.1.1 lists all air permits that have been issued by the OEPA as of December 31, 1989. In addition, 75 sources are in a registration status in lieu of having been issued permits to operate. Another five applications for permits to operate were pending as of year's end. 
Table 2.1.1. Air permits issued by the Ohio Environmental Protection Agency

\begin{tabular}{lll}
\hline Number & Building & \multicolumn{1}{c}{ Description } \\
\hline B001 & X-600 & Coal-fired boiler (No. 1 - south) \\
B002 & X-600 & Coal-fired boiler (No. 2 - middle) \\
B003 & X-600 & Coal-fired boiler (No. 3 - north) \\
P010 & X-700 & $\begin{array}{l}\text { Solvent-contaminated water treatment system } \\
\text { (air stripper) }\end{array}$ \\
L005 & X-700 & Vapor degreaser \\
L002 & X-720 & Compressor shop vapor degreaser \\
L001 & X-720 & Motor shop vapor degreaser \\
F001 & X-73: & Refuse/asbestos handling \\
F002 & X-735 & Roads/parking areas landfill \\
\hline
\end{tabular}

\subsubsection{Emission Monitoring}

This subsection briefly describes major air emission sources and associated emission control and emission-monitoring systems at PORTS. A summary of the total annual emissions and a discussion of the significance of the data and of any anomalies follow.

\subsubsection{Cescription of emission sources and monituring systems}

Three emission-monitoring systems are in place at PORTS, each of which monitors a different class of airborne emissions. In addition, several types of airborne emissions are calculated from process data or purchasing records.

\section{Gaseous radionuclide and fluoride emissions}

Gaseous radionuclide and fluoride emissions from the purge cascade vents, the cold recovery and wet air evacuation vents, the X-345 high-assay sampling area (HASA) vent, the X-344 evacuation vent, and the $\mathrm{X}-333$ seal exhaust vent are sampled continuously by systems developed and built by the PORTS plant laboratory. Additional samplers for the five remaining seal exhaust vents are planned for 1990. Together these vents account for virtually all of the radionuclide and most of the fluoride emissions from PORTS. The "continuous vent samplers" draw a flow-proportional sample of the vent stream through two small alumina traps in series via an isokinetic probe. Routinely, the primary sample traps are replaced weekly, and the secondary traps are replaced quarterly.
Radionuclides known to be present are the isotopes ${ }^{234} \mathrm{U},{ }^{235} \mathrm{U}$, and ${ }^{238} \mathrm{U}$, the two trace impurities from recycled uranium ${ }^{236} \mathrm{U}$ and ${ }^{99} \mathrm{Tc}$, and equilibrium concentrations of short-lived uranium daughters. Fluorides are present as various reactive fluoride gases including $\mathrm{UF}_{6}$ and $\mathrm{HF}$. Alumina from the sampler is analyzed for total uranium, ${ }^{235} \mathrm{U}$, technetium, and total fluorides. The ${ }^{235} \mathrm{U} /$ total uranium ratio (i.e., the "assay") and process data are used to calculate the fractions of ${ }^{234} \mathrm{U}$ and ${ }^{236} \mathrm{U}$ in the emissions.

Because of their short half-lives, uranium daughter emissions cannot be reliably measured in weekly samples and are assumed to be in equilibrium with their parent nuclides. The uranium daughters included in the equilibrium calculations are ${ }^{234} \mathrm{Th}$, ${ }^{231} \mathrm{Th}$, and ${ }^{234 \mathrm{~m}} \mathrm{~Pa}$.

The purge cascades use gaseous diffusion to separate $\mathrm{UF}_{6}$ continuously from light gases (primarily air) that have leaked into or otherwise entered the process. The $\mathrm{UF}_{6}$ is ieturned to the main cascade, and the "lights" are passed through activated alumina traps to remove residual traces of $\mathrm{UF}_{6}$ and other pollutants before sampling and venting to the atmosphere. PORTS uses two purge cascades known as the side purge cascade and the top purge cascade. Both cascades are exhausted by dedicated air jets (i.e., T-jet and S-jet) or a standby air jet (i.e., E-jet) that can be valved in to replace temporarily or to supplement either of the regular air jets. All three jets are sampled by separate continuous samplers. The purge cascades account for the bulk of the routine radionuclide emissions from the cascade. 
The cold recovery and wet air evacuation systems are maintenance support systems in the X-330 and X-333 process buildings (see Fig. 2.1.1). Cold recovery systems are used to evacuate gases from cascade cells that must be opened for maintenance. Refrigerated "cold traps" are used to freeze out $\mathrm{UF}_{6}$, and noncondensable "lights" are passed through sodium fluoride traps and vented to atmosphere. After maintenance the wet air evacuation systems evacuate outside (i.e., "wet") air through alumina traps before the cell is returned to service. In X-330 both systems exhaust through a common roof vent with a single sampler. In X-333 the two systems have separate roof vents and samplers. Normally, these systems are minor emission sources, but a potential for unplanned releases exists.

Another emission source currently in the continuous vent sampler system is the X-345 HASA where cylinders of enriched $U_{6}$ are heated and sampled for quality control. To prevent sample mixing, the sampling manifold must be purged and evacuated after each set of samples. The evacuated gases are cold-trapped to recover residual uranium and vented through alumina traps. In addition, there is a suction device (i.e., a "gulper") behind the sample cylinders to collect any small releases during cylinder disconnects. The combined exhaust from both systems is sampled by a single continuous sampler. Routine emissions from the HASA are small, but because the system handles liquid $\mathrm{UF}_{6}$ of high assay, a potential exists for a significant unplanned release from this vent. The X-344 facility also heats cylinders of UF 6 either for sampling or for transfer between cylinders. Like the X-345 HASA, there is a combined vent for system evacuation and for a "gulper" that is monitored by a single continuous sampler.

The enrichment cascade is divided into six "control areas," each of which has its own control room and seal exhaust station. The seal exhaust stations exhaust "dry air" (i.e., completely dehumidified air as oppose $d$ to normal, or wet, air) from the cascade seal systems. The "dry air" passes through activated alumina traps, vacuum pumps, and oil traps (i.e., mist eliminators) before being vented. The seal exhaust station in X-333 (Area 1) was equipped with a continuous sampler on an experimental basis in early 1989. Present plans are to equip the other five stations with samplers in 1990.

In addition to the continuous samplers, other systems monitor the emissions from the cascade vents. Process control is based on real-time information provided by ionization-chamber-type instruments called "space recorders." Space recorders have limited sensitivity and cannot distinguish between uranium and technetium emissions, but they provide adequate qualitative information for operating purposes. The space recorders are checked (every $4 \mathrm{~h}$ on the purge cascades) by $5-\mathrm{L}$ grab samples that are analyzed on " priority basis. Grab samples provide only limited information, however, and some data suggest that the technetium concentrations in the grab samples may be biased high under some conditions. The 5- $\mathrm{L}$ samples are also less sensitive than the weekly samples, which represent several thousand liters of sampled vent gasus. The PORTS plant laboratory is currently developing a modification of the continuous sampler that would use a pair of gamma detectors to measure uranium accumulation in the traps in real time. Current major problems are background drift and a need to know the assay of the uranium emissions in advance.

\section{Criteria pollutant emissions}

PORTS operates numerous small sources of criteria (or "conventional") air pollutants. The three most significant are the coal-fired boilers at the X-600 steam plant, which supply the plant with 125-psi steam for process and building heat. Typically, only one or two boilers operate at a time depending on outside temperatures. The boilers are permitted by the Ohio Environmental Protection Agency (OEPA) with opacity, particulate, and sulfur dioxide $\left(\mathrm{SO}_{2}\right)$ limits. These permits also specify the required emission monitoring for these parameters, which is reported to the OEPA on a quarterly basis.

Opacity and particulate emissions are controlled by electrostatic precipitators on each of the boilers. Opacity is measured continuously and recorded on 24 -h circular charts and monthly strip 
OANL-DWG 87-10130R

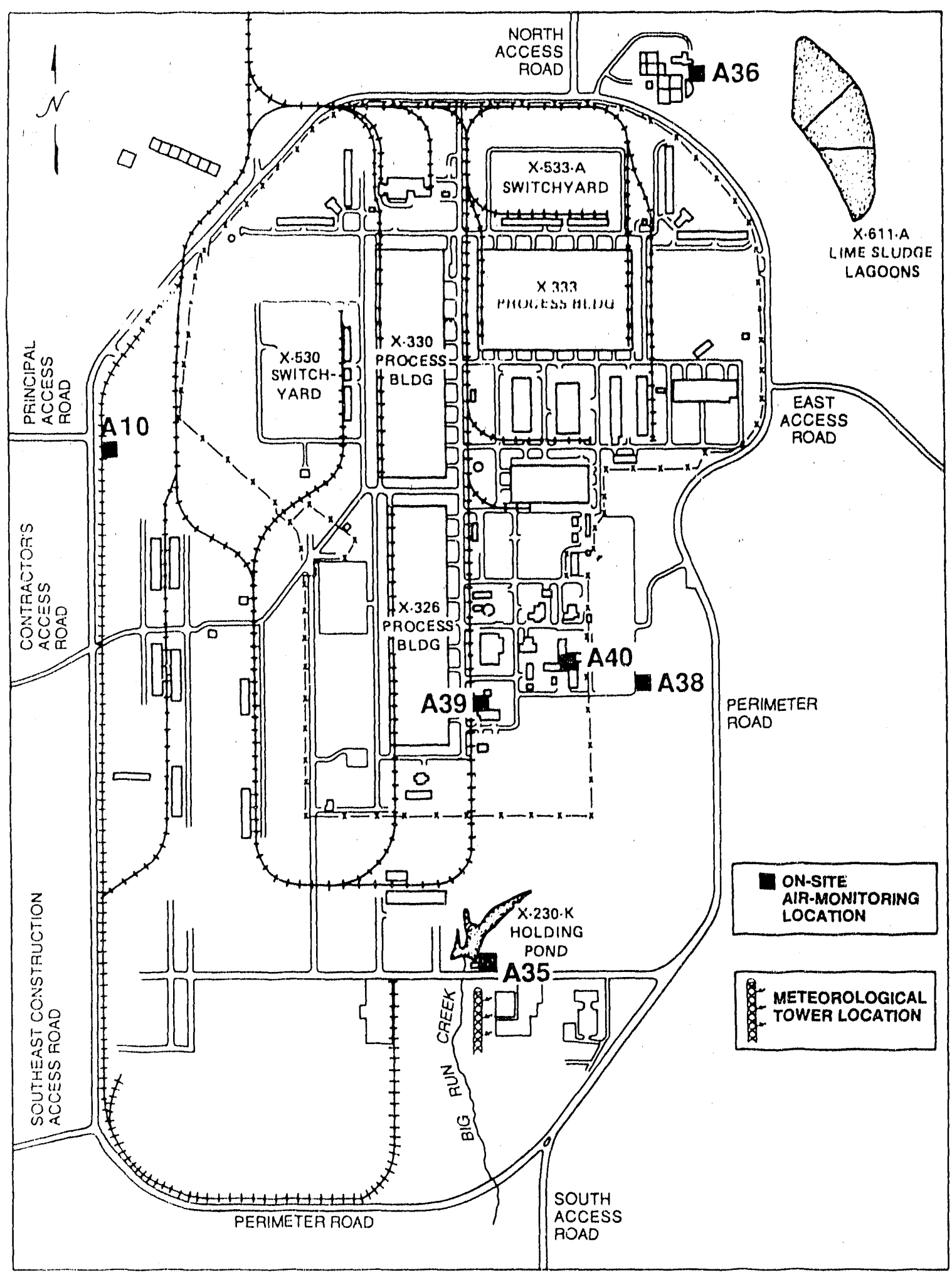

Fig. 2.1.1. PORTS on-site air-monitoring locations and meteorological tower. 
charts. Opacity is a measure of the darkness of a plume of smoke and is measured by shining a beam of light through the smoke and measuring the percentage of light that passes through. Environmental regulations specify a limit of $20 \%$ opacity, which is equivalent to a light haze. Opacity is also used as a daily indicator of particulate emissions; this requires time-consuming and difficult stack sampling to measure directly. Such stack sampling (and 100\% compliance with particulate limitations) is required to renew the OEPA operating permits every 3 years. Boiler 3 passed stack testing in December 1989; boilers 1 and 2 are scheduled for stack testing in 1990.

Sulfur dioxide emissions are calculated from coal analyses rather than direct measurement. All coal deliveries to the plant are sampled and analyzed for calorific value (Btu/pound), ash content, and sulfur content. The quarterly reports to the OEPA summarize the amount of coal purchased, the average results of the coal analyses, and the calculated rate of $\mathrm{SO}_{2}$ emission along with the opacity data.

In addition to $\mathrm{SO}_{2}$ emissions from the $\mathrm{X}-600$ steam plant, PORTS also purchases liquid $\mathrm{SO}_{2}$ in 1 -ton cylinders for treatment of the plant cooling water blowdown at the X-616 liquid effluent treatment facility. The $\mathrm{SO}_{2}$ is injected into the incoming cooling water and is consumed by a reaction that reduces hexavalent chromium (i.e., a corrosion inhibitor in the cooling water) to the less toxic trivalent form. No odor of $\mathrm{SO}_{2}$ is normally present around the X-616 facility; this indicates that essentially all of the $\mathrm{SO}_{2}$ is consumed. In the absence of hard data, however, PORTS assumes that $5 \%$ of the $\mathrm{SO}_{2}$ escapes to the atmosphere.

\section{Other airborne emissions}

Another air pollutant emitted from PORTS is asbestos from the renovation or demolition of plant facilities. Asbestos emissions are controiled to the level of "no visible emissions" by a system of work practices supervised by the Industrial Hygiene and Health Physics Department. The amount of asbestos removed and disposed of is reported to the OEPA on a quarterly basis, but no practical way exists to reliably estimate the amount emitted to the air.
PORTS purchases liquid chlorine $\left(\mathrm{Cl}_{2}\right)$ for water treatment. Chlorine is used to disinfect incoming well water, outgoing treated sewage, and RCW. Very little, if any, chlorine is lost to the air from the incoming water or the sewage, but the $\mathrm{RCW}$ is effectively air-stripped of chlorine in the cooling towers. PORTS assumes that all chlorine fed to the RCW system is lost to the air and that all chlorine used elsewhere is entirely consumed.

The gaseous diffusion process generates a large amount of heat that must be removed by the plant cooling system. Because water is both a nuclear moderator and chemically reactive with $\mathrm{UF}_{6}$, it would be unsafe to cool the process directly with the RCW, so PORTS uses a two-stage system with Freon 114 as the intermediate coolant. Inevitably, given the size of the cooling system, a considerable amount of the Freon leaks into the cascade, the RCW, or Jirectly into the air. A "thermal degrader" destroys the Freon that enters the cascade, but Freon emissions from the RCW system and building ventilation are difficult to control. Currently, estimates of total Freon emissions are based on monthly inventories.

PORTS uses both organic and inorganic solvents for cleaning purposes.

1,1,1-Trichloroethane (TCA) is used to "degrease" equipment prior to maintenance or repair. Freon 113 is used for final cleaning of parts (e.g., piping, gaskets, etc.) that may come in contact with $\mathrm{UF}_{6}$ process gas. Both solvents are usually assumed to evaporate completely eventually. TCA is recycled until it evaporates. Small amounts of other hydrocarbon solvents are also used, and these have been lumped together and are assumed to evaporate completely also. The inorganic solvents used at PORTS (i.e., primarily nitric and citric acids) are not volatile and do not contribute significantly to air emissions.

\subsubsection{Emission data summary}

\section{9 radionuclide emissions}

Routine radionuclide emissions from PORTS (Table 2.1.2) had no significant impact on public health or the environment, and there were no unplanned releases of radionuclides to the environment in 1989. Uranium emissions increased over 1988 levels reflecting, in part, increased 
Table 2.1.2. 1989 PORTS total air emissions

\begin{tabular}{lll}
\hline Pollutant & \multicolumn{1}{c}{$\mathrm{kg} /$ year } & $\mathrm{Ci} /$ year \\
\hline${ }^{234} \mathrm{U}$ & 0.00167 & 0.103 \\
${ }^{235} \mathrm{U}$ & 1.84 & 0.00393 \\
${ }^{236} \mathrm{U}$ & 0.00158 & 0.0000999 \\
${ }^{238} \mathrm{U}$ & 6.50 & 0.00216 \\
${ }^{99} \mathrm{Tc}$ & 0.00561 & 0.0960 \\
Uranium & $1.01 \times 10^{-10}$ & 0.00823 \\
$\quad$ daughters & & \\
\hline
\end{tabular}

operations and, in part, the expanded emissionmonitoring system. Technetium emissions decreased by almost $41 \%$ to the lowest level recorded since technetium was identified in PORTS air emissions in 1977.

Approximately $94.5 \%$ of the uranium activity and $40 \%$ of the uranium mass was released from the top and side purges in the X-326 process building. Another $57 \%$ of the uranium mass (but less than $4 \%$ of the total uranium activity) was released from the $\mathrm{X}-333$ process building vents. Surprisingly, a large part of the mass emissions from the X-333 building were measured in the seal exhaust station vent. This vent was first incorporated into the emission-monitoring network on an experimental basis in 1989 and was expected (based on process knowledge) to prove a small emissions source. In light of the unexpected results, the seal exhaust station vents in the other process buildings are being added to the monitoring network in 1990.

Typically, technetium emissions are marked by very low normal emissions; one or two comparatively large spikes or bubbles from the top and side purge cascades provide the majority of the annual emissions. In 1989, approximately $40 \%$ of the total technetium emissions were released from the purge cascades in January; another 13\%, in June. Over the year the purge cascades released over $88 \%$ of the total technetium emissions.

\section{9 steam plant emissions}

The X-600 steam plant achieved annual permit compliance rates of $99.97 \%$ for opacity and $100 \%$ for $\mathrm{SO}_{2}$ based on the limits in its Permits-ToOperate issued by the OEPA. In April 1990
PORTS was informed by the OEPA that the regulatory limits for its steam plant had been lowered from $7.0 \mathrm{lb} / \mathrm{M}$ Btu to $6.16 \mathrm{lb} / \mathrm{M} \mathrm{Btu}$, a $12 \%$ reduction, effective in November 1989 . The PORTS steam plant did exceed this lower limit in both November $(6.95 \mathrm{lb} / \mathrm{M} \mathrm{Btu})$ and December $(6.24 \mathrm{lb} / \mathrm{M} \mathrm{Btu})$. As a result, PORTS is now pursuing new arrangements with its coal suppliers to ensure deliveries of lower sulfur coal for its steam plant.

A compliance rate for particulate (i.e., fly ash) limits does not have any real meaning because particulate emissions are measured only once every 3 years during renewal of operating permits from the OEPA. Boiler 3 was tested for particulate emissions and was found to be well within permit limits in December. The other boilers are scheduled to be tested in early 1990 .

Causes for opacity violations in 1989 were mechanical failures and coal-handling problems that interrupted smooth boiler operations; an electrical power failure and a power loss while switching supplies; and, on two occasions, very high operating rates during mandatory annual safety testing of the boilers. Under Ohio law opacity exceedances that are caused by malfunctions and are reported at the time to the OEPA are not legally "violations." PORTS reports all opacity exceedances due to malfunctions to the OEPA but normally counts them in the compliance rate listed above anyway if the opacity limit was exceeded. In December a technical "exceedance" occurred that is not included in the compliance rate. A monitor light burned out during the early morning on boiler 3 . The monitor light is located in a locked enclosure on the smokestack, and the key had been misplaced. As a result, the light could not be replaced, and the boiler was not monitored until over $6 \mathrm{~h}$ later. This is legally an opacity "exceedance" since the opacity regulation requires continuous monitoring during operation. However, because it was reported to the OEPA within $24 \mathrm{~h}$, it was not an actual violation of state law. Because no visible smoke was generated during the incident and it is not an actual violation, PORTS has excluded it from the compliance rate cited above. 


\section{Long-term trends}

Figures 2.1.2 through 2.1.4 show the PORTS emission levels over the last 5 years for uranium and technetium. Uranium daughter emissions have ranged from $0.028 \mathrm{Ci} /$ year to $0.002 \mathrm{Ci} /$ year since 1985 and have never made a significant impact on either the environment or public health. Figures 2.1.2 and 2.1.3 show uranium emissions in curies

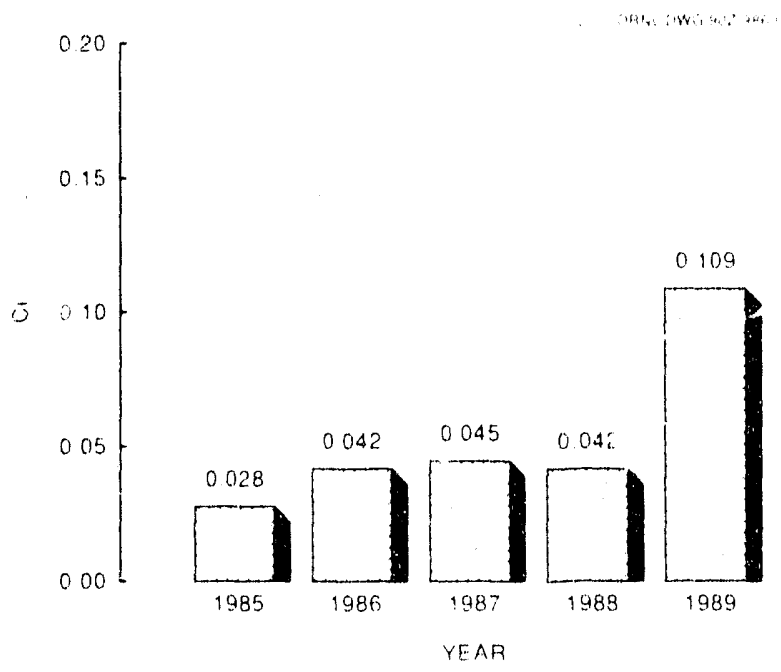

Fig. 2.1.2. Total curies of uranium discharged to the air, 1985-1989.

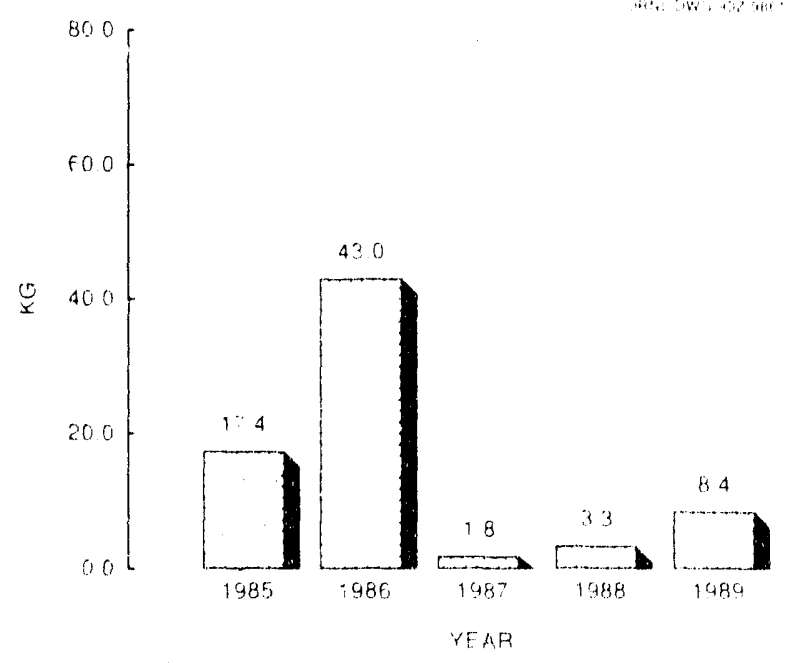

Fig. 2.1.3 Total kilograms of uraniu "lischarged to the air, 1985-1989.

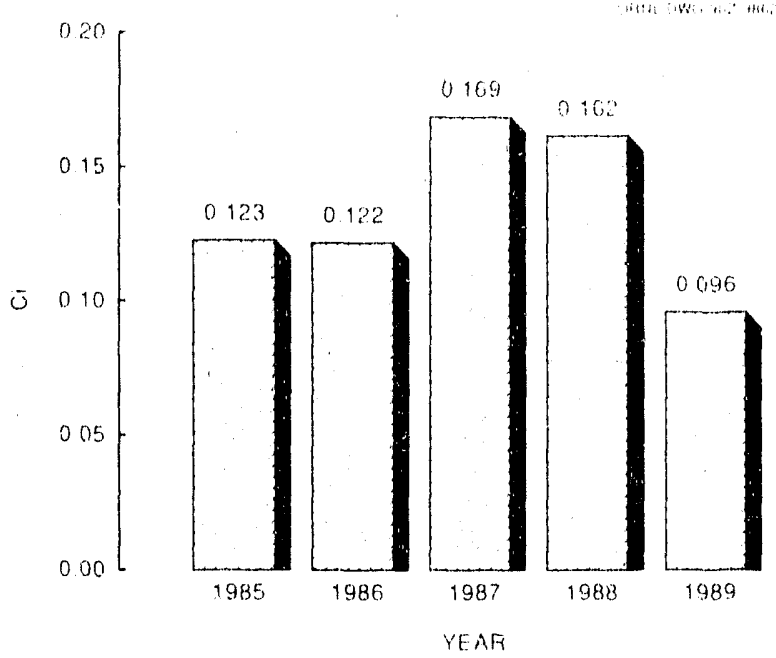

Fig. 2.1.4. Total curies of technetium discharged to the air, 1985-1989.

(i.e., a measure of radioactivity) and kilograms (i.e., a measure of mass) respectively. Both figures are included because uranium is a mixture of three different isotopes $\left({ }^{236} \mathrm{U}\right.$ is a trace contaminant) with widely varying specific activities (i.e., curies per kilogram). At PORTS $1 \mathrm{Ci}$ of uranium can weigh from $9 \mathrm{~kg}$ to almost $3000 \mathrm{~kg}$. Figure 2.1 .4 shows technetium emissions in curies. Because the only isotope of technetium present at PORTS is ${ }^{99} \mathrm{Tc}$, a figure showing mass emissions would only duplicate Fig. 2.1.4.

\subsubsection{Ambient Air Monitoring}

This section describes the ambient-airmonitoring network maintairled by PORTS and presents a summary of the ambient air data at each of the air-monitoring stations. Detailed ambient-air-monitoring data are presented in Tables 2.1.5 and 2.1.6 of Part 2.

\subsubsection{Description of ambient-air-monitoring system}

Since 1964 PORTS has maintained a network of on-site and off-site permanent stations to collect ambient air samples continuously. Figures 2.1.1 and 2.1.5 show the location of the stations in the expanded network. Each station consists of a Teflon particulate filter, a chemically treated filter 


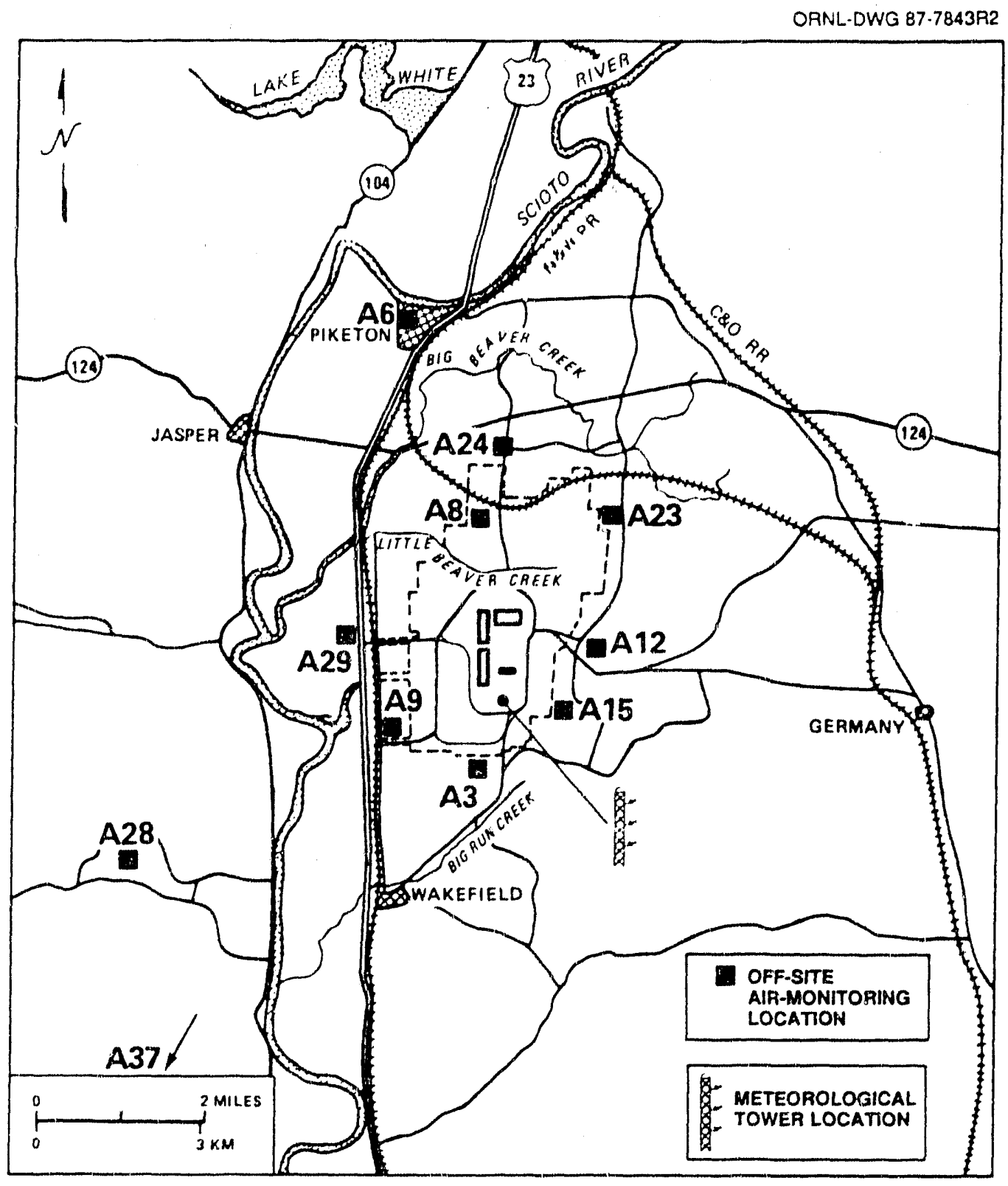

Fig. 2.1.5. Ofi-site air-moaitoring locktions at PORTS.

for gaseous fluorides, a flow controller and gas meter, and a vacuum pump.

The particulate filters are collected monthly and counted for gross alpha and beta-gamma loading. If the gross counts exceed plantestablished limits (i.e., $100 \mathrm{dpm}$ alpha or $200 \mathrm{dpm}$ beta-gamma), the filters are analyzed for specific radionuclides. To date, air samples have never exceeded these limits. All filters that show any positive counts are saved for an annual composite analysis for specific radionuclides to verify that no unexpected radionuclides are being discharged to the air. The treated filters are collected weekly and analyzed for total fluorides. To prevent cross contamination and to simplify handling, the Teflon and treated filters are mounted in separate Teflon filter holders. 


\subsubsection{Ambient air data summary}

Tables 2.1.3 and 2.1.4 present summaries of the airborne gross alpha and beta-gamma radiation concentrations on and around PORTS in 1989.

The tables give the number of samples; the minimum, maximum, and average concentrations; and the standard deviation of the concentrations at each air station. Note that the standard deviations are on the same order of magnitude as the average concentrations. This reflects large variations in concentrations at each station and indicates that small differences in the average concentrations are probably not significant. It also reflects the large predominance of natural background in ambient radioactivity around the PORTS site.

In Table 2.1.3 several of the minimum concentrations listed are negative numbers. Although PORTS does not show actual concentrations of less than zero, this is a consequence of the extremely low airborne alpha concentrations surrounding PORTS. All measuring instruments have some limit of precision (or error) within which the instrument readings waver higher or lower randomly (i.e., better instruments having tighter limits). Usually, these limits are insignificant compared with the parameter being measured and are ignored. The airborne alpha concentrations surrounding PORTS (and most other places) are so close to zero, however, that this imprecision is not insignificant and results in occasional negative "concentration." In such cases, the average of several measurements is more realistic than the individual measurement since the random errors tend to cancel each other.

Typically, station A12, located on the east side of the plant, shows the highest ambient concentration of both alpha and beta-gamma activity by only a slight amount. In 1989 local variation was even greater than usual and caused

Table 2.1.3. 1989 gross alpha concentrations in ambient air

\begin{tabular}{|c|c|c|c|c|c|}
\hline \multirow{2}{*}{ Location } & \multirow{2}{*}{$\begin{array}{l}\text { No. of } \\
\text { samples }\end{array}$} & \multicolumn{3}{|c|}{$\begin{array}{l}\text { Concentration } \\
\left(\mathrm{pCi} / \mathrm{m}^{3}\right)\end{array}$} & \multirow{2}{*}{$\begin{array}{l}\text { Standard } \\
\text { deviation }\end{array}$} \\
\hline & & $\operatorname{Max}$ & Min & Av & \\
\hline \multicolumn{6}{|c|}{ On-site } \\
\hline A 10 & 12 & 0.006 & 0.001 & 0.003 & 0.001 \\
\hline A35 & 11 & 0.008 & 0.000 & 0.003 & 0.002 \\
\hline A 36 & 11 & 0.009 & -0.002 & 0.003 & 0.003 \\
\hline A 38 & 12 & 0.022 & 0.000 & 0.005 & 0.005 \\
\hline A 39 & 12 & 0.040 & 0.006 & 0.016 & 0.010 \\
\hline A40 & 12 & 0.046 & 0.002 & 0.011 & 0.012 \\
\hline \multicolumn{6}{|c|}{ Property line } \\
\hline A3 & 12 & 0.013 & -0.002 & 0.005 & 0.004 \\
\hline A8 & 11 & 0.007 & -0.001 & 0.003 & 0.002 \\
\hline A9 & 12 & 0.011 & 0.001 & 0.003 & 0.003 \\
\hline A 12 & 12 & 0.012 & 0.000 & 0.005 & 0.003 \\
\hline A.15 & 12 & 0.007 & 0.001 & 0.003 & 0.002 \\
\hline A 23 & 12 & 0.013 & -0.003 & 0.003 & 0.004 \\
\hline A24 & 3 & 0.004 & -0.001 & 0.001 & 0.002 \\
\hline A 29 & 12 & 0.005 & -0.000 & 0.002 & 0.002 \\
\hline \multicolumn{6}{|c|}{ Off-site } \\
\hline A6 & 12 & 0.010 & -0.001 & 0.003 & 0.003 \\
\hline A28 & 13 & 0.006 & 0.002 & 0.003 & 0.002 \\
\hline A37 & 11 & 0.007 & $-0.000^{a}$ & 0.004 & 0.003 \\
\hline
\end{tabular}

${ }^{a} \mathrm{~A}-0.000$ number represents a rounding off of a very small measured concentration. 
Table 2.1.4. 1989 gross beta-gamma concentrations in ambient air

\begin{tabular}{|c|c|c|c|c|c|}
\hline \multirow{2}{*}{ Location } & \multirow{2}{*}{$\begin{array}{l}\text { No. of } \\
\text { samples }\end{array}$} & \multicolumn{3}{|c|}{$\begin{array}{l}\text { Concentration } \\
\left(\mathrm{pCi} / \mathrm{m}^{3}\right)\end{array}$} & \multirow{2}{*}{$\begin{array}{l}\text { Standard } \\
\text { deviation }\end{array}$} \\
\hline & & $\operatorname{Max}$ & Min & $A v$ & \\
\hline \multicolumn{6}{|c|}{ Off-site } \\
\hline A 10 & 12 & 0.044 & 0.014 & 0.023 & 0.008 \\
\hline A 35 & 11 & 0.050 & 0.017 & 0.025 & 0.009 \\
\hline A.36 & 11 & 0.040 & 0.001 & 0.016 & 0.012 \\
\hline A 38 & 12 & 0.154 & 0.012 & 0.039 & 0.039 \\
\hline A39 & 12 & 0.196 & 0.023 & 0.051 & 0.046 \\
\hline A40 & 12 & 0.132 & 0.008 & 0.043 & 0.031 \\
\hline \multicolumn{6}{|c|}{ Property line } \\
\hline A. 3 & 12 & 0.089 & 0.006 & 0.035 & 0.020 \\
\hline A8 & 11 & 0.051 & 0.006 & 0.028 & 0.012 \\
\hline A9 & 12 & 0.185 & 0.009 & 0.038 & 0.045 \\
\hline $\mathrm{A} 12$ & 12 & 0.039 & 0.007 & 0.024 & 0.009 \\
\hline A 15 & 12 & 0.036 & 0.009 & 0.020 & 0.007 \\
\hline A23 & 12 & 0.052 & 0.013 & 0.025 & 0.012 \\
\hline A24 & 3 & 0.036 & 0.021 & 0.027 & 0.007 \\
\hline A29 & 12 & 0.092 & 0.004 & 0.028 & 0.022 \\
\hline \multicolumn{6}{|c|}{ Off-site } \\
\hline A6 & 12 & 0.037 & -0.001 & 0.017 & 0.012 \\
\hline A28 & 13 & 0.052 & 0.009 & 0.030 & 0.013 \\
\hline A 37 & 11 & 0.067 & 0.008 & 0.036 & 0.018 \\
\hline
\end{tabular}

pe culiar superricial patterns in the averages. The highest measured alpha concentrations (i.e., 0.005 $\mathrm{pCi} / \mathrm{m}^{3}$ ) were at both $\mathrm{A} 12$ and $\mathrm{A} 3$, located south, and normally upwind, of the plant. The measured beta-gamma concentrations, on the other hand, peaked at stations $\mathrm{A} 9$ ( i.e., $0.038 \mathrm{pCi} / \mathrm{m}^{3}$, southwest corner of the plant site) and $\mathrm{A} 3$ (i.e., $0.035 \mathrm{pCi} / \mathrm{m}^{3}$ ), while station $\mathrm{A} 12$ had the second lowest annual average concentration recorded (i.e., $0.024 \mathrm{pCi} / \mathrm{m}^{3}$ ). Finally, background station $\mathrm{A} 37$, located on Mt. Hope Road approximately $20.9 \mathrm{~km}$ (13 miles) southwest (the prevailing upwind direction) of the plant, recorded average concentrations of both alpha and beta-gamma activity that were higher than most of the concentrations recorded near the plant (i.e., 0.004 $\mathrm{pCi} / \mathrm{m}^{3}$ and $0.036 \mathrm{pCi} / \mathrm{m}^{3}$ respectively). In reality, the different stations were not significantly different from each other, and the "peculiar" patterns are essentially meaningless.

The data in Tables 2.1.3 and 2.1.4 are "gross" concentrations; these include natural radionuclides as well as emissions from PORTS and operations at other facilities including coal-fired power plants. PORTS calculates "net" concentrations due only to its own emissions by subtracting the average background concentration from the highest average concentration measured around the DOE property line. The background concentration is measured at station A37 approximately $20.9 \mathrm{~km}$ ( 13 miles) from the plant near the community of Otway. In 1989 the net average alpha concentration was $0.0011 \mathrm{pCi} / \mathrm{m}^{3}$ above background; the net average beta-gamma concentration, $0.002 \mathrm{pCi} / \mathrm{m}^{3}$. A consequence of the low ambient concentrations is that it is difficult, if not impossible, to actually analyze for specific radionuclides (e.g., uranium, technetium, etc.). PORTS therefore assumes that all the net alpha concentration is from uranium emissions from PORTS and that all the net betagamma concentration is from technetium and uranium daughters in the same proportion as the measured emissions. 


\section{9 ambient gross fluorides}

Table 2.1 .5 presents a summary of the weekly ambient-fluoride-sampling program in the same format as the previous tables. In this table the state ambient air standards from Kentucky and Tennessee are included for comparison only. Although these standards are not legally binding on an Ohio facility, neither the state of Ohio nor the federal EPA has issued standards for ambient gaseous fluorides. There is wide disagreement between states that have issued ambient fluoride standards: Kentucky has set a primary (i.e., public health) standard of $400 \mu \mathrm{g} / \mathrm{m}^{3}$ and a secondary (i.e., public welfare) standard of $0.8 \mu \mathrm{g} / \mathrm{m}^{3}$; Tennessee has set both primary and secondary standards at $1.6 \mu \mathrm{g} / \mathrm{m}^{3}$; and Montana and Idaho do not use airborne concentrations at all (standards are based on fluoride concentrations in local vegetation instead). PORTS also measures vegetation fluoride levels (Subsect. 2.4.5); this may give a more accurate idea of the environmental impact of plant fluoride emissions.

Table 2.1.5 presents a summary of all of the fluoride samples collected during 1989 . The results are consistently lower than the results obtained during 1988 (by two- to three-fold) except station A39. All of the property-line and off-site stations had annual average concentrations below the Kentucky and Tennessee standards. Most of the stations exceeded the Kentucky secondary standard (the tightest in the nation) at least once, and four of the stations exceeded the Tennessee standards at least once. Ironi vally, one of these four was the background station A37. Two other stations that "exceeded" the Tennessee standard at least once, and had the two highest average concentrations, were $\mathrm{A} 39$ and $\mathrm{A} 40$. These two stations are located within the plant area and very near (A39 is directly across the street) to the X-326 process building, the naajor source of fluoride emissions.

Table 2.1.5. 1989 total fluoride concentrations (as HF) in ambient air

\begin{tabular}{|c|c|c|c|c|c|c|}
\hline \multirow{2}{*}{ Location } & \multirow{2}{*}{$\begin{array}{l}\text { No. of } \\
\text { samples }\end{array}$} & \multicolumn{3}{|c|}{$\begin{array}{l}\text { Concentration } \\
\left(\mu \mathrm{g} / \mathrm{m}^{3}\right)\end{array}$} & \multirow{2}{*}{$\begin{array}{l}\text { Standard } \\
\text { deviation }\end{array}$} & \multirow{2}{*}{$\begin{array}{c}\text { Ky. Tenn. } \\
\text { standards } \\
\left(\mu \mathrm{g} / \mathrm{m}^{3}\right)\end{array}$} \\
\hline & & $\operatorname{Max}$ & Min & Av & & \\
\hline \multicolumn{7}{|c|}{ Off-site } \\
\hline A 10 & 43 & 0.67 & 0.03 & 0.21 & 0.14 & $0.8 / 1.6$ \\
\hline A 35 & 41 & 0.97 & 0.05 & 0.24 & 0.20 & \\
\hline A 36 & 46 & 0.79 & 0.00 & 0.27 & 0.17 & \\
\hline A 38 & 45 & 1.02 & 0.05 & 0.35 & 0.21 & \\
\hline A39 & 42 & 3.75 & 0.06 & 1.00 & 0.90 & \\
\hline A40 & 45 & 1.64 & 0.02 & 0.60 & 0.34 & \\
\hline \multicolumn{7}{|c|}{ Property line } \\
\hline A3 & 42 & 2.31 & 0.06 & 0.43 & 0.39 & $0.8 / 1.6$ \\
\hline A8 & 47 & 0.99 & 0.04 & 0.27 & 0.20 & \\
\hline A9 & 48 & 0.72 & 0.05 & 0.21 & 0.13 & \\
\hline$A 12$ & 43 & 1.03 & 0.11 & 0.38 & 0.22 & \\
\hline A 15 & 44 & 0.46 & 0.05 & 0.19 & 0.10 & \\
\hline A23 & 46 & 1.29 & 0.05 & 0.25 & 0.22 & \\
\hline A24 & 15 & 0.82 & 0.03 & 0.22 & 0.21 & \\
\hline A29 & 47 & 0.77 & 0.00 & 0.21 & 0.14 & \\
\hline \multicolumn{7}{|c|}{ Off-site } \\
\hline A6 & 47 & 0.56 & 0.01 & 0.23 & 0.14 & \\
\hline A 28 & 48 & 1.04 & 0.00 & 0.28 & 0.20 & \\
\hline A 37 & 43 & 2.48 & 0.08 & 0.37 & 0.39 & \\
\hline
\end{tabular}


(Ambient air quality standards are defined as applying in areas "accessible to the general public" and hence would not affect A39, A40, or other on-site stations.) The remaining "high maximum" station was A3 (south); also recorded here was the highest off-site annual average fluoride concentration of $0.43 \mu \mathrm{g} / \mathrm{m}^{3}$. This station was followed by $\mathrm{A} 12$ (i.e., $0.38 \mu \mathrm{g} / \mathrm{m}^{3}$ ) and $\mathrm{A} 37$.. (i.e., $0.37 \mu \mathrm{g} / \mathrm{m}^{3}$ ).

As in the case of ambient radionuclides, the off-site stations with the highest average concentrations are A3 (i.e., $0.43 \mu \mathrm{g} / \mathrm{m}^{3}$ ), $\mathrm{A} 12$ (i.e., $0.38 \mu \mathrm{g} / \mathrm{m}^{3}$ ), and $\mathrm{A} 37$ (i.e., $0.37 \mu \mathrm{g} / \mathrm{m}^{3}$ ); the standard deviations of the individual stations approach the annual average concentrations and exceed the standard deviation of the average concentrations. Consequently, the differences between the individual property-line stations is probably not significant.

The general decrease in ambient fluoride concentrations between 1988 and 1989 cannot be explained by any changes in PORTS operations. The most probable explanation for the change is a major fluoride emission source operating somewhere southwest (possibly far southwest) of PORTS during 1987. However, PORTS is aware of no information indicating what this source might be.

\section{Long-term trends}

Figures 2.1.6 thorough 2.1.8 show graphically the net ambient concentrations of alpha and beta-

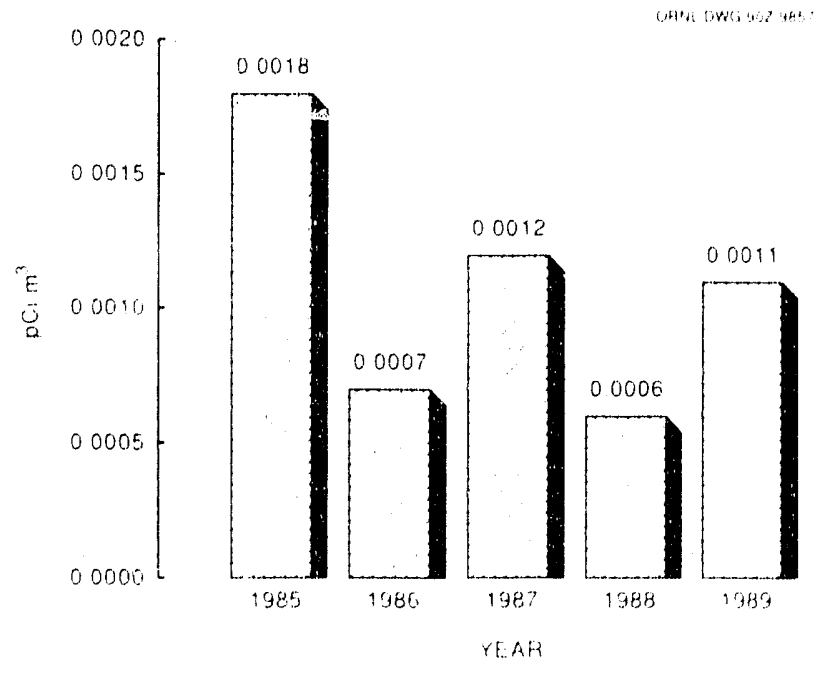

Fig. 2.1.6. Net airborne alpha concentrations, 1985-1989.

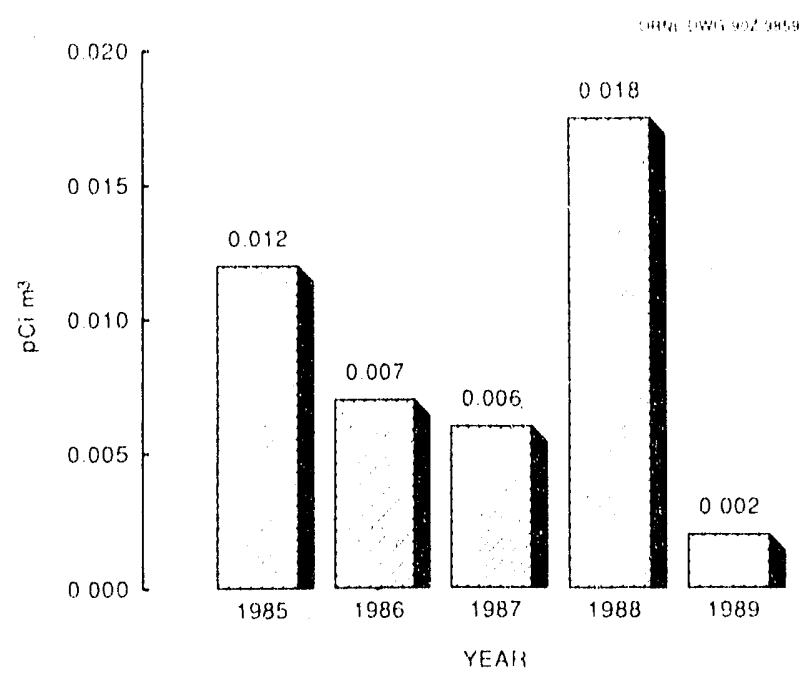

Fig. 2.1.7. Net airborne beta-gamma concentrations, 1985-1989.

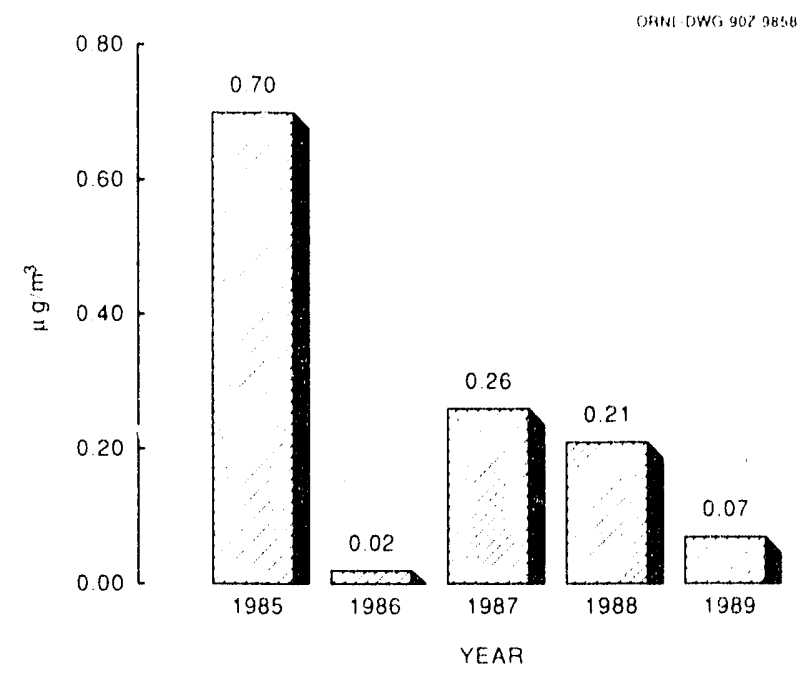

Fig. 2.1.8. Net gaseous nuoride concentrations, 1985-1989.

gamma radioactive particles and gaseous fluorides over the last 5 years around PORTS. The only definite conclusion that can be drawn from these figures is that PORTS' contributions to the ambient concentrations of these pollutants are consistently small. The figures show consistently small net concentrations (relative to the gross concentrations) with considerable random variation within this range. This reflects the fact that the net concentrations are approaching the size of the random-sampling and analytical errors inherent in any real-world sampling system; this is aggravated by the fact that the natural concentrations of these 
pollutants are much larger than the net concentrations and thus mask the numbers of actual interest.

\subsubsection{Meteorological Monitoring}

This subsection describes the meteorologicalmonitoring system in place at PORTS. This system is not part of the National Weather Service network and is designed solely to provide local meteorological data for predicting and evaluating environmental impacts of plant operations. This subsection presents only a graphic summary of wind data directly of interest to the dispersion modeling of pollutants released from the plant.

\subsubsection{Description of meteorological system}

PORTS maintains a comparatively simple meteorological-monitoring system in keeping with a comparatively simple local wind pattern. A single meteorological tower $(X-120)$ is located south of XT-801 (Fig. 2.1.5) and is equipped with instrument packages at the 10 - and $40-\mathrm{m}(32.8$ and 131.2-ft) levels. Air temperature, dew point, and wind speed and direction are measured at both levels. In addition, there is ground-level instrumentation for measuring solar radiation, barometric pressure, precipitation, and soil temperature at $0.30-$ and $0.61-\mathrm{m}(1-$ and $2-\mathrm{ft})$ depths.

A microprocessor located at the foot of the tower receives the analog data from each of the instruments and transmits two digital summaries via telephone lines to the Emergency Preparedness Department. The summaries consist of average and maximum wind speed, average and standard deviation of wind direction, averages of all temperatures, solar radiation, and precipitation over the last interval. One summary is sent at hourly intervals directly to a hardcopy terminal, which also serves as the control terminal of the microprocessor. The other summary is sent at 15-min intervals to a PDP-11 microprocessor and disk drive that operates independently of the rest of the system. The PDT converts wind direction data into compass headings and stability classes and calculates relatively humidity. The converted data are automatically retransmitted to the X-300 process control building and the Environmental Control Department and are averaged hourly for storage on floppy disks. The stored averages are used to generate a printed report at midnight of each day. At the end of the year, the monthly floppy disks are copied onto an annual magnetic tape that is used to generate the annual wind distribution and stability used for dispersion modeling and dose calculations.

\subsubsection{Meteorological data summary}

Figures 2.1.9 and 2.1.10 are standard graphic presentations of wind speed and direction data called "wind roses." The wind rose has 16 arms (representing the standard 16 wind directions), each of which is divided into six segments (representing six wind speed classes). The length of each segment represents the fraction of time wind was blowing from that direction at an average speed within that class. The key at the bottom of the figure gives the maximum speed of each wind speed class except the last. This class includes all wind speeds above the first five classes. The primary use of a wind rose is to provide an easily understood picture of the prevailing wind patterns at a site. The relative length of the arms gives a quick indication of which wind directions predominate and to what extent.

A joint frequency distribution is a set of tables listing the fraction of time in each of 576 wind classes (i.e., 16 directions $\times 6$ wind speeds $\times 6$ stability classes) instead of a wind rose's 96 classes. Atmospheric stability can be roughly described as the tendency of a wind to mix with and dilute a pollutant as opposed to merely transporting it downwind. The stability classes range from A (i.e., extremely unstable, maximum mixing) through $D$ (i.e., neutral) to $F$ (i.e., moderately stable, minimum mixing). Because of their complexity, joint frequency distributions are more useful for input to computer models than for data presentation to humans. Such computer models (known as "air dispersion models") are used to predict the downwind spread of pollutants in greater detail than an ambient-air-monitoring system can. One such model, AIRDOS-EPA, is required by the EPA to make the radiation dose estimates presented in Subsect. 3.1. 
OINL. UWG 9012155

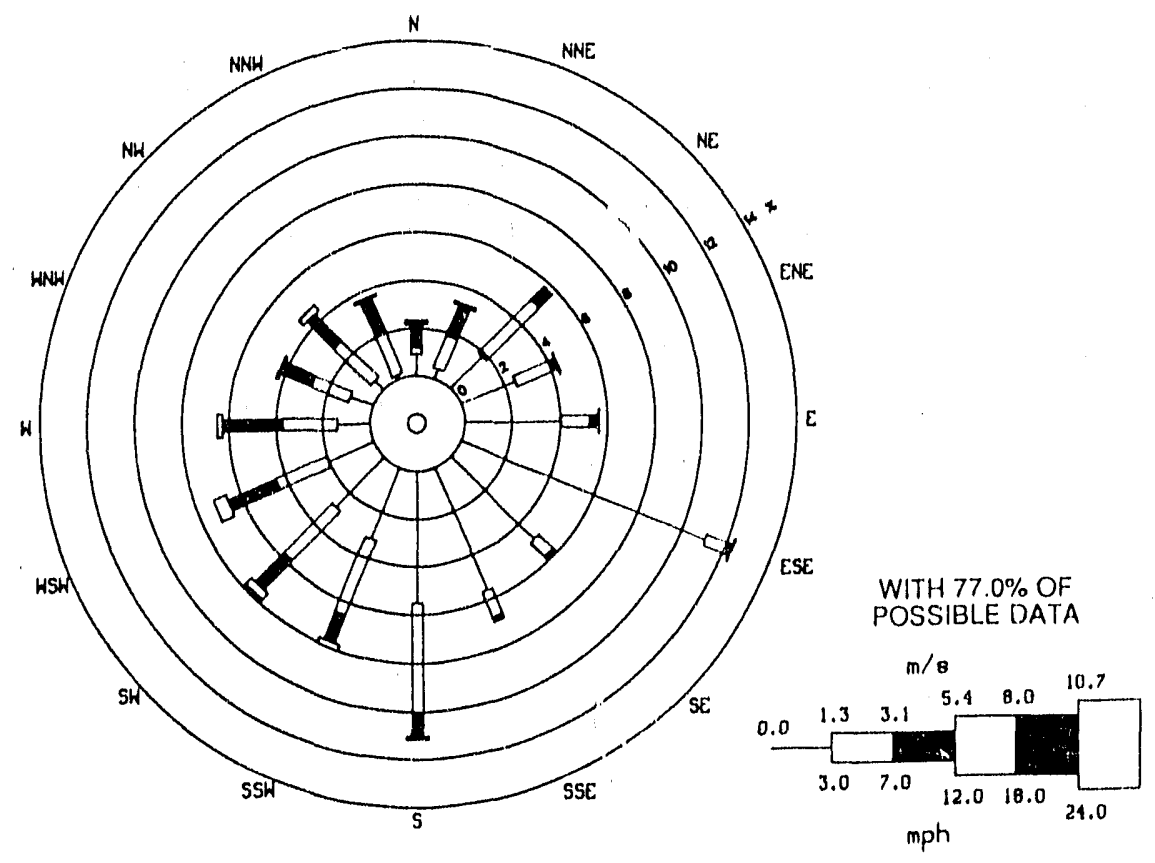

Fig. 2.1.9. 1989 annual wind rose at $10-\mathrm{m}$ level.

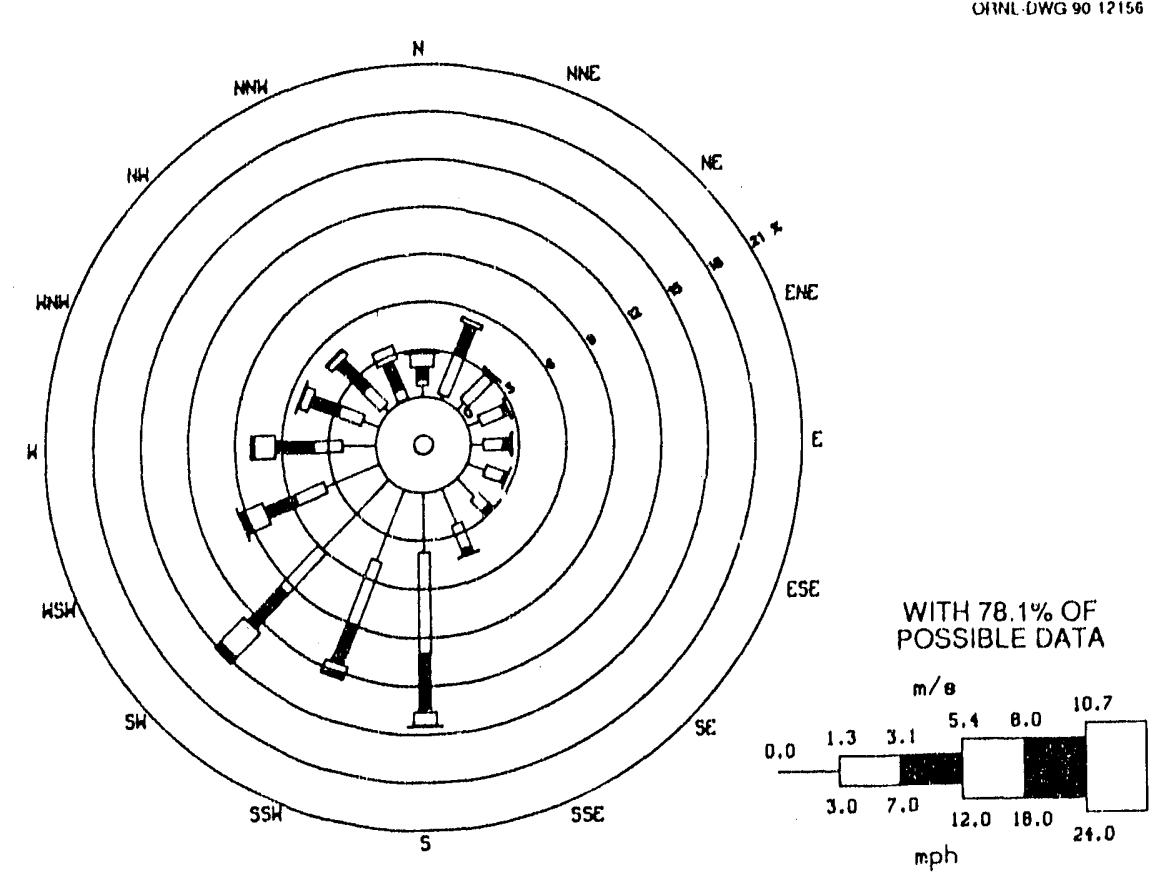

Fig. 2.1.10. 1989 annual wind rose at 40-m level. 


\subsubsection{Findings and Conclusions}

Emission control prograns at PORTS maintained emissions within acceptable limits. Environmental monitoring programs showed no significant impact on human health or the environment attributable to plant operations. Highlights of these programs are summarized below:

- There were no unplanned releases of radionuclides to the environment in 1989.

- Routine uranium emissions increased noticeably in 1989, while technetium emissions decreased. The total emissions (in curies) was almost identical to 1988 emissions and slightly lower than 1987 emissions.

- An experimental expansion of the radionuclide emission-monitoring network discovered unexpectedly high mass emissions of low-assay uranium from the $X-333$ seal exhaust station. Based on this discovery, the remaining seal exhaust stations will be added to the monitoring network in 1990.

- The PORTS steam plant achieved $99.97 \%$ "physical" compliance with the opacity limits and $100 \%$ compliance with the $\mathrm{SO}_{2}$ limits in its environmental operating permits. Legal compliance with the opacity limits is even higher because Ohio regulations exempt opacity exceedances that are due to malfunctions and are reported to the OEPA. Virtually all opacity exceedances at PORTS are due to malfunctions and are reported via the Oak Ridge Operations Center (OROC) to the OEPA as a matter of policy.

- Ambient monitoring of radionuclides consistantly showed no significant difference between concentrations around the plant site and net radionuclide concentrations much smaller than the natural background concentrations.

- Ambient monitoring of gaseous fluorides showed that ambient airborne concentrations around and upwind of the plant site had declined after having been elevated in 1987 and 1988. There was no significant difference in concentrations around the plant site demonstrating that plant emissions were having no significant impact on the surrounding environment.

\subsection{SURFACE WATER}

PORTS practices a progressive environmental strategy for water pollution control in compliance with requirements of the Clean Water Act (CWA). This strategy uses selection of modern pollution abatement technology followed by continual review of treatment facility performance to meet current regulations in the most costeffective manner. Sources of new pollution are first identified and characterized by in-house laboratory studies. A project feasibility study is performed; treatment alternatives are investigated; professional architectural/engineering firms are contracted for design purposes together with continual in-house reviews; and the proposed facility is constructed, operated, and monitored to ensure compliance with all applicable laws.

PORTS' existing collection and treatment facilities are discussed in this subsection together with facility improvements, new treatment facilities, and studies to improve performance. A variety of hazardous liquid wastes (i.e., uraniumcontaminated as well as noncontaminated) results from operations associated with $\mathrm{RCW}$, uranium recovery, and decontamination activities. In addition, a variety of conventional wastes exists such as domestic sewage, steam plant wastewaters, coai pile runoff, and once-through cooling water. Major wastewater sources and systems are given in Fig. 2.2.1.

\subsubsection{Surface Water Monitoring}

The quality of surface waters on the DOE Reservation is affected by wastewater discharges and by groundwater transport of contaminants from land disposal of waste. Although bedrock characteristics differ somewhat among the watersheds of these streams, the observed differences in water chemistry are attributed to different contaminant loadings rather than geologic variation. Water quality, radioactivity, and flow measurements are made at a number of stations operated by PORTS. Water samples were collected and analyzed at various intervals (i.e., weekly, 


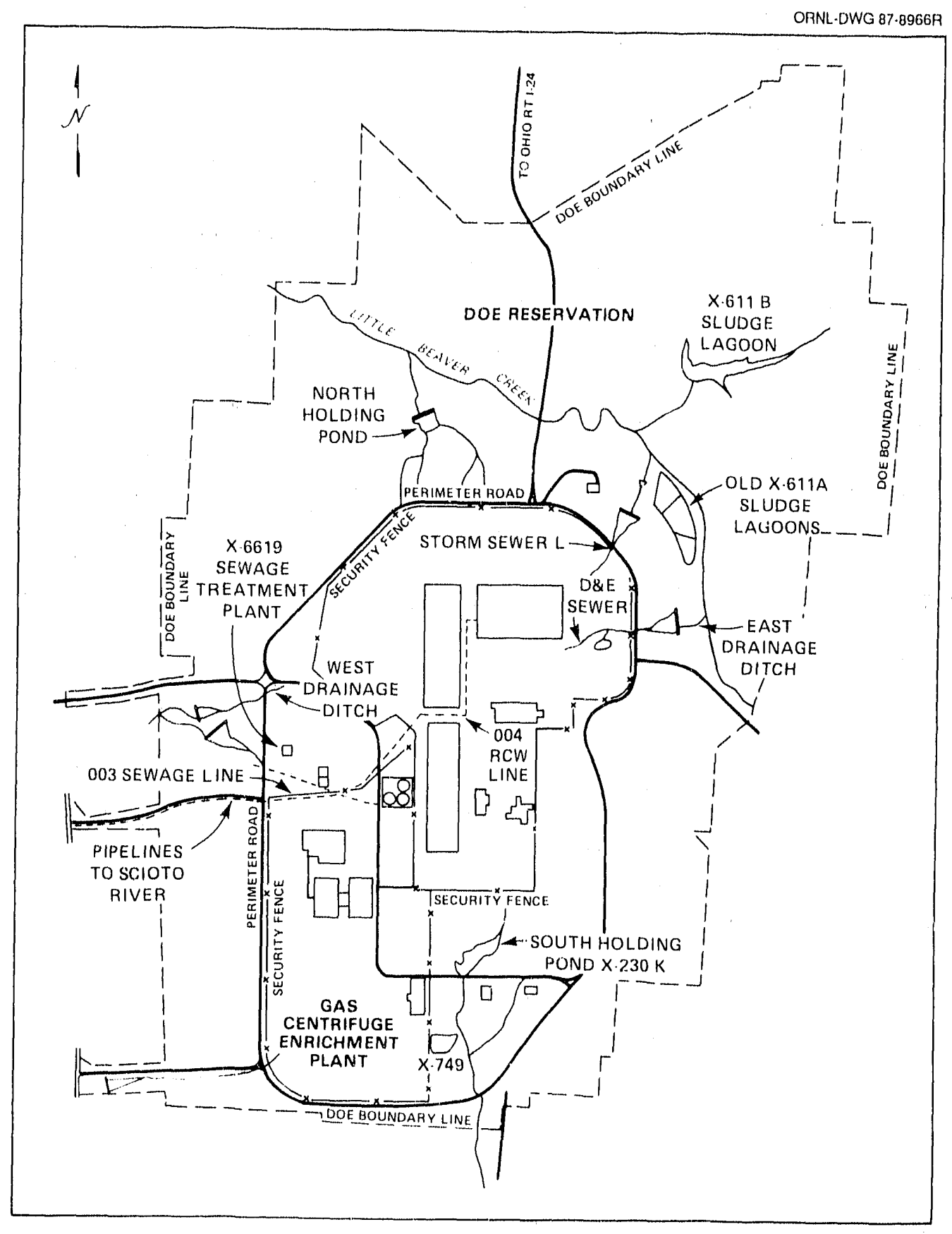

Fig. 2.2.1. Major wastewater sources and systems.

monthly, etc.) for radiological and nonradiological parameters. During 1989 PORTS conducted effluent-stream sampling in accordance vith the National Pollutant Discharge Elimination System (NPDES) permit. (Table 2.2.1 summarizes the parameters and limitations; the locations of the
NPDES stations are shown in Fig. 2.2.2.) In 1989 PORTS also conducted receiving-stream sampling, groundwater sampling and monitoring, and resident sanitary water sampling. The non-NPDES surface-water-monitoring points [i.e., routine water $(\mathrm{RW})$ ] are Little Beaver Creek upstream (RW12), 
Table 2.2.1. NPDES permit-summary effective April 8, 1985

\begin{tabular}{lccc}
\hline $\begin{array}{l}\text { NPDES outfall description } \\
\text { and sample parameters }\end{array}$ & $\begin{array}{c}\text { Required } \\
\text { sampling method }\end{array}$ & $\begin{array}{c}\text { Daily average } \\
\text { limitation }\end{array}$ & $\begin{array}{c}\text { Daily maximum } \\
\text { limitation }\end{array}$ \\
\hline Outfall 00l (east drainage ditch) & \\
Flow & Continuous & & \\
TSS & $24-\mathrm{h}$ composite & $20 \mathrm{mg} / \mathrm{L}$ & $30 \mathrm{mg} / \mathrm{L}$ \\
O\&G & Grab & $10 \mathrm{mg} / \mathrm{L}$ & $15 \mathrm{mg} / \mathrm{L}$ \\
Temperature & Grab & Monitoring only specified \\
TRCL & Grab & $0.02 \mathrm{mg} / \mathrm{L}$ \\
pH & Grab & 6.5 to 9.0
\end{tabular}

Outfall $001 A(601 \times-701 B$ pond effluent)

Flow

Nitrate, as $\mathrm{N}$

Ammonia, as $\mathbf{N}$

Total $\mathrm{Cu}$

Total $\mathrm{Zn}$

Total $\mathrm{Fe}$

Total Ni

Total $\mathrm{Cr}$

$\mathrm{Cr}^{6+}$

TCE

$\mathrm{pH}$

TSS

TSS

TRCL

O\&G

$\mathrm{pH}$

Total Fe

Total Mn

Total $\mathrm{Cu}$

Total $\mathrm{Zn}$

Total $\mathrm{Ni}$

Total As
Continuous

24-h composite

24-h composite

24-h composite

24-h composite

24-h composite

24-h composite

24-h composite

24-h composite

Grab

Continuous
Monitoring only specified

Monitoring only specified

Monitoring anly specified

$0.05 \mathrm{~kg} / \mathrm{d} \quad 0.09 \mathrm{~kg} / \mathrm{d}$

$0.30 \mathrm{~kg} / \mathrm{d} \quad 0.60 \mathrm{~kg} / \mathrm{d}$

Monitoring only specified

$0.007 \mathrm{~kg} / \mathrm{d} \quad 0.014 \mathrm{~kg} / \mathrm{d}$

Monitoring only specified

Monitoring only specified

Monitoring only specified

Outfall 002 (X-230K, south pond effluent)

Continuous

24-h composite

Grab

Grab

Grab

24-h composite

24-h composite

24-h composite

24-h composite

24-h composite

24-h composite

$$
\begin{array}{cr}
20 \mathrm{mg} / \mathrm{L} & 30 \mathrm{mg} / \mathrm{L} \\
& 0.02 \mathrm{mg} / \\
10 \mathrm{mg} / \mathrm{L} & 15 \mathrm{mg} / \mathrm{L} \\
& 6.5 \text { to } 9.0
\end{array}
$$

Monitoring only specified

Monitoring only specified

Monitoring only specified

Monitoring only specified

Monitoring only specified

Monitoring only specified

Outfall $002 A(X-621$ coal pile runoff treatment plant)

Flow

TSS

Total Fe

Total Mn

Total $\mathrm{Cu}$

Total $\mathrm{Zn}$

Total $\mathrm{Ni}$

Total As

$\mathrm{pH}$
Continvous

24-h composite

24-h composite

24-h composite

24-h cumposite

24-h composite

24-h composite

24-h composite

Grab
$50 \mathrm{mg} / \mathrm{L}$ $2.0 \mathrm{mg} / \mathrm{L} \quad 4.0 \mathrm{mg} / \mathrm{L}$

Monitoring only specified

Monitoring only specified

Monitoring only specified

Monitoring only specified

6.5 to 10.0

Outfall 003 (X-6619 sewage treatment plant)

$\begin{array}{llrr}\text { Flow } & \text { Continuous } & & \\ \text { BODs } & 24-\mathrm{h} \text { composite } & 26.3 \mathrm{~kg} / \mathrm{d} & 40.0 \mathrm{~kg} / \mathrm{d} \\ \text { TSS } & 24-\mathrm{h} \text { composite } & 31.8 \mathrm{~kg} / \mathrm{d} & 47.7 \mathrm{~kg} / \mathrm{d} \\ \text { Fecal coliform/100 mL } & \text { Grab } & & 0.5 \mathrm{mg} / \mathrm{L} \\ \text { TRCL } & 24-\mathrm{h} \text { composite } & \text { Monitoring only specified } \\ \text { pH } & \text { Grab } & & 6.5 \text { to } 9.0\end{array}$


Table 2.2.1 (continued)

\begin{tabular}{cccc}
\hline $\begin{array}{c}\text { NPDES outfall description } \\
\text { and sample parameters }\end{array}$ & $\begin{array}{c}\text { Required } \\
\text { sampling methoo }\end{array}$ & $\begin{array}{c}\text { Daily average } \\
\text { limitation }\end{array}$ & $\begin{array}{c}\text { Daily maximum } \\
\text { limitation }\end{array}$ \\
\hline
\end{tabular}

Outfall $003 B$ (604, biodonitrification plant)

Flow

Nitrate, as $\mathrm{N}$

Total $\mathrm{Cu}$

Total $\mathrm{Zn}$

Total Fe

Total $\mathrm{Ni}$

$\mathrm{pH}$

Flow

TDS

TSS

Total $\mathrm{Zn}$

$\mathrm{Cr}^{6+}$

Total $\mathrm{Cr}$

Total Fe

Total $\mathrm{Cu}$

TRCL

TCE

$\mathrm{pH}$

Continuous

24-h composite

24-h composite

24-h composite

24-h composite

24-h composite

Grab

$\begin{array}{ll}11.0 \mathrm{~kg} / \mathrm{d} & 13.0 \mathrm{~kg} / \mathrm{d} \\ 0.007 \mathrm{~kg} / \mathrm{d} & 0.014 \mathrm{~kg} / \mathrm{d} \\ 0.055 \mathrm{~kg} / \mathrm{d} & 0.110 \mathrm{~kg} / \mathrm{d} \\ 0.23 \mathrm{~kg} / \mathrm{d} & 0.45 \mathrm{~kg} / \mathrm{d} \\ 0.018 \mathrm{~kg} / \mathrm{d} & 0.036 \mathrm{~kg} / \mathrm{d} \\ & 6.5 \text { to } 9.0\end{array}$

Outfall 004 (X-616 chromium treatment plant)

Continuous

24-h composite

24-h composite

24-h composite

$3500 \mathrm{mg} / \mathrm{L}$

$75.8 \mathrm{~kg} / \mathrm{d}$

$4000 \mathrm{mg} / \mathrm{L}$

2.4-h composite

$1.9 \mathrm{~kg} / \mathrm{d}$

$113.5 \mathrm{~kg} / \mathrm{d}$

24-h composite

24-h composite

$0.2 \mathrm{~kg} / \mathrm{d}$

$3.8 \mathrm{~kg} / \mathrm{d}$

$1.9 \mathrm{~kg} / \mathrm{d}$

$0.4 \mathrm{~kg} / \mathrm{d}$

$3.8 \mathrm{~kg} / \mathrm{d}$

$3.8 \mathrm{~kg} / \mathrm{d}$

24-h composite

$1.9 \mathrm{~kg} / \mathrm{d}$

$5.7 \mathrm{~kg} / \mathrm{d}$

Grab

$3.8 \mathrm{~kg} / \mathrm{d}$

24-h composite

Grab

$0.02 \mathrm{mg} / \mathrm{L}$

6.5 to 9.0

Outfalls 005, 006, 007, and 008 (X-611 sludge lagoons)

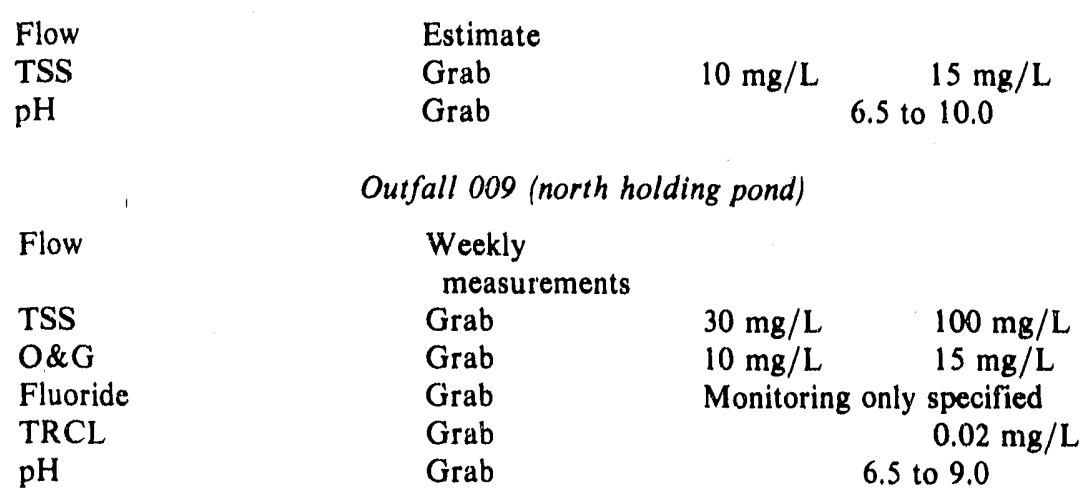

Outfall 009A (609, fluorine generator cleaning)

Flow

TSS (kg/batch)

Fluorine (kg/batch)
Batch

measurement

24-h composite

24-h composite

0.68

0.68

Outfall 010 (west drainage ditch) and Outfall 011 (northeast drainage ditch)

$\begin{array}{llrr}\text { Flow } & \text { Continuous } & \\ \text { O\&G } & \text { Grab } & 10 \mathrm{mg} / \mathrm{L} & 15 \mathrm{mg} / \mathrm{L} \\ \mathrm{pH} & \text { Grab } & & 6.5 \text { to } 9.0\end{array}$


Table 2.2.1 (continued)

\begin{tabular}{|c|c|c|c|}
\hline $\begin{array}{l}\text { NPDES outfall description } \\
\text { and sample parameters }\end{array}$ & $\begin{array}{c}\text { Required } \\
\text { sampling method }\end{array}$ & $\begin{array}{l}\text { Daily average } \\
\text { limitation }\end{array}$ & $\begin{array}{l}\text { Daily maximum } \\
\text { limitation }\end{array}$ \\
\hline \multicolumn{4}{|c|}{ GCEP NPDES Outfalls } \\
\hline \multicolumn{4}{|c|}{$\begin{array}{c}\text { Outfall } 001 X-2230 \mathrm{M} \text { pond effluent, } \\
\text { Outfall } 002 X-2230 \mathrm{~N} \text { pond effluent, and } \\
\text { Outfall } 003 \text { stormwater runoff, south GCEP site }\end{array}$} \\
\hline $\begin{array}{l}\text { Flow } \\
\text { TSS } \\
\text { O\&G } \\
\text { pH }\end{array}$ & $\begin{array}{l}\text { Continuous } \\
\text { Grab } \\
\text { Grab } \\
\text { Grab }\end{array}$ & $\begin{array}{l}30 \mathrm{mg} / \mathrm{L} \\
10 \mathrm{mg} / \mathrm{L}\end{array}$ & $\begin{array}{l}100 \mathrm{mg} / \mathrm{L} \\
20 \mathrm{mg} / \mathrm{L} \\
0.5\end{array}$ \\
\hline
\end{tabular}

Notes: 1. Parameters for which limits are not specified are required to be monitored. Results are to be reported to the U.S. EPA.

2. The $\mathrm{pH}$ limitations were removed at outfall $001 \mathrm{~A}$. A pH range of $6.5 \leqslant$ $\mathrm{pH} \leqslant 9.0$ should be maintained. Continuous $\mathrm{pH}$ monitoring data must be reported monthly to the Ohio EPA.

downstream (RW7), and discharge at the north boundary line (RW8); Big Beaver Creek upstream (RW5) and downstream (RW13); Big Run Creek upstream ( $R$ W33), south boundary line (RW3), and near Wakefield (RW2); and Scioto River upstream (RW6) and down itream (RW1). See Fig. 2.2.3 for the locations of these sampling stations.

All plant-site liquid effluents are regulated by the NPDES permit and either discharge to surface streams that pass through the reservation or are piped directly to the Scioto River. NPDES outfalls $001,005,006,007,008,009$, and 011 discharge to Little Beaver Creek, a tributary of Big Beaver Creek, and the Scioto River. NPDES outfalls 002 and Gas Centrifuge Enrichment Plant (GCEP) 003 discharge to Big Run Creek, which is a tributary of the Scioto River. NPDES 003 and 004 discharge directly to the Scioto River by pipeline. NPDES outfalls 010, GCEP 001, and GCEP 002 discharge to two unnamed tributaries of the Scioto River. NPDES outfalls 001A, 002A, 003B, and 009A are internal outfalls that discharge to NPDES outfalls $001,002,003$, and 009 respectively.

The receiving streams are monitored for radioactive and nonradioactive constituents at designated RW-sampling locations. All RW locations and designated NPDES outfall locations are analyzed for gross alpha and gross beta-gamma radioactivity, ${ }^{99} \mathrm{Tc}$, and uranium concentration. The data are summarized in Tables 2.2.2 through 2.2.4. Part 2 of this report lists detailed sampling and analysis data. The data presented in Tables 2.2.2 through 2.2.4 for Little Beaver Creek, Big Beaver Creek, Big Run Creek, and the Scioto River measure the impact that radioactive discharges have on these receiving streams.

The majority of nonradioactive constituents are regulated under the plant's NPDES permit, which is discussed in detail in Subsect. 2.2.2. The only other contaminants of concern besicies radioactivity and NPDES-regulated parameters are fluorides discharged to surface waters. Fluorides are currently measured at outfalls 001 and 003 and at upstream and downstream locations in the Scioto River. The impact of all fluoride discharges from PORTS operations is neglible, as is graphically shown in Fig. 2.2.4.

\subsubsection{National Pollutant Discharge Elimination System Monitoring}

As is mentioned in Subsect. 2.2.1, the locations of NPDES monitoring points are shown in Fig. 2.2.2. NPDES-regulated effluent parameters 


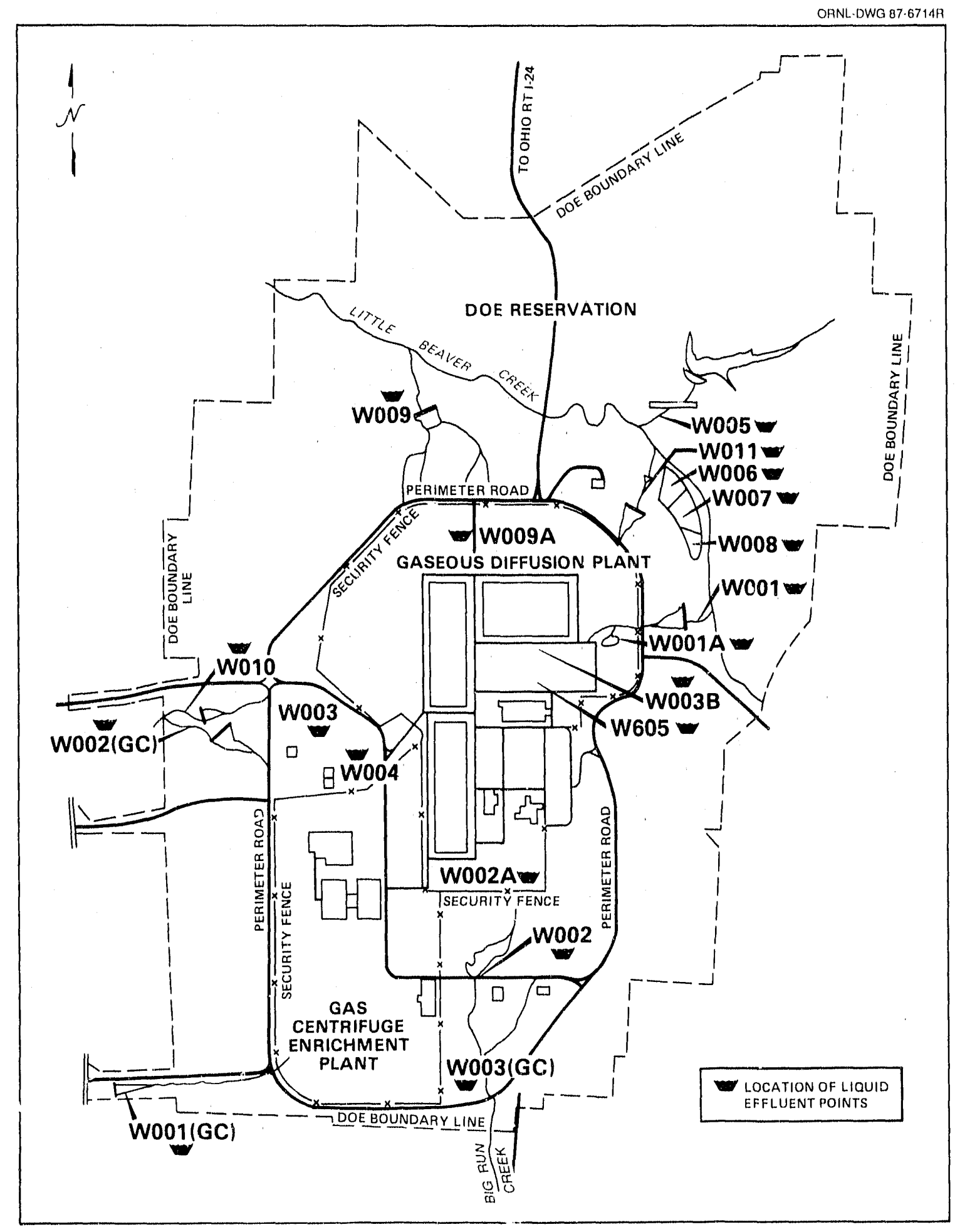

Fig. 2.2.2. Locations of NPDES water-sampling stations. 


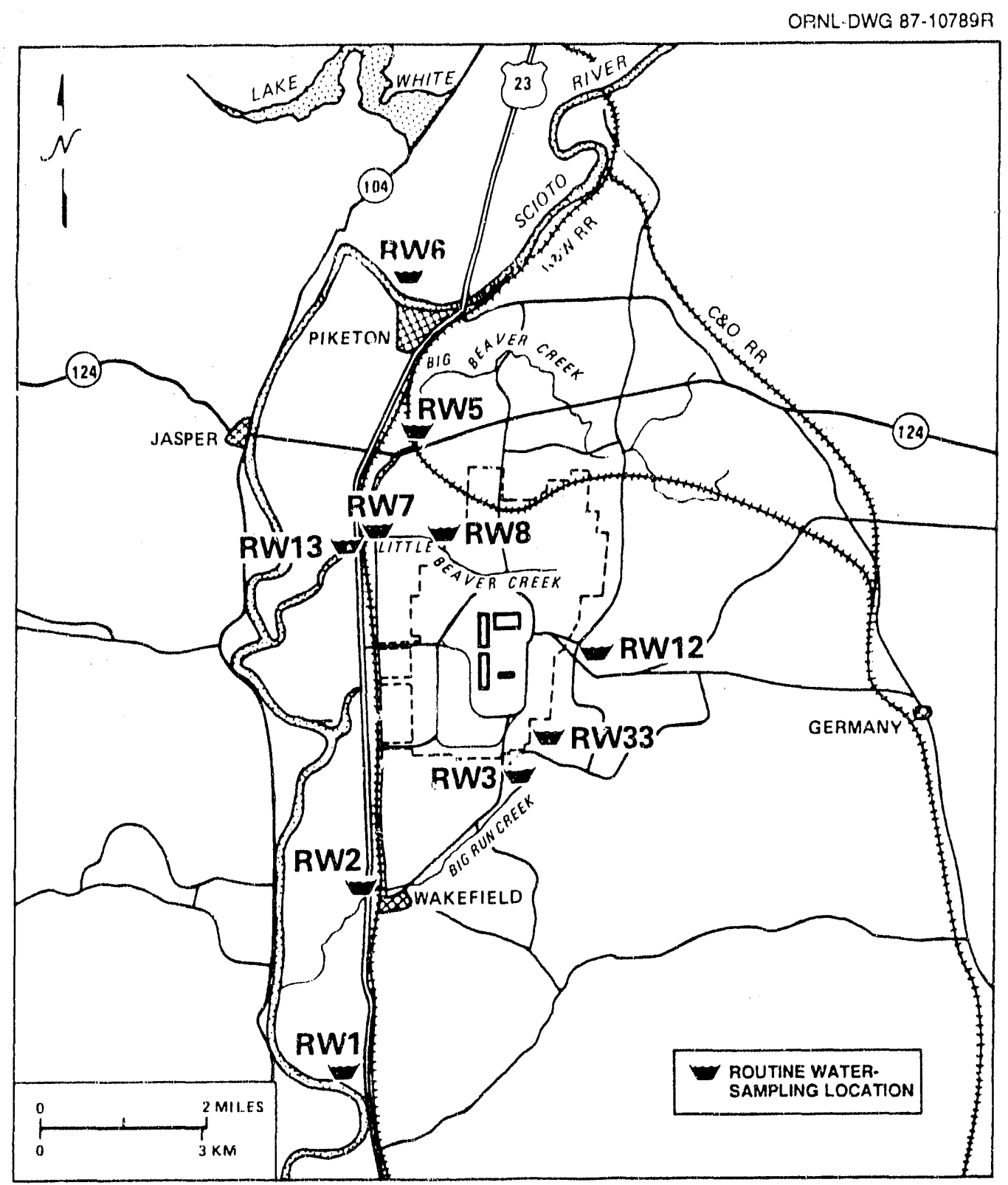

Fig. 2.2.3. Surfuce-water-sampling stations (non-NPDES).

and limitations are given in Table 2.2.1. The OEPA has regulatory authority for the CWA at PORTS. The OEPA, however, did not become active in administering NPDES permits to federal facilities until early 1983. In a letter from the OEPA dated May 25, 1983, PORTS was advised to initiate reporting of monthly self-monitoring NPDES reports directly to the OEPA beginning with the August report.
In December 1983, and again in December 1984, the Southeast District Office of the OEPA conducted compliance inspections of PORTS. These compliance inspections led to the issuance of the Director's Findings and Orders, effective April 8, 1985. The Findings indicated that PORTS has failed to maintain compliance in accordance with the second-round NPDES permit at NPDES outfalls 601 (i.e., $001 \mathrm{~A}$, the X-701B 
Table 2.2.2. 1989 gross alpha radioactivity in water samples

\begin{tabular}{|c|c|c|c|c|c|}
\hline \multirow{2}{*}{$\begin{array}{l}\text { Sample } \\
\text { location }\end{array}$} & \multirow{2}{*}{$\begin{array}{c}\text { No. of } \\
\text { samples }\end{array}$} & \multicolumn{3}{|c|}{$\begin{array}{c}\text { Concentration } \\
(\mathrm{pCi} / \mathrm{L})\end{array}$} & \multirow{2}{*}{$\begin{array}{l}\text { Standard } \\
\text { deviation }\end{array}$} \\
\hline & & Min & $\operatorname{Max}$ & Av & \\
\hline \multicolumn{6}{|c|}{ GDP outfalls } \\
\hline 001 & 60 & 5.00 & 675.00 & 67.67 & 112.99 \\
\hline 002 & 61 & 1.40 & 13.05 & 3.85 & 2.27 \\
\hline 003 & 61 & 17.60 & 778.50 & 182.65 & 148.34 \\
\hline 004 & 62 & 0 & 43.20 & 3.65 & 5.77 \\
\hline 009 & 61 & 3.60 & 27.50 & 9.02 & 3.87 \\
\hline 010 & 61 & 2.30 & 43.70 & 9.71 & 6.61 \\
\hline 011 & 61 & 0.20 & 189.00 & 5.75 & 23.89 \\
\hline 601 & 3 & 640.00 & 1360.00 & 893.33 & 404.64 \\
\hline 605 & 50 & 210.00 & 27400.00 & 5253.00 & 5899.85 \\
\hline \multicolumn{6}{|c|}{ GCEP outfalls } \\
\hline 001 & 19 & $<0.90$ & 43.20 & 7.15 & 12.87 \\
\hline 002 & 21 & 0.90 & 6.30 & 3.22 & 1.88 \\
\hline 003 & 4 & 2.30 & 13.50 & 5.88 & 5.16 \\
\hline \multicolumn{6}{|c|}{ Little Beaver Creek } \\
\hline RW7 & 11 & 1.80 & 8.00 & 4.94 & 1.81 \\
\hline RW8 & 52 & 5.90 & 81.00 & 24.01 & 13.91 \\
\hline $\mathrm{RW} 12$ & 16 & 0.10 & 1.80 & 0.75 & 0.40 \\
\hline \multicolumn{6}{|c|}{ Big Beaver Creek } \\
\hline RW5 & 11 & 0.20 & 6.80 & 2.34 & 1.88 \\
\hline RWI3 & 11 & 0.40 & 6.50 & 2.34 & 1.98 \\
\hline \multicolumn{6}{|c|}{ Big Run Creek } \\
\hline RW2 & 10 & $<0.10$ & 5.90 & $<2.06$ & 1.57 \\
\hline RW3 & 11 & 0.50 & 8.60 & 3.60 & 2.38 \\
\hline RW33 & 11 & 0 & 1.40 & $<0.74$ & 0.47 \\
\hline \multicolumn{6}{|c|}{ Scioto River } \\
\hline RW1 & 50 & 0.50 & 54.00 & 3.86 & 7.41 \\
\hline RW6 & 50 & 0.50 & 45.00 & 3.40 & 6.11 \\
\hline
\end{tabular}

${ }^{a}$ Gaseous Diffusion Plant.

${ }^{b}$ Gas Centrifuge Enrichment Plant.

holding pond) and 604 (i.e., 003B, the biodenitrification pilot plant). The Orders relaxed overly stringent effluent limits at outfall 601 (i.e., the X-701 B holding pond) and outfall 604 (i.e., the biodenitrification facility) and established a schedule for constructing additional treatment facilities at these outfalls.
The current NPDES permit is thus the secondround permit issued in 1980 with revisions described in the Director's Findings and Orders. The first official meeting on the third-round NPDES permit was held with the OEPA in November 1986. Details on EPA's effluent sampling and inspection study, Best Management 
Table 2.2.3. 1989 beta-gamma radioactivity in water samples

\begin{tabular}{|c|c|c|c|c|c|c|}
\hline \multirow{2}{*}{$\begin{array}{l}\text { Sample } \\
\text { location }\end{array}$} & \multirow{2}{*}{$\begin{array}{c}\text { No. of } \\
\text { samples }\end{array}$} & \multirow{2}{*}{$\begin{array}{l}\text { Radio- } \\
\text { nuclide }\end{array}$} & \multicolumn{3}{|c|}{$\begin{array}{l}\text { Concentration } \\
(\mathrm{pCi} / \mathrm{L})\end{array}$} & \multirow{2}{*}{$\begin{array}{l}\text { Standard } \\
\text { deviation }\end{array}$} \\
\hline & & & Min & $\operatorname{Max}$ & Av & \\
\hline \multicolumn{7}{|c|}{ GDP outfalls $s^{a}$} \\
\hline 001 & 56 & ${ }^{234} \mathrm{Th}$ & 8.75 & 60.00 & 12.31 & 13.52 \\
\hline 001 & 64 & ${ }^{99} \mathrm{Tc}$ & 3.00 & 536.00 & 130.92 & 124.47 \\
\hline 002 & 56 & ${ }^{234} \mathrm{Th}$ & 1.00 & 36.00 & 7.45 & 6.43 \\
\hline 002 & 71 & ${ }^{99} \mathrm{Tc}$ & 0 & 173.00 & 15.62 & 33.97 \\
\hline 003 & 54 & ${ }^{234} \mathrm{Th}$ & 0 & 106.00 & 17.90 & 19.15 \\
\hline 003 & 67 & ${ }^{99} \mathrm{Tc}$ & 1.00 & 527.00 & 185.48 & 119.46 \\
\hline 004 & 53 & ${ }^{234} \mathrm{Th}$ & 7.00 & 292.00 & 79.00 & 58.49 \\
\hline 004 & 67 & ${ }^{99} \mathrm{Tc}$ & 7.00 & 718.00 & 145.06 & 151.04 \\
\hline 009 & 49 & ${ }^{234} \mathrm{Th}$ & 1.00 & 45.00 & 9.41 & 8.29 \\
\hline 009 & 68 & ${ }^{99} \mathrm{Tc}$ & 0 & 22.00 & 7.96 & 5.30 \\
\hline 010 & 49 & ${ }^{234} \mathrm{Th}$ & 0 & 39.00 & 8.69 & 7.25 \\
\hline 010 & 66 & ${ }^{99} \mathrm{Tc}$ & 0 & 25.00 & 8.41 & 5.34 \\
\hline 011 & 46 & ${ }^{234} \mathrm{Th}$ & 0 & 114.00 & 9.70 & 16.92 \\
\hline 011 & 68 & ${ }^{99} \mathrm{Tc}$ & 0 & 38.00 & 8.10 & 7.40 \\
\hline 601 & 3 & ${ }^{234} \mathrm{Th}$ & 1.00 & 426.00 & 63.50 & 88.39 \\
\hline 601 & 3 & ${ }^{99} \mathrm{Tc}$ & 1640.00 & 6650.00 & 3410.00 & 2809.93 \\
\hline 605 & 54 & ${ }^{234} \mathrm{Th}$ & 52.00 & 7673.00 & 1016.08 & 1803.84 \\
\hline 605 & 58 & ${ }^{99} \mathrm{Tc}$ & 544.00 & 216057.00 & 27892.57 & 30397.64 \\
\hline \multicolumn{7}{|c|}{ GCEP outfalls $s^{b}$} \\
\hline 001 & 8 & ${ }^{234} \mathrm{Th}$ & 4.00 & 8.00 & 6.12 & 1.25 \\
\hline 001 & 10 & ${ }^{99} \mathrm{Tc}$ & 0 & 14.00 & 7.20 & 4.37 \\
\hline 002 & 8 & ${ }^{234} \mathrm{Th}$ & 1.00 & 30.00 & 9.62 & 10.51 \\
\hline 002 & 9 & ${ }^{99} \mathrm{Tc}$ & 0 & 10.00 & 6.00 & 3.35 \\
\hline 003 & 4 & ${ }^{234} \mathrm{Th}$ & 5.00 & 18.00 & 8.50 & 6.35 \\
\hline 003 & 3 & ${ }^{99} \mathrm{Tc}$ & 1.00 & 15.00 & 8.67 & 7.09 \\
\hline
\end{tabular}

Little Beaver Creek

\begin{tabular}{lrrrrrr} 
RW7 & 10 & ${ }^{234} \mathrm{Th}$ & 4.00 & 68.00 & 30.70 & 19.20 \\
RW7 & 11 & ${ }^{99} \mathrm{Tc}$ & 8.00 & 60.00 & 39.91 & 14.82 \\
RW8 & 12 & ${ }^{234} \mathrm{Th}$ & 9.00 & 62.00 & 38.08 & 18.08 \\
RW8 & 59 & ${ }^{99} \mathrm{Tc}$ & 12.00 & 160.00 & 49.19 & 22.77 \\
RW12 & 15 & ${ }^{234} \mathrm{Th}$ & 0 & 26.00 & 5.07 & 6.71 \\
RW12 & 16 & ${ }^{99} \mathrm{Tc}$ & 2.00 & 14.00 & 6.94 & 3.77 \\
\multicolumn{7}{c}{} \\
RW5 & 11 & ${ }^{234} \mathrm{Th}$ & 1.00 & 13.00 & 4.91 & 3.59 \\
RW5 & 11 & ${ }^{99} \mathrm{Tc}$ & 1.00 & 15.00 & 6.64 & 4.13 \\
RW13 & 8 & ${ }^{234} \mathrm{Th}$ & 3.00 & 55.00 & 23.50 & 17.42 \\
RW13 & 10 & ${ }^{99} \mathrm{Tc}$ & 3.00 & 62.00 & 23.90 & 18.65 \\
& \multicolumn{7}{c}{ Big Beaver Creek } \\
& \multicolumn{7}{c}{ Big Run Creek } & & \\
RW2 & 13 & ${ }^{234} \mathrm{Th}$ & 0 & 20.00 & 7.69 & 6.37 \\
RW2 & 10 & ${ }^{99} \mathrm{Tc}$ & 0 & 14.00 & 6.10 & 495 \\
RW3 & 12 & ${ }^{234} \mathrm{Th}$ & 2.00 & 43.00 & 10.75 & 11.04 \\
RW3 & 11 & ${ }^{94} \mathrm{Tc}$ & 2.00 & 16.00 & 10.82 & 4.47 \\
RW33 & 12 & ${ }^{234} \mathrm{Th}$ & 1.00 & 12.00 & 4.67 & 3.63 \\
RW33 & 9 & ${ }^{999} \mathrm{Tc}$ & 0 & 14.00 & 6.67 & 4.00
\end{tabular}


Table 2.2.3 (continued)

\begin{tabular}{|c|c|c|c|c|c|c|}
\hline \multirow{2}{*}{$\begin{array}{l}\text { Sample } \\
\text { location }\end{array}$} & \multirow{2}{*}{$\begin{array}{c}\text { No. of } \\
\text { samples }\end{array}$} & \multirow{2}{*}{$\begin{array}{l}\text { Radio- } \\
\text { nuclide }\end{array}$} & \multicolumn{3}{|c|}{$\begin{array}{l}\text { Concentration } \\
(\mathrm{pCi} / \mathrm{L})\end{array}$} & \multirow{2}{*}{$\begin{array}{l}\text { Standard } \\
\text { deviation }\end{array}$} \\
\hline & & & Min & $\operatorname{Max}$ & Av & \\
\hline \multicolumn{7}{|c|}{ Scioto River } \\
\hline RWl & 48 & ${ }^{234} \mathrm{Th}$ & 0 & 35.00 & 6.50 & 5.77 \\
\hline RW 1 & 64 & ${ }^{99} \mathrm{Tc}$ & 0 & 17.00 & 5.61 & 4.26 \\
\hline RW6 & 47 & ${ }^{234} \mathrm{Th}$ & 0 & 51.00 & 6.55 & 7.48 \\
\hline RW6 & 60 & ${ }^{99} \mathrm{Tc}$ & 0 & 24.00 & 5.98 & 5.39 \\
\hline
\end{tabular}

${ }^{a}$ Gaseous Diffusion Plant.

${ }^{b}$ Gas Centrifuge Enrichment Plant.

Practice (BMP) Guidelines, and other permitting details were discussed. After much negotiation and coordination, a draft NPDES permit was given public notice in July 1988. All comments to the draft permit have been received, and the OEPA is in the process of responding to all comments. Further negotiations were held in March 1989. A new permit is expected to be issued by mid- 1990 .

\subsubsection{Description of NPDES-regulated outfalls}

The following is a listing of all permitted discharge outfalls at PORTS and a brief description of each.

\section{Portsmouth Gaseous Diffusion Plant (OH0006092)}

NPDES 001 (X-230J-7) east drainage ditch. The X-230J-7 holding pond provides a quiescent zone for settling of suspended solids, dissipation of chlorine, and oil diversion and containment. A flow diagram for 001 is given in Fig. 2.2.5.

NPDES 001 A (601) X-701B holding pond.

The X-701 B holding pond provides $\mathrm{pH}$ adjustment with slaked lime, settling of heavy metals, and containment of oil with use of oil booms. A flow diagram for $001 \mathrm{~A}$ is given in Fig. 2.2.5.

NPDES 002 (X-230K) south holding pond. The south holding pond provides a quiescent zone for settling of suspended solids and dissipation of chlorine, followed by final $\mathrm{pH}$ adjustments before discharge. A flow diagram for 002 is given in Fig. 2.2.6.
NPDES 002A (602) X-621 coal pile treatment plant. The $\mathrm{X}-621$ coal pile treatment plant provides a lagoon for collection and equalization of coal pile runoff and steam plant cooling water. The collected water is then pH adjusted, and suspended solids are removed using a lamella settler. Sludge is dewatered using a pressure filter. Other steam plant waste streams that were originally sent to the $\mathrm{X}-621$ facility are now treated separately and sent directly to the south holding pond. Water-softening regeneration water is treated using $\mathrm{pH}$ adjustment, ash wash water is treated for solids removal using a pressure filter, and steam plant boiler blowdown water is sent to the south holding pond untreated. A flow diagram for $002 \mathrm{~A}$ is given in Fig. 2.2.6.

NPDES $003(X-6619)$ sewage treatment plant.

The X-6619 sewage treatment plant uses screening and a grit chamber as preliminary treatment followed by an activated sludge treatment system. Mixed liquor from the aeration basins is clarified, filtered using multimedia sand filters, and then chlorinated. Sludge is aerobically digested and dried on sludge drying beds. A flow diagram for 003 is given in Fig. 2.2.7.

NPDES 003A (603) X-710 laboratory treatment facility (cancelled). In lieu of constructing a separate treatment facility to treat laboratory wastes, OEPA suggested administrative controls to minimize the discharge of organics and metals. Administrative controls are currently being practiced, and all remaining laboratory wastes are treated at the X-6619 sewage treatment facility. 
Table 2.2.4. 1989 uranium in water samples

\begin{tabular}{|c|c|c|c|c|c|}
\hline \multirow{2}{*}{$\begin{array}{l}\text { Sample } \\
\text { location }\end{array}$} & \multirow{2}{*}{$\begin{array}{c}\text { No. of } \\
\text { samples }\end{array}$} & \multicolumn{3}{|c|}{$\begin{array}{c}\text { Concentration } \\
(\mu \mathrm{g} / \mathrm{l} .)\end{array}$} & \multirow{2}{*}{$\begin{array}{l}\text { Standard } \\
\text { deviation }\end{array}$} \\
\hline & & Min & $\operatorname{Max}$ & $A v$ & \\
\hline \multicolumn{6}{|c|}{ GDP outfalls $s^{a}$} \\
\hline 001 & 61 & 0 & 0.11 & 0.01 & 0.02 \\
\hline 002 & 61 & 0 & 0 & 0 & 0 \\
\hline 003 & 61 & 0 & 0.41 & 0.03 & 0.05 \\
\hline 004 & 62 & 0 & 0.01 & 0 & 0 \\
\hline 009 & 61 & 0 & 0.02 & 0.01 & 0 \\
\hline 010 & 61 & 0 & 0.03 & $<0.01$ & 0.01 \\
\hline 011 & 61 & 0 & 0.01 & 0 & 0 \\
\hline 601 & 3 & 0.41 & 0.98 & 0.16 & 0.29 \\
\hline 605 & 51 & 0.12 & 18.40 & 3.14 & 3.91 \\
\hline \multicolumn{6}{|c|}{ GCEP outfalls } \\
\hline 001 & 21 & 0 & 0 & 0 & 0 \\
\hline 002 & 21 & 0 & 0 & 0 & 0 \\
\hline 003 & 4 & 0 & 0 & 0 & 0 \\
\hline \multicolumn{6}{|c|}{ Little Beaver Creek } \\
\hline RW7 & 11 & 0 & 0.01 & 0.01 & 0 \\
\hline RW8 & 52 & 0 & 0.07 & 0.01 & 0.01 \\
\hline RW12 & 16 & 0 & 0 & 0 & 0 \\
\hline \multicolumn{6}{|c|}{ Big Beaver Creek } \\
\hline RW5 & 11 & 0 & 0 & 0 & 0 \\
\hline RW13 & 11 & 0 & 0.01 & 0 & 0 \\
\hline \multicolumn{6}{|c|}{ Big Run Creek } \\
\hline $\mathrm{RW} 2$ & 10 & 0 & 0 & 0 & 0 \\
\hline RW3 & 11 & 0 & 0 & 0 & 0 \\
\hline RW33 & 11 & 0 & 0 & 0 & 0 \\
\hline \multicolumn{6}{|c|}{ Scioto River } \\
\hline RWl & 50 & 0 & 0 & 0 & 0 \\
\hline RW6 & 50 & 0 & 0.010 & & \\
\hline
\end{tabular}

The OEPA has officially agreed to cancellation of the proposed project. A flow diagram for $003 \mathrm{~A}$ is given in Fig. 2.2.7.

NPDES 003B (604) biodenitrification plant. The existing biodenitrification plant is an actual pilot plant transferred to PORTS from Oak Ridge, Tennessee. Construction of a full-scale plant was completed in early CY 1987, and operational startup was initiated in May 1987. Both the pilot plant and the full-scale plant have continued to operate through CY 1989. Raffinate is first treated to remove heavy metals using a pressure filter, technetium is removed by ion exchange, and the remaining high-nitrate solution is diluted and treated biologically using a fluidized-bed coal media reactor. Bacteria and minor coal fines are 

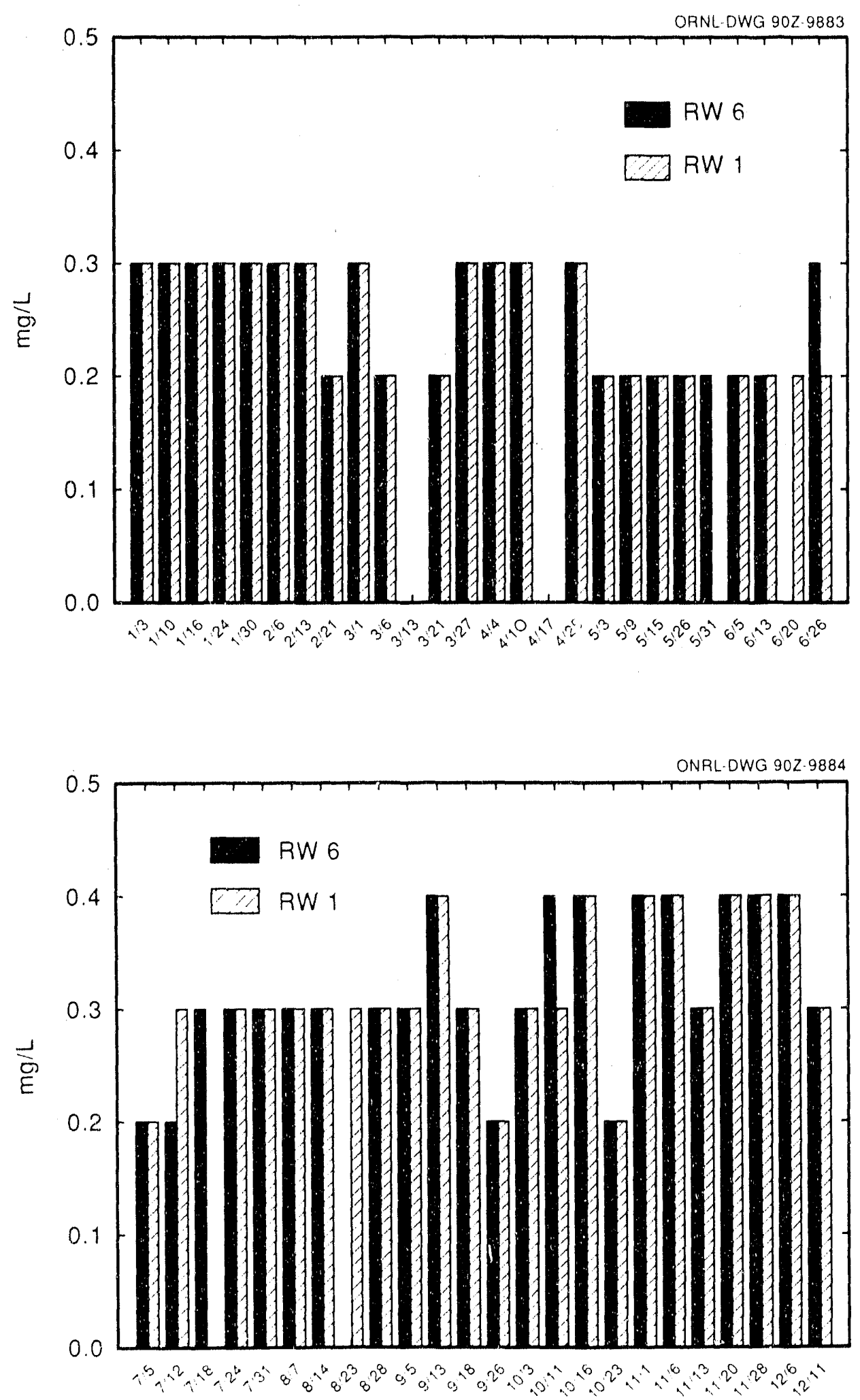

Fig. 2.2.4. 1989 fluoride concentrations. 


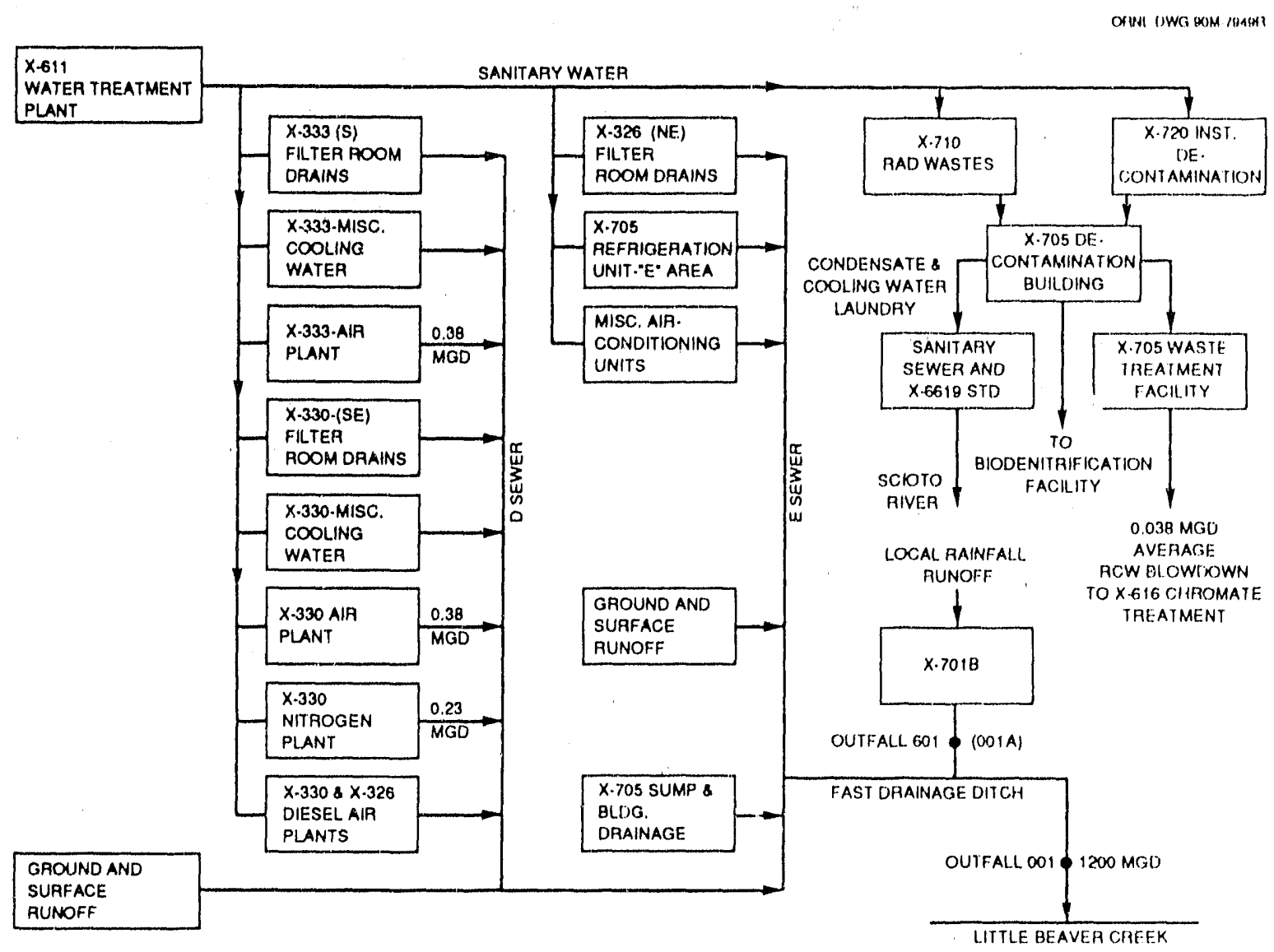

Fig. 2.2.5. Outfalls 001 and $001 \mathrm{~A}$ flow diagram.

discharged to the $\mathrm{X}-6619$ sewage treatment facility. A flow diagram for $003 \mathrm{~B}$ is given in Fig. 2.2.7.

\section{NPDES 004 (X-616) chromate treatment} facility. Recirculating cooling water blowdown containing hexavalent chromium is first reduced to trivalent chromium using sulfurous acid. Slaked lime is added to raise $\mathrm{pH}$, and the trivalent chromium hydroxide sludge is clarified before discharge. Sludge is currently treated, filtered, and stored, and supernatant is returned to the treatment plant influent. Following treatment, the sludge is no longer RCRA hazardous. A flow diagram for 004 is given in Fig. 2.2.8.

NPDES 605 (X-705) waste treatment facility. In November 1988 the X-701 B holding pond (NPDES 601) was eliminated and replaced with the new X-705 waste treatment facility to treat all
X-705 wastewater. All process wastewaters are collected, stored, treated for oil and grease, and then further treated using microfiltration and pressure filtration technology. Discharge is to the RCW blowdown line, which ultimately discharges to the X-616 chromate treatment facility (NPDES 004) and the Scioto River. A flow diagram for 605 is given in Fig. 2.2.8.

NPDES 005 (X-611B) sludge lagoon. The $X-611$ B sludg: lagoon provides a quiescent zone for settling of linis sludge used in the water system softening process and also receives rainfall runoff. Supernatant is returned to the water treatment plant. A discharge from this facility is rare and occurs only during periods of excessive rainfall. A flow diagram for 005 is given in Fig. 2.2.9.

NPDES $006(X-611 A)$ north sludge lagoon, NPDES 007 (X-61/A) middle sludge lagoon, and 


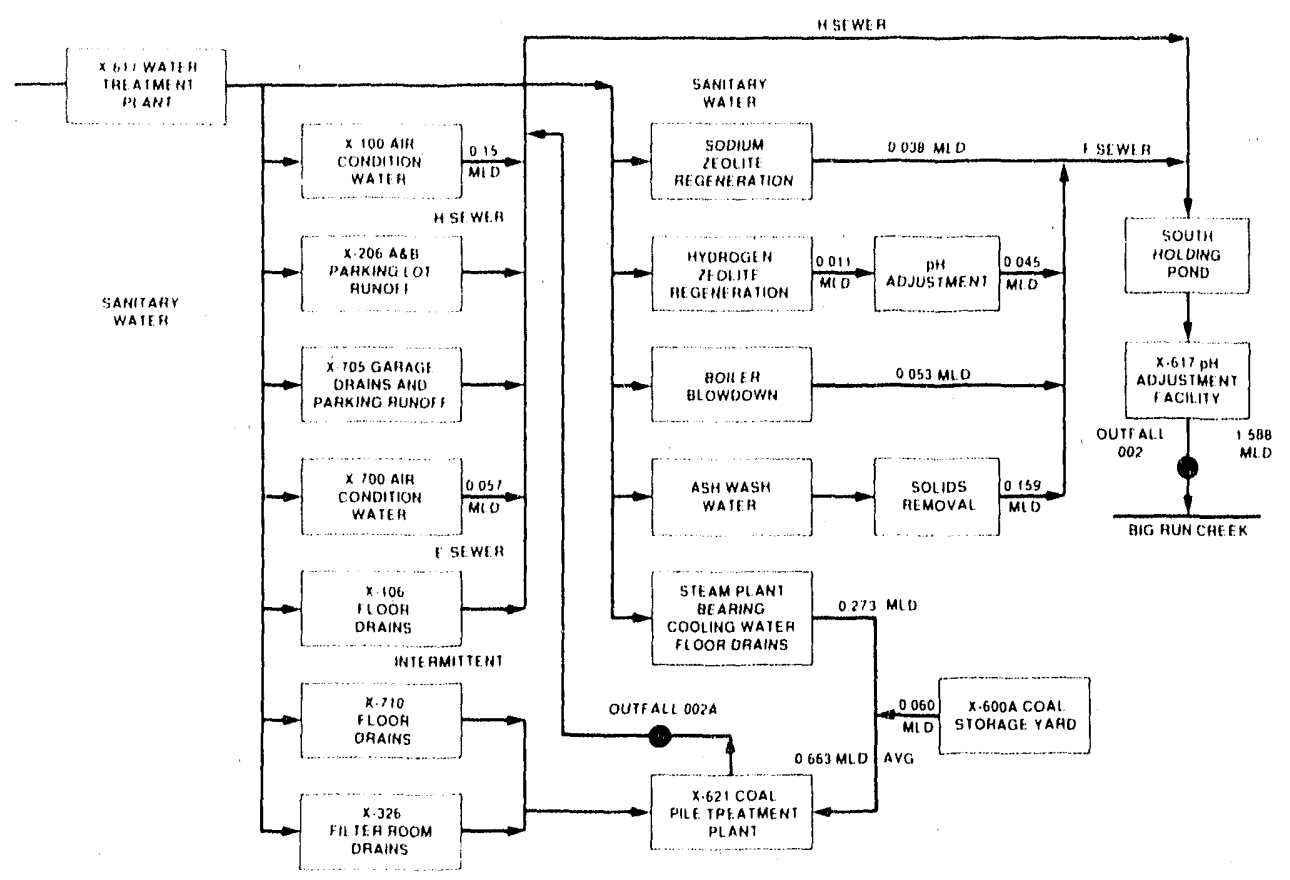

Fig. 2.2.6. Outfalls 002 and $002 \mathrm{~A}$ flow diagram.

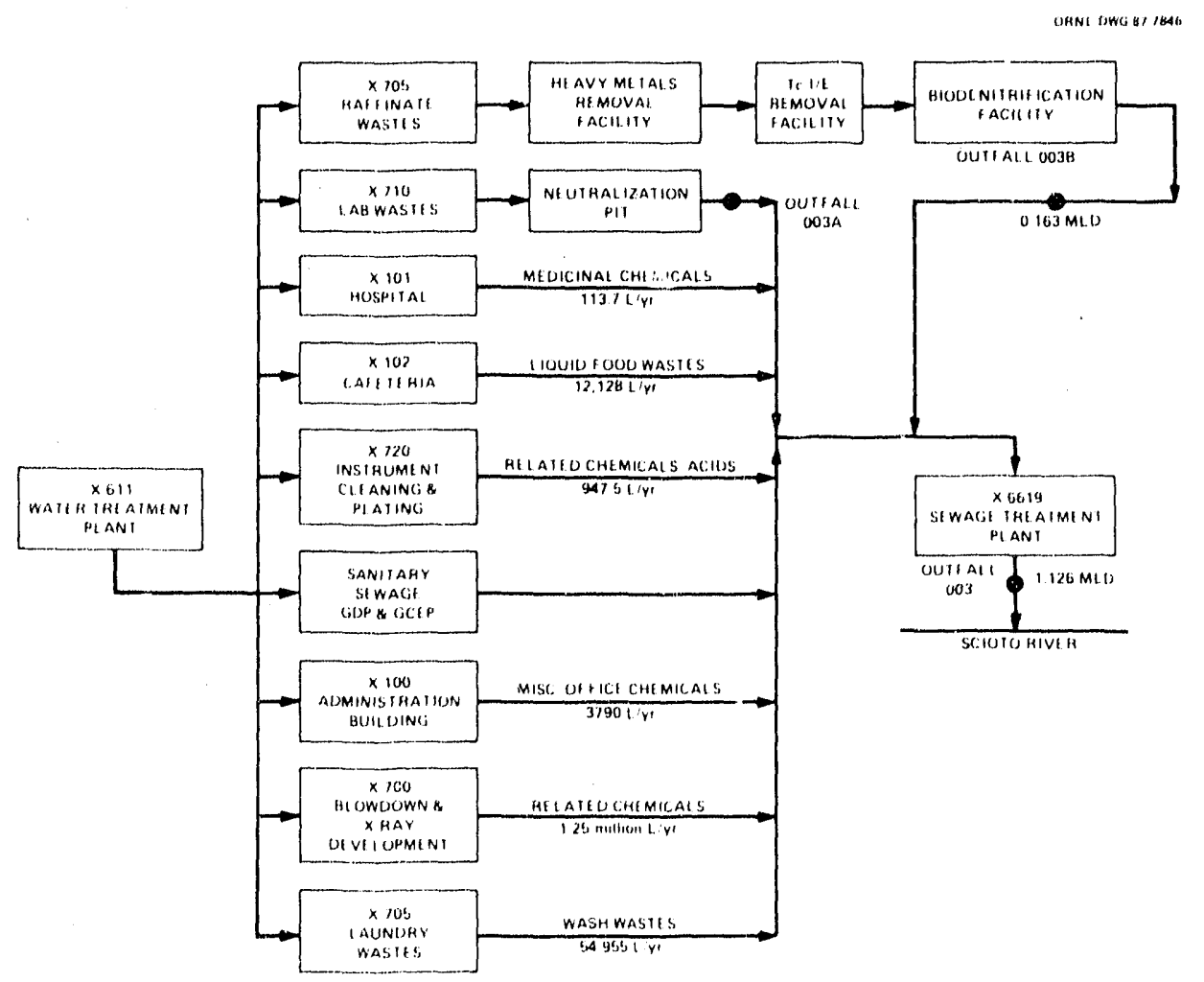

Fig. 2.2.7. Outfalls $003,003 \mathrm{~A}$, and $003 \mathrm{~B}$ now diagram. 


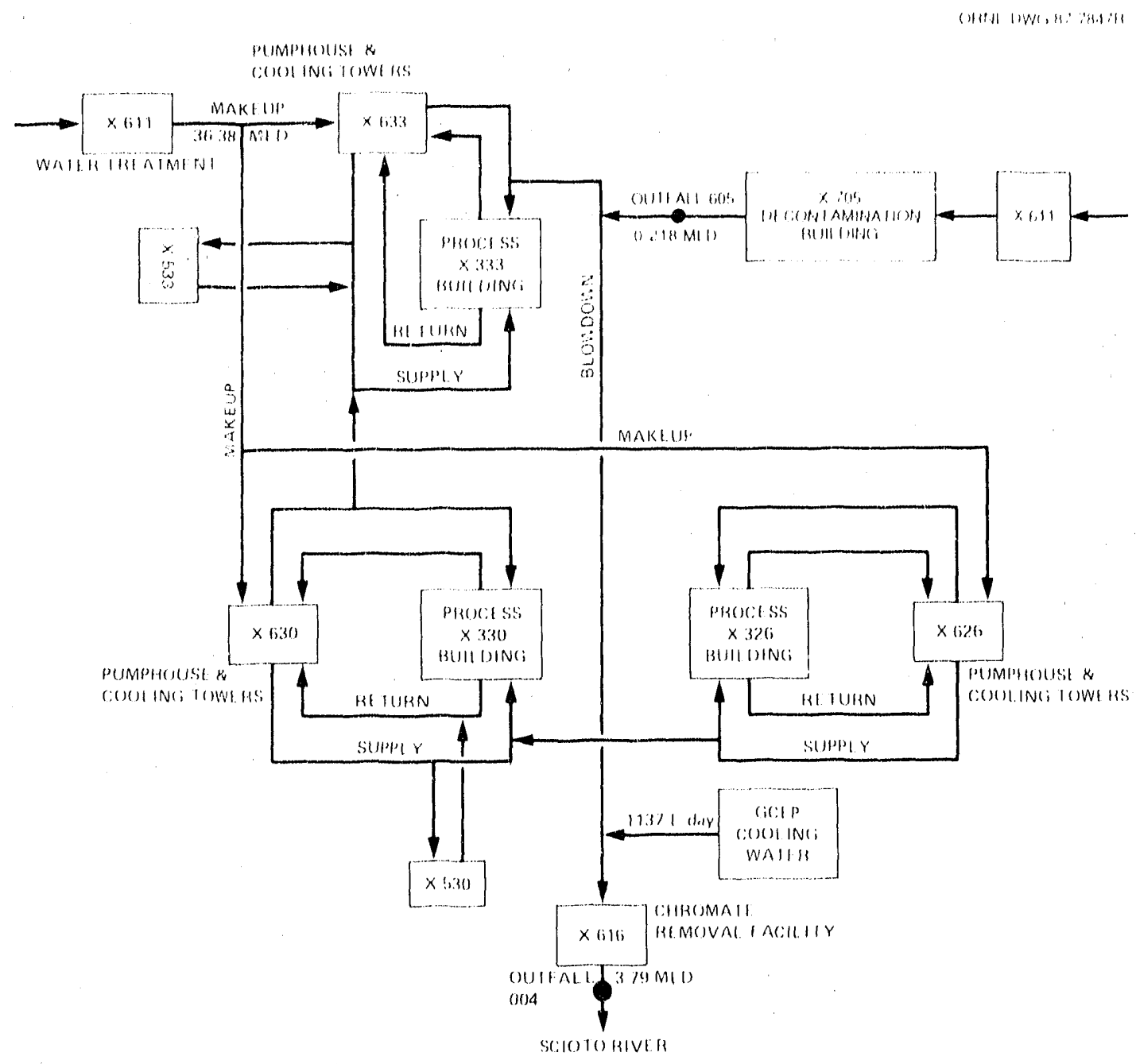

Fig. 2.2.8. Outfalls 004 and 605 flow diagram.

NPDES $008(X-611 A)$ south sludge lagoon. These lagoons once received lime sludge from the plant's water-softening process and are filled to capacity. The lagoons now receive only rainfall runoff. A discharge from these facilities is rare and occurs only during periods of excessive rainfall. Flow diagrams for 006, 007, and 008 are given in Fig. 2.2.9.

NPDES $009(X-230 L)$ north holding pond. The north holding pond provides a quiescent zone for settling of suspended solids and dissipation of chlorine. The pond can be closed for emergency containment of unpermitted discharges. A flow diagram for 009 is given in Fig. 2.2.10.

NPDES 009A (609) fluorine generator cleaning. The fluorine generator-cleaning pit provides a sand- and gravel-settling pit for settling and filtering $\mathrm{pH}$-adjusted cleaning solution solids prior to discharge to the north holding pond. Because of a reduction in plant activity in this area, a discharge from this outfall is very rare. Since 1982 there have been no discharges from this outfall. A flow diagram for $009 \mathrm{~A}$ is given in Fig. 2.2.10,

NPDES $010(X-230 J-5)$ west drainage ditch. The X-230J-5 holding pond provides a quiescent zone for settling of suspended solids, dissipation of chlorine, and oil diversion and containment. A flow diagram for 010 is given in Fig. 2.2.11.

NPDES $011(X-230 J-6)$ northeast drainage ditch. The X-230J-6 holding pond provides a quiescent zone for settling of suspended solids, 


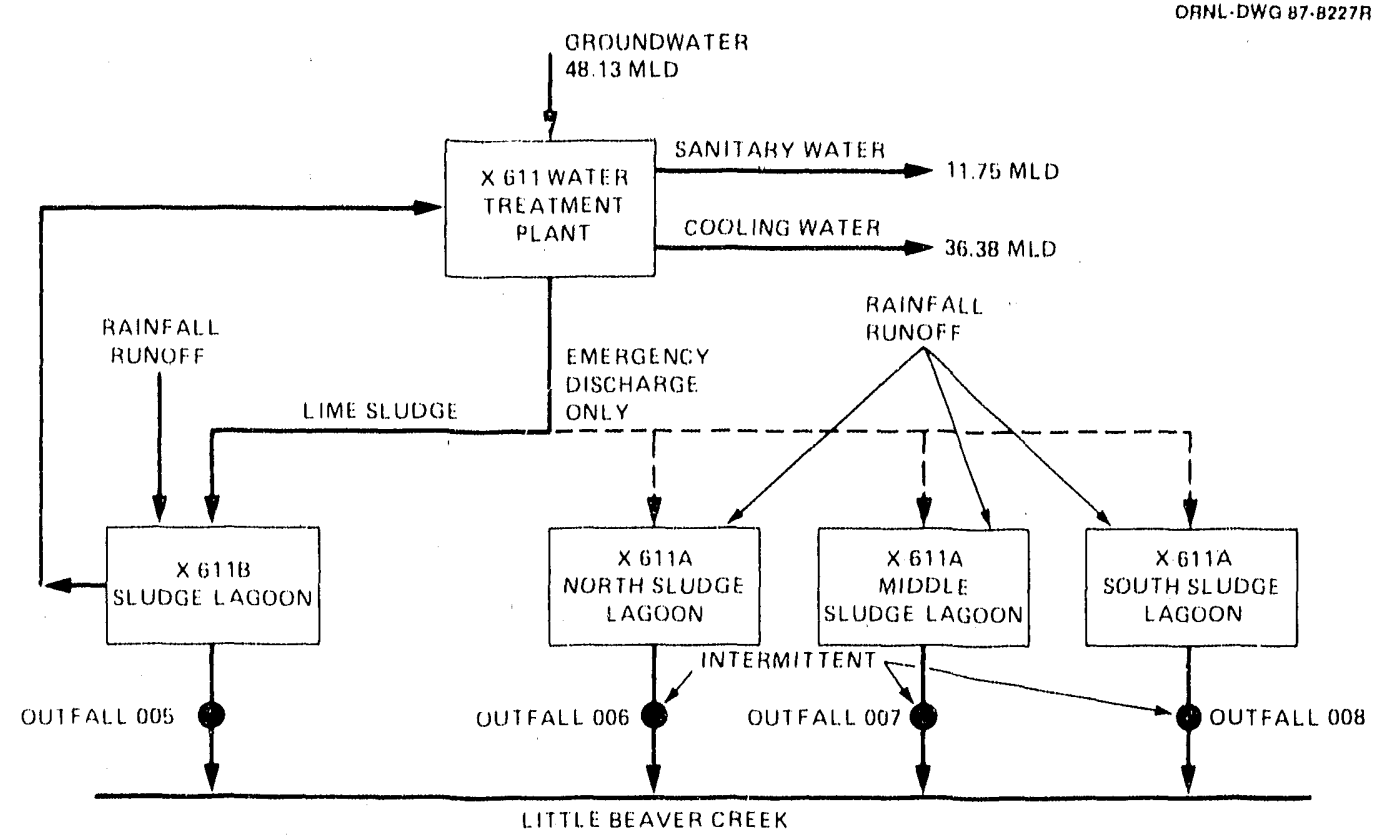

Fig. 2.2.9. Outfalls 005, 006, 007, and 008 flow diagram.

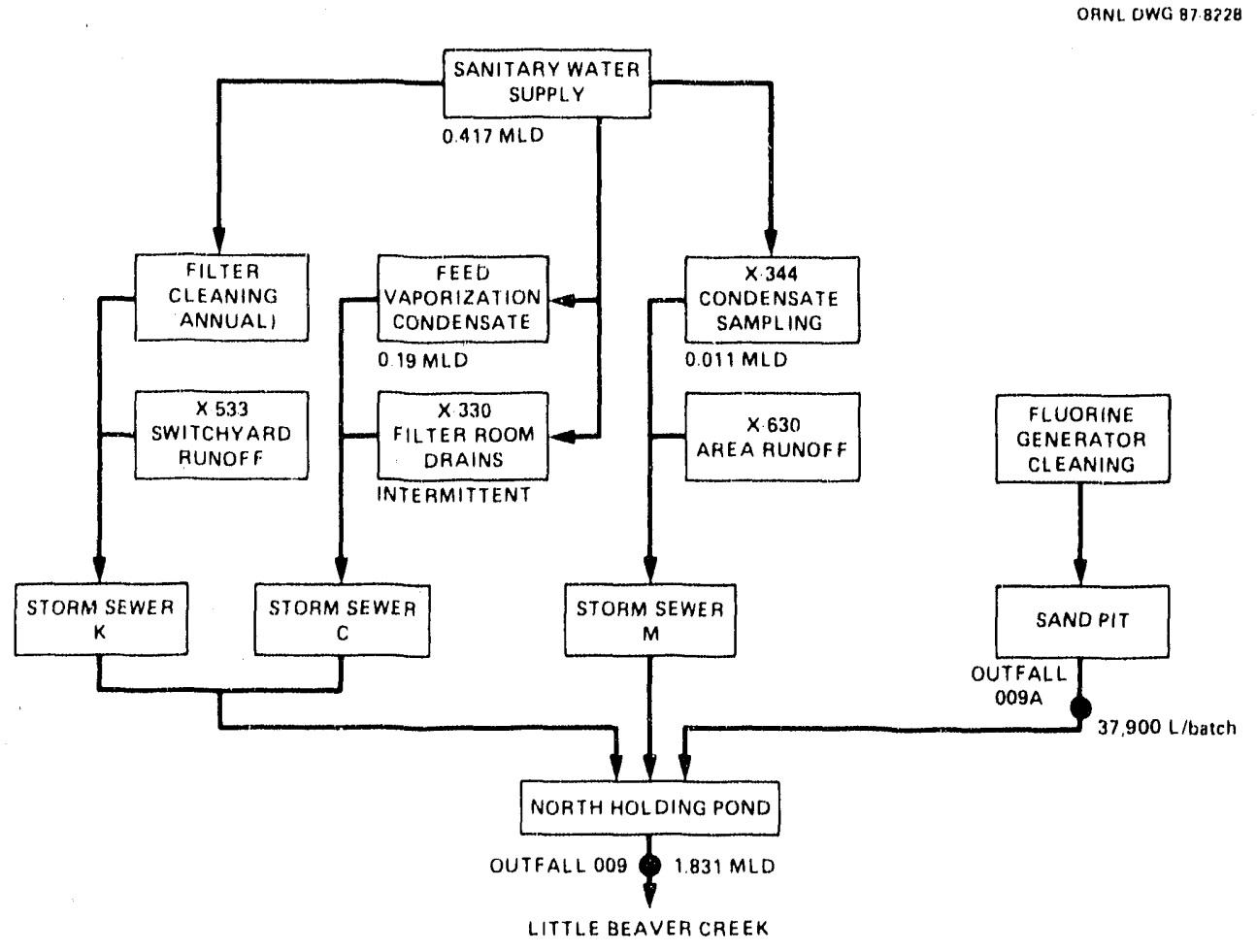

Fig. 2.2.10. Outfalls 009 and 009A now diagram. 


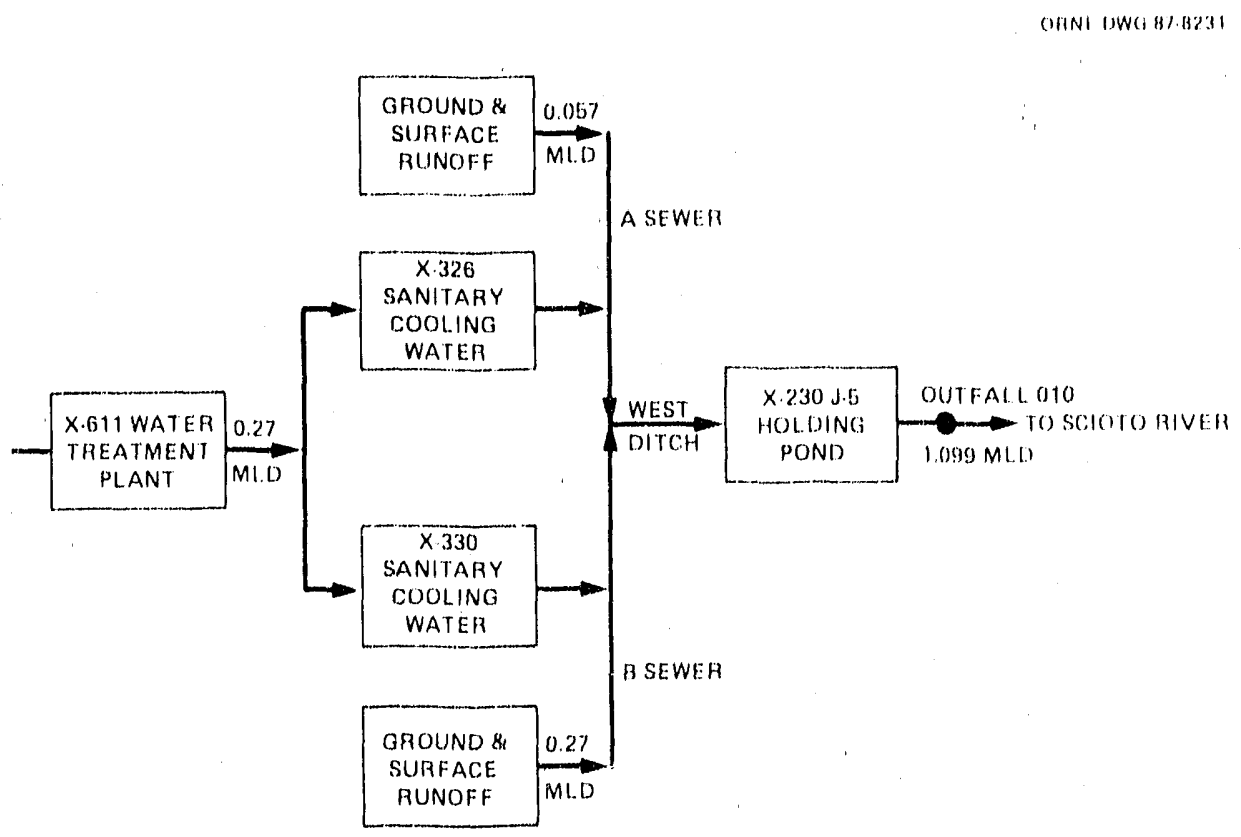

Fig. 2.2.11. Outfall 010 flow diagram.

dissipation of chlorine, and oil diversion and containment. A flow diagram for 011 is given in Fig, 2.2.12.

\section{Portsmouth Gas Centrifuge Enrichment Plant (OH0110931)}

GCEP 001 (X-2230M) holding pond No. 1. The X-2230M holding pond provides a quiescent zone for settling of suspended solids, dissipation of chlorine, and containment of oil with effluent baffling.
GCEP 002 (X-2230N) holding pond No. 2. The $X-2230 \mathrm{~N}$ holding pond provides a quiescent zone for settling of suspended solids, dissipation of chlorine, and containment of oil with effluent baffling.

GCEP 003 (holding pond not constructed). A holding pond originally designated as outfall 003 was never constructed because of significant changes in the area topography. NPDES sampling in the remaining small drainage sector has continued, however, when a measurable flow occurs during periods of excessive rainfall. A flow diagram for these outfalls is given in Fig. 2.2.13.

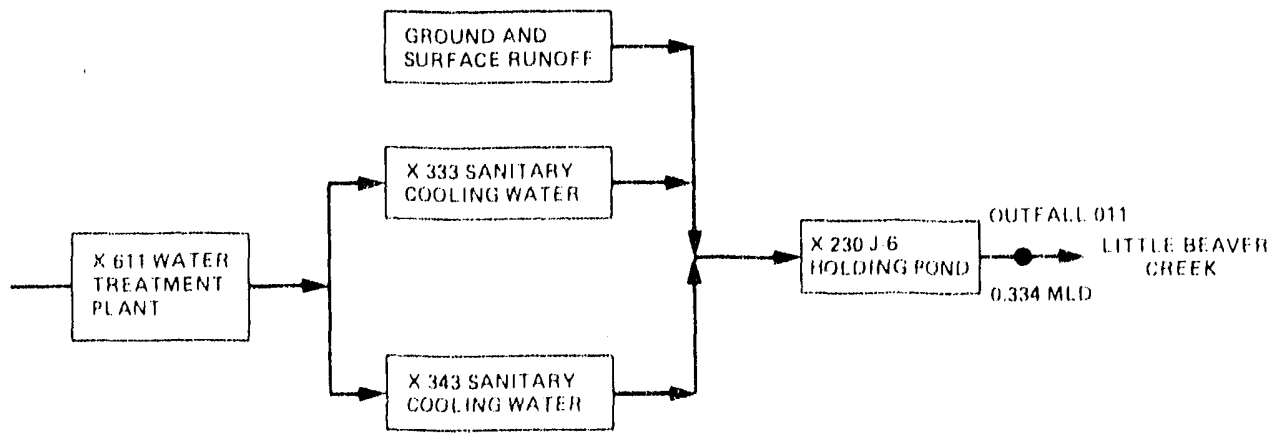

Fig. 2.2.12. Outfall 011 now diagram. 


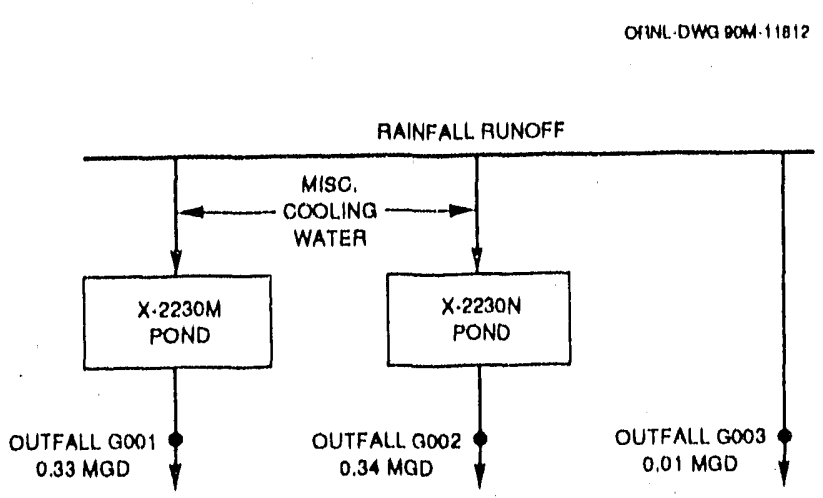

Fig. 2.2.13. Flow diagram for outfalls G001 and $\mathrm{G002}$ and for the drainage sector originally designated as outfall G003.

\subsubsection{NPDES radiological summary}

Summary bar charts for plant-site radioactive discharges are shown in Figs. 2.2.14 through 2.2.17. These charts depict the 5-year trend for the discharge of uranium (in $\mathrm{Ci}$ and $\mathrm{kg} \mathrm{U}$ ), ${ }^{99} \mathrm{Tc}$, and uranium daughters discharged to surface waters through NPDES outfalls. Radiological data by individual outfall locations are also given in Tables 2.2.2 through 2.2.4. These data are given on a concentration basis for total alpha and betagamma radioactivity and total uranium. See Table 2.2.2 of Part 2 of this report for detailed sampling and analyses of all locations.

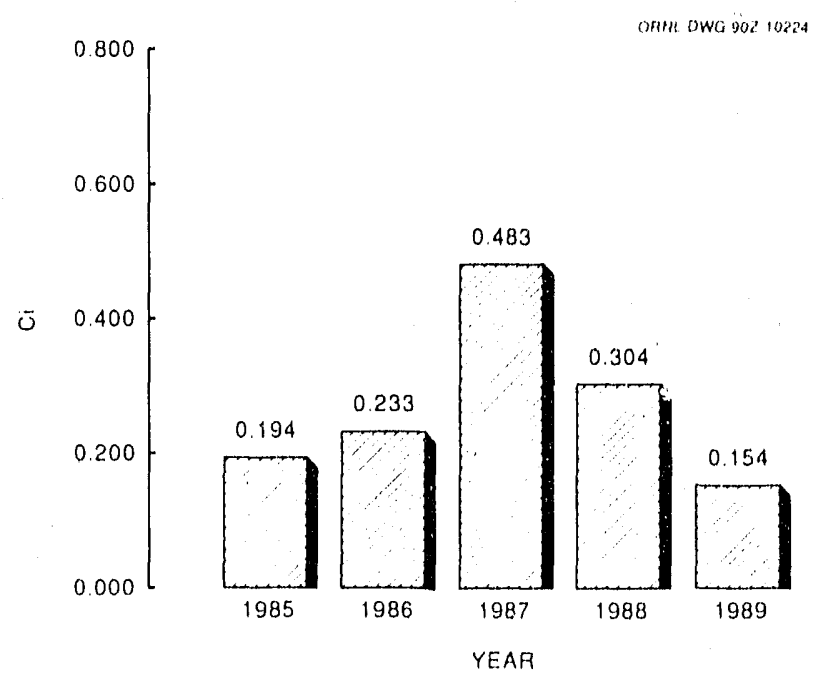

Fig. 2.2.14. Total curies of uranium discharged to surface water.

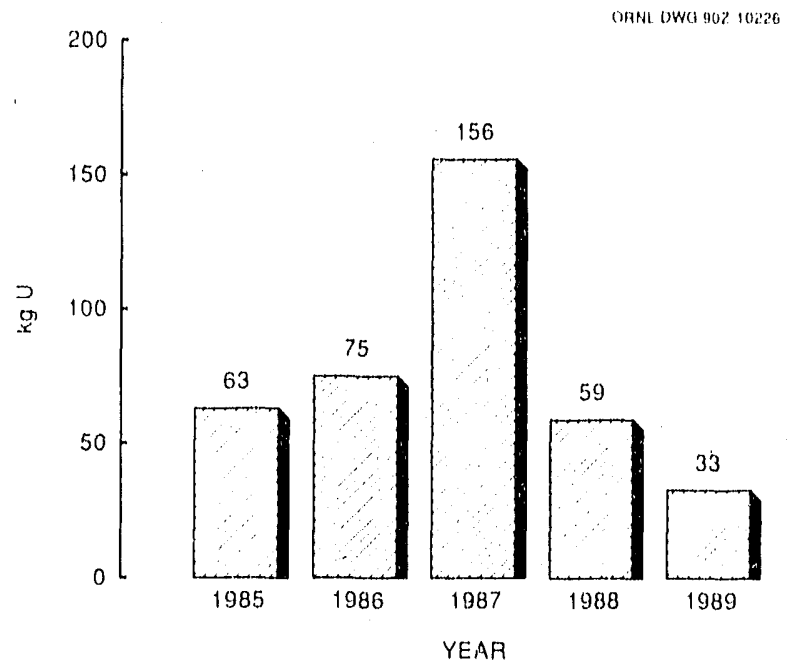

Fig. 2.2.15. Total kilograms of uranium discharged to surface water.

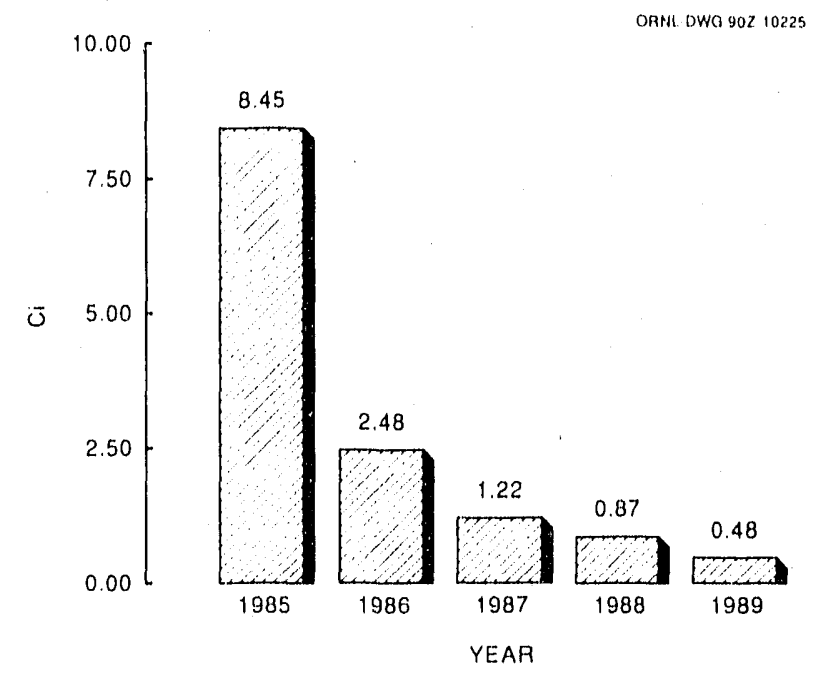

Fig. 2.2.16. Total discharges of ${ }^{99}$ Tc to surface water.

As is shown in Fig. 2.2.14, a total of $0.154 \mathrm{Ci}$ of uranium was discharged in 1989. This was equivalent to $33 \mathrm{~kg}$ of uranium (Fig. 2.2.15). This is a corsiderable decrease from the years 1985 through 1988. The bulk of the uranium discharged was through outfall 001 (52\%) to Little Beaver Creek. The remaining uranium was discharged through outfall 004 (4\%), outfall 003 (sewage treatment plant) (34\%), and outfall $009(3 \%)$; the remaining $7 \%$ was distributed among the remaining outfalls. The decrease in uranium 


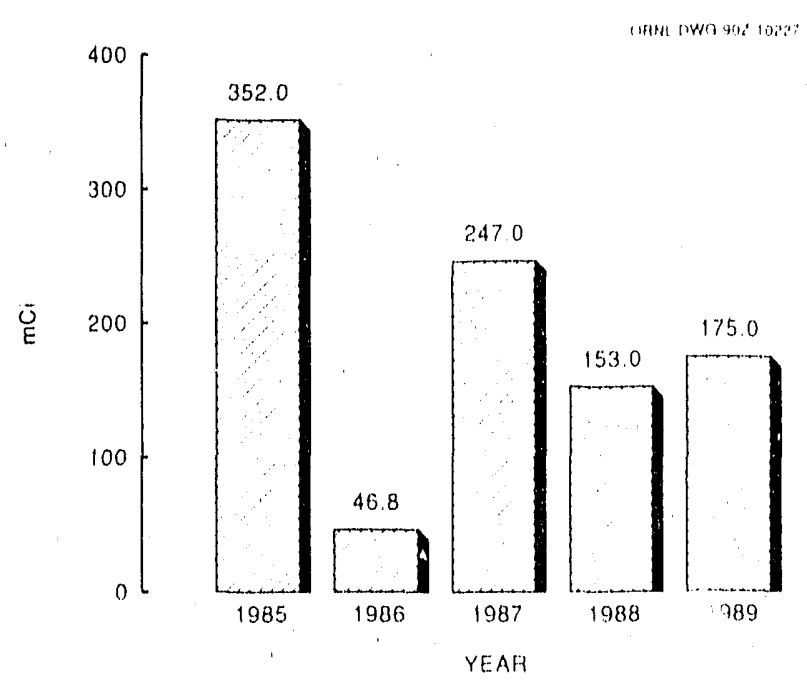

Fig. 2.2.17. Total discharges of uranium daughter products to surface water.

discharges can be attributed to operation of the new X-705 waste treatment facility using microfiltration technology for removal of uranium from X-705 decontamination activities. Because the microfiltration process discharges to the plant's $\mathrm{RCW}$ blowdown line and is further treated at the $\mathrm{X}-616$ chromate treatment facility (004), there is a measurable increase of uranium at the 004 effluent not seen in previous years (see Table 2.2.4). This increase in uranium is responsible for the $4 \%$ of the total plant uranium being discharged at outfall 004.

On a mass basis the major portion of the uranium at PORTS is the isotope ${ }^{238} \mathrm{U}$, but most of the alpha radioactivity comes from the isotope ${ }^{234} \mathrm{U}$. Individual isotopic measurements are not made at NPDES outfalls. They can be calculated from the alpha radioactivity data and the cascade gradient.

As is shown in Fig. 2.2.16, a total of $0.48 \mathrm{Ci}$ of technetium was discharged in 1989. This is a considerable decrease from 1985 through 1988. This decrease can be attributed to improved technetium removal in the ion exchange section of the $\mathrm{X}-705$ raffinate treatment system, to water conservation efforts in X-705, to decontamination efforts, and in part, to operation of the new X-705 microfilter. In addition, the effluent from the ion exchange columns is redirected to the X-700 biodenitrification facility and the X-6619 sewage treatment plant, which may further reduce technetium levels. Technetium, a weak beta ernitter, accounts for the bulk of the radioactivity release $(59 \%)$ from the facility, Of the total technetium released, $32 \%$ was discharged through outfall 001 to Little Beaver Creek; 11\%, through outfall 003 to the Scioto River; and 51\%, through outfall 004 also to the Scioto River. The remaining $6 \%$ was distributed among the remaining outfalls.

As is shown in Fig, 2.2.17, the total millicuries of uranium daughter products discharged to surface water in 1989 was $175 \mathrm{mCi}$. This was a slight increase from 1988 but less than that discharged during the years 1985 and 1987. Uranium daughter products are generated by the natural radioactive decay of uranium and therefore roughly follow uranium emissions. Because ${ }^{238} \mathrm{U}$ generates the bulk of the detectable uranium daughters, the proportion of uranium daughter products to total uranium will vary with the uranium assay. Uranium daughter products account for $22 \%$ of all curies discharged. Seventyseven percent of the daughter products discharged were through outfall 004 ; another $8 \%$, through outfall 001 . The remaining $15 \%$ was divided among the other NPDES outfalls.

Alpha and beta-gamma radioactivity and total uranium data presented in Tables 2.2.2 through 2.2.4 were derived from individual analyses data at each sampling location. NPDES outfall locations that were sampled in 1989 include $001,601,002$, 003, 004, 605, 009, 010, and 011 for Gaseous Diffusion Plant (GDP) outfalls and 001 through 003 for GCEP outfalls. These locations are shown in Fig. 2.2.2.

Outfall 605 (see Fig. 2.2.2) currently has the most concentrated radioactive discharge from plant operations. This outfall replaced the X-701B holding pond (NPDES outfall 601) in November 1988. Treated wastewater from this new X-705 microfilter is discharged to the RCW blowdown line and is further treated at the X-616 chromate treatment facility (NPDES 004) and discharged to the Scioto River. However, effluent flow from this new facility averaged only $5000 \mathrm{gal} / \mathrm{d}$ during 1989. Also included in Tables 2.2.2 through 2.2.4 are radioactivity data at upstream and downstream 
locations in the surface waters (designated as RW loactions) that receive discharges from these NPDES outfalls. These locations are shown in Fig. 2.2.3.

\subsubsection{NPDES nonradiological summary}

The locations of NPDES discharge outfalls are shown in Fig. 2.2.2. The 1985-1989 percentages of compliance at the east drainage ditch (NPDES 001) for total suspended solids (TSS), oil and grease (O\&G), total residual chlorine (TRCL), and pH are given in Figs. 2.2.18 through 2.2.21. Compliance for $\mathrm{O} \& \mathrm{G}$ and $\mathrm{pH}$ was 100\%; compliance for TSS and TRCL was $97 \%$ and $97 \%$ respectively. Two TSS exceedances were the reason for the $97 \%$ compliance and were the results of heavy rainfall runoff. Two chlorine exceedances during 1989 account for the 97\% compliance. Exact reasons for these chlorine exceedances could not be readily determined. Heavy use of chlorinated sanitary drinking ivater and reduced retention times in the X-230J7 pond for dissipation are suspected reasons. An investigation to reduce the use of chromated water is ongoing.

The 1985-1989 percentages of compliance at the X-701B holding pond (NPDES 001A) for total zinc, total iron, and total chromium are given in

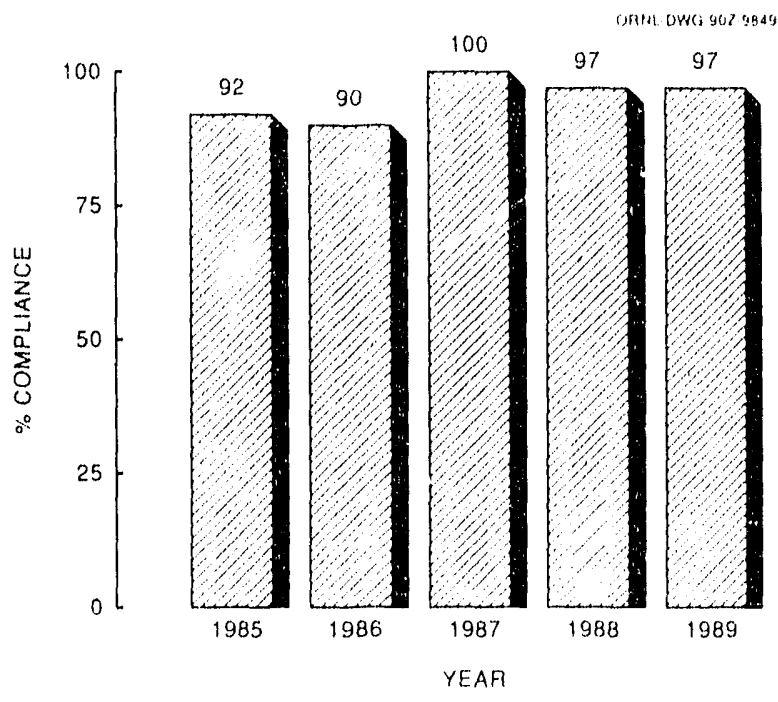

Fig. 2.2.18. NPDES compliance for total suspended solids at the east drainage ditch (001).

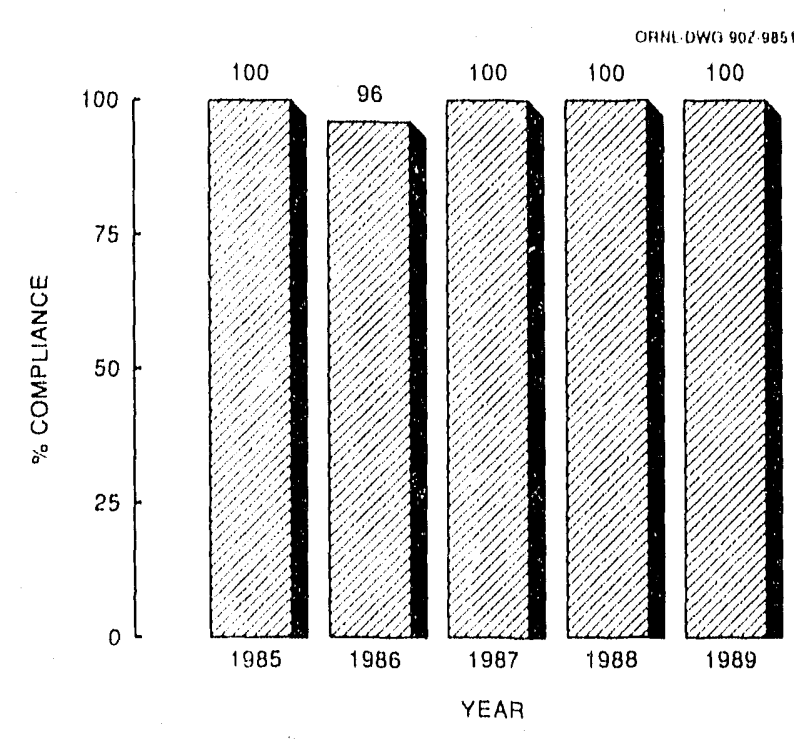

Fig. 2.2.19. NPDES compliance for oil and grease at the east Arainage ditch (001).

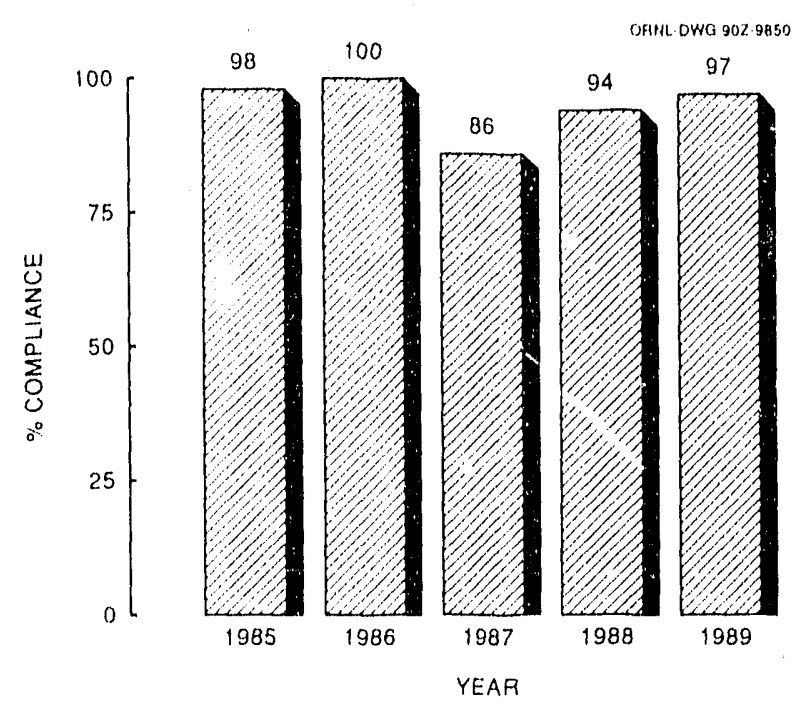

Fig. 2.2.20. NPDES compliance for total residual chlorine at the east drainage ditch (001).

Figs. 2.2.22 through 2.2.24. Effluent from the $\mathrm{X}-701 \mathrm{~B}$ holding pond was sporadic in 1989 as all process discharges to the pond were halted in early November 1988 to comply with RCRA closure requirements. Discharges from the pond were the result of rainfall runoff and/or groundwater infiltration. During days when an effluent flow was observed, compliance with a!l NPDES parameters was $100 \%$ 


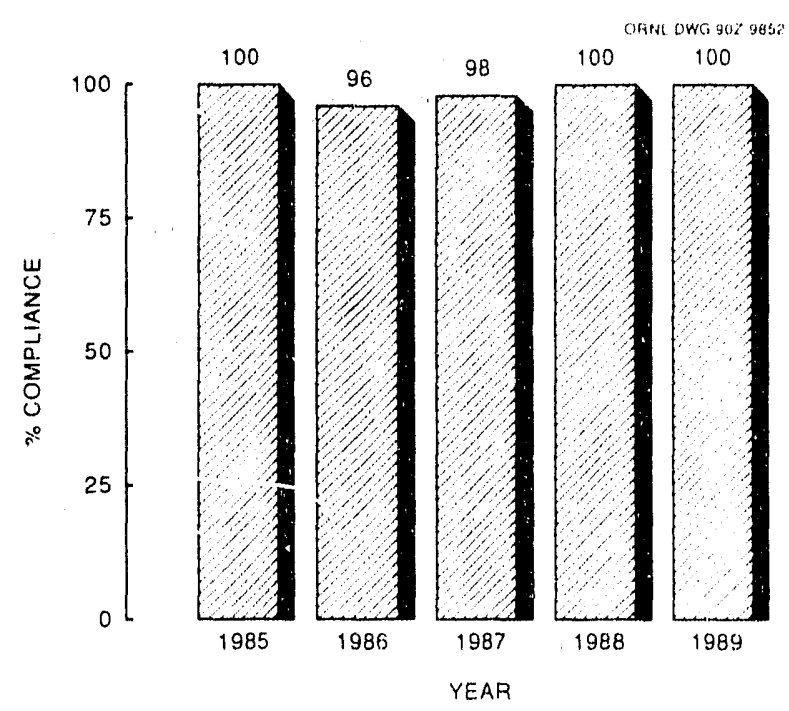

Fig. 2.2.21. NPDES compliance for pH at the east drainage ditch (bwl).

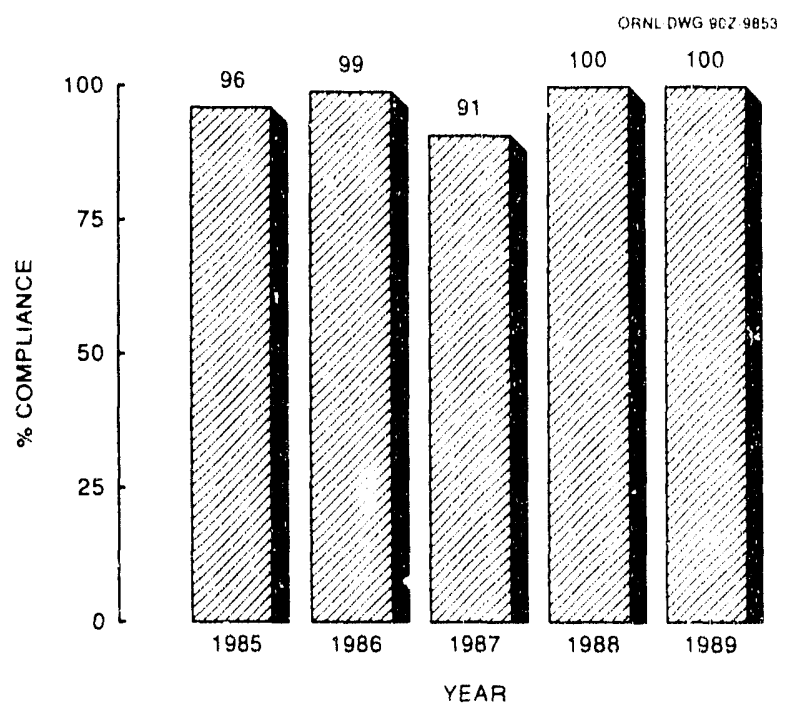

Fig. 2.2.22. NPDES compliance for total zinc at the $X-701 B$ bolding pond (001A).

The 1985-1989 percentages of compliance at the south holding pond (NPDES 002) for TSS, $\mathrm{O} \& \mathrm{C}, \mathrm{TRCL}$, and $\mathrm{pH}$ are given in Figs. 2.2.25 through 2.2.28. Compliance remains high for all parameters except for total suspended solids. The eight exceedances for TSS resulting in the $88 \%$ compliance were caused by frequent rains during

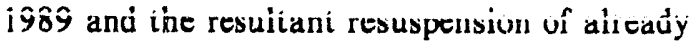

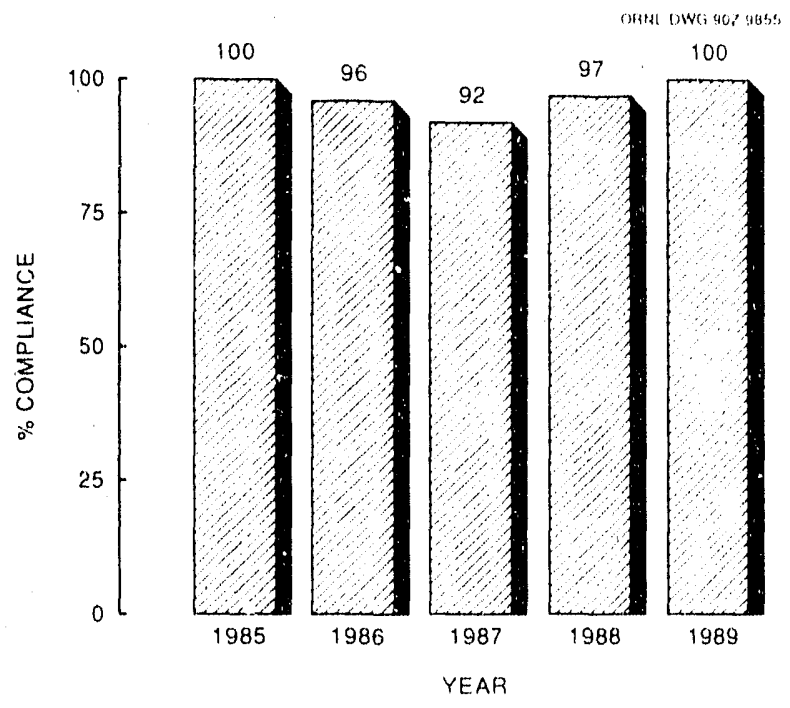

Fig. 2.2.23. NPDES compliance for total iron at the $\mathrm{X}-701 \mathrm{~B}$ holding pond (001A).

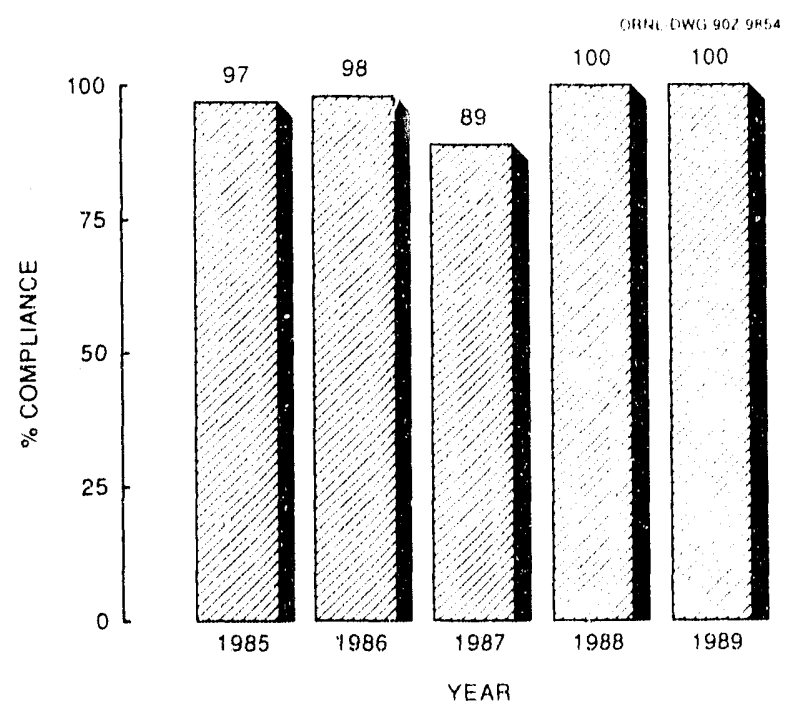

Fig. 2.2.24. NPDES compliance for total chromium at the X-701B holding pond (001A).

settled silt. The one $\mathrm{pH}$ exceedance resulting in the $98 \%$ compliance was caused by algae photosynthesis and diurnal variations of $\mathrm{pH}$.

The 1985-1989 percentages of compliance at the X-621 coal pile treatment plant (NPDES 002A) for TSS, total iron, total manganese, and $\mathrm{pH}$ are given in rigs. 2.2.29 through 2.2.32.

Ôvzraii compiiance improved over 1988 becauste of 


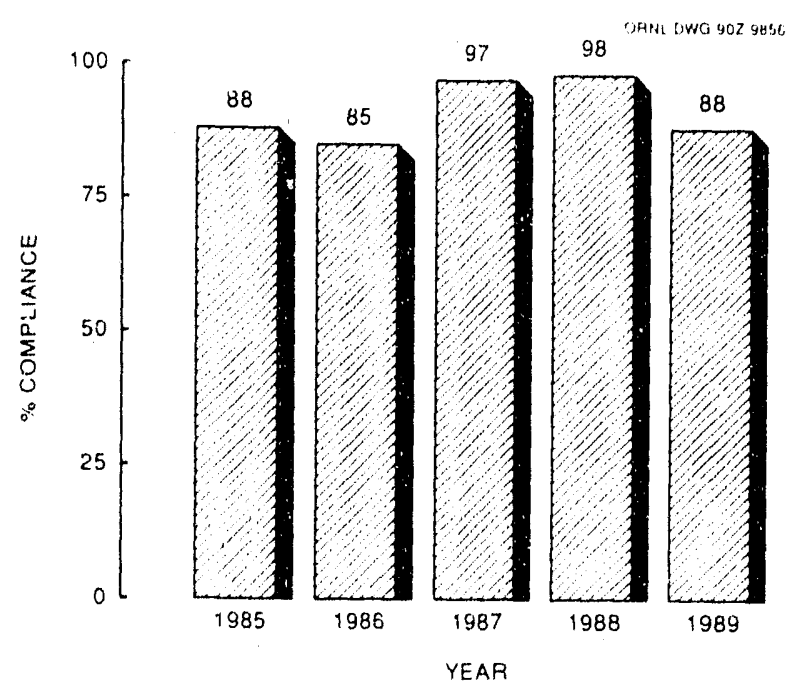

Fig. 2.2.25. NPDES compliance for total suspended solids at the south bolding pond $(002)$.

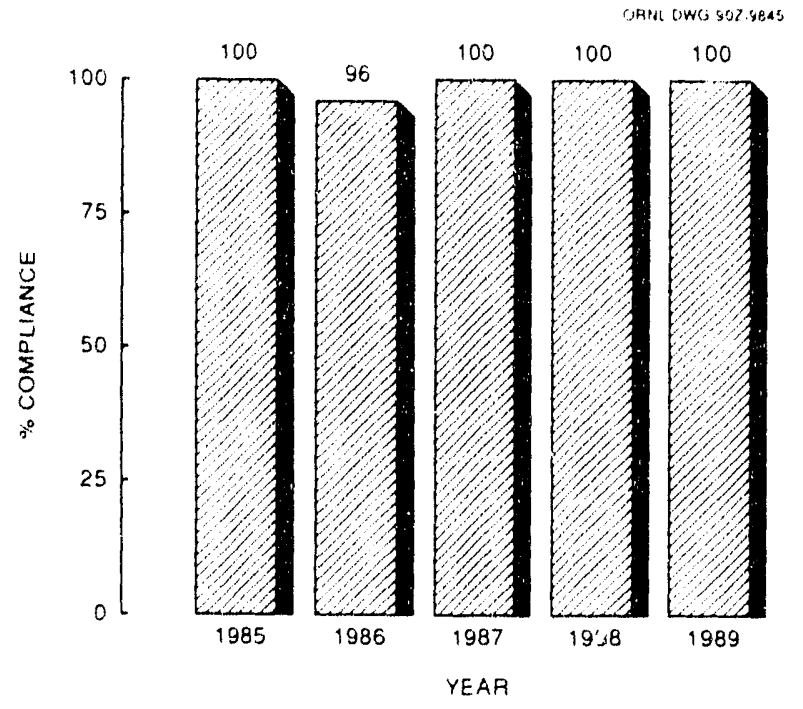

Fig. 2.2.26. NPDES complianice for oil and grease at the south bolding pond (002).

1987 modifications that became fully operational in mid-1988; this improved operation and maintenance. Relaxed limitations are expected in the next NPDES permit in lieu of additional modifications to achieve $100 \%$ compliance.

The 1985 1989 percentages of compliance at the X-6619 sewage treatment plant (NPDES 003) for biochemical oxygen demand, TSS, fecal coliform, pH, and TRCL are given in Figs. 2.2.33

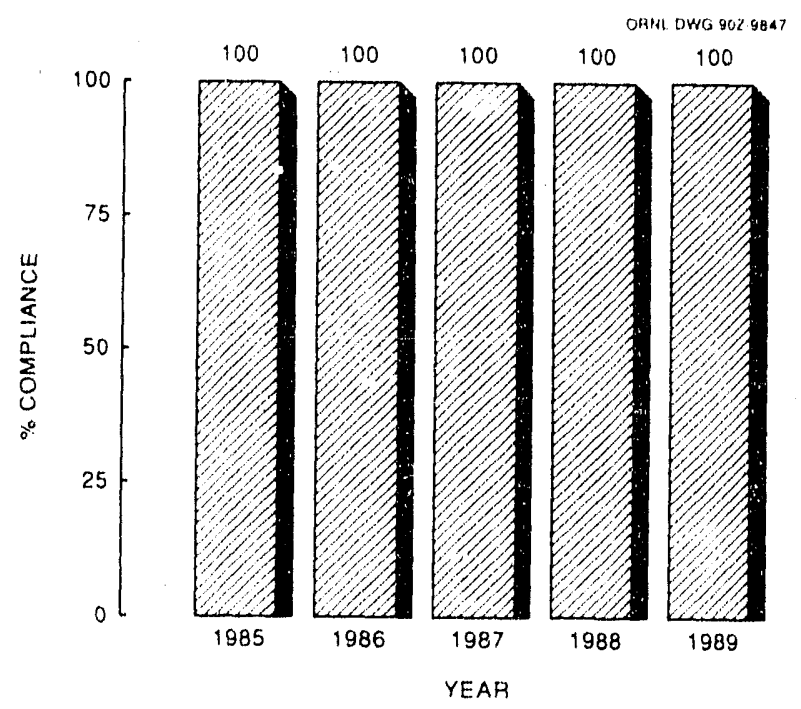

Fig. 2.2.27. NPDES compliance for total residual chlorine at the south holding pond (002).

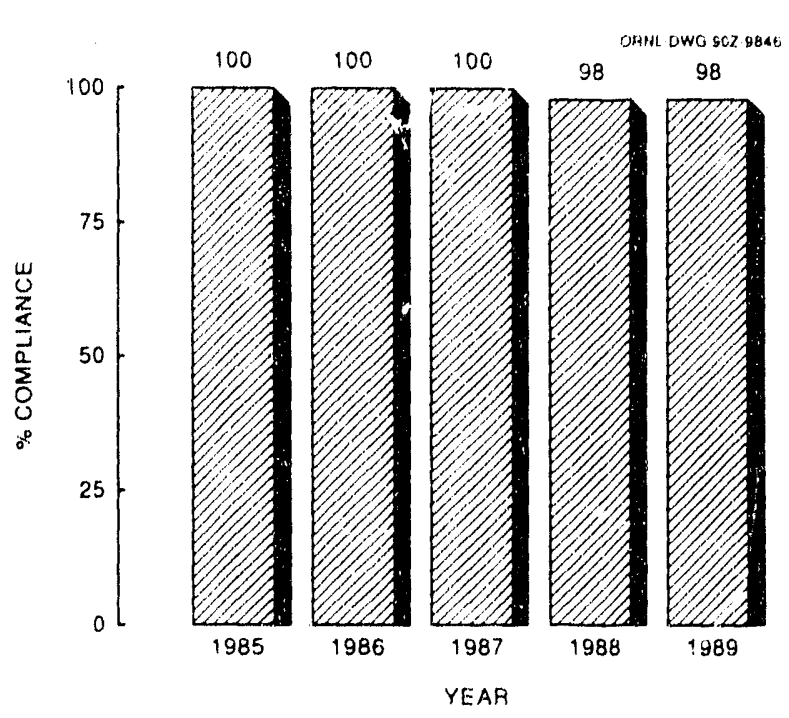

Fig. 2.2.28. NPDES compliance for pH at the south bolding pond (002).

through 2.2.37. Compliance continues to remain high $(100 \%)$ for all parameters. There were no exceedances in 1989 at this outfall.

The 1985-1989 percentages of compliance at the biodenitrification facility (NPDES 003B) for nitrate, total copper, total zinc, total iron, nickel, and $\mathrm{pH}$ are given in Figs. 2.2.38 through 2.2.43. Compliance is high for all parameters. Occasional exceedances of $\mathrm{NO}_{3}-\mathrm{N}$, zinc, iron, and $\mathrm{pH}$ were a 


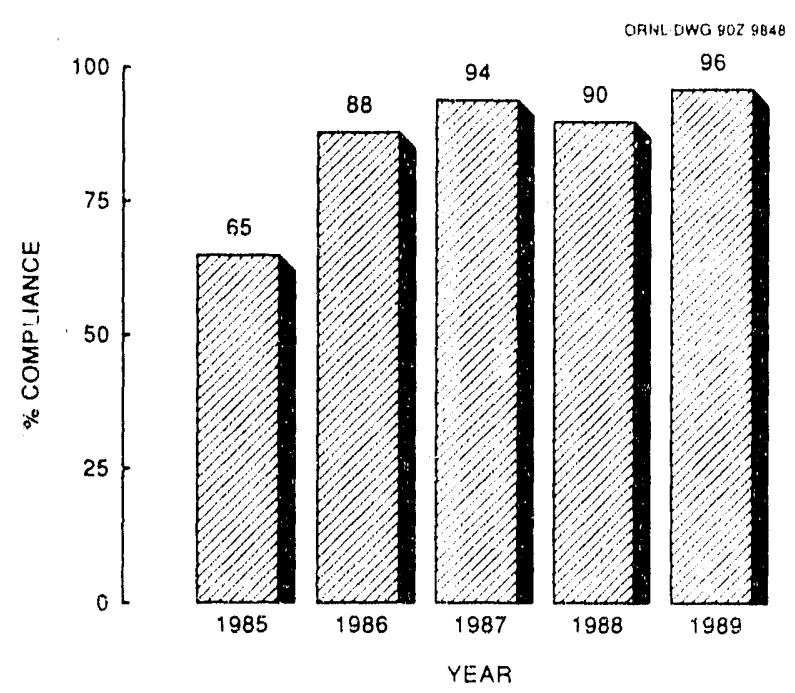

Fig. 2.2.29. NPDFS compliance for tota' suspended solids at the X-621 coal pile treatment plant $(002 \mathrm{~A})$.

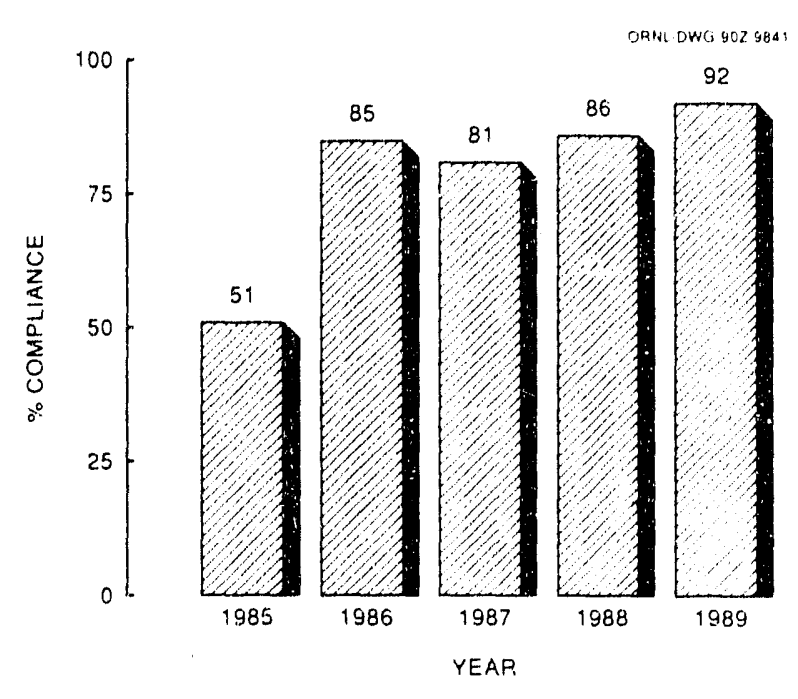

Fig. 2.2.30. NPDES compliance for total iron st the $X-621$ coal pile treatment plant $(002 A)$.

result of either operator error, maintenance activities, or discrepancies between analyses used for NPDES reprorting and those used for operational purposes. Three total zinc exceedances resulted in a $97 \%$ compliance rate and were caused by high use of sanitary drinking water containing zinc additives in the biodenitrification process. Two total iron excecdances resulted in a $98 \%$ compliance and was caused by the optimization

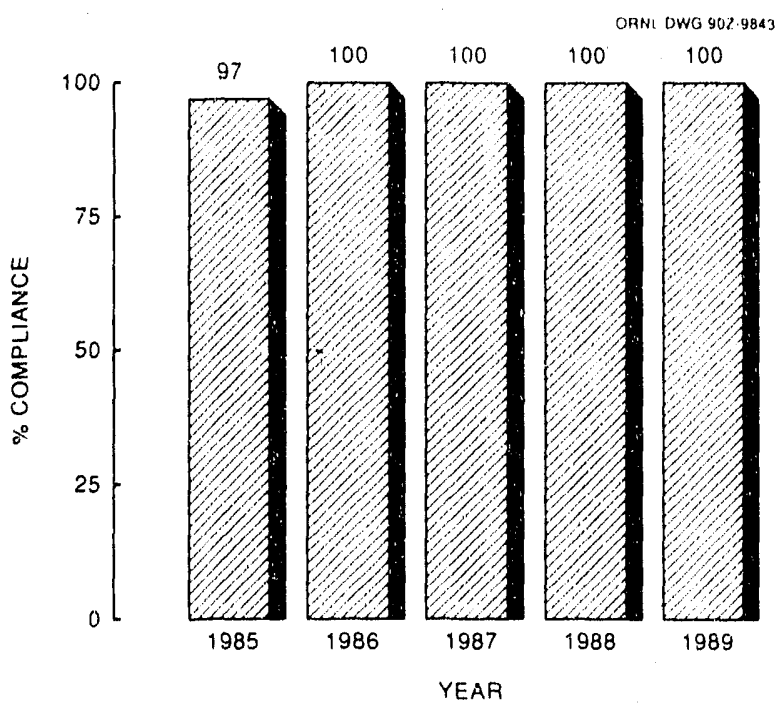

Fig. 2.2.31. NPDES compliance for total manganese at the X-621 coal pile treatment plant (002A).

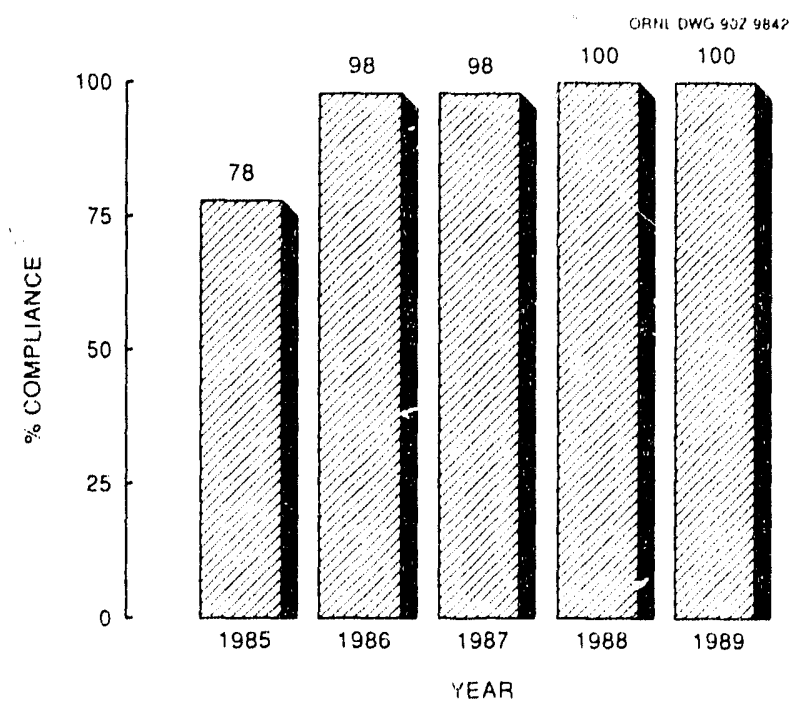

Fig. 2.2.32. NPDES compliance for $\mathrm{pH}$ at the $\mathrm{X}-621$ coal pile treatment plant $(002 \mathrm{~A})$.

efforts described above. One $\mathrm{pH}$ exceedance produced a $98 \%$ compliance rate and was the result of operator error in maintaining a proper system $\mathrm{pH}$ range.

The 1985-1989 percentages of compliance at the X-616 chromate treatment facility (NPDES 004) for total dissolved solids, TSS, total zinc, hexavalent chromi:!m, total chromium, total iron, total copper, TRCL, trichloroethylene (TCE), and 


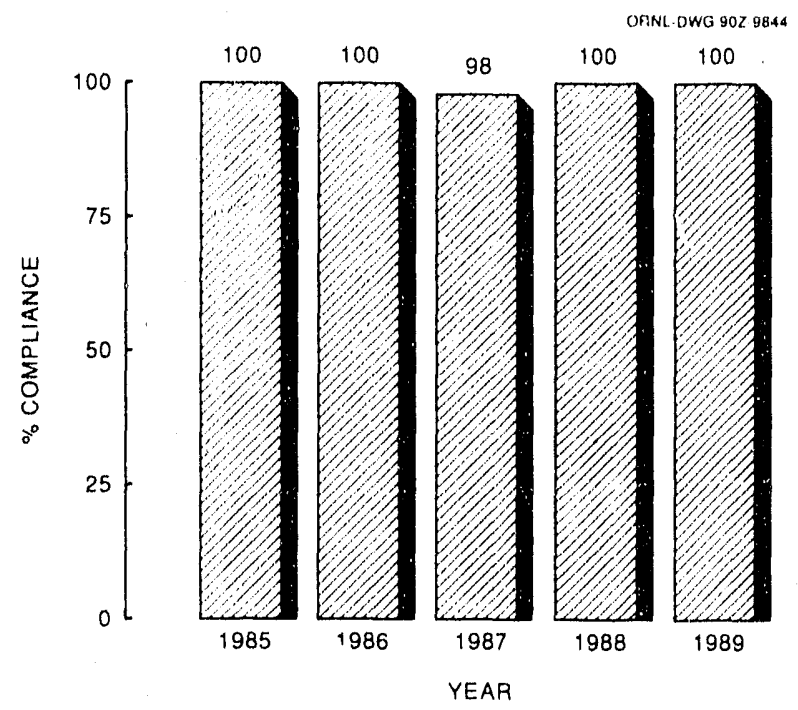

Fig. 2.2.33. NPDES compliance for biochemical oxygen demand at the $X-6619$ sewage treatment plant (003).

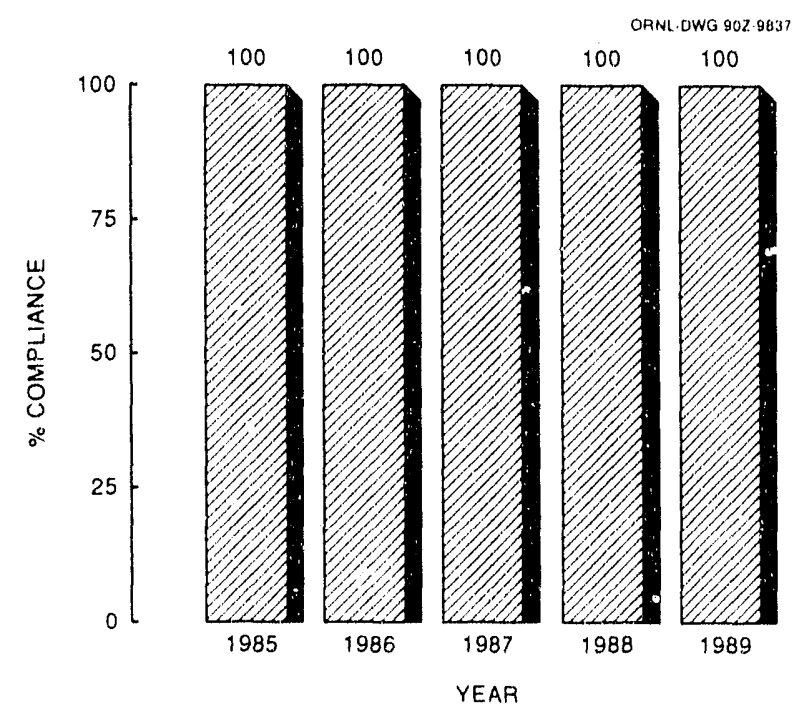

Fig. 2.2.34. NPDES compliance for total suspended solids at the X-6619 sewage treatment plant (003).

$\mathrm{pH}$ are given in Figs. 2.2.44 through 2.2.53. Compliance remains high (100\%) for all parameters except total chromium. Three exceedances for total chromium resulting in $95 \%$ compliance occurred because of high operating flow rates that reduced retention time and treatment efficiency.

Percentage of compliance graphs are not presented for NPDES outfalls 005, 006, 007, 008,

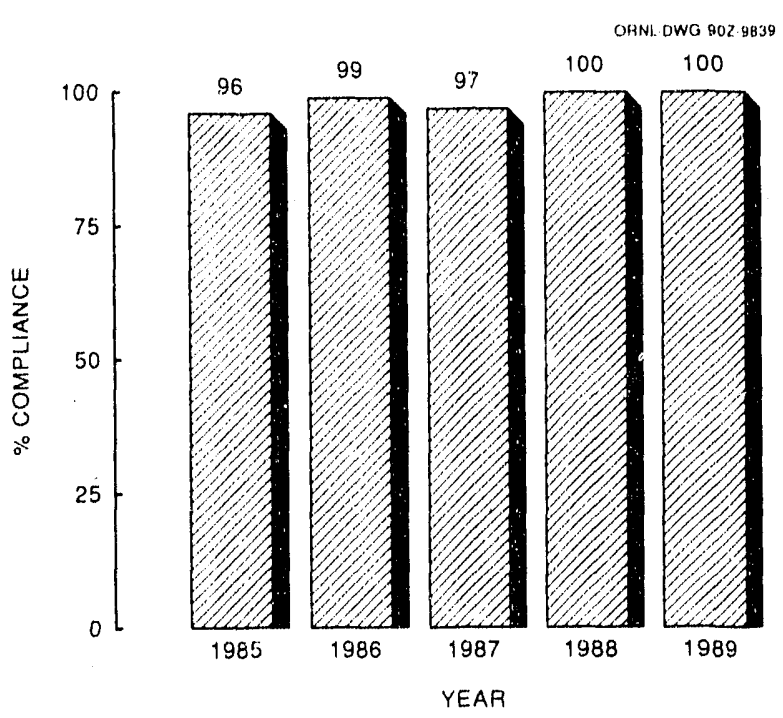

Fig. 2.2.35. NPDES compliance for fecal coliform at the $X-6619$ sewage treatment plant (003).

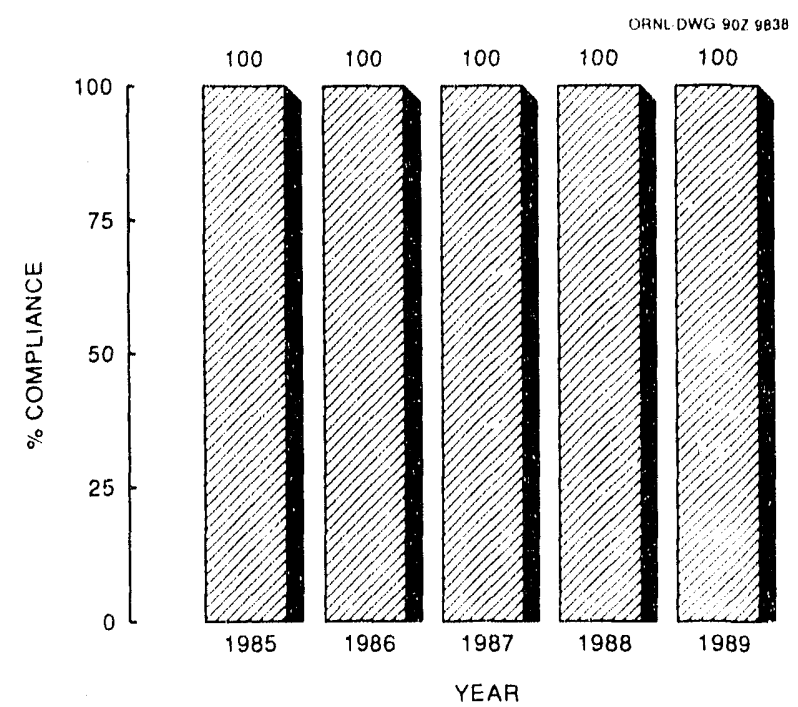

Fig. 2.2.36. NPDES compliance for $\mathrm{pH}$ at the $\mathrm{X}-6619$ sewage treatment plant $(003)$.

009A, and GCEP 003. Effluent flows were very infrequent at these outfalls during 1989.

The 1985-1989 percentages of compliance at the north holding pond (NPDES 009) for TSS, O\&G, TRCL, and $\mathrm{pH}$ are given in Figs. 2.2.54 through 2.2 .57 . One daily maximum and four monthly average exceedances resulted in $92 \%$ TSS compliance at this outfall. All exceedances were the result of seasonal heavy rainfall runoff. 


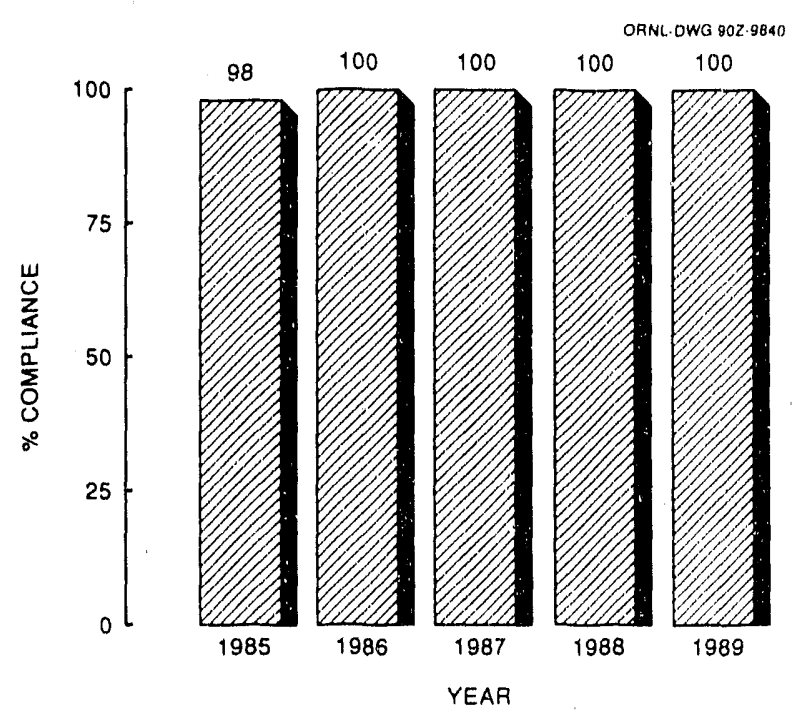

Fig. 2.2.37. NPDES compliance for total residual chlorine at the $\mathrm{X}-6619$ sewage treatment plant (003).

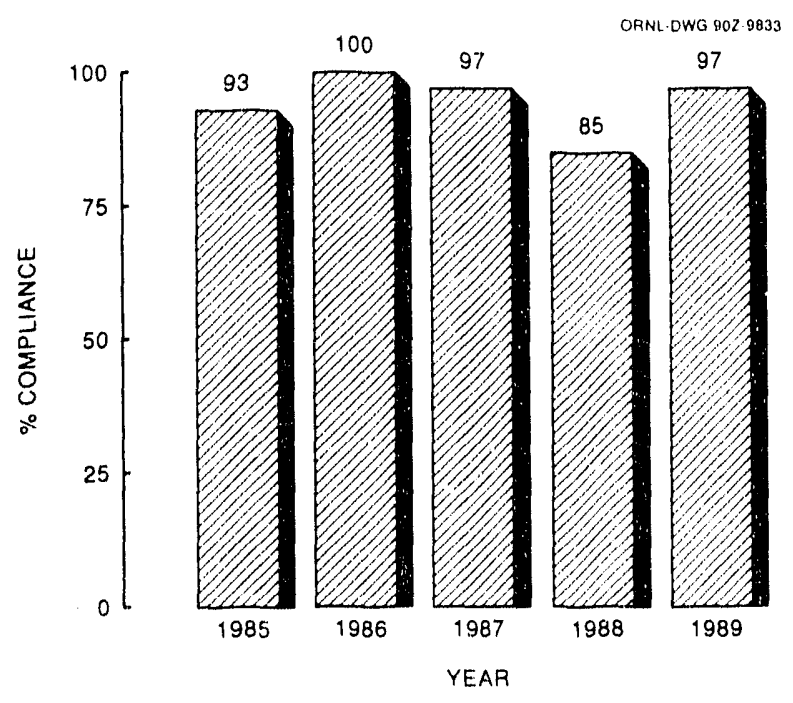

Fig. 2.2.38. NPDES compliance for nitrate at the biodenitrification facility (003B).

Compliance for all other parameters was $100 \%$.

The 1985-1989 percentages of compliance at the west drainage ditch (NPDES 010) and northeast drainage ditch (NPDES 011) for O\&G and $\mathrm{pH}$ are given in Figs. 2.2.58 through 2.2.61. Compliance remains high. Exceedances of one $\mathrm{pH}$ (NPDES 010) and two pH (NPDES 011) resulted in tine 98 and $96 \% \mathrm{pH}$ compliances, respectively, and were the result of algae blooms and the

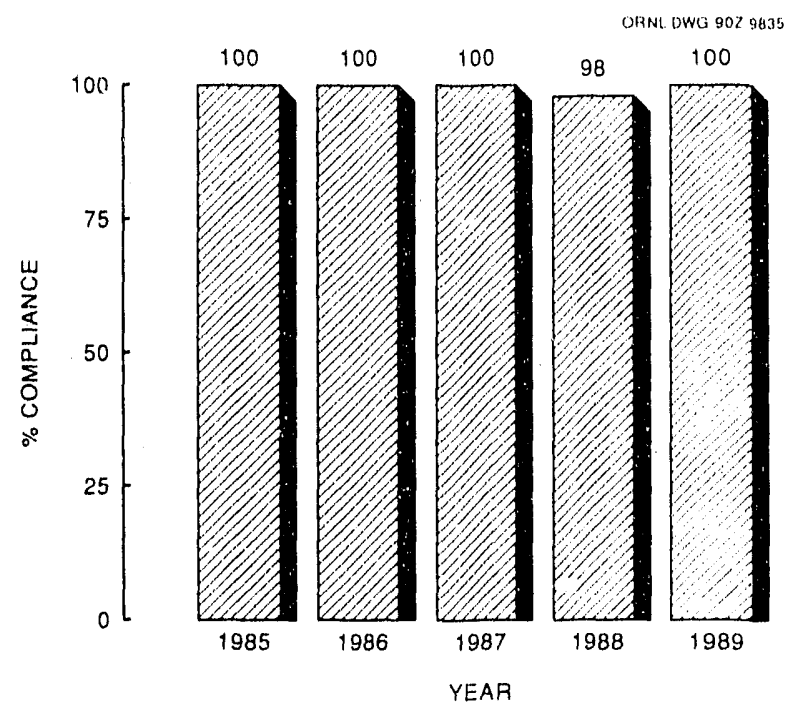

Fig. 2.2.39. NPDES compliance for total copper at the biodenitrification facility (003B).

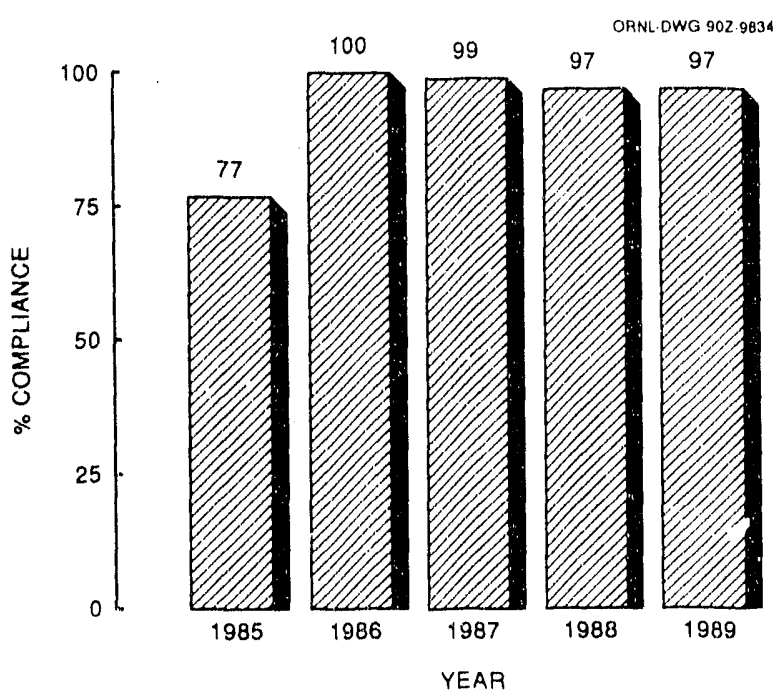

Fig. 2.2.40. NPDES compliance for total zinc at the biodenitrification facility (003B).

associated natural $\mathrm{pH}$ variations that caused the $\mathrm{pH}$ to slightly exceed the upper limit of 9.0 standard units.

The 1985-1989 percentages of compliance at the two GCEP ponds NPDES G001 and NPDES G002 for TSS, O\&G, and $\mathrm{pH}$ are given in Figs. 2.2.62 through 2.2.67. Compliance for TSS and $O \& G$ continues to remain high at G001. Two daily maximum exceedances and three average 


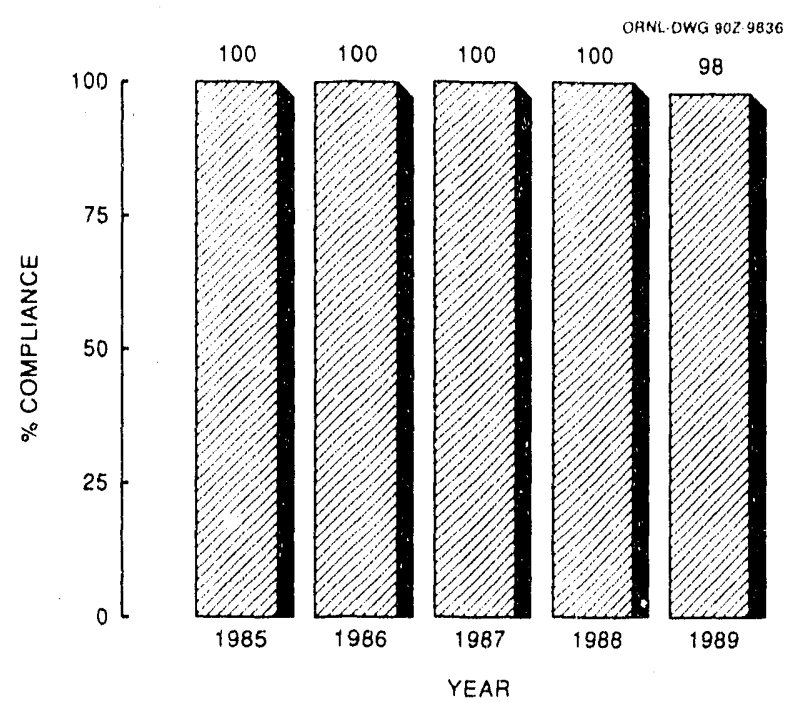

Fig. 2.2.41. NPDES compliance for total iron at the biodenitrification facility (003B).

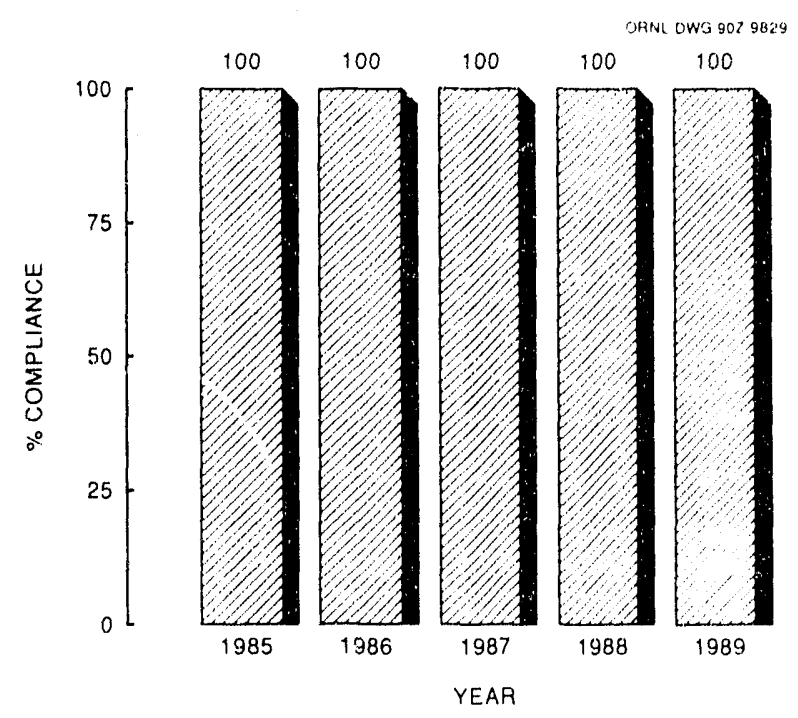

Fig. 2.2.42. NPDES compliance for nickel at the biodenitrification facility (003B).

monthly exceedances at GCEP 001, together with one daily maximum exceedance and two average monthly exceedances at GCEP 002 , were caused by heavy rainfall runoff and algae solids. Oil and grease compliance at both outfalls remained at $100 \%$.

The percentage of $\mathrm{pH}$ compliance at both GCEP ponds was $98 \%$. This is a result of stringent $\mathrm{pH}$ effluent limitations and algae photosynthesis and respiration. The OEPA recognizes the

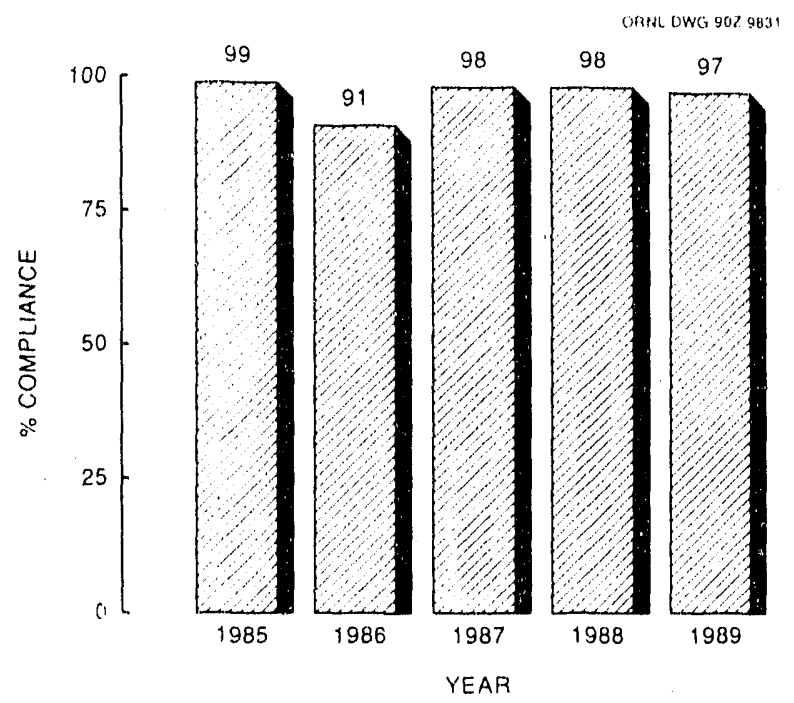

Fig. 2.2.43. NPDES compliance for $\mathrm{pH}$ at the biodenitrification facility (003B).

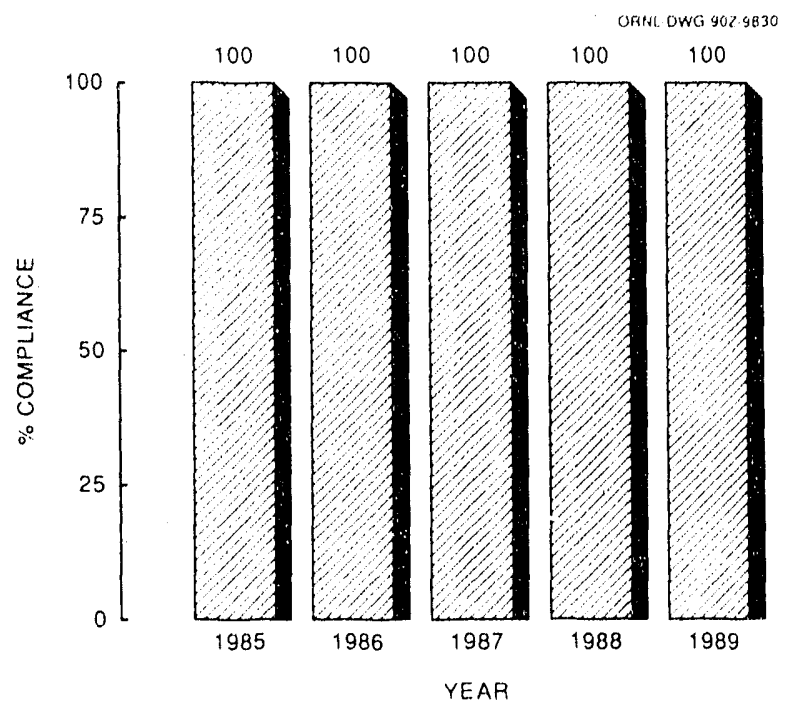

Fig. 2.2.44. NPDES compliance for total dissolved solids at the X-616 chromate treatment facility (004).

stringent $\mathrm{pH}$ limitations and, although it is unable to modify an existing expired permit, it has given the plant a letter stating that the $\mathrm{pH}$ violations are not serious, that no enforcement action will be taken against the plant because of these stringent limits, and that they intend to modify the $\mathrm{pH}$ limitations when the new NPDES permit is issued. Because of plant efforts to control algae growth, $\mathrm{pH}$ compliance has much improved over previous years. 


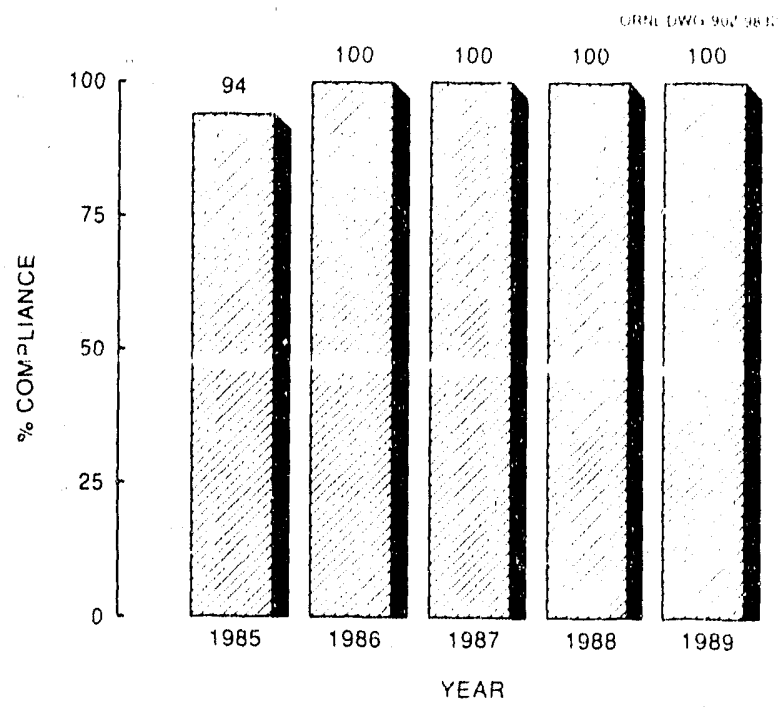

Fig. 2.2.45. NPDES compliance for total suspended solids at the X-616 chromate treatment facility (004).

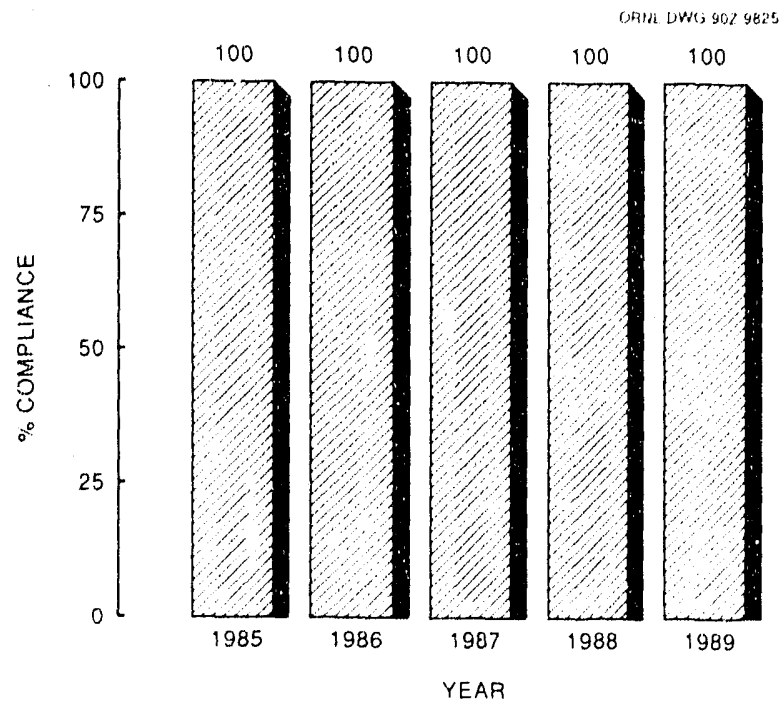

Fig. 2.2.46. NPDES compliance for total zinc at the $\mathrm{X}-616$ chromate treatment facility (004).

\subsubsection{Pollution Abatement Projects and Activities in 1989}

In 1987 construction began on a waste treatment facility to treat all wastewaters currently flowing from the $X-705$ decontamination building to the X-701B holding pond. This project was completed in early November 1988 and placed in operation in mid-November to meet the November

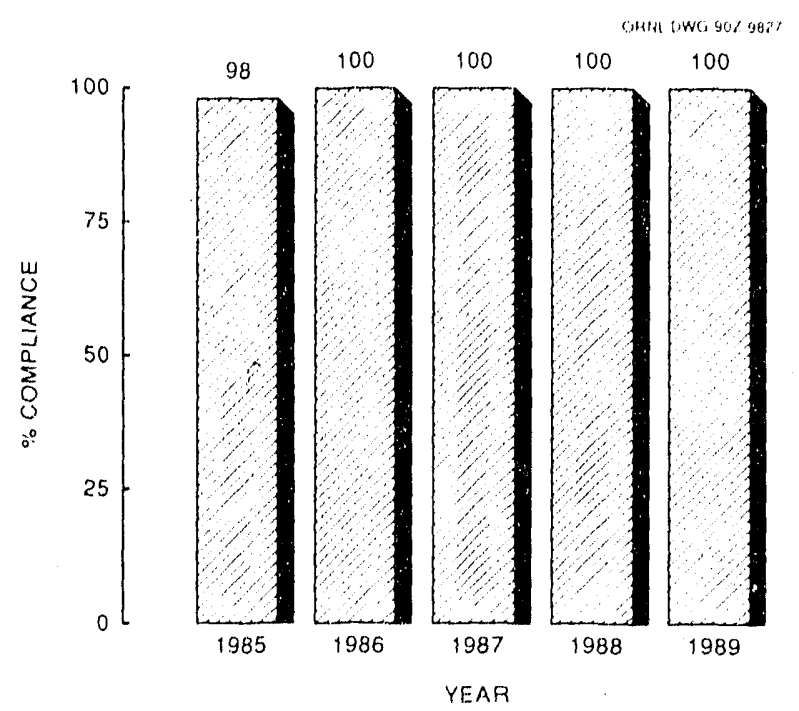

Fig. 2.2.47. NPDES compliance for hexavalent chromium at the X-616 chromate treatment facility (004).

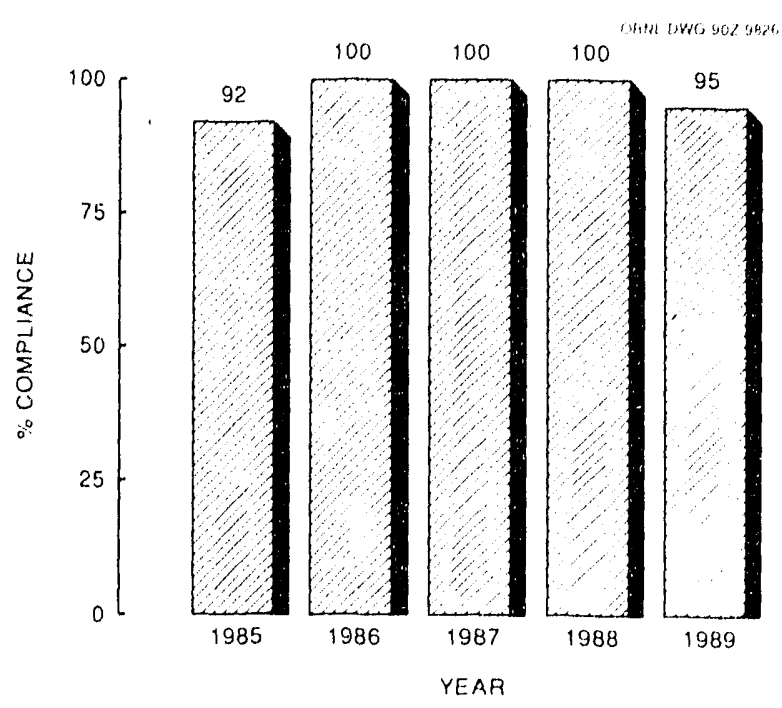

Fig. 2.2.48. NPDES compliance for total chromium at the X-616 chromate treatment facility (004).

8, 1988, RCRA closure requirement for the $X-701 \mathrm{~B}$ holding pond. Operations and optimization of this facility continued through CY 1989. The new X-705 waste treatment facility, which is installed in the X-705 decontamination building, uses microfiltration and pressure filtration technology to treat all process waters produced in the $\mathrm{X}-705$ building. Discharge is to the $\mathrm{RCW}$ blowdown line, which is then further treated at the 


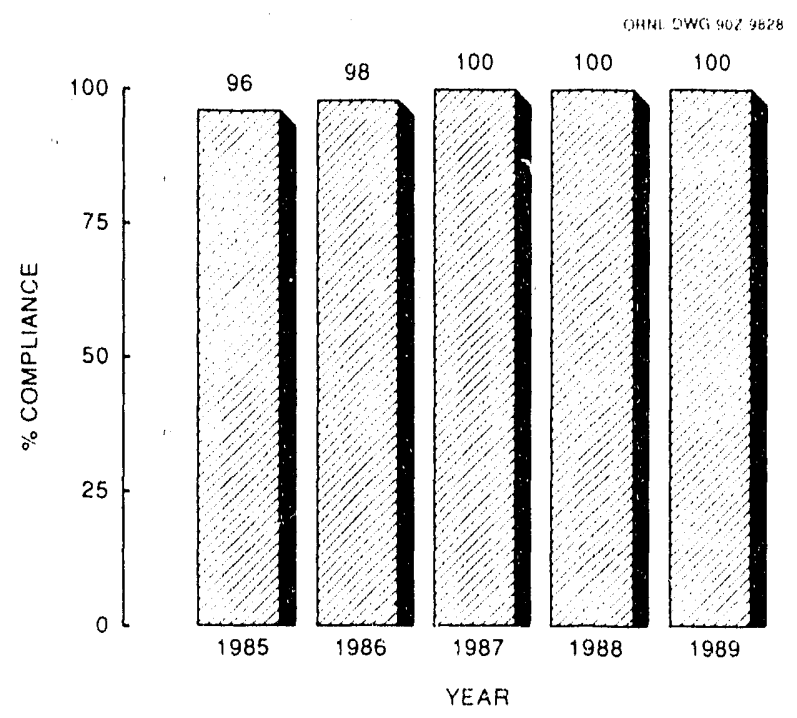

Fig. 2.2.49. NPDES compliance for total iron at the $\mathrm{X}-616$ chromate treatment facility (004).

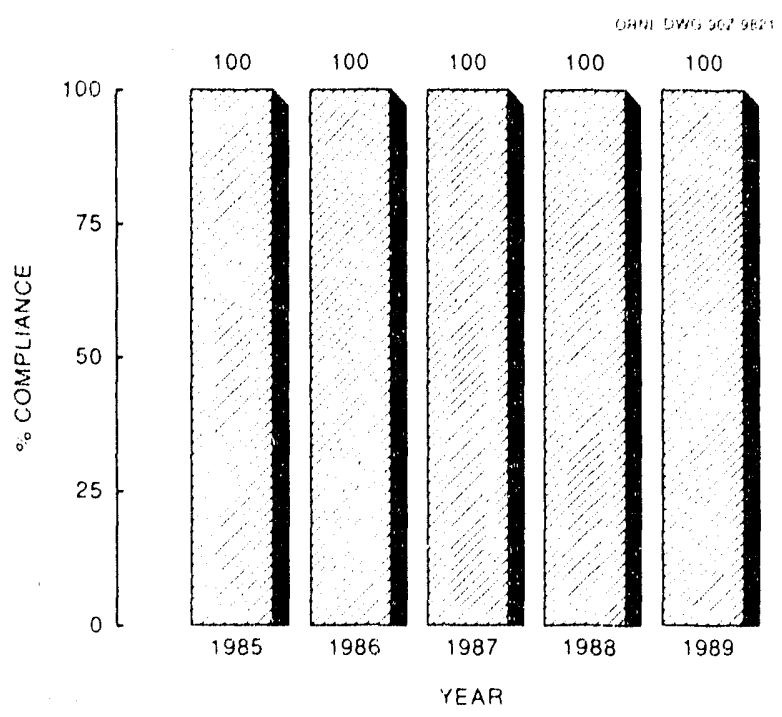

Fig. 2.2.50. NPDES compliance for total copper at the $X-616$ chromate treatment facility (004).

$\mathrm{X}-616$ chromate treatment facility before being discharged to the Scioto River. Building drainage, noncontact cooling water, and evaporator condensate that are uncontaminated from X-70s processes are discharged directly to the east drainage ditch, NPDES outfall 001 , and finally to Little Beaver Creek in accordance with the project OEPA Permit to Install (PTI) agreement.

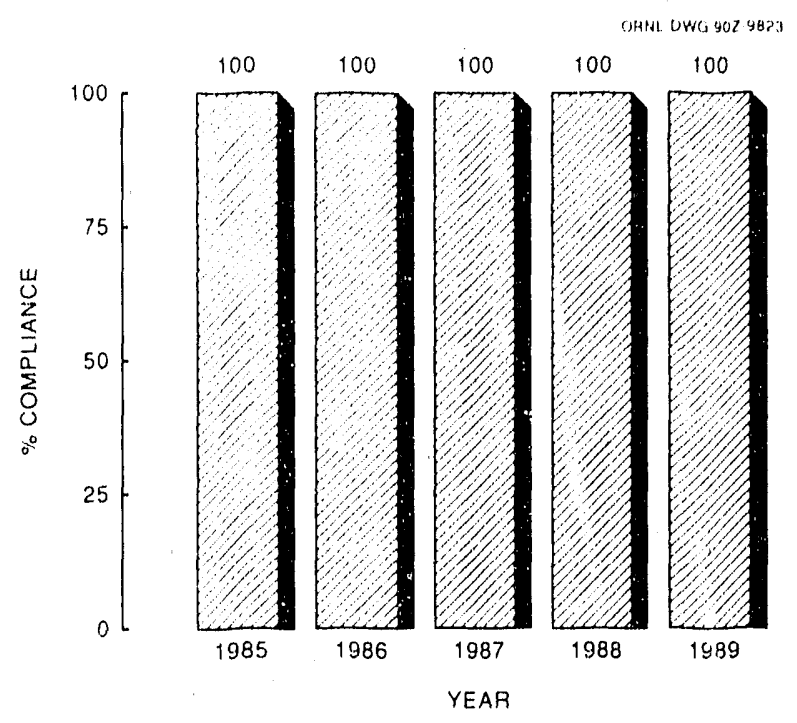

Fig. 2.2.51. NPDES compliance for total residual chlorine at the X-616 chromate treatment facility (004)).

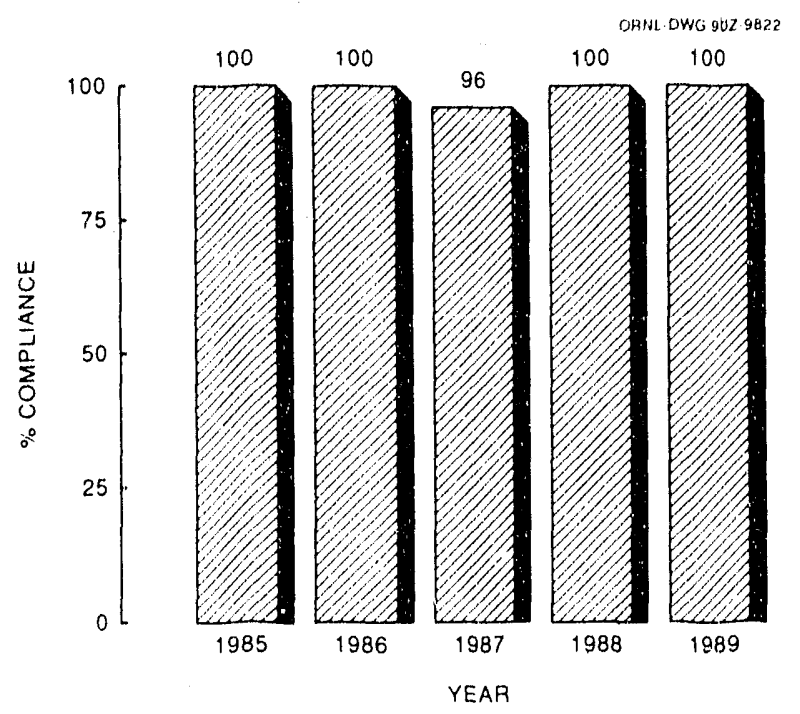

Fig. 2.2.52. NPDES compliance for trichloroethylene at the X-616 chromate treatment facility (004).

Optimization of the full-scale biodenitrification facility located in the X-700 building continued through 1988. Startup began in May 1987; however, the facility experienced numerous startup problems and frequently exceeded effluent limitations. Optimization efforts focused on improving nitrate reduction efficiency and improving metals removal. Detailed reports 


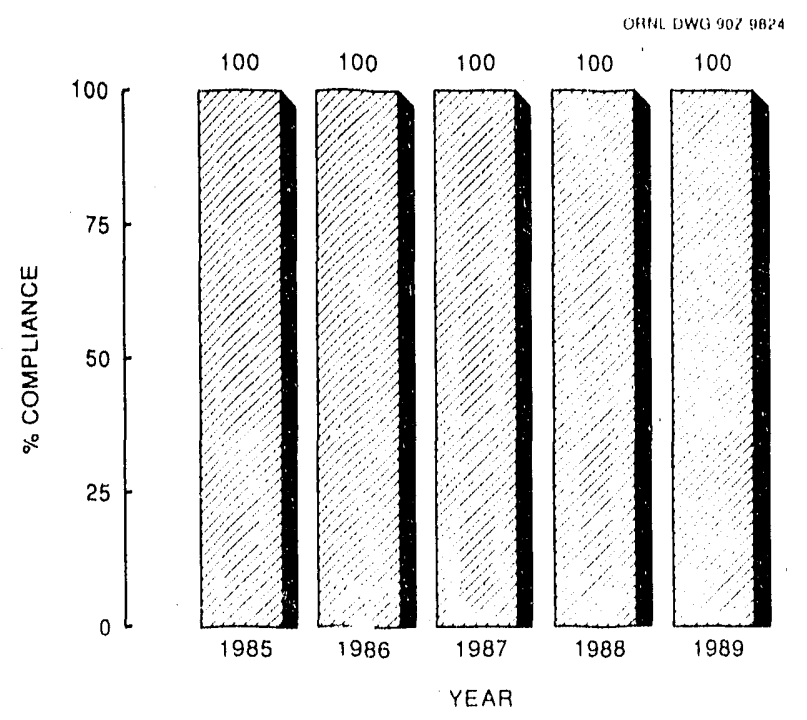

Fig. 2.2.53. NPDES compliance for $\mathrm{pH}$ at the $\mathrm{X}-616$ chromate treatment facility (004).

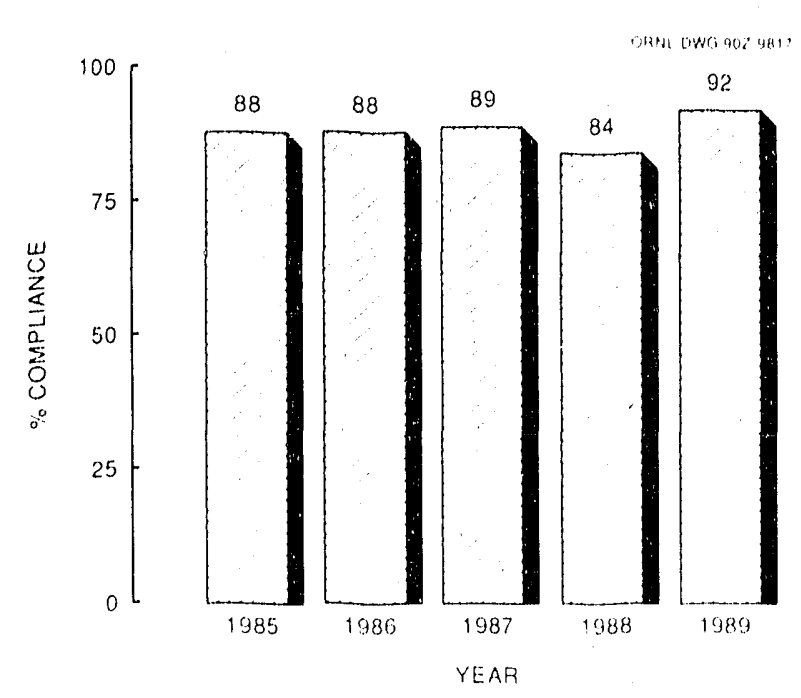

Fig. 2.2.54. NPDES compliance for total suspended solids at the north holding pond (009).

covering the optimization efforts and achievements were completed in December 1988 and in March 1990 for CYs 1988 and 1989 respectively. These reports were forwarded to the OEPA per draft NPDES permit requirements (PORTS December 1988; PORTS March 1990).

A study to replace the chromate-based corrosion inhibitor (i.e., Betz GDP, a proprietary coolant system corrosion inhibitor for gaseous

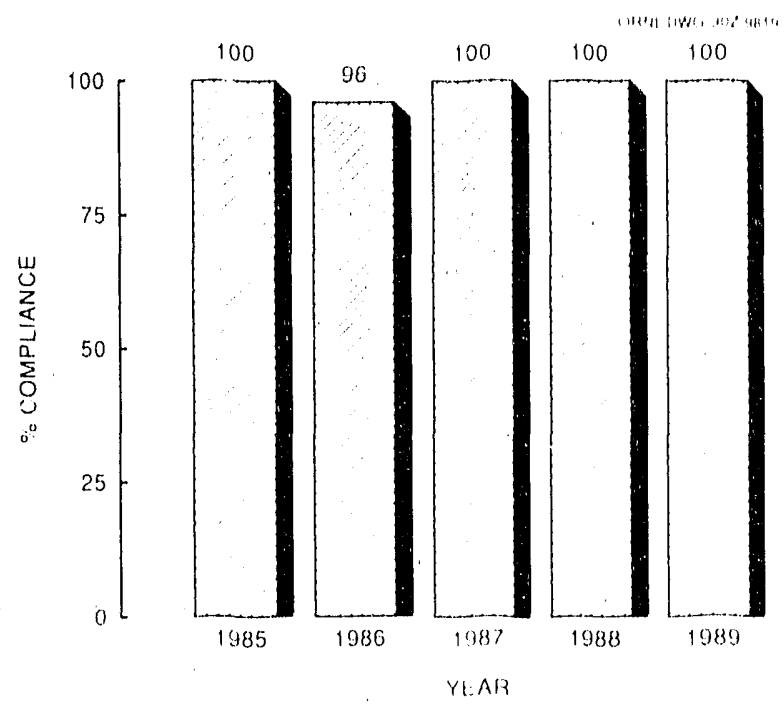

Fig. 2.2.55. NPDES compliance for oil and grease at the north holding pond (009).

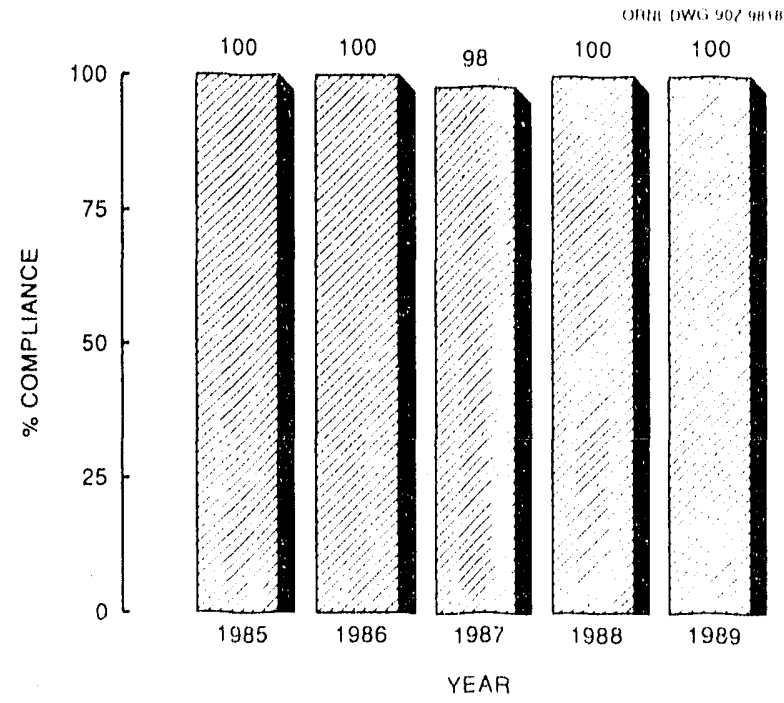

Fig. 2.2.56. NPDES compliance for total residual chlorine at the north holding pond (009).

diffusion plants) used in the RCW system continued through CY 1989. The hexavalent chromium concentration in the RCW, a result of the inhibitor, is considered toxic to aquatic life, and RCW spills lost off-site are considered NPDES violations. Phosphate-based corrosion inhibitors exist that could possibly replace the present formulation. The environmental advantages of replacing the chromate-based inhibitor include 


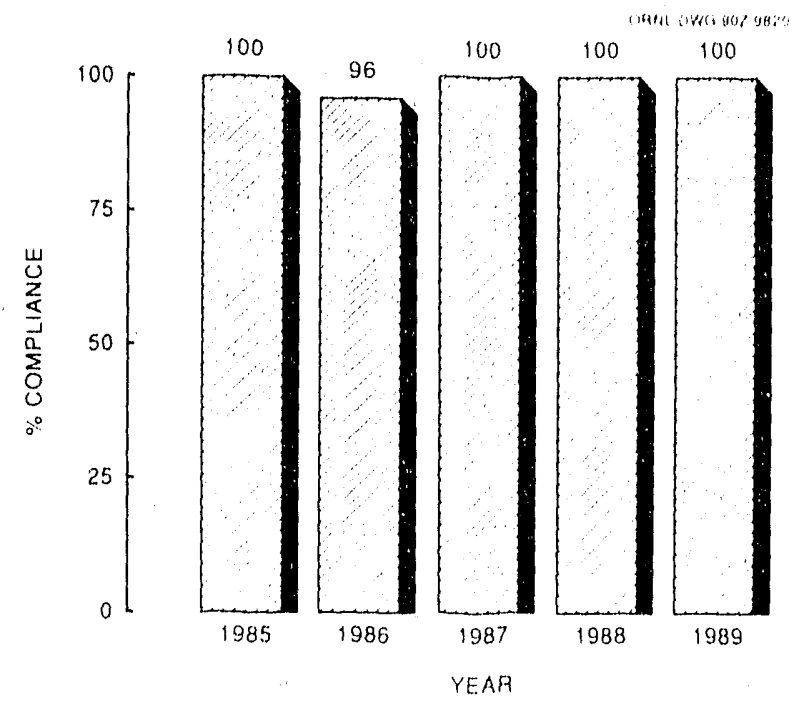

Fig. 2.2.57. NPDES compliance for $\mathrm{pH}$ at the north holding pond (009).

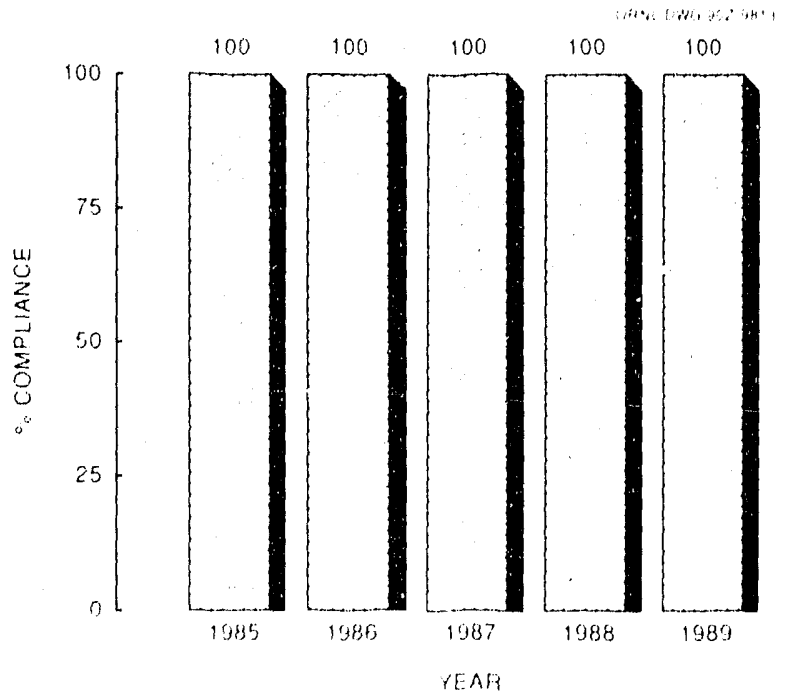

Fig, 2.2.58. NPDES compliance for oil and grease at the west drainage ditch $(010)$.

eliminating the need for X-616 operation and eliminating NPDES violations resulting from RCW spills. The study, which is expected to span several years, will investigate phosphate-based as well as other less toxic corrosion inhibitor systems. The X-626 cooling water system was converted to phosphate in June 1989. The remaining systems (i.e., $X-630$ and $X-633$ ) are scheduled to begin

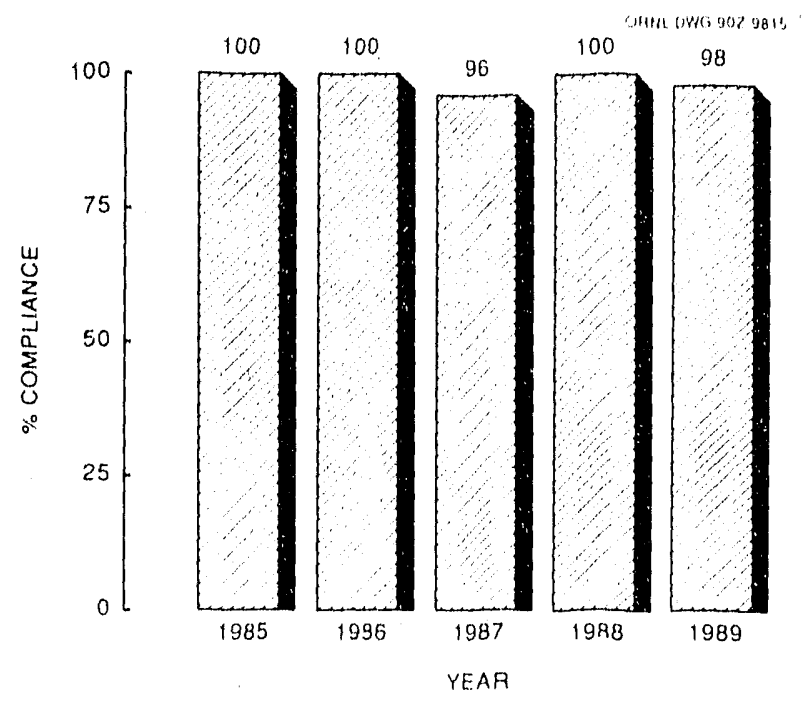

Fig. 2.2.59. NPDES compliance for $\mathrm{pH}$ at the west drainage ditch (010).

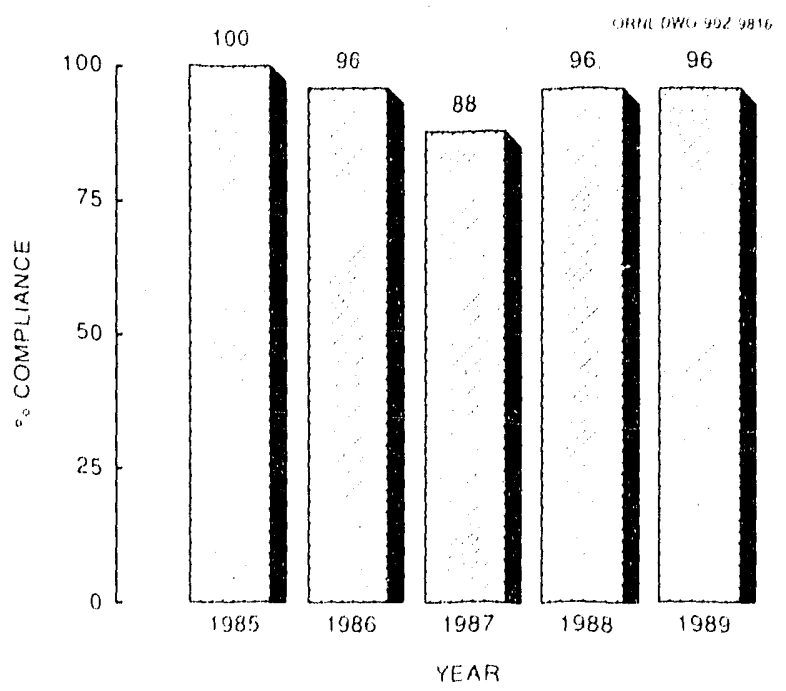

Fig. 2.2.60. NPDES compliance for $\mathrm{pH}$ at the northeast drainage ditch (011).

conversion by June 1990 and June 1991 respectively.

In August 1988 a program to control algae growth and subsequent high $\mathrm{pH}$ exceedances in GCEP ponds was initiated. Stringent NPDES $\mathrm{pH}$ limits of $6.5 \leqslant \mathrm{pH} \leqslant 8.5$ exist at the two GCEP ponds (ouiffalls G001 and G002). Algae and its associated diurnal $\mathrm{pH}$ variations frequently caused 


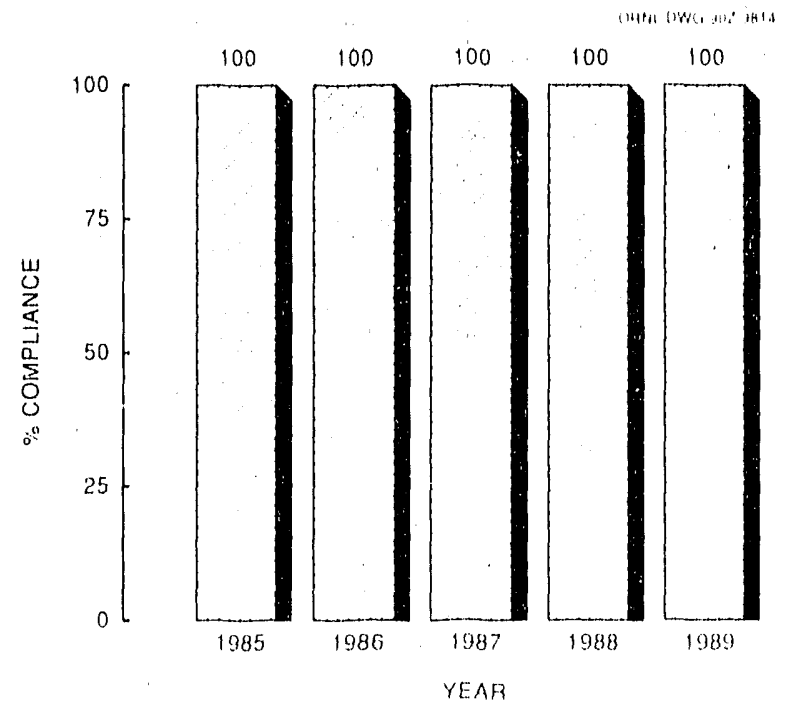

Fig. 2.2.61. NPDES compliance for oil and grease at the northeast drainage ditch (011).

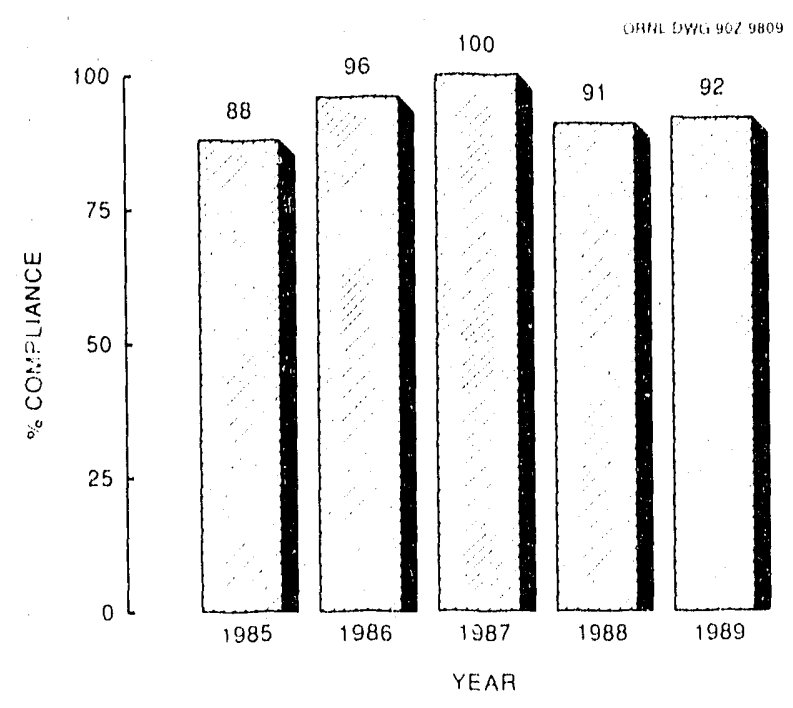

Fig. 2.2.62. NPDES compliance for total suspended solids at GCEP pond G001.

the $\mathrm{pH}$ to exceed the upper limit. In early August the ponds were intentionally drained and refilled to upset the algae growth. From August 1 to December 31,1988 , only $4 \mathrm{pH}$ exceedances occurred compared with 67 exceedances for the same period in 1987. Throughout all of CY 1989, only $11 \mathrm{pH}$ exceedances occurred at these two outfalls. This practice will continue until the

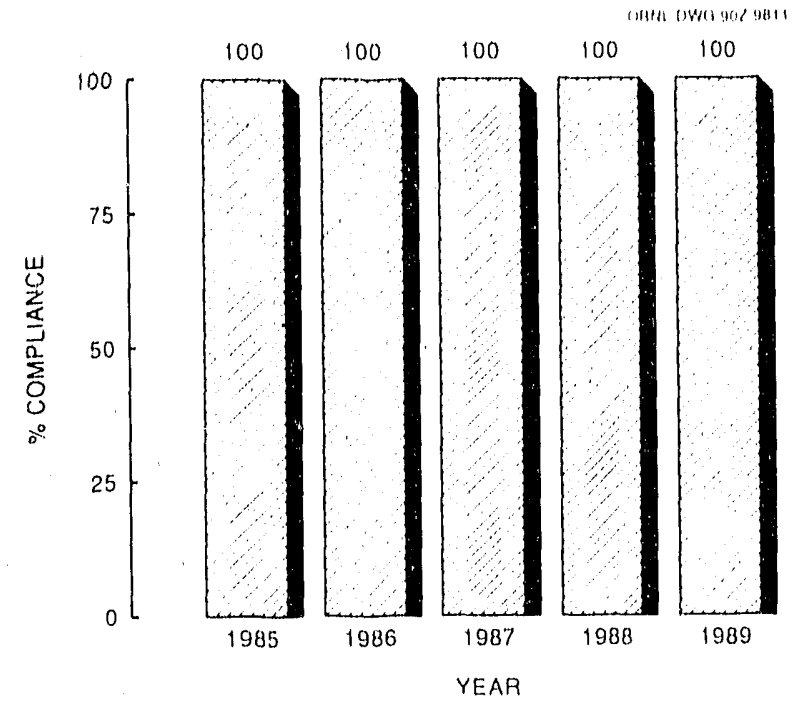

Fig. 2.2.63. NPDES compliance for oil and grease at GCEP pond G001.

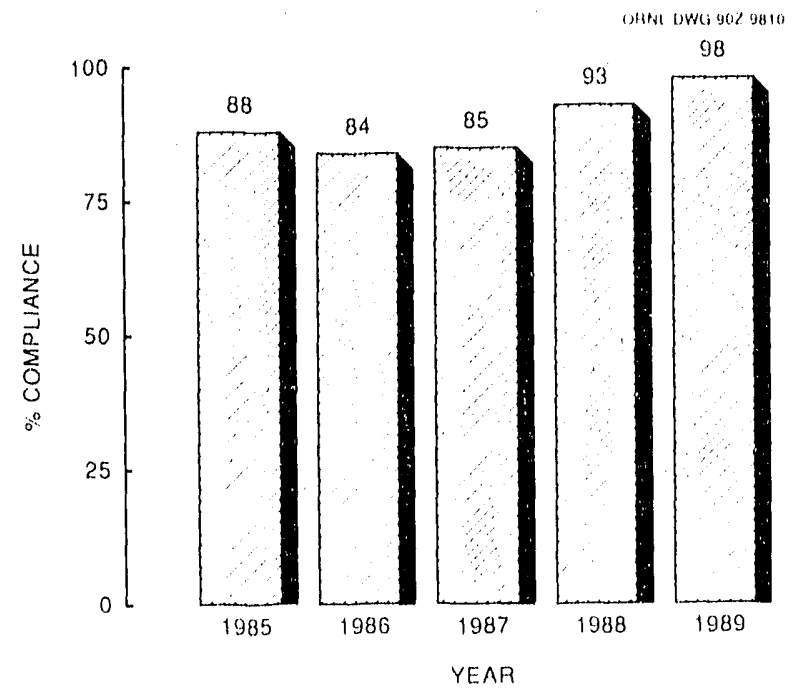

Fig. 2.2.64. NPDES compliance for $\mathrm{pH}$ at GCEP pond $\mathbf{G 0 0 1}$.

stringent $\mathrm{pH}$ limitations are relaxed to a more reasonable $6.5 \leqslant \mathrm{pH} \leqslant 9.0$ when the new NPDES permit is signed.

A new project was initiated in 1988 to dredge the south holding pond (NPDES 002) and to upgrade NPDES monitoring facilities. Settled silt and coal fines are frequently resuspended during heavy rainfall and cause suspended solids and 


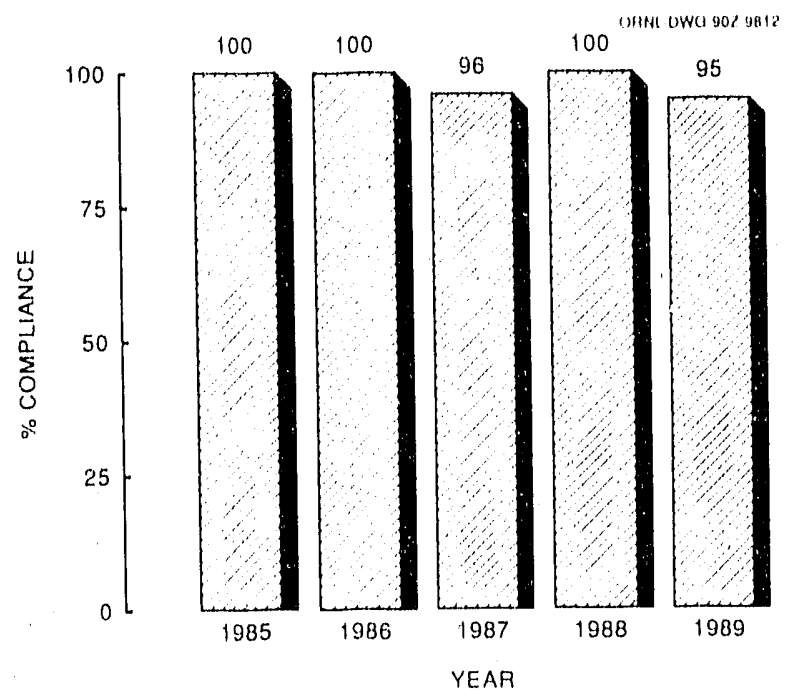

Fig. 2.2.65. NPDES compliance for total suspended solids at GCEP pond G002.

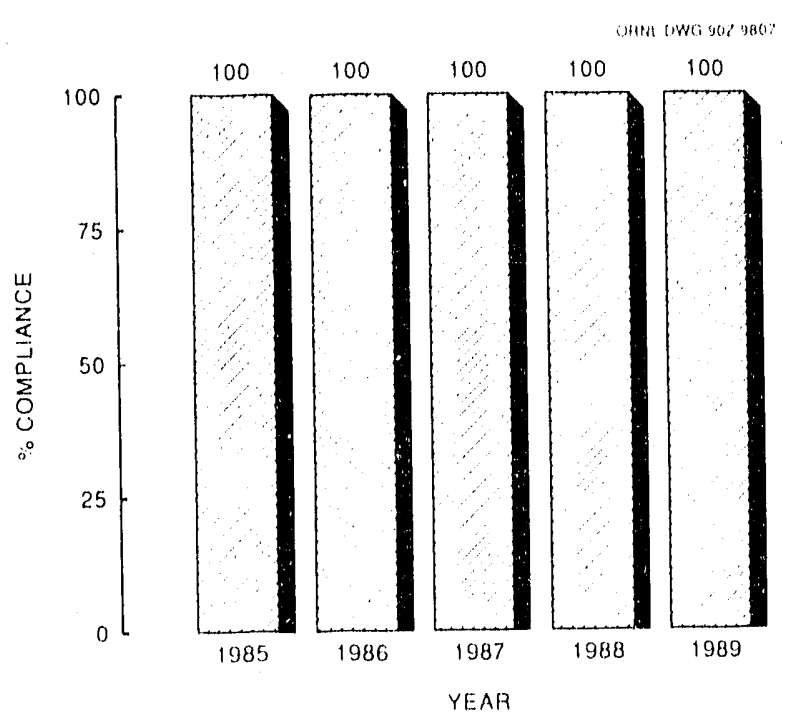

Fig. 2.2.66. NPDES compliance for oil and grease at GCEP pond $\mathrm{GO02}$.

metal concentrations to increase in the pond's effluents. Dredging was one means approved by OEPA to meet new stringent effluent limitations in the new NPDES permit. Construction is expected to begin by mid-summer 1990 . The sludge will be sampled, characterized, and disposed in the X-735 sanitary landfill.

Related to improving NPDES compliances at PORTS and in proactively meeting proposed more

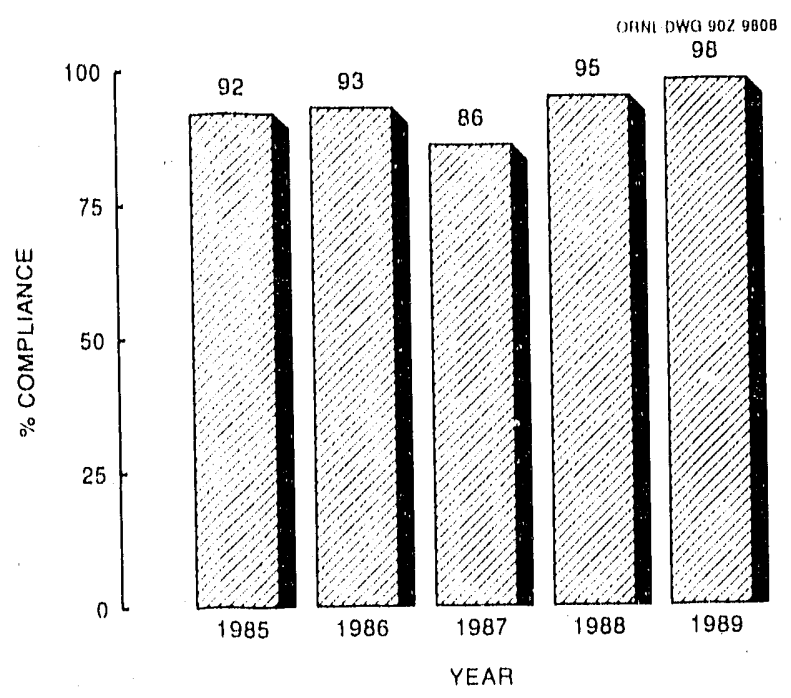

Fig. 2.2.67. NPDES compliance for $\mathrm{pH}$ at GCEP pond $\mathbf{G 0 0 2 .}$

stringent water quality limits at several outfalls, a project has been initiated to construct additional treatment facilities at the south holding pond (NPDES 002) and at the X-621 coal pile treatment facility (NPDES 602). At the south holding pond, additional $\mathrm{pH}$ adjustment facilities (i.e., the capability to adjust $\mathrm{pH}$ downward) will be added. Sand filters will also be installed to improve TSS and metals removal. Flow equalization and containment capabilities will also be installed. Construction is planned for CY 1991. At the $\mathrm{X}-621$ facility, planned improvements include the installation of sand filters, improved $\mathrm{pH}$ adjustment, a new lagoon lift station, an effluent tie to the RCW line as makeup water, improvements to the caustic tank dike, and instrumentation upgrading. Construction is planned for CY 1990.

During the past few years, a significant number of TSS violations have occurred at NPDES outfalls handling surface runoff. The majority of these violations were attributable to extensive construction activities, especially at the 120-ha (300-acre) GCEP site. Although the majority of GCEP areas have been reseeded, a routine semiannual audit program has been developed to monitor site areas for erosion control. This program identifies all site areas with disturbed earth (e.g., construction areas or poorly 
vegetated areas) and ensures that necessary corrective actions are being taken to mitigate soil erosion areas.

In accordance with the PORTS draft NPDES permit, a characterization study of the X-6619 sewage treatment plant was initiated in late 1988. This study was requested by OEPA to determine the quantity and composition of all wastes being discharged to the sanitary sewer other than conventional domestic sewage. The study was completed in March 1989 and submitted to the OEPA.

Negotiations of the plant's new NPDES permit continued through 1988. A draft permit was issued in June 1988. Formal comments from Energy Systems, the EPA, and other concerned parties have been received. Because of other priorities of OEPA, they have delayed further action on the permit. Further negotiations were held in March 1989; issuance of a new permit is expected by mid-1990.

In 1989 an NPDES Task Team was formed, and an NPDES program manager was appointed. The program's objective is $100 \%$ compliance with the NPDES permit at PORTS and elimination of all exceedances. The NPDES Task Team does trend analyses, identifies problem areas, establishes priorities, defines appropriate action, implements projects as necessary, tracks progress, and verifies successful completion of all projects.

\subsubsection{Findings and Conclusions}

Radiological analyses are performed at NPDES sampling locations and RW locations that can better contribute to assessment of the impact of plant discharges on the receiving stream through upstream and downstream measurements. Analyses of Tables 2.2.2 through 2.2.4 and Figs. 2.2.14 through 2.2.17 show that the effects of discharges on the receiving stream have been minimal and are comparable to past annual discharges. Discharges to Little Beaver Creek appear to have only slight noticeable impact on radioactivity levels downstream, primarily because there are no upstream flows during much of the summer (i.e., plant discharges contribute $100 \%$ of the stream flow during dry summer months). Plant discharges appear to have no noticeable effect on radioactivity levels in Big Run Creek or the Scioto River. NPDES compliance by outfall and parameter is graphically presented in Figs, 2.2 .18 through 2.2.67. Compliance by parameter varied from a low of $88 \%$ to a high of $100 \%$. Overall plant-wide NPDES compliance was $97.5 \%$ for CY 1989.

A number of pollution abatement projects and activities were initiated or completed in 1989. They include optimization of the X-705 waste treatment facility, continued optimization of the X-700 biodenitrification facility, conversion of $\mathrm{RCW}$ chromate to phosphate, continued successful algae control at GCEP ponds, implementation of projects to make modifications and improvements to the south holding pond and the X-62l coal pile treatment facility, continuation of the plant's erosion control program, completion of the X-6619 characterization study, continuation of NPDES permit negotiations, and formation of an NPDES Task Team (see Subsect. 2.2.3).

\subsection{GROUNDWATER MONITORING}

Groundwater-monitoring activities at PORTS are directed toward two general efforts. The first defines comprehensively the geology, hydrology, and hydrogeology local to the plant site. Since 1987, efforts have been directed toward a comprehensive definition of the hydrologic flow system in the watershed local to the PORTS site. This effort is necessary to understand the dynamics of the hydrologic cycle and the flow systems that define the occurrence and movement of groundwater and its vulnerability to contamination. Efforts to refine the model of the site hydrologic flow systems will be an ongoing effort of the Groundwater Protection Program as defined by DOE Order 5400.1.

The second general groundwater-monitoring effort defines the effects on groundwater quality due to activities at the DOE site. One of the more probable sources of groundwater contamination has been land disposal sites. The land disposal sites have been given the most attention in the Groundwater Monitoring Program because they have been the major contributors to groundwater contamination on the site. The highest priority of the Groundwater Monitoring Program during the 
last few years has been to identify the contaminants and the extent and rate of movement of the contaminant plumes associated with three inactive RCRA hazardous land disposal units. These efforts were completed in 1989, and it was determined that the contamination is within site boundaries and does not threaten to migrate offsite. Other land disposal units on-site are of secondary priority and are being evaluated as part of investigations that will evaluate the effect of all plant-site operations on groundwater quality.

Much of the groundwater investigative activity and schedules are defined by a Consent Decree between the state of Ohio and the DOE (effective December 1, 1988) and an Adminstrative Consent Order between the EPA and DOE (effective November 1989). The approach is based on guidelines for an RCRA Corrective Action Plan, but because the PORTS site is large, complex, and resource intensive, the Corrective Action Plan is being implemented in a sequence of four parts called quadrants. The quadrants divide the plant site into four geographic areas based roughly on groundwater divides and drainage patterns. The element that provides cohesiveness to this approach is the parallel effort that continues to define the comprehensive geology and the hydrologic flow system local to the plant site. Other programs that have been active for about 10 years are the OffSite Residential Drinking Water Monitoring Program and the X-735 Sanitary Landfill Monitoring Program. Each of these programs is discussed further in Subsect. 2.3.1,

\subsubsection{Groundwater Monitoring Program Descriptions}

\subsubsection{Programs}

\section{RCRA hazardous waste site monitoring}

Groundwater-monitoring requirements applicable to interim status facilities including detection and assessment programs are specified in 40 CFR Part 265, Subpart F-Groundwater Monitoring. Portsmouth has four RCRA land disposal units affected by these requirements: the X-616 chromium sludge surface impoundments, the X-231B oil biodegradation plot, the X-749 low-level waste landfill, and the X-701B holding pond. All four of these sites will be closed as RCRA hazardous waste land disposal units. Detection monitoring has been performed on these sites. Contamination was detected at the X-231B, $X-749$, and X-701 B sites; this was mainly trichloroethene and low concentrations of the lowenergy beta emitter ${ }^{99} \mathrm{Tc}$. Though no solvent or radionuclide contamination has been detected at the X-616 site, the groundwater exceeded indicator parameter thresholds. Groundwater quality assessments were therefore required at all four sites. These groundwater quality assessments were begun in 1988 and were completed in 1989 on three of the sites (i.e., X-231B, X-749, and $X-701 B)$. Assessment monitoring of thes: units will be performed quarterly following the completion of the groundwater quality assessments. The assessments appear to indicate elevated levels of chromium in groundwater at X-616. Until this is resolved, the assessment at $X-616$ is incomplete. A summary of the results of the groundwater quality assessments is provided in the next subsection.

\section{Groundwater Quality Assessment (GWQA) Program}

The goal of interim status assessment monitoring is to determine the rate and extent of migration and the concentration of hazardous waste or hazardous-waste constituents from the facility in groundwater at the site. The purpose of assessment monitoring is to provide information to support future decisions regarding corrective action; characterization of contaminant plumes in terms of Appendix IX (40 CFR Part 264) constituents is developed through the permitting or negotiations process. Assessment monitoring may be triggered by detection monitoring or may be initiated from the outset at interim status sites where groundwater contamination is suspected or known to be present. In either case assessment monitorulg must be conducted quarterly in accordance with a Groundwater Quality Assessment Plan. This plan must specify the monitoring-well network, sampling and analysis procedures, procedures for data evaluation, and a schedule of implementation. Each year the results 
of the GWQA must be summarized and submitted to the appropriate regulatory agency. At the end of CY 1989, PORTS had 242 monitoring wells in 9 monitoring w/al systems and 29 monitoring wells in is loose plant-wide framework. Figure 2.3.1 shows the locations of these systems. The completed GWQA for the four RCRA units at POR.TS was provided to the OEPA in June 1989 and was approved by the OEPA in August 1989 (Geraughty and Miller, Inc. June 1989). A descriptive summary of these assessment activities follows.

In late August 1988 a GWQA investigation was implemented at four RCRA-regulated facilities at PORTS. These facilities include the X-701B holding pond, X-749 low-level waste landfill, X-231B oil biodegradation plot, and X-616 chromium sludge surface impoundments. The results of this investigation indicave that only the Gallia Sand Formation (i.e., the principal waterbearing formation on-site) exhibits significant contamination with trichloroethylene (TCE) at X-231B, X-749, and X-701B. The rate of plume migration, determined by using both field measurements and flow model simulations, ranges from 0.1 to $0.3 \mathrm{ft} / \mathrm{d}$. The leading edge of plumes emanating from the three RCRA facilities is located approximately $300 \mathrm{~m}$ (1000 ft) from $\mathrm{X}-231 \mathrm{~B}, 480 \mathrm{~m}(1600 \mathrm{ft})$ from X-749, and $660 \mathrm{~m}$ $(2200 \mathrm{ft})$ from $X-701 B$. The maximum concentrations of TCE observed in these plumes are on the order of $100 \mathrm{ppb}$ at X-231 B, 10,000 ppb at X-749, and $100,000 \mathrm{ppb}$ at X-701B. The investigation results pointed to some impor ${ }^{*}$ nt factors controlling the groundwater flow anc transport system at these RCRA units: (1) the thickness and tydraulic conductivity of the Gallia Sand Formation, (2) the thickness and low vertical conductivity of the Sunbury Shale Formation, (3) the presence of a storm drain system, and (4) the low hydraulic conductivity of the Minford Clay Formation.

ine water-table and potentiometric-surface maps, the geologic outcrop patterns, and the observ: 1 contamination distribution indicate three principal locations of $d$ 'scharge for groundwater in the $\mathrm{Ga}^{\prime}$ a and the Berea Aquifer ber a 2 POR'TS. These locitions are 'jttle Beaver Creek on the north and east, Big Run Creek on the southeast, and the two unnamed tributaries to the west. Because the waste units are constructed in the Minford, this unit provides a vertical pathway beneath the facilities to the Gallia Sand, the most permeable unit beneath PORTS. The Gallia water-table flow system carries these contaminants downgradient following the thicker sand deposits. The storm drain system depresses the water table, effectively dewaters the Minford silt, and locally captures groundwater and contaminants from the Gallia. Althu agh a downward vertical gradient is observed between the Gallia and tie underlying Berea, the low vertical hydraulic conductivity, the substantial thickness, and the wide areal extent of the Sunbury Shale present an effective barrier that restricts the vertical communication between the Gallia and Berea. This limits the vertical migration of contaminants into the Berea. Only very small concentrations at or near the analytical detection limit for TCE were detected in the Berea. This is consistent with the long-term aquifer test results that exhibited no vertical hydraulic response in the Berea or Minford when pumping from the Gallia.

The GWQA investigation has characterized the contaminant distribution and calculated the rate of plume migration at three RCRA-regulated facilities. At X-701 the thickness of the Gallia controls contaminant migration. A narrow band of thicker Gallia deposits extends from southeast of the holding pond to the northeast corner of the $X-744 G$ building and then eastward across the Perimeter Road to Little Beaver Creek.

At X-701B the groundwater contamination is characterized by a long and narrow plume of predominantly volatile organic compounds (VOCs) stretching from the holding pond to Little Beaver Creek. Contamination was detected in water samp ss taken in Little Bea ver Creek indicating disch.urge from the Gallia. Groundwater velocity calculations in the Gallia estimate a log mean velocity of $0.2 \mathrm{ft} / \mathrm{d}$. Using this $\log$ mean velocity, the travel time for contaminants from the holding pond to the creek is on the order of 25 years. Groundwater velocities in the Berea range from 0.048 to $0.15 \mathrm{ft} / \mathrm{d}$. Particle-tracking results from model simulation exhibit a range of travel times from 15 to 30 years from X-701B to Little Benver 


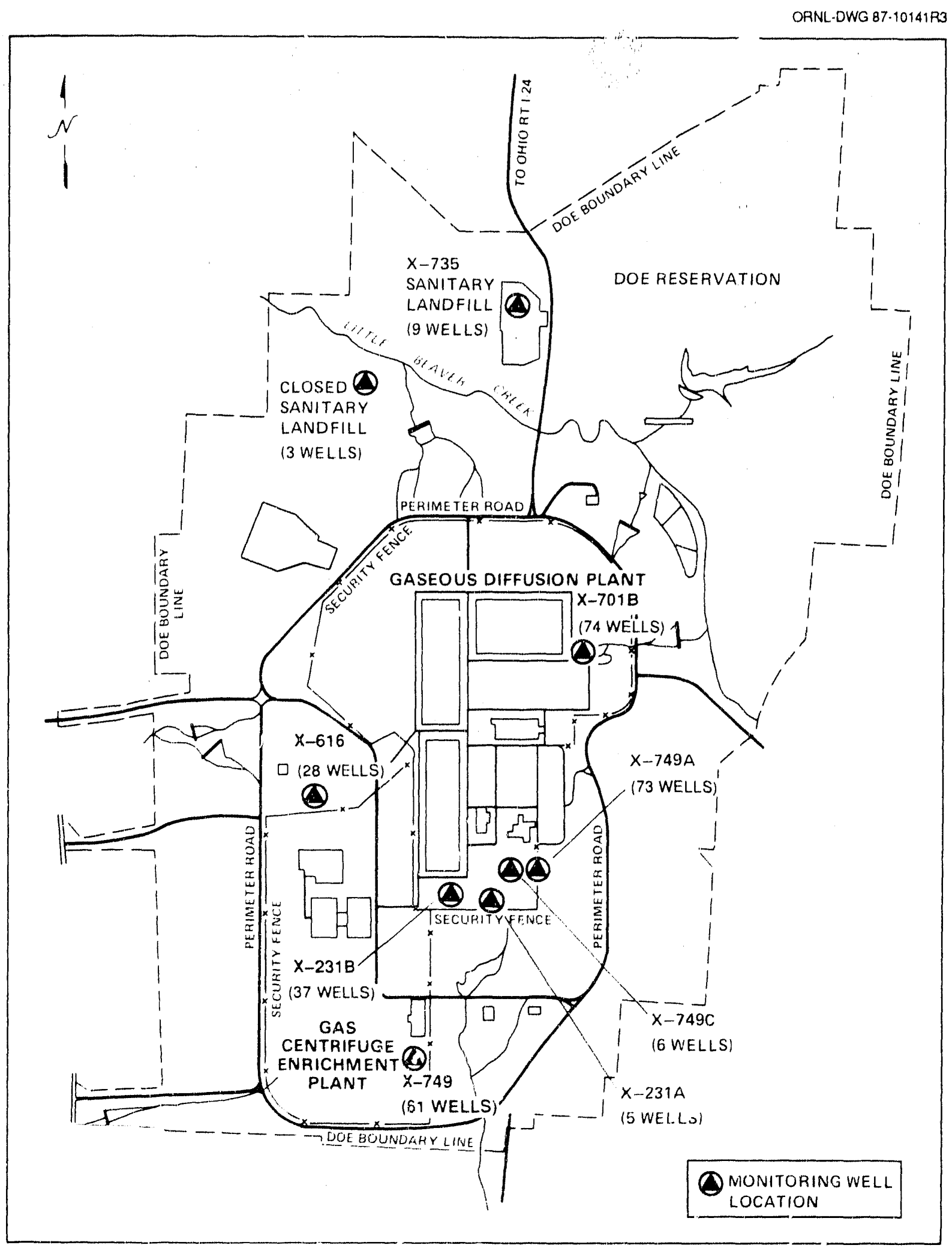

Fig. 2.3.1. Groundwater-monitoring well system at PORTS. 
Creek. One well (BW-2) encountered $1.65 \mathrm{ft}$ of separate phase TCE resting in a closed depression eroded into the Sunbury Shale. A remedial pumping program recovered $1607 \mathrm{~L}$ (425 gal) of separate phase TCE and approximately $321 \mathrm{~L}$ (85 gal) of TCE-water emulsion.

At X-749 groundwater exhibited elevated levels of VOCs. The X-749 area is similar to $\mathrm{X}-701 \mathrm{~B}$ in terms of its hydrogeologic framework. The distribution and thickness of the Gallia control the flow and contaminant migration from this facility. The thickest Gallia deposits occur at the southwest corner of the landfill and extend to the southeast and to the west. To the east the Gallia thins, and contaminant migration has been limited because of the low permeability of the Minford.

The flow system at X-749 is characterized by a north-south-trending divide along the western edge of the landfill. Groundwater flows on the eastern half of the facility to Big Run Creek; on the western half, to the unnamed tributary to the southwest. In the southwestern corner of the landfill, groundwater is influenced by a stormwater runoff drain. In the Berea flow directions are very similar to those in the Gallia. At this facility the Sunbury is approximately $1.5 \mathrm{~m}(5 \mathrm{ft})$ thick over much of the area. Observed head differences between the Gallia and the Berea are still approximately 3 to $4.5 \mathrm{~m}$ ( 10 to $15 \mathrm{ft}$ ).

Contamination of groundwater with VOCs extends to the southwest from the western portion and to the southeast and east from the eastern portion of the facility. Radionuclide plunes at X-749 exhibit the same configuration as the VOCs. Peak concentrations of both technetium (i.e., 766 $\mathrm{pCi} / \mathrm{L}$ ) and uranium (i.e., $700 \mathrm{ppb}$ ) occur on the west side of the unit. Low levels of VOCs have been identified in discharging waters of storm drains. VOC contamination was found in the Minford adjacent and beneath the landfill. Groundwater velocities in the Gallia range from 0.04 to $3.5 \mathrm{it} / \mathrm{d}$; in the Berea, from 0.054 to $1.76 \mathrm{ft} / \mathrm{d}$. Particle-tracking analyses predict complex patterns of contaminant migration. Contarninants on the west side of the landfill migrate to the southwest and discharge to the storm drain in approximatily 20 years. In the southern end particles migrate to the scutheast and Big Run Creek.
The X-231B oil biodegradation plots were used from 1976 to 1985 for the land application of waste oils and degreasing solvents that contained low levels of radioactivity. The Gallia is very thin beneath this facility - generally less than $1.2 \mathrm{~m}$ $(4 \mathrm{ft})$ in thickness. Thicker Gallia deposits occur to the southeast and east of X-231B. These thicker deposits probably represent remnants of the point bar complex associated with the meander of the ancient Portsmouth River.

Groundwater flow in the Gallia, Minford, and Berea exhibits a southeastward gradient. The horizontal gradient in the Gallia is very low because of the close proximity of the major eastwest divide and the higher hydraulic conductivity exhibited by the Gallia to the south and east of the facility. The Sunbury is 3 to $3.6 \mathrm{~m}$ (10 to $12 \mathrm{ft}$ ) thick, thins to the west, and is absent over much of the western portion of PORTS. Vertical gradients between the Gallia and Berea are steep and exhibit a 2.4- to 3-m (8- to 10-ft) head loss across the Sunbury. This head difference and gradient increases to the east, as does the thickness of the Sunbury.

At X-231B, 12 VOC samples were found above detection limits in the Gallia. Exhibiting the highest concentration was TCE at $1800 \mathrm{ppb}$. Also found were 1,1,1-trichloroethane (TCA) and 1,1-DCE in numerous wells with maximum concentrations of $790 \mathrm{ppb}$ and $320 \mathrm{ppb}$ respectively. A storm drain runs north to south through the plume and appears to be influencing the migration of contaminants away from this RCRA unit. Radionuclide contamination is not widespread; technetium exhibited maximum concentrations of $88 \mathrm{pCi} / \mathrm{L}$ (well below the 900-pCi/L drinking water standard). The Berea is unaffected by contamination at X-231B. No VOC contamination was found in three Minford wells.

Groundwater flow is southeast toward the $\mathrm{X}-230 \mathrm{~K}$ holding pond. Ground water velocities in the Gallia at X-231 B range from 0.014 to $5.6 \mathrm{ft} / \mathrm{d}$ with a log mean of $0.28 \mathrm{ft} / \mathrm{d}$. In the Berea the velocities range from 0.054 to $0.33 \mathrm{ft} / \mathrm{d}$ with a mean log velocity of $0.10 \mathrm{ft} / \mathrm{d}$. The particletracking analyses performed indicate contaminant migration to the southeast and contaminant discharge into the X-230K holding pond in 15 to 20 years. 
The X-616 chromium sludge surface impoundments area is used to store precipitant from the treatment of the RCW. Approximately one million gallons of $\mathrm{RCW}$ have been processed daily since 1976 . The Gallia is 1.2 to $1.8 \mathrm{~m}$ (4 to 6 $\mathrm{ft}$ ) thick over much of the facility but thins to the west and the northeast. The Sunbury Shale is absent 'nder X-616, and the bedrock surface is composed of the upper portion of the Berea. This bedrock surface is fairly flat except for a small knob located beneath the northwest corner of the lagoons. Beneath X-616 the Gallia and the Berea essentially act as one unit. The absence of the confining unit minimizes the head difference between the two units. As a result, groundwater flow directions in these units are similar. The Gallia and Berea flow north and west away from the lagoons.

\section{RCRA Facility Investigations (RFI)}

An RFI is the step in an RCRA Corrective Action Plan that investigates contaminant releases and effects on groundwater quality of solid waste management units and land-use contamination associated with industrial activity. An RFI was initiated at PORTS in early 1989 in accordance with the requirements and schedules specified in the Consent Decree issued by the Ohio attorney general's office on August 29,1989, and with the RCRA Sect. 3008(h) Consent Order issued by the EPA, Region V, on September 29, 1989. The objectives of this site-wide RFI are (1) to characterize the environmental seting of the plant, (2) to define and to characterize sources of contamination, (3) to characterize the vertical and horizontal extent and degree of contamination, (4) to assess risk to human health and environment resulting from exposure to contaminants, and (5) to support the Corrective Measures Studies that follow the RFI. During the RFI all possible sources of contamination will be identified and assessed to determine their impac: on groundwater, surface water, soil, sediment, and air. The completion of the RFI will therefore result in a complete assessment of groundwater contamination at PORTS and allow for selection of proper remedial alternatives.
In accordance with the Consent Order and Consent Decree, the PORTS RFI will be conducted using a four-quadrant approach. Each of the four quadrants at the site will be investigated as a separate RFI; however, all data collected during each investigation will be incorporated into the site-wide investigation. To begin the RFI an environmental audit of the site was conducted; this included an extensive record search and review coupled with a detailed site walk-over. Based on the results of the audit, a total of 47 Solid Waste Management Units (SWMUs) have been identified in Quadrants I through III as requiring further investigation (Tables 2.3.1 through 2.3.16 of Part 2). A similar audit is in progress on Quadrant IV and will be submitted to the OEPA and the EPA late in 1990.

\section{Solid waste landfill monitoring (X-735 sanitary landfill)}

Martin Marietta Energy Systems, Inc., has conducted sampling and analyses of wells at X-735 from 1977 to the present. The groundwater monitoring at X-735 is defined and required by the lardfill license in effect for the landfill. The results of these analyses indicate no groundwater contamination from volatile organic compounds or radionuclides. There is some indication of elevated levels of lead in the monitoring wells at the X-735 site. Plans are being developed to respond to these indications and will be implemented. Additiona! plans are being developed to upgrade the monitoring well networ:: and to provide more detailed definition of geology at the X-735 site in response to more stringent requirements (effective in March 1990) by the state of Ohio.

\section{Interim actions: X-668 wellield-well-6B mercury contamination}

The X-608 wellfield is located on the floodplain of the Scioto River. This wellfield provides raw water that is treated in the X-611 water treatment facility. On March 1, 1989, mercury was released into production field well 6-B at the X-608 wellfield (located in the floodplain of the Scioto River) as a result of a 
pump failure. The pump assembly for this well was removed following failure, and traces of mercury were discovered. After pulling the discharge pipe, pump, and motor, it was discovered that the bottom portion of the motor (i.e., the oil reservoir) had separated from the rest of the motor and remained in the well.

The separated oil reservoir was recovered from the well on March 3, 1989. It was determined that the mercury seal failed and that the majority of the $3550 \mathrm{~g}(7.9 \mathrm{lb})$ of mercury was released to the well and to the ground proximal to the well head. Cleanup procedures began immediately including groundwater sampling, soil removal, placement of absorbent pads, and sampling of surrounding wells for contamination. A rough mass balance performed after these initial cleanup actions indicated $2500 \mathrm{~g}(5.5 \mathrm{lb})$ of mercury was lost in well 6-B.

On March 18, 1989, a stainless steel cylinder was inserted into the well as an inner casing to decrease the possible spread of mercury through the well screen during cleanup operations. Approximately $378 \mathrm{~L}$ (100 gal) of liquid and sediment were bailed from the inner casing. These materials were disposed of in an appropriate manner.

On July 18,1989 , an effort was made to purge the well by pumping at a rate of 1890 $\mathrm{L} / \mathrm{min}(500 \mathrm{gal} / \mathrm{min})$. The water pumped from the well was discharged ir to an 18,900-L (5000-gal) tanker. No detectable decrease in the concentration of mercury in the well resulted from these operations.

Additional activity proposed, ill involve the installation of three monitoring wells in a triangular configuration around well 6-B. These wells will be drilled to the base of the alluvial aquifer and will be located approximately 15.2 to $30.5 \mathrm{~m}$ ( 50 to $100 \mathrm{ft}$ ) from the contaminated well. The wells will have PVC casings and $3.05-\mathrm{m}$ (10-ft) screens. Exact placement of the wells will be determined following review of all pertinent information. The wells will be sampled and analyzed for mercury. Data-loggers will be instailed to provide continuous water-level measurement. Additional wells may be required to define the contamination plume precisely. The location and number of additional monitoring wells (if required) will be dictated by the findings generated from the initial monitoring wells.

\section{Off-site monitoring}

Monitoring of the local resident sanitary water systems beg in in 1979. This monitoring is conducted semiannually on springs and private wells that serve as the source of sanitary water for private households near PORTS (see $\mathrm{F}^{\prime}, .2 .3 .2$ and Table 2.3.1). Results of the analyses are outinely communicated to the residents. Parameters typically monitored include uranium, technetium, total alpha, and total beta. Analysis for the presence of organics was added in 1986. The monitored parameters have not been detected above background in any of the sampling events. The results are shown in Tables 2.3.2 and 2.3.3. Drinking water parameters were included in the analyses in 1989 as a baseline characterization of residential water quality (Table 2.3.4). Historical and recent data indicate that plant operations at PORTS have had no adverse chemical or radiological effects on residential drinking water supplies in the area (Keller, April 18, 1989).

\subsubsection{Sampling and analysis}

Groundwater sampling and analysis follows standard procedures for sample collection, sample preservation and handling, chain of custody, sample analysis, quality control (QC)/quality assurance (QA), and data quality evaluation. Sampling and analysis of all groundwater wells is specified in a Groundwater Sampling and Analysis Plan (revision due in August 1990).

\subsubsection{Well construction añu abandonment}

Well installation standards include procedures, specifications, and documentation requirements for monitoring wells at PORTS. Environmental surveillance procedures relating to well installation have been published (Kimbrough, Long, and McMahon Sept. 1, 1988). Generally, monitoring wells installed at Portsmouth have been $5.08-\mathrm{cm}$ (2-in.) stainless steel and constructed with appropriate annular space screen packing and 
ORNL.DWG 89.10466R

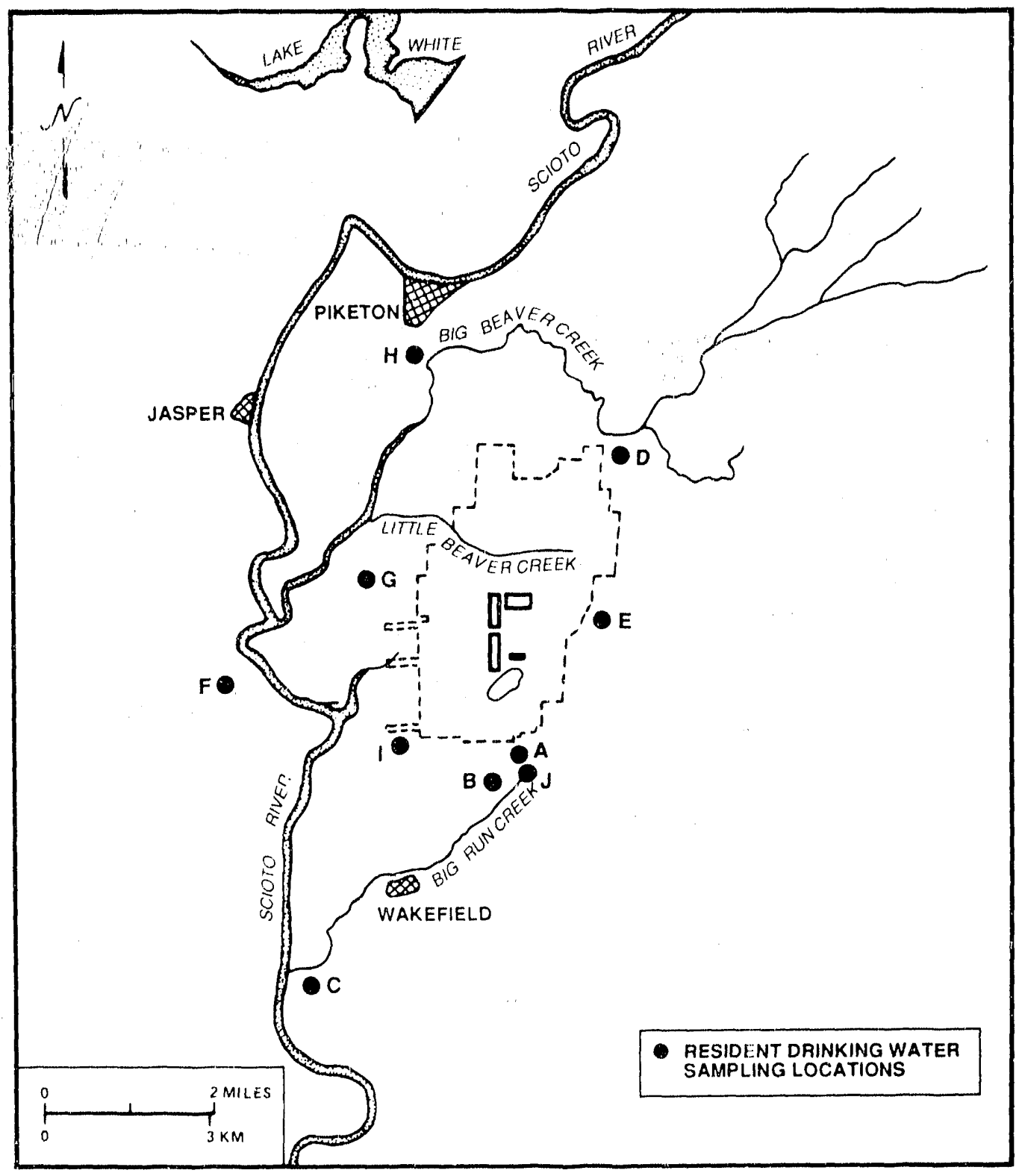

Fig. 2.3.2. Residential drinking-water-monitoring sites.

sealant. Geologic logging and as-built documentation are required for each well construction. Each well is properly developed to restore (as nearly as possible) the flow characteristics representative of the original water-bearing formation being monitored.

Well abandonment procedures apply to the decommissioning of damaged, unusable, or no- longr r-needed groundwater-monitoring and investigation wells. Well inspection and maintenance reports identify wells that are damaged or unusable. Wells must also be removed because of construction or site closure activities. When wells are identified for abandonment, plans are developed that include scheduling plugging and abandonment activities, obtaining 
Table 2.3.1. Residential drinking water location code identification

\begin{tabular}{lll}
\hline \multicolumn{1}{c}{ Location $^{a}$} & Source & Location code \\
\hline Bailey Chapel Road & Well & A \\
Big Run Road & Spring & B \\
Wakefield & Well & C \\
Taylor Hollow Road & Cistern & D \\
McCorkle Road & Well & E \\
Route 104 & Well & F \\
U.S. Highway 23 & Well & G \\
Wakefield Mound Road & Well & H \\
Wakefield Mound Road & Well & I \\
Bailey Chapel Road & 2 Wells & J \\
\hline
\end{tabular}

${ }^{a}$ See Fig. 2.3.2.

Table 2.3.2. Radionuclides tested in residential drinking water

\begin{tabular}{lcccc}
\hline & \multirow{2}{*}{$\begin{array}{c}\text { Number of } \\
\text { Parameter }\end{array}$} & \multicolumn{3}{c}{ Concentration } \\
\cline { 5 - 6 } & samples & Max & Min & Av \\
\hline Uranium (mg/L) & 13 & 0.003 & 0.000 & 0.001 \\
Alpha (pCi/L) & 13 & 21 & -6 & 5 \\
Beta (pCi/L) & 13 & 11 & -7 & 1 \\
Technetium (pCi/L) & 13 & 44 & -7 & 5 \\
Radium (pCi/L) & 13 & 17.4 & 0 & 1.3 \\
\hline
\end{tabular}

Table 2.3.3. Halogenated volatile organics tested in residential drinking water

\begin{tabular}{|c|c|c|c|c|}
\hline \multirow[t]{2}{*}{ Parameter } & \multirow{2}{*}{$\begin{array}{c}\text { Number of } \\
\text { samples }\end{array}$} & \multicolumn{3}{|c|}{$\begin{array}{l}\text { Concentration } \\
(\mu \mathrm{g} / \mathrm{L})\end{array}$} \\
\hline & & Max & Min & Av \\
\hline Methylene chloride & 11 & 0 & 0 & 0 \\
\hline Trichlorofluoromethane & 11 & 0 & 0 & 0 \\
\hline 1,1-Dichloroethene & 11 & 0 & 0 & 0 \\
\hline 1,1-Dichloroethane & 11 & 0 & 0 & 0 \\
\hline trans-1,2-Dichloroethene & 11 & 0 & 0 & 0 \\
\hline Chloroform & 11 & $22^{a}$ & 0 & 2 \\
\hline Freon 113 & 11 & 0 & 0 & 0 \\
\hline 1,2-Dichloroethane & 11 & 0 & 0 & 0 \\
\hline 1,1,1-Trichloroethane & 11 & 0 & 0 & 0 \\
\hline Carbon tetrachloride & 11 & 0 & 0 & 0 \\
\hline Bromodichloromethane & 11 & $21^{a}$ & 0 & 2 \\
\hline Trichloroethene & 11 & 0 & 0 & 0 \\
\hline Dibromochloromethane & 11 & $13^{a}$ & 0 & 1 \\
\hline Bromoform & 11 & $2^{a}$ & 0 & 0 \\
\hline Tetrachloroethene & 11 & 0 & 0 & 0 \\
\hline
\end{tabular}

These maximum values, all of which were from Pike County sanitary water, are by-products of chlorination. 
Table 2.3.4. Inorganics tested in residential drinking water

\begin{tabular}{|c|c|c|c|c|}
\hline \multirow[t]{2}{*}{ Parameter } & \multirow{2}{*}{$\begin{array}{l}\text { Number of } \\
\text { samples }\end{array}$} & \multicolumn{3}{|c|}{$\begin{array}{c}\text { Concentration } \\
\mu \mathrm{g} / \mathrm{L})\end{array}$} \\
\hline & & $\operatorname{Max}$ & Min & Av \\
\hline Arsenic & 11 & 0 & 0 & 0 \\
\hline Chloride & 11 & 123.0 & 2.5 & 31.9 \\
\hline Fluoride & 11 & 0.3 & 0 & 0.1 \\
\hline Mercury & 11 & 0 & 0 & 0 \\
\hline Nitrate & 14 & 20.0 & 0 & 4.9 \\
\hline Selenium & 11 & 0 & 0 & 0 \\
\hline Sulfate & 11 & 176.5 & 5.7 & 60.8 \\
\hline Total dissolved solids & 11 & 830 & 92 & 830 \\
\hline
\end{tabular}

subcontractor services for the plugging and abandonment, obtaining engineering support for field operations oversight, and ensuring that the subcontractor complies with plugging and abandonment procedures. Well 13 at the X-735 sanitary landfill was abandoned in 1989 and replaced with well $13 \mathrm{~A}$. Well 13 was located in the area where a monofill cell was planned for construction.

\subsubsection{Geologic Setting}

\subsubsection{Geology}

PORTS occupies an upland area of southern Ohio with an average land surface elevation of $201 \mathrm{~m}(670 \mathrm{ft})$ above mean sea level. The plant site sits in a mile-wide abandoned river valley

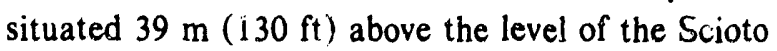
River to the west (Fig. 2.3.3). Five geologic units control groundwater flow beneath the site: the Minford Clay/Silt, Gallia Sand, Sunbury Shale, Berea Sandstone, and Bedford Shale. The upper two units, Minford and Gallia, were deposited during Quaternary time before and during glaciation. The youngest of these unconsolidated units is the Miniford member of the Teays Formatiun. The Gallia member of the Teays Formation lies beneath the Minford and on top of bedrock. The Sunbury Shale of Mississippian age is the upper bedrock unit beneath most of the PORTS site and overlies the Berea Sandstone. The Berea Sandstone overlies the Bedford Shale.
Detailed discussions of the geology and hydrogeology of PORTS have been published (Geraghty and Miller, Inc. June 1989).

\subsubsection{Groundwater flow system}

At POW IS the hydraulic conductivities of all hydrogeologic units are very low. The mean hydraulic conductivity values for each hydrogeologic unit are shown in Table 2.3.5. The most conductive unit is the Gallia with a mean hydraulic conductivity of $3.4 \mathrm{ft} / \mathrm{d}$. The next most permeable formation is the Berea Sandstone with a mean hydraulic conductivity of $0.16 \mathrm{ft} / \mathrm{d}$. These values were estimated from single-well tests, most of wisich were conducted by Geraghty and Miller, Inc. (June 1989).

Numerous laboratory estimates of hydraulic conductivity for the Minford Clay and Silt units were perfor.sed by Law Engineering (1978). The average permeability of the Minford Clay was found to be $0.00023 \mathrm{ft} / \mathrm{d}$. The average permeability of the Minford Silt was found to be $0.0043 \mathrm{ft} / \mathrm{d}$. The mean hydraulic conductivity of the Bedford Shale is $0.059 \mathrm{ft} / \mathrm{d}$ based on 13 packer injection tests performed by Law Engineering (1978).

\subsubsection{Groundwater flow directions}

Groundwater flow at PORTS is controlled by interactions between the Gallia and Berea units. 
OANL.DWG $90 \mathrm{M} \cdot 10433$

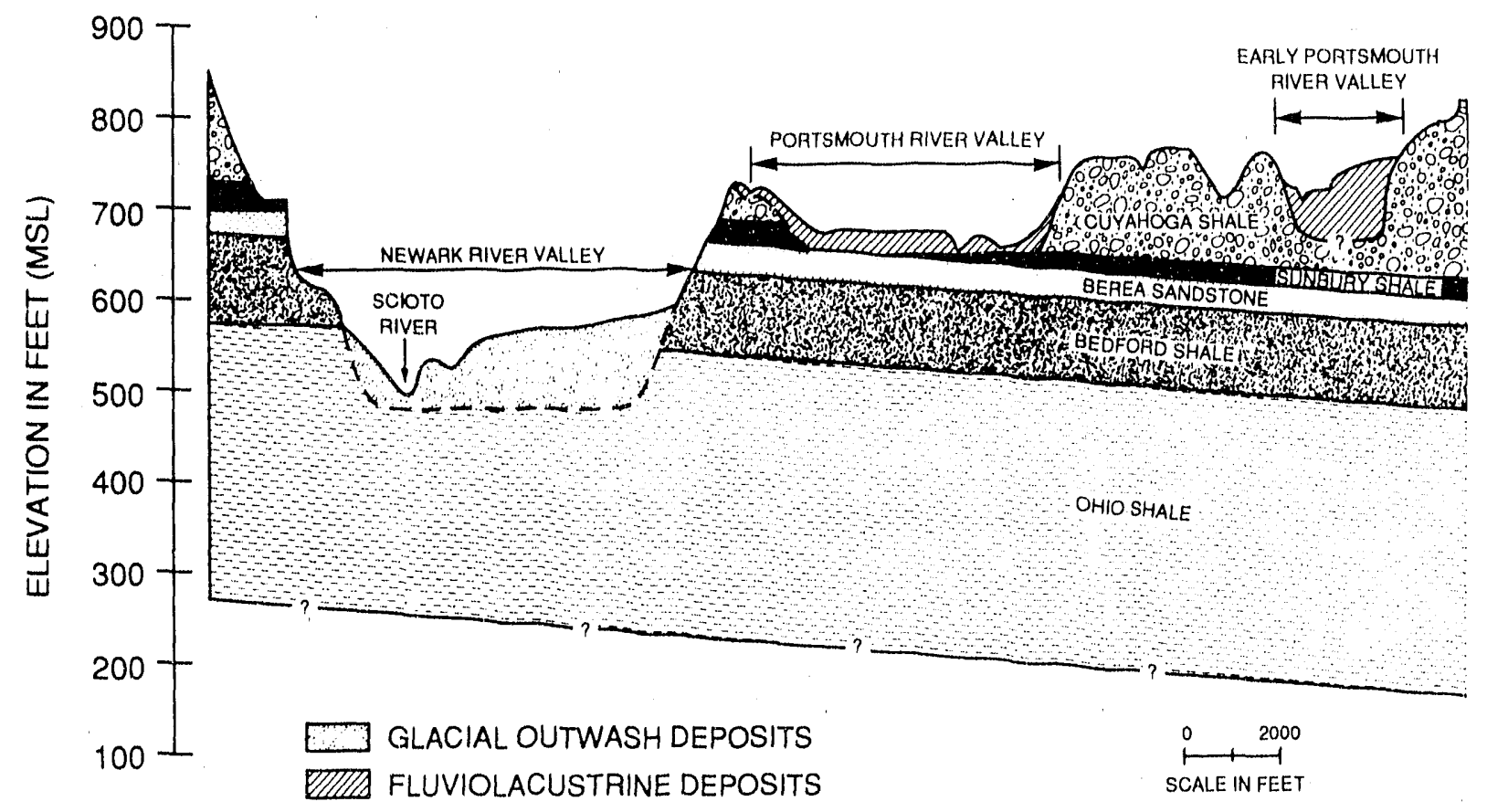

Fig. 2.3.3. Terrain showing geologic units that control groundwater flow beneath the PORTS site.

Table 2.3.5. Hydrogeologic column at Portsmouth Gaseous Diffusion Plant

\begin{tabular}{lllc}
\hline Formation & Lithology & $\begin{array}{c}\text { Permeability } \\
(\mathrm{ft} / \mathrm{d})\end{array}$ & Thickness \\
\hline Minford & Clay & 0.00023 & 16.3 \\
& Silty clay & 0.0043 & 7.60 \\
Gallia & Sand & 3.4 & 5 \\
Sunbury & Shale & Unknown & 10 \\
Berea & Sandstone & 0.16 & 30 \\
Bedford & Shale & 0.059 & 100 \\
\hline
\end{tabular}

The flow patterns are also affected by the presence of the plant storm sewer system and the reduction in recharge caused by buildings and paved areas. The ultimate discharge area for most groundwater is Little Beaver Creek to the north and east, Big Run Creek to the south, and two unnamed drainages to the west. Groundwater flow patterns were analyzed using water-level measurements observed in the Gallia and the Berea in December 1988.
The potentiometric surfaces for both the Berea and Gallia units are characterized by an eastwest-trending groundwater divide. Other groundwater divides are also present; these divide the flow system into four flow cells in the Gallia and three in the Berea. Groundwater flow directions in the Berea roughly parallel those in the Gallia with a few notable exceptions outlined below. Flow directions in the Minford are roughly the same as those in the underlying Gallia. 


\subsubsection{Data Summary and Analysis}

\subsubsection{Data summary}

Data collected during 1989 are provided as summary statistics in Tables 2.3.5 through 2.3.16 of Part 2. Data are provided as indicator parameters, water quality parameters, volatile organic parameters, and radioactivity parameters. The indicator parameters, as the name implies, are gross indicators of contaminants that may be affecting water quality. Water quality parameters are indicators of gross changes in groundwater chemistry. Volatile organic parameters are the principal chemical contaminants of groundwater on the plant site and are usually part of the contaminant "hit list" of parameters monitored to identify and to track groundwater contamination. Radioactivity parameters consist of the two types of radioactivity parameters prevalent on this plant site: alpha and beta. Also monitored are radium and uranium (alpha emitters) and ${ }^{99} \mathrm{Tc}$ (beta emitter).

\subsubsection{Results and interpretation}

Results produced in 1989 for VOCs and radioactivity parameters for $\mathrm{X}-231 \mathrm{~B}, \mathrm{X}-749$, and $X-701 B$ are within expected levels for each of these sites and are comparabl 2 with values produced during investigations in 1988. Additional discussion of the occurrence of contaminants and groundwater contaminant plumes is provided in Subsect. 2.3.1.1.

Unexpectedly, values for chromium were elevated at he X-616 chromium sludge surface impound $e^{4} \uparrow$. There were four data points produc luring Appendix IX analysis of samples from four selected wells that showed elevated levels. Selected wells $8,12,14$ and 16 located at the $\mathrm{X}-616$ chromium sludge surface impoundments were sampled and analyzed for Appendix IX parameters. None of the organic parameters were reported at levels greater than the reported detection limits; however, some of the metal parameters were. Chromium concentrations were repurted at levels nominally greater than the inaximum contaminant level of $0.05 \mathrm{mg} / \mathrm{L}$ total chromium (i.e., $0.06 \mathrm{mg} / \mathrm{L}$ at well $11,0.09 \mathrm{mg} / \mathrm{L}$ at well $14,0.11 \mathrm{mg} / \mathrm{L}$ at well 16 , and $0.32 \mathrm{mg} / \mathrm{L}$ at well 12) but less than maximum contaminant levels for all other metals on the inorganic chemical list (Appendix IX Analyses: X-616 Chromium Sludge Surface Impoundment, 1989). Subsequent monitoring efforts in 1990 will be directed toward confirming or denying these elevated levels and the nature and source of the chromium should the elevated levels be confirmed.

Additional concerns result from elevated levels of lead appearing in multiple wells at the X-735 sanitary landfill during three sampling events in 1989. Monitoring and investigation efforts will be modified to focus attention on the elevated lead levels and to respond appropriately to the findings of the investigations.

\subsection{BIOLOGICAL MONITORING}

Biological monitoring at PORTS is done to directly assess the impact of operations on vegetation growing around the plant, animals that eat the vegetation, and humans who eat the vegetation and animals. This provides a check on indirect assessments based on emission monitoring, atmospheric dispersion modeling, and similar methods. Annual dose assessments are routinely calculated from both direct monitoring and indirect estimation results unless the direct monitoring results do not detect any contamination. Since no contamination implies no radiation dose to the public, the direct assessment is not presented in such cases. A summary of the results of each sampling program is presented here, and analytical results of individual samples are presented in Part 2. The dose assessment is presented in Section 3.

\subsubsection{Food Crops}

Since 1976, PORTS personnel have collected annual samples of food crops from privately owned farms and gardens surrounding the facility. These samples are analyzed for uranium, gross alpha activity, and gross beta activity. Radioactive contamination of crops may result from absorption of radioactive materials from the soil or from deposition of airborne contaminants. 


\subsubsection{Changes in sampling program}

The types of crops analyzed vary from year to year because of availability; however, corn and tomatoes are usually available. Crops analyzed in 1989 were corn, tomatoes, bell peppers, apples, potatoes, sweet potatoes, pumpkins, soybeans, and field corn. These crops were collected from four gardens in the vicinity of the plant and three other gardens several kilometers away that served as a source of background samples.

\subsubsection{Sample collection and analytical procedures}

Samples of garden produce are collected in resealable plastic bags by Environmental Control Department personnel and delivered to the laboratory where the samples are weighed prior to drying for analysis. Reported concentrations are based on the wet weighi because that is the way the produce is consumed. Food crops are analyzed for total uranium and technetium concentrations and for gross alpha and iechnetium activities. They are also analyzed for fluoride concentrations.

Results of the food crop analyses are reported by the laboratory to the PORTS Environmental Surveillance Section. The donors of the crop samples are informed of the results of their samples by letter. Resampling is performed if analytical results are significantly above the normal background range.

\subsubsection{Results}

Out of 17 food crop samples collected in 1989, only one showed any detectable uranium at all. A sample of soybeans grown in a background garden $16 \mathrm{~km}$ (10 miles) north of the plant showed a concentration of $0.4 \mu \mathrm{g} / \mathrm{g}$ of uranium. This is not outside the normal range of uranium concentrations (i.e., soil normally runs 3 to $5 \mu \mathrm{g} / \mathrm{g}$ in the vicinity of the plant site), but it is higher than the other 1989 crop and vegetation samples. Both the distance of the garden from the plant site and the absence of any detectable uranium in crop samples taken uearer to plant site make it very unlikely that this is contamination from PORTS operations. No detectable technetium concentrations or above-normal alpha levels were found in any of the crop samples. (Some crop samples could not be analyzed for alpha activities because of laboratory backlogs. See Part 2 for individual results.)

\subsubsection{Milk and Eggs}

PORTS started a Milk-Sampling and Analysis Program in 1976. The program was terminated after 1984 when several dairies discontinued operations. In 1989 four area dairy farms were found to be operating, and samples were collected. Additionally, an egg sample was collected from a local farm for the first time in 1989.

\subsubsection{Sample collection and analytical procedures}

The milk samples were analyzed for uranium and technetium. The egg sample was analyzed for uranium, gross alpha activity, and technetium.

\subsubsection{Results}

None of the milk or egg samples showed any detectable uranium or technetium. The egg sample showed $0.07 \mathrm{pCi} / \mathrm{g}$ of alpha activity; this is within the normal background range.

\subsubsection{Fish}

In 1983 PORTS started a Fish-Sampling Program in the Sciuto River and in the local creeks downstream of the site. The local creeks are of particular concern because they are small enough that little dilution of plant effluents occurs during dry seasons. Analytical results of fish samples are presented in Part 2.

\subsubsection{Sample collection and analytical procedures}

Fish are collected from all receiv: y streams surrounding PORTS. These streams include Little Beaver Creek, Big Beaver Creek, Big Run Creek, and the Scioto River. None of these streams suppo: 4 anything more than occasional sport fishing; this greatly limits the impact of slight coritamination levels on human beings. In addition, fish are collected from uncontaminated sources to serve as background ramples. Fish samples are collected in fish traps by environmental personnel 
and are analyzed for several known and potential waterborne pollutants. Fish samples are analyzed for several known and potential waterborne pollutants, In 1989, samples were analyzed for total uranium, gross alpha activity, technetium, total fluorides, mercury, chromium, and PCBs.

\subsubsection{Results}

The analyses show no significant pattern of contamination either radioactive or nonradioactive. None of the fish sampled had detectable uranium. Technetium concentrations were all well below the lower limits of analytical confidence (i.e., 10.8 $\mathrm{pCi} / \mathrm{g}$ ). The highest alpha level found was 0.27 $\mathrm{pCi} / \mathrm{g}$ in Big Beaver Creek upstream of plant outfalls.

Average fluoride concentration was $13.6 \mu \mathrm{g} / \mathrm{g}$; the highest concentration was in the Scioto River upstream of plant outfalls. Chromium concentration was less than the detection limit (i.e., $2.0 \mu \mathrm{g} / \mathrm{g}$ ) in all samples. All mercury concentrations were below detection limits (i.e., $0.05 \mu \mathrm{g} / \mathrm{g}$ ). The highest PCB concentration was $2.2 \mu \mathrm{g} / \mathrm{g}$ in Sunfish from Little Beaver Creek. The FDA limit for PCBs in food is $2 \mu \mathrm{g} / \mathrm{g}$.

On August 14, 1989, two Smallmouth bass were submitted to the laboratory for comparative PCB analysis. One fish, submitted whole, had a PCB concentration of $1.4 \mu \mathrm{g} / \mathrm{g}$. The fillets of the other fish showed a less-than-detection level of PCBs. Individual analyses for the fish samples can be found in Tables 2.4.1 through 2.4.4 in Part 2.

\subsubsection{Wildlife}

The analysis of wildlife tissues is not a part of the PORTS surveillance program in 1989.

\subsubsection{Vegetation}

PORT'S has conducted parallel vegetationand soil-sampling programs since 1975 . Vegetation (i.e., wide-blade grass typical of local cattle forage) and soil samples are collected from several locations in and around the PORTS site at semiannual intervals. The parallel programs allow the comparison of soil concentrations with vegetative uptake rates. To date, however, both programs have consistently shown only background levels off-site.

\subsubsection{Sample collection and analytical procedures}

Vegetation (i.e., grass) samples were collected from 31 off-site locations (see Figs. 2.5.2 through 2.5.4); these are divided into three sample groups. Group I includes sample points on or near the DOE Reservation boundary; Group II extends out to $5 \mathrm{~km}$ ( 3 miles) from the site; and Group III extends between 5 and $16 \mathrm{~km}$ ( 3 and 10 miles). Four background locations are located $16 \mathrm{~km}$ (10 miles) from the plant site.

Samples of wide-blade grass are clipped above ground level so that soil is excluded. The sample is placed into resealable plastic bags for transfer to the laboratory. The grass samples are analyzed for uranium, total alpha, and techetium.

The samples are also analyzed for fluoride, which can be taken up from the soil or groundwater or absorbed directly from the air. Some scientists maintain that vegetative uptake of gaseous fluorides is the best indication of undesirable airborne concentrations of these compounds. Because the enrichment cascade emits significant quanitities of gaseous fluorides, vegetative fluoride concentrations are of great interest to PORTS and its neighbors.

\subsubsection{Results}

Vegetation-sampling results showed no significant contamination. Uranium levels were below detection limits in all but one sample collected $16 \mathrm{~km}$ (10 miles) south of the plant site. This uranium concentration was at the lower detection level (i.e., $0.5 \mu \mathrm{g} / \mathrm{g}$ ). Technetium results showed that all samples were near or below the lower detection level. Fluoride analyses showed a very slight elevation in Group I. Group I results averaged $3.6 \mu \mathrm{g} / \mathrm{g}$ fluorides with a maximum level of $5.9 \mu \mathrm{g} / \mathrm{g}$. Other groups average $3.1 \mu \mathrm{g} / \mathrm{g}$. These are all well under the $30-$ to $40 \mu \mathrm{g} / \mathrm{g}$ levels at which vegetative fluoride will begin to damage the teeth of foraging cattle. Individual sample results are presented in Part 2. 


\subsection{SOIL AND SEDIMENT MONITORING}

Soils are analyzed to determine whether airborne emissions from PORTS are influencing the soil surrounding the site. Internal, reservation, off-site, and remote soil-sampling locations are shown in Figs. 2.5.1 through 2.5.4. Sediments are collected from streams that receive liquid discharges from the plant to monitor the effect of plant operations on the streams (see Fig. 2.5.5).

\subsubsection{Soil}

PORTS personnel collect soil and vegetation samples in parallel locations to allow correlations in the two types of samples to be noted.

\subsubsection{Sample collection and analytical procedures}

A representative sample of soil is collected from each sample point and placed into a new container. The container is covered and transported to the laboratory where the sample is dried and ground prior to analysis. Soil samples are analyzed for uranium, total alpha, and technetium. They are not analyzed for nonradioactive parameters.

\subsubsection{Results}

The soil samples showed no significant contamination of the environment. The results were grouped into four sets: Group I (i.e., on or near the DOE property line), Group II (i.e., out to $5 \mathrm{~km}$ ), Group III (i.e., 5 to $16 \mathrm{~km}$ ), and Internal (i.e., within or near the secured plant area). Soil samples are considered contaminated if they show more than $10 \mu \mathrm{g} / \mathrm{g}$ uranium or $10 \mathrm{pCi} / \mathrm{g}$ total alpha or technetium. Typical background levels are 3 to $5 \mu \mathrm{g} / \mathrm{g}$ uranium, 3 to $6 \mathrm{pCi} / \mathrm{g}$ total alpha, and less than $1 \mathrm{pCi} / \mathrm{g}$ teshnetium. For comparison, $10 \mathrm{pCi} / \mathrm{g}$ of technetium corresponds to $0.6 \mathrm{ppb}$. Individual sample results are provided in Tables 2.5.1 and 2.5.2 in Part 2.

The internal samples showed some areas of alpha contamination within the plant site, and one sample showed a slight technetium contamination (i.e., $11 \mathrm{pCi} / \mathrm{g}$ vs a standard of $10 \mathrm{pCi} / \mathrm{g}$ ). The only area to show persistant contamination (i.e., it was contaminated according to both spring and fall samples) was RIS 22 , which is adjacent to the $X-700$ cleaning building. The averages of the internal soil samples showed a slight alpha contamination (i.e., $13.5 \mathrm{pCi} / \mathrm{g}$ vs a standard of $10 \mathrm{pCi} / \mathrm{g}$ ) in the spring and no contamination in the fall. None of the external soil samples showed any contamination except for a very slight alpha contamination (i.e., $10.8 \mathrm{pCi} / \mathrm{g}$ vs a standard of $10 \mathrm{pCi} / \mathrm{g}$ ) in the fall sample taken at sample point $\mathrm{S} ! 1$, which is actually well inside the DOE reservation although outsicle the security lence. Details of individual sample results are presented in Part 2.

\subsubsection{Sediment}

\subsubsection{Sample collection and analytical procedures}

Sediment sample: are collected both upstream and downstream of plant liquid discharge points at semiannual intervals. The samples are placed into new sample containers, capped, and transported to the laboratory where they are dried and ground prior to analysis. Sediment samples are analyzed for uranium, total alpha, and technetium. They are not analyzed for nonradioactive parameters.

\subsubsection{Results}

The 1989 sediment analyses show minor uranium and alpha radiation contamination sediments immediately downstream of plant outfalls; these diminished to normal background levels in Scioto River sedivinents. Minor technetium contamination was also found downstream of the plant; this diminished to near background levels in the Scioto River. None of the concentrations found were high enough to cause health or environmental concerns. These results are consistant with sediment-sampling results of previous years. Individual sample results are presented in Table 2.5.3 of Part 2. 


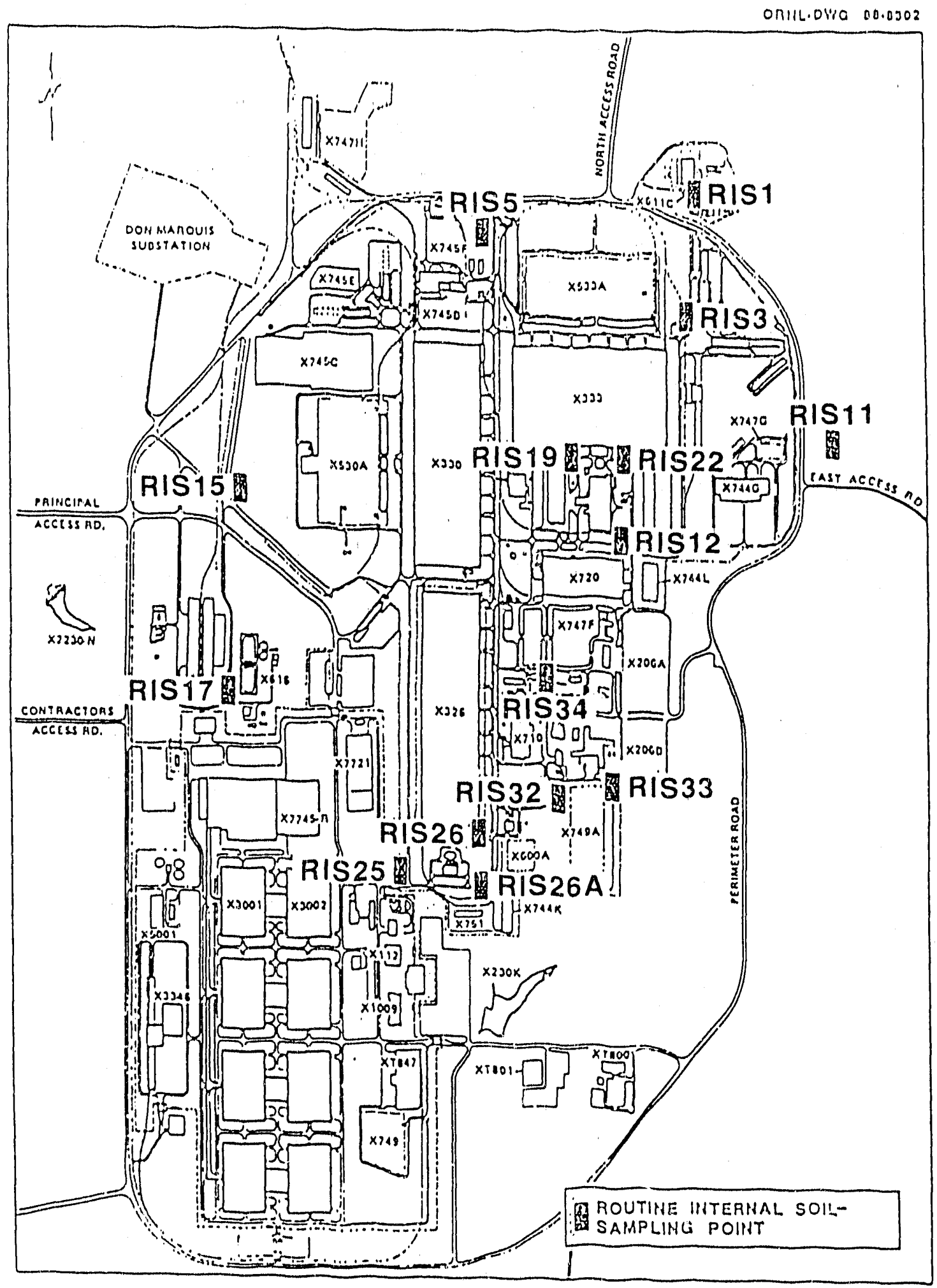

Fig. 2.5.1. Internal soll-sampling locations at PORTS. 
ORNL-DWG $87.7838 R 2$

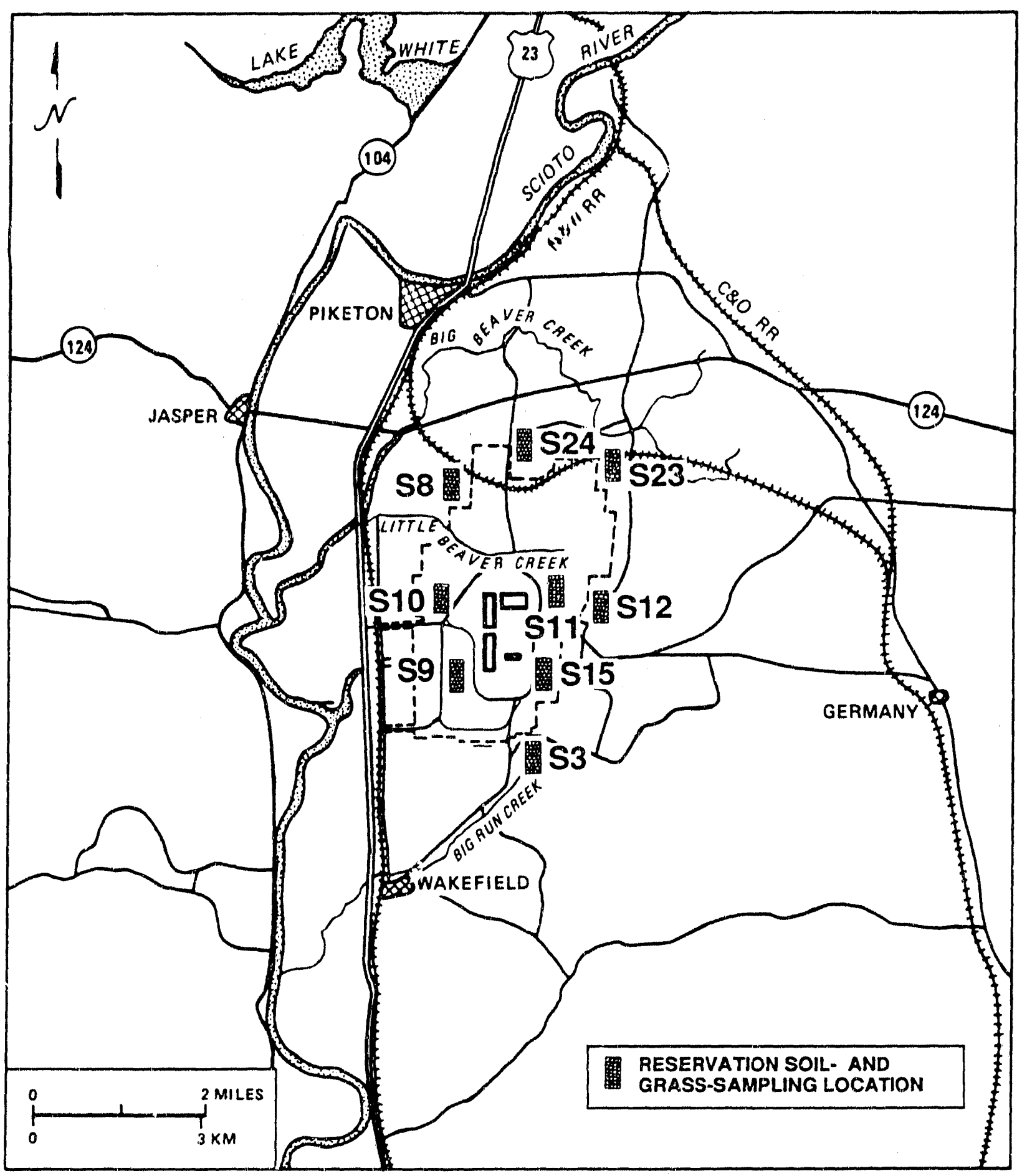

Fig. 2.5.2. Reservation soll- and vegetution-sampling locations at PORTS. 


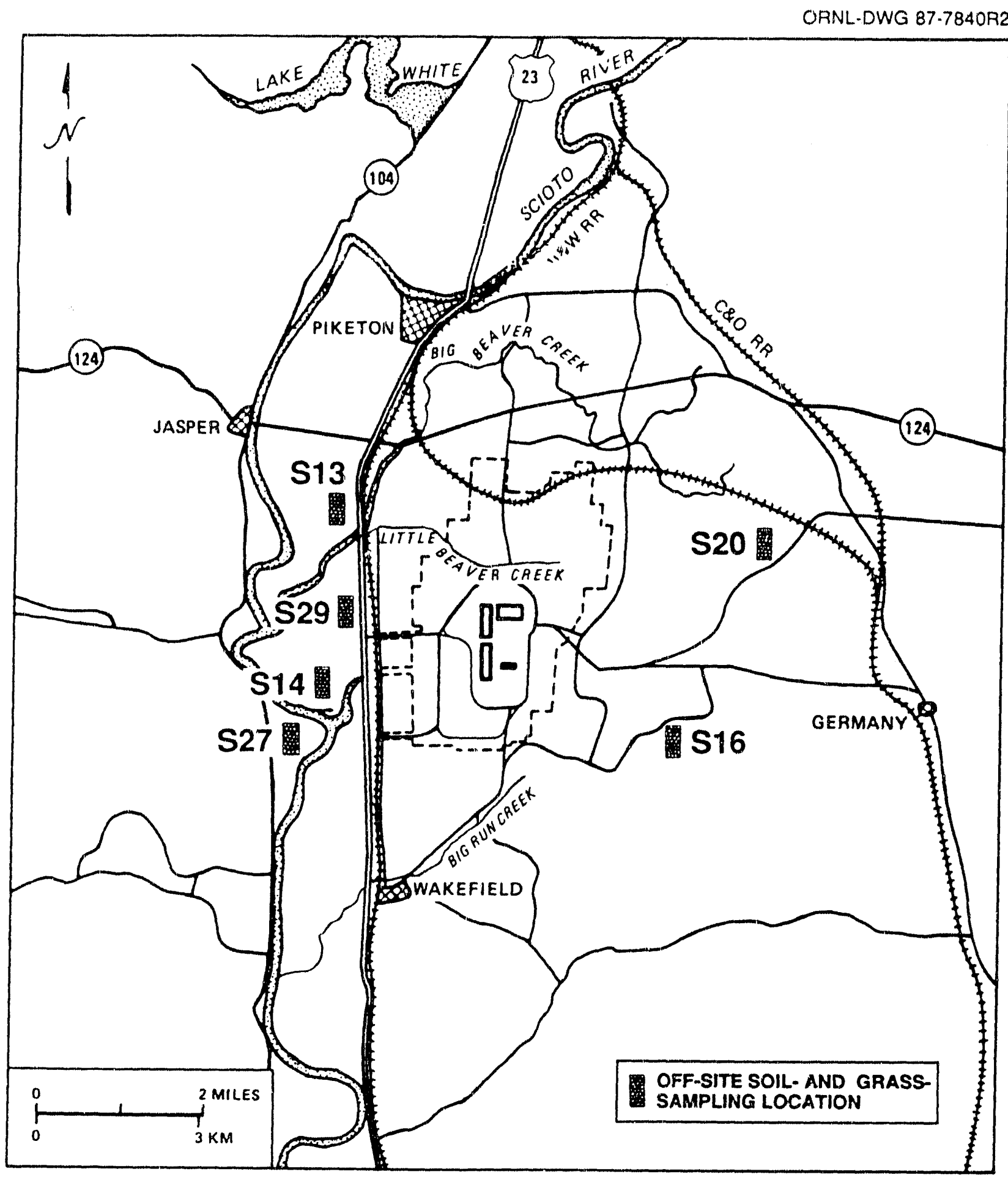

Fig. 2.5.3. Off-site soll- and regetation-sampling locations at PORTS. 
ORNL.DWG 87-7634A

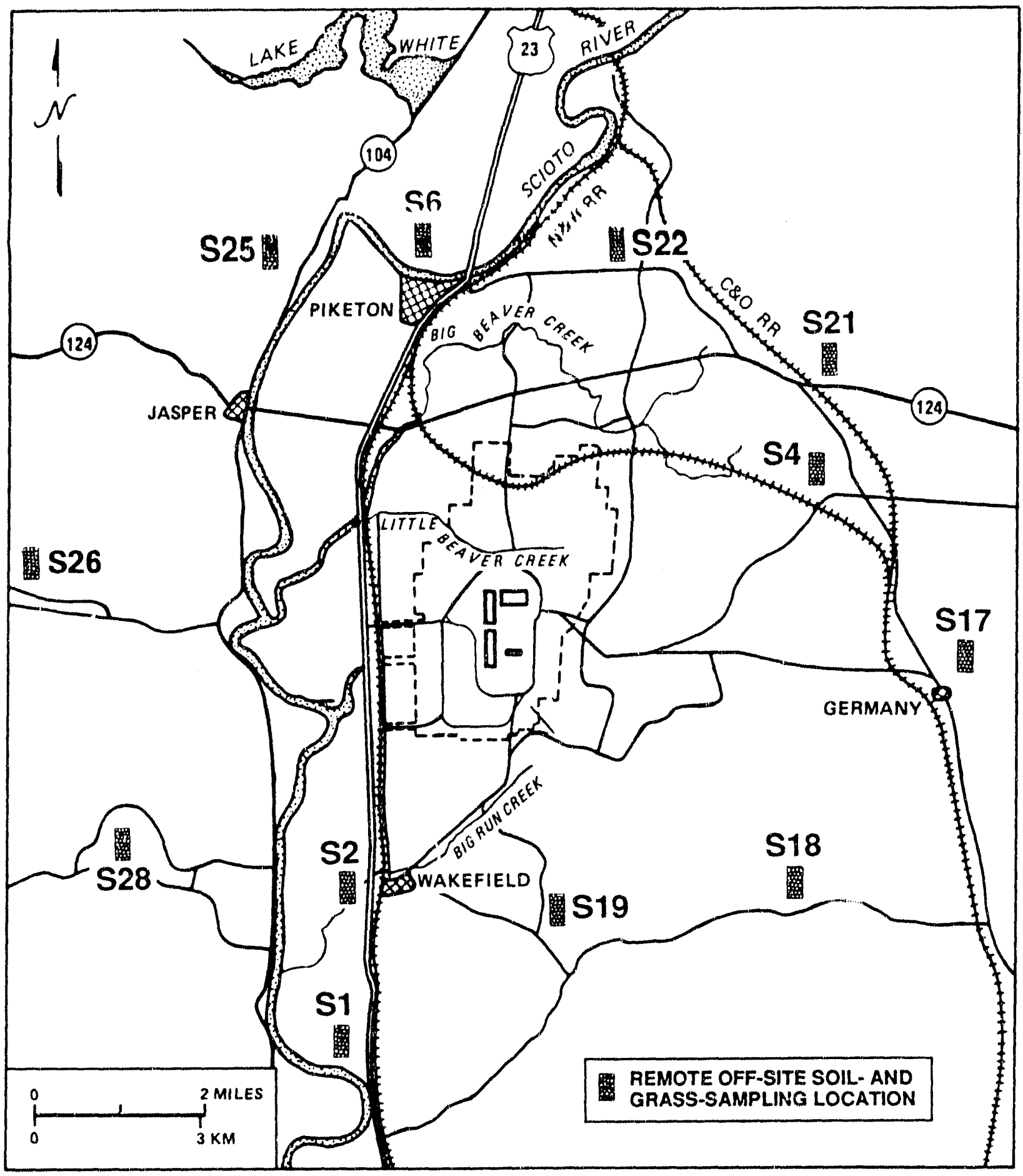

Fig. 2.5.4. Remote soll- and regetation-sampling locations at PORTS. 
IRNL-DWG 87-10318R2

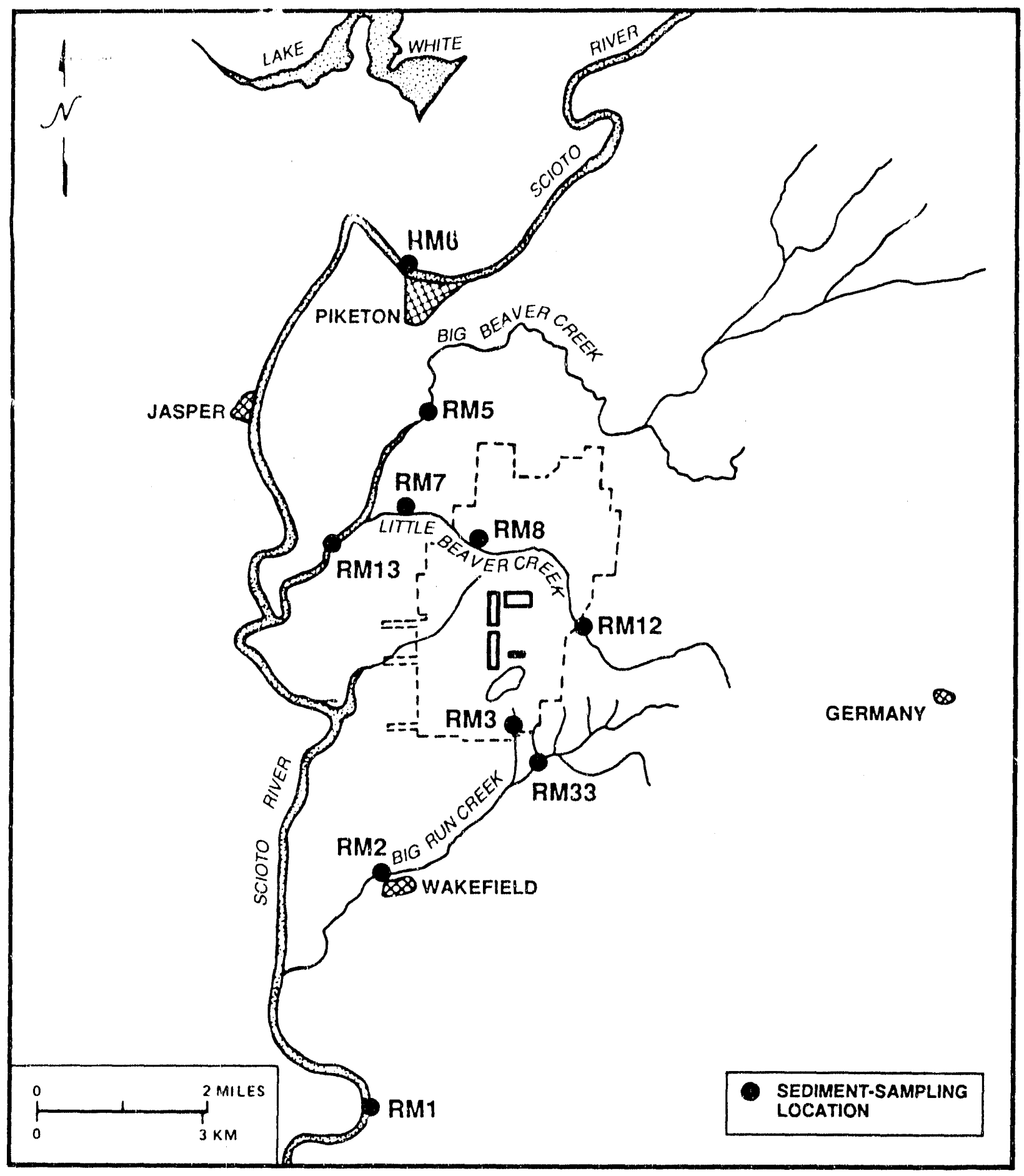

Fig. 2.5.5. Stream-sediment-satupling locations at PORTS. 


\subsection{EXTERNAL GAMMA RADIATION}

External gamma radiation measurements are made to confirm that direct radiation and radioactive effluents from PORTS are not significantly different from natural background levels. The monitoring network may also be useful in assessing the impact of a criticality accident should one occur. This subsection describes the external gamma-radiation-monitoring network at PORTS and presents a summary and discussion of the data collected by that network. A brief summary of the radiation doses that can be estimated from these data is also presented. A more extensive discussion of radiation dose calculations is found in Sect. 3. For a more detailed presentation of the gamma radiation data without discussion, see Subsect. 2.6, Part 2.

\subsubsection{Program Description}

PORTS uses thermoluminescent dosimeters (TLDs) to measure gamma radiation exposure. When exposed to penetrating radiation (such as gamma or cosmic radiation), thermoluminescent materials absorb and store a portion of the radiation energy. If the material is heated later, this energy will be released as light. Although thermoluminescent materials (such as clay) are fairly common, only a handful are sufficiently accurate for use in radiation measuring devices (i.e., dosimeters). Because TLDs can be reused 50 or more times, they have almost completely replaced the older film-badge system of dosimetry.

The TLDs used in the PORTS environmental program are based on two chips of a commercial thermoluminescent material (i.e., $\mathrm{CaF}_{2}: \mathrm{Dy}$ ) permanently mounted in a Teflon and aluminum card. The card is mounted inside a plastic and cadmium holder for field use. These TLDs respond to gamma and neutron radiation and, to some extent, to cosmic radiation. Normally, no significant levels of neutron radiation exist around PORTS, but all uranium-handling facilities have some theoretical chance of experiencing a criticality or nuclear chain reaction. Such an event has never occurred at PORTS, and the chances of its happening in the future are remote.
Nevertheless, the TLD network is designed to assist in evaluating the environmental impact of such an event should it occur.

The usual concern of the TLD network is the level of ambient or external gamma radiation around PORTS. The term "external" is used because the source of the radiation is external to the body, so only penetrating radiation is of concern. In contrast, internal sources that have been inhaled or swallowed involve nonpenetrating alpha and beta radiation. The only significant source of gamma radiation at PORTS is the uranium isotope ${ }^{235} \mathrm{U}$.

External gamma measurements are made in the relatively small area of the PORTS site accessible to the public, around the edge of the PORTS site, and out to about $8 \mathrm{~km}$ ( 5 miles) from the plant center. Nine on-site field TLDs (Site Group I) are on or near Perimeter Road, which runs around the secure, or Q-cleared, area of the plant. In addition, a group of six reference TLDs is on top of the X-100 administration building, and another group of six control TLDs is kept in a lead "pig" in the Environmental Control Department. Reference and control TLDs are used in calibrating the field TLDs. Eight TLDs (Site Group II) are collocated with the fence-post airmonitoring stations discussed in Subsect. 2.1, and 15 off-site TLDs (Site Group III) are located from 3 to $8 \mathrm{~km}$ ( 2 to 5 miles) out from the plant center. The TLD locations are shown in Figs. 2.6.1 and 2.6.2.

PORTS maintains three complete sets of TLDs (i.e., 32 field, 6 reference, 6 control, and spares) that are exposed alternately on a quarterly basis. At the beginning of each calendar quarter, an unused set is annealed (i.e., zeroed by heating) and exchanged for the current set. The current set is read with a manual TLD reader. TLDs that give unusually high or low readings or that give significantly different readings between the individual chips are set aside as suspect. All TLDs are then annealed, exposed to a calibrated gamma radiation source (i.e., ${ }^{137} \mathrm{Cs}$ ), and reread. The nonsuspect TLDs are used to construct a calibration curve for the set, and the suspect TLDs are tested for accuracy against this curve. Suspect 
ORNL-DWG 87-10788R

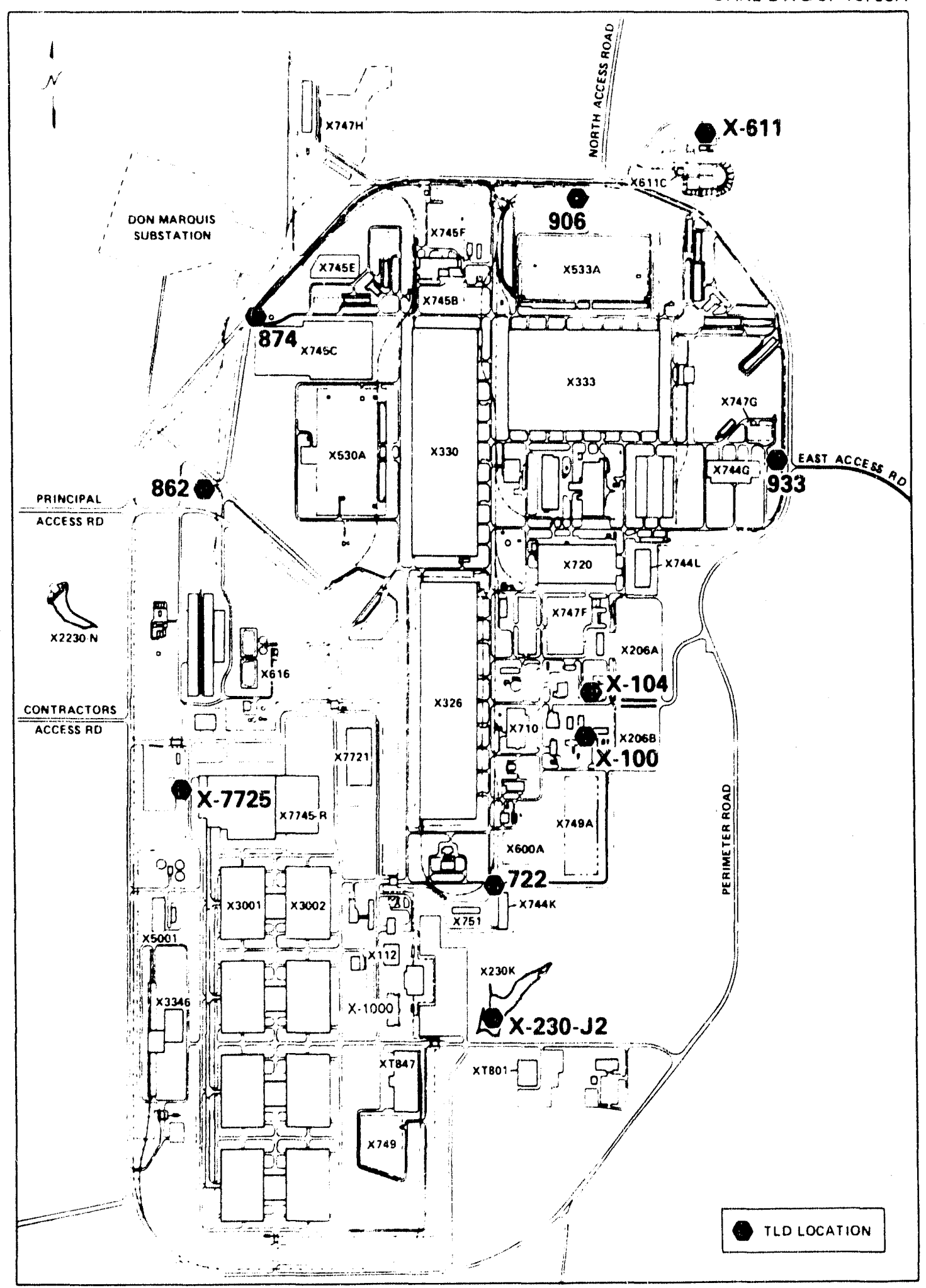

Fig. 2.6.1. On-site TLD locations (Site Group I). 


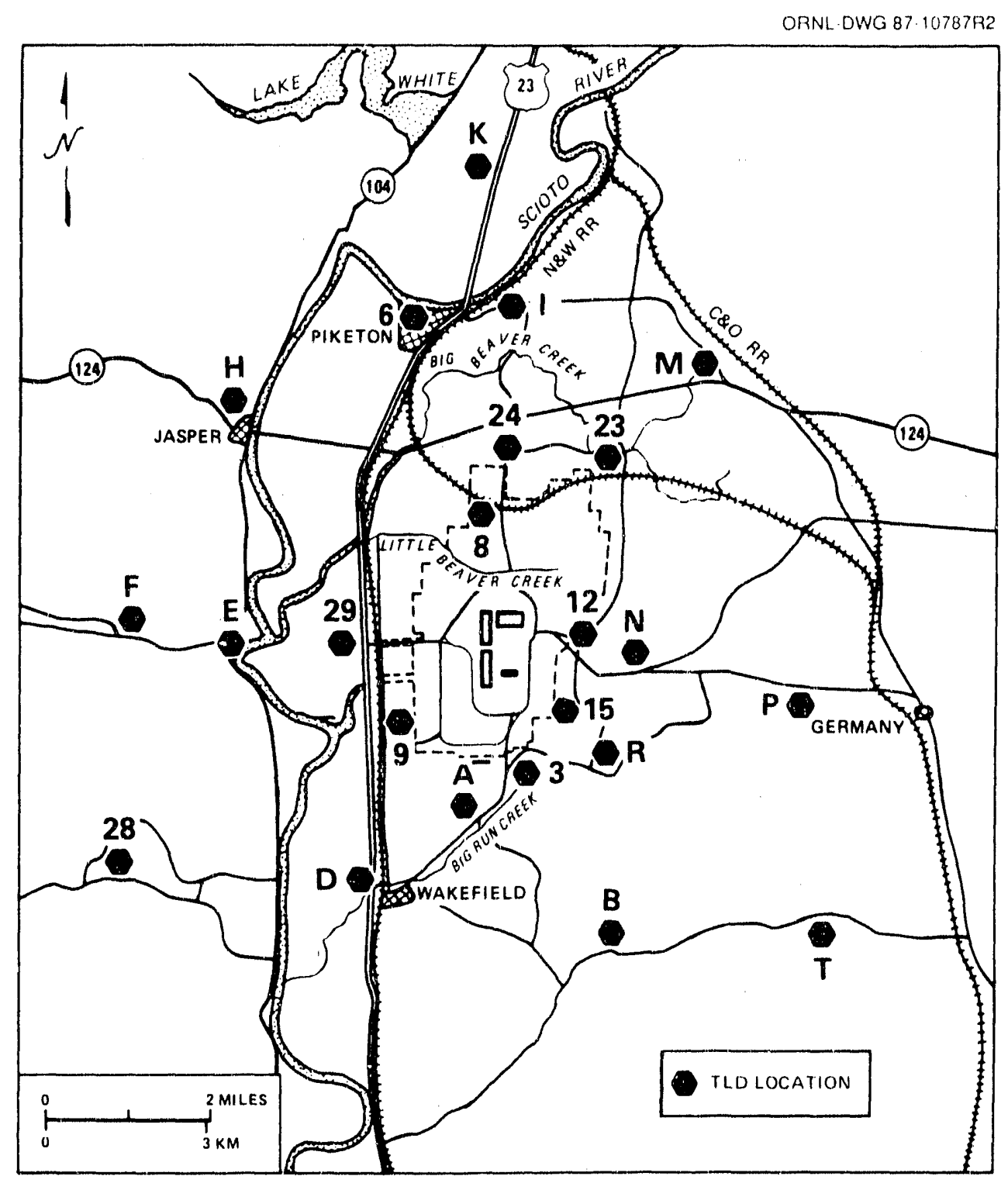

Fig. 2.6.2. Off-site TLD locations (Site Groups II and III).

TLDs that fail this test are discarded along with their readings.

The external gamma radiation levels are reported as quarterly site-group averages with one exception in Site Group I: TLD Location 874 is at the corner of a uranium storage yard, and gamma levels there are sharply higher than those on the rest of Perimeter Road. This location is reported separately to emphasize that a single hot spot exists and that the Perimeter Road in general is not an area of elevated gamma levels. This procedure is particularly important when estimating radiation dose from radiation levels. In Site Groups II and III this calculation is made by multiplying the annual average gamma levels by the time exposed (i.e., $24 \mathrm{~h} / \mathrm{d}, 365 \mathrm{~d} /$ year) because a continuous exposure in those areas is a reasonable assumption.

Public exposure to Site Group I gamma levels is limited to through traffic on Perimeter Road and actual plant business and is much lower than the 
$24 \mathrm{~h} / \mathrm{d}, 365 \mathrm{~d} /$ year assumed for the other site groups. Because it is impossible to measure accurately how much lower the public exposure is and because the group average gamma level is not significantly higher than background, a conservative worst case of continuous exposure is assumed for the group average. Since location 874 is away from any access roads or security fence gates, it is possible to estimate an annual exposure to members of the general public for this area. Public traffic is not allowed to stop in this area, and tests by environmental personnel have shown that a car traveling slightly under the posted speed limit passes completely through the hot spot in less than $20 \mathrm{~s}$. Assuming two trips per day (coming and going) and $5 \mathrm{~d} /$ week gives a public annual exposure of $3 \mathrm{~h} /$ year. The Site Group I public dose is therefore estimated as the annual average gamma level at location 874 times $3 \mathrm{~h}$ /year plus the annual average gamma level for the rest of the group times $24 \mathrm{~h} / \mathrm{d}, 365 \mathrm{~d} /$ year. Worker exposures in this and other areas of elevated gamma radiation are measured directly by a separate TLD system operated by the Health Physics Department.

\subsubsection{Thermoluminesient Dosimeter Data Summary}

Except for TLD location 874, external gamma levels around PORTS are not significantly different from natural background. Table 2.6.1 shows the average external gamma level (in $\mu \mathrm{rad} / \mathrm{h}$ ) plus or minus twice the standard deviation by site group and calendar quarter. One microrad of gamma radiation will produce $1 \mu \mathrm{rem}$ of dose equivalent if a person is exposed. Quarterly average gamma levels at location 874 are shown in parentheses under the data for the rest of Site Group I. The last column shows the estimated gross annual external gamma dose within each of the site groups. For Site Group I the annual dosage is estimated assuming the 3-h/year exposure (see previous discussion) to location 874 radiation levels and otherwise continuous exposure to the group average radiation levels.

The numbers in Table 2.6.1 represent gross gamma levels and doses including gamma radiation from PORTS activities and effluents, local natural sources (terrestrial gamma), and some cosmic radiation. No effort has been made to separate the natural radiation from the PORTS contribution. In theory this could be done by subtracting the external gamma level at a background location from external gamma levels at all the other locations. In practice the off-site TLDs around PORTS consistently record higher gamma levels than the supposedly more exposed TLDs on and within the site boundary.

The cause of this radiation level pattern lies in the geology of the PORTS site. As is described in Sect. 1, the PORTS plant is located within an ancient river bed that was filled with clay and silt during the glacieral period. The surrounding hills and ridges, on the other hand, are largely composed of much older shale that are significantly richer in natural radioactive minerals. Most of the off-site TLDs are located among these hills and ridges where they are exposed to a higher

Table 2.6.1. Ambient gamma levels and doses

\begin{tabular}{lccccc}
\hline \multirow{2}{*}{$\begin{array}{c}\text { Site } \\
\text { groups }\end{array}$} & \multicolumn{4}{c}{ Ambient gamma levels $(\mu \mathrm{rad} / \mathrm{h})$} & \multirow{2}{*}{$\begin{array}{c}\text { Annual dose } \\
\text { (mrem) }\end{array}$} \\
\cline { 2 - 6 } & First quarier & Second quarter & Third quarter & Fourth quarter & \\
\hline I $^{a}$ & $9.3 \pm 2.0$ & $13.1 \pm 3.0$ & $10.3 \pm 1.6$ & $9.3 \pm 1.4$ & 92 \\
$(874)$ & $(131)$ & $(156)$ & $(118)$ & $(110)$ & \\
II & $9.0 \pm 1.8$ & $11.5 \pm 4.0$ & $10.1 \pm 1.1$ & $8.6 \pm 3.0$ & 86 \\
III & $10.4 \pm 3.8$ & $12.2 \pm 3.2$ & $10.9 \pm 2.2$ & $9.7 \pm 2.4$ & 95 \\
\hline
\end{tabular}

${ }^{a}$ Excludes TLD location 874 (see text). 
level of radiation than TLDs located in the old Portsmouth, Teays, or Newark River valleys or even the on-site TLDs. Consequently, no legitimate background level of radiation can be designated. On the other hand, plant emissions that are consistently lower than the local variation in natural background radiation can hardly be called significant.

For comparison, Fig. 2.6.3 shows graphically the annual gross external radiation dose for each of the three site groups along with average annual doses for (terrestrial) gamma radiation and for gamma plus cosmic (total) radiation for the state of Olio and for the United States. For a more general comparison, Fig. 2.6.4 shows average annual doses from terrestrial gamma, cosmic, and total radiation for several states and for the entire country. In Fig. 2.6.3 both types of doses (gamma

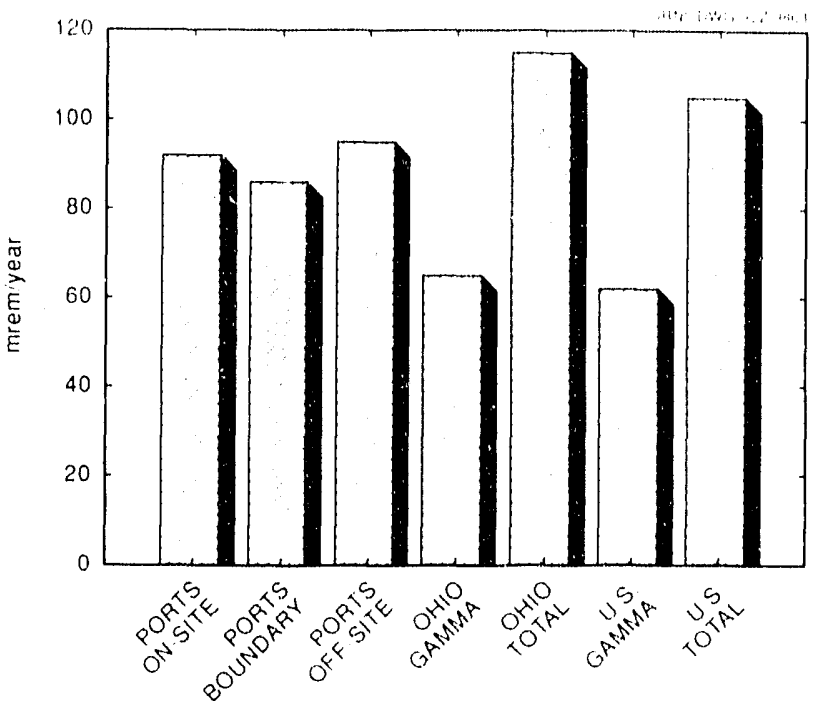

Fig. 2.6.3. External gamma exposure on, off, and around the PORTS site.

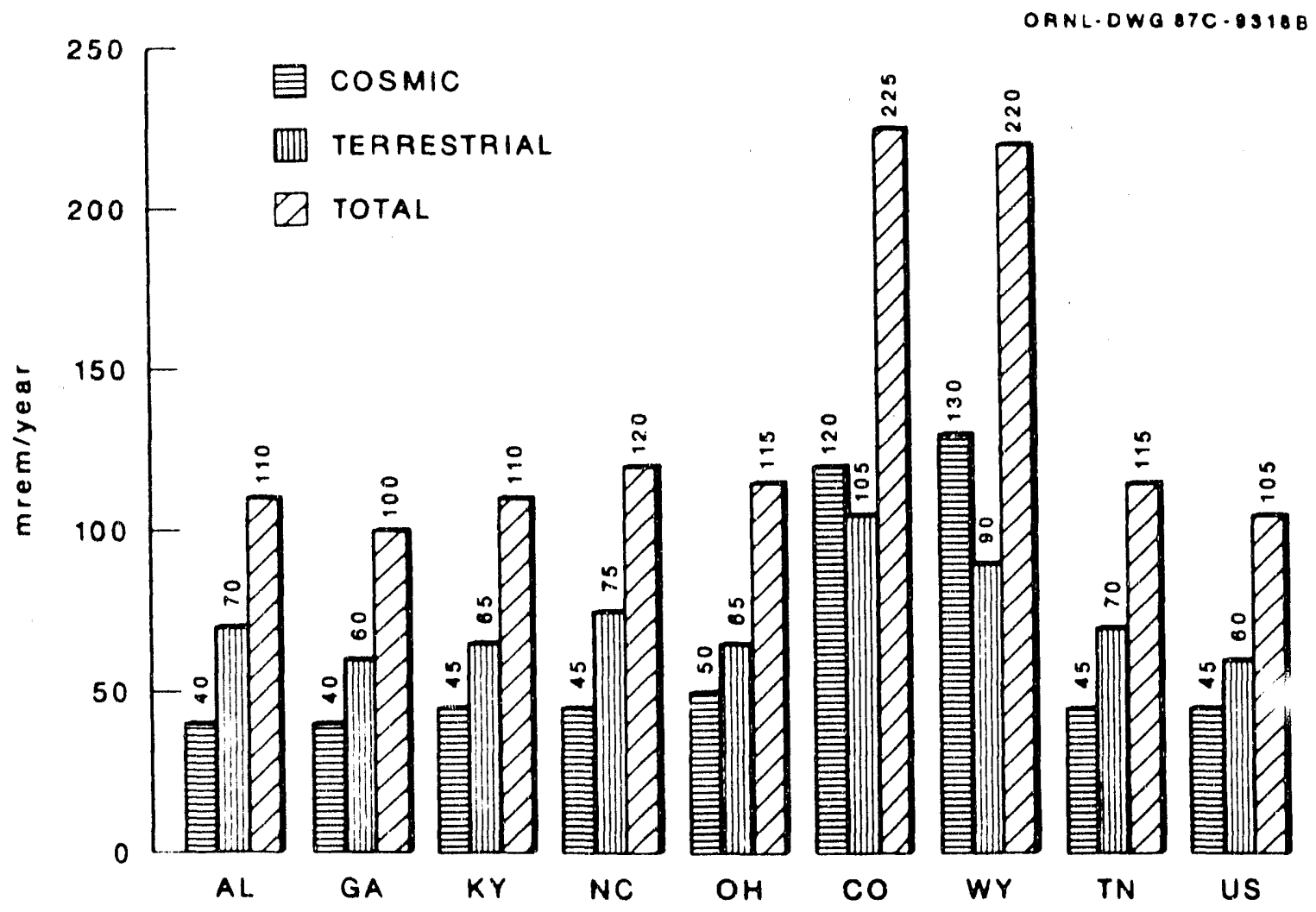
States.

Fig. 2.6.4. External cosmic, terrestrial, and total gamma exposure for several states and for the United 
and total) are included because the PORTS TLD system does not completely exclude cosmic radiation. Consequently, the recorded site-group average doses, exclusive of any plant emissions, should be somewhat above the state average gamma dose and below the state average total dose. As can be seen in Fig. 2.6.3, the 1989 gamma radiation doses in the vicinity of PORTS are within this range. 


\section{POTENTIAL RADIATION AND CHEMICAL.DOSE TO THE PUBLIC}

\subsection{RADIATION DOSE}

Small quantities of radionuclides were released to the environment from operations at PORTS during 1989. These releases are quantified and characterized in Sect. 2. This section summarizes estimates of the potential consequences of the releases and describes the methods used to make the estimates. More detailed information on different exposure pathways can be found in Part 2 of this report.

\subsubsection{Terminology}

Most consequences associated with radionuclides released to the environment are caused by interactions between radiations emitted by the radionuclides and human tissue. These interactions involve the transfer of energy from the radiations to tissue, a process that may damage the tissue. The radiation may come from radionuclides located outside the body (in or on environmental media or objects) or from radionuclides deposited inside the body (via inhalation, ingestion, and, in a few cases, absorption through the skin). Exposures to radiation from nuclides outside the body are called external exposures; exposures to radiation from nuclides deposited inside the body are called internal exposures. This distinction is important because external exposures occur only when a person is near the external radionuclides; simply leaving the area of the source will stop the exposure. Internal exposures will continue as long as the radionuclides remain inside the person.

A number of specialized units have been defined for characterizing exposures to ionizing radiation. Because the damage associated with such exposures results primarily from the deposition of radiant energy in tisc:ae, the units are defined in terms of the amount of incident radiant energy absorbed by tissue and the biological consequences of that absorbed energy. Some of these units are:

- . bsorbed dose. A physical quantity that defines the amount of incident radiant energy absorbed per unit mass of an irradiated material; its unit of measure is the rad. The absorbed dose depends on the type and energy of the incident radiation and on the atomic number of the absorbing material.

- Dose equivalent. A quantity that expresses the biological effectiveness of an absorbcd dose in a specified human organ or tissue; its unit of measure is the rem. The dose equivalent is numerically equal to the absorbed dose multiplied by modifying factors that relate the absorbed dose to biological effects. In this report, as in many others, the term "dose equivalent" often is shortened to "dose."

- Effective dose equivalent. A measure of overall carcinogenic and genetic risk resulting from exposures to radiation. It is a weighted sum of dose equivalents to specified organs. The weighting factors and identification of these specific organs have been published (ICRP 1977; ICRP 1978).

- Committed (effective) dose equivalent. The total (effective) dose equivalent that will be received over a specified time period (i.e., 50 years in this document) because of current exposures (internal) to radionuclides.

- Collective (committed) effective dose equivalent. The sum of (committed) effective dose equivalents to all individuals in an exposed population. The unit of measure is the personrem.

- Dose conversion factor $(D C F)$. The dose equivalent received from exposure to a unit 
quantity of a radionuclide via a specific exposure pathway. Two types of DCFs exist. One type gives the committed dose equivalent (rem) resulting from intake (via inhalation and ingestion) of a unit activity $(1.0 \mu \mathrm{Ci})$ of a radionuclide. The second type gives the dose equivalent rate (mrem/year) per unit activity $(1.0 \mu \mathrm{Ci})$ of a radionuclide in a unit $\left(\mathrm{cm}^{3}\right.$ or $\mathrm{cm}^{2}$ ) of an environmental compartment (air or ground surface). DCFs for inhalation and ingestion of radionuclides, for immersion in contaminated air, and for exposure to a contaminated yround surface are listed in Table 3.1 .1 of Part 2 of this report.

\subsubsection{Methods of Evaluation}

\subsubsection{Airborne radionuclides}

Characterization of radiological consequences of radionuclides rel sased to the atmosphere from PORTS during 1989 was accomplished by calculating dose equivalents to the maximally exposed off-site individual, to the population of the nearest community, and to the population residing within $80 \mathrm{~km}$ (50 miles) (Part 2, Table 3.1.2). Airborne releases from the plant are characterized in Subsect. 2.1.

Emissions of radionuclides from PORTS to the atmosphere are regulated under the National Emission Standards for Hazardous Air Pollutants (NESHAP). For 1989, NESHAP limits annual radionuclide emissions to a level that will not cause any member of the general public to receive total radiation dose equivalents that are equal to or greater than 25 mrem to the whole body or 75 mrem to any organ. Beginning in 1990 the NESHAP limit will be expressed in the currently accepted units of radiation dose, the effective dose equivalent. The new limit will be based on a total effective dose equivalent of less than $10 \mathrm{mrem}$ to any member of the public. Since use of the new limit is conservative with respect to the old limits and it uses contemporary units, all dose estimates are presented as effective dose equivalents. Calculated whole-body and maximum organ dose equivalents were only $0.01 \%$ and $1 \%$, respectively, of their limits and were reported to the EPA (Mitchell Apr. 18, 1990).

Doses were calculated using a suite of computer codes (Moore et al. 1979; Begovich et al. 1981; Dunning et al. 1980; Sjoreen and Miller 1984) developed under sponsorship of the EPA for use in demonstrating compliance with NESHAP - Radionuclides (40 CFR 61, subpart H). The atmospheric transport code, AIRDOSEPA, calculates concentrations of released radionuclides in air, on the ground, and in foodstuffs (i.e., meat, milk, and vegetables). Through the DARTAB computer code, the DCFs in the RADRISK data base are applied to the calculated concentrations to give estimates of individual and collective 50-year committed effective dose equivalents from inhalation oî and immersion in contaminated air, from exposure to contaminated ground surfaces, and from ingestion of locally grown foodstuffs (i.e., milk, meat, and vegetables).

Plant-specific meteorological data, population distributions, and source terms were used in all calculations. Beef, milk, and food crop production were assumed to be the maximum possible for the available ground area, an assumption that overstates these activities in the area. It was further assumed that one-third of the foodstuffs consumed by the local population was grown locally; the remaining two-thirds were assumed to be imported from outside an $80-\mathrm{km}$ (50-mile) radius of PORTS.

Releases from PORTS essentially were from two stacks, a 50-m-high (165-ft-high) stack and a 20 -m-high (65.5-ft-high) stack, that were assumed to be located at the plant center. The calculated 50-year committed effective dose equivalent rate to the maximally exposed resident located $1770 \mathrm{~m}$ $(5800 \mathrm{ft})$ east-northeast of the plant is given in Table 3.1.1 (also see Part 2, Tables 3.1.2 and 3.1.3). The 0.07-mrem effective dose equivalent is well below the new NESHAP limit of $10 \mathrm{mrem}$. The 50-year collective committed effective dose equivalent to the approximately 600,000 persons residing within $80 \mathrm{~km}$ ( 50 miles) of the plant was calculated to be 0.4 person-rem (Part 2, Table 
Table 3.1.1. Summary of estimated radiation doses to an adult during 1989 at locations of maximum exposure

\begin{tabular}{ccc}
\hline $\begin{array}{c}\text { Emission } \\
\text { pathway }\end{array}$ & Location & $\begin{array}{c}\text { Effective dose } \\
\text { (mrem) }\end{array}$ \\
\hline Gaseous efnuents & $\begin{array}{c}\text { Maximally ixposed } \\
\text { individual } \\
(1779 \mathrm{~m} \text { ENE) } \\
\text { Liquid efnuents }\end{array}$ & 0.07 \\
\hline
\end{tabular}

3.1.2). The collective dose commitment to the nearest community was calculated to be 0.01 person-rem.

\subsubsection{Waterborne radionuclides}

Water is sampled at all plant outfalls and in the receiving streams. Sample results for the Scioto River show no significant difference in radionuclide concentrations between upstream and downstream locations (see Subsect. 2.2). A hypothetical dose was calculated using measured radionuclide discharges from plant outfalls and the average annual flow rate of the Scioto River [i.e., $4.64 \times$ $10^{12} \mathrm{~L} /$ year $\left(1.2 \times 10^{12} \mathrm{gal} /\right.$ year $\left.)\right]$. If a person should eat $21 \mathrm{~kg}(157 \mathrm{lb})$ of fish caught in the Sciotc River and drink $730 \mathrm{~L}$ (190 gal) of river water during the yeur, that hypothetical person could receive a 50-year committed effective dose equivalent of 0.002 mrem (Part 2, Tables 3.1.1 and 3.1.4). This is a conservative exposure scenario because the river is not used for drinking water downstream of PORTS. About $79 \%$ of the calculated dose from liquid effluents is from drinking water.

\subsubsection{Radionuclides in other environmental media}

No radionuclide contamination has been found in milk produced within $16.1 \mathrm{~km}$ (10 miles) of PORTS. The pasture-cow-milk pathway is included in AIRDOS calculations of dose from airborne radionuclides. Assuming that one-third of the milk consumed by the maximally exposed individual is produced locally, about $5 \%$ of the effective dose reported in Table 3.1.1 is from ingestion of milk. Assuming that one-third of the vegetables and meat consumed are produced locally, about $27 \%$ of the effective dosc is from ingestion of these food items.

\subsubsection{Current Year Summary}

A summary of 1989 maximum doses to an individual is given in Table 3.1.1. It is unlikely that any one real person could be exposed to maximum doses from both gaseous and liquid effluents because the points of maximum exposure are on opposite sides of the plant. Furthermore, no one is believed tc get drinking water from the Scioto River. Nevertheless, the maximum, hypothetical, 30-year committed effective dose equivalent rate to an off-site individual from $1 \geqslant 89$ emissions could be $0.07 \mathrm{mrem} /$ year.

\subsubsection{Findings and Conclusions}

The 50-year committed effective dose equivalent to the maximally exposed off-site individual from airborne effluents was calculated to be $0.07 \mathrm{mrem}$. The effective dose is well within the new dose limits (i.e., 10 mrem) specified in the Clean Air Act (CAA) for DOE facilities. Doses to individuals in the nearest community (Piketon) are about 10 times less.

The estimated 50-year committed effective dose equivalent to the approximately 600,000 persors living within $80 \mathrm{~km}$ ( 50 miles) of PORTS is 0.4 person-rem for 1989 emissions. This represents about $0.0002^{\%}$, of the $2 \times 10^{5}$ personrem the surrounding population would receive from all sources of background radiation. The estimated committed effective dose to the population of the nearest community is 0.01 person-rem.

\subsection{CHEMICAL DOSE}

Varying amounts of chemicals were released to the environment from operations at PORTS during 1989. Those releases are quantified in tables included in this subsection and compared with acceptable levels of exposure for humans to each chemical. Methodology used for determining acceptable chemical exposure levels for humans is described.

\subsubsection{Terminology}

Chemicals gain access to the human body by inhalation, ingestion, absorption, and other routes. Whether or not these chemicals produce a toxic effect in the body is dependent on a nuliser of 
factors including the physical and chemical properties of the compound, the exposure conditions (i.e., duration and frequency), and susceptibility of the human. Lack of available environmental measurement data precludes an analysis of all pathways of concern for human exposure to chemicals. For example, no measurements of organics in air have been made precluding an analysis of the inhalation pethway. However, a relevant and practical analysis of the ingestion pathway via drinking water is possible; therefore, a characterization of human exposure to chemicals via drinking water is provided. It is necessary to define pertinent terms. They are as follows:

- Acceptable daily intake (ADI). Intake of a toxic chernical (measured in micrograms per day) that is not anticipated to result in any adverse health effect following exposure. Represents an EPAestablished value.

- Calculated daily intake (CDI). Intake of a toxic chemical (in milligrams per day) based on the assumption that humans drink $2 \mathrm{~L}(0.5 \mathrm{gal})$ of water per day (an overestimate).

- Carcinogen potency facior (CPF). An estimate based on : lifetime probability that a carcinogenic chemical will cause cancer at a dose of $1 \mathrm{mg} / \mathrm{kg} /$ day. The actual risk is probably lower than the predicted risk or may even be zero.

- National Pollutant Discharge Elimination System. A permit that requires evaluation of area source discharges from within and around the facilities to determine their impact on water quality.

\subsubsection{Methods of Evaluation}

\subsubsection{Airborne chemicals}

The release of airborne chemicals into the atmosphere at PORTS is minimized by avoiding unnecessary generation of wastes and by applying effective control technology to meet all pery it conditions and DOE requirements. Emission sources may release permitted quantities of chemicals into the atmosphere. Further discussion cäit be found in Subsect. 2.!.

\subsubsection{Waterborne chemicals}

Health criteria for water were set so that chemical intake from consumption of $2 \mathrm{~L}$ of wacer per day would not exceed the acceptable daily intake. For noncarcinogenic toxic chemicals, the $\mathrm{ADII}$ is the intake of a toxicant that is not anticipated to result in any adverse health effects after chronic exposure to the general human population including sensitive subgroups (Hoffman et al. 1984). For carcinogenic chemicals, there is no accepted threshold limit. For the purposes of this document, a speciffc risk of developing cancer over a human lifetime of 1 in 100,000 was used to establish acceptable levels of exposure to carcinugesess (Hoffman et al. 1984). The ADI for carcinogenic chemicals was derived using the formula

$$
A D I=\frac{1 \times 10^{-5} \times B W}{C P F},
$$

where

$$
\begin{aligned}
B W= & 70 \mathrm{~kg} \text { (reference adult, healthy male), } \\
C P F= & \text { carcinogen potency factor, which is the } \\
& \text { upper limit on the lifetime probability } \\
& \text { that the carcinogen will cause cancer at a } \\
& \text { dose of } 1 \mathrm{mg} / \mathrm{kg} / \mathrm{d} . \text { The term "upper } \\
& \text { limit" means that the actual risk is } \\
& \text { probably lower than the predicted risk or } \\
& \text { may even be zero. }
\end{aligned}
$$

All ADIs were derived primarily from the EPA Integrated Risk Information System data base, which contains updated ADIs for 1988. For those chemicals not included in the data base, the most current ADIs from other available sources were used and are referenced in Table 3.2.1.

The term ADI represents an allowable daily intake for both carcinogens and noncarcinogens. For example, in establishing water quality criteria for the chemicals of greatest concern, the EPA used the following relationship:

$$
C_{w}=A D I / I_{w},
$$

where

$$
\begin{aligned}
C_{w^{\prime}}= & \text { water quality criteria level }(\mu \mathrm{g} / \mathrm{L}), \\
A D I= & \text { EPA-established value for an "acceptable } \\
& \text { daily intake" }(\mu \mathrm{g} / \mathrm{d}), \\
I_{w^{\prime}}= & \text { EPA-assumed value for daily water } \\
& \text { consumntion }(2 \mathrm{~L} / \mathrm{d}) .
\end{aligned}
$$


Table 3.2.1. Acceptable daily intakes for selected contaminants

\begin{tabular}{|c|c|c|}
\hline Contaminant & $\begin{array}{c}\text { Acceptable daily } \\
\text { intake } \\
(\mathrm{mg} / \text { day })\end{array}$ & Reference \\
\hline Antimony (Sb) & 0.028 & 1 (RfD) \\
\hline Arsenic (As) & 0.100 & 2 \\
\hline Barium (Ba) & 3.50 & $1(\mathrm{RfD})$ \\
\hline Benzene & 0.0241 & 1 (CPF) \\
\hline Benzo-a-pyrene & 0.0001 & $1(\mathrm{CPF})$ \\
\hline Beryllium ( $\mathrm{Be})$ & 0.0002 & 3 \\
\hline Bis(2-chloroethyl)ether & 0.0006 & $1(\mathrm{CPF})$ \\
\hline Bromoform & 0.004 & 3 \\
\hline Cadmium (Cd) & 0.0574 & 3 \\
\hline Carbon tetrachloride & 0.0054 & $1(\mathrm{CPF})$ \\
\hline Chlorobenzene & 0.04 & 3 \\
\hline Chloroethane & 930.0 & 3 \\
\hline Chloroform & 0.1148 & $1(\mathrm{CPF})$ \\
\hline Chromium (Cr) & 0.100 & 3 \\
\hline Copper (Cu) & 2.6 & 4 \\
\hline Cyanide (HCN) & 1.4 & $1(\operatorname{RfD})$ \\
\hline 1,1-Dichloroethane & 290.0 & 3 \\
\hline 1,1-Dichloroethene & 0.0012 & $1(\mathrm{CPF})$ \\
\hline 1,2-Dichloroethane & 0.0077 & 1 (CPF) \\
\hline 2,4-Dichlorophenol & 0.21 & 1 (RfD) \\
\hline 1,2-Dichloropropane & 0.42 & 3 \\
\hline 2,4-Dini -roluene & 0.002 & 1 (CPF) \\
\hline Ethylbe ene & 1.6 & 3 \\
\hline Hexachiorobenzene & 0.0004 & 1 (CPF) \\
\hline Hexachlorobutadiene & 0.009 & $1(\mathrm{CPF})$ \\
\hline Hexachloroethane & 0.05 & 1 (CPF) \\
\hline Lead $(\mathrm{Pb})$ & 0.100 & 3 \\
\hline Mercury $(\mathrm{Hg})$ & 0.0235 & 3 \\
\hline Methyl chloroform & 6.3 & $1(\mathrm{RfD})$ \\
\hline Methylene chloride & 0.093 & 1 (CPF) \\
\hline Nickel $(\mathrm{Ni})$ & 1.4 & $1(\mathrm{RfD})$ \\
\hline N-Nitrosodimethylamine & 0.00001 & $1(\mathrm{CPF})$ \\
\hline N-Nitrosodi-N-propyiamire & 0.0001 & $1(\mathrm{CPF})$ \\
\hline N-Nitrosodiphenylamine & 0.1429 & 1 (CPF) \\
\hline PCBs-total & 0.0002 & 1 (CPF) \\
\hline Aroclor 1016 & 0.0002 & 3 \\
\hline Aroclor 1221 & 0.0002 & 3 \\
\hline Arailor 1232 & 0.0002 & 3 \\
\hline Aroclor 1242 & 0.0002 & 3 \\
\hline Aroclor 1248 & 0.0002 & 3 \\
\hline Aroclor 1254 & 0.0002 & 3 \\
\hline Aroclor 1260 & 0.0002 & 3 \\
\hline Selenium (Se) & 0.21 & 4 \\
\hline Silver ( $\mathrm{Ag})$ & 0.21 & $1(\mathrm{RfD})$ \\
\hline 1,1,2,2-Tetrachloroethane & 0.0035 & 1 (CPF) \\
\hline Tetrachloroethyiene (PCE) & 0.014 & 1 (CPF) \\
\hline Thallium $(\mathrm{Tl})$ & 0.0049 & $1(\mathrm{CPF})$ \\
\hline 1,1,2-Trichlorocthane & 0.0123 & $1(\mathrm{CPF})$ \\
\hline Trichloroethylene (TCE) & 0.064 & 1 (CPF) \\
\hline
\end{tabular}


Table 3.2.1 (continued)

\begin{tabular}{lcc}
\hline \multicolumn{1}{c}{ Contaminant } & $\begin{array}{c}\text { Acceptable daily } \\
\text { intake } \\
(\mathrm{mg} / \text { day })\end{array}$ & Reference \\
\hline 2,4,6-Trichlorophenol & 0.035 & 1 (CPF) \\
Vinyl chloride & 0.0003 & 1 (CPF) \\
Zinc $(\mathrm{Zn})$ & 14.7 & 4 \\
\hline
\end{tabular}

Sources:

1. U.S. EPA 1988. Integrated Risk Information System (IRIS) Data Base. Based on oral reference dose (RfD) for noncarcinogens. For carcinogens, an oral carcinogen potency factor (CPF) was used to calculate an acceptable level of exposure using a 1 in 100,000 lifetime risk of developing cancer.

2. Munro, N. B., and Travis, C. C. 1986, "Drinking-Water Standards," Environ. Sci. Technol. 20(8): 768-69.

3. Hoffman, F. O. et al. 1984. Preliminary Screening of Contaminants in Sediments. ORNL/TM-9370. Union Carbide Corp. Nuclear Div., Oak Ridge Natl. Lab. Using EPA standard values and criteria as explained in this document.

4. U.S. EPA 1986. Superfund Public Health Evaluation Manual, EPA/540/1-86/060, Office of Emergency and Remedial Response.

A review of water quality criteria documents appears in Sittig (1980). Table 3.2.2 lists the calculated daily intake of chemicals from surface water on and off the PORTS site. If the CDI/ADI ratio is $>1$, then an unacceptable level of risk would result from consumption of water from PORTS discharge points. This means the chemical is designated as one that warrants further investigation. If the CDI/ADI ratio is expressed as less than (<) a particular numerical value, this is based on a "less-than" concentration for the CDI because of the detection limitations of the instruments used to measure for chemicals. Information compiled in Table 3.2.2 was obtained from the NPDES outfall data on surface water quality. If one NPDES outfall discharged into another NPDES outfall, only the applicable data associated with the second outfall were reviewed.

Estimates of CDI are based on the assumption that total daily intake of drinking water (i.e., 2 $\mathrm{L} / \mathrm{d}$ ) comes from the stream (which is unlikely). In reality, outfalls are generally located within areas of DOE facilities not readily accessible to the general public; thus, public consumption of water from the outfalls is highly unlikely. Further, as the pollutant moves downstream from the outfall and the volume of water increases, the concentration of the pollutant decreases. A dilution factor can thus be determined. For example, the flow rate at discharge point 004 outfall (see Fig. 2.2.2) is $2.73 \times 10^{6} \mathrm{~L} / \mathrm{d}$. This flow rate is divided by the flow rate of the Scioto River $\left(6.01 \times 10^{9} \mathrm{~L} / \mathrm{d}\right)$ at Higby, Ohio, where public consumption is more likely, and then multiplied by the CDI for chromium of $0.186 \mathrm{mg} / \mathrm{d}$. This calculation yields a new CDI for chromium of $0.0001 \mathrm{mg} / \mathrm{d}$. A dilution factor of $1.86 \times 10^{3}$ is, therefore, determined. Thus, the values given in Table 3.2.2 represent overestimates of intake.

\subsubsection{Chemicals in other environmental media}

An important pathway of concern for human exposure to chemicals is t.rough atmospheric deposition onto vegetation and subsequent transfer into beef and milk. Direct measurements for 
Table 3.2.2. Potential chemical dose comparison for PORTS surface waters, 1989 annual average values

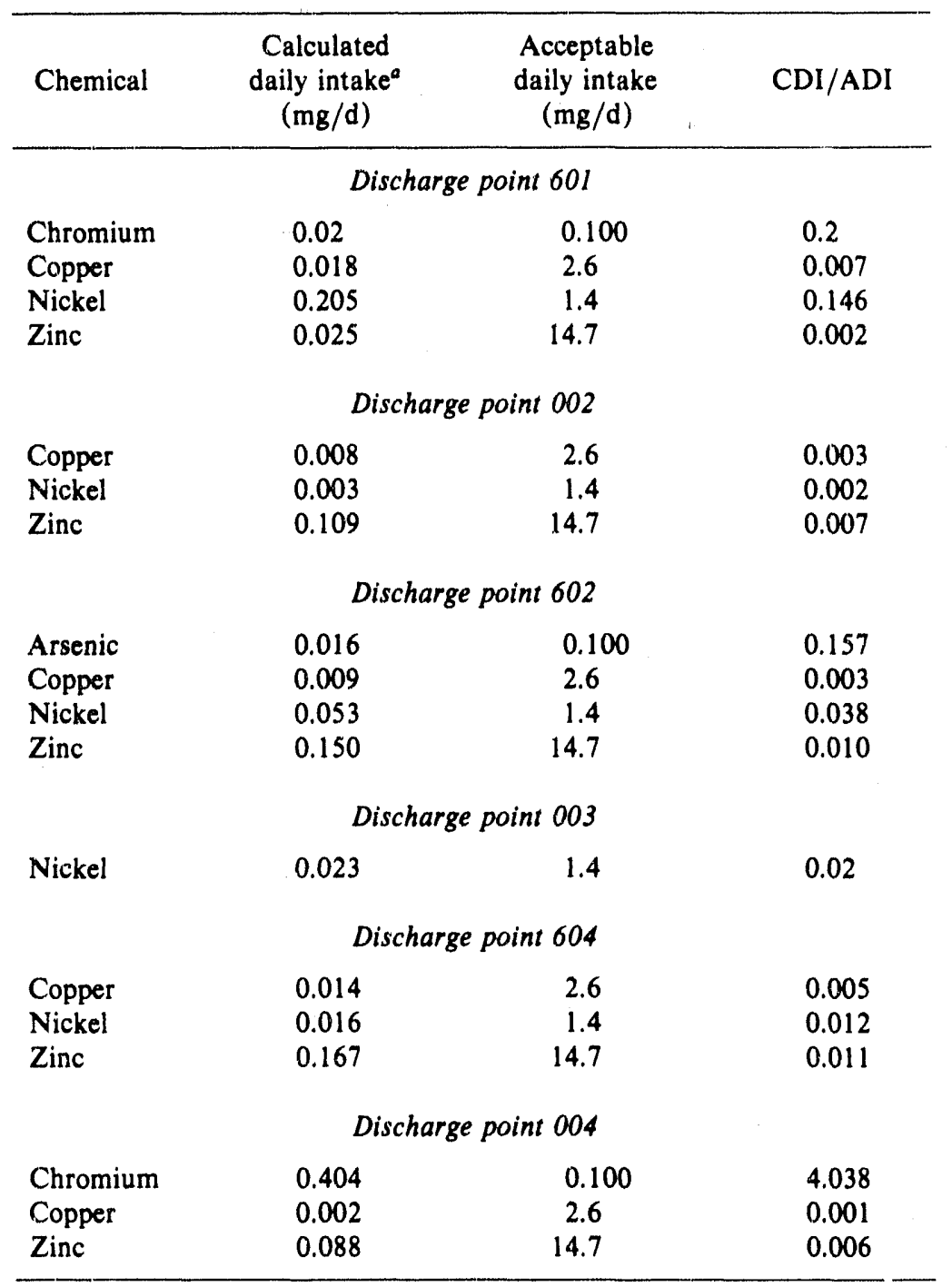

"Values represent annual averages

concentrations of organics in vegetation, beef, or milk in the vicinity of PORTS have not been made. Capabilities to make predictions of impacts on the food chain pathway are being developed.

\subsubsection{Direct exposure}

Direct exposure to chemicals does not represent a likely pathway of exposure at the PORTS facility. For airborne releases, concentrations off-site are too small to be a problem through the dermal exposure pathway. For aquatic releases, outfalls are generally located within areas of DOE facilities that are not readily accessible to the general public. Public consumption of water from the outfalls is highly unlikely; however, ingestion of chemicals in water downstream could occur and thus is addressed in Subsect. 3.2.2.2.

\subsubsection{Current year summary}

Chemicals where the CDI/ADI ratio exceeds 1 warrant further investigation. At the PORTS facility, the CDI/ADI ratio for chromium at discharge point 004 (see Fig. 2.2.2) was found to be 4.04. However, taking into consideration a dilution factor for chromium, a new CDI of 0.0004 $\mathrm{mg} / \mathrm{d}$ was determined (see Subsect. 3.2.2); this yielded a new CDI/ADI screening ratio of 0.004 . Thus, concentrations of chromium downstream of PORTS are within acceptable guidelines. 


\section{ENVIRONMENTAL RESTORATION PROGRAM}

\subsection{OBJECTIVES}

The objectives of the PORTS Environmental Restoration Program (ERP) are to apply a systematic approach that will follow the methodology prescribed by the RCRA under 3008(h) corrective actions. These are:

- A preliminary assessment of each facility is made to identify releases or possible releases of hazardous wastes justifying in-depth investigation of the facility. However, neither the terms of the Ohio Consent Decree nor the Administrative Consent Order requires that an RCRA Facility Assessment (RFA) be completed.

- An RCRA Facility Investigation (RFI). Each facility undergoes a detailed investigation through sampling and physical examination to fully define whether the facility and/or the adjacent environment is contaminated with hazardous wastes.

- A Corrective Measures Study. Data and information from the RFI are evaluated to determine the need for, and the extent of, remedial action at each facility.

- A Corrective Measures Implementation. Appropriate remedial action for each facility is selected and implemented.

- Interim Remedial Measures (IRM). If at any time during the course of the above steps conditions are found that could present an impact to human health and the environment, immediate steps are taken to implement remedial measures.

The methodology of problem definition is two-fold. First, a generalized understanding is obtained of the dynamics of the relationships among the various media in the ecosystem local to the PORTS site. This understanding is based on knowledge available through the Ohio Department of Natural Resources, the USGS, the U.S. Climatological Center, the Portsmouth Site Environmental Impact Statement, and the many past studies and ongoing monitoring efforts of Energy Systems and DOE. Second, the effects of plant operations on the various media (i.e., air, surface water, groundwater, soil, vegetation, and animal life) are characterized. These characterizations typically center on the most probable causes (based on experience) of unacceptable degradative effects on the quality of the environment. If samples representative of a medium of interest reveal that quality standards have been exceeded, these results must be evaluated in the context of a dynamic and integrated ecosystem. Other media may also have been affected at levels exceeding the media-specific quality standards.

\subsection{CURRENT STATUS}

Following a hazardous waste inspection by the OEPA in December 1988, the state of Ohio's attorney general issued a Notice of Violation 3 and Actions, and the director of the OEPA issucd their Findings and Orders. In August 1989 the Fintings and Orders were superceded by a Consent Decree between the state of Ohio and DOE. In October 1989 an Administrative Consent Order was signed by DOE and the EPA. The scope of work of these two documents is generally the same and follows an RCRA corrective action approach (see Subsect. 4.1).

The approach of the ERP at PORTS for the next several years will be to follow the RCRA Corrective Action Plan guidance as detailed in EPA documents and Consent Orders between DOE and the OEPA. By following the EPA guidance, 
the ERP will be oriented toward a site-wide comprehensive approach that will (1) build on information gathered during previous studies, (2) fulfill regulatory requirements, and (3) maintain consistency with established objectives.

\subsubsection{RCRA Facility Investigation}

Through environmental audits and internal reviews, PORTS has identified 77 SWMUs distributed widely across the reservation but occurring more densely where there has been greater industrial activity. The SWMUs will be investigated during their respective quadrant RFI. The action taken to correct identified problems will depend heavily on the defined limits and character of each contaminant. Continued surveillance will document the effectiveness of the actions taken.

The site was divided into four quadrants based on a radial groundwater flow pattern from a high located near the center of PORTS (Fig. 4.2.1). The quadrants are numbered in clean-up priority based on preliminary reviews (Quadrant I being the most important). The site will be investigated on this quadrant basis to allow for the most economical use of resources and to ensure that environmental restoration priorities are properly assigned. An RFI general work plan was issued to the regulatory agencies and approved by the OEPA. A draft work plan for Quadrant I containing the scope of work, site maps, and schedules was issued in May 1989. The OEPA submitted comments in September 1989, and comments are expected from the EPA. The Quadrant I RFI is anticipated to start during late summer 1990. The Quadrant II Work Plan was submitted to both agencies in December 1989, and comments are expected in June 1990. The Quadrant III Draft Work Plan was submitted in May, and the Quadrant IV draft is scheduled for November 1990. Figures showing the location of each quadrant, the SWMUs in each quadrant, a description of tine SWMUs, and proposed schedules will be available in Quadrants I, II, III, and IV RFIs after approval by the EPA and the OEPA.

\subsubsection{Correcive Measures Study (CMS)}

A contractor has been signed to conduct the CMS. Work is not expected to begin until late 1990.

\subsubsection{Corrective Measures Implementation (CMI)}

No action has taken place yet on the CMI phase of the ERP. Work is not expected to begin on this phase until 1992.

\subsubsection{Interim Remedial Measures}

In the OEPA Consent Decree, four RCRA sites were identified for immediate activity. These sites were the X-701B holding pond, the X-749 contaminated materials disposal facility, the X-231B oil biodegiadation plot, and the X-616 chromium sludge lagoons. A GWQA for the four RCRA sites was issued in May 1989 and was approved by the OEPA in August 1989. The GWQA is still under review by the EPA.

In May 1988, closure plans for the four RCRA sites were submitted to the OPEA, and comments were received in November 1988. The OEPA approved all four closure plans in July 1989. Closure option studies (COSs) were submitted on the X-749 and the X-231B in November 1989. Approval of these COSs is expected. The next phase would be to submit a work plan containing detailed schedules for design and implementation of closure of the sites.

The COS for X-701B is to be reviewed by the OEPA in 1990. The first phase of this closure will be sludge removal. No COS was requested by the OEPA on the X-616. Detailed design and a request for a PTI for a monocell to hold the processed chromium sludge (i.e., filter cake) is being reviewed by the OEPA.

A closur plan for the X-749A classified material disposal facility was submitted to the OEPA in May 1989. Comments were received on the plan in Lecember 1989; responses are to be forwarded to the OEPA. No COS is required; the etore once approval is received on the closurc 


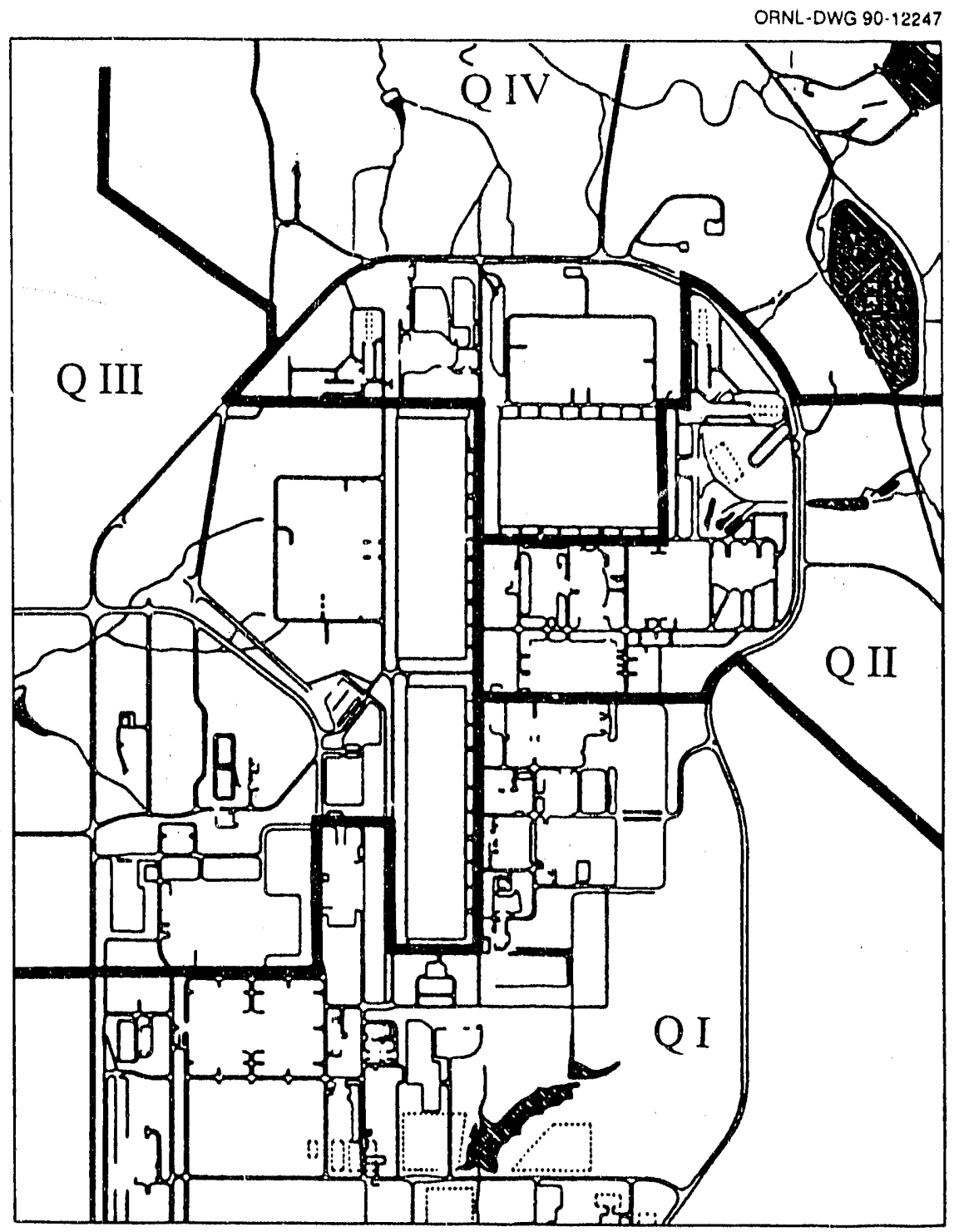

Fig. 4.2.1. Quadrant map of PORTS showing order of priority in which resources will be utilized to ensure environmental restoration.

plan, detailed design and construction is expected to begin.

One of the wastewater treatment systems at the PORTS site is the X-701B holding pond and associated total containment ponds. This holding pond was used for settling and $\mathrm{pH}$ adjustments of wastewater before discharge. Groundwater monitoring around this area has identified contamination by organic solvents, predominantly TCE. Groundwater-monitoring data in the area indicated the presence of pure TCE in one of the monitoring wells. Other wells in the area also indicated the presence of TCE. PORTS decided to take interim corrective actions with respect to the pure TCE in advance of an expected closure of the $\mathrm{X}-701 \mathrm{~B}$ holding pond and also in advance of any larger scale groundwater corrective action in the vicinity of the pond. This action was taken to reduce the potential for the pure TCE to act as a continuing source of contamination for the surrounding groundwater.

The TCE Removal Program began in 1987. Phase I was completed in August 1989, and phase II will begin on completion of the sludge removal 
at X-701B (expected to be completed in May 1990); a report covering this phase is scheduled for release in September 1990.

Other IRMs accomplished or started during 1989 were as follows:

- a waste stream evalu stion for the PORTS site,

- an analysis of the runoff from the X-749 contaminated materials disposal unit,

- a revised Facility Contingency Plan (submitted to the OEPA and local municipalities),

- a TCE Removal and Control Plan for X-701 B (approved by the OEPA and implemented in June 1989),

- an Off-Site Residential-Well-Sampling Plan (submitted to the OEPA in April 1989 and approved in May 1989), and

- a plan for investigation and remediation of areas around approximately 76 above-ground storage tanks located on the plant site (submitted to the OEPA for approval).

\subsection{UNDERGROUND STORAGE TANKS (USTs)}

Responsibilities for the Underground Storage

Tank Program are split between the Environmental Control and Remedial Action Departments. The two elements of the program are compliance with the applicable regulations and corrective actions when the environment has been contaminated. The UST Program is managed in accordance with EPA, OEPA, Ohio Division of State Fire Marshal, and DOE regulations. By the end of 1989 , substantial efforts had been made to bririg the UST Program into compliance. These efforts (including a management plan, an inventory control program, tightness testing, and tank removals) are discussed in more detail below.

Forty-cne USTs are registered by PORTS with the Ohio Division of State Fire Marshal. An additional five USTs are located on the reservation but are maintained by the Ohio Army National Guard through a tenant agreement with DOE.

During 1989 a survey was conducted of all below-grade petroleum storage tanks greater than $189 \mathrm{~L}$ ( $50 \mathrm{gal})$ in volume. The survey listed locations, status, capacity, installation date, material of construction, product stored, and leakdetection requirement dates. Furthermore, the survey listed recommendations for upgrade, removal, or consolidation of tanks.

A UST Management Plan was prepared to ensure that daily operations of the USTs remain in compliance with EPA, OEPA, Ohio Division of State Fire Marshal, and DOE regulations. The plan has three major sections: Regulations, Facility Inventory Description, and Recommended Activities. The Regulations Section discusses the notification requirements, the minimum technical requirements, and additional DOE requirements. The Facility Inventory Description Section provides an overview of the tank inventory at PORTS including tanks currently in use, temporarily or permanently out of service, or exempted by the regulations. The Recommended Activities Section addresses tank closure and consolidation, release monitoring, replacement and upgrading, and operation and maintenance. The objective of the plan is implementation of a program that reduces the risks associated with USTs. This will be accomplished through the prevention of leaks by proper design and installation, early detection of leaks that do occur, and rapid remediation after a release is detected.

In October 1989 an Inventory Control Program was instituted to establish a record of daily operations at each tank and to indicate any abnormal operating conditions requiring further investigation. Tank operators are required to take daily inventory level measurements each day of tank operation. If a tank is not in use every day, as a minimum, inventory level measurements are required once a week. "Inches to gallons" conversion charts are to be prepared for each UST. The conversion charts will be used to interpret the inventory level measurement data. Standard operating and fuel-handling procedures are to be developed by the tank operators to ensure that inventory level measurements are taken correctly and consistently.

During the fall of 1989 , four USTs (i.e., X-104, X-611, X-640-1, and X-710), and their associated piping were integrity-tested by the tracer leak detection method. No tracer or 
hydrocarbons were detected in the vicinity of three of the tanks (i.e., X-104, X-640-1, and X-710) indicating that these tanks were not leaking. Although test results indicated that the X-104 UST did not leak, further investigation indicated that the associated piping leaked. Following a second integrity test and remediation, the tank is scheduled to be placed back in service. Test results for the fourth tank, X-611, indicated a product leak.

A fifth UST, X-720, was air-pressure-tested in October 1989 by Energy Systems personnel because water was discovered in the tank. Test results indicated that the tank did not leak. It was determined that the water inleakage was a result of the associated piping. The piping is to be repaired, and the UST will be placed back in service following site remediation and a second tightness tesi.

Five USTs were removed from the ground for various reasons. The $\mathrm{X}-611 \mathrm{C}(\mathrm{W})$ and the $\mathrm{X}-611 \mathrm{C}$ (NW) were removed because they had been out of service more than 2 years. One X-750 UST and the X-611 UST failed integrity tests. The results for the second X-750 tank ' ere inconclusive, indicating a potential for leakage. Repair and/or upgrading at these three USTs was not feasible because of the extent of the site remediation required. A sixth UST, X-605G, was to have been removed in December 1989 because it has been out of service more than 2 years. However, the actual removal was delayed until January 1990.

Corrective actions were undertaken at those UST sites where contamination occurred. Where possible, the contaminated soil has been removed for treatment and disposal. In some instances remediation is still ongoing. All sites will be cleaned up and returned to a stable condition.

UST activities planned in 1990 include removal of three additional USTs and a tightness test of one UST. The three tanks to be removed were temporarily closed in 1989 and will be permanently closed in 1990. The tightness test is required because of the age of the tank and is consistent with the EPA underground storage tank release detection requirements. 


\section{SOLID WASTE MANAGEMENT}

\subsection{DESCRIPTION}

\subsubsection{Purpose}

PORTS has developed an extensive solid waste program to ensure compliance with applicable state and federal regulations. Under these regulations a solid waste is defined to include any solid, liquid, semisolid, or contained gas that is discarded. Therefore, the solid waste program at PORTS includes contained gaseous waste and liquid wastes that are not regulated under Ohio NPDES regulations.

The common objective to all PORTS solid waste management programs is prudent waste management. The underlying policy of all waste management programs declares that the protection of employees, the public, and the environment is the primary objective of waste management practices. To comply with this policy, equipment, practices, and procedures must be continually improved.

The PORTS waste management program is designed to provide a "cradle-to-grave" system that supplies management services for all types of wastes from the point of generation through ultimate disposal. The system ensures that all wastes are managed in accordance with all state and federal regulations and accepted industry practices. Waste minimization is an integral part of the waste management program and includes efforts to minimize waste generation and to reduce the toxicity of the wastes that are generated.

\subsubsection{Environmental Regulatory Review}

\subsubsection{Orders and regulations governing radioactive wastes}

Management of radioactive wastes and radioactively contaminated facilities is governed by DOE Order 5820.2A and the Atomic Energy Act of 1954 as amended. These regulations provide guidelines for characterization, storage, and disposal of all categories of radioactive wastes. Radioactive wastes that also contain RCRA hazardous wastes are subj;:ct to RCRA requirements in addition to radioactive waste requirements. Prior to June 1987, PORTS mixed radioactive and hazardous wastes were considered to be exempt from the RCRA regulations according to the "by-product" rule. These wastes had been regulated by DOE Order 5480.2. In June 1987 DOE and EPA agreed that mixed waste formerly characterized as by-product would henceforth be subject to RCRA rules as well. Consequently, the exemptions in DOE Order 5480.2 for mixed waste were cancelled.

\subsubsection{Orders governing classified wastes}

Portions of the materials, equipment, and information at the PORTS facility are classified for security reasons by the U.S. government. DOE Order 5632.1 prescribes DOE requirements for the physical protection of classified material.

\subsubsection{Orders and regulations governing hazardous wastes}

Hazardous waste regulations at PORTS include the RCRA of 1976 and the Ohio Hazardous Waste Rules. Additionally, DOE Order 5480.4 requires that hazardous waste generated by DOE-funded activities be managed in an environmentally acceptable manner. The Atomic Energy Act of 1954 as amended establishes the regulations governing processing and use of source, by-product, and special nuclear materials. The provisions of the Atomic Energy Act, as well as RCRA, are applied to those wastes that are hazardous and radioactive (i.e., mixed wastes). 
RCRA of 1976 and the Hazardous and Solid Waste Amendments of 1984 regulate the generation, transportation, treatment, and disposa! of hazardous wastes. RCRA establishes the following PORTS requirements:

1. standards for hazardous waste identification;

2. standards for the operation and management of hazardous waste treatment, storage, and disposal facilities;

3. requirements for obtaining permits for treatment, storage, or disposal of hazardous wastes;

4. inspections;

5. federal enforcement;

6. hazardous waste site inventory.

The Hazardous and Solid Waste Amendments of 1984 reauthorize and expand RCRA through 1988. The federal RCRA Program now establishes land disposal criteria for hazardous wastes, new analytical testing requirements, and corrective action criteria for solid waste management units.

\subsubsection{Regulations governing conventional waste}

Regulations promulgated under the Solicl Waste Disposal Act, the Ohio Solid Waste Disposal Act, and codified in OAC-3745-27 and -37 govern the planning, design, construction, operation, and maintenance of solid waste processing and disposal facilities. Domestic sewage and industrial wastewater flows subject to NPDES permits are excluded. Waste materials such as RCRA wastes, PCB wastes, asbestos, and other specified wastes cannot be disposed of in a conventional disposal or treatment facility unless granted a variance under this act. Prior to construction or modification of a treatment or disposal facility, detailed design drawings, specifications, operating plans, and closure plans must be approved by Ohio and must comply with the requirements of the National Environmental Policy Act (NEPA). Operating records, future plans, and capacities must also be reported to the OEPA.

\subsubsection{Compliance Activities}

Hazardous waste facilities at PORTS are currently operated in accordance with the interim status standards stated in 40 CFR 265 and Ohio Administrative Code (OAC) 3745-50 to -69 . The Part A Permit Application, originally submitted in April 1984, is updated as hazardous waste management needs dictate or as the regulatory climate changes. The latest revision was submitted on July 22,1988 , and included the X-700 chromic acid cleaning solution storage tank and deleted a waste pile containing a treated chromium sludge. A Part B Permit Application has been submitted for three treatment and storage facilitits located at PORTS. Those facilities included in the hazardous waste permit application are (1) the X-616 chromium sludge lagoons (surface impoundments), (2) the X-752 hazardous waste storage facility, and (3) the X-701B holding pond and containment area (treatment and storage surface impoundments).

Closure plans have been submitted to the OEPA and the EPA for the X-616 and X-701B surface impoundments, the $X-231 B$ oil biodegradation plot, and the X-749 contaminated materials disposal facility. The OEPA approved the closure plans in July 1989. At the X-701 B facility waste removal began in December 1989 and will be completed in early 1990 . Closure option studies are under way for all facilities.

The Part B A pplication is currently being revised to include two additional facilities as a result of the DOE Final Interpretative Rule. These facilities are (1) the X-326 radioactive waste storage area and (2) the X-744G radioactive bulk storage building. The $\mathrm{X}-616$ chromium sludge surface impoundments and the X-701B holding pond area are to be removed from the revised Part B Hazardous Waste Permit Application. The OEPA and Region V of EPA are in the process of reviewing the latest version of Part $B$, which was submitted April 27, 1987. Until a full permit is issued, PORTS will continue to operate according to interim status standards.

The latest hazardous waste inspection was performed by the OEPA in December 1987. Areas of noncompliance include (1) operation of 
hazardous waste facilities without a permit (Part A was submitted after April 9, 1981), (2) contingency plans, (3) container management in storage areas, (4) landfill and land treatment area noncompliances, and (5) incomplete recordkeeping and reporting. As a result, revised and updated contingency plans were issued to the appropriate agencies in June 1988, and new waste management procedures were issued in October 1988.

The X-735 sanitary landfill is the only permitted solid waste facility at PORTS. A license to operate the landfill is obtained from the state of Ohio annually. The types of wastes permitted in the X-735 facility include cafeteria wastes, industrial wastes, disinfected medical wastes (except drugs), construction/demolition debris, and, in designated locations, asterstos. No hazardous wastes, Toxic Substances Ciontrol Act (TSCA) wastes, or radioactive wastes are permitted in this facility.

The construction spoils area, located adjacent to the X-735 sanitary landfill, is an operating landfill for materials that are not regulated as solid wastes by the Ohio Solid Waste Regulations. Materials of this type include construction and demolition debris and wastes from brush and treeclearing operations.

\subsubsection{Program Strategy}

The goals of PORTS solid waste management are (1) to ensure compliance with all federal, state, and local regulations; (2) to minimize the volume and hazard of all hazardous and radioactive wastes produced; (3) to protect the environment from unintentional releases of hazardous or radioactive materials; (4) to protect personnel from hazards associated with handling solid wastes; and (5) to minimize the long-term risks and liability from waste disposal. These goals are implemented through the combined efforts of PORTS operations and maintenance personnel with coordination, strategy, technology, and regulatory interpretation provided by Waste Management and Environmental Control. Compliance assurance is provided by employee training and by periodic inspections and audits performed by supervision and by Waste Management and Environmental Control.

Long-range planning, new technolngy investigation, and new program strategies for waste management are developed under the combined Low-Level Waste Disposal Development and Demonstration (LLWDDD) and the Hazardous Waste Development, Demonstration, and Disposal (HAZWDDD) Programs. These programs are designed to provide for government and private industry cooperation in the development of technologies and strategies in waste disposal. Waste minimization strategy includes five current options:

- segregation,

- material substitution,

- process changes,

- mechanical compiession volume reduction, and

- recycle/reuse.

Environm $m^{\prime n t a l}$ protection is provided through the use of specification packaging and by storage in properly engineered and permitted facilities. An extensive monitoring and surveillance program monitors NPDES discharges and checks for unintentional releases. Personnel protection is provided through standard industrial protective equipment applied in accordance with requirements determined from Material Safety Data Sheets and Industrial Hygiene/Health Physics recommendations. Long-term risks and liabilities for disposals are minimized by destroying or removing the hazardous constituent whenever possible as opposed to burial or storage.

\subsection{WASTE GENERATION}

\subsubsection{Types of Waste Generated}

PORTS solid waste is managed in five different categories determined by the regulatory requirements for solid wastes. The first category is radioactive waste and does not contain materials regulated by RCRA or TSCA. They are low-level radioactively contaminated wastes. Subsect. 5.3.1.1 
describes the wastes in this category and the measures used to manage them. The second category, classified wastes, consists of discarded articles that reveal classified information. Subsect. 5.3.1.2 discusses classified waste management. The third category is hazardous wastes as defined by RCRA. Since June 1987, radioactive wastes that also contain RCRA hazardous wastes are subject to RCRA requirements in addition to radioactive waste requirements. Subsect. 5.3.1.3 describes the hazardous waste characterization process and management programs.

Toxic Substances Control Act wastes are the fourth categury of waste. These wastes include PCBs and asbestos. PCBs are used as a dielectric fluid in PORTS electrical equipment, are present is a contaminant in some waste materials, and are an original component in the gasket material in ventilation ducts. Asbestos materials are used as insulation on water and steam lines and other heat-related processes. Subsect. 5.3.1.4 discusses the waste management programs for these wastes.

Conventional solid waste is the fifth and final category of PORTS solid waste. The PORTS sanitary landfill, X-735, is the principal means of sanitary waste disposal. Asbestos waste is managed as a special subcategory of sanitary waste. Conventional solid wastes, disinfected wastes, construction spoils, and fly ash are included in this waste category (see Subsect. 5.3.1.5). PORTS' waste generation data for 1989 are given in Table 5.2.1 of Part 2 of this report.

\subsubsection{Waste-Generating Activities}

\subsubsection{Radioactive waste}

Scrap metal and other nonburnable waste is generated as a result of modification or replacement of equipment and may be radioactively contaminated depending upon the usage of the equipment or the location of the maintenance work. Nonradioactive wastes are carefully segregated to minimize the total volume of radioactive wastes. Other radioactive wastes that do not contain hazardous constituents are generated by the various processes used to enrich the uranium, from the trapping media used by the gaseous diffusion industry, and from decontamination of radioactively contaminated equipment.

\subsubsection{Classified waste}

Classified wastes are generated because of classification of the equipment and materials used in the gaseous diffusion plant. This category also includes magnetic media (e.g., computer disks and tapes) and classified documents requiring disposal.

\subsubsection{Hazardous waste}

Hazardous wastes are generated on the plant site during various cleaning and degreasing operations as well as during the required analytical work done by the laboratory. Hazardous wastes contaminated with uranium are typically generated as a result of degreasing and cleaning of components that are contaminated with uranium or from treatment of the uranium recovery system, which generates a sludge that is characteristically hazardous because of leachable cadmium and lead.

\subsubsection{TSCA wastes}

PCB wastes of greater than 500 ppm PCB are typically generated as a direct result of work with the electrical switch gear. PCB wastes of less than 500 ppm PCB are a direct result of the replacement of oils in some of the small equipment or lubricating-oil spills and leaks in the systems contaminated with PCBs. PCB wastes contaminated with uranium are typically generated as a result of the process building floor sweepings and the amelioration of leaks of electrical equipment in the process buildings. Asbestos wastes are generated as a result of removing asbestos insulation from water and steam lines and other heat-related processes throughout the plant site.

\subsubsection{Conventional solid waste}

Conventional solid waste consists of normal industrial wastes that contain no radioactive, hazardous, or toxic constituents. These wastes are reguiated by the Ohio Solid Waste Act or are 
specifically exempted by the act. Typical of regulated waste examples are industrial waste, sanitary waste, and sterilized medical and infectious waste. Examples of nonregulated wastes are fly ash and construction spoils. Fly ash waste is generated by burning coal for heating and process needs. Sanitary waste is generated by the administrative duties, cafeteria, and similar activities on the plant site. Sterilized medical and infectious wastes are considered to be conventional solid waste and are generated at the medical facility in small quantities. Construction sfoils are a direct result of the ongoing construction activities on the plant site.

\subsection{WASTE MANAGEMENT ACTIVITIES}

\subsubsection{Waste Management System}

\subsubsection{Low-level Radioactive Waste Management Program}

DOE Order 5820.2A addresses the management of radioactive waste and radioactively contaminated surplus facilities. Disposal site requirements include site selection, design, operation, waste acceptance, closure, and postclosure. Each year, productior ctivities at PORTS generate tons of nonhazardous solid waste contaminated by low levels of radioactivity. Disposal of low-level radioactive solid wastes has been by shallow land burial at the X-749 contaminated materials burial facility. Closure of $\mathrm{X}-749$ has been mandated by the OEPA and is scheduled for FY 1990. The very small remaining portion will be used under the most recent technology for double-lined, leacheate-collected oisposal cells. A new disposal facility is planned for operation in FY 1996 that will meet established criteria from the EPA and DOE and will meet the LLWDDD strategy for disposal of low-level radioactive waste.

PORTS has been placing those radioactive wastes that are not disposed of in the X-749 facility into canisters that are constructed from excess converter shells and in carbon steel boxes that are approximately $3 \mathrm{yd}^{3}$ in volume. These canisters are constructed of nickel-lined steel with steel tops and bottoms welded to the shell. I-beams are welded to the bottom of the canisters for bracing and to allow movement by a forklift. Contaminated burnable materials (formerly incinerated on-site) and contaminated nonburnable scrap are placed in these steel boxes. The boxes of burnable waste will be stored until the new disposal facility or incinerator becomes operational. All materials in canisters or strong, tight containers are placed in indefinite storage pending final disposition.

\subsubsection{Classified Waste Management Program}

Portions of the materials, equipment, and information at the PORTS facility are classified for security reasons by the U.S. government. DOE Order 5632.1 prescribes DOE requirements for the physical protection of classified material. The Atomic Energy Act h.s provisions for protection of classified nuclear materials. Classified wastes are stored on-site in secure storage areas or disposed of by shallow land burial if appropriate.

\subsubsection{Hazardous Waste Management Program}

Hazardous wastes (as defined by the RCRA) contain constituents that are described by one or more of the following four categories:

- The waste possesses one of the following characteristic hazards: toxicity, ignitability, corrosivity, reactivity, extraction procedure (EP) toxicity (i.e., leachable $\mathrm{Ag}, \mathrm{As}, \mathrm{Ba}, \mathrm{Cd}, \mathrm{Cr}, \mathrm{Pb}$, $\mathrm{Hg}$, or Se concentration(s) or certain pesticides exceeding specified threshold values).

- The waste is generated from a nonspecific listed source or process (e.g., spent halogenated solvents or wastewater treatment sludges from electroplating operations).

- The waste is generated from a specific listed source. PORTS does not use any specific listed processes and thus does not have any wastes characterized as hazardous by this criterion.

- The waste contains specifically listed, discarded, commercial chemical products. 
A vaste is a mixed waste if it is a listed hazardous waste or possesses a hazardous characteristic and is contaminated with radioactive materials. A waste mixture of nonhazardous material with listed hazardous waste becomes a hazardous waste regardless of the relative amounts of each material in the mixture.

Waste solvents: All waste solvents, flammable and nonflammable, are stored in containers meeting Department of Transportation (DOT) specifications. The waste solvents are stored in the $X-752$ warehouse, which has interim status as a RCRA storage facility. The tacility is locked except when in use.

Lab packs and waste chemicals: Small quantities of miscellaneous waste chemicals are stored in hazardous material storage lockers in the X-710 laboratory facility or are packaged in small quantities with inert absorbent in DOT Specification $17 \mathrm{C}$ drums and stored in the X-752 hazardous waste storage facility. Small quantities of nonradioactive miscellaneous waste chemicals are stored in several hazardous material storage lockers at various storage locations in the X-710 laboratory facility and await disposal. Separate unit price cor:tracts are also planner' to allow disposal of hazardous waste chemicals approximately as generated; shipments are projected every 90 days.

Sludges: Some sludges resulting from the treatment of process wastes are listed or are characteristically hazardous as well as radioactive. Storage is, and will continue to be, needed for these sludges. Management of sludges may include treatment, delisting, and stabilization. Treatment of chromium sludges from the X-616 chromium sludge surface impoundments has been performed on a limited basis with the approva! of the OEPA and EPA. The treatment immobilizes : $:$ : chromium, which would otherwise be leachable above the specified limits. After treatment, the waste can generally be managed as a nonhazardous waste. Commerciai waste incinerators are used to the extent possible to treat and dispose of PORTS nonradioactive listed sludges.

PORTS generates a radioactive waste, "heavy metal sludge, " that has been found to be hazardous because of leachable cadmium and occasionally lead exceeding the specified limits. Plans are to immobilize the toxic metals by encapsulation. The process has not been selected at this time.

Corrosive wastes: Acid and caustic wastes are generated from plating, cleaning, and laboratory operations. These wastes may either be treated onsite or disposed commercially. The X-701B holding pond and the $\mathrm{X}-701 \mathrm{C}$ neutralization pit can treat acid and caustic wastes (the X-701B holding pond was closed in November 1988). Sludges resulting from treatment are managed as previously described. Certain coriosive wastes may be storty in the X-752 hazardous waste storage facility before shipment off-site.

Ignitable wastes: Ignitable wastes generated at PORTS include nonchlorinated solvents and waste fuels. Solvents are stored in drums within the $\mathrm{X}-752$ hazardous waste storage facility. This storage is in compliance with environmental regulations and provides waste segregation of incompatible materials.

Reactive wastes: I.eactive wastes have one or more of the following properties: unstable, react with water either violently or to form explosive or toxic gases, or can be readily detonated. Spent cyanide-bearing solutions and laboratory chemicals comprise the majority of reactive wastes generated at PORTS. Interim storage is provided by the $\mathrm{X}-752$ hazardous waste storage facility or, for small-quantity lab chemicals, by hazardous material lockers in X-710.

Extraction procedure toxic wastes: Solid wastes contain certain metals or pesticides that will leach out in concentrations above specified limits. At PORTS wastewater treatment sludges have been found to contain toxic levels of metals. At the $\mathrm{X}-616$ chromium sludge lagoons chromium is the toxic constituent, whereas the X-705 heavy metals treatment process and the $X-705$ microfiltration process produce sludges that contain leachaile cadmiurn and occasionally lead.

Delisting: Delisting allows for the exemption of RCRA-listed wastes from further regulation under RCRA. Comprehensive lab analysis and documentation must be provided proving that the waste no longer meets the criteria for which it was listed and that any hazardous constituent potentially present $\mathrm{z}$ es not pose a threat to the 
environment. Delisting is required after treatment for any waste that contained a listed hazardous constituent. After delisting, the waste can generally be managed as a nonhazardous waste.

Disposal: Commercial waste incinerators are used to the extent possible to destroy and to dispose of PORTS nonradioactive hazardous materials that are amenable to this type of treatment. No existing commercial sites are licensed to manage hazardous waste that is also radioactive. PORTS generates large quantities of hazardous sludges that are also radioactive as a result of wastewater treatment facilities and remedial action programs. Current plans are to ship these sludges for removal of the listed constituent by incineration in an incinerator located at the Oak Ridge Gaseous Diffusion Plant (ORGDP) facility in Oak Ridge, Tennessee. The incinerator underwent startup testing during 1988 and 1989 and will begin productive operation during 1991. The incinerator has been designed to destructively incinerate most listed organic materials as well as PCBs, ani it will be capable of handling radioactive materials. However, an approximate 5-year backlog of this type of material has been identified for this incinerator, so considerable storage capacity will still be required.

\subsubsection{Wastes governed by the TSCA}

PCBs are regulated by the TSCA. Sources of PCBs at PORTS are primarily dielectric fluids used in electrical equipment. Ventilation duct gaskets have also been PCB-impregnated during installation to enhance pliability and sealing properties. Contamination from these sources has spread during past operations to include lube and hydraulic oils, solvents, and floor sweepings. Steps have been taken to prevent further crosscontamination; however, several lube oil systems had become contaminated in excess of $50 \mathrm{ppm}$ PCB. All PCB transformers at PORTS are diked. Nonradioactive PCB materials are shipped off-site for destructive incineration. Radioactive PCB materials are being stored and will be incinerated at the ORGDP TSCA incinerator in the future. However, long-term storage is required because at least a 5-year backlog of solids and a 2-year backlog of liquids has accrued for the incinerator.

PCB transformers: PORTS has 159

transformers in service that are filled with PCBs. These transformers are located throughout the plant, mostly within the three process buildings. Current regulations allow the continued operation in industrial service of any transformer containing PCBs as long as that use does not pose any risk of contamination to the food chain. PORTS plans to continue to use these PCB transformers. Dikes and concrete pads prevent inadvertent release of the transformer oils to the environment in case a failure of the casing occurs.

$P C B$-contaminated waste oils, solvents, and solids: Liquid and solid PCB wastes are stored in drums at PORTS. PCB-contaminated solids (e.g., soil, sludge, floor sweepings, rags, paper, etc.) are stored in DOT specification drums in the X-333, $X-330$, and $X-326$ process buildings and in the X-334 PCB transfer building. For those wastes that are not radioactive, off-site treatment using incineration for PCB destruction is arranged with a commercial disposal facility. To manage these wastes, the TSCA incinerator has been constructed at ORGDP in Oak Ridge, Tennessee. This facility will incinerate $\mathrm{PCB}$ and other hazardous organic wastes that also may be radioactive. It will receive wastes from all Oak Ridge Operations (ORO) facilities including PORTS. A dedicated bulk liquid trailer and a dedicated cargo van trailer are available for transport of materials. Each trailer has been equipped with antileak and leak detection devices. Shipping facilities are responsible for proper packaging and manifesting wastes. Tractors and drivers are also the responsibility of the shipping facility. Environmental permits for incineration of TSCA and RCRA wastes have been requested, and trial burns as required by the RCRA Part B Permit are imminent. The facility has $340,000 \mathrm{~L}$ of liquid storage capacity and segregation capability for PCB and non-PCB wastes. Ash is continuously discharged from the incinerator to a quench-water pit and conveyed from the pit to drums for storage. Ash will be analyzed, delisted if possible, stabilized if 
necessary, and stored. Plans call for returning the ash to the generator for disposal.

\subsubsection{Conventional Solid Waste Management Program}

Ohio Solid Waste Disposal Act: The Ohio Solid Waste Disposal Act and associated promulgated regulations govern the planning, design, construction, operation, and maintenance of solid waste-processing and disposal facilities. Solid wastes are defined by this act as any garbage, refuse, or sludge from water and wastewater treatments or air pollution control, and any other discarded material including solid, liquid, semisolid, or contained gaseous material resulting from industrial, commercial, and agricultural operations. Solid or dissolved material in domestic sewage flows are excluded. Special wastes (such as low-level radioactive wastes and RCRA and TSCA wastes) cannot be disposed of in a conventional facility. A feasibility study including system and site evaluations must be submitted to Ohio for approval for any contemplated construction or modification to a solid waste disposal or processing facility. Detailed design drawings, specifications, operating plans, and closure plans must be approved by Ohio prior to construction and operation. Records, plans, and capacities must also be provided to the state during operation.

Sanitary wastes: PORTS currently operates the X-735 sanitary landfill for the disposal of conventional (i.e., nonradioactive, nonhazardous, and non-PCB) solid wastes. X-735 is located on the North Access Road approximately $0.8 \mathrm{~km}$ north of the Perimeter Road. Disposial is by shallow land burial using the trench-and-fill method. The site $1 . s$ permits from the OEPA and the Pike County Department of Health. This permit allows disposal of asbestos and certain semisolid sludges.

Construction spoils: Construction spoils generated at PORTS have been disposed of in a construction spoils area. Near the X-735 sanitary landfill, a new spcils area was opened in 1989.

Waste oils: The X-740 facility is used to store drummed and bulk waste oils. The waste oils managed at X-740 are sampled and segregated for disposal. These oils are normally disposed of commercially. Oils believed to be contaminated by radioactivity or hazardous or toxic wastes are not stored at X-740. Waste oil stored at X-740 that is subsequently found to contain hazardous or toxic constituents is moved to appropriate storage prior to disposal. Waste oil found to be radioactive will be disposed at the ORGDP incinerator rather than commercially (see Subsect. 5.6.2).

Fly ash disposal: PORTS disposes of fly ash and bottom ash generated at the X-600 steam plan in the X-735 sanitary landfill. OEPA approved a request to increase the amount of waste disposed at the X-735 landfill to allow the disposal of the ash. The old construction spoils landfill will be formally closed. A new spoils area was opened in 1989.

Clean scrap: Tons of uncontaminated or "clean" scrap metal are currently stored in designated areas at PORTS. The scrap is primarily ferrous and has resale value for outside commercial scrap dealers. Storage of the clean scrap presents very little impact in the way of environmental pollution. For security reasons the salvage yard is not located inside the secured area so that uncleared buyers and other personnel may have access to examine and to transport scrap metal.

Medica! and infectious waste: PORTS generates three types of medical and infectious waste at the X-101 medical facility. These are (1) human blood and blood products, (2) contaminated sharps, and (3) expired drugs. The blood and blood products and the contaminated sharps are autoclaved prior to disposal to destroy any infectious content. Sharps are placed in a puncture-proof container prior to disposal. Disinfected and containerized blood and blood products and sharps are then disposed of in the sanitary landfill. Expired drugs are disposed of by commercial incineration.

Asbestos wastes: Asbestos wastes are regulated by the NESHAP regulations. Sources of asbestos are insulation around water and steam lines and other heat-related processes. When removed, the asbestos must be properly packaged and landfilled in a designated area of the X-735 sanitary landfill or the X-749 contaminated materials disposal facility if contaminated with 
radioactivity. The areas where asbestos has been disposed of are specifically identified.

\subsubsection{Waste Management Facilities}

The primary waste management storage facilities at the PORTS site are shown in Fig. 5.3.1 and described below:

- The X-752 hazardous waste storage facility contains the RCRA wastes generated on the plant site that will be disposed of off-site either at a commercial disposal site or, if the waste has unacceptable uranium concentrations, at the TSCA incinerator at Oak Ridge, Tennessee.

- Hazardous mixed wastes are stored in the X-744G bulk storage building and the X-326 process building. Areas in each of these buildings have been upgraded for the storage of hazardous wastes by installation of dikes and sealing of the floor.

- PCB wastes are stored in the X-333, X-330, and $\mathrm{X}-326$ process buildings and in the X-334 PCB transfer building.

- Wastes oils that do not contain hazardous, toxic, or radioactive constituents are stored in the $\mathrm{X}-740$ waste oil storage facility and subsequently disposed of off-site.

- Scrap metal, if radioactively contaminated, is stored at the $\mathrm{X}-747 \mathrm{H}$ salvage yard after it has been partially decontaminated (i.e., removable contamination has been removed). Nonradioactively contaminated scrap metal is stored near the surplus and salvage facility and is offered for sale to the general public. Scrap precious metals such as copper and nickel are stored at X-747G precious metal storage yard.

The waste management disposal facilities (primarily burial yarits) are as follows:

- X-735 is the sanitary landfill and is used for sanitary waste, and no free liquids, hazardous constituents, or radioactive materials are allowed in this facility. Asbestos is also disposed of at this facility in designated and segregated cells.
- X-749A and X-749C are the classified material burial facilities, and only materials that are classified and not contaminated with hazardous or radioactive materials or free liquids are allowed to be buried in these burial facilities. There are no facilities on-site for classified radioactive or hazardous constituents burial. X-749 is to be closed by EPA mandate in 1990 .

- X-749 is the radioactively contaminated burial facility, and disposed material must not contain hazardous or classified material. Contaminated asbestos is also buried at this site.

- The X-736 construction spoils landfill, a new construction spoils area, was opetied in 1989. Nonregulated materials such as construction and demolition debris are buried here to conserve the area dedicated for use by the X-735 sanitary landfill. These spoils must not contain any type of regulated waste.

\subsubsection{Off-site Disposal Activities}

Incineration is the preferred method for offsite disposal of waste, particularly hazardous and PCB wastes; however, landfills and other types of disposal are used as needed. For example, PCBcontaminated transformer carcasses cannot be incinerated and must be sent to an approved landfill. All commercial sites used for disposal have been inspected and approved by Martin Marietta Energy Systems ESA personnel prior to shipping wastes to that site. These inspections are used to examine processes and review management systems, permits, and insurance information. Offsite disposals, as listed in Table 5.3.1 of Part 2, are arranged through the PORTS Waste Management, Purchasing, and Traffic Departments. Unless special circumstances dictate otherwise, all such disposals are awarded to the lowest qualified bidder. All waste-shipping containers must meet DOE specifications. Table 5.3.1 (Part 2) lists wastes shipped to ORGDP in Oak Ridge, Tennessee. These wastes are intended for eventual incineration at ORGDP; however, at present the wastes are being stored until the ORGDP 
ORNL-DWG 87.8755R2

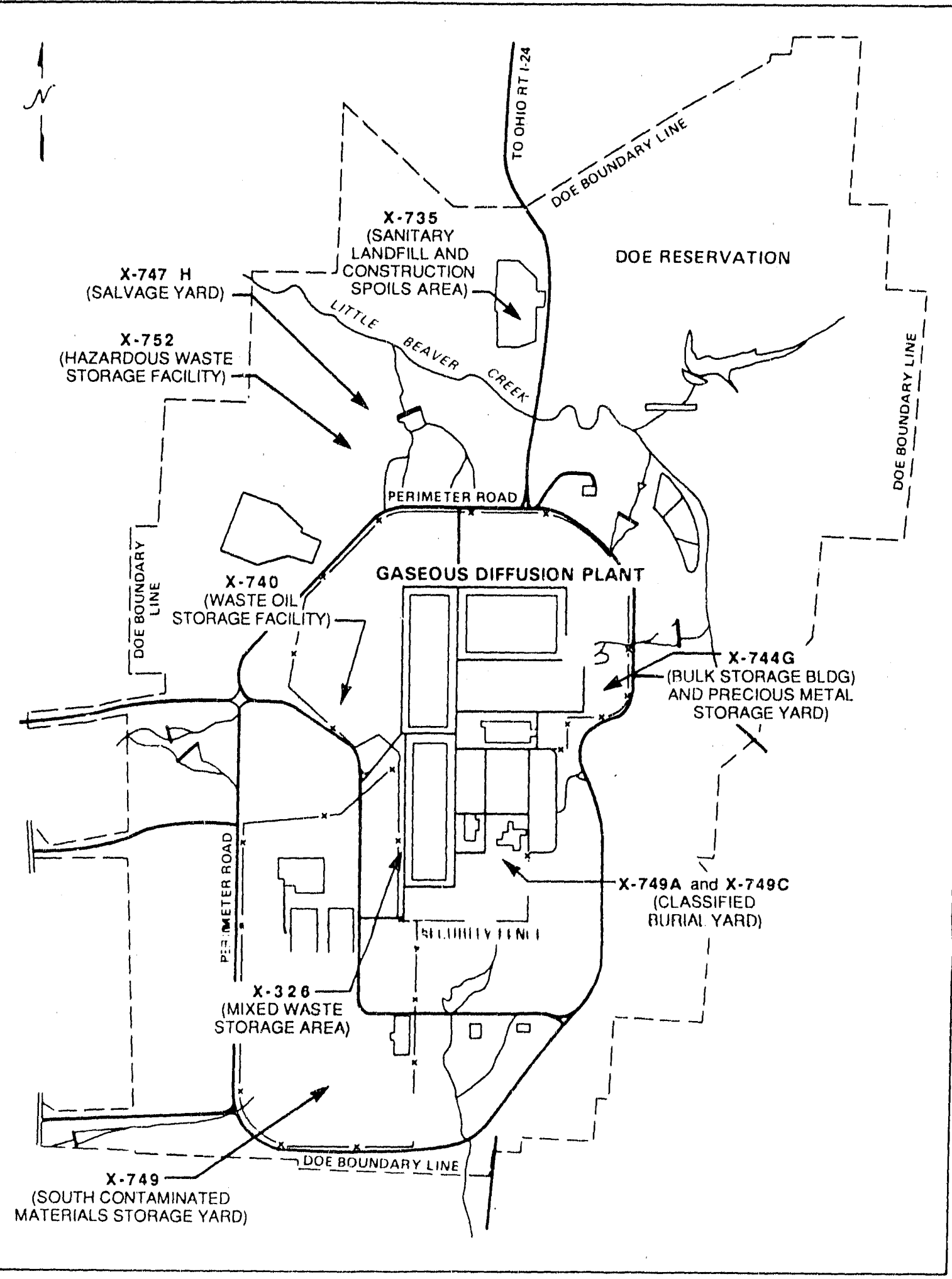

Fig. 5.3.1. Waste management storage facilities at PORTS. 
incinerator is operational. During CY 1989 , off-site shipments of six different types of wastes totaling 1.434 million $\mathrm{kg}$ (Part 2, Table 5.3.1) were made to three different sites. All of the disposals were, or will be, incinerated except for the landfilling of transformer carcasses.

\subsubsection{Waste Placed in Storage}

Low-level radioactive waste. Low-level radioactively contaminated wastes are currently segregated and handled as follows:

- Radioactively contaminated burnable trash (nonhazardous) is currently stored in retrievable storage canisters made of surplus converter shells. The supply of shells is nearly exhausted. These shells will be replaced with strong, tight containers purchased for this use and stored on a concrete pad. Examples of this material include contaminated wood, rags, plastic shoe covers, paper coveralls, other clothing articles, mop heads, plastic and paper sheeting, plastic bags, and boundary tapes. This burnable trash is being held for possible future incineration. If storage space becomes a severe problem, this trash will be volume-reduced and buried in X-749 until closure in 1990.

- Radioactively contaminated nonburnable trash (nonhazardous) is currently stored in retrievable storage canisters made of surplus converter shells. The supply of shells is nearly exhausted. These shells will be replaced with strong, tight containers purchased for this use and stored on a concrete pad. Examples of this waste include contaminated metal turnings, concrete and asphalt, dirt and other construction spoils, drums, cans, and other miscellaneous nonburnables.

- Asbestos with known radioactive contamination is stored in 6-mil, double-walled polyethylene bags until disposal.

- Nonhazardous chemical trap and filter wastes that have too little uranium to warrant uranium recovery have been disposed in X-749 using trench and fill methods. Samples of this material are periodically checked for EP toxicity to ensure that no RCRA hazardous materials are buried. No free liquids are permitted. This material is specially packaged in plastic and inserted in wooden crates before burial. Burial practices comply with sanitary landfill regulations until closure in 1990.

- Wastes containing highly mobile radioactive isotopes (e.g., technetium) in significant concentrations are stored.

A total of $722,844 \mathrm{~kg}$ of radioactively contaminated waste was generated and managed during CY 1989.

Contaminated PCB (mixed) wastes from process buildings. This waste is generated in the $X-333, X-330$, and $X-326$ process buildings through the collection of floor sweepings, cleanup of lube oil leaks, and collection of ventilation duct seam seepage. All waste generated in the process buildings is assumed to be uranium contaminated and is placed into DOT specification drums for indefinite storage. Disposal of these materials is through incineration at the TSCA incinerator at ORGDP in Oak Ridge, Tennessee. A total of $175,093 \mathrm{~kg}$ of mixed PCB waste was entered into storage during CY 1989.

Contaminated hazardous (mixed) wastes. The majority of this waste is generated from the raffinate treatment process in the $\mathrm{X}-705$ building. The neutralization of the raffinate results in a sludge that is EP toxic with regard to cadmium and lead and contains low-level quantities of uranium and techentium. During CY 1989 , $117,734 \mathrm{~kg}$ of mixed hazardous waste was placed into storage.

Radioactively contaminated bulk scrap. A variety of scrap metal is stored at the existing $\mathrm{X}-747 \mathrm{H}$ salvage yard located at the northwest corner of the perimeter road. This yard is used for the storage of radioactively contaminated scrap metal and equipment. Scrap intended for storage in this yard is decontaminated of removable radioactive material before movement to the storage yard. This salvage yard will continue to operate as a storage area for radioactive scrap metal.

Lithium hydroxide storage. A supply of lithium hydroxide is currently stored in warehouses 
at PORTS. The containers had deteriorated, and the warehouses had degraded. A projest to repair the buildings and to improve drainage was completed. A project to repackage the deteriorated drums into overpack drums has been completed, and additional storage warehouses have been constructed to contain the larger volume that resulted from the repackaging. These projects will provide safe, long-term storage.

Hazardous waste storage and disposal. The $\mathrm{X}-752$ hazardous waste storage facility is an interim status facility for the temporary storage of hazardous waste. During CY 1989, 182,798 kg of hazardous waste was manifested and entered into storage. During the year hazardous waste amounting to $58,687 \mathrm{~kg}$ was shipped to commercial incinerators for destructive disposal.

PCB waste storage and disposal. During CY 1989 a total of 38,481 L of PCB-contaminated liquids and $168,196 \mathrm{~kg}$ of PCB equipment (e.g., capacitors, light ballasts) were placed into storage for disposal. A total of $658,496 \mathrm{~kg}$ of PCB and PCB-contaminated wastes was shipped for incineration, and two PCB transformer carcasses were shipped to a secure chemical landfill.

\subsubsection{Special Activities}

Low-level waste disposal demonstration and development. The facilities administered by the DOE-ORO, which include PORTS, have undertaken an LLWDDD Program. The objective of this program is to develop, to demonstrate, and to evaluate the low-level waste disposal options for the future. The LLWDDD Program is defining performance parameters that must be achieved by the future waste disposal facilities. These are being developed consistent with Nuclear Regulatory Commission regulations and with the advice of area EPA regulators. PORTS is planning to evaluate each of the previously listed waste streams and to arrange for development and demonstration as required to provide the necessary options for the future. Technologies selected for future disposals will depend primarily on capability to meet performance requirements and secondarily on cost effectiveness and convenience.
Hazardous and radioactively contaminated waste. The HAZWDDD Program is responsible for identifying and categorizing mixed and hazardous waste streams from installations operated by Energy Systems; assessing technology, facility requirements, equipment, and program needs to handle these waste streams; determining suitable waste streams as candidates for technology demonstrations; and evaluating these demonstrations. Required facilities will need environmental studies based on NEPA. Permitting support for this activity will be provided by the program. The general philosophy and strategy derived to perform these series of tasks are given in the August 1987 Central Waste Management Organization's Strategic Hazardous and Mixed Waste Management Plan for the Department of Energy Installations Operated by Martin Marietta Energy Systems, Inc. Management and technical direction of the HAZWDDD Program is the responsibility of the Energy Systems Central Waste Management Office (CWMO). The individual Energy Systems installations provided a plan to the CWMO in December 1988 for incorporation into the Energy Systems overall plan for DOE-ORO.

Radioactively contaminated bulk scrap metal. The metal scrap stored at X-747H salvage yard consumes a major storage space. This waste contains radioactivity that prohibits its sale as scrap metal. However, the DOE-ORO, which has similar yards at each facility, is seeking to implement a program with private industry that will process this material. An initial demonstration contract, which was used to qualify potential vendors and processes, was completed in 1987, and details of the results of the demonstrations are available. Some of the private firms were generally successful in demonstrating methods to enable reuse of the metals. A bidding process was initiated in 1989 among the qualified vendors for processing the scrap from all facilities.

DOE Tiger Team Audit. The Doe Tiger Team performed an audit at PORTS from October 24, 1989, to November 13,1989. The audit team identified a total of 35 compliance-related findings, five of which specifically addressed solid waste 
management functions. The compliance-related items identified were storage of land-banned wastes beyond 1 year, deficiencies in two RCRA storage facilities, improper storage of hazardous waste drums, and inadequate storage of low-level waste.

Three of the five findings were corrected prior to the enci of 1989. Of the two remaining items, the storage of nonradioactive land-banned wastes will be corrected by mid-1990. The last item, deficiencies in RCRA storage areas, is the subject of a new audit procedure instituted at PORTS. All RCRA storage areas are surveyed on a monthly basis to identify and to correct any deficiencies within a short time frame. The surveys will also help determine if additional process or wastehandling training is required. 


\section{SPECIAL STUDIES}

\subsection{PORTSMOUTH GASEOUS DIFFUSION PLANT SPILL REPORT}

The PORTS plant had a total of 104 spills and releases of various types during 1989 (see Table 6.1.1). Each of the spills or releases was investigated by PORTS shift superintendents, PORTS Environmental Safety and Health (ES\&H) staff, and other staff to determine the environmental impact, to provide input for reducing any harmful effects, and to monitor any potential health effects. Cleanup activities were performed in accordance with PORTS standard operating procedures and were overseen by members of the ES\&H stiff. The PORTS shift superintendents report all spills or releases to various levels of PORTS management and to DOE-ORO of ficials as appropriate.

Forty PCB-contaminated oil spills account for almost half of the reported spills. Most of the reported spills amounted to new locations where drips from the ventilation ducts were found. There
Table 6.1.1. Spills and releases at PORTS during 1989

\begin{tabular}{lr}
\hline Acid (including raffinate) & 9 \\
Mercury & 6 \\
RCW/RHW & 8 \\
Sanitary water & 12 \\
PCB-contaminated oil & 40 \\
Other oil and petroleum & 9 \\
$\quad$ products & \\
Miscellaneous & 20 \\
$\quad$ Total & 104 \\
\hline
\end{tabular}

were 12 sanitary water breaks that resulted in the release of chlorine to the environment and eight $\mathrm{RCW} / \mathrm{RHW}$ line breaks that resulted in chromium being released. Additionally, there were nine releases of petroleum products including releases from leaking underg:ound storage tanks.

The magnitude of the spills ranged from gram quantities to an estimated 2000 gal. Efforts to enhance spill/release prevention of all products are ongoing. 


\section{QUALITY ASSURANCE AND GENERAL REVIEWS}

Quality assurance (QA), an integral part of the environmental surveillance effort, requires systematic control of the processes involved in sampling the environment and analyzing the samples. To achieve the desired level of control, PORTS uses the following major types of planned and systematic activities:

- implementation of standard operating procedures for sample collection and analysis,

- surveyor and analyst training and qualification,

- implementation of sample tracking and chainof-custody procedures to ensure traceability and integrity of samples and data,

- participation in external control programs,

- frequent calibration and routine maintenance of measuring and test equipment,

- maintenance of internal control programs, and

- implementation of good measurement techniques and good laboratory practices.

At PORTS environmental sampling is the responsibility of the Environmental Control Department. That organization prepares sampling plans and procedures and selects the appropriate sampling instruments or devices in accordance with use and clcaning practices recommended by the American Society for Testing and Materials, the EPA, or other authorities. Environmental Control also prepares chain-of-custody documentation from the point of sampling and properly protects the samples until they are transferred to the sampl custodian at the laboratory.

The PORTS analytical laboratory has a QA coordinator who has general oversight for all phases of laboratory QA and who is administratively independent of the laboratory. In addition, the coordinator of the Laboratory Controls and Standards Group (LCSG) also functions as a quality control (QC) officer administratively independent of the analytical laboratory. The LCSG operates the laboratory's central sample-receiving hain-of-custody facility and administers external and internal control programs. It generates internal QC samples using materials from the National Institute for Standards and Testing (NIST) or other reliable source materials and submits them to the laboratories on an established periodic basis. The LCSG performs statistical evaluations and issues QC reports to laboratory supervision and Environmental Control. The LCSG also generates control charts for the analytical laboratories. Good measurement practices in the laboratory include the use of matrix spikes, replicate analyses, check samples, and internal controls with the analysis of every batch of samples. Along with good measurement practices the extensive internal QC Program ensures the reliability of the analytical data on a day-to-day basis.

Treatment and interpretation of analytical results are the responsibility of the Environmental Control Department. Because the volume of data generated in the environmental surveillance is very large, the task for each monitoring program is compartmentalized to maintain responsible control. With the major objectives of protection of the public and protection of the environment, the data are promptly reviewed as soon as they are available to establish regulatory compliance and to determine whether remedial action is needed. Periodically, the $\mathrm{d} z$ ' a are reviewed for overall interpretation and, where relevant, for their interprogram relationships. The documentation of the overall effort in periodic publications, such as this report, serves as a resource for future activity. 


\subsection{FIELD SAMPLING AND MONITORING}

Personnel involved in field sampling and monitoring are trained, qualified, and use approved procedures developed from guidelines and regulations promulgated by $\mathrm{DOE}$ and regulatory organizations (such as the EPA) that exercise authority over activities on the plant site. These procedures spell out sampling protocol, sampling devices, and containers and preservatives to be used. Chain-of-custody procedures (in force for all sampling) are documented, and samples are controlled and protected from the point of sampling to the generation of analytical results.

\subsubsection{Basic Concepts and Practices}

Because data generated from field sampling can be greatly influenced by the methods used to collect and to transport the samples, it is imperative that a QA Program is in place to guarantee that the data are generated accurately and that the data reveal the conditions that exist in the environment at the time of sampling. The PORTS QA Program involves following written sampling procedures, using clean sampling devices and containers, employing approved samplepreservation techniques, and submitting field blanks and duplicate samples. Chain-of-custody procedures are strictly adhered to in order to ensure that sample integrity is maintained. Samples are delivered to the laboratory as soon as practicable after collection because some characteristics of the sample may be altered over time.

\subsubsection{Air Monitoring}

The PORTS air-monitoring network uses a pretreated filter-paper fluoride collector behind a Teflon particulate filter for collecting radionuclides and fluorides. The sample train uses materials that will not react with fluorides; this ensures that all gaseous fluoride drawn into the sample train will reach the collector. The flow calibration of the devices that measure total air drawn through the sample trains during the sampling period are checked semiannually to ensure that results accurately reflect airborne concentrations. Written procedures are in place to guide personnel in the collection and analysis of the samples and to check the calibration of the air-metering device.

\subsubsection{Surface Water Monitoring}

Liquid effluent streams from PORTS are sampled and analyzed in compliance with the NPDES discharge permit. Written procedures are used by personnel for both sampling and analysis of these effluent streams. Flow and $\mathrm{pH}$ are continuously measured and recorded at several discharge points. The calibration of the instrumentation is checked weekly to ensure that gond data are being collected. Weekly composite samples are collected at two of the NPDES sample sites and one receiving stream site and measured for radioactive constituents. Once a month two of these composite samples and an upstream sample from the receiving stream are split and shared with the Ohio Department of Health, Radiological Health Division, for analysis in its laboratory. Comparisons of data from the two laboratories show close correlation. Agreement with the state laboratory is another indicator of good performance by the PORTS analytical laboratories.

\subsubsection{Groundwater Monitoring}

The extensive PORTS Groundwater Monitoring Program is accomplished using individually dedicated pumps and delivery lines to purge and pump the wells. Dedicated equipment eliminates cross contamination of wells and samples. Written procedures are used by personnel for both sample collection and analysis. Field blanks and duplicate samples are also submitted to the laboratory to ensure that sampling techniques are not detrimentally affecting the data being collected.

\subsubsection{Biological Monitoring}

Biological monitoring at PORTS in 1989 included fish from receiving streams, food crops grown outside the reservation, and grass from outside the reservation. Randomly selected 
duplicate samples are submitted to verify the reliability of sampling and analytical procedures. Written procedures are used by personnel in the collection and analysis of these samples.

\subsubsection{Soil and Sediment}

Soil from the area surrounding the PORTS reservation and sediment from streams that receive liquid plant discharges are collected semiannually and analyzed for parameters that would indicate the influence that plant operations have on the sampled media. These samples are collected by field technicians who follow written procedures for sample collection. Duplicate samples are collected at randomly selected sites to verify the integrity of the sampling techniques.

\subsubsection{External Gamma Radiation}

Measurements of external gamma levels on and around the PORTS site are made according to a written procedure based on American National Standards Institute guidelines (ANSI 1975). Thermoluminescent dosimeters (TLDs) are installed, collected, and evaluated by Environmental Control Department personnel. Each TLD contains two TL chips that are treated as duplicate samples, and any TLD whose individual chips show significantly different results is tested for accuracy and validity before either result is used. All TLDs not being tested for validity are used to reestablish a group-specific calibration curve after each exposure cycle. Each calibration curve and data set is compared with previous calibration curves and filed with TLD results as an ongoing check against deterioration. Control TLDs are used to document extraneous exposures, and reference TLDs are used to measure TLD fading and to establish a correction factor using the midcycle exposure method.

\subsubsection{Waste-Monitoring Program}

Because solid wastes exist in various forms, several sampling and monitoring methods have been developed and instituted. The sampling and analyses of solid wastes at PORTS are accomplished using approved EPA procedures
(EPA November 1986). These methods include the hitsign and use of sampling equipment; method for calculating the number and pattern of samples to be collected to ensure that the average properties of the waste are represented, and analytical methods to be used. When feasible, duplicate samples are collected or replicate analyses are run to ensure reliability of the analytical data.

\subsection{ANALYTICAL QUALITY ASSURANCE}

The PORTS analytical laboratory continues a long tradition of QA and has a well-established QA Program. Integral to this program are a highly trained, well-qualified staff; use of approved and clocumented procedures and current analytical methodology; availability of excellent equipment and facilities; and routine use of good laboratory practices and good measurement techniques. As part of the QA effort, the analytical laboratory maintains comprehensive internal QC programs, participates in a number of external QC programs, and uses statistical interpretation extensively to evaluate its performance.

The laboratory meets the QA/QC requirements mandated by the OEPA, the EPA, and DOE. These requirements are an integral part of the laboratory QA programs. Analyses are performed using EPA-approved methods or other approved methods when available. Listings of analytical methods and lowest concentrations reported are given in Tables 7.2.1-7.2.5 of Part 2.

\subsubsection{Internal Quality Control}

The internal QC programs are the basis for ensuring reliable analytical results on a day-to-day and batch-to-batch basis. The total effort in these programs averages 10 to $20 \%$ of the laboratory effort (in accordance with EPA expectations). In 1989 PORTS analyzed 3000 internal environmental controls. The QC programs also frequently serve as the basis for on-the-job training and qualification of laboratory analysts performing environmental analyses. The internal QC programs are administered by the LCSG independent of the analytical laboratories. The LCSG is responsible 
for the preparation of known and blind controls and the statistical evaluation of the QC programs.

All analytical activities are supported by the routine use of either standard or reference materials from the NIST, EPA, other DOE laboratories, or other reliable sources. Quality control is accomplished through the use of such materials for instrument standardization; preparation of known, blind, and double blind controls; yield/efficiency determinations; and spike recoveries. The results of the blind-control programs, as well as a number of known-control programs, are statistically evaluated and reported to the laboratories and their customers. These reports assist in assessing the adequacy of analytical programs and procedures. If serious deviations are noted by the QC groups, the operating laboratories are promptly notified so that corrective actions can be initiated. Quality control data are stored retrievably so they can be related to the analytical results they support. The analytical laboratory has well-established QC for routine measured parameters in all types of environmental samples, and these parameters are shown in Table 7.2.1.

\subsubsection{External Quality Control}

In addition to the internal QC programs, PORTS, like all Energy Systems installations, is directed by DOE and by EPA regulators to participate in external QC programs. These programs generate data that serve as a periodic indicator of performance. Although participation is expected, the degree of participation by each laboratory is voluntary; thus, the analytical parameters selected are of particular interest to that plant. These control programs are administered by the EPA, DOE, and commercial laboratories.

\subsubsection{Radiological quality control}

In 1989 the PORTS analytical laboratory participated in two external radiological QC programs: the EPA Intercomparison Radionuclide Control Program and the DOE Environmental Measurements Laboratory (EML) Radionuclide Quality Assessment Program. The EPA Radionuclide Control Program is administered by the EPA Environmental Monitoring System Laboratory at Las Vegas. During 1989, in conjunction with this control program, the PORTS laboratory performed a total of seven analyses on four parameters (i.e., two analyses each on alpha and beta, two analyses on uranium, and one analysis on plutonium). As is shown in Table 7.2.6 of Part 2, five results were within the acceptable range, while two results were unacceptable.

The DOE-EML Quality Assessment Program is administered by DOE's EML in New York. Various matrix samples such as water, air filters, soil, tissue, and vegetation are analyzed semiannually for a variety of radioactive isotopes; a statistical report is submitted by EML for each period. PORTS results for this program, reported

Table 7.2.1. PORTS laboratory quality control

\begin{tabular}{lcc}
\hline & Parameters & \\
\hline Water monitoring & Vent monitoring & Air monitoring \\
Trace metals (19 elements) & Uranium & Sulfur (from coal) \\
Oil and grease & Technetium & Technetium \\
Alpha activity & Fluoride & Asbestos fibers \\
Beta activity & & Alpha activity \\
Uranium & Soil & Beta activity \\
Technetium & & \\
Suspended solids & Uranium & Vegetation \\
Dissolved solids & Alpha activity & \\
Nutrients & & Uranium \\
& & Alpha \\
\hline
\end{tabular}


in EML 525, are shown in Table 7.2.7 of Part 2. Of the 16 analyses performed in this program, 15 results were in the acceptable range. One result was unacceptable, but because all of the program participants reported unacceptable results in this case, the reference value is suspect.

\subsubsection{Nonradioactive quality control}

Nonradiological QC programs in which the PORTS laboratory participated during 1989 included the Proficiency Environmental Testing (PET) Program, the EPA Discharge Monitoring Report (DMR) QA Study, the Pittsburgh Energy Technology Center (PETC) Coal Program, the National Institute of Occupational Safety and Health (NIOSH) Proficiency Analytical Testing (PAT) Program, and the NIST National Voluntary Laboratory Accreditation Program (NVLAP).

PET Program. The PET Program is a commercial control program for envirormental analysis sponsored by Analytical Products Group (APG), a commercial laboratory. Samples at two concentration levels representing a wide variety of environmental parameters are distributed monthly to over 50 laboratories nationwide. Results are statistically evaluated by APG, and reports are then issued to each participating laboratory approximately 3 weeks after submission of data to APG. For each analysis the report includes two evaluations as a measure of performance: percent recovery of the reference value (which is based on APG's reference value for the analyte) and deviation from the mean result of all reporting laboratories in the program (which provides a performance comparison with all participants).

Data generated by the PORTS analytical laboratory in the PET Program during 1989 are given in Table 7.2.8 of Part 2. For each of the two performance measures (i.e., percent recovery and standard deviation from the mean), the results of both high and low concentrations for each parameter are averaged. The performance evaluation of acceptable results, however, is not based on averages. Instead, it is based on the individual standard deviation of the mean of all participating laboratories for each analysis.
Therefore, for a parameter analyzed each month at two concentrations, there are 24 performance indicators (i.e., acceptable, marginal, or unacceptable results) for the year. One thousand and one analyses representing 40 parameters were performed. Of these analyses, results for 978 were acceptable, 14 were marginal, and 9 were unacceptable. Because there were no more than three unacceptable values for any parameter, no trends could be identified to explain unacceptable results.

EPA DMR QA Study. The EPA conducts a national QA Program in support of the NPDES Program. All holders of major NPDES permits are required to participate. The EPA furnishes the QC samples and evaluates the results. Data representing PORTS participation in this program during 1989 are shown in Table 7.2.9 of Part 2. Results were obtained for 18 analytes. Two marginal results were obtained for nickel and nitrate as nitrogen; all other results were acceptable.

DOE/PETC Coal Program. In the DOE/PETC Coal Program coal is analyzed for the following parameters: volatile matter, fixed carbon, ash, sulfur, and heating value. From an environmental standpoint the ash, sulfur, and heating values results are of prime importance. The results of PORTS participation in the PETC Program during 1989 are shown in Table 7.2.10 of Part 2. Three samples were analyzed-each for five parameters. Acceptable results were obtained in all cases.

NIOSH PAT Program. Over 620 laboratories nationwide participate in NIOSH's PAT Program. Although its primary purpose is to support safety and liealth programs, the PAT Program includes a number of parameters that also represent environmental concerns. Therefore, PORTS 1989 PAT Program data are presented in Table 7.2.11 of Part 2 as a further indicator of performance in environmental analyses. During the course of the year, there were four rounds of program participation. In each round, four analyses were performed for each of eight parameters (i.e., lead, cadmium, zinc, silica, asbestos, and three organic solvents). Of the 128 results, only 6 were designated an "outliers" by NIOSH. Because of 
high performance, PORTS maintained its proficiency rating in the PAT Program for all parameters tested during 1989.

NIST-NVLAP Program for Bulk Asbestos. In 1989 the PORTS laboratory was accredited by the NIST for the analysis of bulk asbestos by polarized light microscopy under the NVLAP. Laboratory accreditation is based on the implementation of an extensive QA/QC Program, assessment of laboratory operations by the NVLAP technical representative, and satisfactory completion of al! proficiency samples. Accreditation is granted for one year and is subject to concinual demonstrated proficiency. PORTS has satisfactorily completed all proficiency checks conducted by NIST during 1989.

\subsection{AUDITS AND REVIEWS}

The PORTS Environmental Protection Program undergoes rigorous annual appraisals, audits, and reviews by organizations both internal and external. All appraisals, audits, and reviews may result in recommendations requiring operating contractor management attention.

Recommendations on environmental issues result in the development and implementation of corrective actions formulated to improve overall site environmental protection. Internal organizations that conduct periodic reviews of the Environmental Protection Program include Contractor SelfAppraisal Program coordinators and the Internal Audit and QA Department. Although the appraisals, audits, and reviews performed by these organizations might seem redundant, many areas addressed are reviewed with different criteria by various organizations.

Appraisals, audits, and/or reviews of the site Environmental Protection Program conducted during 1989 included an Environmental Protection Appraisal by the DOE-ORO Environmental Protection Division in April, a review of the 1986 Environmental Survey by the Office of Environmental Audit in May, a pre-Tiger Team review by Lee Wan Associates in July, a follow-up to the Lee Wan Associates review by Analysas Corporation in August, and the DOE Tiger Team Appraisal in October and November. The
Environmental Protection Appraisal was conducted April 24-28. The appraisal evaluated the effectiveness of Energy Sysiems implementation of DOE environmental protection requirements in the following areas: (1) the CAA (including asbestos), (2) the NEPA, (3) the Groundwater Program, (4) the TSCA (polychlorinated biphenyls), (5) internal audits, (6) the RCRA, and (7) the ERP.

In addition, PEER Consultants performed a field-sampling QC/QA audit of the Environmental Monitoring Program. This audit includes sampling in surface water, soil, vegetation, groundwater, and stand and ambient air. A review of the 1986 Environmental Survey by the Office of Environmental Audit was conducted in May. This review was a prioritization process to develop the relative ranking of the Category II and III findings from the Environmental Surveys conducted at DOE's 35 major operating facilities based on potential impacts to public health and the environment. To date no final report has been received from this review.

The pre-Tiger Team review was conducted by Lee Wan Asscciates in July. This review covered all the environmental programs, and field surveys were conducted to identify any environmental deficiencies in any of the facilities or plant site. A follow-up review to the Lee Wan Associates audit was conducted by Analysas Corporation. This was a verification review to see that Energy Systems had followed through on the Lee Wan Associates audit findings.

In October and November an extensive audit was conducted by a DOE Tiger Team. This environmental assessment was a comprehensive, facility-wide evaluation of all environmental media and adherence to applicable federal, state, and local regulations, requirements, and best management practices. The environmental areas addressed include air, surface water/drinking water, groundwater/soils, active waste management/underground storage tanks, toxic and hazardous materials, radioactive materials management, QA, inactive waste sites/emergency response, and the NEPA. Team specialists in each of the disciplines evaluated their respective concerns facility-wide through extensive reviews of the Portsmouth plant production, processes, 
training, recordkeeping, and procedures. In addition to environmental concerns, the Tiger Team review also addressed management and health and safety disciplines. The Environmental
Control Department has submitted and implemented corrective action plans to address all deficiencies noted in the audits or appraisals conducted in 1989. 


\section{PART 2}

\section{Data Presentation}


2.1 AIR 


\subsection{AIRBORNE DISCHARGES AND AIR}

\subsubsection{Emission-Monitoring Data}

Table 2.1.1 presents a quarterly summary of the X-600 steam plant opacity compliance. Particulate compliance is assumed to follow opacity compliance. Table 2.1.1 does not include the 395-min incident on December 2 (see Part 1).

Table 2.1.2 presents a monthly summary of coal deliveries and analysis data for the X-600 steam plant. Coal sampling and analysis data indicate that the X-600 steam plant was in $100 \%$ $\mathrm{SO}_{2}$ compliance.

Tables 2.1 .3 and 2.1 .4 present monthly summaries of total emissions from the stacks and vents monitored by the continuous sampler system. Emissions from the 50-m stack serving the purge cascades in X-326 are presented in Table 2.1.3, and emissions from the $20-\mathrm{m}$ roof vents comprising the rest of the system are shown in Table 2.1.4.

\subsubsection{Ambient Monitoring Data}

Tables 2.1.5 and 2.1.6 list the results of the monthly radioactive particulate samples from the ambient-air-monitoring stations. The results are for gross alpha and gross beta-gamma radiation respectively. Note that limits of detection for ambient samples will vary inversely with sample size. Small samples (i.e., less than $65 \%$ of design volume) are considered questionable. Very small samples (i.e., less than $50 \%$ of design volume) are considered invalid.

Table 2.1.7 lists the results of the weekly gaseous fluoride samples from the ambient air stations by station and sample collection date. These results are from chemically treated filter papers. Samples that did not contain detectable fluorides are reported as less than $(<)$ a concentration based on the analytical limit of detection.

Table 2.1.8 lists the ambient air station designations with their locations and directions from the plant center.

Table 2.1.1. 1989 X-600 steam plant opacity compliance

\begin{tabular}{cccc}
\hline Quarter & $\begin{array}{c}\text { Total minutes } \\
\text { of operation }\end{array}$ & $\begin{array}{c}\text { Total minutes } \\
\text { out of compliance }\end{array}$ & $\begin{array}{c}\text { Percent of time } \\
\text { in compliance }\end{array}$ \\
\hline 1 & 259,194 & 84 & 99.97 \\
2 & 262,062 & 102 & 99.96 \\
3 & 169,860 & 12 & 99.99 \\
4 & 253,020 & 84 & 99.97 \\
\hline
\end{tabular}


Table 2.1.2. 1989 X-600 steam plant coal delivery and analysis data

\begin{tabular}{lcccc}
\hline Month & $\begin{array}{c}\text { Tons of coal } \\
\text { delivered }\end{array}$ & $\begin{array}{c}\text { Percent } \\
\text { ash }\end{array}$ & $\begin{array}{c}\text { Percent } \\
\text { sulfur }\end{array}$ & $\begin{array}{c}\text { Tons } \mathbf{S O}_{2} \\
\text { generated }\end{array}$ \\
\hline January & 5842 & 12.4 & 3.16 & 350 \\
February & 5320 & 12.5 & 3.18 & 322 \\
March & 4558 & 10.9 & 2.99 & 250 \\
April & 3186 & 11.5 & 4.10 & 248 \\
May & 3477 & 11.4 & 3.36 & 223 \\
June & 3036 & 11.1 & 3.51 & 213 \\
July & 1215 & 12.0 & 3.75 & 87 \\
August & 1128 & 10.5 & 3.35 & 71 \\
September & 2277 & 12.2 & 3.62 & 157 \\
October & 3148 & 9.8 & 3.74 & 224 \\
November & 3689 & 11.6 & 4.53 & 319 \\
December & 4270 & 12.3 & 4.08 & 332 \\
\hline
\end{tabular}

Table 2.1.3. 1989 PORTS (50-m) radionuclide emissions

\begin{tabular}{lrllllll}
\hline \multicolumn{1}{c}{ Month } & $\begin{array}{r}{ }^{234} \mathrm{U} \\
(\mathrm{mCi})\end{array}$ & $\begin{array}{c}{ }^{235} \mathrm{U} \\
(\mathrm{mCi})\end{array}$ & $\begin{array}{c}{ }^{236} \mathrm{U} \\
(\mathrm{mCi})\end{array}$ & $\begin{array}{c}{ }^{238} \mathrm{U} \\
(\mathrm{mCi})\end{array}$ & $\begin{array}{c}\text { Uranium } \\
(\mathrm{kgU})\end{array}$ & $\begin{array}{c}{ }^{99} \mathrm{Tc} \\
(\mathrm{mCi})\end{array}$ & $\begin{array}{c}\text { Uranium daughters } \\
(\mathrm{mCi})\end{array}$ \\
\hline January & 4.41 & 0.178 & 0.0050 & 0.032 & 0.181 & 38.19 & 0.2425 \\
February & 7.71 & 0.295 & 0.0075 & 0.044 & 0.271 & 3.68 & 0.3823 \\
March & 9.81 & 0.381 & 0.0100 & 0.058 & 0.354 & 2.29 & 0.4965 \\
April & 13.45 & 0.504 & 0.0124 & 0.065 & 0.434 & 1.42 & 0.6335 \\
May & 7.27 & 0.240 & 0.0042 & 0.008 & 0.138 & 5.03 & 0.2564 \\
June & 6.28 & 0.235 & 0.0057 & 0.027 & 0.193 & 12.94 & 0.2894 \\
July & 4.99 & 0.192 & 0.0050 & 0.032 & 0.186 & 4.47 & 0.2556 \\
August & 13.32 & 0.511 & 0.0129 & 0.073 & 0.461 & 1.93 & 0.6565 \\
September & 6.53 & 0.252 & 0.0066 & 0.038 & 0.235 & 6.28 & 0.3288 \\
October & 9.49 & 0.362 & 0.0092 & 0.056 & 0.339 & 1.59 & 0.4737 \\
November & 7.39 & 0.281 & 0.0070 & 0.035 & 0.237 & 5.85 & 0.3504 \\
December & 7.27 & 0.291 & 0.0079 & 0.047 & 0.278 & 1.10 & 0.3841 \\
Misc. & 0.83 & $\underline{0.034}$ & $\underline{0.0010}$ & $\underline{0.007}$ & $\underline{0.037}$ & $\underline{0.17}$ & $\underline{0.0479}$ \\
\multicolumn{1}{c}{ Total (50-m) } & 98.74 & $\mathbf{3 . 7 5 6}$ & 0.0944 & 0.521 & 3.344 & 84.93 & 4.7974 \\
\hline
\end{tabular}

${ }^{a}$ Miscellaneous emissions are those from rinse solutions of inlet lines, crossover lines, and primary and secondary traps associated with each vent sampler. 
Table 2.1.4. 1989 PORTS (20-m) racionuclide emissions

\begin{tabular}{lccccccc}
\hline Month & $\begin{array}{c}{ }^{234} \mathrm{U} \\
(\mathrm{mCi})\end{array}$ & $\begin{array}{c}{ }^{235} \mathrm{U} \\
(\mathrm{mCi})\end{array}$ & $\begin{array}{c}{ }^{236} \mathrm{U} \\
(\mathrm{mCi})\end{array}$ & $\begin{array}{c}{ }^{238} \mathrm{U} \\
(\mathrm{mCi})\end{array}$ & $\begin{array}{c}\text { Uranium } \\
(\mathrm{kgU})\end{array}$ & $\begin{array}{c}{ }^{99} \mathrm{Tc} \\
(\mathrm{mCi})\end{array}$ & $\begin{array}{c}\text { Uranium daughters } \\
(\mathrm{mCi})\end{array}$ \\
\hline January & 0.095 & 0.005 & 0.00016 & 0.032 & 0.098 & 0.56 & 0.0684 \\
February & 0.326 & 0.017 & 0.00050 & 0.177 & 0.542 & 0.79 & 0.3717 \\
March & 0.209 & 0.010 & 0.00023 & 0.101 & 0.307 & 0.98 & 0.2113 \\
April & 0.220 & 0.012 & 0.00026 & 0.155 & 0.472 & 0.72 & 0.3215 \\
May & 0.097 & 0.005 & 0.00014 & 0.064 & 0.194 & 0.86 & 0.1327 \\
June & 0.324 & 0.013 & 0.00030 & 0.078 & 0.240 & 0.92 & 0.1683 \\
July & 0.116 & 0.005 & 0.00016 & 0.057 & 0.174 & 0.65 & 0.1192 \\
August & 0.444 & 0.012 & 0.00020 & $0.05 ;$ & 0.173 & 0.70 & 0.1227 \\
Septemter & 0.537 & 0.027 & 0.00089 & 0.263 & 0.805 & 1.28 & 0.5535 \\
October & 0.482 & 0.025 & 0.00085 & 0.271 & 0.828 & 1.25 & 0.5680 \\
November & 0.761 & 0.039 & 0.00155 & 0.322 & 0.987 & 0.98 & 0.6833 \\
December & 0.147 & 0.006 & 0.00020 & 0.052 & 0.161 & 0.92 & 0.1111 \\
Misc. ${ }^{a}$ & 0.093 & 0.004 & 0.00008 & 0.014 & $\underline{0.044}$ & 0.50 & $\underline{0.0316}$ \\
$\quad$ Total (20-r. $)$ & 3.85 & 0.179 & 0.0055 & 1.642 & 5.024 & 11.10 & 3.4631 \\
Total (50-1n) & 98.74 & 3.756 & 0.0944 & 0.521 & 3.344 & 84.93 & 4.7974 \\
\hline
\end{tabular}

${ }^{a}$ Rinse solutions from vent sampler components. 
Table 2.1.5. 1989 airborne gross alpha radiation concentrations $\left(\mathrm{pCi} / \mathrm{m}^{3}\right)$

\begin{tabular}{|c|c|c|c|c|c|c|c|c|c|}
\hline Month & $\begin{array}{c}\mathrm{A} 3 \\
\mathrm{~S}\end{array}$ & $\begin{array}{c}\text { A15 } \\
\text { SE }\end{array}$ & $\begin{array}{c}\mathrm{A} 12 \\
\mathrm{E}\end{array}$ & $\begin{array}{l}\text { A23 } \\
\mathrm{NE}\end{array}$ & $\underset{N}{\mathrm{~A} 24}$ & $\begin{array}{l}\text { A8 } \\
\text { NW }\end{array}$ & $\begin{array}{l}\text { A29 } \\
\text { W }\end{array}$ & $\begin{array}{l}\text { A9 } \\
\text { SW }\end{array}$ & $\begin{array}{l}\text { A28 } \\
\text { Bkg }\end{array}$ \\
\hline January & 0.005 & 0.002 & 0.009 & 0.002 & & 0.002 & 0.005 & 0.005 & 0.002 \\
\hline February & 0.005 & 0.006 & 0.006 & 0.013 & & 0.007 & 0.003 & 0.002 & -0.002 \\
\hline \multirow[t]{2}{*}{ March } & 0.005 & & & -0.003 & & 0.004 & & & 0.006 \\
\hline & 0.001 & 0.001 & 0.000 & 0.002 & & 0.003 & 0.000 & 0.001 & $-0.000^{a}$ \\
\hline April & 0.004 & 0.001 & 0.001 & 0.002 & & -0.001 & 0.001 & 0.002 & 0.002 \\
\hline May & -0.001 & 0.003 & 0.006 & 0.002 & & & 0.003 & 0.003 & 0.003 \\
\hline June & & 0.002 & 0.003 & & & & 0.003 & 0.002 & 0.004 \\
\hline July & -0.002 & 0.004 & 0.004 & 0.003 & & 0.004 & 0.003 & 0.003 & 0.000 \\
\hline August & 0.005 & 0.003 & 0.004 & 0.004 & & 0.004 & 0.002 & 0.003 & 0.006 \\
\hline September & 0.013 & 0.003 & 0.003 & 0.001 & & 0.002 & 0.001 & 0.003 & 0.004 \\
\hline October & 0.007 & 0.001 & 0.012 & 0.007 & 0.001 & 0.006 & 0.004 & 0.011 & 0.002 \\
\hline November & 0.004 & 0.007 & 0.002 & 0.002 & -0.001 & 0.001 & 0.003 & 0.001 & 0.003 \\
\hline December & 0.008 & 0.001 & 0.007 & 0.005 & 0.004 & 0.002 & -0.000 & 0.001 & 0.003 \\
\hline Month & $\begin{array}{c}\text { A6 } \\
\text { Pike }\end{array}$ & $\begin{array}{c}\mathrm{A} 10 \\
\mathrm{X}-230 \mathrm{~J}-3\end{array}$ & $\begin{array}{c}\text { A35 } \\
\text { X-230J-2 }\end{array}$ & $\begin{array}{c}\text { A36 } \\
\text { X-61! }\end{array}$ & $\begin{array}{c}\text { A38 } \\
\text { X-230J-8 }\end{array}$ & $\begin{array}{c}\text { A39 } \\
\times-760\end{array}$ & $\begin{array}{c}\text { A40 } \\
X-100\end{array}$ & $\begin{array}{l}\text { A37 } \\
\text { Bkg }\end{array}$ & \\
\hline January & 0.003 & 0.001 & 0.000 & 0.000 & 0.002 & 0.017 & 0.046 & 0.006 & \\
\hline February & 0.010 & 0.001 & 0.004 & -0.001 & 0.003 & 0.010 & 0.008 & -0.000 & \\
\hline \multirow[t]{2}{*}{ March } & 0.001 & & & -0.002 & & & & & \\
\hline & -0.001 & 0.003 & 0.002 & & 0.000 & 0.013 & 0.002 & 0.000 & \\
\hline April & -0.000 & 0.003 & & 0.001 & 0.002 & 0.015 & 0.005 & 0.006 & \\
\hline May & 0.001 & 0.002 & 0.005 & 0.005 & 0.005 & 0.016 & 0.004 & -0.000 & \\
\hline June & & 0.004 & 0.004 & & 0.004 & 0.019 & 0.003 & & \\
\hline July & 0.001 & 0.006 & 0.005 & 0.004 & 0.003 & 0.008 & 0.008 & 0.003 & \\
\hline August & 0.001 & 0.004 & 0.001 & 0.001 & 0.002 & 0.040 & 0.022 & 0.006 & \\
\hline September & 0.000 & 0.003 & 0.004 & 0.002 & 0.001 & 0.010 & 0.006 & 0.005 & \\
\hline October & 0.005 & 0.002 & 0.008 & 0.009 & 0.022 & 0.036 & 0.017 & 0.004 & \\
\hline November & 0.002 & 0.002 & 0.003 & 0.004 & 0.008 & 0.006 & 0.003 & 0.007 & \\
\hline December & 0.008 & 0.004 & 0.001 & 0.006 & 0.004 & 0.008 & 0.007 & 0.004 & \\
\hline
\end{tabular}

${ }^{a}$ Although PORTS does not show actual concentrations of less than zero, the negative numbers shown here are a consequence of the extremely low airborne alpha concentrations surrounding PCRTS. A -0.000 number, for example, represents a rounding off of a very small measured concentration. 
Table 2.1.6. 1989 airborne gross beta-gayama radiation concentrations ( $\mathrm{pCl} / \mathrm{m}^{3}$ )

\begin{tabular}{|c|c|c|c|c|c|c|c|c|c|}
\hline Month & $\begin{array}{c}\mathrm{A} 3 \\
\mathrm{~S}\end{array}$ & $\begin{array}{l}\text { A15 } \\
\text { SE }\end{array}$ & $\underset{\mathrm{E}}{\mathrm{A} 12}$ & $\begin{array}{l}\mathrm{A} 23 \\
\mathrm{NE}\end{array}$ & $\begin{array}{c}\mathrm{A} 24 \\
\mathrm{~N}\end{array}$ & $\begin{array}{l}\text { A8 } \\
\text { NW }\end{array}$ & $\begin{array}{c}A 29 \\
W\end{array}$ & $\begin{array}{l}\text { A9 } \\
\text { SW }\end{array}$ & $\begin{array}{l}\text { A28 } \\
\text { Bkg }\end{array}$ \\
\hline January & 0.035 & 0.025 & 0.032 & 0.036 & & 0.041 & 0.039 & 0.039 & 0.036 \\
\hline February & 0.032 & 0.019 & 0.029 & 0.052 & & 0.028 & 0.008 & 0.025 & 0.011 \\
\hline \multirow[t]{2}{*}{ March } & 0.016 & & & 0.017 & & 0.006 & & & 0.043 \\
\hline & 0.029 & 0.017 & 0.027 & 0.016 & & 0.027 & 0.012 & 0.033 & 0.009 \\
\hline April & 0.030 & 0.009 & 0.007 & 0.014 & & 0.008 & 0.004 & 0.009 & 0.015 \\
\hline May & 0.006 & 0.014 & 0.011 & 0.018 & & & 0.024 & 0.012 & 0.013 \\
\hline June & & 0.015 & 0.018 & & & & 0.027 & 0.019 & 0.028 \\
\hline July & 0.022 & 0.022 & 0.024 & 0.018 & & 0.028 & 0.027 & 0.028 & 0.034 \\
\hline August & 0.035 & 0.015 & 0.032 & 0.022 & & 0.029 & 0.024 & 0.026 & 0.032 \\
\hline September & 0.031 & 0.017 & 0.024 & 0.013 & & 0.031 & 0.092 & 0.026 & 0.040 \\
\hline October & 0.046 & 0.036 & 0.035 & 0.036 & 0.021 & 0.051 & 0.037 & 0.185 & 0.052 \\
\hline November & 0.089 & 0.026 & 0.016 & 0.018 & 0.025 & 0.026 & 0.028 & 0.023 & 0.040 \\
\hline December & 0.046 & 0.020 & 0.039 & 0.041 & 0.036 & 0.030 & 0.012 & 0.028 & 0.038 \\
\hline Month & $\begin{array}{l}\text { A6 } \\
\text { Pike }\end{array}$ & $\begin{array}{c}\mathrm{A} 10 \\
\mathrm{X}-230 \mathrm{~J}-3\end{array}$ & $\begin{array}{c}\text { A35 } \\
\mathrm{X}-230 \mathrm{~J}-2\end{array}$ & $\begin{array}{c}\text { A36 } \\
X-611\end{array}$ & $\begin{array}{c}\text { A38 } \\
\mathrm{X}-230 \mathrm{~J}-8\end{array}$ & $\begin{array}{c}\text { A39 } \\
X-760\end{array}$ & $\begin{array}{c}A 40 \\
X-100\end{array}$ & $\begin{array}{l}\text { A37 } \\
\text { Bkg }\end{array}$ & \\
\hline January & 0.029 & 0.032 & 0.017 & 0.006 & 0.017 & 0.067 & 0.132 & 0.067 & \\
\hline February & 0.037 & 0.044 & 0.028 & 0.001 & 0.012 & 0.023 & 0.046 & 0.024 & \\
\hline \multirow[t]{2}{*}{ March } & -0.001 & & & 0.004 & & & & & \\
\hline & 0.007 & 0.015 & 0.019 & & 0.073 & 0.027 & 0.018 & 0.023 & \\
\hline April & 0.014 & 0.017 & & 0.004 & 0.013 & 0.196 & 0.028 & 0.020 & \\
\hline May & 0.009 & 0.014 & 0.021 & 0.017 & 0.020 & 0.029 & 0.008 & 0.008 & \\
\hline June & & 0.019 & 0.019 & & 0.017 & 0.037 & 0.027 & & \\
\hline July & 0.022 & 0.021 & 0.031 & 0.021 & 0.035 & 0.031 & 0.033 & 0.020 & \\
\hline August & 0.013 & 0.019 & 0.019 & 0.012 & 0.025 & 0.033 & 0.044 & 0.045 & \\
\hline September & 0.005 & 0.021 & 0.024 & 0.013 & 0.052 & 0.033 & 0.041 & 0.036 & \\
\hline October & 0.027 & 0.023 & 0.050 & 0.040 & 0.154 & 0.065 & 0.070 & 0.050 & \\
\hline November & 0.009 & 0.021 & 0.021 & 0.023 & 0.022 & 0.033 & 0.034 & 0.040 & \\
\hline December & 0.034 & 0.029 & 0.025 & 0.035 & 0.030 & 0.032 & 0.033 & 0.063 & \\
\hline
\end{tabular}


Tuble 2.1.7. 1989 Gross airborne gaseous fluoride concentrations $\left(\mu \mathrm{g} / \mathrm{m}^{3}\right)$

\begin{tabular}{|c|c|c|c|c|c|c|c|c|c|c|c|c|c|c|c|c|c|}
\hline Date & $\begin{array}{c}A 3 \\
S\end{array}$ & $\begin{array}{c}A 15 \\
\text { SE }\end{array}$ & $\begin{array}{c}A 12 \\
E\end{array}$ & $\begin{array}{l}\mathrm{A} 23 \\
\mathrm{NE}\end{array}$ & $\stackrel{\mathrm{A} 24}{\mathrm{~N}}$ & $\begin{array}{l}\text { A8 } \\
\text { NW }\end{array}$ & $\begin{array}{c}\text { A } 29 \\
W\end{array}$ & $\begin{array}{l}\text { A9 } \\
\text { SW }\end{array}$ & $\begin{array}{l}128 \\
B k_{8}\end{array}$ & $\begin{array}{c}\text { A6 } \\
\text { Piketon }\end{array}$ & $\begin{array}{c}\text { Al0 } \\
\times-2303-3\end{array}$ & $\begin{array}{c}A 35 \\
X .230 \mathrm{~J} \cdot 2\end{array}$ & $\begin{array}{c}A 36 \\
X .611\end{array}$ & $\begin{array}{c}\text { A38 } \\
X \cdot 230 \mathrm{~J}-8\end{array}$ & $\begin{array}{c}A 39 \\
\times .760\end{array}$ & $\begin{array}{c}A 40 \\
x-100\end{array}$ & $\begin{array}{l}\text { A37 } \\
\text { Bkg }\end{array}$ \\
\hline $01 / 04 / 89$ & 0.19 & 0.10 & 0.28 & 0.13 & & 0.15 & 0.13 & 0.12 & 0.12 & 0.08 & 0.13 & 0.11 & 0.00 & 0.57 & & 0.11 & 0.20 \\
\hline $01 / 11 / 89$ & 0.30 & 0.20 & 0.27 & 0.21 & & 0.17 & 0.19 & 0.13 & 0.48 & 0.22 & 0.67 & 0.05 & 0.27 & 0.47 & & 0.04 & 0.31 \\
\hline $01 / 17 / 89$ & 0.21 & 0.21 & 0.28 & 0.10 & & 0.11 & 0.24 & 0.12 & 0.19 & 0.19 & 0.34 & 0.12 & 0.05 & 025 & & 0.18 & 0.2 .3 \\
\hline $01 / 23 / 89$ & 0.29 & 0.14 & 0.20 & 0.17 & & 0.12 & 0.26 & 0.09 & 0.16 & 0.13 & $0.1 i$ & 0.06 & 0.19 & 0.67 & & 0.35 & 0.19 \\
\hline $02 / 01 / 89$ & 0.27 & 0.11 & 0.20 & 0.10 & & 0.14 & 0.12 & 0.10 & 0.18 & 0.12 & 0.15 & 0.07 & 0.49 & 0.45 & 0.31 & 0.46 & 0.34 \\
\hline $02 / 07 / 89$ & 0.24 & 0.15 & & & & 0.09 & 0.14 & 0.07 & 0.10 & 0.15 & & 0.07 & 0.42 & 0.22 & 0.66 & 0.52 & 0.10 \\
\hline $02 / 20 / 89$ & 0.09 & 0.07 & 0.15 & 0.06 & & 0.20 & 0.06 & 0.26 & 0.11 & 0.10 & 0.08 & 0.11 & 0.04 & 0.36 & 0.65 & 0.39 & 0.15 \\
\hline $02 / 27 / 89$ & 0.36 & 0.13 & 0.49 & 0.08 & & 0.21 & 0.48 & 0.21 & 0.59 & 0.13 & 0.22 & 0.10 & 0.06 & 0.36 & 0.13 & 0.36 & 0.16 \\
\hline $03 / 08 / 89$ & 0.25 & 0.15 & 0.18 & 0.14 & & 0.36 & 0.15 & 0.05 & 0.13 & 0.14 & 0.32 & 0.11 & 0.09 & 0.05 & 0.24 & 0.35 & \\
\hline $03 / 14 / 89$ & 0.23 & 0.19 & 0.44 & 0.19 & & 0.33 & 0.00 & 0.19 & 0.35 & 0.25 & 0.50 & 0.25 & 0.10 & 0.09 & 0.29 & 2.31 & 0.17 \\
\hline $03 / 22 / 89$ & 0.16 & 0.14 & 0.21 & 0.38 & & 0.16 & 0.18 & 0.24 & 0.43 & 0.36 & 0.13 & 0.13 & 0.34 & 0.13 & 0.33 & 0.29 & 0.17 \\
\hline $03 / 29 / 89$ & 0.37 & 0.16 & 0.33 & 0.36 & & 0.35 & 0.14 & 0.14 & 0.02 & 0.27 & 0.18 & 0.12 & 0.28 & 0.27 & 0.90 & & 0.18 \\
\hline $04 / 03 / 89$ & 0.29 & 0.34 & 0.22 & 0.21 & & 0.21 & 0.22 & 0.15 & 0.70 & 0.18 & 0.16 & & 0.37 & & 0.49 & 0.02 & 0.18 \\
\hline $04 / 10 / 89$ & 0.29 & 0.25 & 0,38 & 0.13 & & 0.41 & 0.16 & 0.26 & 0.44 & 0.24 & 0.21 & & 0.11 & 0.66 & 0.06 & 0.99 & 0.54 \\
\hline $04 / 18 / 89$ & 0.31 & 0.28 & & 0.30 & & 0.29 & 0.12 & 0.20 & 0.16 & $0.0 \varsigma$ & 0.14 & & 0.11 & 0.33 & 0.09 & 0.58 & 0.18 \\
\hline $04 / 26 / 89$ & 0.30 & & 0.18 & 0.36 & & 0.21 & 0.19 & 0.20 & 0.16 & 0.45 & 0.11 & & 0.16 & 0.27 & 9.26 & 0.44 & 0.31 \\
\hline $05 / 02 / 89$ & 0.41 & 0.31 & 0.64 & 0.36 & & 0.20 & 0.20 & 0.17 & 0.52 & 0.47 & 0.16 & & 0.28 & 0.34 & 0.42 & 0.41 & 0.32 \\
\hline $05 / 08 / 89$ & 0.16 & 0.15 & 0.34 & 0.11 & & 0.39 & 0.58 & 0.15 & 0.20 & 0.56 & 0.55 & 0.25 & 0.27 & 1.02 & 0.56 & 0.58 & 0.12 \\
\hline $05 / 16 / 89$ & 0.14 & 0.26 & & 0.10 & & 0.26 & 0.31 & 0.15 & 0.14 & 0.17 & 0.12 & 0.25 & 0.26 & 0.32 & 0.72 & 0.36 & 0.12 \\
\hline $05 / 23 / 89$ & 0.16 & 0.34 & & 0.18 & & 0.28 & 0.15 & 0.29 & 0.42 & 0.26 & 0.25 & 0.36 & 0.18 & 0.26 & 1.30 & 0.59 & 0.39 \\
\hline $05 / 31,89$ & 0.53 & 0.23 & 0.71 & 0.12 & & 0.35 & 0.10 & 0.22 & 0.25 & 0.27 & & 0.97 & 0.27 & 1.01 & 0.66 & 1.04 & 0.33 \\
\hline $06 / 08 / 89$ & & 0.43 & 1.03 & 0.58 & & 0.55 & 0.57 & 0.35 & 1.04 & 0.38 & 0.23 & 0.68 & 0.79 & 0.08 & 1.57 & 1.12 & 0.33 \\
\hline $06 / 12 / 89$ & & 0.25 & 0.77 & 0.27 & & 0.99 & & 0.45 & 0.39 & 0.38 & 0.24 & 0.25 & 0.22 & 0.48 & 1.13 & 0.59 & \\
\hline $06 / 20 / 89$ & & & & & & & 0.25 & 0.18 & 0.25 & 0.21 & & & & & & & \\
\hline $06 / 29 / 89$ & & 0.15 & 0.53 & 0.16 & & 0.13 & 0.32 & 0.72 & 0.32 & 0.27 & 0.16 & 0.25 & 0.55 & 0.42 & 3.14 & 1.25 & 1.06 \\
\hline $07 / 12 / 89$ & 0.65 & 0.10 & 0.60 & 0.20 & & 0.18 & 0.19 & 0.25 & 0.26 & 0.25 & 0.30 & 0.22 & 0.21 & 0.43 & 0.51 & 0.58 & 0.17 \\
\hline $07 / 18 / 89$ & 0.59 & 0.11 & 0.34 & 0.42 & & 0.30 & 0.15 & 0.24 & 0.31 & 0.19 & 0.19 & 0.68 & 0.47 & 0.31 & 1.11 & 0.82 & 2.48 \\
\hline $07 / 24 / 89$ & 0.77 & & 0.52 & 1.29 & & 0.35 & 0.21 & 0.10 & 0.30 & 0.27 & & 0.32 & 0.52 & 0.74 & 2.32 & 1.33 & 0.57 \\
\hline $08 / 02 / 89$ & 1.05 & 0.15 & 0.45 & 0.62 & & 0.50 & 0.14 & 0.19 & 0.20 & 0.16 & 0.30 & 0.24 & 0.54 & 0.50 & 2.71 & 0.85 & 0.36 \\
\hline $08 / 08 / 89$ & 0.81 & 0.46 & 0.48 & 0.37 & & 0.30 & 0.34 & 0.29 & 0.37 & 0.39 & 0.20 & 0.64 & 0.37 & 0.49 & 3.75 & 1.64 & 0.45 \\
\hline $08 / 15 / 89$ & 1.11 & 0.29 & 0.90 & 0.19 & & 0.77 & 0.20 & 0.25 & 0.32 & 0.18 & 0.38 & 0.18 & 0.34 & 0.35 & 1.56 & 1.64 & 0.38 \\
\hline $08 / 22 / 89$ & 2.31 & 0.33 & 0.41 & 0.40 & & 0.23 & 0.19 & 0.27 & 0.28 & 0.50 & 0.25 & 0.24 & 0.57 & 0.30 & 2.13 & 0.75 & 0.35 \\
\hline $08 / 28 / 89$ & 0.74 & 0.43 & 0.34 & 0.70 & & 0.22 & 0.77 & 0.38 & 0.46 & 0.50 & 0.13 & 0.31 & 0.45 & 0.38 & 3.24 & 1.16 & 0.66 \\
\hline $09 / 06 / 89$ & $1.6 j$ & 0.21 & 0.80 & 0.26 & 0.27 & 0.78 & 0.31 & 0.59 & 0.74 & 0.32 & & 0.31 & 0.41 & 0.46 & 0.64 & 0.55 & 0.78 \\
\hline $09 / 14 / 89$ & 0.55 & 0.22 & 0.77 & 0.55 & 0.60 & 0.69 & 0.30 & 0.36 & 0.14 & 0.48 & 0.30 & 0.40 & 0.41 & 0.20 & 0.62 & 0.48 & 0.68 \\
\hline $09 / 19 / 89$ & 0.49 & 0.14 & 0.27 & 0.17 & 0.13 & 0.23 & 0.23 & 0.29 & 0.34 & 015 & 0.18 & 0.22 & & 0.07 & 1.60 & 0.38 & 0.41 \\
\hline $10 / 03 / 89$ & 0.32 & 0.07 & 0.24 & 0.10 & 0.82 & 0.16 & 0.03 & 0.25 & 0.43 & 0.26 & 0.13 & 0.21 & 0.13 & 0.19 & 0.73 & 0.70 & 0.72 \\
\hline $10 / 10 / 89$ & 0.26 & 0.20 & 0.23 & 0.21 & 0.19 & 0.13 & 0.23 & 0.06 & 0.31 & 0.14 & 0.10 & 0.63 & 0.21 & & & & 0.28 \\
\hline $10 / 18 / 89$ & 0.31 & 0.17 & 0.21 & 0.15 & 0.18 & 0.10 & 0.15 & 0.05 & 0.12 & 0.18 & 0.15 & 0.24 & 0.20 & 0.15 & 0.49 & 0.40 & 0.28 \\
\hline $10 / 24 / 89$ & 0.41 & 0.17 & 0.35 & 0.26 & 0.32 & 0.24 & 0.14 & 0.13 & 0.38 & 0.39 & 0.10 & 0.18 & 0.17 & 0.12 & 0.80 & 0.89 & 0.12 \\
\hline $10 / 31 / 89$ & 0.29 & 0.00 & 0.28 & 0.13 & 0.13 & 0.36 & 0.16 & 0.26 & 0.00 & 0.14 & 0.55 & & 0.16 & 0.16 & 0.51 & 0.45 & \\
\hline $11 / 07 / 89$ & 0.21 & 0.12 & 0.25 & 0.11 & 0.17 & 0.11 & 0.10 & 0.05 & 0.18 & 0.01 & 0.14 & 0.10 & 0.15 & 0.31 & 0.62 & 0.49 & 0.16 \\
\hline $11 / 13 / 89$ & & 0.17 & 0.42 & 0.16 & 0.13 & 0.14 & 0.20 & 0.22 & 0.23 & 0.11 & 0.16 & 0.16 & 0.25 & 0.31 & 0.68 & 0.59 & 0.17 \\
\hline $11 / 26 / 89$ & 0.15 & 0.09 & 0.14 & 0.08 & 0.07 & 0.11 & 0.09 & 0.06 & 0.08 & 0.05 & 0.10 & 0.09 & 0.15 & 0.29 & 0.96 & 0.76 & 0.11 \\
\hline $12 / 05 / 89$ & 0.25 & 0.09 & 0.17 & 0.09 & 0.07 & 0.09 & 0.16 & 0.10 & 0.14 & 0.13 & 0.13 & 0.06 & 0.11 & 0.21 & 2.02 & 0.96 & 0.24 \\
\hline $12 / 14 / 89$ & 0.23 & 0.07 & 0.11 & 0.09 & 0.08 & 0.07 & 0.11 & 0.14 & 0.16 & 0.12 & 0.14 & 0.12 & 0.35 & 0.18 & 0.45 & 0.22 & \\
\hline $12 / 18 / 89$ & & 0.09 & 0.16 & 0.10 & 0.14 & 0.12 & 0.11 & 0.12 & 0.00 & & 0.10 & 0.11 & 0.18 & 0.22 & 0.29 & 0.73 & 0.18 \\
\hline $01 / 04 / 90$ & 0.12 & 0.05 & 0.13 & 0.05 & 0.03 & 0.04 & 0.09 & 0.05 & 0.04 & 0.03 & 0.03 & 0.06 & 0.07 & 0.23 & 0.45 & 0.43 & 0.08 \\
\hline
\end{tabular}


Table 2.1.8. PORTS ambient air station locations

\begin{tabular}{|c|c|c|}
\hline Designation & Location & Direction \\
\hline \multicolumn{3}{|c|}{ On-site air-sampling stations } \\
\hline A40 & $\mathrm{X}-100$ administration building penthouse & NA \\
\hline A35 & $\begin{array}{l}\mathrm{X}-230-\mathrm{J} 2 \text { south (holding pond) environmental } \\
\text { monitoring station }\end{array}$ & $\mathbf{S}$ \\
\hline A10 & $\begin{array}{l}\text { X-230-J3 west (ditch) environmental monitoring } \\
\text { station }\end{array}$ & W \\
\hline A38 & $\begin{array}{l}\mathrm{X}-230-\mathrm{J} 8 \text { environmental storage building, } \\
\text { southeast corner of main parking lot }\end{array}$ & ESE \\
\hline A36 & $\begin{array}{l}\text { X-611 water treatment plant, southeast corner } \\
\text { of facility parking lot }\end{array}$ & ENE \\
\hline A39 & $\begin{array}{l}\text { X-760 chemical engineering facility, } \\
\text { northwest corner of outside wall }\end{array}$ & NA \\
\hline \multicolumn{3}{|c|}{ Off-site (fence-line) air-sampling stations } \\
\hline A3 (S) & South Access Rd., south of DOE property line & SSW \\
\hline A8 (NW) & $\begin{array}{l}\text { DOE power pole } 74 \text { on dirt patrol road starting } \\
\text { north of } X-735\end{array}$ & NW \\
\hline A9 (SW) & Southwest Access Rd. and Old Rt. 23 & SW \\
\hline $\mathrm{A} 12(\mathrm{E})$ & McCorkle Rd. north of East Access Rd. & ENE \\
\hline A 15 (SE) & North end of Cemetery Rd. & SE \\
\hline A23 (NE) & Barlow Rd, and Taylor Hollow Rd. & NE \\
\hline $\mathrm{A} 24(\mathrm{~N})$ & North Access Rd. north of Shyville Rd. & $N$ \\
\hline A29 (W) & U.S. Rt. 23 on near leg of old GAT sign & W \\
\hline \multicolumn{3}{|c|}{ Off-site air-sampling stations } \\
\hline A6 PK & $\begin{array}{l}\text { DOE power pole } 6 \text { on access road to X-608 pump } \\
\text { house in Piketon }\end{array}$ & NNW \\
\hline A28 CC & Mt. Zion Church on Camp Creek Rd. & SW \\
\hline A37 OT & AT\&T booster station on Mt. Hope Rd. west of Otway & SW \\
\hline
\end{tabular}


2.2 SURF ICE WATER 
Table 2.2.1. NPDES chemical data summary

\begin{tabular}{|c|c|c|c|c|c|}
\hline Parameters & Min & $\operatorname{Max}$ & Av & $\begin{array}{c}\text { Std. } \\
\text { deviation }\end{array}$ & $\begin{array}{c}\text { No. of } \\
\text { samples }\end{array}$ \\
\hline \multicolumn{6}{|c|}{ NPDES 001} \\
\hline Temperature $\left({ }^{\circ} \mathrm{C}\right)$ & 0 & 27 & $\cdot 17.37$ & 5.61 & 51 \\
\hline $\mathrm{pH}$ (std, units) & 7.00 & 8.60 & 7.98 & NA & 53 \\
\hline TSS $(\mathrm{mg} / \mathrm{L})$ & $b$ & 64.0 & 7.45 & 10.35 & 52 \\
\hline Oil $(\mathrm{mg} / \mathrm{L})$ & $b$ & $b$ & $b$ & 0.0 & 51 \\
\hline Chlorine $(\mathrm{mg} / \mathrm{L})$ & $b$ & 0.06 & 0.01 & 0.01 & 52 \\
\hline Flow (Mgd) & 0.05 & 1.57 & 0.85 & 0.39 & 12 \\
\hline \multicolumn{6}{|c|}{ NPDES 601} \\
\hline pH (std. units) & 7.30 & 10.30 & 8.44 & NA & 9 \\
\hline Flow (Mgd) & 0.00 & 0.01 & 0.0011 & 0.00280 & 8 \\
\hline Ammonia (mg/L) & $b$ & 0.30 & 0.09 & 0.12 & 8 \\
\hline Nitrate $(\mathrm{mg} / \mathrm{L})$ & $b$ & 7.00 & 1.60 & 2.57 & 8 \\
\hline Chromium $(\mu \mathrm{g} / \mathrm{L})$ & $b$ & 80 & 10 & 28.28 & 8 \\
\hline Copper $(\mu \mathrm{g} / \mathrm{L})$ & $b$ & 30 & 8.75 & 12.46 & 8 \\
\hline Iron $(\mu \mathrm{g} / \mathrm{L})$ & 110 & 6,000 & $1,071.25$ & $2,011.33$ & 8 \\
\hline Nickel $(\mu \mathrm{g} / \mathrm{L})$ & 60 & 120 & 102.50 & 19.82 & 8 \\
\hline $\operatorname{Zinc}(\mu \mathrm{g} / \mathrm{L})$ & $b$ & 100 & 12.50 & 35.36 & 8 \\
\hline TCE $(\mu \mathrm{g} / \mathrm{L})$ & $b$ & 276 & 66.11 & 97.41 & 9 \\
\hline Chromhex $(\mu \mathrm{g} / \mathrm{L})$ & $b$ & $b$ & $b$ & 0.0 & 8 \\
\hline \multicolumn{6}{|c|}{ NPDES 002} \\
\hline pH (std. units) & 7.0 & 9.20 & 7.88 & NA & 52 \\
\hline $\mathrm{TSS}(\mathrm{mg} / \mathrm{L})$ & 1.40 & 190.0 & 16.46 & 28.26 & 52 \\
\hline $\operatorname{Oil}(\mathrm{mg} / \mathrm{L})$ & $b$ & $b$ & $b$ & 0.0 & 52 \\
\hline Chlorine $(\mathrm{mg} / \mathrm{L})$ & $b$ & $b$ & $b$ & 0.0 & 52 \\
\hline Flow (Mgd) & 0.44 & 0.81 & 0.56 & 0.11 & 12 \\
\hline Copper $(\mu \mathrm{g} / \mathrm{L})$ & $b$ & 80 & 4.04 & 14.04 & 52 \\
\hline Iron $(\mu \mathrm{g} / \mathrm{L})$ & 80 & 7,800 & $1,416.92$ & $1,326.74$ & 52 \\
\hline Nickel $(\mu \mathrm{g} / \mathrm{L})$ & $b$ & 20 & 1.54 & 5.38 & 52 \\
\hline Zinc $(\mu \mathrm{g} / \mathrm{L})$ & $b$ & 400 & 54.51 & 55.00 & 51 \\
\hline Arsenic $(\mu \mathrm{g} / \mathrm{L})$ & $b$ & 20 & 0.58 & 3.08 & 52 \\
\hline Manganese $(\mu \mathrm{g} / \mathrm{L})$ & $b$ & 1,700 & 174.81 & 291.34 & 52 \\
\hline \multicolumn{6}{|c|}{ NPDES 602} \\
\hline pH (std. units) & 7.10 & 9.50 & 8.46 & NA & $\$ 2$ \\
\hline TSS (mg/L) & 1.40 & 390.0 & 16.07 & 54.97 & 52 \\
\hline Flow (Mgd) & 0.02 & 0.07 & 0.03 & 0.01 & 13 \\
\hline Copper $(\mu \mathrm{g} / \mathrm{L})$ & $b$ & 12,0 & 4.42 & 18.62 & 52 \\
\hline Iron $(\mu \mathrm{g} / \mathrm{L})$ & 60 & 144,000 & $5,142.31$ & $20,415.99$ & 52 \\
\hline Nickel $(\mu \mathrm{g} / \mathrm{L})$ & $b$ & 300 & 26.54 & 50.44 & 52 \\
\hline Zinc $(\mu \mathrm{g} / \mathrm{L})$ & $b$ & 940 & 75.19 & 138.15 & 52 \\
\hline Arsenic $(\mu \mathrm{g} / \mathrm{L})$ & $b$ & 80 & 7.84 & 16.65 & 51 \\
\hline Manganese $(\mu \mathrm{g} / \mathrm{L})$ & $b$ & 340 & 52.12 & 59.16 & 52 \\
\hline \multicolumn{6}{|c|}{ NPDES 003} \\
\hline pH (std. units) & 7.30 & 8.40 & 7.76 & NA & 53 \\
\hline $\mathrm{TSS}(\mathrm{mg} / \mathrm{L})$ & $b$ & 23.0 & 1.75 & 3.34 & 53 \\
\hline Chlorine $(\mathrm{mg} / \mathrm{L})$ & 0.02 & 0.43 & 0.18 & 0.08 & 52 \\
\hline Flow (Mgd) & 0.01 & 0.28 & 0.21 & 0.07 & 13 \\
\hline Nickel $(\mu \mathrm{g} / \mathrm{L})$ & $b$ & 90 & 11.73 & 17.46 & 52 \\
\hline BOD $(\mathrm{mg} / \mathrm{L})$ & 0.39 & 5.17 & 2.15 & 1.23 & 52 \\
\hline Coliform (\#/100 mg) & $b$ & 1,898 & 82.29 & 311.93 & 52 \\
\hline
\end{tabular}


Table 2.2.1 (continued)

\begin{tabular}{|c|c|c|c|c|c|}
\hline Parameters & Min & $\operatorname{Max}$ & Av & $\begin{array}{c}\text { Std. } \\
\text { deviation }\end{array}$ & $\begin{array}{l}\text { No. of } \\
\text { samples }\end{array}$ \\
\hline \multicolumn{6}{|c|}{ NPDES 604} \\
\hline pH (std. units) & 7.10 & 9.40 & 8.39 & NA & 103 \\
\hline Flow (Mgd) & 0.01 & 0.07 & 0.03 & 0.02 & 12 \\
\hline Nitrate $(\mathrm{mg} / \mathrm{L})$ & $b$ & 250 & 53.25 & 49.51 & 101 \\
\hline Copper $(\mu \mathrm{g} / \mathrm{L})$ & $b$ & 600 & 6.86 & 59.54 & 102 \\
\hline Iron $(\mu \mathrm{g} / \mathrm{L})$ & $b$ & 5,800 & 271.76 & 659.72 & 102 \\
\hline Nickel $(\mu \mathrm{g} / \mathrm{L})$ & $b$ & 70 & 8.04 & 16.23 & 102 \\
\hline $\operatorname{Zinc}(\mu \mathrm{g} / \mathrm{L})$ & $b$ & 1,400 & 83.73 & 156.84 & 102 \\
\hline \multicolumn{6}{|c|}{ NPDES 004} \\
\hline pH (std. units) & 7.40 & 8.70 & 8.11 & NA & 52 \\
\hline TSS (mg/L) & $b$ & 14.0 & 3.52 & 3.27 & 52 \\
\hline Chlorine $(\mathrm{mg} / \mathrm{L})$ & $b$ & $b$ & $b$ & 0.0 & 51 \\
\hline Flow (Mgd) & 0.92 & 1.45 & 1.23 & 0.16 & 12 \\
\hline Chromium $(\mu \mathrm{g} / \mathrm{L})$ & 20 & 860 & 201.92 & 210.12 & 52 \\
\hline Copper $(\mu \mathrm{g} / \mathrm{L})$ & $b$ & 20 & 0.77 & 3.88 & 52 \\
\hline Iron $(\mu \mathrm{g} / \mathrm{L})$ & $b$ & 70 & 19.23 & 19.39 & 52 \\
\hline $\operatorname{Zinc}(\mu \mathrm{g} / \mathrm{L})$ & $b$ & 320 & 44.23 & 60.27 & 52 \\
\hline $\operatorname{TCE}(\mu \mathrm{g} / \mathrm{L})$ & $b$ & $b$ & $b$ & 0.0 & 51 \\
\hline Chromhex $(\mu \mathrm{g} / \mathrm{L})$ & $b$ & 20 & 0.77 & 3.34 & 52 \\
\hline Disol & 2,300 & 3,750 & $2,872.69$ & 277.62 & 52 \\
\hline \multicolumn{6}{|c|}{ NPDES 605} \\
\hline $\mathrm{pH}$ (std. units) & 7.00 & 10.00 & 8.25 & $\mathrm{NA}$ & 99 \\
\hline TSS $(\mathrm{mg} / \mathrm{L})$ & $b$ & 770 & 57.37 & 134.30 & 102 \\
\hline Oil $(\mathrm{mg} / \mathrm{L})$ & $b$ & 45.0 & 15.58 & 11.06 & 101 \\
\hline Flow (Mgd) & 0.002 & 0.01 & 0.00467 & 0.00187 & 12 \\
\hline Ammonia (mg/L) & $b$ & 4.30 & 0.47 & 0.55 & 102 \\
\hline Nitrate (mg/L) & 0.80 & 1,280 & 471.78 & 289.02 & 102 \\
\hline Chromium $(\mu \mathrm{g} / \mathrm{L})$ & $b$ & 1,500 & 92.79 & 217.34 & 102 \\
\hline Copper $(\mu \mathrm{g} / \mathrm{L})$ & $b$ & 4,000 & 480.69 & 754.84 & 102 \\
\hline $\operatorname{Iron}(\mu \mathrm{g} / \mathrm{L})$ & $b$ & 166,000 & $14,055.40$ & $33,107.70$ & 102 \\
\hline Nickel $(\mu \mathrm{g} / \mathrm{L})$ & 20 & 6,200 & 822.84 & $1,214.75$ & 102 \\
\hline $\operatorname{Zinc}(\mu \mathrm{g} / \mathrm{L})$ & $b$ & 3,600 & 400.49 & 735.39 & 102 \\
\hline $\operatorname{TCE}(\mu \mathrm{g} / \mathrm{L})$ & $b$ & 705 & 20.63 & 103.72 & 99 \\
\hline Nitrite (mg/L) & $b$ & 31 & 5.32 & 5.25 & 102 \\
\hline Nitrogen $(\mathrm{mg} / \mathrm{L})$ & $b$ & 11 & 0.52 & 1.18 & 102 \\
\hline \multicolumn{6}{|c|}{ NPDES 005} \\
\hline Flow (Mgd) & 0.00 & 0.00 & 0.00 & 0.00 & 12 \\
\hline $\mathrm{pH}$ (std. units) & NA & NA & $\mathrm{NA}$ & NA & 0 \\
\hline TSS (mg/L) & NA & $\mathrm{NA}$ & NA & NA & 0 \\
\hline \multicolumn{6}{|c|}{ NPDES 006} \\
\hline pH (std. units) & 9.20 & 9.80 & 9.54 & NA & 14 \\
\hline $\mathrm{TSS}(\mathrm{mg} / \mathrm{L})$ & 6.40 & 41.0 & 13.34 & 9.56 & 13 \\
\hline Flow (Mgd) & 0.00 & 0.03 & 0.00383 & 0.01 & 12 \\
\hline \multicolumn{6}{|c|}{ NPDES 007} \\
\hline p니 (std units) & 8.00 & 0.60 & 0.20 & NA & 35 \\
\hline $\operatorname{TSS}\left(\mathrm{mE}_{\mathrm{E}} / \mathrm{L}\right)$ & 1.00 & 7.20 & 3.05 & 1.18 & 35 \\
\hline Flow (Mgd) & 0.00 & 0.04 & 0.004 & 0.01 & 12 \\
\hline
\end{tabular}


Table 2.2.1 (continued)

\begin{tabular}{|c|c|c|c|c|c|}
\hline Parameters & Min & $\operatorname{Max}$ & Av & $\begin{array}{c}\text { Std. } \\
\text { deviation }\end{array}$ & $\begin{array}{l}\text { No. of } \\
\text { samples }\end{array}$ \\
\hline \multicolumn{6}{|c|}{ NPDES 008} \\
\hline $\begin{array}{l}\text { pH (std. units) } \\
\text { TSS (mg/L) } \\
\text { Flow (Mgd) }\end{array}$ & $\begin{array}{l}7.40 \\
b \\
0.00\end{array}$ & $\begin{array}{c}10.40 \\
230.0 \\
0.02\end{array}$ & $\begin{array}{c}9.38 \\
47.62 \\
0.002\end{array}$ & $\begin{array}{r}\text { NA } \\
83.38 \\
0.01\end{array}$ & $\begin{array}{r}10 \\
9 \\
12\end{array}$ \\
\hline \multicolumn{6}{|c|}{ NPDES 009} \\
\hline $\begin{array}{l}\text { pH (std. units) } \\
\text { TSS (mg/L) } \\
\text { Oil (mg/L) } \\
\text { Chlorine (mg/L) } \\
\text { Flow (Mgd) } \\
\text { Fluoride (mg/L) }\end{array}$ & $\begin{array}{l}6.80 \\
b \\
b \\
b \\
0.24 \\
0.20\end{array}$ & $\begin{array}{c}8.90 \\
300.0 \\
b \\
0.02 \\
0.61 \\
0.50\end{array}$ & $\begin{array}{l}7.86 \\
28.43 \\
b \\
0.011 \\
0.37 \\
0.38\end{array}$ & $\begin{array}{l}\text { NA } \\
45.00 \\
0.0 \\
0.00471 \\
0.11 \\
0.07\end{array}$ & $\begin{array}{l}52 \\
52 \\
52 \\
52 \\
11 \\
13\end{array}$ \\
\hline \multicolumn{6}{|c|}{ NPDES 010} \\
\hline $\begin{array}{l}\text { pH (std. units) } \\
\text { Oil (mg/L) } \\
\text { Flow (Mgd) }\end{array}$ & $\begin{array}{l}7.30 \\
b \\
0.09\end{array}$ & $\begin{array}{l}9.20 \\
b \\
0.27\end{array}$ & $\begin{array}{l}7.95 \\
b \\
0.20\end{array}$ & $\begin{array}{l}\text { NA } \\
0.0 \\
0.06\end{array}$ & $\begin{array}{l}52 \\
52 \\
12\end{array}$ \\
\hline \multicolumn{6}{|c|}{ NPDES 011} \\
\hline $\begin{array}{l}\text { pH (std. units) } \\
\text { Oil (mg/L) } \\
\text { Flow (Mgd) }\end{array}$ & $\begin{array}{l}6.80 \\
b \\
0.01\end{array}$ & $\begin{array}{l}9.50 \\
b \\
0.09\end{array}$ & $\begin{array}{l}7.93 \\
b \\
0.05\end{array}$ & $\begin{array}{l}\text { NA } \\
0.0 \\
0.02\end{array}$ & $\begin{array}{l}52 \\
51 \\
11\end{array}$ \\
\hline \multicolumn{6}{|c|}{ GCEP NPDES 001} \\
\hline $\begin{array}{l}\text { pH (std. units) } \\
\text { TSS (mg/L) } \\
\text { Oil (mg/L) } \\
\text { Flow (Mgd) }\end{array}$ & $\begin{array}{l}6.80 \\
1.20 \\
b \\
0.06\end{array}$ & $\begin{array}{c}9.20 \\
450.0 \\
8.50 \\
1.04\end{array}$ & $\begin{array}{r}8.07 \\
29.13 \\
0.27 \\
0.33\end{array}$ & $\begin{array}{r}\mathrm{NA}^{a} \\
64.45 \\
1.40 \\
0.30\end{array}$ & $\begin{array}{r}229 \\
50 \\
50 \\
12\end{array}$ \\
\hline \multicolumn{6}{|c|}{ GCEP NPDES 002} \\
\hline $\begin{array}{l}\text { pH (std. units) } \\
\text { TSS (mg/L) } \\
\text { Oil (mg/L } \\
\text { Flow (Mgd) }\end{array}$ & $\begin{array}{l}7.20 \\
1.20 \\
b \\
0.05\end{array}$ & $\begin{array}{c}9.20 \\
200.0 \\
6.40 \\
1.64\end{array}$ & $\begin{array}{r}8.07 \\
16.79 \\
0.34 \\
0.34\end{array}$ & $\begin{array}{r}\text { NA } \\
28.90 \\
1.38 \\
0.45\end{array}$ & $\begin{array}{r}231 \\
51 \\
51 \\
12\end{array}$ \\
\hline \multicolumn{6}{|c|}{ GCEP NPDES 003} \\
\hline $\begin{array}{l}\text { pH (std. units) } \\
\text { TSS (mg/L) } \\
\text { Oil (mg/L) } \\
\text { Flow (Mgd) }\end{array}$ & $\begin{array}{l}7.60 \\
6.60 \\
b \\
0.00\end{array}$ & $\begin{array}{c}8.40 \\
1,290.0 \\
b \\
0.02\end{array}$ & $\begin{array}{c}8 \\
233.43 \\
b \\
0.01\end{array}$ & $\begin{array}{c}\text { NA } \\
517.68 \\
0.0 \\
0.01\end{array}$ & $\begin{array}{r}14 \\
6 \\
6 \\
12\end{array}$ \\
\hline
\end{tabular}

${ }^{a} \mathrm{NA}=$ not applicable.

${ }^{b}$ Less than detection limit. 
Table 2.2.2. 1989 radiological data summary

\begin{tabular}{|c|c|c|c|c|c|}
\hline \multirow{2}{*}{ Parameter } & \multicolumn{4}{|c|}{ Concentration } & \multirow{2}{*}{$\begin{array}{l}\text { Number of } \\
\text { samples }\end{array}$} \\
\hline & Min & $\operatorname{Max}$ & Av & Standard deviation & \\
\hline \multicolumn{6}{|c|}{ NPDES 001} \\
\hline $\mathrm{U}(\mathrm{mg} / \mathrm{L})$ & 0 & 0.11 & 0.01 & 0.02 & 61 \\
\hline Alpha (pCi/L) & 5 & 675 & 67.67 & 112.99 & 60 \\
\hline${ }^{99} \mathrm{Tc}(\mathrm{pCi} / \mathrm{L})$ & 3 & 536 & 130.92 & 124.47 & 64 \\
\hline Beta $(\mathrm{pCi} / \mathrm{L})$ & -8.75 & 60 & 12.31 & 13.52 & 56 \\
\hline \multicolumn{6}{|c|}{ NPDES $601(001 A)$} \\
\hline $\mathrm{U}(\mathrm{mg} / \mathrm{L})$ & 0.41 & 0.98 & 0.16 & 0.29 & 3 \\
\hline Alpha $(\mathrm{pCi} / \mathrm{L})$ & 640 & 1,360 & 893.33 & 404.64 & 3 \\
\hline${ }^{99} \mathrm{Tc}(\mathrm{pCi} / \mathrm{L})$ & 1,640 & 6,650 & 3,410 & $2,809.92$ & 3 \\
\hline Beta $(\mathrm{pCi} / \mathrm{L})$ & 1 & 126 & 63.50 & 88.39 & 2 \\
\hline \multicolumn{6}{|c|}{ NPDES 605} \\
\hline $\mathrm{U}(\mathrm{mg} / \mathrm{L})$ & 0.12 & 18.40 & 3.14 & 3.91 & 51 \\
\hline Alpha (pCi/L) & 210 & 27,400 & $5,252.80$ & $5,899.85$ & 50 \\
\hline${ }^{99} \mathrm{Tc}(\mathrm{pCi} / \mathrm{L})$ & 544 & 216,057 & $27,892.57$ & $30,397.64$ & 58 \\
\hline Beta $(\mathrm{pCi} / \mathrm{L})$ & 52 & 9,673 & $1,016.08$ & $1,803.84$ & 54 \\
\hline \multicolumn{6}{|c|}{ NPDES 002} \\
\hline $\mathrm{U}(\mathrm{mg} / \mathrm{L})$ & 0 & 0 & 0 & 0 & 61 \\
\hline Alpha $(\mathrm{pCi} / \mathrm{L})$ & 1.40 & 13.05 & 3.85 & 2.27 & 61 \\
\hline Beta, total $(\mathrm{pCi} / \mathrm{L})$ & 1 & 36 & 7.45 & 6.43 & 56 \\
\hline${ }^{99} \mathrm{Tc}(\mathrm{pCi} / \mathrm{L})$ & 0 & 173 & 15.62 & 33.97 & 71 \\
\hline \multicolumn{6}{|c|}{ NPDES 003} \\
\hline$U(\mathrm{mg} / \mathrm{L})$ & 0 & 0.41 & 0.03 & 0.05 & 61 \\
\hline Alpha $(\mathrm{pCi} / \mathrm{L})$ & 17.60 & 778.50 & 182.65 & 148.34 & 61 \\
\hline${ }^{99} \mathrm{Tc}(\mathrm{pCi} / \mathrm{L})$ & 1 & 527 & 185.48 & 119.46 & 67 \\
\hline Beta $(p C i / L)$ & 0 & 106 & 17.90 & 19.15 & 54 \\
\hline \multicolumn{6}{|c|}{ NPDES 004} \\
\hline $\mathrm{U}(\mathrm{mg} / \mathrm{L})$ & 0 & 0 & 0 & 0 & 62 \\
\hline Alpha $(\mathrm{pCi} / \mathrm{L})$ & 0 & 43.20 & 3.65 & 5.77 & 62 \\
\hline Beta, total ( $\mathrm{pCi} / \mathrm{L})$ & 7 & 292 & 79 & 58.49 & 53 \\
\hline${ }^{99} \mathrm{Tc}(\mathrm{pCi} / \mathrm{L})$ & 7 & 718 & 145.06 & 151.04 & 67 \\
\hline \multicolumn{6}{|c|}{ NPDES 009} \\
\hline $\mathrm{U}(\mathrm{mg} / \mathrm{L})$ & 0 & 0.02 & 0.01 & 0 & 61 \\
\hline Alpha $(\mathrm{pCi} / \mathrm{L})$ & 3.60 & 27.50 & 9.02 & 3.87 & 61 \\
\hline Beta, total $(\mathrm{pCi} / \mathrm{L})$ & 1 & 45 & 9.41 & 8.29 & 49 \\
\hline${ }^{99} \mathrm{Tc}(\mathrm{pCi} / \mathrm{L})$ & 0 & 22 & 7.95 & 5.29 & 68 \\
\hline \multicolumn{6}{|c|}{ NPDES 010} \\
\hline$U(m g / L)$ & 0 & 0.03 & $<0.01$ & 0 & 61 \\
\hline Alpha (pCi/L) & 2.30 & 43.70 & 9.71 & 6.61 & 61 \\
\hline Beta, total $(\mathrm{pCi} / \mathrm{L})$ & 0 & 39 & 8.69 & 7.25 & 49 \\
\hline${ }^{99} \mathrm{Tc}(\mathrm{pCi} / \mathrm{L})$ & 0 & 25 & 8.41 & 5.34 & 66 \\
\hline
\end{tabular}


Table 2.2.2 (continued)

\begin{tabular}{|c|c|c|c|c|c|}
\hline \multirow{2}{*}{ Parameter } & \multicolumn{4}{|c|}{ Concentration } & \multirow{2}{*}{$\begin{array}{l}\text { Number of } \\
\text { samples }\end{array}$} \\
\hline & Min & $\operatorname{Max}$ & Av & Standard deviation & \\
\hline \multicolumn{6}{|c|}{ NPDES 011} \\
\hline $\mathrm{U}(\mathrm{mg} / \mathrm{L})$ & 0 & 0.01 & 0 & 0 & 61 \\
\hline Alpha (pCi/L) & 0.20 & 189 & 5.75 & 23.89 & 61 \\
\hline Beta, total $(\mathrm{pCi} / \mathrm{L})$ & 0 & 114 & 9.69 & 16.92 & 46 \\
\hline${ }^{99} \mathrm{Tc}(\mathrm{pCi} / \mathrm{L})$ & 0 & 38 & 8.10 & 7.40 & 68 \\
\hline \multicolumn{6}{|c|}{ GCEP NPDES 001} \\
\hline $\mathrm{U}(\mathrm{mg} / \mathrm{L})$ & 0 & 0 & 0 & 0 & 21 \\
\hline Alpha (pCi/L) & 0.90 & 43.20 & 7.15 & 12.87 & 19 \\
\hline Beta, total $(\mathrm{pCi} / \mathrm{L})$ & 4.00 & 8.00 & 6.12 & 1.25 & 8 \\
\hline${ }^{99} \mathrm{Tc}(\mathrm{pCi} / \mathrm{L})$ & 0 & 14.00 & 7.20 & 4.37 & 10 \\
\hline \multicolumn{6}{|c|}{ GCEP NPDES 002} \\
\hline$U(\mathrm{mg} / \mathrm{L})$ & 0 & 0 & $<0.00$ & 0 & 21 \\
\hline Alpha (pCi/L) & 0.90 & 6.30 & 3.22 & 1.87 & 21 \\
\hline Beta, total $(\mathrm{pCi} / \mathrm{L})$ & 1.00 & 30.00 & 9.62 & 10.51 & 8 \\
\hline${ }^{99} \mathrm{Tc}(\mathrm{pCi} / \mathrm{L})$ & 0 & 10.00 & 6.00 & 3.35 & 9 \\
\hline \multicolumn{6}{|c|}{ GCEP NPDES 003} \\
\hline $\mathrm{U}(\mathrm{mg} / \mathrm{L})$ & 0 & 0 & 0 & 0 & 4 \\
\hline Alpha $(\mathrm{pCi} / \mathrm{L})$ & 2.30 & 13.50 & 5.88 & 5.16 & 4 \\
\hline Beta, total ( $\mathrm{pCi} / \mathrm{L})$ & 5.00 & 18.00 & 8.50 & 6.35 & 4 \\
\hline${ }^{99} \mathrm{Tc}(\overline{\mathrm{CC}} / \mathrm{L})$ & 1.00 & 15.00 & 8.67 & 7.09 & 3 \\
\hline \multicolumn{6}{|c|}{$R W-1$} \\
\hline $\mathrm{U}(\mathrm{mg} / \mathrm{L})$ & 0 & 0 & $<0.00$ & 0 & 50 \\
\hline Alpha $(\mathrm{pCi} / \mathrm{L})$ & 0.50 & 54.00 & 3.86 & 7.41 & 50 \\
\hline Beta, total $(\mathrm{pCi} / \mathrm{L})$ & 0 & 35.00 & 6.50 & 5.77 & 48 \\
\hline${ }^{99} \mathrm{Tc}(\mathrm{pCi} / \mathrm{L})$ & 0 & 17.00 & 5.61 & 4.26 & 64 \\
\hline \multicolumn{6}{|c|}{$R W-2$} \\
\hline$U(\mathrm{mg} / \mathrm{L})$ & 0 & 0 & 0 & 0 & 10 \\
\hline Alpha (pCi/L) & $<0.10$ & 5.90 & $<2.06$ & 1.57 & 10 \\
\hline Beta, total $(\mathrm{pCi} / \mathrm{L})$ & 0 & 20.00 & 7.69 & 6.37 & 13 \\
\hline${ }^{99} \mathrm{Tc}(\mathrm{pCi} / \mathrm{L})$ & 0 & 14.00 & 6.10 & 4.95 & 10 \\
\hline \multicolumn{6}{|c|}{$R W-3$} \\
\hline $\mathrm{U}(\mathrm{mg} / \mathrm{L})$ & $<0.00$ & $<0.01$ & 0.00 & 0 & 11 \\
\hline Alpha $(\mathrm{pCi} / \mathrm{L})$ & 0.50 & 8.60 & 3.60 & 2.38 & 11 \\
\hline Beta, total $(\mathrm{pCi} / \mathrm{L})$ & 2.00 & 43.00 & 10.75 & 11.04 & 12 \\
\hline${ }^{99} \mathrm{Tc}(\mathrm{pCi} / \mathrm{L})$ & 2.00 & 16.00 & 10.82 & 4.47 & 11 \\
\hline \multicolumn{6}{|c|}{$R W-5$} \\
\hline$U(\mathrm{mg} / \mathrm{L})$ & $<0.60^{a}$ & 0.00 & 0 & 0 & 11 \\
\hline Alpha $(\mathrm{pCi} / \mathrm{L})$ & 0.20 & 6.80 & 2.34 & 1.88 & 11 \\
\hline Beta, total $(\mathrm{pCi} / \mathrm{L})$ & 1.00 & 13.00 & 4.91 & 3.59 & 11 \\
\hline${ }^{99} \mathrm{Tc}(\mathrm{pCi} / \mathrm{L})$ & 1.00 & 15.00 & 6.64 & 4.13 & 11 \\
\hline
\end{tabular}


Table 2.2.2 (continued)

\begin{tabular}{|c|c|c|c|c|c|}
\hline \multirow{2}{*}{ Parameter } & \multicolumn{4}{|c|}{ Concentration } & \multirow{2}{*}{$\begin{array}{c}\text { Number of } \\
\text { samples }\end{array}$} \\
\hline & Min & $\operatorname{Max}$ & Av & Standard deviation & \\
\hline \multicolumn{6}{|c|}{$R W-6$} \\
\hline$U(\mathrm{mg} / \mathrm{L})$ & 0 & 0.01 & 0 & 0 & 50 \\
\hline Alpha (pCi/L) & 0.50 & 45.00 & 3.40 & 6.11 & 50 \\
\hline Beta, total ( $\mathrm{pCi} / \mathrm{L})$ & 0 & 51.00 & 6.55 & 7.48 & 47 \\
\hline${ }^{99} \mathrm{Tc}(\mathrm{pCi} / \mathrm{L})$ & 0 & 24.00 & 5.98 & 5.39 & 60 \\
\hline \multicolumn{6}{|c|}{$R W-7$} \\
\hline $\mathrm{U}(\mathrm{mg} / \mathrm{L})$ & 0 & 0.01 & 0.01 & 0 & 11 \\
\hline Alpha (pCi/L) & 1.80 & 8.00 & 4.94 & 1.81 & 11 \\
\hline Beta, total (pCi/L) & 4.00 & 68.00 & 30.70 & 19.20 & 10 \\
\hline${ }^{99} \mathrm{Tc}(\mathrm{pCi} / \mathrm{L})$ & 8.00 & 60.00 & 39.91 & 14.82 & 11 \\
\hline \multicolumn{6}{|c|}{$R W=8$} \\
\hline $\mathrm{U}(\mathrm{mg} / \mathrm{L})$ & 0 & 0.07 & 0.01 & 0.01 & 52 \\
\hline Alpha (pCi/L) & 5.90 & 81.00 & 24.01 & 13.91 & 52 \\
\hline${ }^{99} \mathrm{Tc}(\mathrm{pCi} / \mathrm{L})$ & 12.00 & 160.00 & 49.19 & 22.77 & 59 \\
\hline \multicolumn{6}{|c|}{$R W-12$} \\
\hline $\mathrm{U}(\mathrm{mg} / \mathrm{L})$ & 0 & 0 & 0 & 0 & 16 \\
\hline Alpha (pCi/L) & 0.10 & 1.80 & 0.75 & 0.40 & 16 \\
\hline Beta, total (pCi/L) & 0 & 26.00 & 5.07 & 6.71 & 15 \\
\hline${ }^{99} \mathrm{Tc}(\mathrm{pCi} / \mathrm{L})$ & 2.00 & 14.00 & 6.94 & 3.77 & 16 \\
\hline \multicolumn{6}{|c|}{$R W-13$} \\
\hline$U(\mathrm{mg} / \mathrm{L})$ & 0 & 0.01 & 0 & 0 & 11 \\
\hline Alpha $(p C i / L)$ & 0.40 & 6.50 & 2.34 & 1.98 & 11 \\
\hline Beta, total $(\mathrm{pCi} / \mathrm{L})$ & 3.00 & 55.00 & 23.50 & 17.42 & 8 \\
\hline${ }^{99} \mathrm{Tc}(\mathrm{pCi} / \mathrm{L})$ & 3.00 & 62.00 & 23.90 & 18.65 & 10 \\
\hline \multicolumn{6}{|c|}{$R W \cdot 33$} \\
\hline $\mathrm{U}(\mathrm{mg} / \mathrm{L})$ & 0 & 0 & 0 & 0 & 11 \\
\hline Alpha $(\mathrm{pCi} / \mathrm{L})$ & 0 & 1.40 & $<0.74$ & 0.47 & 11 \\
\hline Beta, total (pCi/L) & 1.00 & 12.00 & 4.67 & 3.63 & 12 \\
\hline${ }^{99} \mathrm{Tc}(\mathrm{pCi} / \mathrm{L})$ & 0 & 14.00 & 6.67 & 4.00 & 9 \\
\hline
\end{tabular}

${ }^{a} \mathrm{~A}<0.00$ number represents a rounding off of a very small measured concentration. 


\subsection{GROUNDWATER}


Table 2.3.1 Groundwater monitoring parameters and standards

\begin{tabular}{|c|c|c|}
\hline Parameter & Standard & Reference \\
\hline $\mathrm{TOC}(\mathrm{mg} / \mathrm{L})$ & None & \\
\hline $\operatorname{TOX}(\mu \mathrm{g} / \mathrm{L})$ & None & \\
\hline Specific conductance $(\mu \mathrm{mhos} / \mathrm{cm})$ & None & \\
\hline pH (standard units) & $6.5-8.5$ & 40 CFR 143.3 \\
\hline $\mathrm{Cl}(\mathrm{mg} / \mathrm{L})$ & $250 \mathrm{mg} / \mathrm{L}$ & 40 CFR 143.3 \\
\hline $\mathrm{Fe}(\mathrm{mg} / \mathrm{L})$ & $0.3 \mathrm{mg} / \mathrm{L}$ & 40 CFR 143.3 \\
\hline $\mathrm{Mn}(\mathrm{mg} / \mathrm{L})$ & $0.05 \mathrm{mg} / \mathrm{L}$ & 40 CFR 143.3 \\
\hline $\mathrm{Na}(\mathrm{mg} / \mathrm{L})$ & None & 40 CFR 143.3 \\
\hline $\mathrm{SO}_{4}(\mathrm{mg} / \mathrm{L})$ & $250 \mathrm{mg} / \mathrm{L}$ & 40 CFR 143.3 \\
\hline $\mathrm{Ba}(\mathrm{mg} / \mathrm{L})$ & $1.0 \mathrm{mg} / \mathrm{L}$ & 40 CFR 141.11 \\
\hline $\mathrm{Cd}(\mathrm{mg} / \mathrm{L})$ & $0.010 \mathrm{mg} / \mathrm{L}$ & 40 CFR 141.11 \\
\hline $\mathrm{Cr}(\mathrm{mg} / \mathrm{L})$ & $0.05 \mathrm{mg} / \mathrm{L}$ & 40 CFR 141.11 \\
\hline $\mathrm{Pb}(\mathrm{mg} / \mathrm{L})$ & $0.05 \mathrm{mg} / \mathrm{L}$ & 40 CFR 141.11 \\
\hline$\alpha(\mathrm{pCi} / \mathrm{L})$ & $15 \mathrm{pCi} / \mathrm{L}$ & 40 CFR 141.15 \\
\hline$\beta(\mathrm{pCi} / \mathrm{L})$ & $\begin{array}{l}4 \mathrm{mrem} / \text { year } \\
(\sim 1000 \mathrm{pCi} / \mathrm{L})\end{array}$ & 40 CFR 141.16 \\
\hline $\mathrm{Tc}(\mathrm{pCi} / \mathrm{L})$ & $900 \mathrm{pCi} / \mathrm{L}$ & EPA-570/9-76-003 \\
\hline $\mathrm{Ra}(\mathrm{pCi} / \mathrm{L})$ & $5 \mathrm{pCi} / \mathrm{L}$ & 40 CFR 141.15 \\
\hline Trichlorsethene $(\mu \mathrm{g} / \mathrm{L})$ & $5.0 \mu \mathrm{g} / \mathrm{L}$ & 40 CFR 141.62 \\
\hline $1,1,1$-Trichloroethane $(\mu \mathrm{g} / \mathrm{L})$ & $5.0 \mu \mathrm{g} / \mathrm{L}$ & 40 CFR 141.62 \\
\hline 1,2-Dichloroethane $(\mu \mathrm{g} / \mathrm{L})$ & $5.0 \mu \mathrm{g} / \mathrm{L}$ & 40 CFR 141.62 \\
\hline 1,1-Dichloroethane $(\mu \mathrm{g} / \mathrm{L})$ & $7.0 \mu \mathrm{g} / \mathrm{L}$ & 40 CFR 141.62 \\
\hline
\end{tabular}

Table 2.3.2. X-701B indicator parameters

\begin{tabular}{lcccc}
\hline & $\begin{array}{c}\text { TOX } \\
(\mu \mathrm{g} / \mathrm{L})\end{array}$ & $\begin{array}{c}\text { TOC } \\
(\mathrm{mg} / \mathrm{L})\end{array}$ & $\begin{array}{c}\mathrm{pH} \\
(\text { standard units })\end{array}$ & $\begin{array}{c}\text { Specific conductance } \\
(\mu \mathrm{mhos} / \mathrm{cm})\end{array}$ \\
\hline Av & 24.262 & 2.90 & 6.0 & 368 \\
Max & 31.830 & 4.90 & 6.6 & 486 \\
Min & 17.550 & 1.95 & 5.5 & 160 \\
& \multicolumn{5}{c}{ Upgradient } \\
Av & $82,505.211$ & 67.90 & 5.6 & 539 \\
Max & $199,700.0$ & 198.70 & 6.9 & 758 \\
Min & 3.180 & 0.51 & 4.8 & 172 \\
& \multicolumn{5}{c}{ Downgradient } \\
Av & 2.071 & 0.12 & \\
Max & 3.280 & 0.24 & \\
Min & 0.350 & & \\
\hline
\end{tabular}


Table 2.3.3. X-701 nolding pond parameters

\begin{tabular}{|c|c|c|c|c|c|c|c|}
\hline Filtration ${ }^{a}$ & Gradient & Parameter & Max & Min & Av & Standard & Count $t^{b}$ \\
\hline \multicolumn{8}{|c|}{ Water quality $(\mathrm{mg} / \mathrm{L})$} \\
\hline $\mathrm{F}$ & Down & Iron & $<0.050$ & 0.11 & $<0.055$ & 0.014 & 28 \\
\hline $\mathrm{F}$ & Down & Manganese & $<0.030$ & 0.56 & $<0.18$ & 0.14 & 28 \\
\hline $\mathrm{F}$ & Up & Iron & $<0.050$ & 0.070 & $<0.055$ & 0.010 & 4 \\
\hline $\mathrm{F}$ & $U_{p}$ & Manganese & $<0.030$ & 0.030 & $<0.030$ & 0 & 4 \\
\hline$U$ & Down & Iron & 0 & 840 & 63 & 170 & 28 \\
\hline $\mathrm{U}$ & Down & Manganese & $<0.030$ & 2.8 & $<0.521$ & 0.65 & 28 \\
\hline $\mathrm{U}$ & Down & $\mathrm{SO}_{4}$ & $<1$ & 260 & $<120$ & 95 & 25 \\
\hline $\mathrm{U}$ & Down & Chlorine & $<2$ & 42.5 & $<23$ & 16 & 22 \\
\hline$U$ & Up & Iron & $<0.050$ & 2 & $<0.8$ & 0.9 & 4 \\
\hline $\mathrm{U}$ & $U_{p}$ & Manganese & $<0.030$ & 0.050 & $<0.038$ & 0.010 & 4 \\
\hline U & Up & $\mathrm{SO}_{4}$ & 29 & 120 & 72 & 39 & 4 \\
\hline U & Up & Chlorine & 9 & 43 & 22 & 16 & 4 \\
\hline \multicolumn{8}{|c|}{ Drinking water $(m g / L)$} \\
\hline $\mathrm{F}$ & Down & Cadmium & $<0.005$ & 0.005 & $<0.0050$ & 0 & 28 \\
\hline $\mathrm{F}$ & Up & Cadmium & $<0.005$ & $<0.050$ & $<0.0500$ & 0 & 4 \\
\hline U & Down & Cadmium & $<0.005$ & 0.050 & $<0.014$ & 0.02 & 28 \\
\hline $\mathrm{U}$ & Up & Cadmium & $<0.005$ & 0.006 & $<0.005$ & 0.001 & 4 \\
\hline \multicolumn{8}{|c|}{ Radioactivity $(p C i / L)$} \\
\hline $\mathrm{F}$ & Down & Uranium & $<0.001$ & $<0.003$ & $<0.001$ & 0.001 & 19 \\
\hline $\mathrm{F}$ & Up & Uranium & $<0.00$ & 0.001 & $<0.001$ & 0 & 3 \\
\hline U & Down & Uranium & $<0.001$ & 0.060 & $<0.009$ & 0.01 & 44 \\
\hline $\mathrm{U}$ & Down & Alpha & -5 & 320 & 32 & 52 & 45 \\
\hline $\mathrm{U}$ & Down & Beta & -9 & 1078 & 123.518 & 230 & 45 \\
\hline$U$ & Down & Tc & -7 & 1490 & 164.296 & 340 & 44 \\
\hline$U$ & Up & Uranium & $<0.001$ & 0.001 & $<0.001$ & 0 & 4 \\
\hline U & Up & Alpha & -2 & 12 & 3.4 & 6 & 5 \\
\hline$U$ & Up & Beta & -8 & 15 & 5 & 9 & 5 \\
\hline$U$ & Up & Tc & -2 & 18 & 9.2 & 8 & 5 \\
\hline
\end{tabular}

${ }^{a} \mathrm{~F}$-filtered; U-unfiltered.

${ }^{b}$ Count-the number of samples. 
Table 2.3.4. Well field X-701B halogenated volatile organics data [ppb ( $\mu \mathrm{g} / \mathrm{L})$ ]

\begin{tabular}{|c|c|c|c|}
\hline Chemical name & $\operatorname{Max}$ & Min & Av \\
\hline \multicolumn{4}{|c|}{ Upgradient wells (6 samples) } \\
\hline Methylene chloride & 0 & 0 & 0 \\
\hline 1,1-Dichlorocthene & 0 & 0 & 0 \\
\hline Trans-1,2-dichloroethene & 0 & 0 & 0 \\
\hline 1,1-Dichloroethane & 0 & 0 & 0 \\
\hline 1,2-Dichloroethane & 0 & 0 & 0 \\
\hline Chloroform & 0 & 0 & 0 \\
\hline Trichlorofluoromeihane & 0 & 0 & 0 \\
\hline Freon-113 & 0 & 0 & 0 \\
\hline 1,1,1-Trichlorocthane & 0 & 0 & 0 \\
\hline Trichloroethene & 61.0 & 0 & 13.0 \\
\hline \multicolumn{4}{|c|}{ Downgradient wells (24 samples) } \\
\hline Methylene chloride & 0 & 0 & 0 \\
\hline 1,1-Dichloroethene & 0 & 0 & 0 \\
\hline Trans-1,2-dichloroethene & 0 & 0 & 0 \\
\hline 1,1-Dichloroethane & 0 & 0 & 0 \\
\hline 1,2-Dichloroethane & 0 & 0 & 0 \\
\hline Chloroform & 0 & 0 & 0 \\
\hline Trichlorofluoromethane & 0 & 0 & 0 \\
\hline Freon-113 & 0 & 0 & 0 \\
\hline 1,1,1-Trichloroethane & 0 & 0 & 0 \\
\hline Trichloroethene & $356,000.0$ & 0 & $104,384.79$ \\
\hline \multicolumn{4}{|c|}{ Sample blanks (6 samples) } \\
\hline Methylene chloride & 0 & 0 & 0 \\
\hline 1,1-Dichloroethene & 0 & 0 & 0 \\
\hline Trans-1,2-dichloroethene & 0 & 0 & 0 \\
\hline 1,1-Dichloroethane & 0 & 0 & 0 \\
\hline 1,2-Dichloroethane & 0 & 0 & 0 \\
\hline Chloroform & 8.0 & 0 & 1.3 \\
\hline Trichlorofluoromethane & 0 & 0 & 0 \\
\hline Freon-113 & 0 & 0 & 0 \\
\hline 1,1,1-Trichloroethane & 0 & 0 & 0 \\
\hline Trichloroethene & 0 & 0 & 0.3 \\
\hline
\end{tabular}


Table 2.3.5. X-616 indicator parameters

\begin{tabular}{|c|c|c|c|c|}
\hline & $\begin{array}{c}\text { TOX } \\
(\mu \mathrm{g} / \mathrm{L})\end{array}$ & $\begin{array}{c}\text { TOC } \\
(\mathrm{mg} / \mathrm{L})\end{array}$ & $\begin{array}{c}\mathrm{pH} \\
\text { (standard units) }\end{array}$ & $\begin{array}{l}\text { Specific conductance } \\
(\mu \mathrm{mhos})\end{array}$ \\
\hline \multicolumn{5}{|c|}{ Upgradient } \\
\hline Av & 10.3 & 1.2 & $\mathrm{NA}^{a}$ & 2100 \\
\hline Max & 13 & 1.3 & 7.3 & 3600 \\
\hline Min & 8.3 & 1.0 & 5.4 & 1500 \\
\hline \multicolumn{5}{|c|}{ Downgradient } \\
\hline Av & 57.0 & 3.4 & 6.3 & 2200 \\
\hline Max & 101.4 & 4.6 & 7.1 & 3500 \\
\hline Min & 41.0 & 2.6 & 5.3 & 100 \\
\hline \multicolumn{5}{|c|}{ Blanks } \\
\hline Av & 5.7 & 0.60 & & \\
\hline Max & 17.2 & 1.9 & & \\
\hline Min & 1.5 & 0 & & \\
\hline
\end{tabular}


Table 2.3.6. $X-616$ chromium sludge surface impoundment parameters

\begin{tabular}{|c|c|c|c|c|c|c|c|}
\hline Filtration ${ }^{a}$ & Gradient & Parameter & $\operatorname{Max}$ & Min & Av & Standard & Count \\
\hline \multicolumn{8}{|c|}{ Water quality $(\mathrm{mg} / \mathrm{L})$} \\
\hline $\mathrm{F}$ & Down & Iron & $<0.050$ & 0.130 & $<0.059$ & 0.022 & 23 \\
\hline $\mathbf{F}$ & Down & Manganese & $<0.030$ & 0.090 & $<0.035$ & 0.014 & 23 \\
\hline U & Down & Iron & $<0.050$ & 2.490 & $<0.549$ & 0.649 & 23 \\
\hline U & Down & Manganese & $<0.030$ & 0.070 & $<0.033$ & 0.009 & 23 \\
\hline U & Down & $\mathrm{SO}_{4}$ & $<1$ & 1651.300 & $<986.736$ & 585.575 & 22 \\
\hline $\mathbf{U}$ & Down & Chlorine & $<2$ & 655 & $<304.476$ & 208.497 & 41 \\
\hline \multicolumn{8}{|c|}{ Drinking water $(\mathrm{mg} / \mathrm{L})$} \\
\hline $\mathbf{F}$ & Down & Barium & $<0.200$ & $<0.200$ & $<0.200$ & 0 & 17 \\
\hline $\mathrm{F}$ & Down & Cadmium & $<0.005$ & 0.014 & $<0.008$ & 0.003 & 23 \\
\hline$F$ & Down & Chromium & $<0.030$ & $<0,030$ & $<0.030$ & 0 & 17 \\
\hline $\mathbf{F}$ & Down & Arsenic & $<0.010$ & $<0.020$ & $<0.017$ & 0.005 & 15 \\
\hline $\mathbf{F}$ & Down & Selenium & $<0.020$ & $<0.020$ & $<0.020$ & 0 & 14 \\
\hline$F$ & Down & Mercury & $<0.001$ & $<0.001$ & $<0.001$ & 0 & 15 \\
\hline $\mathrm{U}$ & Down & Barium & $<0.200$ & $<0.200$ & $<0.200$ & 0 & 17 \\
\hline $\mathrm{U}$ & Down & Cadmium & $<0.005$ & 0.012 & $<0.008$ & 0.003 & 23 \\
\hline$U$ & Down & Chromium & $<0.030$ & 0.280 & $<0.046$ & 0.061 & 17 \\
\hline U & Down & Arsenic & $<0.010$ & $<0.020$ & $<0.017$ & 0.005 & 17 \\
\hline U & Down & Selenium & $<0.020$ & $<0.020$ & $<0.020$ & 0 & 17 \\
\hline $\mathbf{U}$ & Down & Mercury & $<0.001$ & $<0.001$ & $<0.001$ & 0 & 17 \\
\hline \multicolumn{8}{|c|}{ Radioactivity $(p C l / L)$} \\
\hline $\mathrm{F}$ & Down & Uranium & $<0.001$ & $<0.002$ & $<0.001$ & 0.001 & 18 \\
\hline U & Down & Uranium & $<0.001$ & 0.020 & $<0.002$ & 0.004 & 26 \\
\hline U & Down & Alpha & -19 & 49 & 1.923 & 16.606 & 26 \\
\hline$U$ & Down & Beta & -15 & 43 & 4.423 & 13.648 & 26 \\
\hline $\mathrm{U}$ & Down & Technetium & -11 & 59 & 9.769 & 14.917 & 26 \\
\hline
\end{tabular}

${ }^{a} \mathrm{~F}$-filtered; U-unfiltered. 
Table 2.3.7. X-749 indicator parameters

\begin{tabular}{|c|c|c|c|c|}
\hline & $\begin{array}{c}\mathrm{TOX} \\
(\mu \mathrm{g} / \mathrm{L})\end{array}$ & $\begin{array}{c}\mathrm{TOC} \\
(\mathrm{mg} / \mathrm{L})\end{array}$ & $\begin{array}{c}\mathrm{pH} \\
\text { (standard units) }\end{array}$ & $\begin{array}{c}\text { Specific conductance } \\
(\mu \mathrm{mhos} / \mathrm{cm})\end{array}$ \\
\hline \multicolumn{5}{|c|}{ Upgradient } \\
\hline Av & & & 6.0 & 300 \\
\hline Max & & & 6.8 & 330 \\
\hline Min & & & 5.5 & 280 \\
\hline \multicolumn{5}{|c|}{ Downgradient } \\
\hline Av & 2500 & 2.2 & 5.9 & 400 \\
\hline Max & 5500 & 5.8 & 6.8 & 460 \\
\hline Min & 680 & 1.2 & 5.5 & 340 \\
\hline \multicolumn{5}{|c|}{ Blanks } \\
\hline Av & 13 & 0.44 & & \\
\hline Max & 43 & 1.0 & & \\
\hline Min & 0.36 & 0.13 & & \\
\hline
\end{tabular}


Table 2.3.8. X-749 low-level-waste landrill parameters

\begin{tabular}{|c|c|c|c|c|c|c|c|}
\hline Filtration ${ }^{a}$ & Gradient & Parameter & $\operatorname{Max}$ & Min & Av & Standard & Count \\
\hline \multicolumn{8}{|c|}{ Water quality $(\mathrm{mg} / L)$} \\
\hline$F$ & Down & Iron & $<0.050$ & $<0.500$ & $<0.069$ & 0.090 & 25 \\
\hline $\mathrm{F}$ & Down & Manganese & $<0.030$ & 0.090 & $<0.035$ & 0.013 & 25 \\
\hline $\mathrm{F}$ & Up & Iron & $<0.050$ & $<0.050$ & $<0.050$ & 0 & 4 \\
\hline $\mathrm{F}$ & Up & Manganese & $<0.030$ & $<0.030$ & $<0.030$ & 0 & 4 \\
\hline $\mathrm{U}$ & Down & Iron & $<0.050$ & 39 & $<3.039$ & 7.476 & 27 \\
\hline $\mathrm{U}$ & Down & Manganese & $<0.030$ & 0.200 & $<0.043$ & 0.035 & 27 \\
\hline $\mathrm{U}$ & Down & $\mathrm{SO}_{4}$ & $<1$ & 25.800 & $<14.552$ & 8.431 & 23 \\
\hline $\mathrm{U}$ & Down & Chlorine & $<2$ & 101 & $<58.064$ & 31.568 & 28 \\
\hline $\mathrm{U}$ & Up & Iron & $<0.050$ & 2.500 & $<0.740$ & 1.182 & 4 \\
\hline$U$ & $U_{p}$ & Manganese & $<0.030$ & $<0.030$ & $<0.030$ & 0 & 4 \\
\hline $\mathrm{U}$ & Up & $\mathrm{SO}_{4}$ & 19.100 & 23.500 & 21.267 & 2.201 & 3 \\
\hline $\mathrm{U}$ & $U_{p}$ & Chlorine & 41.500 & 44 & 43.167 & 1.443 & 3 \\
\hline \multicolumn{8}{|c|}{ Drinking water $(\mathrm{mg} / L)$} \\
\hline $\mathrm{F}$ & Down & Cadmium & $<0.005$ & $<0.005$ & $<0.005$ & 0 & 25 \\
\hline $\mathrm{F}$ & Up & Cadmium & $<0.005$ & 0.005 & $<0.005$ & 0 & 4 \\
\hline $\mathrm{U}$ & Down & Cadmium & $<0.005$ & 0.006 & $<0.035$ & 0 & 27 \\
\hline U & Up & Cadmium & $<0.005$ & $<0.005$ & $<0.005$ & 0 & 4 \\
\hline \multicolumn{8}{|c|}{ Radioactivity $(p C i / L)$} \\
\hline $\mathrm{F}$ & Down & Uranium & $<0.001$ & $<0.001$ & $<0.0010$ & 0 & 22 \\
\hline $\mathrm{F}$ & Up & Uranium & $<0.001$ & $<0.001$ & $<0.0010$ & 0 & 4 \\
\hline $\mathrm{U}$ & Down & Uranium & $<0.001$ & 0.081 & $<0.0116$ & 0.019 & 40 \\
\hline $\mathrm{U}$ & Down & Alpha & -4 & 116 & 20.9512 & 30.835 & 41 \\
\hline $\mathrm{U}$ & Down & Beta & -10 & 450 & 81.9512 & 107.076 & 41 \\
\hline $\mathrm{U}$ & Down & Technetium & -3 & 625 & 108.6585 & 148.576 & 41 \\
\hline U & Up & Uranium & $<0.001$ & 0.015 & $<0.0045$ & 0.007 & 4 \\
\hline $\mathrm{U}$ & Up & Alpha & -2 & 14 & 3.6000 & 6.580 & 5 \\
\hline U & Up & Beta & -10 & 23 & 8.2000 & 14.721 & 5 \\
\hline $\mathrm{U}$ & Up & Technetium & 5 & 28 & 14.2000 & 8.871 & 5 \\
\hline
\end{tabular}

${ }^{a} \mathrm{~F}$-filtered; U-unfiltered. 
Table 2.3.9. Well neld X-749 halogenated volatile organics data Ippb $(\mu \mathrm{g} / \mathrm{L}) \boldsymbol{H}^{\mu}$

\begin{tabular}{lccr}
\hline \multicolumn{1}{c}{ Chemical name } & Max & Min & Av \\
\hline & Downgradient wells (33 samples) & \\
Methylene chloride & 56.0 & 0 & 4.3 \\
1,1-Dichloroethene & 2910.0 & 0 & 647.8 \\
Trans-1,2-dichloroethene & 16.0 & 0 & 0.7 \\
1,1-Dichloroethane & 920.0 & 0 & 348.6 \\
1,2-Dichloroethane & 247.0 & 0 & 68.7 \\
Chloroform & 253.0 & 0 & 49.5 \\
Trichlorofluoromethane & 71.0 & 0 & 8.1 \\
Freon-113 & 409.0 & 0 & 116.5 \\
1,1,1-Trichloroethane & 7120.0 & 153.0 & 1435.3 \\
Trichloroethene & 2880.0 & 50.0 & 924.8 \\
& & & \\
& 0 & & \\
Methylene chloride & 0 & 0 & 0 \\
1,1-Dichloroethene & 0 & 0 & 0 \\
Trans-1,2-dichloroethene & 0 & 0 & 0 \\
1,1-Dichloroethane & 0 & 0 & 0 \\
1,2-Dichloroethane & 0 & 0 & 0 \\
Chloroform & 0 & 0 & 0 \\
Trichlorofluoromethane & 0 & 0 & 0 \\
Freon-113 & 0 & 0 & 0 \\
1,1,1-Trichloroethane & 0 & 0 & 0 \\
Trichloroethene & 0 & samples) & \\
\hline
\end{tabular}

aNo upgradient wells sampled.

Table 2.3.10. X-231B indicator parameters"

\begin{tabular}{lcccc}
\hline & $\begin{array}{c}\text { TOX } \\
(\mu \mathrm{g} / \mathrm{L})\end{array}$ & $\begin{array}{c}\text { TOC } \\
(\mathrm{mg} / \mathrm{L})\end{array}$ & $\begin{array}{c}\mathrm{pH} \\
(\text { standard units })\end{array}$ & $\begin{array}{c}\text { Specific conductance } \\
(\mu \mathrm{mhos} / \mathrm{cm})\end{array}$ \\
\hline \multicolumn{4}{c}{ Downgradient } \\
Av & 250.460 & 1.56 & 5.3 & 1425 \\
Max & 250.460 & 1.56 & 6.5 & 1972 \\
Min & 250.460 & 1.56 & 4.9 & 512 \\
\hline
\end{tabular}

${ }^{a}$ No upgradient wells sampled. 
Table 2.3.11. X-231 oil biodegration plot parameters

\begin{tabular}{|c|c|c|c|c|c|c|}
\hline Filtration $^{a}$ & Gradient & Parameter & $\operatorname{Max}$ & Min & Av & Count \\
\hline \multicolumn{7}{|c|}{ Water quality $(\mathrm{mg} / L)$} \\
\hline $\mathbf{F}$ & Down & Iron & $<0.050$ & 0.22 & 0.22 & 27 \\
\hline $\mathrm{F}$ & Down & Manganese & $<0.030$ & 0.58 & 0.04 & 27 \\
\hline $\mathrm{U}$ & Down & Iron & $<0.050$ & 1.50 & $<0.25$ & 26 \\
\hline$U$ & Down & Manganese & $<0.030$ & 0.57 & $<0.11$ & 26 \\
\hline$U$ & Down & $\mathrm{SO}_{4}$ & 4.1 & 1100 & 630 & 27 \\
\hline $\mathrm{U}$ & Down & Chlorine & $<2$ & 143 & 143 & 31 \\
\hline \multicolumn{7}{|c|}{ Drinking water $(m g / L)$} \\
\hline $\mathrm{F}$ & Down & Cadmium & $<0.005$ & 0.008 & $<0.005$ & 27 \\
\hline $\mathrm{U}$ & Down & Cadmium & $<0.005$ & 0.010 & $<0.006$ & 26 \\
\hline \multicolumn{7}{|c|}{ Radioactivity $(p C i / L)$} \\
\hline $\mathrm{F}$ & Down & Uranium & $<0.001$ & $<0.001$ & $<0.001$ & 27 \\
\hline $\mathbf{F}$ & Down & Alpha & 100 & 100 & 100 & 1 \\
\hline$F$ & Down & Beta & 64 & 64 & 64 & 1 \\
\hline$F$ & Down & Technetium & 4 & 4 & 4 & 1 \\
\hline $\mathrm{U}$ & Down & Uranium & $<0.001$ & 0.073 & 0.005 & 38 \\
\hline $\mathrm{U}$ & Down & Alpha & -39 & 59 & 4 & 36 \\
\hline $\mathrm{U}$ & Down & Beta & -22 & 54 & 4 & 36 \\
\hline $\mathrm{U}$ & Down & Technetium & -6 & 98 & 14 & 36 \\
\hline
\end{tabular}

${ }^{\circ} \mathrm{F}$-filtered; U-unfiltered. 
Table 2.3.12. Well field X-231B halogenated volatile organics data lppb $(\mu \mathrm{g} / \mathrm{L}) \mathrm{P}^{\circ}$

\begin{tabular}{lccc}
\hline \multicolumn{1}{c}{ Chemical name } & Max & Min & Av \\
\hline & Downgradient wells & (22 samples) & \\
Methylene chloride & 8.0 & 0 & 0.9 \\
1,1-Dichloroethene & 48.0 & 0 & 12.6 \\
Trans-1,2-dichloroethene & 8.0 & 0 & 0.3 \\
1,1-Dichloroethane & 1.0 & 0 & 0 \\
1,2-Dichloroethane & 0 & 0 & 0 \\
Chloroform & 5.0 & 0 & 0.5 \\
Trichlorofluoromethane & 0 & 0 & 0 \\
Freon-113 & 0 & 0 & 0 \\
1,1,1-Trichloroethane & 143.0 & 0 & 34.8 \\
Trichloroethene & 298.0 & 2.0 & 76.0 \\
& & & \\
& & 0 & \\
Methylene chloride & 0 & 0 & 0 \\
1,1-Dichloroethene & 0 & 0 & 0 \\
Trans-1,2-dichloroethene & 0 & 0 & 0 \\
1,1-Dichloroethane & 0 & 0 & 0 \\
1,2-Dichloroethane & 0 & 0 & 0 \\
Chloroform & 0 & 0 & 0 \\
Trichlorofluoromethane & 0 & 0 & 0 \\
Freon-113 & 0 & 0 & 0 \\
1,1,1-Trichloroethane & 0 & 0 & 0 \\
Trichloroethene & &
\end{tabular}

${ }^{\circ}$ No upgradient wells sampled.

Table 2.3.13. X-735 indicator parameters

\begin{tabular}{|c|c|c|c|c|}
\hline & $\begin{array}{c}\text { TOX } \\
(\mu \mathrm{g} / \mathrm{L})\end{array}$ & $\begin{array}{c}\text { TOC } \\
(\mathrm{mg} / \mathrm{L})\end{array}$ & $\begin{array}{c}\mathrm{pH} \\
\text { (standard units) }\end{array}$ & $\begin{array}{l}\text { Specific conductance } \\
(\mu \mathrm{mhos} / \mathrm{cm})\end{array}$ \\
\hline \multicolumn{5}{|c|}{ Upgradient } \\
\hline $\mathrm{Av}$ & 2.1 & 0.78 & 6.5 & 410 \\
\hline Max & 3.7 & 1.1 & 6.5 & 450 \\
\hline Min & 0.45 & 0.46 & 6.4 & 380 \\
\hline \multicolumn{5}{|c|}{ Downgradient } \\
\hline Av & 4.4 & 1.5 & 6.3 & 570 \\
\hline Max & 9.9 & 4.0 & 6.6 & 2200 \\
\hline Min & 0.36 & 0.42 & 5.1 & 72 \\
\hline \multicolumn{5}{|c|}{ Blanks } \\
\hline$A v$ & 3.6 & 0.15 & & \\
\hline Max & 8.3 & 0.45 & & \\
\hline Min & 0.1 & 0.02 & & \\
\hline
\end{tabular}


Table 2.3.1A, $X-735$ sanitary landfill parameters

\begin{tabular}{|c|c|c|c|c|c|c|c|}
\hline Filtration ${ }^{a}$ & Gradient & Parameter & $\operatorname{Max}$ & Min & Av & Standard & Count \\
\hline \multicolumn{8}{|c|}{ Water quality (mg/L) } \\
\hline $\mathbf{F}$ & Down & Iron & $<0.050$ & 1.780 & $<0.214$ & 0.494 & 12 \\
\hline $\mathrm{F}$ & Down & Manganese & $<0.030$ & 0.230 & $<0.068$ & 0.068 & 12 \\
\hline $\mathrm{F}$ & Up & Iron & $<0.050$ & $<0.050$ & $<0.050$ & 0 & 2 \\
\hline $\mathrm{F}$ & $U_{p}$ & Manganese & $<0.030$ & $<0.030$ & $<0.030$ & 0 & 2 \\
\hline $\mathrm{U}$ & Down & Iron & $<0.050$ & 54 & $<15.507$ & 16.992 & 19 \\
\hline $\mathrm{U}$ & Down & Manganese & $<0.030$ & 1.350 & $<0.281$ & 0.417 & 19 \\
\hline$U$ & Down & $\mathrm{SO}_{4}$ & $<1$ & 402 & $<103.290$ & 124.243 & 31 \\
\hline$U$ & Down & Chlorine & $<2$ & 234 & $<29$ & 65.746 & 31 \\
\hline $\mathrm{U}$ & $\mathrm{Up}_{\mathrm{p}}$ & Iron & 1.520 & 58 & 23.007 & 30.568 & 3 \\
\hline $\mathrm{U}$ & $U_{p}$ & Manganese & $<0.030$ & 0.070 & $<0.043$ & 0.023 & 3 \\
\hline $\mathrm{U}$ & Up & $\mathrm{SO}_{4}$ & 31.100 & 34.700 & 32.400 & 1.998 & 3 \\
\hline $\mathrm{U}$ & $U_{p}$ & Chlorine & $<2$ & 2 & $<2$ & 0 & 3 \\
\hline \multicolumn{8}{|c|}{ Drinking water $(m g / L)$} \\
\hline $\mathrm{F}$ & Down & Barium & $<0.200$ & $<0.200$ & $<0.200$ & 0 & 12 \\
\hline $\mathrm{F}$ & Down & Cadmium & $<0.005$ & $<0.005$ & $<0.005$ & 0 & 12 \\
\hline $\mathbf{F}$ & Down & Chronium & $<0.030$ & $<0.030$ & 0.030 & 0 & 12 \\
\hline F & Down & Arsenic & $<0.010$ & 0.020 & $<0.015$ & 0.005 & 12 \\
\hline $\mathrm{F}$ & Down & Selenium & $<0.020$ & $<0.020$ & $<0.020$ & 0 & 9 \\
\hline $\mathrm{F}$ & Down & Mercury & $<0.001$ & $<0.001$ & $<0.001$ & 0 & 12 \\
\hline $\mathrm{F}$ & Up & Barium & $<0.200$ & $<0.200$ & $<0.200$ & 0 & 2 \\
\hline $\mathrm{F}$ & $U_{p}$ & Cadmium & $<0.005$ & $<0.005$ & $<0.0 \% 5$ & 0 & 2 \\
\hline $\mathrm{F}$ & $U_{p}$ & Chromium & $<0.030$ & $<0.030$ & $<0.030$ & 0 & 2 \\
\hline $\mathrm{F}$ & Up & Arsenic & $<0.010$ & $<0.020$ & $<0.015$ & 0.007 & 2 \\
\hline $\mathrm{F}$ & $U_{p}$ & Selenium & $<0.020$ & $<0.020$ & $<0.020$ & 0 & 2 \\
\hline $\mathrm{F}$ & $U_{p}$ & Mercury & $<0.001$ & $<0.001$ & $<0.001$ & 0 & 2 \\
\hline $\mathrm{U}$ & Down & Barium & $<0.200$ & $<0.200$ & $<0.200$ & 0 & 19 \\
\hline $\mathrm{U}$ & Down & Cadmium & $<0.005$ & 0.008 & $<0.005$ & 0.001 & 19 \\
\hline $\mathrm{U}$ & Down & Chronium & $<0.030$ & 0.080 & $<0.035$ & 0.014 & 19 \\
\hline $\mathrm{U}$ & Down & Arsenic & $<0.010$ & 0.020 & $<0.017$ & 0.005 & 20 \\
\hline $\mathrm{U}$ & Down & Selenium & $<0.020$ & 0.020 & $<0.020$ & 0 & 19 \\
\hline $\mathrm{U}$ & Down & Mercury & $<0.001$ & $<0.001$ & $<0.001$ & 0 & 20 \\
\hline $\mathrm{U}$ & $U_{p}$ & Barium & $<0.200$ & $<0.200$ & $<0.200$ & 0 & 3 \\
\hline $\mathrm{U}$ & $U_{p}$ & Cadmium & $<0.005$ & 0.005 & $<0.005$ & 0 & 3 \\
\hline $\mathrm{U}$ & $U_{p}$ & Chromium & $<0.03 C$ & $<0.030$ & $<0.030$ & 0 & 3 \\
\hline$U$ & $U_{p}$ & Arsenic & $<0.010$ & $<0.02 i$ & $<0.017$ & 0.006 & 3 \\
\hline$U$ & Up & Selenium & $<0.020$ & $<0.020$ & $<0.020$ & 0 & 3 \\
\hline$U$ & $U_{p}$ & Mercury & $<0.001$ & $<0.001$ & $<0.001$ & 0 & 3 \\
\hline
\end{tabular}

${ }^{a} \mathrm{~T}$-filtered; U-unfiltered. 
Table 2.3.15. Well field X-734 halogenated volatile organics data [ppb $(\mu \mathrm{g} / \mathrm{L})]$

\begin{tabular}{|c|c|c|c|}
\hline Chemical name & $\operatorname{Max}$ & Min & Av \\
\hline \multicolumn{4}{|c|}{ Upgradient wells (I sample) } \\
\hline Methylene chloride & 0 & 0 & 0 \\
\hline 1,1-Dichloroethene & 0 & 0 & 0 \\
\hline Trans-1,2-dichloroethene & 0 & 0 & 0 \\
\hline 1,1-Dichloroethane & 0 & 0 & 0 \\
\hline 1,2-Dichloroethane & 0 & 0 & 0 \\
\hline Chloroform & 0 & 0 & 0 \\
\hline T'richlorofluoromethane & 0 & 0 & 0 \\
\hline Freon-113 & 0 & 0 & 0 \\
\hline 1,1,1-Trichloroethane & 0 & 0 & 0 \\
\hline Trichloroethene & 8.0 & 8.0 & 8.0 \\
\hline \multicolumn{4}{|c|}{ Downgradient wells (I sample) } \\
\hline Methylene chloride & 0 & 0 & 0 \\
\hline 1,1-Dichloroethene & 0 & 0 & 0 \\
\hline Trans-1,2-dichloroethene & 0 & 0 & 0 \\
\hline 1,1-Dichloroethane & 0 & 0 & 0 \\
\hline 1,2-Dichloroethane & 0 & 0 & 0 \\
\hline Chloroform & 0 & 0 & 0 \\
\hline Trichlorofluoromethane & 0 & 0 & 0 \\
\hline Freon-113 & 0 & 0 & 0 \\
\hline 1,1,1-Trichloroethane & 0 & 0 & 0 \\
\hline Trichloroethene & 7.0 & $? . .9$ & 7.0 \\
\hline \multicolumn{4}{|c|}{ Sample blanks (3 samples) } \\
\hline Methylene chloride & 0 & 0 & 0 \\
\hline 1,1-Dichloroethene & 0 & 0 & 0 \\
\hline Trans-1,2-dichloroethene & 0 & 0 & 0 \\
\hline 1,1-Dichloroethane & 0 & 0 & 0 \\
\hline 1,2-Dichloroethane & 0 & 0 & 0 \\
\hline Chloroform & 0 & 0 & 0 \\
\hline Trichlorofluoromethane & 0 & 0 & 0 \\
\hline Freon-113 & 0 & 0 & 0 \\
\hline 1,1,1-Trichloroethane & 0 & 0 & 0 \\
\hline Trichloroethene & 0 & 0 & 0 \\
\hline
\end{tabular}


Table 2.3.16. X-734 indicator parameters

\begin{tabular}{|c|c|c|c|c|}
\hline & $\begin{array}{c}\text { TOX } \\
(\mu g / L)\end{array}$ & $\begin{array}{c}\text { TOC } \\
(\mathrm{mg} / \mathrm{L})\end{array}$ & $\begin{array}{c}\mathrm{pH} \\
\text { (standard units) }\end{array}$ & $\begin{array}{c}\text { Specific conductance } \\
(\mu \mathrm{mhos} / \mathrm{cm})\end{array}$ \\
\hline \multicolumn{5}{|c|}{ Upgradient } \\
\hline Av & 17.450 & 1.86 & $\mathrm{NA}^{a}$ & 538 \\
\hline Max & 17.450 & 2.52 & 7.2 & 546 \\
\hline Min & 17.450 & 1.21 & 7.1 & 535 \\
\hline \multicolumn{5}{|c|}{ Downgradient } \\
\hline Av & 84.997 & 5.66 & NA & 939 \\
\hline $\operatorname{Max}$ & 141.440 & 12.89 & 6.2 & 939 \\
\hline Min & 9.750 & 1.30 & 6.2 & 939 \\
\hline \multicolumn{5}{|c|}{ Blanks } \\
\hline Av & 3.653 & 0.15 & & \\
\hline $\operatorname{Max}$ & 8.280 & 0.45 & & \\
\hline Min & 0.150 & 0.02 & & \\
\hline
\end{tabular}


Table 2.3.17. Well field X-735 halogenated volatile organics data [ppb $(\mu \mathrm{g} / \mathrm{L})$ ]

\begin{tabular}{|c|c|c|c|}
\hline Chemical name & Max & Min & Av \\
\hline \multicolumn{4}{|c|}{ Upgradient wells (2 samples) } \\
\hline Methylene chloride & 0 & 0 & 0 \\
\hline 1,1-Dichloroethene & 0 & 0 & 0 \\
\hline Trans-1,2-dichloroethene & 0 & 0 & 0 \\
\hline 1,1-Dichloroethane & 0 & 0 & 0 \\
\hline 1,2-Dichloroethane & 0 & 0 & 0 \\
\hline Chloroform & 0 & 0 & 0 \\
\hline Trichlorofluoromethane & 0 & 0 & $\mathbf{0}$ \\
\hline Freon-113 & 0 & 0 & 0 \\
\hline 1,1,1-Trichloroethane & 0 & 0 & 0 \\
\hline Trichloroethene & 5.0 & 0 & 2.5 \\
\hline \multicolumn{4}{|c|}{ Downgradient wells (5 samples) } \\
\hline Methylene chloride & 0 & 0 & 0 \\
\hline 1,1-Dichloroethene & 1.0 & 0 & 0 \\
\hline Trans-1,2-dichloroethene & 0 & 0 & 0 \\
\hline 1,1-Dichloroethane & 1.0 & 0 & 0 \\
\hline 1,2-Dichloroethane & 0 & 0 & 0 \\
\hline Chloroform & 0 & 0 & 0 \\
\hline Trichlorofluoromethane & 0 & 0 & 0 \\
\hline Freon-113 & 1.0 & 0 & 0 \\
\hline 1,1,1-Trichloroethane & 0 & 0 & 0 \\
\hline Trichloroethene & 21.0 & 0 & 6.8 \\
\hline \multicolumn{4}{|c|}{ Sample blanks (3 samples) } \\
\hline Methylene chloride & 0 & 0 & 0 \\
\hline 1,1-Dichloroethene & 0 & 0 & 0 \\
\hline Trans-1,2-dichloroethene & 0 & 0 & 0 \\
\hline 1,1-Dichloroethane & 0 & 0 & 0 \\
\hline 1,2-Dichloroethane & 0 & 0 & 0 \\
\hline Chloroform & 0 & 0 & 0 \\
\hline Trichlorofluoromethane & 0 & 0 & 0 \\
\hline Freon-113 & 0 & 0 & 0 \\
\hline 1,1,1-Trichloroethane & 0 & 0 & 0 \\
\hline Trichloroethene & 0 & 0 & 0 \\
\hline
\end{tabular}




\subsection{BIOLOGICAL}




\subsection{BIOLOGICAL MONITORING}

Table 2.4.1 shows the individual analytical results of the vegetable, fruit, egg, and milk samples collected in 1989. Sample locations are private farms and residences surrounding the site and are not published.

Tables 2.4.2 and 2.4.3 present the individual analytical results of the fish samples collected in 1989. Sample locations are given as the stream from which the sample was collected and (if off- site) marked as upstream (U) or downstream (D) from PORTS effluents.

Table 2.4.4 presents the analytical results of the 1989 vegetation (grass) sampling. Locations of the individual vegetation samples are the same as the soil-sampling locations shown in Figs. 2.5.2 through 2.5.4 in Sect. 2.5 of Part 1. Sample locations designated as a number plus " $\mathrm{A}$ " are replicate sample locations in the near vicinity of that sample location number and are not shown separately.

Table 2.4.1. 1989 vegetables, fruit, eggs, and milk

\begin{tabular}{lcccc}
\hline \multicolumn{1}{c}{ Sample } & $\begin{array}{c}\text { Date } \\
\text { sampled }\end{array}$ & $\begin{array}{c}\text { Uranium } \\
(\mu \mathrm{g} / \mathrm{g})\end{array}$ & $\begin{array}{c}\text { Alpha } \\
(\mathrm{pCi} / \mathrm{g})\end{array}$ & $\begin{array}{c}\text { Technetium } \\
(\mathrm{pCi} / \mathrm{g})\end{array}$ \\
\hline Tomatoes & $11-06-89$ & $<0.05$ & 0.01 & $<0.45$ \\
Tomatoes & $11-02-89$ & $<0.05$ & 0.05 & $<0.45$ \\
Tomatoes & $11-02-89$ & $<0.05$ & 0.05 & $<0.45$ \\
Tomatoes & $10-24-89$ & $<0.05$ & 0.02 & $<0.45$ \\
Tomatoes & $10-31-89$ & $<0.05$ & 0.02 & $<0.45$ \\
Peppers & $11-02-89$ & $<0.05$ & 0.04 & $<0.45$ \\
Peppers & $11-06-89$ & $<0.05$ & 0.01 & $<0.45$ \\
Sweet potatoes & $11-02-89$ & $<0.05$ & 0.07 & $<0.45$ \\
Pumpkin & $11-06-89$ & $<0.05$ & 0.02 & $<0.45$ \\
Apples & $10-24-89$ & $<0.05$ & 0.01 & $<0.45$ \\
Potatoes & $10-24-89$ & $<0.05$ & 0.02 & $<0.45$ \\
Soybeans & $10-26-89$ & 0.40 & - & $<0.45$ \\
Soybeans & $10-25-89$ & $<0.05$ & - & $<0.45$ \\
Corn & $10-26-89$ & $<0.05$ & - & $<0.45$ \\
Corn & $10-25-89$ & $<0.05$ & - & $<0.45$ \\
Corn & $10-24-89$ & $<0.05$ & - & $<0.45$ \\
Corn & $10-31-89$ & $<0.05$ & - & $<0.45$ \\
Eggs & $10-30-89$ & $<0.05$ & 0.07 & $<0.45$ \\
Milk & $10-30-89$ & $<0.01$ & - & $<0.45$ \\
Milk & $10-30-89$ & $<0.01$ & - & $<0.45$ \\
Milk & $10-31-89$ & $<0.01$ & - & $<0.45$ \\
Milk & $11-07-89$ & $<0.01$ & - & $<0.45$ \\
\hline
\end{tabular}


Table 2.4.2. Radiological analyses of 1989 fish samples

\begin{tabular}{ccrc}
\multicolumn{1}{c}{ Location } & $\begin{array}{c}\text { Uranium } \\
(\mu \mathrm{g} / \mathrm{g})\end{array}$ & $\begin{array}{c}\text { Alpha } \\
(\mathrm{pCi} / \mathrm{g})\end{array}$ & $\begin{array}{c}\text { Technetium } \\
(\mathrm{pCi} / \mathrm{g})\end{array}$ \\
\hline Plant outfalls & & & \\
X-230K (NPDES 002) & & & \\
Receiving streams (immediate) & & & \\
Little Beaver Creek (A) & $<0.25$ & -0.40 & 0.23 \\
Little Beaver Creek (A) & $<0.25$ & -0.30 & 0.03 \\
Big Beaver Creek (U) & $<0.25$ & 0.22 & -0.32 \\
Big Beaver Creek (U) & $<0.25$ & 0.04 & -0.05 \\
Big Beaver Creek (U) & $<0.25$ & 0.27 & -0.05 \\
Big Beaver Crtek (U) & $<0.25$ & -0.27 & -0.05 \\
Big Beaver Creek (D) & $<0.25$ & -0.36 & -0.18 \\
Big Beaver Creek (D) & $<0.25$ & 0.04 & -0.05 \\
Receiving stream (ultimate) & & & \\
Scioto River (U) & $<0.25$ & -0.30 & -0.01 \\
Background & & & \\
Sunfish Creek & $<0.25$ & 0.04 & 0.05 \\
\hline
\end{tabular}

${ }^{\circ} \mathrm{A}$-At first plant outfall.

¿U-Upstream of plant outfalls.

' $\mathcal{L}$-Downstream of plant outfalls.

Table 2.4.3. Nonradiological analyses of 1989 fish samples

\begin{tabular}{lcccr}
\multicolumn{1}{c}{ Location } & $\begin{array}{c}\text { Fluorides } \\
(\mu \mathrm{g} / \mathrm{g})\end{array}$ & $\begin{array}{c}\text { Mercury } \\
(\mu \mathrm{g} / \mathrm{g})\end{array}$ & $\begin{array}{c}\text { Chromium } \\
(\mu \mathrm{g} / \mathrm{g})\end{array}$ & $\begin{array}{c}\text { PCB } \\
(\mu \mathrm{g} / \mathrm{g})\end{array}$ \\
\hline $\begin{array}{l}\text { Plant outfalls } \\
\text { X-230K (NPDES 002) }\end{array}$ & & & & \\
Receiving streams (immediate) & & & - & 1.4 \\
Little Beaver Creek (whole) (D) & - & - & - & $<0.2$ \\
Little Beaver Creek (fillets) (D) & - & & & \\
Little Beaver Creek (A) & & & & \\
Little Beaver Creek (A) & 32.0 & $<0.05$ & $<2.0$ & 2.2 \\
Big Beaver Creek (U) & 7.3 & $<0.05$ & $<2.0$ & 2.1 \\
Big Beaver Creek (U) & 28.0 & $<0.05$ & $<2.0$ & $<0.2$ \\
Big Beaver Creek (U) & 3.4 & $<0.05$ & $<2.0$ & $<0.2$ \\
Big Beaver Creek (U) & 7.4 & $<0.05$ & $<2.0$ & $<0.2$ \\
Big Beaver Creek (D) & & & $<.0$ \\
Big Beaver Creek (D) & 0.6 & $<0.05$ & $<2.0$ & $<0.2$ \\
Receiving stream (ultimate) & 7.4 & $<0.05$ & $<2.0$ & $<0.2$ \\
Scioto River (U) & 18.0 & $<0.05$ & $<2.0$ & 1.4 \\
Background & & & & \\
Sunfish Creek & 25.0 & $<0.05$ & $<2.0$ & 1.1 \\
\hline
\end{tabular}

${ }^{a}$ A-At first plant outfall. Entering stream.

'U-Upstrearn of plant outfalls.

'D-Downstream of plant outfalls. 
Table 2.4.4. 1989 vegetation sampling data

\begin{tabular}{|c|c|c|c|c|c|c|c|c|}
\hline \multirow{2}{*}{ Location } & \multicolumn{2}{|c|}{ Uranium $(\mu \mathrm{g} / \mathrm{g})$} & \multicolumn{2}{|c|}{ Total alpha $(\mathrm{pCi} / \mathrm{g})$} & \multicolumn{2}{|c|}{ Technetium $(\mathrm{pCi} / \mathrm{g})$} & \multicolumn{2}{|c|}{ Fluorides $(\mu \mathrm{g} / \mathrm{g})$} \\
\hline & Spring & Fall & Spring & Fall & Spring & Fall & Spring & Fall \\
\hline \multicolumn{9}{|c|}{ Group I (DOE property line) } \\
\hline 3 & $<0.5$ & $<0.5$ & 0.23 & 0.09 & $<0.45$ & $<0.45$ & 3.6 & 3.0 \\
\hline 8 & $<0.5$ & $<0.5$ & 0.09 & 0.02 & $<0.4 \xi$ & $<0.45$ & 4.8 & 4.6 \\
\hline 9 & $<0.5$ & $<0.5$ & -0.02 & 0.09 & $<0.45$ & $<0.45$ & 3.4 & 2.2 \\
\hline $9 \mathrm{~A}$ & $<0.5$ & $<0.5$ & 0.23 & 0.18 & $<0.45$ & $<0.45$ & 1.5 & 2.8 \\
\hline 10 & $<0.5$ & $<0.5$ & 0.23 & 0.18 & 0.46 & 0.45 & 2.8 & 4.6 \\
\hline $10 \mathrm{~A}$ & $<0.5$ & $<0.5$ & 0.45 & 0.23 & $<0.45$ & $<0.45$ & 2.6 & 3.4 \\
\hline 11 & $<0.5$ & $<0.5$ & 0.41 & 0.02 & 0.80 & 0.45 & 2.8 & 2.2 \\
\hline 12 & $<0.5$ & $<0.5$ & 0.09 & 0.09 & $<0.45$ & 0.90 & 5.6 & 2.8 \\
\hline $12 \mathrm{~A}$ & $<0.5$ & $<0.5$ & 0.09 & 0.32 & $<0.45$ & 0.90 & 2.9 & 3.8 \\
\hline 15 & $<0.5$ & $<0.5$ & -0.09 & 0.18 & $<0.45$ & $<0.45$ & 2.5 & 4.4 \\
\hline $15 \mathrm{~A}$ & $<0.5$ & $<0.5$ & 0.27 & 0.27 & $<0.45$ & 0.45 & 2.5 & 6.4 \\
\hline 23 & $<0.5$ & $<0.5$ & 0.09 & 0 & 0.28 & $<0.45$ & 4.0 & 6.7 \\
\hline 24 & $<0.5$ & $<0.5$ & 0.68 & 0.18 & 0.27 & $<0.45$ & 5.9 & 5.9 \\
\hline \multicolumn{9}{|c|}{ Group II (up to $5 \mathrm{~km}$ off-site) } \\
\hline 13 & $<0.5$ & $<0.5$ & 0.23 & 0.09 & 0.39 & $<0.45$ & 1.8 & 4.0 \\
\hline 14 & $<0.5$ & $<0.5$ & 0.32 & 0.09 & 0.38 & $<0.45$ & 1.2 & 3.8 \\
\hline 16 & $<0.5$ & $<0.5$ & 0.18 & 0.36 & $<0.45$ & 0.90 & 1.8 & 1.2 \\
\hline 20 & $<0.5$ & $<0.5$ & 0.32 & 0.09 & 0.21 & $<0.45$ & 3.1 & 2.8 \\
\hline 27 & $<0.5$ & $<0.5$ & 0.32 & 0.09 & 0.41 & $<0.45$ & 2.4 & 2.5 \\
\hline 29 & $<0.5$ & $<0.5$ & 0.45 & 0.09 & 0.43 & $<0.45$ & 2.8 & 3.4 \\
\hline \multicolumn{9}{|c|}{ Group III (5 to $16 \mathrm{~km}$ off-site) } \\
\hline 1 & $<0.5$ & $<0.5$ & -0.02 & 0.18 & 0.27 & $<0.45$ & 3.1 & 7.0 \\
\hline 2 & $<0.5$ & $<0.5$ & 0.18 & 0.18 & $<0.45$ & $<0.45$ & 4.2 & 1.0 \\
\hline 4 & $<0.5$ & $<0.5$ & 0.09 & -0.02 & 0.41 & 0.45 & 1.6 & 1.4 \\
\hline 6 & $<0.5$ & $<0.5$ & 0.41 & 0.09 & 0.54 & $<0.45$ & 3.9 & 4.2 \\
\hline 17 & $<0.5$ & $<0.5$ & 0.45 & -0.02 & $<0.45$ & $<0.45$ & 2.6 & 1.6 \\
\hline 18 & $<0.5$ & $<0.5$ & 0.45 & 0.99 & $<0.45$ & $<0.45$ & 1.2 & 1.8 \\
\hline 19 & $<0.5$ & $<0.5$ & 0.09 & 0.09 & $<0.45$ & 0.45 & 2.1 & 2.7 \\
\hline 21 & $<0.5$ & $<0.5$ & 0.18 & 0.05 & 0.42 & $<0.45$ & 3.2 & 4.2 \\
\hline 22 & $<0.5$ & $<0.5$ & 0.03 & -0.02 & 0.29 & 0.45 & 1.8 & 4.2 \\
\hline 25 & $<0.5$ & $<0.5$ & 0.05 & 0 & 0.36 & $<0.45$ & 2.0 & 3.5 \\
\hline 26 & $<0.5$ & $<0.5$ & 0.05 & 0 & 0.16 & $<0.45$ & 3.8 & 3.5 \\
\hline 28 & $<0.5$ & $<0.5$ & -0.02 & 0.45 & 0.22 & $<0.45$ & 2.9 & 2.4 \\
\hline \multicolumn{9}{|c|}{ Background (16 km off-site) } \\
\hline SAV $10 \mathrm{~W}$ & $<0.5$ & $<0.5$ & 0.27 & 0.45 & $<0.45$ & $<0.45$ & 3.7 & 3.4 \\
\hline SAV 10E & $<0.5$ & $<0.5$ & 0.23 & 0.36 & $<0.45$ & $<0.45$ & 5.2 & 3.0 \\
\hline SAV ION & $<0.5$ & $<0.5$ & 0.32 & 1.04 & 0.50 & $<0.45$ & 4.6 & 4.4 \\
\hline SAV IOS & 0.5 & $<0.5$ & 0.54 & 0.45 & $<0.45$ & $<0.45$ & 5.2 & 4.6 \\
\hline
\end{tabular}




\subsection{SOIL AND SEDIMENT}




\subsection{SOIL AND SEDIMENT SAMPLING}

The following tables give individual analytical results for soil and sediment samples taken both on and off the Portsmouth site. Note that the internal soil samples are a separate sampling network from the external soil samples and that the sample location numbers overlap (i.e., RISI is located on site at the X-611 water treatment plant, but external soil sample $\mathrm{S} 1$ is downstream on the Scioto River). Individual sample locations are shown in Figs, 2.5.1 through 2.5.4 in Part 1. Sample locations that are designated as a number plus " $A$ " are replicate locations in the immediate vicinity of that sample location number and are not shown separately.

The 1989 stream-sediment-sampling results are given in Table 2 5.3. Sample points are grouped according to watercourse and listed from upstream to downstream. Sediment-samipling locations are shown in Fig. 2.5.5 in Part 1.

Table 2.5.1. 1989 routine internal soil (RIS) samples

\begin{tabular}{|c|c|c|c|c|c|c|}
\hline \multirow{2}{*}{ Location } & \multicolumn{2}{|c|}{ Uranium $(\mu \mathrm{g} / \mathrm{g})$} & \multicolumn{2}{|c|}{ Alpha (pCi/g) } & \multicolumn{2}{|c|}{ Technetium ( $\mathrm{pCi} / \mathrm{g}$ ) } \\
\hline & Spring & Fall & Spring & Fall & Spring & Fall \\
\hline 1 & 3.1 & 3.1 & 3.0 & 1.8 & 0.29 & $<0.45$ \\
\hline 3 & 2.2 & $<2.0$ & 1.8 & $<0.90$ & 0.67 & 0.90 \\
\hline 5 & 4.4 & 7.0 & 6.8 & 4.05 & 0.23 & $<0.45$ \\
\hline 12 & 3.6 & 8.2 & 3.3 & 6.31 & 0.31 & 2.25 \\
\hline 15 & 5.7 & 4.4 & $<2.3$ & 2.7 & 0.45 & 0.45 \\
\hline 17 & 4.7 & 2.6 & 3.2 & 0.9 & 0 & $<0.45$ \\
\hline 19 & 5.2 & 6.4 & 4.5 & 5.4 & 1.8 & 9.5 \\
\hline 22 & 7.3 & 9.4 & 13.1 & 16.2 & 11.7 & 11.3 \\
\hline 25 & 3.9 & $<2.0$ & 3.15 & $<0.9$ & 0.45 & $<0.45$ \\
\hline 26 & 5.5 & 4.7 & 11.7 & 3.15 & 1.62 & 0.90 \\
\hline $26 \mathrm{~A}$ & 4.8 & 10.0 & 3.8 & 20.7 & 1.17 & 4.95 \\
\hline 32 & 6.4 & 4.3 & 4.95 & 2.70 & 0.9 & 1.35 \\
\hline 33 & 7.1 & $<2,0$ & 26.1 & 2.70 & 1.76 & 0.45 \\
\hline 34 & 7.0 & 4.7 & 121.6 & 4.05 & 2.03 & 0.90 \\
\hline 35 & $<2.0$ & 4.6 & $<0.90$ & 3.15 & $<0.45$ & $<0.45$ \\
\hline 36 & 10.4 & 3.5 & 6.31 & 1.80 & $<0.45$ & $<0.45$ \\
\hline
\end{tabular}


Table 2.5.2. 1989 external soil samples

\begin{tabular}{|c|c|c|c|c|c|c|}
\hline \multirow{2}{*}{ Location } & \multicolumn{2}{|c|}{ Uranium $(\mu \mathrm{g} / \mathrm{g})$} & \multicolumn{2}{|c|}{ Alpha $(\mathrm{pCl} / \mathrm{g})$} & \multicolumn{2}{|c|}{ Technetium $(\mathrm{pCi} / \mathrm{g})$} \\
\hline & Spring & Fall & Spring & Fall & Spring & Fall \\
\hline \multicolumn{7}{|c|}{ Group I (DOE property line) } \\
\hline S3 & 3.6 & 3.6 & 1.80 & 1.80 & $<0.45$ & $<0.45$ \\
\hline S8 & 9.8 & 6.4 & $<0,9$ & 3.60 & $<0.45$ & 0.45 \\
\hline S9 & 5.4 & 4.3 & 3.15 & 1.8 & $<0.45$ & $<0.45$ \\
\hline S9A & 6.8 & 2.0 & 3.15 & 1.35 & $<0.45$ & $<0.45$ \\
\hline S10 & 4.0 & 5.0 & 1.80 & 3.2 & $<0.45$ & $<0.45$ \\
\hline S10A & 6.5 & 3.8 & 3.15 & 1.8 & $<0,45$ & $<0.45$ \\
\hline S11 & 2.0 & 3.5 & 2.25 & 10.8 & 0.9 & $<0.45$ \\
\hline $\mathrm{S} 12$ & 2.0 & $<2.0$ & 3.60 & $<0.9$ & $<0.45$ & $<0.45$ \\
\hline $\mathrm{S} 12 \mathrm{~A}$ & 4.0 & 5.2 & 2.25 & 1.8 & $<0.45$ & $<0.45$ \\
\hline $\mathrm{S} 15$ & 3.0 & 4.0 & 1.35 & 2.3 & $<0.45$ & $<0.45$ \\
\hline S15A & 3.0 & 6.0 & 1.35 & 3.6 & $<0.45$ & $<0.45$ \\
\hline S23 & 4.2 & 3.2 & 5.4 & 2.6 & $<0.45$ & $<0.45$ \\
\hline S24 & 4.4 & $<2.0$ & 6.3 & 0.9 & 0 & $<0.45$ \\
\hline \multicolumn{7}{|c|}{ Group II (up to $5 \mathrm{~km}$ off-site) } \\
\hline S12 & 1.7 & 3.3 & 2.9 & 1.35 & 0 & $<0.45$ \\
\hline S14 & 3.8 & 3.9 & $<1.5$ & 3.2 & $<0.45$ & $<0.45$ \\
\hline S16 & 5.4 & 2.6 & 3.15 & 4.05 & $<0.45$ & $<0.45$ \\
\hline S20 & 1.3 & $<2.0$ & 4.4 & $<0.90$ & $<0.45$ & $<0.45$ \\
\hline$\$ 27$ & 4.7 & 5.7 & 6.4 & 3.15 & 0 & $<0.45$ \\
\hline S29 & 1.4 & 5.4 & 2.1 & 3.15 & 0 & $<0.45$ \\
\hline \multicolumn{7}{|c|}{ Group III (5-16 km off-site) } \\
\hline S1 & 5.1 & 3.8 & 5.0 & 5.25 & $<0.45$ & $<0.45$ \\
\hline S2 & 4.6 & $<2.0$ & 3.15 & $<0.9$ & $<0.45$ & $<0.45$ \\
\hline S4 & 5.0 & 4.3 & 3.5 & 2.6 & 0.13 & $<0.45$ \\
\hline S6 & 4.6 & 5.1 & 3.9 & 3.15 & $<0.45$ & $<0.45$ \\
\hline S17 & 4.3 & 4.0 & 2.70 & 2.25 & $<0.45$ & $<0.45$ \\
\hline S18 & $<2.0$ & 5.1 & $<0.90$ & 2.70 & $<0.45$ & $<0.45$ \\
\hline S19 & 4.4 & 4.9 & 2.25 & 4.05 & $<0.45$ & $<0.45$ \\
\hline S21 & 3.5 & 4.0 & 4.5 & 2.25 & $<0.45$ & $<0.45$ \\
\hline S22 & 4.5 & 6.2 & 3.8 & 3.60 & 0 & $<0.45$ \\
\hline S25 & 4.7 & 3.0 & 5.4 & 2.25 & $<0.45$ & $<0.45$ \\
\hline S26 & 3.0 & $<2.0$ & 2.5 & 1.80 & 0 & $<0.45$ \\
\hline S28 & 3.3 & 6.4 & 3.5 & 3.60 & $<0.45$ & $<0.45$ \\
\hline \multicolumn{7}{|c|}{ Background (16 km off-site) } \\
\hline RS10W & 6.2 & 4.0 & 8.1 & 2.3 & 0 & $<0.45$ \\
\hline RS10E & 3.6 & 5.0 & $<1.5$ & $<2$ & 0 & 0.45 \\
\hline RS10N & 5.8 & 3.9 & 5.4 & 2.25 & 0 & $<0.45$ \\
\hline RS10S & 2.2 & 5.0 & $<9.0$ & 3.2 & 0 & $<0.45$ \\
\hline
\end{tabular}


Table 2.5.3. 1989 stream sediment sampling results

\begin{tabular}{|c|c|c|c|c|c|c|}
\hline \multirow{2}{*}{ Location } & \multicolumn{2}{|c|}{ Uranium $(\mu \mathrm{g} / \mathrm{g})$} & \multicolumn{2}{|c|}{ Alpha (pCi/g) } & \multicolumn{2}{|c|}{ Technetium (pCi/g) } \\
\hline & Spring & Fall & Spring & Fall & Spring & Full \\
\hline \multicolumn{7}{|l|}{ On-site outfalls } \\
\hline $\begin{array}{l}\text { Southwest holding } \\
\text { pond }\end{array}$ & 5.4 & 2.4 & $<3.0$ & 1.4 & $<0.45$ & $<.0 .45$ \\
\hline $\begin{array}{l}\text { West drainage } \\
\text { ditch }\end{array}$ & 4.6 & $<2,0$ & 3.6 & $<0.90$ & 0.45 & $<0.45$ \\
\hline $\begin{array}{l}\text { East drainage } \\
\text { ditch }\end{array}$ & 16.0 & 8.4 & 103.6 & 37.8 & 96.2 & 39.2 \\
\hline \multicolumn{7}{|l|}{ Little Beaver Creek } \\
\hline RM12 & 5.1 & 2.4 & 10.4 & 1.35 & $<0.45$ & $<0.45$ \\
\hline RM8 & 14.0 & 9.7 & 48.6 & 9.5 & 53.8 & 36.5 \\
\hline RM7 & 4.3 & 10.0 & 3.6 & 15.3 & 2.25 & 50.5 \\
\hline \multicolumn{7}{|l|}{ Big Beaver Creek } \\
\hline RM5 & 4.1 & 3.6 & 5.4 & 1.80 & 0 & $<0.45$ \\
\hline RM13 & 8.8 & 8.0 & 29.3 & 16.7 & 39.3 & 35.6 \\
\hline \multicolumn{7}{|l|}{ Big Run Creek } \\
\hline RM33 & $<0.6$ & 3.1 & $<2.5$ & 2.25 & 0 & $<0.45$ \\
\hline RM3 & 5.1 & 6.2 & 0.6 & 3.60 & 0.83 & $<0.45$ \\
\hline RM3A & 8.3 & 6.5 & 11.7 & 3.15 & 2.22 & $<0.45$ \\
\hline RM2 & 3.4 & 3.7 & 3.6 & 2.25 & 0 & 0.45 \\
\hline \multicolumn{7}{|l|}{ Scioto River } \\
\hline RM6 & 3.8 & 4.1 & 7.2 & 2.25 & 0 & $<0.45$ \\
\hline RM1 & 4.4 & $<2.0$ & 4.0 & $<0.9$ & $<0.45$ & 0.45 \\
\hline \multicolumn{7}{|l|}{ Background } \\
\hline RM10W & 8.0 & 2.3 & 6.8 & 1.35 & C & $<0.45$ \\
\hline RM10S & 4.6 & 3.1 & 3.5 & 1.80 & $<0.45$ & $<0.45$ \\
\hline RM10E & 4.4 & $<2.0$ & $<1.8$ & $<0.90$ & $<0.45$ & $<0.45$ \\
\hline RM10N & 2.4 & $<2.0$ & $<1.0$ & $<0.90$ & 0 & $<0.45$ \\
\hline
\end{tabular}


2.6 EXTERNAL GAMMA 


\subsection{EXTERNAL GAMMA RADIATION MONITORING}

The external gamma radiation measurements on site, on the boundry line, and off-site are given in Table 2.6.1. The table shows the TLD series numbers of the TLDs used, the gamma radiation levels measured, the storage and transit exposure calculated from the control TLDs, and the percent of fading calculated from the reference TLDs. Blank entries indicate no data for a location in a quarter. A listing of TLD locations is given in Table 2.6.2. 
Table 2.6.1. Quarterly external gamma radiation leveis $(\mu \mathrm{rad} / \mathrm{h})$ for 1989

\begin{tabular}{|c|c|c|c|c|c|}
\hline $\begin{array}{c}\text { Date } \\
\text { (Series No.) }\end{array}$ & $\begin{array}{c}\text { Jan-Mar } \\
(486)\end{array}$ & $\begin{array}{l}\text { Apr-Jun } \\
(506)\end{array}$ & $\begin{array}{l}\text { Jul-Sept } \\
(486)\end{array}$ & $\begin{array}{c}\text { Oct-Dec } \\
(506)\end{array}$ & $\begin{array}{l}\text { Annual } \\
\text { dosages } \\
\text { (mrem) }\end{array}$ \\
\hline \multicolumn{6}{|l|}{ On-site } \\
\hline$X-104$ & 8.1 & 10.9 & 8.8 & 7.0 & \\
\hline $\mathrm{X}-230-\mathrm{J} 2$ & 10.6 & 13.4 & 8.4 & 8.9 & \\
\hline$X-611$ & 9.4 & 15.7 & 10.8 & 9.1 & \\
\hline$X-7725$ & 10.1 & 11.8 & 11.0 & 9.2 & \\
\hline 722 & 10.5 & 13.0 & & 10.6 & \\
\hline 862 & 8.1 & 14.8 & 12.6 & 9.8 & \\
\hline 906 & 8.4 & 11.8 & 9.7 & 11.7 & \\
\hline 933 & 8.8 & 13.4 & 11.1 & 8.0 & \\
\hline Mean & 9.3 & $! 3.1$ & 10.3 & 9.3 & 92 \\
\hline Standard deviation & 1.0 & 1.5 & 1.3 & 1.4 & \\
\hline $874^{a}$ & 130.7 & 155.5 & 118.1 & 110.1 & \\
\hline \multicolumn{6}{|l|}{ Boundary line } \\
\hline No. 3 & 8.0 & 9.8 & 9.3 & 7.4 & \\
\hline No. 8 & 10.3 & 15.9 & 11.5 & 11.3 & \\
\hline No. $9(\mathrm{C})$ & 8.6 & 9.7 & 9.1 & 7.7 & \\
\hline No. 12 & 8.6 & 11.6 & 10.9 & 8.2 & \\
\hline No. 15 & 9.5 & 12.8 & 10.0 & 10.7 & \\
\hline No. $23(\mathrm{~L})$ & 9.5 & 10.6 & 11.1 & 8.5 & \\
\hline No. 24 & 10.2 & 11.8 & 10.5 & 8.2 & \\
\hline No. 29 & 7.6 & 9.6 & 8.0 & 6.7 & \\
\hline Mean & 9.0 & 11.5 & 10.1 & 8.6 & 86 \\
\hline Standard deviation & 0.9 & 2.0 & 1.1 & 1.5 & \\
\hline \multicolumn{6}{|l|}{ Off-site } \\
\hline A & 11.0 & 12.6 & 11.7 & 9.1 & \\
\hline B & 9.3 & 13.9 & & 10.7 & \\
\hline $\mathrm{D}$ & 8.2 & 11.0 & 10.2 & 8.2 & \\
\hline $\mathrm{E}$ & 10.4 & 12.3 & 12.2 & 10.6 & \\
\hline $\mathrm{F}$ & 14.2 & & & & \\
\hline $\mathrm{H}$ & 14.0 & 11.7 & & 9.6 & \\
\hline I & 10.2 & 14.3 & 12.6 & 9.0 & \\
\hline $\mathbf{K}$ & 10.7 & 12.7 & & 10.5 & \\
\hline $\mathbf{M}$ & & & 11.0 & & \\
\hline $\mathbf{N}$ & 9.6 & 11.0 & 10.3 & 8.4 & \\
\hline $\mathbf{P}$ & 12.6 & 16.0 & & 12.5 & \\
\hline $\mathbf{R}$ & 8.5 & 11.0 & 9.4 & 9.6 & \\
\hline$T$ & 9.5 & 11.9 & & & \\
\hline No. 6 & 9.5 & 10.6 & 9.5 & 9.6 & \\
\hline No. 28 & 8.2 & 9.9 & & 8.5 & \\
\hline Mean & 10.4 & 12.2 & 10.9 & 9.7 & 95 \\
\hline Standard deviation & 1.9 & 1.6 & 1.1 & 1.2 & \\
\hline Control & 0.0 & 0.3 & 0.0 & 0.0 & \\
\hline Fading (\%) & 10.4 & 24.6 & 10.0 & -2.5 & \\
\hline
\end{tabular}

${ }^{a}$ Not included in Site Group I averages or standard deviations. 
Table 2.6.2. Environmental TLD locations

\begin{tabular}{|c|c|}
\hline Designation & Location \\
\hline \multicolumn{2}{|l|}{ Reference } \\
\hline$X-100$ & $\begin{array}{l}\text { Administration building penthouse } \\
\text { (on air sampler weather hood) }\end{array}$ \\
\hline \multicolumn{2}{|c|}{$\begin{array}{l}\text { Site Group I } \\
\text { (in or inside security fence) }\end{array}$} \\
\hline $\mathrm{X}-104$ & Guard headquarters \\
\hline $\mathrm{X}-230-\mathrm{J} 2$ & South holding pond monitoring station \\
\hline$X-611$ & Water treatment plant \\
\hline$X-7725$ & $\begin{array}{l}\text { GCEP R/A building (on security fence north } \\
\text { of vehicle portal }\end{array}$ \\
\hline 722 & $\begin{array}{l}\text { DOE power pole } 722 \text { at south end of Pike Ave } \\
\text { between X-231 oil biodegradation plots }\end{array}$ \\
\hline 862 & DOE power pole 862 opposite main access road \\
\hline 874 & $\begin{array}{l}\text { DOE power pole } 874 \text { at NW corner of } \mathrm{X}-745 \mathrm{C} \\
\text { tails storage yard }\end{array}$ \\
\hline 906 & DOE power pole 906 opposite North Access Road \\
\hline 933 & DOE power pole 933 opposite East Access Road \\
\hline \multicolumn{2}{|c|}{$\begin{array}{l}\text { Site Group II } \\
\text { (on or near DOE property line) }\end{array}$} \\
\hline 3 & South air sampler on South Access Road \\
\hline 8 & Northwest air sampler at DOE power pole 74 \\
\hline 9 & $\begin{array}{l}\text { Southwest air sampler at Southwest Access Road } \\
\text { and Old Rt. } 23 \text { (old location C) }\end{array}$ \\
\hline 12 & East air sampler on McCorkle Road \\
\hline 15 & Southeast air sampler it north end of Cemetery Road \\
\hline 23 & $\begin{array}{l}\text { Northeast air sampler at Barlow Road and } \\
\text { Taylor Hollow Road (replaces old location L) }\end{array}$ \\
\hline 24 & $\begin{array}{l}\text { North air sampler on North Access Road } \\
\text { at Shyville Road }\end{array}$ \\
\hline 29 & West air sampler on U.S. Rt. 23 at Old GAT Sign \\
\hline \multicolumn{2}{|l|}{$\begin{array}{l}\text { Site Group III } \\
\text { (off site) }\end{array}$} \\
\hline A & South Access Road and Rapp Hollow Road \\
\hline B & Caldwell Road and Cockrell Road \\
\hline $\mathrm{D}$ & Big Run Creek and Old Rt. 23 (RW2) \\
\hline $\mathrm{E}$ & Sunfish Creek and State Rt. 104 \\
\hline $\mathbf{F}$ & River Run Road opposite church \\
\hline$H$ & State Rt. 124 west of Jasper \\
\hline 1 & North Access Road and Market Road \\
\hline K & Prairie Road (north end) and Rt. 23 \\
\hline M & State Rt. 124 at Hammond Church \\
\hline $\mathbf{N}$ & Bobo Road, Dutch Run Road, and Loop Road \\
\hline $\mathbf{P}$ & Dutch Run Road and Gravel Road \\
\hline $\mathbf{R}$ & Loop Road and Baily Chapel Road \\
\hline $\mathrm{T}$ & Owl Creek Road and Hunting Road \\
\hline 6 & $\begin{array}{l}\text { DOE power pole } 6 \text { on access road to X-608 } \\
\text { pump house in Piketon }\end{array}$ \\
\hline 28 & Camp Creek air ampler \\
\hline
\end{tabular}




\section{RADIATION AND CHEMICAL DOSE}


Table 3.1.1. Effective dose rate conversion factors"

\begin{tabular}{|c|c|c|c|c|}
\hline $\begin{array}{l}\text { Radionuclide } \\
\text { (soituritiy) }\end{array}$ & $\begin{array}{l}\text { Inhalation } \\
(\mathrm{rem} / \mu \mathrm{Ci})\end{array}$ & $\begin{array}{l}\text { Ingestion } \\
(\mathrm{rem} / \mu \mathrm{Ci})\end{array}$ & $\begin{array}{c}\text { Immersion } \\
(\mathrm{mrem} / \text { year } \\
\left.\text { per } \mu \mathrm{Ci} / \mathrm{cm}^{3}\right)\end{array}$ & $\begin{array}{l}\text { Ground surfaces } \\
\text { (mrem/year } \\
\text { per } \mu \mathrm{Ci} / \mathrm{cm}^{2} \text { ) }\end{array}$ \\
\hline${ }^{99} \mathrm{~T} \in(D)^{4}$ & $1.00 \times 10^{-3}$ & $1.40 \times 10^{-3}$ & $2.50 \times 10^{3}$ & $5.91 \times 10^{-1}$ \\
\hline${ }^{234} \mathrm{U}$ (D) & 2.67 & $2.74 \times 10^{-1}$ & $7.36 \times 10^{5}$ & $7.94 \times 10^{2}$ \\
\hline${ }^{235} \mathrm{U}$ (D) & 2.54 & $2.63 \times 10^{-1}$ & $7.37 \times 10^{8}$ & $1.64 \times 10^{5}$ \\
\hline${ }^{236} \mathrm{U}(\mathrm{D})$ & 2.53 & $2.60 \times 10^{-1}$ & $5.80 \times 10^{5}$ & $7.20 \times 10^{2}$ \\
\hline${ }^{238} U$ (D) & 2.40 & $2.47 \times 10^{-2}$ & $5.00 \times 10^{5}$ & $6.5 \times 10^{2}$ \\
\hline${ }^{231} \mathrm{Th}(\mathrm{W})$ & $9.30 \times 10^{-4}$ & $1.23 \times 10^{-3}$ & $5.52 \times 10^{-7}$ & $1.83 \times 10^{4}$ \\
\hline${ }^{234} \mathrm{Th}(\mathrm{W})$ & $3.00 \times 10^{-2}$ & $1.30 \times 10^{-2}$ & $3.65 \times 10^{7}$ & $9.53 \times 10^{3}$ \\
\hline${ }^{234 m p a ~(W) ~}$ & $3.76 \times 10^{-6}$ & $5.80 \times 10^{-6}$ & $5.83 \times 10^{7}$ & $1.11 \times 10^{4}$ \\
\hline
\end{tabular}

aFactors taken from the EPA Clean Air Act data tapes.

${ }^{b} \mathrm{D}$-soluble in blood; W-moderately soluble in blood.

Table 3.1.2. Predicted doses from 1989 airborne releases at Portsmouth

\begin{tabular}{ll}
\hline \multicolumn{1}{c}{ Dose to } & Effective \\
\hline Nearest resident $^{a}$ & $7 \times 10^{-2}$ \\
Population $^{b}$ & $4 \times 10^{-1}$ \\
Nearest community $^{c}$ & $1 \times 10^{-2}$ \\
\hline${ }^{a}$ Nearest resident & is $1770 \mathrm{~m}$
\end{tabular}

ENE. Dose in mrem.

${ }^{b}$ Dose to population within $80 \mathrm{~km}$. Dose in person-rem.

'Piketon is nearest community.

Dose in person-rern. 
Table 3.1.3. 1989 annual report of radionuclides released to the atmosphere (40 CFR 61.94)"

Radionuclide Activity (Ci/year)

Section I: Air emissions

$\begin{array}{ll}{ }^{234} \mathrm{U} & 0.10 \\ { }^{235} \mathrm{U} & 0.0039 \\ { }^{236} \mathrm{U} & 0.0001 \\ { }^{238} \mathrm{U} & 0.0022 \\ { }^{99} \mathrm{Tc} & 0.096 \\ { }^{231} \mathrm{Th} & 0.0039 \\ { }^{234} \mathrm{Th} & 0.0022 \\ { }^{234 m} \mathrm{~Pa} & 0.0022\end{array}$

Section II: Methods for dose assessment

Dose equivalents were estimated using the DARTAB computer code, which uses the atmospheric concentrations predicted by the AIRDOS-EPA atmospheric dispersion model and the dose conversion factors contained in the RADRISK data base.

Section III: Dose equivalent estimates (mren ।

\begin{tabular}{|c|c|c|}
\hline & EPA standard & Facility estimate \\
\hline Effective & $\leq 10$ & 0.07 \\
\hline
\end{tabular}

${ }^{\circ}$ Owner: U.S. Department of Energy; Operations Office: Oak Ridge, Tennessee;

Site Operator: Martin Marietta Energy Systems, Inc;

Site Address: Portsmouth Gaseous Diffusion Plant, P.O. Box 628, Piketon, OH 45661.

Table 3.1.4. Doses from aquatic pathways in the Scioto River

\begin{tabular}{lc}
\hline & $\begin{array}{c}\text { Effective dose equivalent } \\
\text { (mrem) }\end{array}$ \\
\hline Eating fish $^{a}$ & 0.0004 \\
Drinking water $^{b}$ & $\frac{0.002}{0.002}$ \\
Total & 0.00 \\
\hline
\end{tabular}

${ }^{a}$ Assumed consumption of $21 \mathrm{~kg}$ of fish per year.

${ }^{b}$ Assumed consumption of $730 \mathrm{~L}$ of water per year. 
5. SOLID WASTE MANAGEMENT 
Table 5.2.1. Waste generation report for 1989

\begin{tabular}{|c|c|c|c|c|c|c|c|c|c|c|c|c|c|}
\hline & Jan & Feb & Mar & Apr & May & Jun & Jul & Aug & Sep & Oct & Nov & Dec & Total \\
\hline Sanitary waste, solid $\left(\mathrm{m}^{3}\right)$ & 849 & 516 & 691 & 690 & 505 & 638 & 706 & 768 & 742 & 258 & 303 & 275 & 6,941 \\
\hline $\begin{array}{r}\left.\text { Hazardous waste, liquid ( } k_{8}\right) \\
\text { (L) }\end{array}$ & $\begin{array}{l}859 \\
819\end{array}$ & $\begin{array}{l}3,475 \\
3,331\end{array}$ & $\begin{array}{l}3,169 \\
3,022\end{array}$ & $\begin{array}{l}73 \\
69\end{array}$ & $\begin{array}{l}276 \\
263\end{array}$ & $\begin{array}{l}2,969 \\
2,832\end{array}$ & $\begin{array}{l}9,585 \\
9,141\end{array}$ & $\begin{array}{l}14,223 \\
13,564\end{array}$ & $\begin{array}{l}5,284 \\
5,039\end{array}$ & $\begin{array}{l}5,107 \\
4,870\end{array}$ & $\begin{array}{l}3,453 \\
3,293\end{array}$ & $\begin{array}{l}1,054 \\
1,005\end{array}$ & $\begin{array}{l}49,527 \\
47,248\end{array}$ \\
\hline $\begin{array}{r}\text { Hazardous waste, solid }(\mathrm{kg}) \\
\qquad\left(\mathrm{m}^{3}\right)\end{array}$ & $\begin{array}{r}29,801 \\
24\end{array}$ & $\begin{array}{l}0 \\
0\end{array}$ & $\begin{array}{r}197 \\
0\end{array}$ & $\begin{array}{r}6,029 \\
5\end{array}$ & $\begin{array}{r}232 \\
0\end{array}$ & $\begin{array}{r}49 \\
0\end{array}$ & $\begin{array}{r}12,163 \\
10\end{array}$ & $\begin{array}{r}59,326 \\
48\end{array}$ & $\begin{array}{r}4,937 \\
4\end{array}$ & $\begin{array}{r}19,295 \\
16\end{array}$ & $\begin{array}{r}1,014 \\
1\end{array}$ & $\begin{array}{r}227 \\
0\end{array}$ & $\begin{array}{r}133,271 \\
108\end{array}$ \\
\hline \multicolumn{14}{|l|}{ Polychlorinated biphenyl waste } \\
\hline (L) & 779 & 0 & 0 & 0 & 211 & 0 & 0 & 0 & 0 & 0 & 0 & 0 & 990 \\
\hline $50-500$ ppm PCB liquid (kg) & 0 & 1,090 & 0 & 1,386 & 2,586 & 3,567 & 13,507 & 4,738 & 2,488 & 2,413 & 1,081 & 1,525 & 34,381 \\
\hline (L) & 0 & 1,189 & 0 & 1,512 & 2,820 & 3,890 & 14,733 & 5,168 & 2,714 & 2,632 & 1,180 & 1,663 & $37 ; 502$ \\
\hline$>500$ ppm PCB liquid $(\mathrm{kg})$ & 0 & 0 & 0 & 0 & 0 & 0 & 0 & 0 & 0 & 0 & 0 & 0 & \\
\hline$(\mathrm{L})$ & 0 & 0 & 0 & J & 0 & 0 & 0 & 0 & 0 & 0 & 0 & 0 & \\
\hline Total PCB liquid ( $\mathrm{kg}$ ) & 714 & 1,090 & 0 & 1,386 & 2,779 & 3,567 & 13,507 & 4,738 & 2,488 & 2,413 & 1,081 & 1,525 & 35,288 \\
\hline (L) & 779 & 1,189 & 0 & 1,512 & 3,031 & 3,890 & 14,733 & 5,168 & 2,714 & 2,632 & 1,180 & 1,663 & $38,49 !$ \\
\hline PCB solids $\left(k_{g}\right)$ & 0 & 0 & 0 & 2,771 & 3,726 & 11,716 & 16,869 & 122,410 & 1,749 & 7,344 & 831 & 780 & 168,196 \\
\hline$\left(\mathrm{m}^{3}\right)$ & 0 & 0 & 0 & 41 & 55 & 172 & 248 & 1,799 & 26 & 108 & 12 & 11 & 2,472 \\
\hline Empty drums & 0 & 0 & 0 & 0 & 0 & 0 & 3 & 0 & 17 & 43 & 0 & 3 & 66 \\
\hline \multicolumn{14}{|l|}{ Mixed wastes } \\
\hline Hazardous, solid ( $\mathrm{kg}$ ) & 0 & 0 & 3,770 & 1,588 & 1,225 & 6,632 & 1,529 & 2,515 & 6,747 & 48,778 & 6,508 & 2,091 & 81,382 \\
\hline$\left(m^{3}\right)$ & 0 & 0 & 4 & 2 & 1 & 7 & 2 & 3 & 7 & 54 & 7 & 2 & 90 \\
\hline Hazardous, liquid $(\mathrm{kg})$ & 0 & 0 & 4.38 & 0 & 282 & 0 & 0 & 5,841 & 500 & 16,437 & $\mid 2,081$ & 772 & 36,352 \\
\hline (L) & 0 & 0 & 418 & 0 & 269 & 0 & 0 & 5,570 & 477 & 15,675 & 11,521 & 736 & 34,667 \\
\hline PCB, liquids $(\mathrm{kg})$ & 0 & 0 & 0 & 0 & 227 & 0 & 0 & 1,528 & 2,956 & 3,499 & 970 & 117 & 9,297 \\
\hline (L) & 0 & 0 & 0 & 0 & 247 & 0 & 0 & 1,667 & 3,224 & 3,817 & 1,058 & 128 & 10,141 \\
\hline PCB, solid $(\mathrm{kg})$ & 0 & 0 & 19,843 & 31,199 & 3,738 & 3,485 & 7.012 & 34,496 & 7,633 & 26,158 & $2.1,822$ & 10,411 & 165,796 \\
\hline$\left(\mathrm{m}^{3}\right)$ & 0 & 0 & 19 & 31 & 4 & 3 & 7 & 34 & 7 & 26 & 21 & 10 & 163 \\
\hline \multicolumn{14}{|l|}{ Scrap metal } \\
\hline Radioactive (kg) & 2,722 & 3,629 & 1,633 & 12,247 & 7,167 & 52,617 & 2,812 & 93,078 & 438,628 & 44,725 & 43,583 & 8,146 & 710,986 \\
\hline Nonradioactive $\left(k_{8}\right)$ & 15,422 & 1,814 & 5,443 & 6,350 & 5,443 & 0 & 1,021 & 1,021 & 0 & 1,905 & 0 & 0 & 38,420 \\
\hline \multicolumn{14}{|l|}{ Waste oils } \\
\hline Radicactive (L) & 0 & 0 & 905 & 0 & 1,665 & 208 & 208 & 208 & 0 & 4,987 & 461 & 0 & 8,643 \\
\hline Nonradioactive (L) & 208 & 208 & 2,672 & 6,113 & 2,086 & 1,457 & 6,339 & 2,120 & 5,256 & 14,635 & 2,961 & 3,663 & 47,718 \\
\hline Fly ash, solid $\left(\mathrm{m}^{3}\right)$ & 559 & 541 & 513 & 450 & 442 & 496 & 511 & 459 & 432 & 450 & 618 & 518 & 5,990 \\
\hline Classified wastes, solid $\left(\mathrm{m}^{3}\right)$ & 0 & 0 & 0 & 0 & 0 & 0 & 0 & 0 & 0 & 0 & 0 & 0 & 0 \\
\hline \multicolumn{14}{|l|}{ Asbestos } \\
\hline Radioactive (kg) & 211 & 109 & 0 & 85 & 23 & 44 & 452 & 1.325 & 5,051 & 927 & 469 & 86 & 8,781 \\
\hline$\left(\mathrm{m}^{3}\right)$ & 3 & 1 & 0 & 1 & 0 & 1 & 6 & 17 & 63 & 12 & 6 & 1 & 110 \\
\hline Nonradioactive (kg) & 166 & 181 & 211 & 0 & 217 & 248 & 515 & 1,373 & 811 & 286 & 325 & 343 & 4,734 \\
\hline$\left(\mathrm{m}^{3}\right)$ & 2 & 2 & 3 & 0 & 3 & 3 & 6 & 17 & 10 & 4 & 4 & 4 & 58 \\
\hline $\begin{array}{l}\text { Radioactive wastes }\left(\mathrm{m}^{3}\right) \\
\quad \text { (not listed above) }\end{array}$ & 0 & 1 & 1 & 39 & 7 & 448 & 163 & 254 & 94 & 1,406 & 94 & 570 & 3,077 \\
\hline
\end{tabular}




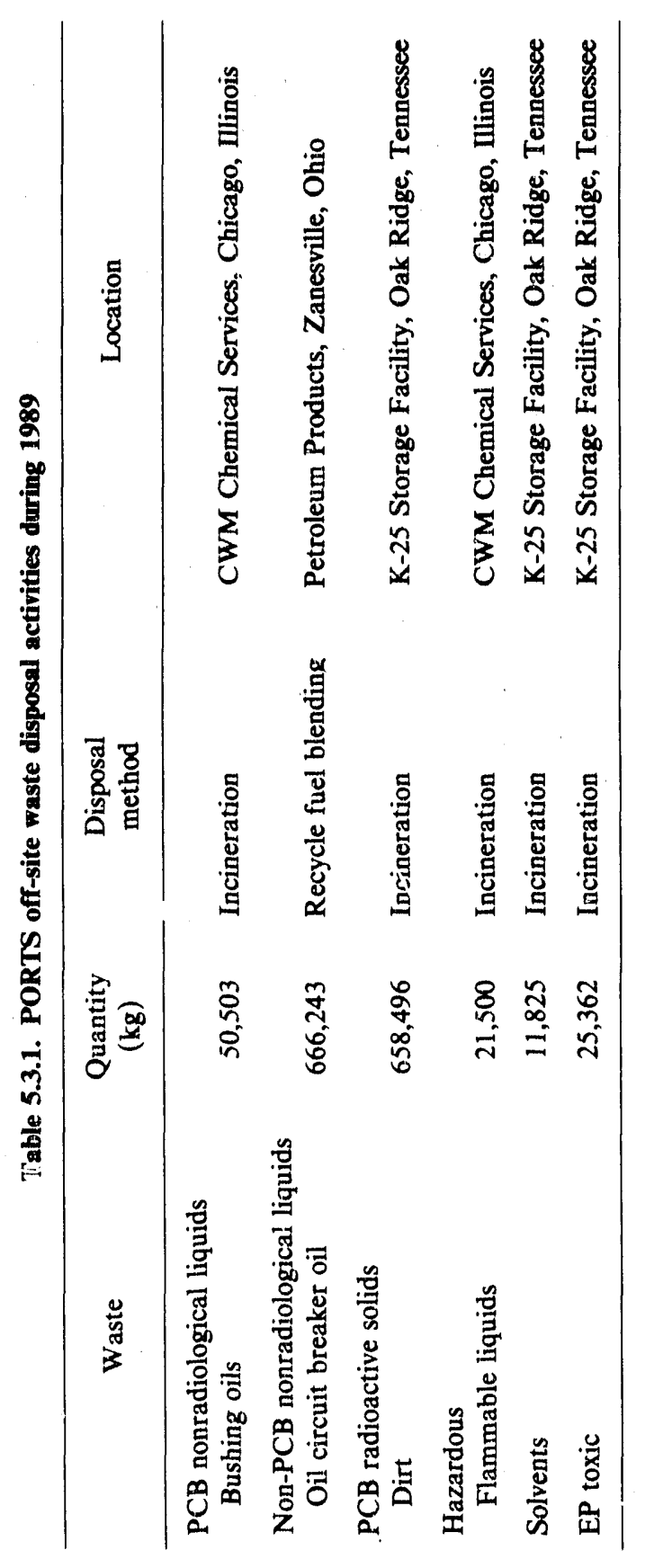




\section{QUALITY ASSURANCE}


Table 7.2.1. Martin Marietta Energy Systems, Inc. (PORTS), environmental analysis procedures for water

\begin{tabular}{|c|c|c|c|}
\hline \multirow{2}{*}{ Parameter } & \multicolumn{2}{|c|}{ Procedure } & \multirow{2}{*}{$\begin{array}{l}\text { Lowest concen- } \\
\text { tration reported } \\
(\text { LCR })^{b}\end{array}$} \\
\hline & $\mathrm{ES}^{a}$ & EPA & \\
\hline Alpha activity (uranium) & EC-033-PORTS & & $2.0 \mathrm{pCl} / \mathrm{L}$ \\
\hline Alpha activity (gross) & EC-034 PORTS & & $2.0 \mathrm{pCi} / \mathrm{L}$ \\
\hline Beta activity (gross) & EC-035 PORTS & & $4.0 \mathrm{pCi} / \mathrm{L}$ \\
\hline Arsenic & & 206.2 & 0.02 \\
\hline Asbestos & & $600 / 4-83-043$ & $1.34 \times 10^{5}$ fibers $/ \mathrm{L}$ \\
\hline Barium & & $208.1 / 200.7 / 6010$ & $0.4 / 0.2 / 0.2 \mathrm{mg} / \mathrm{L}$ \\
\hline $\mathrm{BOD}(5 \mathrm{~d})$ & QTS-511E-029-0 & & $5 \mathrm{mg} / \mathrm{L}$ \\
\hline Cadmium (total) & & $213.1 / 200.7 / 6010$ & $0.02 / 0.01 / 0.01 \mathrm{mg} / \mathrm{L}$ \\
\hline Carbitol & & 8270 & $10 \mu \mathrm{g} / \mathrm{L}$ \\
\hline Carbon (organic, total) & & 9060 & $1 \mathrm{mg} / \mathrm{L}$ \\
\hline Chloride & QTS-511E-032.0 & & $1 \mathrm{mg} / \mathrm{L}$ \\
\hline Chlorine (total, residual) & QTS-511E-058-0 & & $0.02 \mathrm{mg} / \mathrm{L}$ \\
\hline Chlorinated solvent scan & & 8010 & $1 \mu \mathrm{g} / \mathrm{L}$ \\
\hline Chromium (total) & & $218.1 / 200.7 / 6010$ & $0.1 / 0.02 / 0.03 \mathrm{mg} / \mathrm{L}$ \\
\hline Chromium (VI) & EC-009 PORTS & & $0.01 \mathrm{mg} / \mathrm{L}$ \\
\hline Coliform bacteria (fecal) & QTS-511E-043-0 & & $<1$ colony $/ 100 \mathrm{~mL}$ \\
\hline Coliform bacteria & QTS-511E-028-0 & & $<1$ colony $/ 100 \mathrm{~mL}$ \\
\hline Copper (total) & & $220.1 / 200.7$ & $0.05 / 0.01 \mathrm{mg} / \mathrm{L}$ \\
\hline Endrin & & 8080 & $1 \mu \mathrm{g} / \mathrm{L}$ \\
\hline Fluoride & & 340.2 & $0.1 \mathrm{mg} / \mathrm{L}$ \\
\hline Freon-113 & & 8010 & $1 \mu \mathrm{g} / \mathrm{L}$ \\
\hline Gamma emitters & EC-039 PORTS & & $2.5 \mathrm{pCi} / \mathrm{L}$ \\
\hline Halogen (organic, total) & & 9020 & $5 \mu \mathrm{g} / \mathrm{L}^{\prime}$ \\
\hline Iron (total) & & $236.1 / 200.7 / 6010$ & $0.05 / 0.02 / 0.02 \mathrm{mg} / \mathrm{L}$ \\
\hline Lead (total) & & $239.1 / 200.7 / 6010$ & $0.1 / 0.05 / 0.05 \mathrm{mg} / \mathrm{L}$ \\
\hline Lindane & & 8080 & $1 \mu \mathrm{g} / \mathrm{L}$ \\
\hline Manganese (total) & & $243.1 / 200.7 / 6010$ & $0.03 / 0.02 / 0.03 \mathrm{mg} / \mathrm{L}$ \\
\hline Mercury & & 245.1 & $0.5 \mu \mathrm{g} / \mathrm{L}$ \\
\hline $\begin{array}{l}\text { Methoxychlor } \\
{ }_{237} \mathrm{~Np}\end{array}$ & EC-038 PORTS & 8080 & $\begin{array}{l}1 \mu \mathrm{g} / \mathrm{L} \\
1.0 \mathrm{pCi} / \mathrm{L}\end{array}$ \\
\hline Nickel (total) & & $249.1 / 200.7 / 6010$ & $0.1 / 0.02 / 0.05 \mathrm{mg} / \mathrm{L}$ \\
\hline Nitrogen (ammonia) & & 350.3 & $0.1 \mathrm{mg} / \mathrm{L}$ \\
\hline Nitrogen (nitrate-nitrite) & & 353.3 & $0.2 \mathrm{mg} / \mathrm{L}$ \\
\hline Nitrogen, Kjeldahl (total) & & 351.4 & $0.1 \mathrm{mg} / \mathrm{L}$ \\
\hline Oil and grease & & 413.1 & $5 \mathrm{mg} / \mathrm{L}$ \\
\hline PCB (scan) & & 8080 & $1 \mu \mathrm{g} / \mathrm{L}$ \\
\hline $\mathrm{pH}$ & & 150.1 & $\begin{array}{l}\text { Nearest } 0.1 \mathrm{pH} \\
\text { unit }\end{array}$ \\
\hline Phosphate (all forms) & & 365.2 & $0.1 \mathrm{mg} / \mathrm{L}$ \\
\hline Phenols & & 8040 & $1.10 \mu \mathrm{g} / \mathrm{L}$ \\
\hline Plutonium & EC-038 PORTS & & $1.0 \mathrm{pCi} / \mathrm{L}$ \\
\hline Radium & & 900.1 & $4.0 \mathrm{pCi} / \mathrm{L}$ \\
\hline
\end{tabular}


Table 7.2.1 (Continued)

\begin{tabular}{|c|c|c|c|}
\hline \multirow{2}{*}{ Parameter } & \multicolumn{2}{|c|}{ Procedure } & \multirow{2}{*}{$\begin{array}{l}\text { Lowest concen- } \\
\text { tration reported } \\
(\mathrm{LCR})^{b}\end{array}$} \\
\hline & $\mathrm{ES}^{a}$ & EPA & \\
\hline Selenium (total) & & $270.2 / 200.7$ & $0.02 / 0.2 \mathrm{mg} / \mathrm{L}$ \\
\hline Silver (total) & & $272.1 / 200.7 / 6010$ & $0.06 / 0.05 / 0.1 \mathrm{mg} / \mathrm{L}$ \\
\hline Sodium (total) & & $273.1 / 200.7 / 6010$ & $0.01 / 0.1 / 0.1 \mathrm{mg} / \mathrm{L}$ \\
\hline Solids (dissolved) & & 160.1 & $1 \mathrm{mg} / \mathrm{L}$ \\
\hline Solids (suspended) & & 160.2 & $1 \mathrm{mg} / \mathrm{L}$ \\
\hline Solids (total) & & 160.3 & $10 \mathrm{mg} / \mathrm{L}$ \\
\hline Sulfate & QTS-511E-049-1 & & $1 \mathrm{mg} / \mathrm{L}$ \\
\hline${ }^{89} \mathrm{Tc}$ & EC-038-PORTS & & $300 \mathrm{pCi} / \mathrm{L}$ \\
\hline Thorium & EC-037 PORTS & & $4 \mathrm{pCi} / \mathrm{L}$ \\
\hline Toxaphene & & 8080 & $10 \mu \mathrm{g} / \mathrm{L}$ \\
\hline Tributyl phosphate & & 8270 & $10 \mu \mathrm{g} / \mathrm{L}$ \\
\hline Trichloroethylene & & $8010 / 601$ & $1 \mu \mathrm{g} / \mathrm{L}$ \\
\hline 2,4,5 TP Silvex & & 8150 & $1 \mu \mathrm{g} / \mathrm{L}$ \\
\hline Turbidity & & 180.1 & $0.05 \mathrm{NTU}$ \\
\hline Uranium (total) & EC.031 PORTS & & $1 \mu \mathrm{g} / \mathrm{L}$ \\
\hline Zinc (total) & & $289.1 / 200.7 / 6010$ & $0.02 / 0.02 / 0.05 \mathrm{mg} / \mathrm{L}$ \\
\hline
\end{tabular}

"Procedures described in:

1. Works Laboratory Procedures Manual, GAT-115, rev. 3, Vols. I and II, Goodyear Atomic Corp., Piketon, Ohio.

2. Determination of Trace Elements in Uranium Compounds-Specification Analyses. GAT-507, Goodyear Atomic Corp., Piketon, Ohio, 1968.

3. Hesse, P. R., A Textbook of SOIL Chemical Analysis, Chemical Publishing Co., New York, 1972.

4. Environmental Control-Analytical Procedure Manual, POEF 1192, Vol, 3, Martin Marietta Energy Systems, Portsmouth Gaseous Diffusion Plant, Piketon, Ohio.

${ }^{b}$ The LCR may vary among specific samples, depending on interferences in the sample matrix. However, these LCRs have been assigned to accommodate most minor interferences. Some of the Energy Systems LCRs are higher than the method detection limits (MDLs) listed by EPA. This is consistent with guidance from EPA; however, any data reported below the MDLs must be supported by sound documentation. The LCRs applied by Energy Systems laboratories meet the needs of the programs they support. 
Table 7.2.2. Martin Marietta Energy Systems, Inc. (PORTS), environmental analysis procedures for air

\begin{tabular}{|c|c|c|c|}
\hline \multirow{2}{*}{ Parameter } & \multicolumn{2}{|l|}{ Procedure } & \multirow{2}{*}{$\begin{array}{l}\text { Lowest concen- } \\
\text { tration reported } \\
\qquad(\mathrm{LCR})^{b}\end{array}$} \\
\hline & $\mathrm{ES}^{a}$ & EPA & \\
\hline \multicolumn{4}{|c|}{ AA membrane filters } \\
\hline Alpha activity & EC-102 PORTS & & $1.2 \mathrm{pCi} / \mathrm{sample}$ \\
\hline Beta activity & EC-102 PORTS & & $1.8 \mathrm{pCi} /$ sample \\
\hline Fluoride & EC-100 PORTS & & $2.5 \mu \mathrm{g} / \mathrm{sample}$ \\
\hline Asbestos & $\begin{array}{l}\text { NIOSH Method } 7400 \\
\quad(5-15-89)\end{array}$ & & $\begin{array}{l}7 \text { fibers } / \mathrm{mm}^{2} \\
\text { filter area }\end{array}$ \\
\hline \multicolumn{4}{|l|}{ Cascade vents } \\
\hline Alpha activity & $2-\mathrm{G}-35-0$ & & $20 \mathrm{pCi} /$ sample \\
\hline Fluoride & $2-\mathrm{J}-302-1$ & & $5 \mu \mathrm{g} /$ sample \\
\hline \multirow[t]{2}{*}{${ }^{99} \mathrm{Tc}$} & $2-\mathrm{G}-25-2$ & & $300 \mathrm{pCi} /$ sample \\
\hline & $2-\mathrm{G}-34-0$ & ' & $0.02 \mu \mathrm{g} / \mathrm{sample}$ \\
\hline \multirow[t]{2}{*}{ Uranium } & $2-G-34-0$ & & $2.5 \mu \mathrm{g} / \mathrm{sample}$ \\
\hline & $2-J-22-1$ & & $0.25 \mu \mathrm{g} /$ sample \\
\hline${ }^{235} \mathrm{U}$ & $2-\mathrm{G} \cdot 34-0$ & & $2.0 \mu \mathrm{g} / \mathrm{sample}$ \\
\hline
\end{tabular}

"Procedures described in:

1. Works Laboratory Procedures Manual, GAT-115, rev. 3, Vols. I and II, Goodyear Atomic Corp., Piketon, Ohio.

2. Determination of Trace Elements in Uranium Compounds-Specification Analyses. GAT-5n7, Goodyear Atomic Corp., Piketon, Ohio, 1968.

3. Hesse, P. R., A Textbook of SOIL Chemical Analysis, Chemical Publishing Co., New York, 1972.

4. Environmental Control-Analytical Procedure Manual, POEF 1192, Vol, 2 , Martin Marietta Energy Systems, Portsmouth Gaseous Diffusion Plant, Piketon, Ohio.

${ }^{6}$ The LCR may vary among specific samples, depending on interferences in the sample matrix. However, these LCRs have been assigned to accommodate most minor interferences. Some of the Energy Systems LCRs are higher than the method detection limits (MDLs) listed by EPA. This is consistent with guidance from EPA; however, any data reported below the MDLs must be supported by sound documentation. The LCRs applied by Energy Systems laboratories meet the needs of the programs they support. 
Table 7.2.3. Martin Marietta Energy Systems, lnc. (PORTS), environmental analysis procedures for soii and sediment

\begin{tabular}{|c|c|c|c|}
\hline \multirow{2}{*}{ Parameter } & \multicolumn{2}{|c|}{ Procedure } & \multirow{2}{*}{$\begin{array}{l}\text { Lowest concen- } \\
\text { tration reported } \\
(\text { LCR })^{b}\end{array}$} \\
\hline & $\mathrm{ES}^{a}$ & EPA & \\
\hline Alpha activity (uranium) & $\begin{array}{l}2-G-35-0 \\
\text { EC-204-PORTS }\end{array}$ & & $\begin{array}{l}1.4 \times 10^{3} \mathrm{pCi} / \mathrm{kg} \\
1 \times 10^{4} \mathrm{pCi} / \mathrm{kg}\end{array}$ \\
\hline $\begin{array}{l}\text { Fluoride } \\
\text { Mercury (total) } \\
\text { Metals (Al, Ba, Be, Ca, } \\
\text { Col, Co. Cr, Cu, Fe, } \\
\text { Pb, Mg, Mo, Ni, K, } \\
\text { Na, Tl, V, Zn) }\end{array}$ & $\begin{array}{l}\text { EC-201-PORTS } \\
\text { EC-202 PORTS } \\
3050 / 6010\end{array}$ & & $\begin{array}{l}\text { NA } \\
\text { NA } \\
\text { NA }\end{array}$ \\
\hline Nitrates & $a(3)$ & & NA \\
\hline PCB (scan) & & 8080 & $0.4 \mathrm{mg} / \mathrm{kg}$ \\
\hline Phosphate & $a(3)$ & & NA \\
\hline $\mathrm{pH}$ & & 9040 & $\begin{array}{l}\text { Nearest } 0.1 \mathrm{pH} \\
\text { unit }\end{array}$ \\
\hline${ }^{90} \mathrm{Tc}$ & $2-G-26-2$ & & $2 \times 10^{4} \mathrm{pCi} / \mathrm{kg}$ \\
\hline Uranium (total) & EC-203-PORTS & & $1 \mathrm{mg} / \mathrm{kg}$ \\
\hline
\end{tabular}

"Procedures described in:

1. Works Laboratory Procedures Manual, GAT-115, rev. 3, Vols. I and II, Goodyear Atomic Corp., Piketon, Ohio.

2. Determination of Trace Elements in Uranium Compounds-Specification Analyses. GAT-507, Goodyear Atomic Corp., Piketon, Ohio, 1968.

3. Hesse, P. R., A Textbook of SOIL Chemical Analysis, Chemical Publishing Co., New York, 1972.

4. Environmental Control-Analytical Procedure Manual, POEF 1192, Vol. 2, Martin Marietta Energy Systems, Portsmouth Gaseous Diffusion Plant, Piketon, Ohio.

'The LCR may vary among specific samples, depending on interferences in the sample matrix. Howeler, these LCRs have been assigned to accommodate most minor interferences. Some of the Energy Systems LCRs are higher than the method detection limits (MDLs) listed by EPA. This is consistent with guidance from E.PA; however, any data reported below the MDLs must be supported by sound documentation. The LCRs applied by Energy Systems laboratories meet the needs of the programs they support. 
Table 7.2.4. Martin Marietta Energy Systems, Inc. (PORTS), environmental analysis procedures for biota

\begin{tabular}{|c|c|c|c|}
\hline \multirow{2}{*}{ Parameter } & \multicolumn{2}{|l|}{ Procedure } & \multirow{2}{*}{$\begin{array}{l}\text { Lowest concen- } \\
\text { tration reported } \\
\qquad(\text { LCR })^{b}\end{array}$} \\
\hline & $\mathrm{ES}^{a}$ & EPA & \\
\hline \multicolumn{4}{|l|}{ Vegetation and vegetables } \\
\hline Alpha activity (uranium) & EC-204-PORTS & & $5.3 \times 10^{2} \mathrm{pCi} / \mathbf{k g}$ \\
\hline Fluoride & EC-300-PORTS & & $1 \mathrm{mg} / \mathrm{kg}$ \\
\hline${ }^{99} \mathrm{Tc}$ & $2-G-26-2$ & & $3.5 \times 10^{3} \mathrm{pCi} / \mathrm{kg}$ \\
\hline Uranium (total) & EC-301-PORTS & & $0.5 \mathrm{mg} / \mathrm{kg}$ \\
\hline \multicolumn{4}{|l|}{ Milk } \\
\hline Alpha activity (uranium) & EC-204-PORTS & & $10 \mathrm{pCi} / \mathrm{L}$ \\
\hline Techrietium & $2-G-26-2$ & & $4.5 \times 10^{2} \mathrm{pCi} / \mathrm{L}$ \\
\hline Uranium (total) & EC-302-PORTS & & $0.01 \mathrm{mg} / \mathrm{L}$ \\
\hline \multicolumn{4}{|l|}{ Fish } \\
\hline Arsenic & EC-303-PORTS & & $0.5 \mathrm{mg} / \mathrm{kg}$ \\
\hline Mercury & EC-304-PORTS & & $0.05 \mathrm{mg} / \mathrm{kg}$ \\
\hline PCB (scan) & & 8080 & $0.2 \mathrm{mg} / \mathrm{kg}$ \\
\hline Selenium & EC-305-PORTS & & $0.1 \mathrm{mg} / \mathrm{kg}$ \\
\hline Uranium & $2 \cdot \mathrm{J}-145-1$ & & $0.25 \mathrm{mg} / \mathrm{kg}$ \\
\hline
\end{tabular}

"Procedures described in:

1. Works Laboratory Procedures Manual, GAT-115, rev, 3, Vols. I and II, Goodyear Atomic Corp., Piketon, Ohio.

2. Determination of Trace Elements in Uranium Compounds-Specification Analyses. GAT-507, Goodyear Atomic Corp., Piketon, Ohio, 1968.

3. Hesse, P. R., A Textbook of SOIL Chemical Analysis, Chemical Publishing Co., New York, 1972.

4. Environmental Control-Analytical Procedure Manual, POEF 1192, Vol. 2, Martin Marietta Energy Systems, Portsmouth Gaseous Diffusion Plant, Piketon, Ohio.

The LCR may vary among specific samples, depending on interferences in the sample matrix. However, these LCRs have been assigned to ascommodate most minor interferences. Some of the Energy Systems LCRs are higher than the method detection limits (MDLs) listed by EPA. This is consistent with guidance from EPA; however, any data reported below the MDLs must be supported by sound documentation. The LCRs applied by Energy Systems laboratories meet the needs of the programs they support. 
Table 7.2.5. Martin Marietta Energy Systems, Inc. (PORTS), eavironmental analysis procedures for miscellaneous materials

\begin{tabular}{|c|c|c|c|}
\hline \multirow{2}{*}{$\begin{array}{l}\text { Sample } \\
\text { type/ } \\
\text { Parameter }\end{array}$} & \multicolumn{2}{|c|}{ Procedure } & \multirow{2}{*}{$\begin{array}{l}\text { Lowest concen- } \\
\text { tration reported } \\
\qquad(\text { LCR })^{b}\end{array}$} \\
\hline & $\mathrm{ES}^{a}$ & EPA & \\
\hline \multicolumn{4}{|l|}{$\begin{array}{l}\text { Radioactive Waste Materials } \\
\text { (alurnina, sodium } \\
\text { fluoride, solutions) }\end{array}$} \\
\hline $\begin{array}{l}\text { Chromium (total) } \\
\text { Chromium (VI) }\end{array}$ & $\begin{array}{l}2 \cdot \text { E-30-0 } \\
\text { GAT } 507\end{array}$ & 6010 & $\begin{array}{c}0.2 / 0.03 \mathrm{mg} / \mathrm{L} \\
\mathrm{NA}\end{array}$ \\
\hline Iron (total) & $2-J-212-0$ & 6010 & $0.05 / 0.05 \mathrm{mg} / \mathrm{L}$ \\
\hline Nickel (tutal) & & 6010 & $0.05 \mathrm{mg} / \mathrm{L}$ \\
\hline Nitrogen (ammonia) & $2-E-32-0$ & & $0.2 \mathrm{mg} / \mathrm{L}$ \\
\hline Nitrogen (nitrate-nitrate & $2-E-32-0$ & & $0.2 \mathrm{mg} / \mathrm{L}$ \\
\hline Technetium & $\begin{array}{l}\text { 2-G-24-4 } \\
2-G-27-0\end{array}$ & & $\begin{array}{l}2 \times 10^{4} \mathrm{pCi} / \mathrm{kg} \\
300 \mathrm{pCi} / \mathrm{L}\end{array}$ \\
\hline Uraniurn & $\begin{array}{l}2-E-21-1 \\
2-E-24-1\end{array}$ & & $\begin{array}{l}\text { NA } \\
\text { NA }\end{array}$ \\
\hline Uranium daughter beta & $2-G-24-4$ & & NA \\
\hline $\begin{array}{l}{ }^{233} \mathrm{U} \\
\text { Zinc }\end{array}$ & $\begin{array}{l}2-G-29-1 \\
2 \cdot J-222-0\end{array}$ & 6010 & $\begin{array}{c}\mathrm{NA} \\
0.02 / 0.05 \mathrm{mg} / \mathrm{L}\end{array}$ \\
\hline \multicolumn{4}{|l|}{$\begin{array}{l}\text { Special Annual Composites } \\
\text { (air filters, cylinder } \\
\text { cleaning solutions) }\end{array}$} \\
\hline Alpha activity & $\begin{array}{l}2-J-144-1 \\
2-G-30-2\end{array}$ & & $\begin{array}{l}2.0 \mathrm{pCi} / \mathrm{L} \\
2.0 \mathrm{pCi} / \text { filter }\end{array}$ \\
\hline Beta activity & $\begin{array}{l}2-G-27-0 \\
2-G-30-2\end{array}$ & & $\begin{array}{l}300 \mathrm{pCi} / \mathrm{L} \\
100 \mathrm{pCi} / \text { filter }\end{array}$ \\
\hline Transuranics & $2 \cdot G \cdot 21-1$ & & $2.0 \mathrm{pCi} / \mathrm{L}$ \\
\hline Technetium & $\begin{array}{l}2-G-27-2 \\
2-G-30-2\end{array}$ & & $\begin{array}{l}300 \mathrm{pCi} / \mathrm{L} \\
100 \mathrm{pCi} / \text { filter }\end{array}$ \\
\hline Uranium & $\begin{array}{l}2-E-21-1 \\
2-E-24-1 \\
2-J-145-1\end{array}$ & & $\begin{array}{r}\mathrm{NA} \\
\mathrm{NA} \\
0.001 \mathrm{mg} / \mathrm{L}\end{array}$ \\
\hline \multicolumn{4}{|l|}{$\begin{array}{l}\text { Waste Management } \\
\text { Samples (EP Extracts, } \\
\text { organic liquids, oils) }\end{array}$} \\
\hline Barium & EC-401-PORTS & 6010 & $0.2 \mathrm{mg} / \mathrm{L}$ \\
\hline Cadmium & EC-401-PORTS & 6010 & $0.05 \mathrm{mg} / \mathrm{L}$ \\
\hline Chromium & EC-401-PORTS & 6010 & $0.03 \mathrm{mg} / \mathrm{L}$ \\
\hline Lead & EC-401-PORTS & 6010 & $0.05 \mathrm{mg} / \mathrm{L}$ \\
\hline
\end{tabular}


Table 7.2 .5 (continued)

\begin{tabular}{lccc}
\multicolumn{1}{c}{ Parameter } & \multicolumn{1}{c}{ Procedure } & $\begin{array}{c}\text { Lowest concen- } \\
\text { tration reported } \\
(\text { LCR) }\end{array}$ \\
\hline $\begin{array}{l}\text { Sewage Sludge } \\
\text { Alpha activity }\end{array}$ & $E S^{a}$ & EPA & $1.4 \times 10^{3} \mathrm{pCi} / \mathrm{kg}$ \\
$\begin{array}{l}\text { Beta activity } \\
\text { PCB (scan) }\end{array}$ & $2-\mathrm{J}-144-1$ & & $2 \times 10^{4} \mathrm{pCi} / \mathrm{kg}$ \\
$\begin{array}{l}\text { Mercury } \\
\text { Uranium (total) }\end{array}$ & $2-\mathrm{G}-26-2$ & 8080 & $0.4 \mathrm{mg} / \mathrm{kg}$ \\
\hline
\end{tabular}

aprocedures described in:

1. Works Laburatory Procedures Manual, GAT-115, rev. 3, Vols. I and II, Goodyear Atomic Corp, Piketon, Ohio.

2. Determination of Trace Elements in Uranium Compounds-Specification Analyses. GAT-507, Goodyear Atomic Corp., Piketon, Ohic, 1968.

3. Hesse, P. R., A Textbook of SOIL Chemical Analysis, Chemical Publishing Co., New York, 1972.

4. Environmental Control-Analytical Procedure Manual, POEF-1192, Vol. 2, Martin Marietta Energy Systems, Portsmouth Gaseous Diffusion Plant, Piketon, Ohio.

'The LCR may vary among specific samples, depending on interferences in the sample matrix. However, these LCRs have been assigned to accommodate most minor interferences. Some of the Energy Systems LCRs are higher than the method detection limits (MDLs) listed by EPA. This is consistent with guidance from EPA; however, any data reported below the MDLs must be supported by sound documentation. The LCRs applied by Energy Systems laboratories meet the needs of the programs they support. 
Table 7.2.6. EPA EMSL-LV intercomparison study results for PORTS-1989

\begin{tabular}{|c|c|c|c|c|c|c|}
\hline \multirow{2}{*}{$\begin{array}{l}\text { Analysis and } \\
\text { sample date }\end{array}$} & \multicolumn{2}{|c|}{$\begin{array}{c}\text { Value } \\
(\mathrm{pCi} / \mathrm{L})\end{array}$} & \multirow{2}{*}{$\begin{array}{l}\text { Normalized } \\
\text { deviation }\end{array}$} & \multicolumn{3}{|c|}{ Performance evaluation ${ }^{a}$} \\
\hline & EPA & PORTS & & Acceptable & Marginal & Unarceptable \\
\hline \multicolumn{7}{|c|}{ Water } \\
\hline \multicolumn{7}{|l|}{ Alpha } \\
\hline January & 8.0 & 9.3 & 0.5 & 1 & & \\
\hline May & 30.0 & 31.9 & 0.4 & 1 & & \\
\hline \multicolumn{7}{|l|}{ Beta } \\
\hline January & 4.0 & 7.3 & 1.2 & 1 & & \\
\hline May & 50.0 & 61.7 & 4.0 & & & 1 \\
\hline \multicolumn{7}{|l|}{ Uranium } \\
\hline March & 5.0 & 8.3 & 1.0 & 1 & & \\
\hline July & 41.0 & 40.3 & 0.2 & 1 & & \\
\hline \multicolumn{7}{|l|}{ Plutonium } \\
\hline January & 4.2 & 3.4 & 3.6 & & & 1 \\
\hline & & & & 5 & 0 & 2 \\
\hline
\end{tabular}


Table 7.2.7. EPA EML intercomparison study results for PORTS-1989

EML report 525 (August 1989)

\begin{tabular}{|c|c|c|c|c|c|c|}
\hline \multirow{2}{*}{ Analysis } & \multicolumn{2}{|c|}{$\begin{array}{c}\text { Value } \\
\text { (pCi/unit) }\end{array}$} & \multirow{2}{*}{$\begin{array}{c}\text { Ratio } \\
\text { PORTS/EML }\end{array}$} & \multicolumn{3}{|c|}{ Performance evaluation ${ }^{\circ}$} \\
\hline & EML & PORTS & & Acceptable & Marginal & Unacceptable \\
\hline \multicolumn{7}{|c|}{ Water } \\
\hline${ }^{54} \mathrm{Mn}$ & 0.34 & $0.30 \pm 0.15$ & 1.1 & 1 & & \\
\hline${ }^{57} \mathrm{Co}$ & 0.91 & $0.88 \pm 0.13$ & 1.0 & 1 & & \\
\hline${ }^{60} \mathrm{Co}$ & 0.87 & $0.94 \pm 0.12$ & 0.9 & 1 & & \\
\hline${ }^{134} \mathrm{Cs}$ & 2.45 & $2.73 \pm 0.12$ & 0.9 & i & & \\
\hline${ }^{137} \mathrm{Cs}$ & 2.53 & $2.55 \pm 0.13$ & 1.0 & 1 & & \\
\hline${ }^{144} \mathrm{Ce}$ & 1.94 & $1.89 \pm 0.12$ & 1.0 & 1 & & \\
\hline Total uranium $(\mu \mathrm{g})$ & 0.013 & $0.013 \pm 1.0$ & 1.0 & $i$ & & \\
\hline \multicolumn{7}{|c|}{ Air } \\
\hline${ }^{7} \mathrm{Be}$ & 1840 & $1950 \pm 0.11$ & 0.9 & 1 & & \\
\hline${ }^{60} \mathrm{Co}$ & 112 & $126 \pm 0.10$ & 0.9 & 1 & & \\
\hline${ }^{134} \mathrm{Cs}$ & 141 & $158 \pm 0.10$ & 0.9 & 1 & & \\
\hline${ }^{137} \mathrm{Cs}$ & 184 & $189 \pm 0.11$ & 1.0 & 1 & & \\
\hline${ }^{144} \mathrm{Ce}$ & 368 & $327 \pm 0.14$ & 1.1 & $i$ & & \\
\hline Total uranium $(\mu \mathrm{g})$ & 0.46 & $0.27 \pm 0.20$ & 1.7 & & & $1^{b}$ \\
\hline \multicolumn{7}{|c|}{ Soil } \\
\hline${ }^{137} \mathrm{Cs}$ & 22.8 & $20.8 \pm 0.12$ & 1.1 & 1 & & \\
\hline \multicolumn{7}{|c|}{ Vegetation } \\
\hline${ }^{137} \mathrm{Cs}$ & 1.79 & $1.60 \pm 0.13$ & 1.1 & 1 & & \\
\hline Total uranium $(\mu \mathrm{g})$ & 0.034 & $0.033 \pm 0.18$ & 1.0 & 1 & & \\
\hline Total & & & & 15 & 0 & 1 \\
\hline
\end{tabular}


Table 7.2.8. Average performance of PORTS PET controls-1989

\begin{tabular}{|c|c|c|c|c|c|}
\hline \multirow{2}{*}{ Parameter } & \multirow{2}{*}{$\begin{array}{l}\text { Average } \\
\text { percent } \\
\text { recovery }\end{array}$} & \multirow{2}{*}{$\begin{array}{l}\text { Average number of } \\
\text { SDs }^{\circ} \\
\text { from the mean } \\
\text { of all participants }\end{array}$} & \multicolumn{3}{|c|}{ Performance evaluation ${ }^{b}$} \\
\hline & & & $\begin{array}{c}\text { No. } \\
\text { acceptable }\end{array}$ & $\begin{array}{c}\text { No. } \\
\text { marginal }\end{array}$ & $\begin{array}{c}\text { No. } \\
\text { unacceptable }\end{array}$ \\
\hline Biochemical oxygen demand & 70 & 1.25 & 17 & 2 & 1 \\
\hline Chemical oxygen demand ${ }^{c}$ & 93 & 0.22 & 4 & 0 & 0 \\
\hline Total organic carbon & 100 & 0.28 & 48 & 0 & 0 \\
\hline Ammonia nitrogen & 107 & 0.63 & 24 & 0 & 0 \\
\hline Nitrate nitrogen & 94 & 0.63 & 24 & 0 & 0 \\
\hline Total Kjeldahl nitrogen ${ }^{c}$ & 83 & 1.03 & 12 & 0 & 0 \\
\hline Orthophosphate as $\mathrm{P}$ & 102 & 0.22 & 27 & 0 & 0 \\
\hline Total phosphorus & 102 & 0.37 & 24 & 0 & 0 \\
\hline Suspended solids & 96 & 0.68 & 24 & 0 & 0 \\
\hline Dissolved solids & 100 & 0.61 & 24 & 0 & 0 \\
\hline Oil and grease & 93 & 0.39 & 24 & $\mathbf{0}$ & 0 \\
\hline Alkalinity & 98 & 0.35 & 24 & $\mathbf{0}$ & 0 \\
\hline Calcium & 109 & 1.13 & 20 & 2 & 2 \\
\hline Chloride & 105 & 0.93 & 23 & 1 & 0 \\
\hline Conductivity & 89 & 0.30 & 24 & 0 & 0 \\
\hline Magnesium $^{c}$ & 101 & 0.82 & 16 & 0 & 0 \\
\hline Sulfate & 97 & 0.70 & 23 & 1 & 0 \\
\hline Total hardness as $\mathrm{CaCO}_{3}$ & 102 & 0.38 & 24 & 0 & 0 \\
\hline $\mathrm{pH}$ & 104 & 0.52 & 24 & 0 & 0 \\
\hline Aluminum & 99 & 0.64 & 24 & 0 & 0 \\
\hline Arsenic & 86 & 0.71 & 22 & 1 & 1 \\
\hline Barium & 103 & 0.41 & 24 & 0 & 0 \\
\hline Beryllium & 108 & 1.10 & 23 & 0 & 1 \\
\hline Cadmium & 108 & 1.04 & 24 & 0 & 0 \\
\hline Chromium & 104 & 0.45 & 38 & 0 & 0 \\
\hline Copper & 104 & 0.75 & 24 & 0 & 0 \\
\hline Iron & 100 & 0.62 & 23 & 1 & 0 \\
\hline Lead & 94 & 0.57 & 24 & 0 & 0 \\
\hline Manganese & 102 & 0.64 & 24 & 0 & 0 \\
\hline Mercury & 96 & 0.42 & 24 & 0 & 0 \\
\hline Nickel & 109 & 0.89 & 23 & 1 & 0 \\
\hline Selenium ${ }^{c}$ & 89 & 0.72 & 14 & 0 & 0 \\
\hline Silver & 106 & 1.12 & 31 & 2 & 3 \\
\hline Vanadium $^{c}$ & 103 & 0.32 & 18 & 0 & 0 \\
\hline Zinc & 107 & 0.56 & 23 & 1 & 0 \\
\hline Residual chlorine & 88 & 0.37 & 24 & 0 & 0 \\
\hline Fluoride & 99 & 0.54 & 24 & 0 & 0 \\
\hline Total organic halides & 100 & 0.41 & 48 & 0 & 0 \\
\hline Hexavalent chromium ${ }^{d}$ & 100 & 0.52 & 23 & 0 & 1 \\
\hline Uranium & 104 & 0.92 & 46 & $\underline{2}$ & $\underline{0}$ \\
\hline Total & & & 978 & 14 & 9 \\
\hline
\end{tabular}

${ }^{\circ} \mathrm{SD}=$ standard deviation from the mean.

${ }^{b}$ Individual performance was evaluated for both the high- and low-concentration samples on the basis of SD of all participating laboratories: acceptable $=$ less than $1.96 \mathrm{SD}$; marginal $=1.96$ to $2.58 \mathrm{SD}$; and unacceptable $=$ greater than $2.58 \mathrm{SD}$.

'Participation in the program for the parameter indicated was limited to part of the year; therefore, fewer than 24 samples were analyzed.

'One unacceptable result deleted from data. 
Table 7.2.9. EPA Performance Evaluation DMR-QA Study 609 for PORTS

\begin{tabular}{|c|c|c|c|c|c|}
\hline Analytes & $\begin{array}{l}\text { Reported } \\
\text { value }\end{array}$ & $\begin{array}{l}\text { True } \\
\text { value }^{a}\end{array}$ & $\begin{array}{c}\text { Acceptance } \\
\text { limits }\end{array}$ & $\begin{array}{l}\text { Warning } \\
\text { limits }\end{array}$ & $\begin{array}{c}\text { Performance } \\
\text { evaluation }\end{array}$ \\
\hline \multicolumn{6}{|c|}{ Trace metals $(\mu \mathrm{g} / \mathrm{L})$} \\
\hline Arsenic & 189 & 226 & $171-272$ & $184-259$ & Acceptable \\
\hline Chromium & 165 & 150 & $118-179$ & $125 \cdot 171$ & Acceptable \\
\hline Copper & 79 & 76.2 & $65.4-860$ & $67.9-83.4$ & Acceptable \\
\hline Iron & 763 & 749 & $652 \cdots 859$ & $678-833$ & Acceptable \\
\hline Manganese & 1020 & 970 & $886-1050$ & $906-1030$ & Acceptabie \\
\hline Nickel & 682 & 622 & $547-691$ & $565-673$ & Check for error \\
\hline Zinc & 227 & 210 & $181-237$ & $188-230$ & Acceptable \\
\hline \multicolumn{6}{|c|}{ Miscellanecus analytes } \\
\hline $\mathrm{pH}$ (units) & 7.75 & 7.80 & $7.55 \cdots 7.97$ & $7.60-7.92$ & Acceptable \\
\hline Total suspended solids (mg/L) & 42.9 & 41.9 & $33.3-46.6$ & $34.9-45.0$ & Acceptable \\
\hline Oil and grease $(\mathrm{mg} / \mathrm{L})$ & 18.8 & 19.8 & $10.7-24.8$ & $12.5-23.0$ & Acceptable \\
\hline \multicolumn{6}{|c|}{ Nutrients (mg/L.) } \\
\hline Ammonia as nitrogen & 12.6 & 13.0 & $10.2 \cdots 15.5$ & $10.9 \ldots 14.9$ & Acceptable \\
\hline Nitrate as nitrogen & 7.0 & 8.50 & $6.94 \cdots 10.1$ & $7.32-9.72$ & Check for Error \\
\hline Kjeldahl as nitrogen & 15.10 & 15.0 & $11.4 \cdots 18.2$ & $12.2-17.4$ & Acceptable \\
\hline \multicolumn{6}{|c|}{ Demands, $(m g / L)$} \\
\hline $5 \cdot d \mathrm{BOD}$ & 41.5 & 59.7 & $41.7 \cdot 85.7$ & $47.2-80.3$ & Not acceptable \\
\hline \multicolumn{6}{|c|}{ Additional miscellaneous analytes } \\
\hline Total residual chlorine $(\mathrm{mg} / \mathrm{L})$ & 1.83 & 2.00 & $1.35 \cdots 2.53$ & $1.51-2.38$ & Acceptable \\
\hline $\mathrm{pH}$ (units) & 5.82 & 5.80 & $5.66-5.91$ & $5.69 \cdots 5.88$ & Acceptable \\
\hline Total suspended solids (mg/L) & 32.2 & 29.7 & $24.2-33.3$ & $25.3 \cdots 32.2$ & Acceptable \\
\hline Oil and grease $(\mathrm{mg} / \mathrm{L})$ & 11.2 & 12.0 & $6.32 \cdots 16.4$ & $7.57-15.1$ & Acceptable \\
\hline
\end{tabular}

${ }^{a}$ Based on theoretical calculations or a reference value when necessary.

${ }^{b}$ The performance evaluation shown is the one reported by the EPA. For the purpose of this report, EPA acceptance limits are considered acceptable limits, the EPA notation "check for error" is indicative of a marginal value, and "not acceptable" is considered an outlier. 
Table 7.2.10. DOE intercomparison results for PORTS in the PETC coal program-1989

\begin{tabular}{|c|c|c|c|c|}
\hline \multirow{2}{*}{ Sample ID } & \multicolumn{2}{|c|}{ Value, moisture free } & \multirow{2}{*}{$\begin{array}{l}\text { PORTS/ } \\
\text { PETC }\end{array}$} & \multirow{2}{*}{ Performance evaluation ${ }^{a}$} \\
\hline & PETC & PORTS & & \\
\hline \multicolumn{5}{|c|}{ Volatile matter (\%) } \\
\hline January & 37.09 & 37.95 & 1.0 & Acceptable \\
\hline March & 18.17 & 20.74 & 1.1 & Acceptable \\
\hline May & 38.49 & 38.07 & 1.0 & Acceptable \\
\hline \multicolumn{5}{|c|}{ Fixed carbon (\%) } \\
\hline January & 53.85 & 52.99 & 1.0 & Acceptable \\
\hline March & 76.75 & 74.19 & 1.0 & Acceptable \\
\hline May & 57.11 & 57.51 & 1.0 & Acceptable \\
\hline \multicolumn{5}{|c|}{ Ash (\%) } \\
\hline January & 9.06 & 9.06 & 1.0 & Acceptable \\
\hline March & 5.08 & 5.07 & 1.0 & Acceptable \\
\hline May & 4.40 & 4.42 & 1.0 & Acceptable \\
\hline \multicolumn{5}{|c|}{ Sulfur (\%) } \\
\hline January & 1.63 & 1.84 & 1.1 & Acceptable \\
\hline March & 0.53 & 0.67 & 1.3 & Marginal \\
\hline May & 1.11 & 1.27 & 1.1 & Acceptable \\
\hline \multicolumn{5}{|c|}{ Heating value (Btu/lb) } \\
\hline January & 13,238 & 13,149 & 1.0 & Acceptable \\
\hline March & 14,834 & 14,889 & 1.0 & Acceptable \\
\hline May & 14,446 & 14,415 & 1.0 & Acceptable \\
\hline
\end{tabular}

aperformance evaluation is based on a ratio of the PORTS analytical results to the PETC reference value: acceptable $=0.8$ to 1.2 ; marginal $=$ 0.5 to 1.5 ; unacceptable $=$ outside marginal limits. 
Table 7.2.11. NIOSH intercomparison results for PORTS in the Proficiency Analytical Testing Program (PAT)-1989

\begin{tabular}{|c|c|c|c|c|}
\hline \multicolumn{5}{|c|}{ Rounds 96-99 } \\
\hline \multirow{2}{*}{ Analysis } & \multicolumn{2}{|c|}{ Value (mg) } & \multirow{2}{*}{$\begin{array}{c}\text { Ratio } \\
\text { PORTS/PAT }\end{array}$} & \multirow{2}{*}{ Performance evaluation ${ }^{\circ}$} \\
\hline & PAT & PORTS & & \\
\hline & & & Lead & \\
\hline \multirow[t]{4}{*}{$2 / 28$} & 0.0395 & 0.0425 & 1.1 & Acceptable \\
\hline & 0.0224 & 0.0240 & 1.1 & Acceptable \\
\hline & 0.0785 & 0.0771 & 1.0 & Acceptable \\
\hline & 0.0523 & 0.0544 & 1.0 & Acceptable \\
\hline \multirow{4}{*}{$6 / 01$} & 0.0175 & 0.0150 & 0.9 & Acceptable \\
\hline & 0.0310 & 0.0282 & 0.9 & Acceptable \\
\hline & 0.0585 & 0.0570 & 1.0 & Acceptable \\
\hline & 0.0434 & 0.0440 & 1.0 & Acceptable \\
\hline \multirow[t]{4}{*}{$8 / 28$} & 0.0774 & 0.0820 & 1.1 & Acceptable \\
\hline & 0.0622 & 0.0675 & 1.1 & Acceptable \\
\hline & 0.0515 & 0.0548 & 1.1 & Acceptable \\
\hline & 0.0348 & 0.0358 & 1.0 & Acceptable \\
\hline \multirow[t]{4}{*}{$11 / 21$} & 0.0485 & 0.0490 & 1.0 & Acceptable \\
\hline & 0.0658 & 0.0664 & 1.0 & Acceptable \\
\hline & 0.0334 & 0.0325 & 1.0 & Acceptable \\
\hline & 0.0462 & 0.0426 & 1.0 & Acceptable \\
\hline \multicolumn{5}{|c|}{ Cadmium } \\
\hline \multirow[t]{4}{*}{$2 / 28$} & 0.0079 & 0.0079 & 1.0 & Acceptable \\
\hline & 0.0125 & 0.0126 & 1.0 & Acceptable \\
\hline & 0.0176 & 0.0175 & 1.0 & Acceptable \\
\hline & 0.0101 & 0.0101 & 1.0 & Acceptable \\
\hline \multirow[t]{4}{*}{$6 / 01$} & 0.0187 & 0.0155 & 0.8 & Outlier \\
\hline & 0.0139 & 0.0120 & 0.9 & Outlier \\
\hline & 0.0090 & 0.0091 & 1.0 & Acceptable \\
\hline & 0.0069 & 0.0072 & 1.0 & Acceptable \\
\hline \multirow[t]{4}{*}{$8 / 28$} & 0.0129 & 0.0139 & 1.1 & Acceptable \\
\hline & 0.0060 & 0.0065 & 1.1 & Acceptable \\
\hline & 0.0098 & 0.0106 & 1.1 & Acceptable \\
\hline & 0.0167 & 0.0175 & 1.0 & Acceptable \\
\hline \multirow[t]{4}{*}{$11 / 21$} & 0.0119 & 0.0123 & 1.0 & Acceptable \\
\hline & 0.0148 & 0.0152 & 1.0 & Acceptable \\
\hline & 0.0090 & 0.0091 & 1.0 & Acceptable \\
\hline & 0.0100 & 0.0103 & 1.0 & Acceptable \\
\hline \multicolumn{5}{|c|}{ Zinc } \\
\hline \multirow[t]{4}{*}{$2 / 28$} & 0.0880 & 0.0834 & 0.9 & Acceptable \\
\hline & 0.1222 & 0.1171 & 1.0 & Acceptable \\
\hline & 0.2091 & 0.1988 & 1.0 & Acceptable \\
\hline & 0.1521 & 0.1478 & 1.0 & Acceptable \\
\hline \multirow[t]{4}{*}{$6 / 01$} & 0.1328 & 0.1054 & 0.8 & Outlier \\
\hline & 0.1718 & 0.1412 & 0.8 & Outlier \\
\hline & 0.1031 & 0.1006 & 1.0 & Acceptable \\
\hline & 0.1589 & 0.1548 & 1.0 & Acceptable \\
\hline \multirow[t]{4}{*}{$8 / 28$} & 0.1022 & 0.1053 & 1.0 & Acceptable \\
\hline & 0.1369 & 0.1386 & 1.0 & Acceptable \\
\hline & 0.0762 & 0.0781 & 1.0 & Acceptable \\
\hline & 0.1807 & 0.1808 & 1.0 & Acceptable \\
\hline
\end{tabular}


Table 7.2.11 (continued)

\begin{tabular}{|c|c|c|c|c|}
\hline \multirow{2}{*}{ Analysis } & \multicolumn{2}{|c|}{ Value (mg) } & \multirow{2}{*}{$\begin{array}{c}\text { Ratio } \\
\text { PORTS/PAT }\end{array}$} & \multirow{2}{*}{ Performance evaluation ${ }^{a}$} \\
\hline & PAT & PORTS & & \\
\hline \multirow[t]{5}{*}{$11 / 21$} & 0.0885 & 0.0895 & 1.0 & Aeceptable \\
\hline & 0.1170 & 0.1179 & 1.0 & Acceptable \\
\hline & 0.1589 & 0.1578 & 1.0 & Acceptable \\
\hline & 0.1909 & 0.1917 & 1.0 & Acceptable \\
\hline & \multicolumn{3}{|c|}{ Silica } & \\
\hline \multirow[t]{4}{*}{$2 / 28$} & 0.1050 & 0.1002 & 1.0 & Acceptable \\
\hline & 0.0740 & 0.0687 & 0.9 & Acceptable \\
\hline & 0.0401 & 0.0442 & 1.1 & Acceptable \\
\hline & 0.0534 & 0.0264 & 0.5 & Acceptable \\
\hline \multirow[t]{4}{*}{$6 / 01$} & 0.0863 & 0.0857 & 1.0 & Acceptable \\
\hline & 0.0805 & 0.0788 & 1.0 & Acceptable \\
\hline & 0.1332 & 0.1554 & 1.2 & Acceptable \\
\hline & 0.0449 & 0.0416 & 0.9 & Acceptable \\
\hline \multirow[t]{4}{*}{$8 / 28$} & 0.0817 & 0.0787 & 1.0 & Acceptable \\
\hline & 0.0676 & 0.660 & 1.0 & Acceptable \\
\hline & 0.1074 & 0.1012 & 0.9 & Acceptable \\
\hline & 0.1674 & 0.1523 & 0.9 & Acceptable \\
\hline \multirow[t]{4}{*}{$11 / 21$} & 0.0603 & 0.0489 & 0.8 & Acceptable \\
\hline & 0.0887 & 0.0738 & 0.8 & Acceptable \\
\hline & 0.1962 & 0.1625 & 0.8 & Acceptable \\
\hline & 0.1341 & 0.1132 & 0.8 & Acceptable \\
\hline \multicolumn{5}{|c|}{ Asbestos } \\
\hline \multirow[t]{4}{*}{$2 / 28$} & 466.9 & 393.1 & 0.8 & Acceptable \\
\hline & 666.6 & 637.4 & 1.0 & Acceptable \\
\hline & 305.9 & 325.5 & 1.1 & Acceptable \\
\hline & 944.3 & 1341.4 & 1.4 & Acceptable \\
\hline \multirow[t]{4}{*}{$6 / 01$} & 247.1 & 329.3 & 1.3 & Acceptable \\
\hline & 731.1 & 1445.2 & 2.0 & Acceptable \\
\hline & 549.4 & 932.5 & 1.7 & Acceptable \\
\hline & 795.8 & 1034.4 & 1.3 & Acceptable \\
\hline \multirow[t]{4}{*}{$8 / 28$} & 802.5 & 1194.3 & 1.5 & Acceptable \\
\hline & 1130.9 & 1264.3 & 1.1 & Acceptable \\
\hline & 602.2 & 681.5 & 1.1 & Acceptable \\
\hline & 371.7 & 522.3 & 1.4 & Acceptable \\
\hline \multirow[t]{4}{*}{$11 / 21$} & 148.5 & 223.5 & 1.5 & Acceptable \\
\hline & 399.6 & 145.9 & 0.4 & Acceptable \\
\hline & 823.6 & 1117.8 & 1.4 & Acceptable \\
\hline & 241.4 & 364.0 & 1.5 & Acceptable \\
\hline \multicolumn{5}{|c|}{ Solvent I } \\
\hline \multirow[t]{4}{*}{$2 / 28$} & 0.6518 & 0.6440 & 1.0 & Acceptable \\
\hline & 0.8600 & 0.8720 & 1.0 & Acceptable \\
\hline & 1.0786 & 1.0600 & 1.0 & Acceptable \\
\hline & 0.3852 & 0.3820 & 1.0 & Acceptable \\
\hline \multirow[t]{4}{*}{$6 / 01$} & 0.6971 & 0.7200 & 1.0 & Acceptable \\
\hline & 0.9318 & 0.9250 & 1.0 & Acceptable \\
\hline & 0.3610 & 0.3610 & 1.0 & Acceptable \\
\hline & 0.5036 & 0.5150 & 1.0 & Acceptable \\
\hline
\end{tabular}


Table 7.2.11 (continued)

\begin{tabular}{|c|c|c|c|c|}
\hline \multirow{2}{*}{ Analysis } & \multicolumn{2}{|c|}{ Value (mg) } & \multirow{2}{*}{$\begin{array}{c}\text { Ratio } \\
\text { PORTS/PAT }\end{array}$} & \multirow{2}{*}{ Performance evaluation ${ }^{a}$} \\
\hline & PAT & PORTS & & \\
\hline \multirow[t]{4}{*}{$8 / 28$} & 0.1418 & 0.1690 & 1.2 & Acceptable \\
\hline & 0.1754 & 0.1650 & 0.9 & Acceptable \\
\hline & 0.2704 & 0.2950 & 1.1 & Acceptable \\
\hline & 0.2337 & 0.2580 & 1.1 & Acceptable \\
\hline \multirow[t]{5}{*}{$11 / 21$} & 0.3184 & 0.2910 & 0.9 & Acceptable \\
\hline & 1.0516 & 1.0650 & 1.0 & Acceptable \\
\hline & 0.8143 & 0.8020 & 1.0 & Acceptable \\
\hline & 0.6019 & 0.5560 & 0.9 & Acceptable \\
\hline & & & Solvent 2 & \\
\hline \multirow[t]{4}{*}{$2 / 28$} & 0.6848 & 0.6660 & 1.0 & Acceptable \\
\hline & 0.8276 & 0.8230 & 1.0 & Acceptable \\
\hline & 0.5403 & 0.5170 & 1.0 & Acceptable \\
\hline & 1.1063 & 1.1100 & 1.0 & Acceptable \\
\hline \multirow[t]{4}{*}{$6 / 01$} & 0.7702 & 0.8210 & 1.1 & Acceptable \\
\hline & 0.3670 & 0.3750 & 1.0 & Acceptable \\
\hline & 1.0115 & 1.0500 & 1.0 & Acceptable \\
\hline & 0.8082 & 0.8290 & 1.0 & Acceptable \\
\hline \multirow[t]{4}{*}{$8 / 28$} & 1.0565 & 1.1900 & 1.1 & Outlier \\
\hline & 1.4097 & 1.3300 & 0.9 & Acceptable \\
\hline & 0.9372 & 0.9950 & 1.1 & Acceptable \\
\hline & 1.5765 & 1.7100 & 1.1 & Acceptable \\
\hline \multirow[t]{4}{*}{$11 / 21$} & 1.0249 & 1.0510 & 1.0 & Acceptable \\
\hline & 0.5183 & 0.5360 & 1.0 & Acceptable \\
\hline & 0.7673 & 0.7930 & 1.0 & Acceptable \\
\hline & 0.6428 & 0.6430 & 1.0 & Acceptable \\
\hline \multicolumn{5}{|c|}{ Solvent 3} \\
\hline \multirow[t]{4}{*}{$2 / 28$} & 1.1675 & 1.1500 & 1.0 & Acceptable \\
\hline & 0.7561 & 0.7570 & 1.0 & Acceptable \\
\hline & 1.0490 & 1.0300 & 1.0 & Acceptable \\
\hline & 0.5883 & 0.5900 & 1.0 & Acceptable \\
\hline \multirow[t]{4}{*}{$6 / 01$} & 1.0080 & 1.0500 & 1.0 & Acceptable \\
\hline & 1.1524 & 1.1000 & 1.0 & Acceptable \\
\hline & 0.9205 & 0.9300 & 1.0 & Acceptable \\
\hline & 0.7835 & 0.8070 & 1.0 & Acceptable \\
\hline \multirow[t]{4}{*}{$8 / 28$} & 1.0315 & 1.1800 & 1.1 & Outlier \\
\hline & 1.3212 & 1.2500 & 0.9 & Acceptable \\
\hline & 1.5831 & 1.6900 & 1.1 & Acceptable \\
\hline & 0.8665 & 0.9540 & 1.1 & Acceptable \\
\hline \multirow[t]{4}{*}{$11 / 21$} & 0.4452 & 0.4590 & 1.0 & Acceptable \\
\hline & 0.7930 & 0.8340 & 1.1 & Acceptable \\
\hline & 0.5760 & 0.5910 & 1.0 & Acceptable \\
\hline & 0.9006 & 0.9100 & 1.0 & Acceptable \\
\hline
\end{tabular}




\section{REFERENCES}

American National Standards Institute (ANSI) 1975. Performance Testing and Procedural Specifications for Thermoluminescent Dosimetry (Environmental Applications).

Begovich, C. L. et al August 1981. DART AB: A Program to Combine Airborne Radionuclide Environmental Exposure Data with Dosimetric and Health Effects Data To Generate Tabulations of Predicted Health Impacts, ORNL-5692, Union Carbide Corp. Nuclear Div., Oak Ridge Natl. Lab.

Dunning, D. E., Jr., Leggett, R. W., and Yalcintas, M. G. 1980. A Combined Methodology for Estimating Dose Rates and Health Effects from Radioactive Pollutants, ORNL/TM-7105, Union Carbide Corp. Nuclear Div., Oak Ridge Natl. Lab.

Geraghty and Miller, Inc. May 1989. Quadrant I Description of Current Conditions, Dublin, Ohio.

Geraghty and Miller, Inc. June 1989. Groundwater Quality Assessment of Four RCRA Units. Dublin, Ohio.

Hoffman, F. O., et al. 1984. Preliminary Screening of Contaminants in Sediments, ORNL/TM-9370, Union Carbide Corp. Nuclear Div., Oak Ridge Natl. Lab.

International Commission on Radiologica! Protection (ICRP) 1977. Annals of the ICRP l, ICRP Publication 26, Pergamon, Oxford, N.Y.

International Commission on Radiological Protection (ICRP) 1978. Publication 30, Pergamon, Oxford, N.Y.

Keller, K. K. Apr. 18, 1989. Off-site Residential Drinking Water Quality Monitoring Plan, Martin Marietta Energy Systems, Portsmouth Gaseous Diffusion Plant.
Kim'srough, C. W., Long, L. W., and McMahon, L. W., eds. Sept. 1, 1988. Environmental Surveillance Procedures Quality Control Program, ESH/Sub/87-21706/1, Advanced Sciences, Oak Ridge, Tenn.

Law Engineering 1978. Gas Centrifuge Enrichment Plant-Geotechnical Investigation, Vols. 1-4, Marietta, Ga.

Mitchell, M. E. Apr. 18, 1990. Environmental and Safety Activities, Martin Marietta Energy Systems, Inc., Oak Ridge Gaseous Diffusion Plant, Oak Ridge, Tenn., letter to P. J. Gross, Environmental Protection Division, Oak Ridge Operations Office, Oak Ridge, Tenn., "Annual Radionuclide Air Emissions Report for the Oak Ridge Reservation (ORR), Paducah Gaseous Diffusion Plant (PGDP), and the Portsmouth Gaseous Diffusion Plant (PORTS) for CY 1989.-40 CFR 61.94(ع)."

Moore, R. E. et al. 1979. AIRL JS-EPA: A Computerized Methodology for Estimating Environmental Concentrations and Dose to Man from Airborne Releases of Radionuclides, EPA-520/1-79.009.

Munro, N. B., and Travis, C. C. 1986. "DrinkingWater Standards," Environ. Sci. Technol. 20(8), 768-69.

Portsmouth Gascous Diffusion Plant (PORTS) December 1988. Biodenitrification Optimization Report, POEF-P-128.

Portsmouth Gaseous Diffusion Plant (PORTS) March 1990. Biodenitrification Optimization Report.

Sittig, M. 1980. Priority Toxic Pollutants: Health Impact and Allowable Limits, Noyes Data Corp., Parkridge, N.J., p. 370.

Sjoreen, A. L. and Miller, C. W. 1984. PREPAR-A User-Friendly Preprocessor To 
Create AIRDOS-EPA Input Data Sets, ORNL.5952, Martin Mariet:a Energy

Systems, Oak Ridge Natl. Lab.

U.S. Department of Energy (DOE). DOE Manual, DOE Order 5400.1.

U.S. Department of Energy (DOE), DOE Manual, DOE Order 5480.2 .

U.S. Department of Energy (DOE). DOE Manual, DOE Order 5632.1.

U.S. Department of Energy (DOE). DOE Manual, DOE Order 5820.2A.
U.S. Environmental Protection Agency (EPA) November 1986. Test Methods for Evaluating Solid Wastes, Rev. 3, SW-846.

U.S. Environmental Protection Agency (EPA) 1986. Superfund Public Health Evaluation Manual, EPA/540/1-86/060, Office of Emergency and Remedial Response.

U.S. Environmental Protection Agency (EPA) 1988. Integrated Risk Information System (IRIS) Data Base.

U.S. Geological Survey (USGS) 1986. Water Resource Data for Ohio. 


\section{Appendix}

\section{PORTSMOUTH GASEOUS DIFFUSION PLANT CHEMICAL RELEASE DATA}

In addition to indicating the concentrations of various chemicals present in the environment near U.S. Department of Energy (DOE) facilities, in recent years the annual Environmental Reports have contained an estimate of the quantities of certain chemicals being en itted to the environment. This appendix contains an expanded list of chemicals with additional information regarding the types of releases, the estimated quantities released, the major processes contributing to the releases, and a brief description of the basis of estimates for calendar year (CY) 1989. Radiological chemical releases ${ }^{\sim}$ CY 1989 are not included in this appendix; the are reported in the applicable sections in this report.

Three categories of chemical releases at each Portsmouth Gaseous Diffusion Plant (PORTS) facility are reported in this appendix: (1) SARA 313, (2) Other Large Inventory Chemicals, and (3) Steam Plant Emissions. The SARA 313 chemicals are summarized from information currently being compiled for the SARA Title III, Section 313 report, required by the Superfund Amendments and Keauthorization Act of 1986 (SARA). This report is submitted on July 1 of each year for the previous calendar year and contains chemicals on the Environmental Protection Agency's (EPA's) toxic substance list. Currently, 309 specific chemicals and 20 chemical categories must be reviewed and possibly reported under SARA Sect. 313. If any of these chemicals were manufactured in excess of $25,000 \mathrm{lb}$, processed in excess of $25,000 \mathrm{lb}$, or "otherwise used" in excess of $10,000 \mathrm{lb}$ at a facility during CY 1989, the chemical must be reported. In many instances the estimate of quantities released was obtained via material balance calculations, monitoring data, or engineering calculations. In some cases no quantitative monitoring data, inventory estimates, or emission factors were readily available, and release estimates were based on "best engineering judgement." "Best engineering judgement" was the principal method used to derive "Quantity released." Information obtained from air permits, rate of operation, quantities used, and known treatment efficiencies were used to estimate quantities released into the environment. Typically, ascumptions based on "best engineering judgement" were required in order to perform the calculations when all variables were not known. Considerable manpower was expended reviewing chemical inventory information and estimating the quintities released to the environment.

The second category of chemicals reported in this appendix is "Other large inventory chemicals." This listing is included to provide additional chemical information not reportable under SARA 313. Note that this is not a complete listing of all chemicals that may have been released at the site. This list was developed to better inform the reader of the additional chemicals (i.e., those that may be of interest to the general public) used and released at PORTS.

The third category, "Steam plant emissions," is release estimates of certain chemicals from the coal-fired steam plants.

Information contained in this appendix may not coincide for all chemicals with the information to be reported under SARA, Title III, Sect. 313. The SARA 313 report must be submitted to the EPA and the Ohio Environmental Protection Agency no later than July 1, 1990. The information for this appendix was generated in the March and April time frame; additional refinements are under way. It is imperative that May and June be utilized to ensure compliance under SARA Title III, the community right-toknow law. The following table summarizes the chemical release information for PORTS. 
Portsmouth Gaseons Diffusion Plant 1989 chemical retease information

\begin{tabular}{|c|c|c|c|c|}
\hline Chemical name & $\begin{array}{l}\text { Type of } \\
\text { release }\end{array}$ & $\begin{array}{l}\text { Quantity } \\
\text { released } \\
\left(\mathrm{Ib} / \mathrm{kg}_{\mathrm{g}}\right)\end{array}$ & Major release sources & $\begin{array}{l}\text { Basis of } \\
\text { estimate }\end{array}$ \\
\hline \multicolumn{5}{|c|}{$S A R A 3 / 3$} \\
\hline Acetone & Air: fugitive & $8,000 / 3,600$ & $\begin{array}{l}\text { Miscellaneous equipment } \\
\text { cleaning }\end{array}$ & Material balance \\
\hline \multirow[t]{3}{*}{ Chlorine } & Water: Scioto River & $<1$ & Sewage Treatment Facility & Monitoring \\
\hline & Little Beaver Creek & $<1$ & Water treatment & Monitoring \\
\hline & Air: point source & $100,000 / 46,000$ & Cooling tower & Other \\
\hline \multirow[t]{3}{*}{ Chromium $^{\circ}$} & Air: stack & $1,800 / 8.30$ & $\begin{array}{l}\text { Recirculating cooling water } \\
\text { system }\end{array}$ & Material balance \\
\hline & Water: Scioto River & $750 / 340$ & $\begin{array}{l}\text { Recirculating cooling water } \\
\text { system }\end{array}$ & Monitoring \\
\hline & Land: other & $420 / 180$ & $\begin{array}{l}\text { Spill-XX-752 hazardous } \\
\text { waste storage }\end{array}$ & Monitoring \\
\hline \multirow[t]{4}{*}{ Ethylene glycol } & Air: fugitive & $3,400 / 1,500$ & $\begin{array}{l}\text { Air-conditioning systems/ } \\
\text { steam plant }\end{array}$ & Other \\
\hline & Water: Big Run Creek & $16,000 / 7,300$ & $\begin{array}{l}\text { Air-conditioning systems/ } \\
\text { steam plant }\end{array}$ & Other \\
\hline & Water: unnàmed tributary & $720 / 330$ & Air-conditioning systems & Other \\
\hline & Land: other & $9.200 / 4,200$ & Steam plant & Other \\
\hline Freon 113 & Air: fugitive & $15,000 / 6,800$ & $\begin{array}{l}\text { Miscellaneous equipment } \\
\text { cleaning }\end{array}$ & Material balance \\
\hline Hydrogen fluoride & Air: stack & $24,000 / 11,000$ & Production vents & Monitoring \\
\hline \multirow[t]{2}{*}{ Methanol } & Air: fugitive & $4,000 / 1,800$ & Biodenitrification Facility & Other \\
\hline & Air: stack & $110 / 48$ & Biodenitrification Facility & Other \\
\hline $\begin{array}{l}\text { Sodium hydroxide } \\
\text { (solution) }\end{array}$ & Land: other & $5 / 2$ & Spill & Other ${ }^{b}$ \\
\hline Sulfuric acid & Land: other & $5 / 2$ & Spill-acid storage areas & Others \\
\hline \multirow[t]{3}{*}{ 1,1,1-Trichloroethane } & Air: fugitive & $26,000 / 12,000$ & $\begin{array}{l}\text { Miscellaneous equipment } \\
\text { cleaning }\end{array}$ & Material balance \\
\hline & Air: stack & $230 / 100$ & $\begin{array}{l}\text { Miscellaneous oquipment } \\
\text { cleaning }\end{array}$ & Other \\
\hline & Water: Little Beaver Creek & $<1 /<0.5$ & Contaminated groundwater & Monitoring \\
\hline \multicolumn{5}{|c|}{ Other large inventory chemicals } \\
\hline Freon 12 & Air: fugitive & $4,400 / 2,000$ & Air-conditioning systerns & Other \\
\hline Freon 22 & Air: fugitive & $1,700 / 770$ & Air-conditioning systems & Other \\
\hline Freon 114 & Air: fugitive & $180,000 / 81,000$ & Air-conditioning systems & Other \\
\hline \multicolumn{5}{|c|}{ Steam plant } \\
\hline Sulfur dioxide & Air: stack & $5,600,000 / 2,500,000$ & $\begin{array}{l}\text { Steam plant } \\
\text { ernissions }\end{array}$ & Other \\
\hline $\mathrm{NO}_{x}$ & Air: stack & $620,000 / 280,000$ & $\begin{array}{l}\text { Fossil fuels } \\
\text { combustion }\end{array}$ & Emission factors ${ }^{c}$ \\
\hline $\mathrm{CO}$ & Air: stack & $83,000 / 38,000$ & $\begin{array}{l}\text { Fossil fuels } \\
\text { combustion }\end{array}$ & Emission factors \\
\hline Particulates & Air: stack & $1,800 / 820$ & $\begin{array}{l}\text { Fossil fuels } \\
\text { combustion }\end{array}$ & Emission factors ${ }^{c}$ \\
\hline
\end{tabular}

"Quantity calculated as chromium; chemical compound is sodium bichromate

${ }^{b}$ Based on best engineering judgement.

'Based on AP.42 emission factors. 
ES/ESH-13/V4

POEF-2025

\section{INTERNAL DISTRIBUTION}

1. M. R. Aaron

2. R. E. Anderson

3. A. D. Arms

4. L. D. Bates

5. B. A. Berven

6-26. R. E. Blake

27. W. J. Boegly

28. D. J. Bostock

29. J. B. Cannon

30. W. W. Chance

31. R. B. Clapp

32. M. S. Dill

33. R. S. Eby

34. J. S. Eldridge

35. M. W. Francis

36. D. W. Frazier

37. A. H. Geisler

38. C. G. Giltner

39. S. T. Goodpasture

40. G. Goslow

41-91. R. L. Grant

92. C. C. Hill

93. D. D. Huff

94. V. A. Jacobs

95. T. G. Jett

96. S. V. Kaye

97. R. H. Ketelle

98. R. M. Keyser

99. C. W. Kimbrough

100. F. C. Kornegay

101. E. H. Krieg

102. W. R. Laing

103. J. M. Loar

104. P. Y. Lu

105. F. C. Maienschein

106. L. W. McMahon

107. L. J. Mezga

108. J. R. Merriman
109. M. E. Mitchell

110. R. W. Morrow

111. T. E. Myricí

112. F. R. ODonnell

113. G. V. Pierce

114. H. Pulley

115. J. G. Rogers

116. J. E. Shoemaker

117. S. P. N. Singh

118. M. A. Smith

119. G. W. Snyder

120. K. W. Sommerfeld

121. A. J. Stewart

122. S. H. Stow

123. D. W. Swindle

124. M. F. Tardiff

125. F. G. Taylor, Jr.

126. C. C. Travis

127. J. W. Turner

128. L. D. Voorhees

129. G. E. Ward

130. C. W. Weber

131-190. D. C. West

191. M. C. Wiest

192. J. K. Williams

193. W. R. Williams

194. A. R. Wilson

195. J. P. Witherspoon

196. S. W. Wohlfort

197. Central Research Library

198-199. ORNL Laboratory Records

200. ORNL Laboratory Records-RC

201. Y-12 Technical Library

202-206. PGDP Library

207-213. PORTS Library

214. ORGDP Library

215. Patent Section 


\section{EXTERNAL DISTRIBUTION}

216. Assistant Manager for Energy Research and Development, U.S. Department of Energy/Oak Ridge Operations, P.O. Box E, Oak Ridge, TN 37831

217-226. Office of Scientific and Technical Information

227-400. This report is distributed widely by the Department of Energy's Oak Ridge Operations Office to local, state, and federal government agencies, the Congress, the public, and the news media. 


\section{ERRATA}

The following corrections should be made to the 1988 report.

\section{Part 1}

Pages 21 and 23, Subsect. 2.1.2.2. The two paragraphs under "1988 ambient gaseous fluorides" should be as follows:

Table 2.1.4 presents a summary of the weekly ambient-fluoride-sampling program in the same format as the previous tables. In this table the standards used are state ambient air standards from Kentucky and Tennessee. Although these standards are not legally binding on an Ohio facility, neither the state of Ohio nor the federal EPA has issued standards for ambient gaseous fluorides. Note that only gaseous fluorides are included. The standards exclude particulate fluorides. There is wide disagreement between states that have issued ambient fluoride standards: Kentucky has set a primary (i.e., public health) standard of 400 $\mu \mathrm{g} / \mathrm{m}^{3}$ and a secondary (i.e., public welfare) standard of $0.8 \mu \mathrm{g} / \mathrm{m}^{3}$; Tennessee has set both primary and secondary standards at $1.6 \mu \mathrm{g} / \mathrm{m}^{3}$ (i.e., a 7-day average); and Montana and Idaho do not use airborne concentrations at all (standards are based on fluoride concentrations in local vegetation instead). PORTS also measures vegetation fluoride levels (Sect. 2.4.5); these measurements may give a more accurate idea of the environmental impact of plant fluoride emissions.

Table 2.1 .4 reflects a summary of all of the fluoride samples collected during 1988. These results are consistently higher than those obtained during 1987 in that 2 of the 17 air stations (i.e., A39 and A40) had average fluoride concentrations that exceeded the Kentucky standard of $0.8 \mu \mathrm{g} / \mathrm{m}^{3}$, and one air station (i.e., A24) had concentrations that equaled it. No definite reason for this was identified, but the most plausible possibilities are (1) a pervasive analytical or sampling error or (2) an unidentified source of fluoride emissions upwind of the PORTS site. The two with the highest results, A39 and A40, are located on-site at $\mathrm{X}-760$ and X-100 respectively. Both of these stations are located in close proximity to the X-326 process building, the major fluoride emitter on the plant site. A24 is located due north of the plant directly upwind of the most prevalent wind direction. However, the 1988 fluoride level exceeded the 1987 levels at 11 of the 13 stations at which fluorides were sampled in 1987. The levels at two stations, A6 and A8, both of which were downwind of the prevailing wind direction, actually decreased.

Page 23, Table 2.1.4. This table should be replaced with that shown in this Errata.

\section{Part 2}

Page 129, Table 2.1.7. This table should be replaced with that shown in this Errata. 
Table 2.1.4. 1988 total fluoride concentrations (ws HF) in ambient air

\begin{tabular}{|c|c|c|c|c|c|c|}
\hline \multirow{2}{*}{ Location } & \multirow{2}{*}{$\begin{array}{l}\text { No. of } \\
\text { samples }^{a}\end{array}$} & \multicolumn{3}{|c|}{$\begin{array}{c}\text { Concentration } \\
\left(\mu \mathrm{g} / \mathrm{m}^{3}\right)\end{array}$} & \multirow{2}{*}{$\begin{array}{l}\text { Standard } \\
\text { deviation }\end{array}$} & \multirow{2}{*}{$\begin{array}{c}\text { Ky./Tenn } \\
\text { standards } \\
\left(\mu g / \mathrm{m}^{3}\right)\end{array}$} \\
\hline & & Max & Min & Av & & \\
\hline \multicolumn{7}{|c|}{ Off-site } \\
\hline A3 & 38 & 3.38 & 0.04 & 0.74 & 0.91 & $0.8 / 1.6$ \\
\hline A6 & 37 & 2.30 & 0.05 & 0.42 & 0.45 & \\
\hline A8 & 34 & 2.33 & 0.07 & 0.46 & 0.52 & \\
\hline A9 & 35 & 3.23 & 0.08 & 0.59 & 0.79 & \\
\hline$A 12$ & 34 & 3.47 & 0.08 & 0.69 & 0.87 & \\
\hline A 15 & 38 & 3.33 & 0.09 & 0.57 & 0.65 & \\
\hline A23 & 38 & 1.90 & 0.07 & 0.43 & 0.42 & \\
\hline A24 & 31 & 6.36 & 0.14 & 0.80 & 1.22 & \\
\hline A28 & 39 & 2.97 & 0.08 & 0.68 & 0.78 & \\
\hline A29 & 40 & 2.75 & 0.09 & 0.57 & 0.60 & \\
\hline A37 & 35 & 2.84 & 0.09 & 0.56 & 0.67 & \\
\hline \multicolumn{7}{|c|}{ On-site } \\
\hline A 10 & 30 & 2.37 & 0.12 & 0.65 & 0.59 & $0.8 / 1.6$ \\
\hline A35 & 30 & 3.48 & 0.14 & 0.68 & 0.79 & \\
\hline A 36 & 37 & 3.28 & 0.08 & 0.53 & 0.55 & \\
\hline A38 & 26 & 1.57 & 0.15 & 0.57 & 0.39 & \\
\hline A39 & 31 & 2.88 & 0.23 & 0.93 & 0.58 & \\
\hline A40 & 36 & 3.14 & 0.08 & 0.97 & 0.66 & \\
\hline
\end{tabular}

${ }^{o}$ The $<52$ samples per station reflects missing data.

${ }^{b}$ These standards are for gaseous fluorides only, while PORTS data include both particulate and gaseous fluorides. 


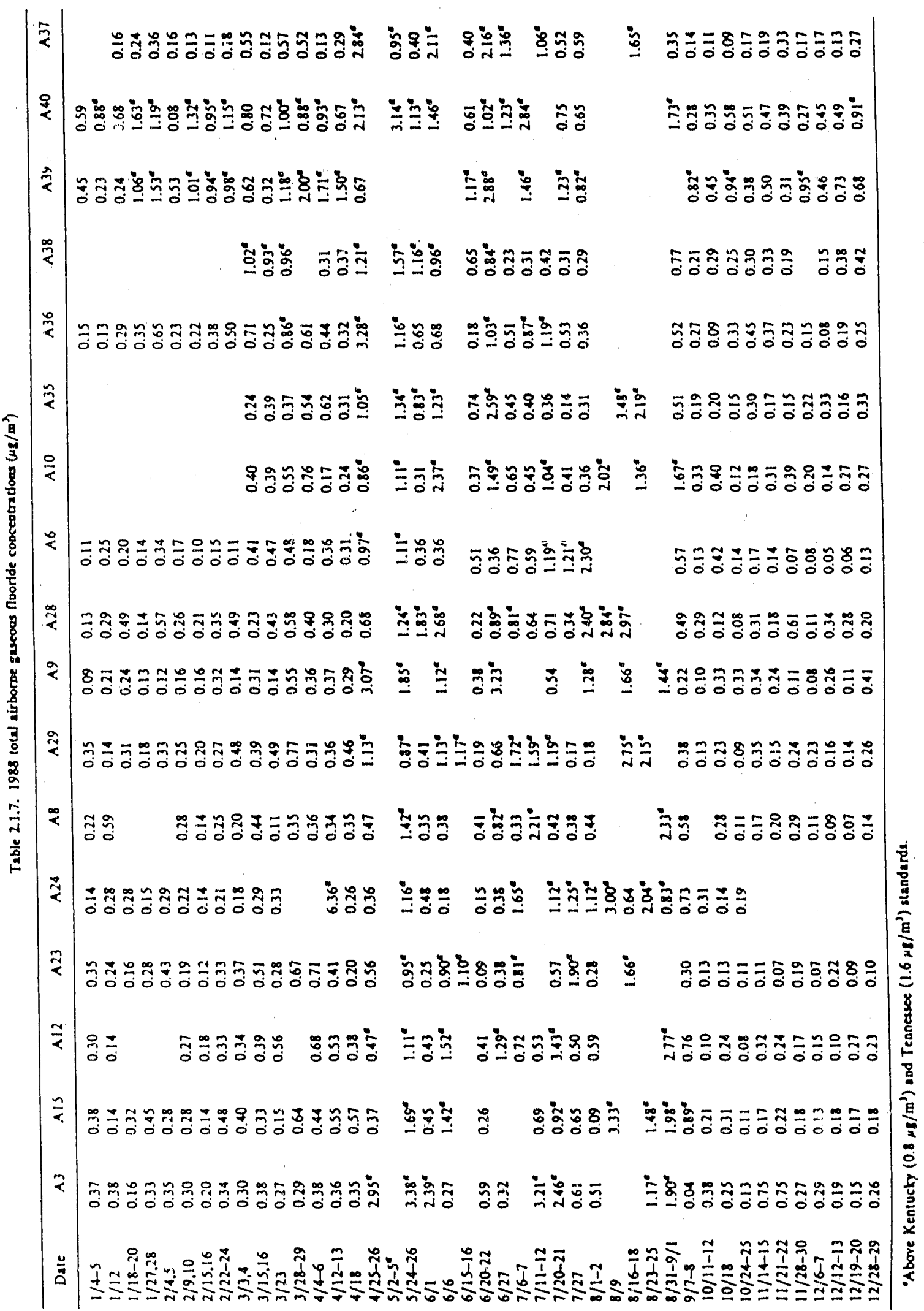



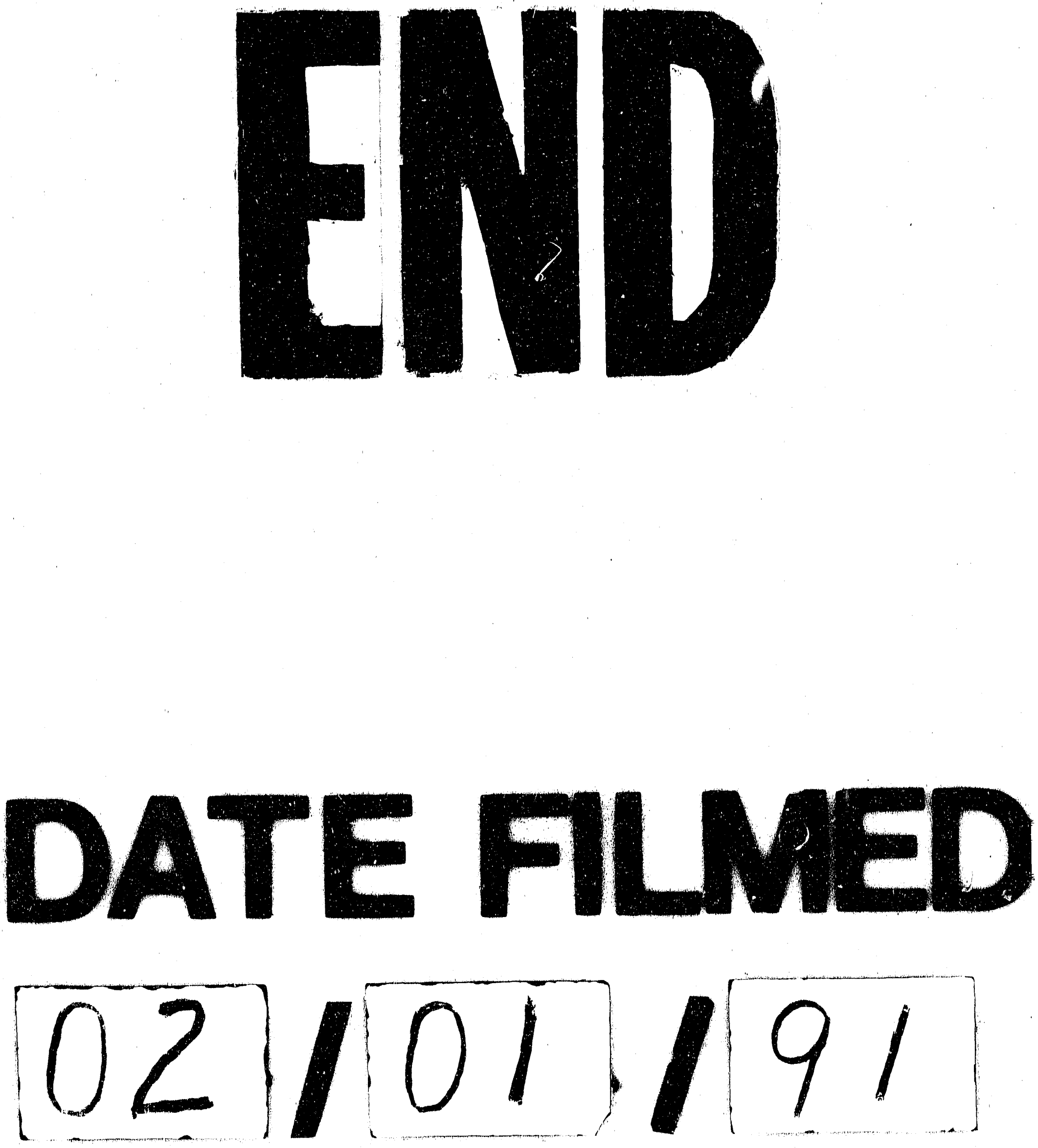
